hes

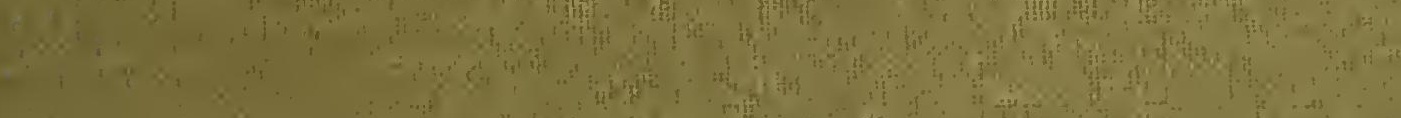
ate

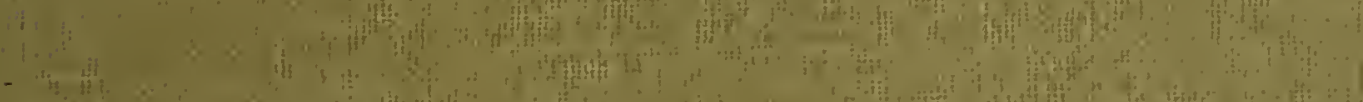
-

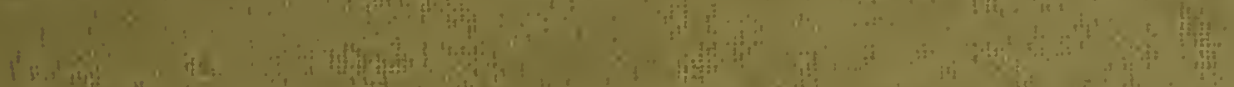
(4) $a_{1}^{2}$

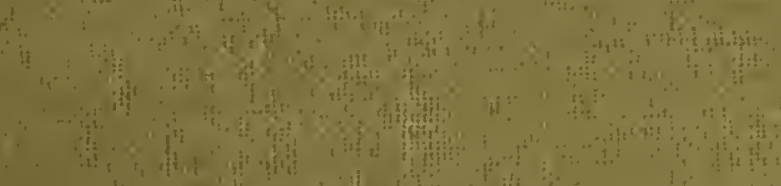

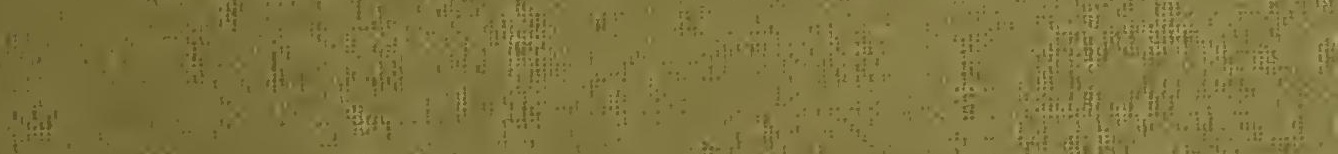
a

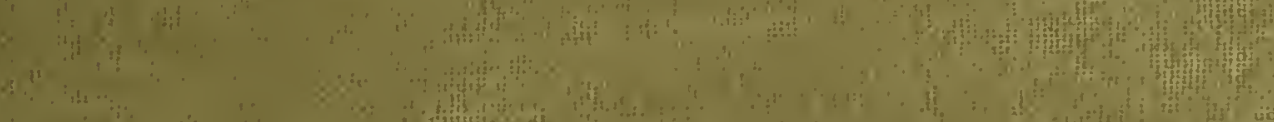

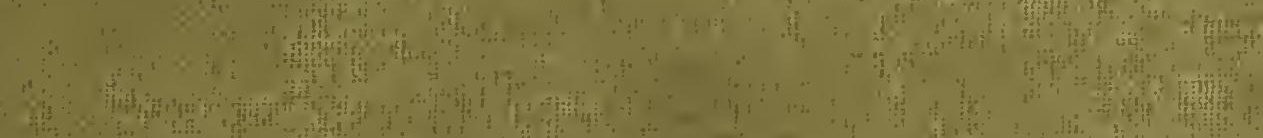
1: $=$ a d ,

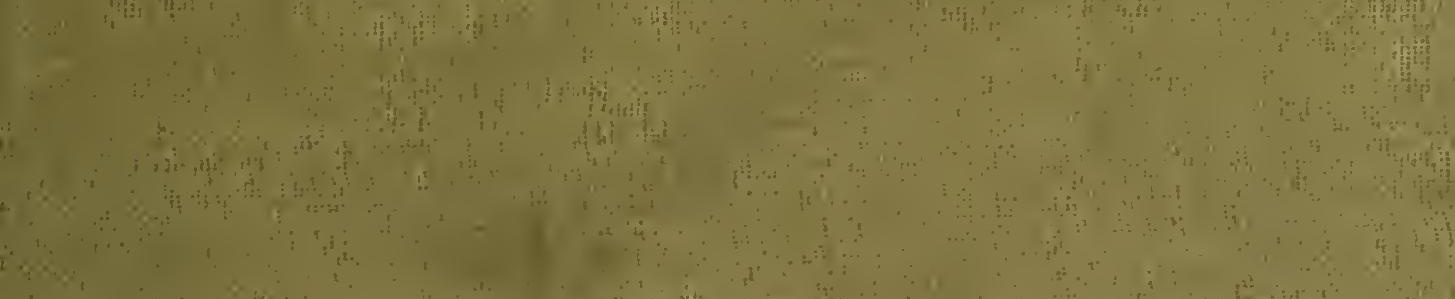
ats

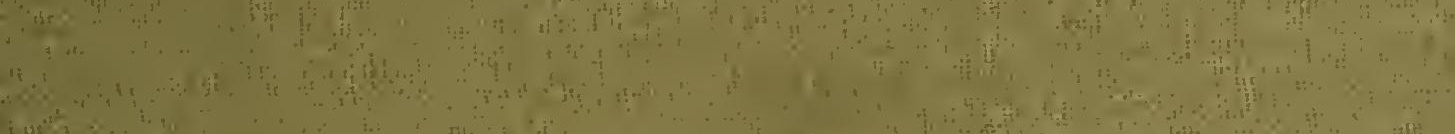

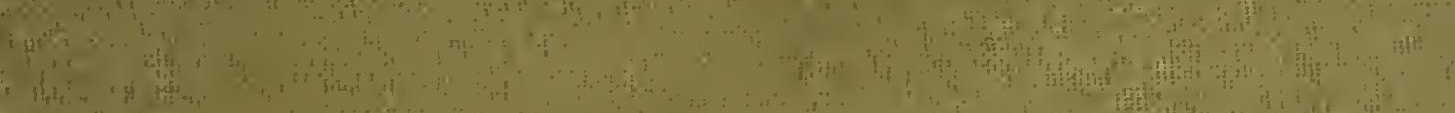

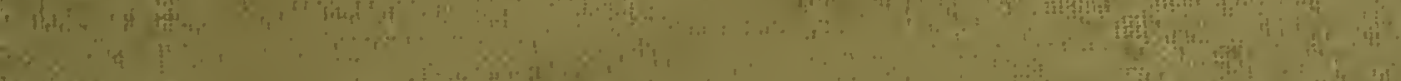

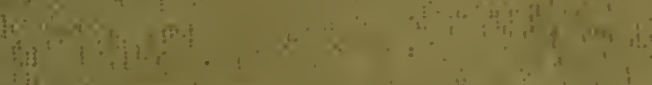




\section{THE D. H. HILL LIBD,APY}

NORTH CAOLINA STATE COLLEGE

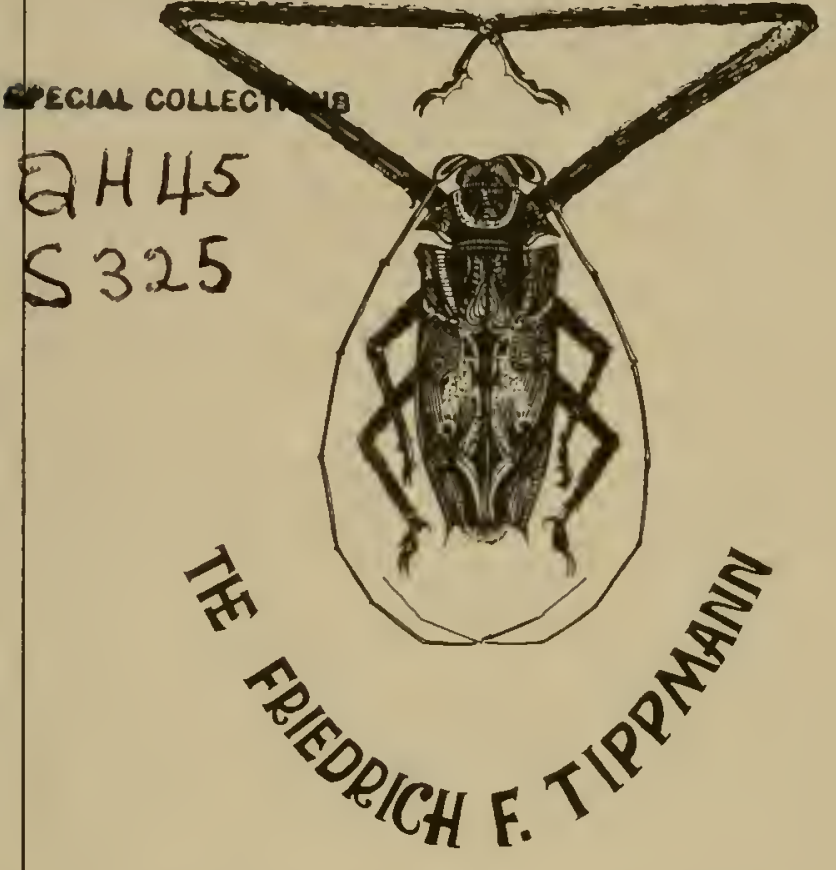

ENTOMOLOGICAL COLLECTION 


\section{7}

This book must not be taken from the Library building.

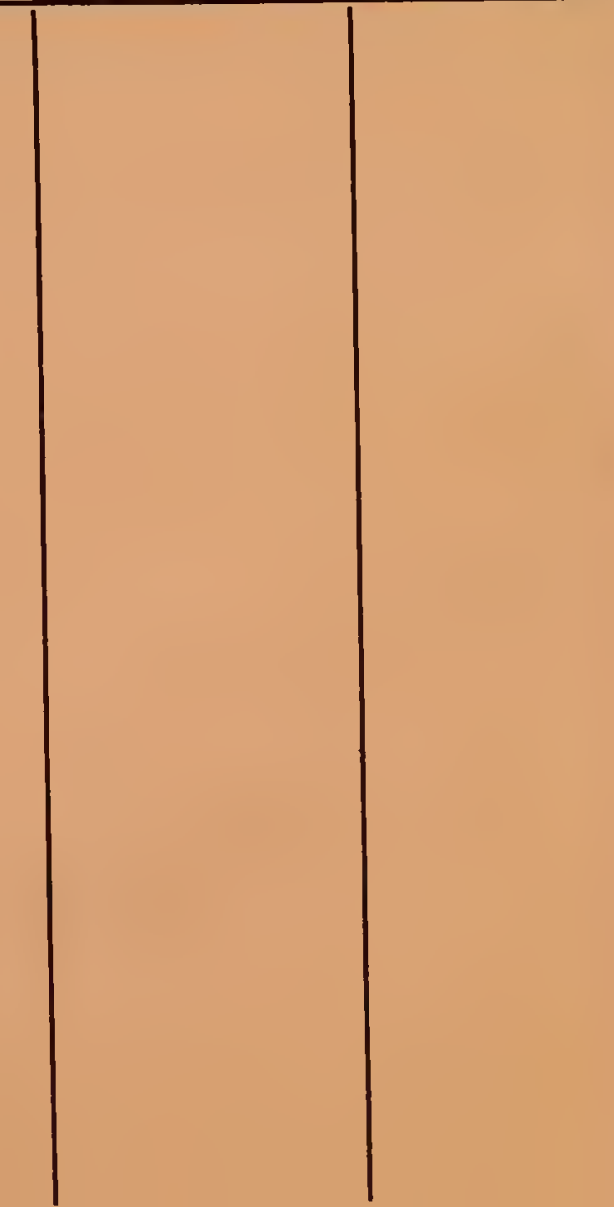





\section{Naturbiftorifbe}

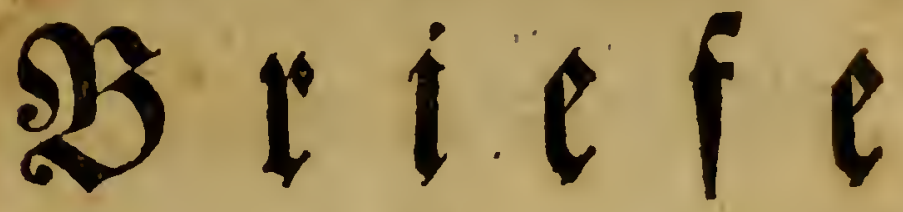

úber

\section{Deftrei(f), Salzburg, Pafau und Berôteşgaden,}

\section{von}

\section{Franz von Paula Sarank,}

ber Theologie Doctor, Surpfalzbairidten wirfl. geiftliben

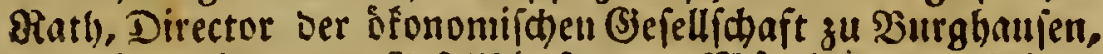
Der âfiseemien uno Giejellid)nten zu Mnundern, zu Erfurt,

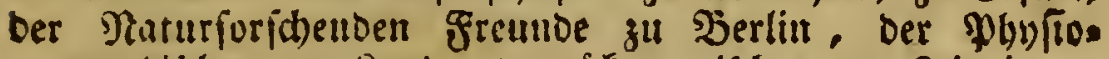

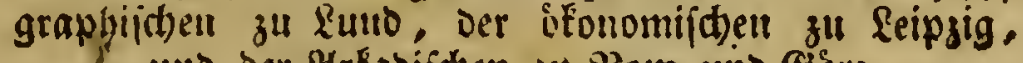

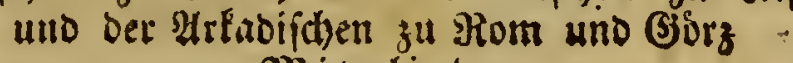

$$
\text { 2hitgliebe, }
$$

\section{uno}

\section{Raul Efrenbert Slitter von Miol,}

Deftreichidyen fanbumann, פRitglied ber sfonomiden Gepellibait ju biugianujeu, Der Naturjorichenden

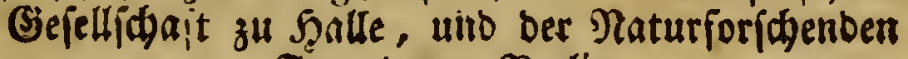
Freunde zu $\mathfrak{B e r l i n}$,

\section{L̈rfter Laano.}

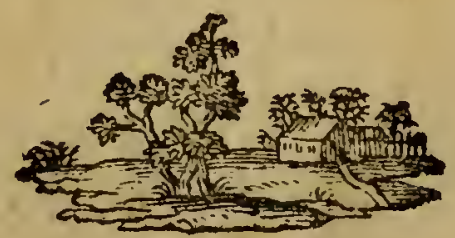

\section{Salzburg, I 785 .}

in Der Jof̣. Jof. Maners feel.Erbinn Butf̧̨andlung. 
gatudatirese 


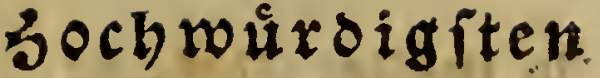

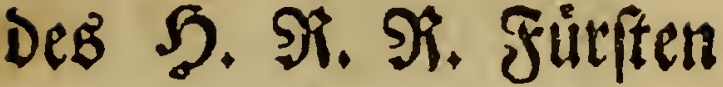

Siteroninntit: Sofepf

Eitzbifitof zu Sallaturg,

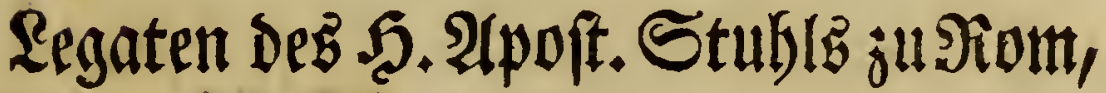

Primas von Deutfitaland ic. 2t. 
In tiefefter LEgufutdt

\section{sugeeignet}

bon bem Şerausgeber Sarl Erentert yon Modl. 


\section{Sorberidft.}

Pैal liefere bier den Sreundent der Naturs - geffbidfte ein Die zwif fon menem verellyungsinúrdigen Freunde, Dem Şerrn Direttor S(f)tank, und mir von Burghaufen nad) Bell, und won Bell nach Sargs baufen getaufen find. Durd die Sherausgabe Derferben glaubte id) ithen um fo weniget einer 


\section{Pas o}

unangenchmen Dienft zut erweifen, ba fie groffen. theils die Naturgefdidste ciniger, won biefer Sei te noch ganz unbefannter, Giegenden betreffen.

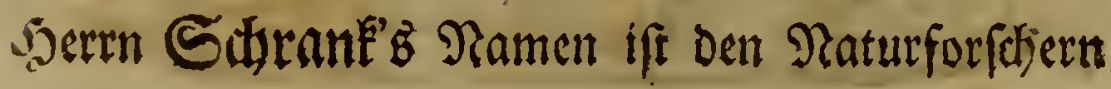
olinedem aus mefrern widtigen Sd)riften rulbm

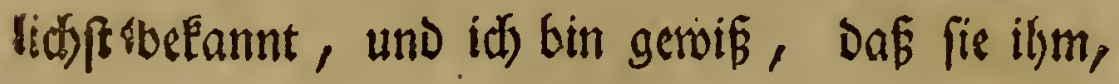
fir Die mir gutig gegebene Erlaubnif, feine ß3riefe iffentlid) befannt zu mad)en, Dane wiffen werden. Goll biefer crfite Band gluctich) genug feyn, mit Beyfall aufgenommen zu werden, fo foll lángftens bis zur Oftermeffe I 785. Der Bweite nadyfolgen.

(ङefdrieben ju Bell im Zillerţ̧all, Den I. Şerbftmon. I 784 .

Satl Erentert von Mtoll. 


\section{Şnbalt bes eriften Şants.}

\section{Erfter Brief.}

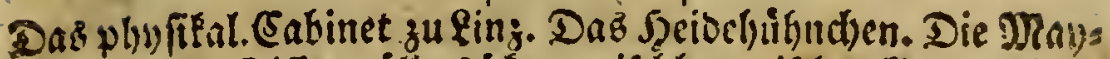
ferd)e, J2. Siffermillers d fonomifhbotanifher (S) Drten. S. I, - Breenter Brief.

Sherr Solmann'z Raturalienjammlung. Salmling. Bon ten

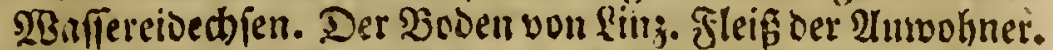

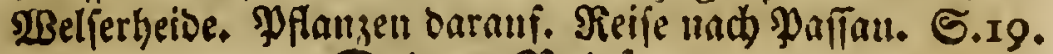

\section{Dritter $\mathfrak{B r i e f}$.}

smabreporiten von $\mathfrak{B o l f f i t e i n . ~ B r i n e r ~ M a r m o r . ~ P o r c e l l a n : ~}$ erbe. Sinferhley. Znlf. Soldanella alpina. YJerlen:

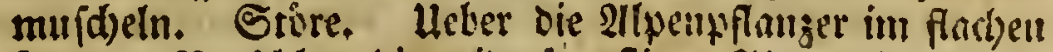
Rande. Borichlug bie miderffenftigen Altenpllanzen ju zábmen. Baierns frudhtharteit. STitif eincr Etrile in Den. Sriefen eines Franzo[en. Spitindifher Delbant. Rontenber Steinlurudi.

\section{Bierter Dirief.}

S.26.

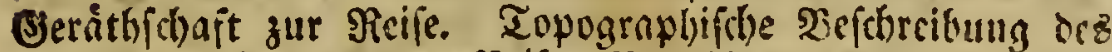

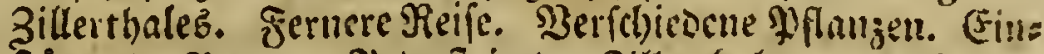

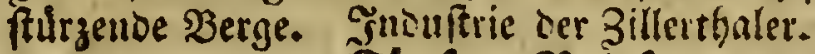

\section{Finnfter SBrief.}

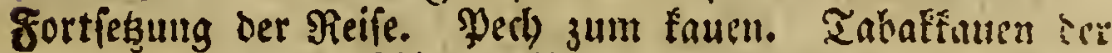

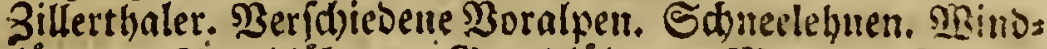
Iáhnen. Staublåhuen. Grumblåbnen. MRurren. Fernere Reife. Råjerlůr. Brcitlåner. Edłwenen. Getłster : b̂rief.

Fine Alpenicene am 2Rorgen. Fine Snäcabe. Alpenfflan: zen. (5rawanber Sdinter. Sonterbarer Bergneg.

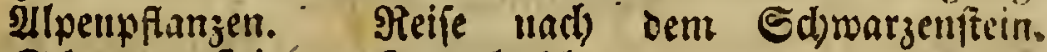
Sdwwargenfein. J̧orngletid)er. Siebenter SOrief.

Stunbrechnung ber 2lpenbenolnner. 2(nd) etwas you ibret

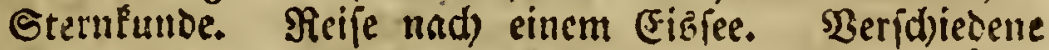
2llpentplanzen. Ein neuer Sd)metterling. Einige firiper.

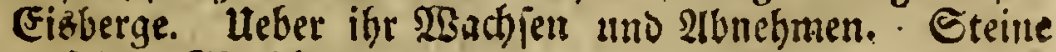
auf Den (Slet/d)ern.

Idfter Brief.

S. 93.

Reife son Saliburg nad) Reid)enlyall. Ulber die vielfpizis

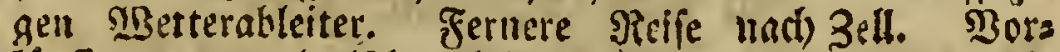
lintige entomologifhe Erimerungen.. Die falzburgis (rb)en Sdjoterarteu. 


\section{Neunter SBrief.}

Borláufige Eritmerung. Salzburgi îde Solbenlåfer. S.153* Bebnter Brief.

Sortíekgung Der Solbentáfer.

Eilfter Brief.

ธ. 188 .

Reife nadh Saljburg. Papilio Lucilla. (Befäfrlid)e (Bee băube in Sulzburg. Dentwoirbigfeiten in Salzburg. Der Siapujineriverg. গieife von Salzburg nadt Berdteg.

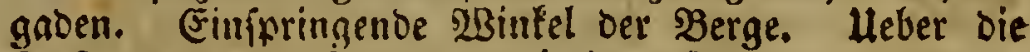
Eyfteme von Entiferyuna der iegigen $23 e l t . \quad$ S. x94. Zwoifter Szrief.

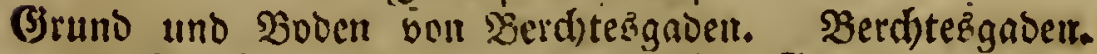
Echóltenberg. Ramian: 2lngenelyme Gienenden. Rands

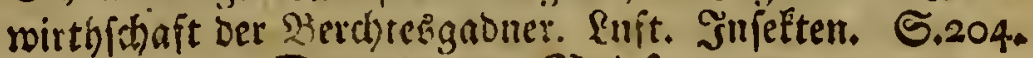
(Drewjel)nter Brief.

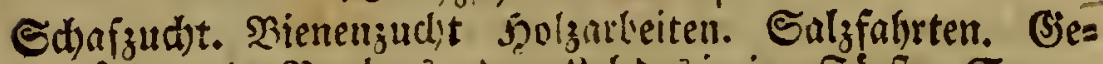

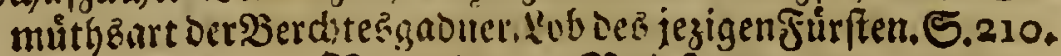
Bicrzelynter Birief.

Der Salzberg. Urfpramig Der Berghthilen. Ealzquéle. S.23r. Finnfzelynter Brief.

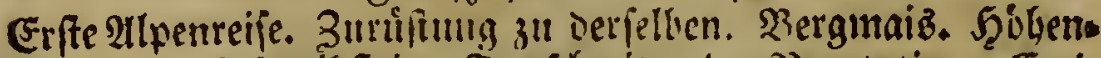

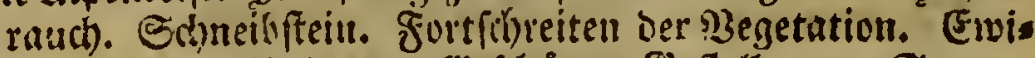

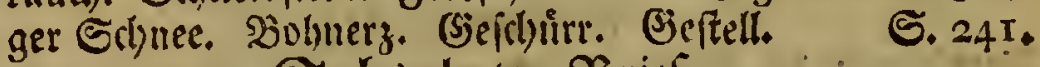
Sect)şzelinter SBrief.

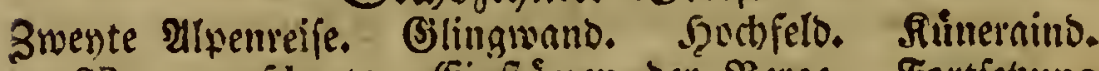
23azmannid)arte. (Einffitrzen Dee Berge. Fortifezung

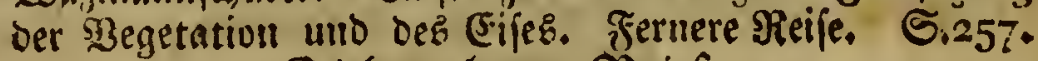
Giebenzchnter Brief.

Dritte Mtpenteilic: und) Demi Geclion. 2(t)tzethuter Srief.

S. 275

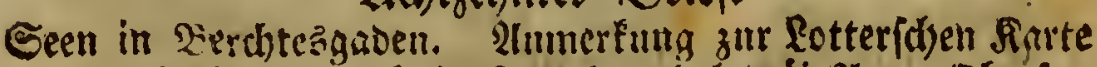

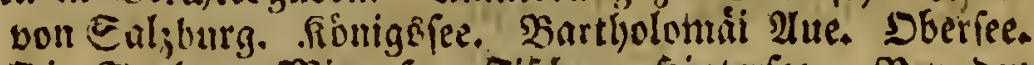
Die Sanhat. Mitteriee. Gifid)e. Szinterfee. Bey) Dent 3etlern. Brrunfee. Fountenfee. Meunzelunter B̧ricf.

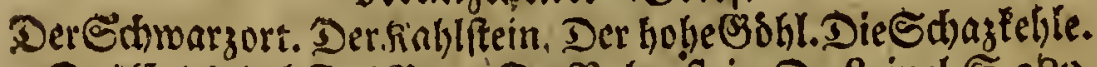

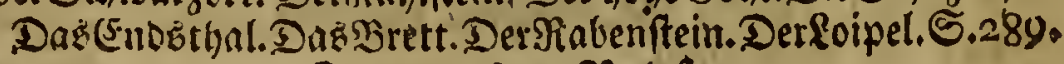
Bwanzigfter Brief.

Eghere it \$erditešgaben.

Ein uno jwangigfter Bortef.

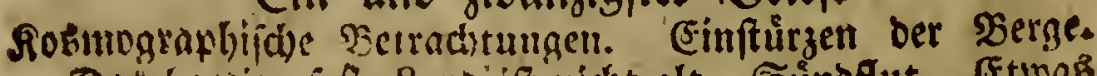

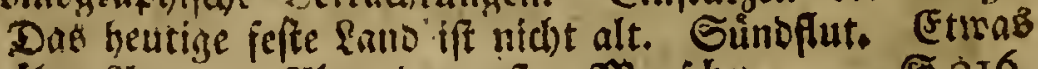

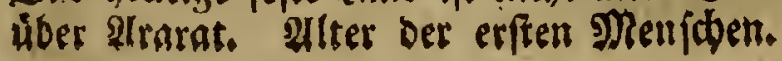

S. 296 . อ. 316. Enter 
feinen 2lugen, ba ex felleft baju nidjt Mufe ḩat, bet. fertiget merben, begleiten laffen.

Eie foonnen fich Daber wob̨l vorffellen, dab

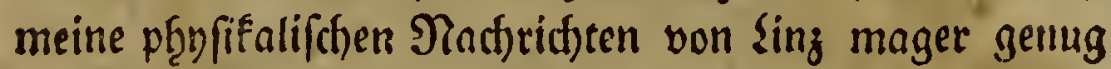
ausfallen muiffen. Dennoch) mill idf) Şrrem $\mathfrak{B e r}$ langen nach) Bermogen (Jenưge zu leiften fudjen.

Rommen Sie cinnzal nad) $\sin _{3}$, fo verfåumen

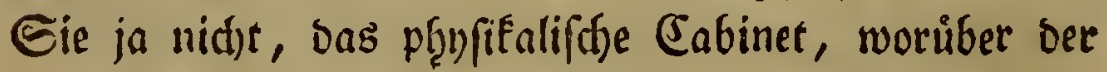

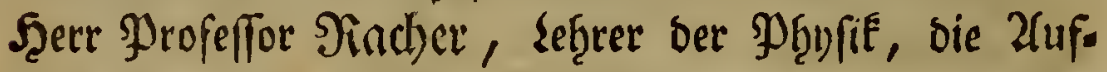
fid)t haat, ju befudsen. Begenwårtig bat ber Şof orei) groß̧e Bimmer in Sd)lofe bnju eingeràumt. Zluffer ber großen Menge Der Mafffinen zu allen

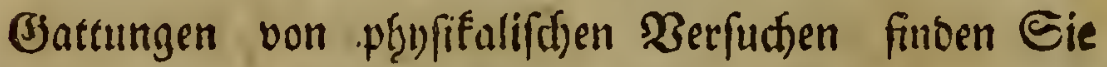

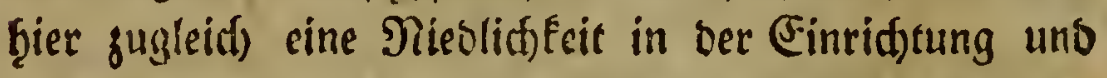
Znoronung berfelben, bie ifgres gleid)en nict) bुat. Gie wersen aber nod) mef̧e erfaunen, wenn Sie fid Daran erinnern follten, oaß̃ id) J̧̧nen in gegenwarti.

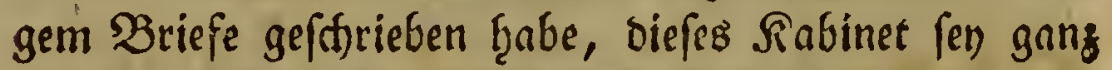
Das WerÉ diefes unermilibeten Belef̧rten, Der bie meiften Maftsinen groß̧entheils mit eigener J̧ano ver.

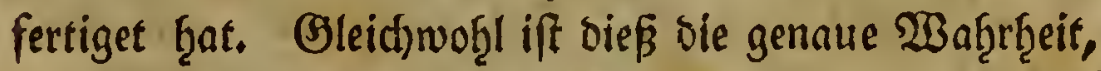
uno id) Fann midh von einer beträdtlidfen Menge folcher $\mathfrak{B e r f e r t i g u n g e n ~ f e l l o f t ~ a l s ~ 2 l u g e n z e n g e n ~ a n g e b e n . ' ~}$ Sing ḩat wirflid) Urfacje nuf biefes (Eabinet, und

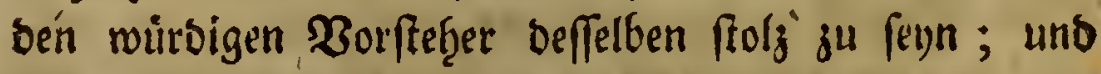
Sie múrben gemiß eine ber grooften Suinden wiber

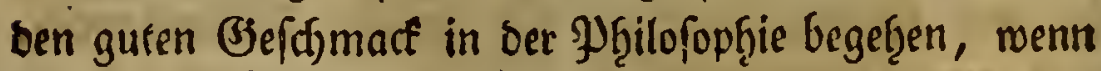
Sie bel) (J)elegenheit irgend einer Reife nad) Deftreid biefem Sabinete zu siebe fiid nid)t einen ₹ag lang in sing auffielten. 


\section{Đaffa, Den 16. May, 1783 .}

Bon ben gang vortrefflid)en Sammlingen ber

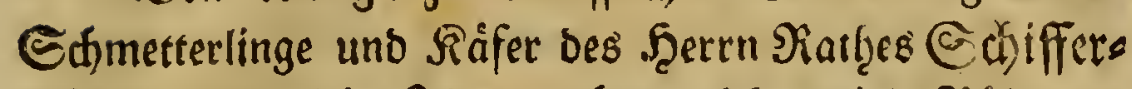

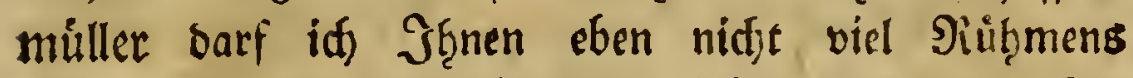
machen, sa fie ganz liber mein Sob hinnus finto; Eie fennen ofgnef̧in bie exftere aus feinem Bergeidyniffe ber Ed)metterlinge ber $\mathfrak{B i e n e r g e g e n d ~ G ̧ i n l a ́ n g l i d , ~ u n o ~}$ mein Eupplement wird fie in ben Etand fercen von

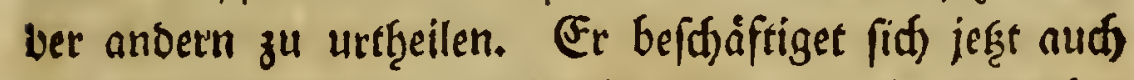
mit einer -niedliden $\mathfrak{B o g}$ gelfammlung, bie mandjes feltene Ștůc entf̧ălt.

Eben zur Beit meiner 2furvefenţeit bradite mant

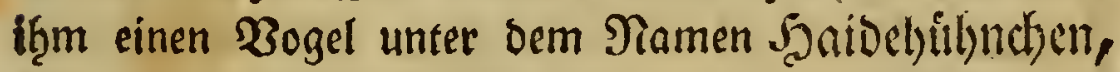
beffen $\mathfrak{B} e f t i m m u n g$ uns beyse viele Mrüge Éftete.

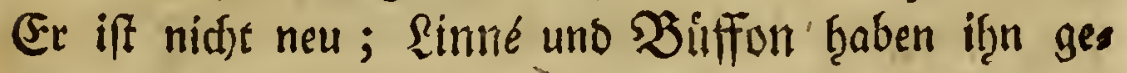
fanut; aber Sinné bat in Den Rennzeiden ber (Jats tung einen Edreibfefler gemadte, weldher Urfadje

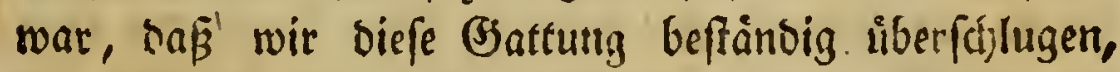
uno wie fdrwer es fen, ein ₹̧̧ier, das man nod)

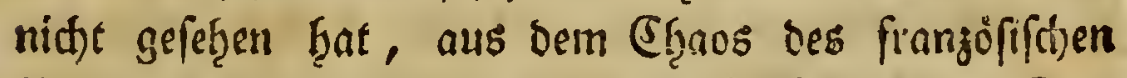
Plinius ḩcrauszufinoen, wiffen Sie felbft mob̨l. Dies fer Boget ift das $\mathfrak{B}$ Beibdien von Otis Tetrax Lin. S. N. p. 264. Jp. 3. Der Ritter fagt in Den Rern. zeichen biefer Siattung: pedes curforii tetradictyli; nun fanben roir immer nur brey Bef̧en; aber um eine Srappe zu fenn, foll aud) ber $\mathfrak{B}$ ogel nidft metrrere Ģaben : bie grofe Trappe (Otis Tarda Lin.), die man vor einigen Jaḩren bier uno ba in Baiern gea

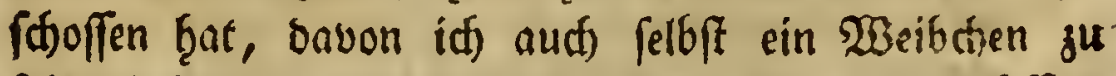

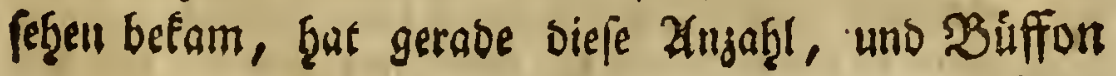




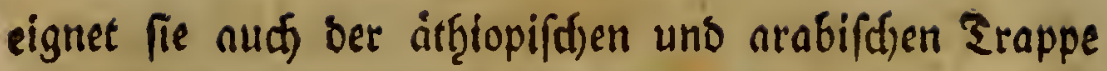

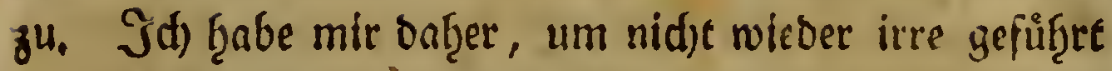
zu merben, mit bem slenfififte bas tetradactyli in tridactyli veränbert. Silffon bat alfo unrérit), wenn er biefen $\mathfrak{B o g e l}$, Den er petite outarde trennet, weld)es

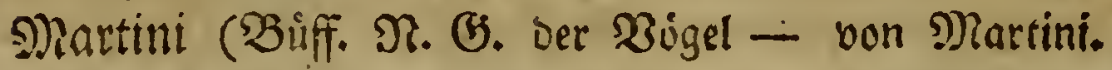
4. 2. 5. 48.) Durd) Srappenz!verg überfeget ţat, unferm Deurfdilande gerne abfprechen módtfe. WBenig. fenns foll er eben nidt) unter bie feltenften 3 ógel ber sinzergegento geţòten, weldes faton genug aus bem erţelllet, onß î̧n bie Einwor̂ner mit einem eigenen Namen, ber nod) baju ziemlid) paffeno iff, zu bes zelidnnell wiffen; fie wiffen es fogar eben foroogl, als

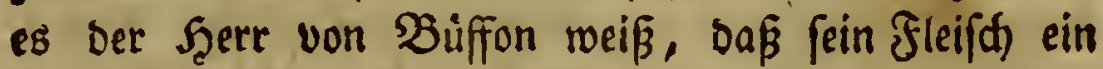
ganz nieblicter secferbiffen fen.

Saf fönnte Gie nod) mit ter niebliden Mays ferthe unterţalten, bie mir ber ફ̧err Siath, ber eben eine erhalten thatte, borwies; fie war fo groß als

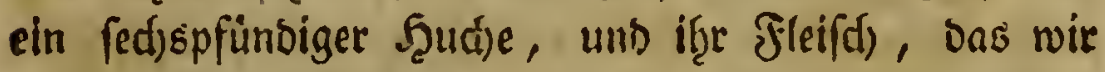
nod) benfelben $\mathcal{T}_{\mathrm{ng}}$ in einer simonienbrühe genoffen,

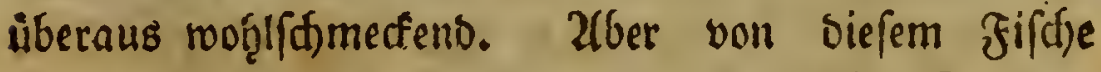

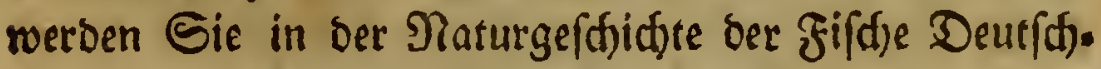
lanbes des Şertn Dr. Sllochs, Dem Der Şert Siath nảd)ffens ein Eremplar fenচen wiro, of̣neţin genug. famen Unterridft finten $(*)$.

2lber

(") Şert Dr. Şlod) madht in cinem briefe an Den f̧erth abt Gchiffermúller vom 18. Dftob. 1783. Die are

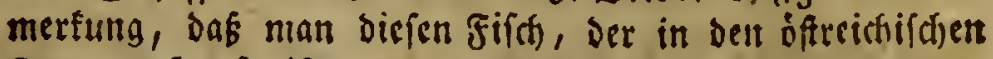
Geen in Saulfe if, in Det Dffee, wo man ifn mit bem गeanien StIberlachs befeiajnet, miederfinde. 
2fGer witroen Sie fichs wob̨l jemal vergeben förnen, wenn Sie einmal im Eommer eine Sieife in biefe (S)egend madien, und mieber abreifen follten,

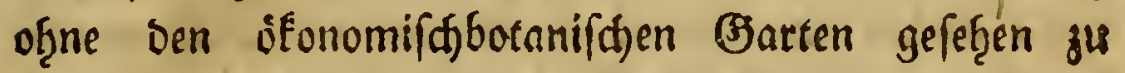

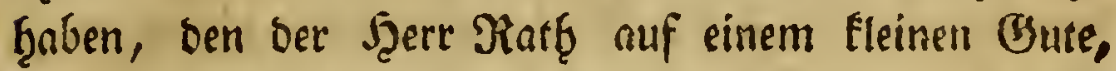
Das ungefáf̧r eine halbe Grunde auffer ber Erabs liegt, uno bem norbifthen Etifte zugehiorret, angeleget hat? Dhne ber übrigen Brauchbarteit biefes Drtes bas geringfte ju benef̧nen, fat er geroufte eine grope Menge forwoḅt inlänoifdjer als ausländif́cher baaume uno Etràucher f̧ię̧er ju verpflanjen, baßj es felbff

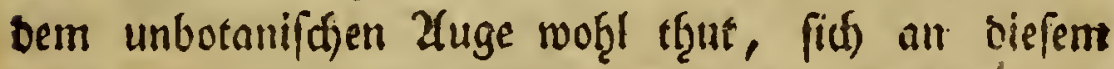
fleinen Parabiefe zu weiben. $\mathfrak{U m}$ ben $\mathfrak{N u G ̧ e n ~ b i e f e r ~}$ PPlangung nodh allgemeiner ju madien, if bey jeoem Diefer $\mathfrak{B}$ åume und Eträuffer ber finftematifahe Tame nad) Simné, Niller, ober. Sacquin auf einer Sledja platte gemali angefradfet worden.

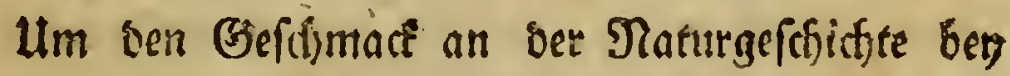
ber untergebenen Jugeno, zu Seren Beluftigungsous biefer Barten eigentlich bienet, nod) melgr zu reizen,

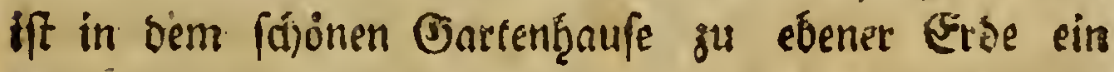
Eleiner Epcifefnal angebradt, Dabon bie Deffe fowoht

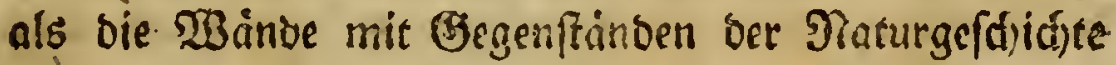

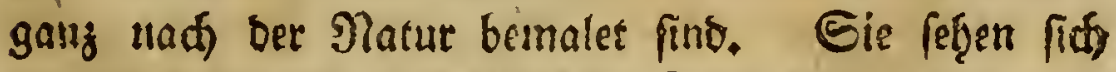
Gier suf einmal nad benoen Inbien yerfesęet, uno fino nur baruber verlegen', ob Gie meg̨r sen freisigen Pinfel bes Malers ober sen vortrefflitt)en (beffimars

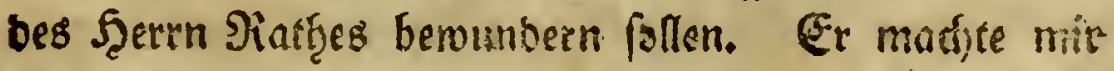
einmal bas Bergnügen minf in biefem Saăle gu ba 
wirtţen, aber id meis es in ber ₹̧̧at nidft, wie mir bie Speifen gefdmeçt Ģnben, weil alle meine Sinne bey bem lauten Befüḩle ber $\mathfrak{W}$ onne, das mir meine 2lugen gewaihtrten, in biefem fleinen Eson ver. ftummten. Siod) ift mir ber Besanfen Daran felige 23olluft, und ons wird er nod) lange feun.

2lber nidft bie Naturgefdichte allein foll bie Jugend mit ifren Sieizen bejaubern. Frevlid) ift nidfts to berrlich als bie 2 Berfe ber 2(llmat)t, un' Die Runft ift nirgendos fo vollfummen, als in ber

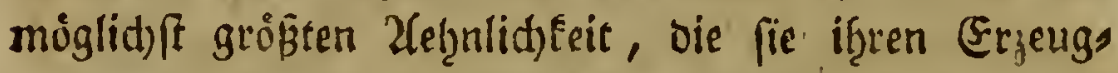
niffen mit oicfen Mieifterftucten bes Sidjopfers giebt. Zaber all einem Orte, ber- fúr eine zallireid)e Jugeno erbaut ift, miffen nidjt biefe Meifterfticfe allein zur Ed)au aufgefứrzer werben; fie muß fd)maç an ben bilbenden Siuniften befommen, ein (J)efdmate, ber nidje nur bem fopfe beffen, ber if̧n befiget, Elzre mad)t, fontern zugleid) unvermerft bas Şerg beffert, und bie Sitten auf milbere Sone ftimmt.

Der f̧err Siath ift gu gut in ber gropenen uns wid)tigen Runft ber Ergief̧ung erfaţren, alz onß er

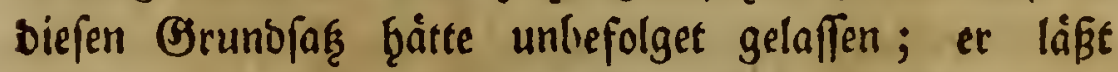

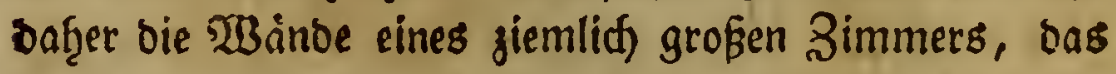
ùber bem Saale angebradte ift, mit gewåg̨lten Ģiftori. fdeen Stưfen nad) ben beften Meiftern bemalen, uns Damit bie Jugeno zugleid) bie Manieren lfennen lerne, bie Namen biefer Meifter, 3. B. mads van DycF, Darunter feģen. 
20̉enn Gie nun biéz alles vielleidfe einftens fetcen werben, biéz alles bewunbern werben, fo era innern Gie fid) zuglein), onßs bießs alles, fo gemeins nülidi) es aud immer feln mag, nid)t auf Soften bes Collegiums veranftaltet, fonbern aus bem eigenen Beutel Des Szerrn Siatţs bejab̨lt werbe.

Jof fomme nod) einmal ju ben feltenen Stolgs arten jurůcf, weld)e in biefem Barten gepfleget merben,

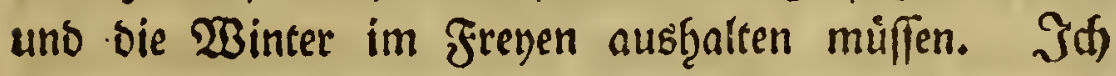
thabe fie mir aufgefdrieben, aber mit allen fonnte ids bas nidft thun:- fie bratten nod) feinen bengee esten Niamen, und zum Unglioffe nudf feine Slumen. J゙' werbe alfo nur einige nambiaft mad)en foinnen.

I. Ptelea trifoliata. Lin. Jpec. plant. p. I 73 . $S p$. . . Nod) ein gand junges baiumidjen, Das bey meiner afbreife exft $\mathfrak{B l a t t e r ~ t r i e b . ~}$

2. Lonicera tatarica. Lin. Sp. plant. p. 247. Sp. 5. Simné neunt bie $\mathfrak{B l a t t e r}$ biefer Sonicere fóledf)to weg cordata; on fie aber fomal uno lang find, fo sứrben fie vielleid)t beffer cordatolanceolata bzeiffer.

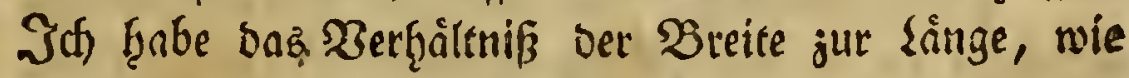
8 zu 33 gefunden. Die SBlàtter fino úbrigenz jart, glatt, am Rande foft unmerflid ausgefofmeift; fie figen auf ủberaus furzen Gtield)en, berer (ánge id́) nidjt ùGer $\mathrm{x} \frac{\mathrm{T}}{2}$ Sinie gefunben habe.

f̧err $\mathfrak{Y}$ Bcigel (Obferv. botan. p. 25. obf. 8.)

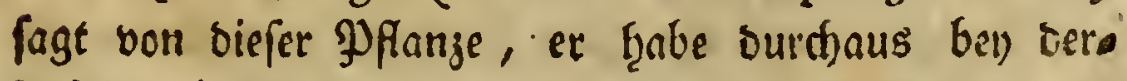
jenigen Sonicere, bie im afabemiftsen Barten ju

$$
24
$$

(ื)⿺廴 


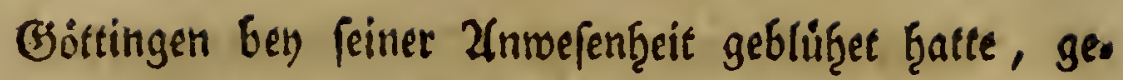
funben, Daß Der Reldfftheile, ber Blumentḩeile, uno Der Staubfäben nur viere waren; id habe aber ben

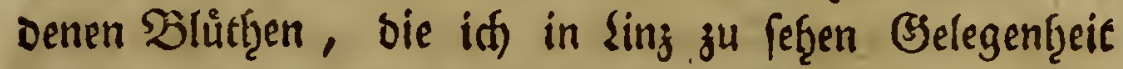

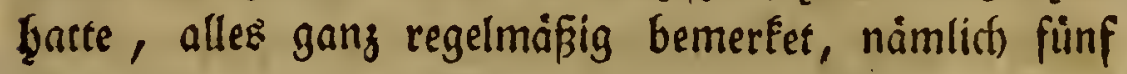
Relddlappen, fünf B̉lumeniappen, fünf Stnubfäben.

3. Lycium barbarum. Lin.jp.plant.p.277.

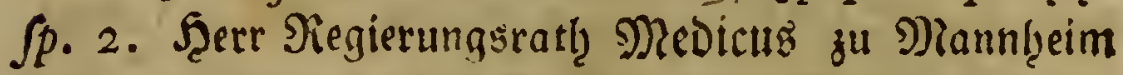
hat bas 2 Bort crafjucculis aus ben linnaifiten Renna zeiden ausgefffloffen, weldfes er audy mit bem Enno. nymum aus Flufenet

Rhamnus peregrinus rofmarini folio candidior,

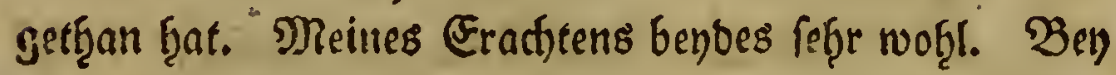

Jasminoides aculeatum polygoni folio, floribus parvis albidis Shaw. zweifle id) nid)t, oấ man das ifragejeidten weglaffen Dirfte, bas stunté bingefeģt hat; Denn fo wentg bie Blatter einem Siusmarinblatte âfinlid) fino, fo gut frellen fie bie bes $\mathfrak{B}$ egtrittes vor.

Diefer Straud, ber auch des Jeern Medictis Iycium barbarum (Pfälo. Bemerf. für i 777, ) iff, bat fdjon cinige $23 i n t e r$ gang vortrefflict) in sing aus. gef̧ntten. Unterdeffen habe id immer einen fleinen Berbad)t, sinné burrfte woh̨l ganz ein anderes Lycium vor 2lugen gef̧abt ḩaben, als bie benben 2frten fino, sie man gemeiniglidi für das linnaildhe Lycium barbarum failt, unb bie Sgerr Medicus a. a. Drten fo gut befdyreibet. 
4. Ribes nigrum. Lin. Spec.pl.p. 2 gr. Sp.3. Die Slàtter fleiner uno rauţer, die Blumentrauben fürzer aber zaḩireid)er als an Ribes rubrum.

5. Rbus glabrum. Lin. Jpec.pl.p. 380 . Jp.4.

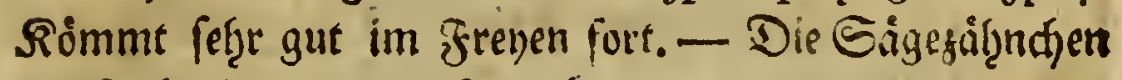
ber SBlättdjen fino tief, aber weit aubeinander.

6. Rhus Toxcicodendrum. Lin.Jp.pl.p.38 r. Sp.6. Die wisinfel ber Blätter gef̧én nidtet tief, fins aber zab̧lreid), uno feţr verfthieden.

7. Rbus Cotimus. Lin. $p p . p l . p \cdot 383 \cdot \int p .15$.

8. Sambucus lacinato folio. C B P. 456 '.

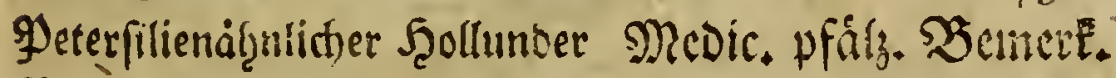

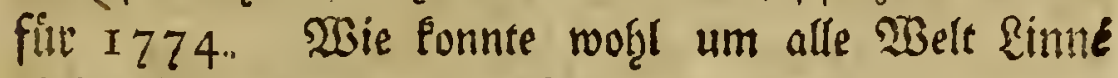
biefen Etrand) für eine 2 (bart yon Sambucus nigra Ģalten ? Sönnen SPfanzen fo feţr abåndern, fo eennen wir in ber ganjen Botanif feine einjige 2lrt, felloft viele Enttungen nid)t mit Berwi b̧eit.

9. Stapbylea trifolia. Lin. Jp. plant. p. 387. Sp. 2. Römmt bortrefflid) fort.

10. Hydrangea arborefcens. Lin. $\int p . p l$. p. 568. $\int p$. I.

11. Amygdalus nana. Lin. Sp. plant. p. $67 \%$. Sp. 3. Fin vorjiglid) fdoines bàumden, bas faum

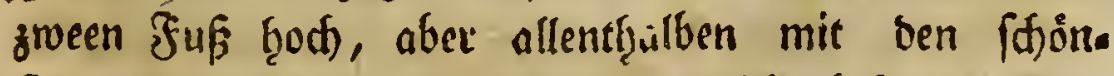
ften, fȩ̣r furzftieligten Blüţ̧en bidst befleibet war. Die Bhimenblätter baben an ber Spige eine feidfte Einferbung.

$$
\text { at } 5 \text { 32. Prumus }
$$


12. Prumus Mahaleb. Lin. Spec. plant.

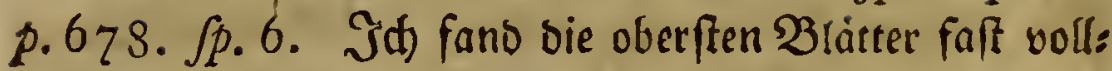
fommen runo, mit einer ftumpfen Spige (mucrone obtufo.)

13. Crataegus Aria. Lin. Spec. plant. p. $68 \mathrm{x}$. Sp. i.

14. Crataegus lucida. Miller DiEt. 6. Die. fes Baium(t)en f̧at lánglid) elforrmige, am Sianbe

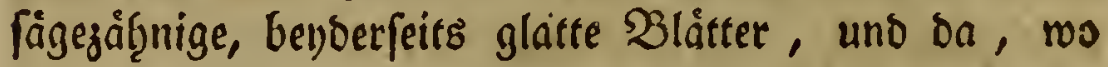
junge Triebe Ģervorf́omnien, eingelne, fȩ̣r lange, ftarfe, rơtf̧lidht fotwarze Stadjeln.

I5. Sorbus virginiana. - Die Blüţ̧en auf einer wolligten 2 lffterbolve, bie Reldie finfmal einges fofnitten, welligt; ' fünf Blumenblätter, weiß, am Ranbe ganz; brel) Briffel aus einem wolligten fructst: fnoten. Die Slätter lánglid)t elfởrmig, oben fef̧e wenig, unten fark wolligt, uno bavon gans meiß, am Sanbe lappidyt (gefiebert) eingefdnitten, bie Zuffenfeite ber Sappen fägejåţnig : bie Einfánitte, weldje biefe sappen bilben, reerben immer feidter, ie

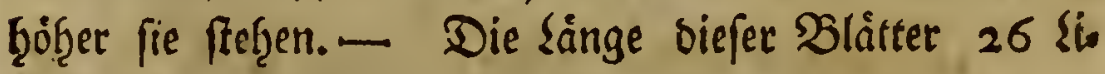
nien, bie Breite 13 Sinien.

Эd) reiß nidts, woţer ber Name fey, ben

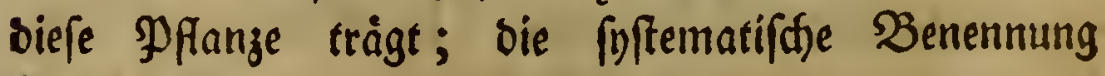
tönnte fenn :

Sorbus foliis lanceolatis fubtus tomentofis pinnatifidis : lobis ferratis.

2Bie aus bem গlamen erbellet, ift bas Bater lans Birginien. 
16. Mefpilus Pyracantha. Lin. Spec. plant. p. $685 \cdot \int p .2$. Zllerbings laufen die Slâtter gegen Den ઝlattifiel fpisig ju, viel fpişiger, als fie oben fino. Isth muste diefes anmerfen, weil oer frere Siegierungstath Medicus bem Sitter bon Sinmé als einen frebler angerectnet hat, baß̧ er fie unten lanzets fortmig nennt. (sigentlid) fraben bevoe rest)t: Die unterften $\mathfrak{B l a ́ t t e r ~ b e r ~} 3$ weige fino bollfommen oval, aber biefe vertrocfnen, uno fallen $a b$; die folgenoen fino fo, rie fie Sintié befdrieben ḩat.

I7. Pyrus crataegoides. - Id) glaube, Şerr Bergrath Jacquin habe siefem Baume bett Namen gegeben. - Gein Blatt bat fȩ̣r grof̧e (eø̧nlichfeit mit bem von Crataegus Aria.

18. Spiraea falicifolia. Lin. Sp. plant. p. 700. p. 1.

19. Spiraea chamaedryfolia. Lin. spec. pl. p. 70I $\int p .4$. Die Blátter Ģaben cine fef̧r ent. fernte a (eb̨nlid)teit mit Teucrium Chamaedrys (benn waḩrfdeinlid) biefe Pflanje, nid)t Veronica Chamaedrys, batten Ifmmann und sinné im Gebanten, als fie bie gegenmåntige Spiraea faben): fie fino aber viel feiner uno glatt. - Wber Umbellas mơthte idg mit sinné bie afferbolben (Cymas) diefer Pflanje nithe nennen.

20. Spiraea opulifolia. Lin. Jpec. plant. p. $702 . \sqrt{p} .6$.

21. Rofa alpina. Lin. Spec.plant. p. . $03 \cdot \int p . r$. Diefe Shofe grat nirgenos Dorne, als am Jrudjtfnoten. Die Relde fino ungefiebert; die Jrudtfnoten evförmig. 22. RoJa 
22. Rofa villofa. Lin. Jpec. plant. p. $704 \cdot \int p .6$.

23. Rofa pendula. Lin. Spec. plant. p. 705 . Jp. r 3. Sm Naturfyfteme aber freift fie Rofa pendulina. - Die Reldje ungefiebert, an ber Spige fadenfớrmig, rotf̧.

24. Rofa pumila. Limn. Silpplem. plant. p. 262. Die FructutEnoten enfürmig; bie S̉lätter aús fünf glatten Slä́ttchen jufaummenḡeferçet.

25. Potentilla fruticofa. Lin. Spec. plant. p. $709 \cdot$. p. I.

26. Tilia americana. Lin. Spec. plant. p. 733. Sp.2. 2luffer ben Frudtutheilen bat biejer Baum fein Rennzeirgen, Das ifgn von unferer sinbe unterfdeiden fónnte.

27. Liriodendron Tulipifera. Lin. Jp. plant. p. $\because 55 \cdot \int p$. I. Diefer $\mathfrak{B}$ num ift foton feit einigen Jahreen im Barten, aber alle WBinter gånzlids) abges

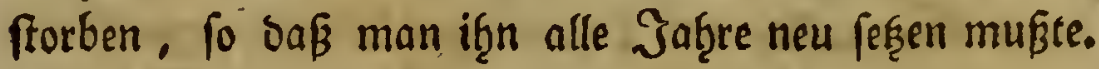
Das gegenwartige 3 Baumden uberfano enolid) ben Winter fegre gut.

28. Robinia Caragana. Lin. Spec. plant. p. 1044.Jp.4. Diefer Straud), ober Bauln (bann er fann bendes fenn) ift in feiner Jugeno fef̧r zartlid. Id) ţatte im vorigen Jab̧re Eaamen bavon ven seipsig. erţalten, und, weil id) if̧n in unferer (Biegent gerne einf̧eimifd) martien wollte. fo vertheilte id) fie, indem id) einen Theil $n$ nd) Sorfau an faerrn Brafen von 2tuerperg, einen Sgeil nach Bern in ben fojonen Edjlopgarten 
Der Frenfrau von Gngenheim fdyiffte. Bergeblid fuchte idf an biefem lef̧tern Orfe nad) ben jungen PPfaingchen meiner Baiume; endlid Eamen fie herbor, unb wudfien eben fo longfam, als fie aufgegangerf

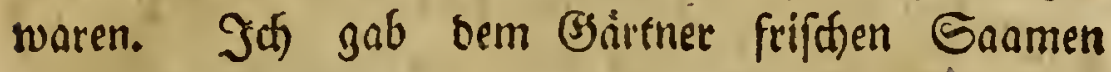
mit oem $\mathfrak{B}$ efefle, fie in cinem tiefen $\mathfrak{B}$ lumentopfe anjubauen, weil id) beforgte, ber $\mathfrak{Z}$ Ginter birffe bie anbern, weldje im falten Boben ftanben, tobten; bie Baiumctien famen freubiger, uns erreidten un. gefähre die Sănge bes fleinen Fingers, aber ba fie ben 23inter unter ben Dranienbaiumen jubrad)ten, fo tfa. ten if̧nen, wie id f̧ơre, bie Draniengallinfeften (Coccus Hefperidum) to meţe, daß fie ganj fränf.

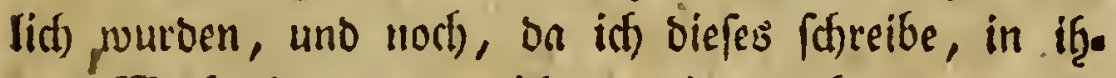
ren Wadfstţume um nichts weiter gefommen finis.

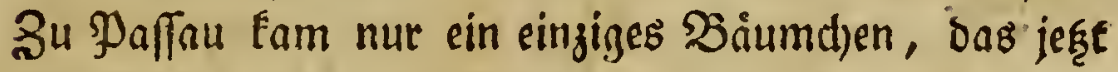

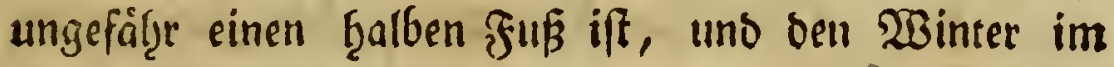
Jrenen fę̧r gut überftanden f̧at. - WBann aber bie.

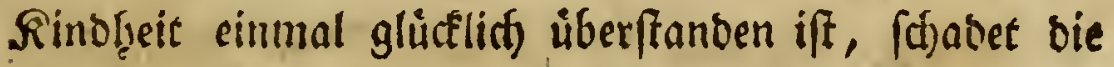

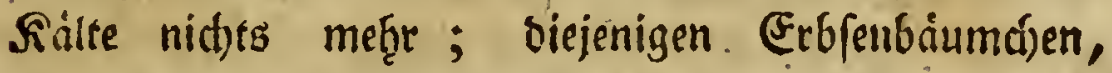

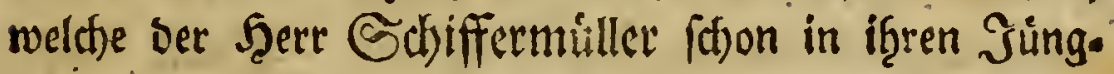
lingsjabten befommen bat, mact)en vortreffilid) fort, uno jino voll Blutthen. Dod fiehte man es Der Pflanje ganz roof̧l all, baf fie viel geneigter fey buffjigt und ftraud)artig, als zu einem Baum, zu wats)en, in weldhem zalle es if̧e ber Rleebaum (Cytifus Laburnum), ben man um Burgḩaufen unter bem গiamen Der fibirifthen Erbfenbaumes fojon lange einf̧eimif zu madjen verfudft b̧at, viel zuvorţ̧ut. 
29. Robinia frutefcens. Lin./pec.pl.p. 1044 , Sp. 5. Die Slättctien fteḩen balo ju oreyen, balb ju vieren benfammen.

\section{Robinia hifpida. Lin. Syft. Nat. p.490.}

$\int p$. 7. Die Stengel fino bidft mit tơtţlid)ten f̧aaren befestet, die an der Epiz̧e eine Driffe tragen, reldje cinen bicfen Eaft abfonvert. Die Blattfiele fino gleidffalls haarid)t, aud) bie Stielthen ber Blattthen,

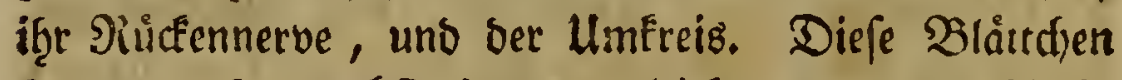
figen ju adten nebft einem ungleidfen an gemein/d)aft. liden Etiele, nidgt genau gegenúber, fonbern etroas

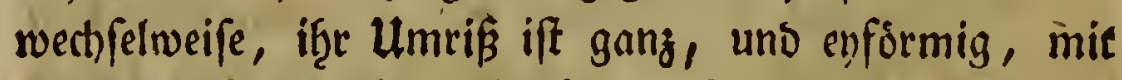

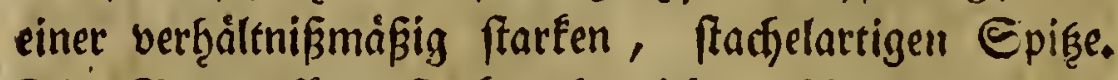

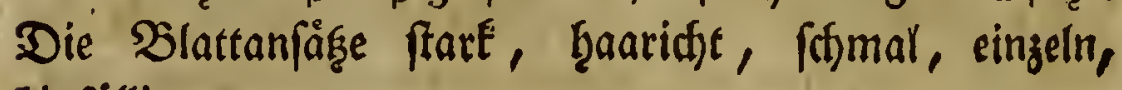
ந̧infállig.

31. Coronilla Emerus. Lin. Spec. plant. p. 1046. Sp.r.

32. Coryius Colurna. Lin. Jpec. plant. p. 14 I $\%$. Sp. 2. Die $\mathfrak{B l a t t f e r ~ f i n d ~ e c f i g t , ~ D i e ~ h e r v o r : ~}$ ppringenden (Ec(en ungleid) fägezåñnig. Dursh) biefes Rennzeid)en iff biefer Etrauch viel leid)ter von ber gemeinen Şafelftaube wegzufennen, als burd) bie Blattanfäkęe, bie eben nid̦t allemal linienförmig, fons bern lanjefformig fins, uns viel reniger in bie 2lugen fallen. Jonfton ţat in feiner Hiftoria naturali de arboribus auf ber 39. Fafel unter bem Namen Avellanae Bizantinae ramus sine fę̧r gufe $266 b i l$. sung cines Broeiges geliefert. 


\section{Эaffa, Den I 6. May, I 783 .}

33. Platamus orientalis. Lin. Jpec. planc. p. I 418. Jp. I. Diefer Platanus andert. Jef̧r ab; feine Blátter find richtig allemal in brel) ooer fuinf Sape pell getf̧eilt, weld)e abermal, wie bel einigen 2(rten

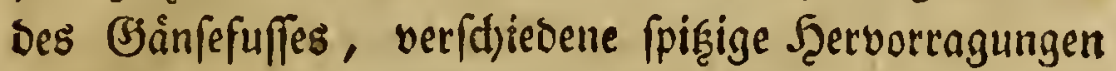
நृaben. Die Einfdanitfe, meld)e bie Lappen bilben, finto weber tief not) (d)arf, fondern frummlinigt, und Paum von dell Berticfungen zwifd)en ben fpifigen J̧erborragungen zul unterffheioen; bie mittlere $\mathfrak{B l a t t}$ fláche ift Dab̧er allemal im Berţåleniffe mit bie.

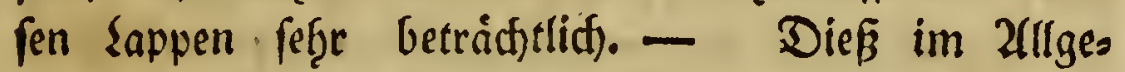
meinen; iff) muß 3 Jgnen aber noch befonbere 2(nmers fungen ůber brel) Z(rten ober Zabarten biefes $\mathfrak{B a u m e s}$ madjen, weld)e idf in siefem Barsen zu beobadjten Ëelegenţeit ந̧atte.

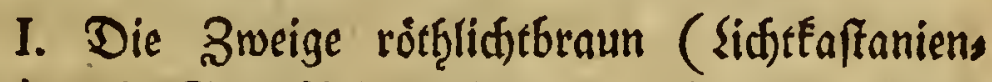
farben); bie Blattfiele wolligt; aud bie grooffern Nerben ber Unterfeite bes Slattes, und b̧ier und ba Gtellen im Slatte felbft; die Sberfeite fef̧r wenig mit mollenartigen Şäărctien befę̧et; bie Ģerborfprin.

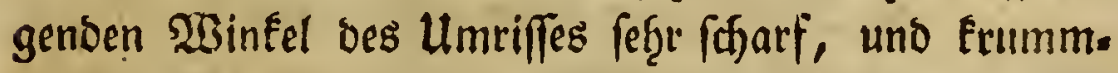
linigt; Der untere ₹̧̧eil Des Utmriffes, no Der. Etiel in bas Slatt ḩineingeget, getabe veggefdjnitten, ro Daß Der $B$ lattfiel mit dem Girunde bes Blattes zween red)te Wintel madt.

II. Die Sweige rơtflichter, oft mit einem zer. riffenen weiffen Dberf̧autchen bebect', bie Blattfiele wie oben; Die 'Blåtter Fleiner, unten ourcfanı ftarÊ rollid)t, oben aber armer; ber Utmriß beutlid) nue 
brenlappictit ; ber Grunb bes SBlattes madit mit Dem Blattfitele zu benoen Seiten eiren frumpfen ISinfel; bie $\mathfrak{B l a ̆ t t e r ~ f e l b f t ~ f i n d ~ b i c f e r ~ a l s ~ a m ~ v o r i g e n ~}$ Baume.

III. Z(lles, wie in nåd)ftworfergeţenben Baumre; aber bie Blätter groffer, weniger biff, uns nid)t fo mollenreict), wie int leşten, nber bod) mef̧r, als im erften.

IV. Die Blátter faft, wie an ber zwenten । $2(1 \mathrm{t}$; aber nod) fleiner, uno fefre menig wolligt. Die Grunblinie madt) mit bem $\mathfrak{B l a t t f t i e l e ~ n i d f t ~ n u r ~ b e n b e r s ~}$

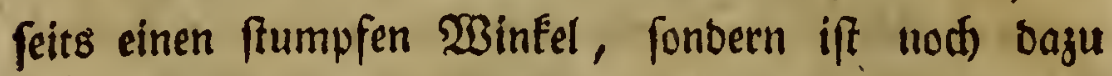
nidite gerahlinigt, fondern ausgebogen. - Diefe 2 ret fat ben Tamen Plantanus nana erf̧alten, und ift in

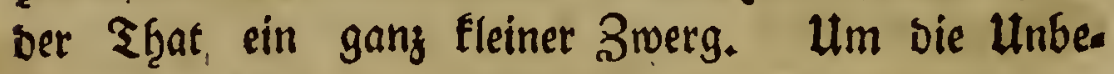
frimmtḩeit ber limnaiifhen Sezeid)nung anfzuţeben, wurrbe man biefe vier Baiume füglid folgendermaffen unterfdjeiben fónnen.

x. Plantanus rectangula petiolo folium ad angulos rectos ingrediente. I.

2. Plantanus obliquangula petiolo folium ad angulos obliquos rectilineos ingrediente. II. III.

3. Plantanus naná petiolo folium ad angulos obliquos curvilineos indiente.

34. Thuja orientalis. Lin.Jp.pl.p. I 422. Sp 2.

35. Salix babylonica. Lin. Spec. plant. p. 1443 . Sp. 9 . 
36. Hippopbä̈ rhamnoides. Lin. Spec plant. p. 1452 . $\int$. I. Die fonberbare Sefleibung ter Biato ter madjt diefen Straudy vorzuglid) fenntlid). Die unterften $\mathfrak{B l a t t t e r}$ ber jungen Sriebe werben, eb̨e fie abfallen, auf der Unterfeite gang weiß mit roftgelben Puncten. Die Breige felbft fino meţr ectigi als gefurdst.

37. Populus Tacamahaca. Miller. Dict. Un ter biefem Namen befinbet fich in biefem Barten ein Baum, ber allerbings einen fark riechenden Rleber ausfdfwiget, aber weber mit ben Rennjeichen, bie Einné in feinem Species plantarum von Populus balfamifera angiebt :

Populus foliis, fubcordatis denticulatis,

zufammenpapit, no(t) mit benen, bie im Syftema Naturae Edit. I 3. angegeben werben:

foliis ovatis ferratis fubtus albidis, ftipulis refinofis,

genau paffend ift; viel treffender ift Ģmeling $\mathfrak{B e}$ fofreibung, fo furg fie nud ift:

Populus foliis ovatis acutis ferratis.

Die Blätter find feicf)t, aber nidt ungleid, geferbet, wie fie Şerr, Mredicus angiebt.

38. Menifpermum canadenfe. Lin. Sp.plant. p. 1468 . $\int p .1$. 


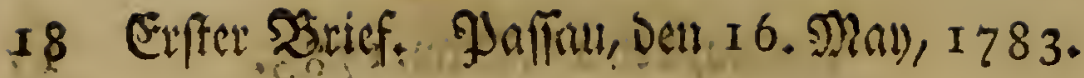

39: Celtis occidentalis. Lin. Spec. plant. p. $1479 . \int p .3$. उd zmeifle fegre, ob biejenige

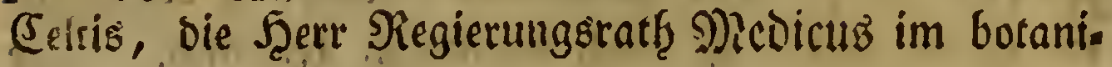
f(t)en Barten zu Mannf̧ein unter biefem Namen er: jief̨e, bie wafgre Celtis occidentalis fen; wenigftens tömmt bie, weldhe J̧ere Rath Gdyiffermúller befiget, vollfommen mit ber Beftreibung Des Siltters von Silmé úberein, ber weit entfernt ift, bie Blatter bollisminen cyrund ju nennen, fonbern fie bielmegre fdicf curumb, jugefpigt, u. f. F. nennt, weldes fich

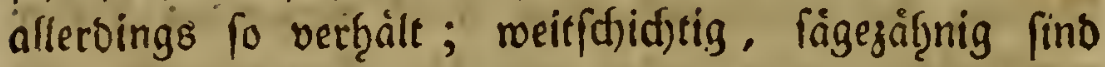
fie noḥl aud); aud) fino sie Bák̨̧ne feģr fdjarf, uno einnártళ nach bem Ratto ţingebogen.

40. Acer rubrum. Lin. Spec. planc. p. I 496. $\int p \cdot 3$.

41. Acer Negurido. Lin. Spec. pl. p. 1497. Sp. 10.

I(t) berfpare mirs auf eine anbere Belegenţeit, Sie mit meinen úbrigen Bemerfungen, die ith auf biefer Reife gemadt)t habe, zu unterb̧altell. Ith vers

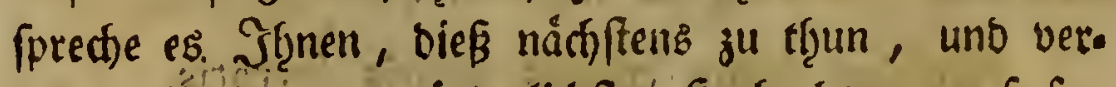
b̧arre mit ber unberånberlidffen f̧odjact)tung u. f. f.

Sd)rank.

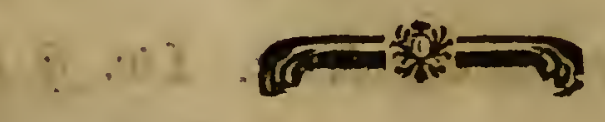


3weuter 20rief. Jaffau, Den 25.May, I 783, 19

\section{3wenter Srief.}

ग̧affau, ben 25. May x783.

Sherr Gólmann's Praturalicnfanmmlung. Gatms

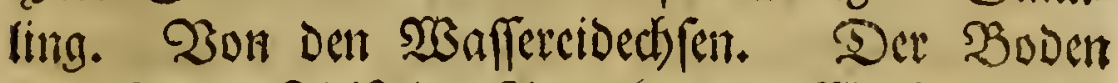

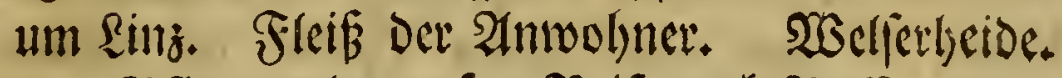

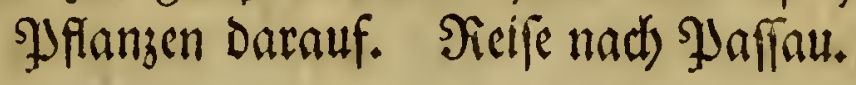

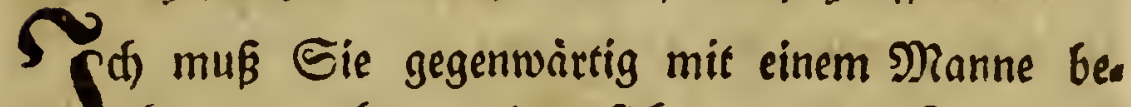
$\checkmark$ Eannt madien, ber fidf aus ben Taufenden

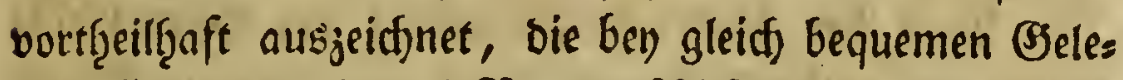
genf̧eiten vieles in Der פaturgeffdidfre zu arbeiten, fie gleidwoobl unbenuģet Iaffen. Ess iff biefes Şerr

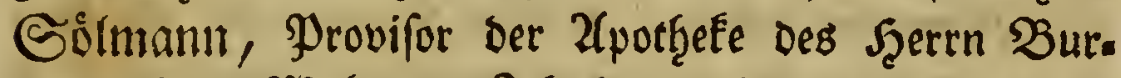

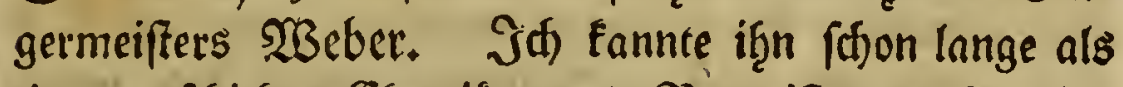
einen geffdirften Cḩenifer uno Botaniffen; aber bey

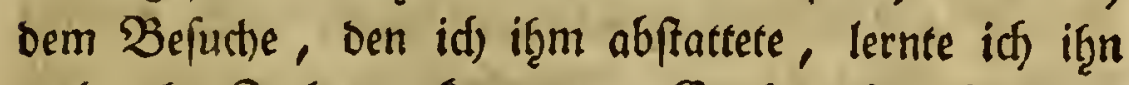
oudb als Boologen feemnen. Er that eine feğr bes tràdtllidfe Infectenfaimmlung, bie er mit einer Tieos.

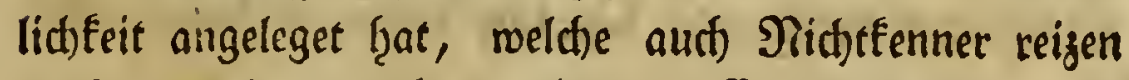

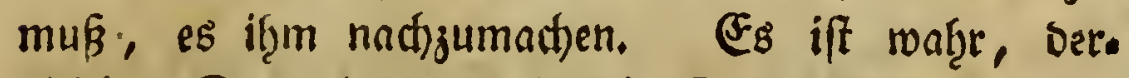

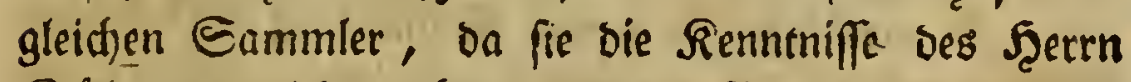
Sớtnaant nidft haben, werden für if̧re perfon ber

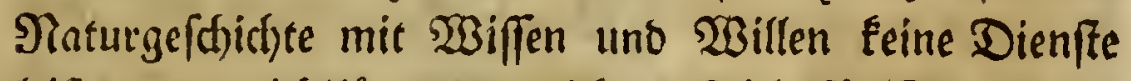

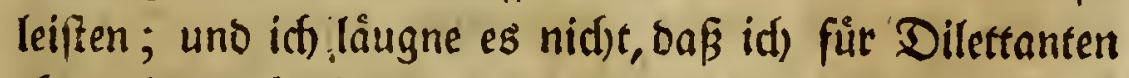

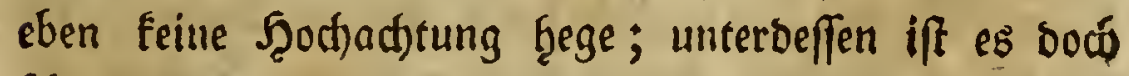

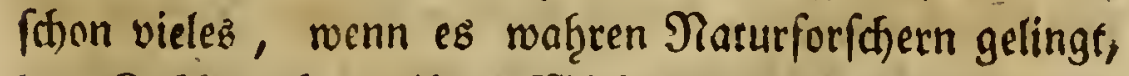

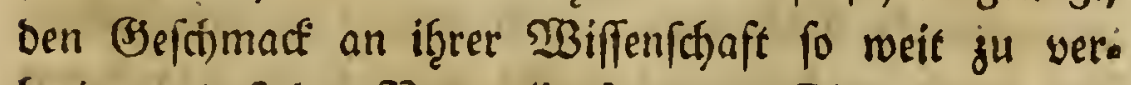

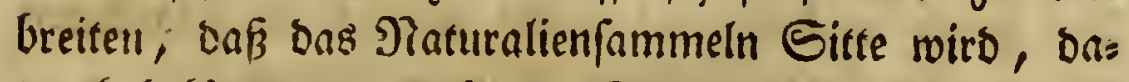
burdf) befómmt gar oft Der Renner, ber biefe Samm

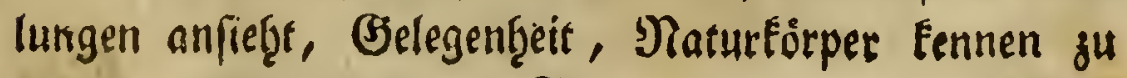


leruen, bie if̧in, auffer biefem Falle nođ) lange, viels leicht immer, wurben unbefonnt geblieben fenn. -

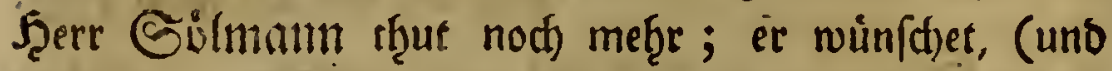

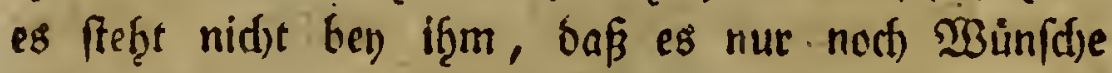

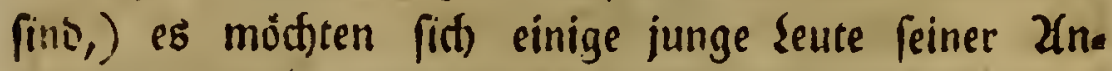
fithrung in ben verfdiebenen Stgeilen ber शatur. gefthidte ùberlaffen. Sie mürben fid) gemis nidft betrůgen, wenn fie es ţ̧ăten.

Was ber Naturalienfammlung bes Şerrn Göl.

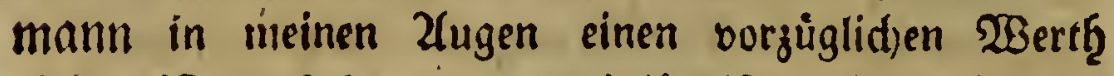
giebt, ift, oak fie ganz aus inländifden Taturfurpern Oberơfterreictso beftę̧t. (Einige Räfer ḩabe id oar. aus in meinem Supplemente angeführtet, uno idh) ḩätte aud) unter ben ùbrigen Jnfecten, wenn fith meine Sthrift auf bieferbigen erfirectet ḩäte, nod) mandse sůcfe ausfüflen förnen. Merfmùrbig war mirs, baßs idf) Ģier einen Papilio Memnofyna fah, 'ben Şerr

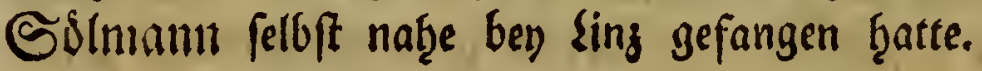

Er martit fid jezt eine Sammlung inlänbifdyer Fifde, uno ba ber Salmling bom (s)munderfee faton

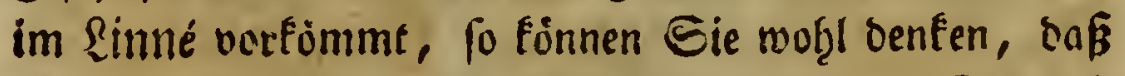
(id) ig̨n bafelbft meroe angetroffent baben. Eeit id biefen Fifd) bet) if̧m gefergen ţabe, bin id) begierig, bie von Berditesgaben, bavon id) vor einigen Jaf̧ren ein paar Eticfe befdrieben, nod) einmal zu uncerfuchen. Jd) Denfe immer, es müffe menigftens zroo Zarten geben, bie bende einerley Namen füb̧ren. 


\section{ЭJaffau, Den 25. Maus, 1 783.}

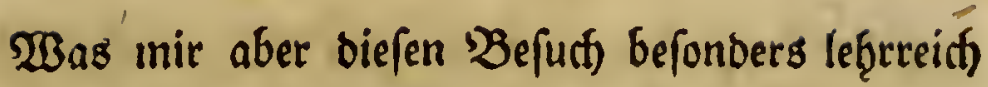
machte, war bie artige Eammlung offreidifiner $2 \mathrm{~m}$.

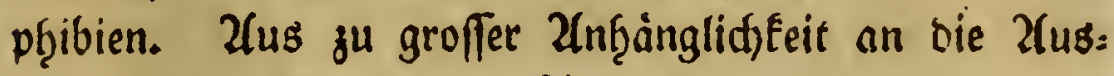
fprüd)e bes ritters bon simue fielt id) bisher alle europaifd)e $\mathfrak{W a f f e t e i b e d ) f e n , ~ b e n ~ g e f l e c t e n ~ M o l ( t ) ~ n e b f t ~}$ nod) zrooen 2lrten ausgenommen, für Sarben berjeni. gen, weldie mir aus feinem Naturfinfteme befannt waren. Notf̧wendig mußte fith baḩer die 2unjab̨l mir befannter 2 reten an oen fingern berzăb̨len laffen. 2aber Serr Golmann verfidserte mid), er habe feine fifd). forrmiggen (Fibed)jen zu allen Jabrgeseiten in bem (Se. wäffer um sing angetroffen, ḩabe ib̨re Evyer gefef̧en, uno fely aus feinen Erfag̨rungen vollEommen ubergeugt, baßs es vollfommene Tgiere fenn, die-if̧res gleidjen zeugen. Fin wienerifther $\mathfrak{Z}_{\boldsymbol{r}} \mathrm{t}$, Seerr Sautenti, Gatte fthon vorf̧er über biefen (S)genftand viele unb mühfamé Beobad)tungen angeftellet, uno fie in einem eigenen, aber nad) feinen Berbienften viel ju renig befanuten, Butje befanut gemadt. (*)

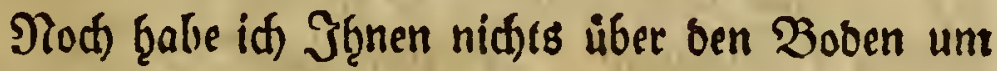
Sing gefagt, ber ein ftarter Beneis ift, wie viel eine bolfreid)e Etnot auf bie Urbarmadung ber unfrucht.

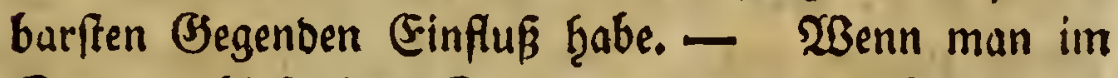
Sommer blok einen Spajiergang aus ver Stabt fin. aus auf loie naid)fit gelegenen Felber macts, fo follte

$$
\mathfrak{B} 3
$$

man

(*) Jofephi Nicolai Laurenti Specimen medicum exhibens Synopfin reptilium emendatum, cum experimentis circa venena et antidota reptillum auftriacorum. - Typis $J$. F. Nob. de Trattnern 1768. in 8v0. 214. Seitch mit 5. Pupfertafeln. 
man benfert, Sing lage in Der frud)tbarften Bsegens von her Welt; alle Betreibarten wadfen ungenrein

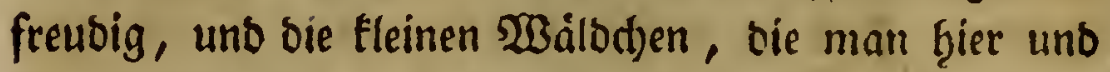
da antriffe, bienen bloß̧ baju, bie gar ju großje (sin= formigfeit zu unterbrect)en, uno ben 2lugen immer neue suftrevieren zu offnen. Unterbeffen zeigen eben

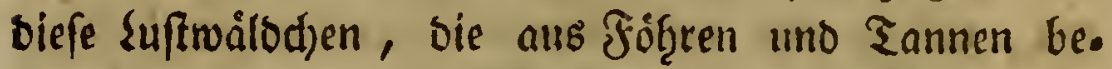
ftef̧en, an, was biefe Begeno efiemals war, und waz fie weifer von ber Stnot noch) ift, eine Freibe, bie fid) von $\mathfrak{W a}$ ald loings ber Traun bis an Die Donau, in

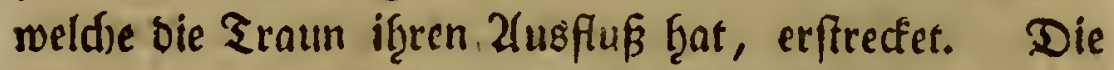
Begend an ber Donau, meldse wahtrid)einlid) am ers

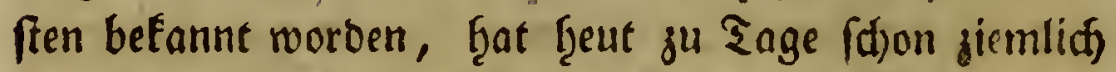
tief bortreffid)es (Eroreid); aber bie etroas entferntere, burd) weldye ber $\mathfrak{B}$ eg von sing nad) E.ferfperg und

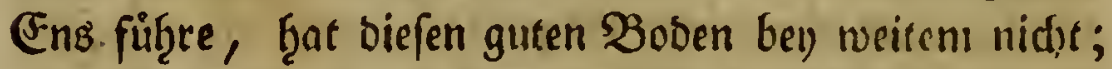
alles if nod) ein unermeßlid)er Ed)ut Eleiner Siefel, ober wenn man lieber. rill, groben Sanbez; uno wenn

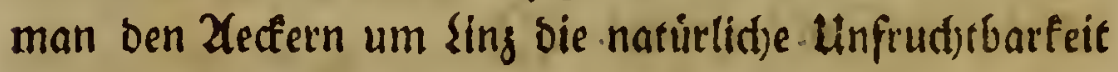

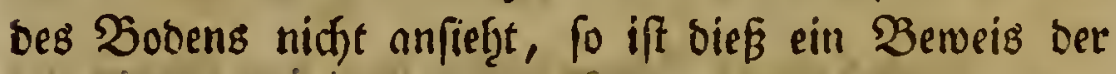
großen Betriebfamfeit ber 2fnmoḩner, bie allemal eine Folge ber frarfen Bevolferung ift. Man' lägrt fier nidfts weg, was gefdicft ift bie frudftbarfeit ber 2lecter zu vermetren. Die benad)barten Bauern, um nid)t auf Roften if̧rer Felber bie Miefent vermeţren zu múffen, baben die heimlidsen (Bemáther in der Etaot gemietḩet, uno führen biefen foftharen Dinger auf iņre Grunbe, den man anberwátts aus nidtigen, uns zum SGgeile ládjerlidgen Urfacten in vorbenflieffende ฌaffer leitet. 
Eelbft bie benad)barteu İnģöben fino auf oicfer Eeite der Donau nichts als ungeģeure Ganbţaufen. Man fann fid) bavon zur (Benuge ůbergetigen; wenn

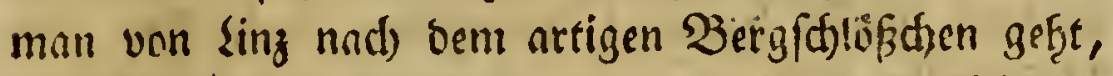
bas Dem norbifden Collegium gef̨oret, uno ben fajören

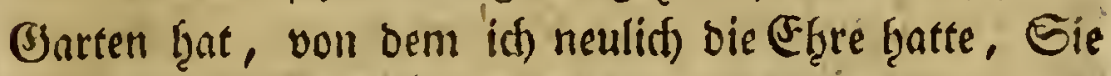
fu unterḩalten. Man formmt nuf biefem Ziege ei.

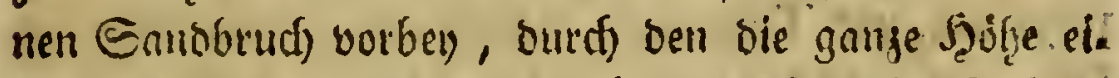
neE ziemlid) anfergnlidfen sanbberges entblóffet ift, unt eine noturliche Sicfertuand vorfeflet. Jim $\mathfrak{B o r b e y s}$ gef̧en muß id) J̧̧nen f̧ier fagen, daßs man in biefent

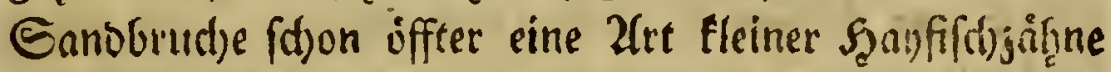
entbecfet laabe, weld)e von den 2(rbeitern einiger ente

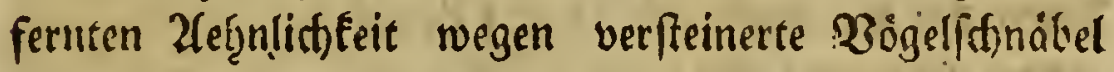
genennet werden, uno den $\mathfrak{B a u m e i f t e r ~ b i e f e r ~ S a n d = ~}$ baufen uno ber umb̨erliegenden $\mathfrak{h}$ aibe beutlid) gu er: fennen geben. Unterbeffen verbienet biefe (5) gens biefen Namen nisthe meł̧r, uno menn man oben auf biefen ungeb̧euten Sandḩaufen feţe, fo vergibe inan barauf, was man unter feinen friffen fiabe; allent.

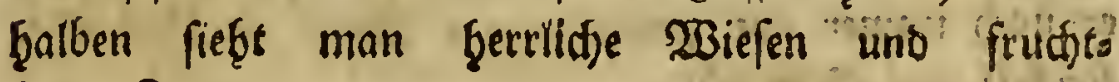
bare Jelber.

Den Namen formof̧l als bie Eigenjinafft ciner

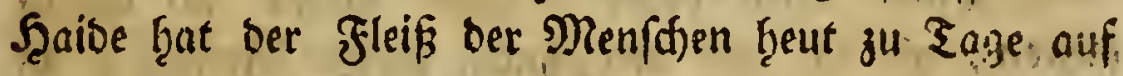
cinen viel fleinern $\mathfrak{B}$ egirk eingefdränfet; es ift bief bie (⿹)geno, durd) meldbe man fonmt, wenn man von ber WBienerfitraffe ablenft, uno nad) WBels zufägrt, von welcher Gtabt man ig̨r aud) ben गamen ber Welferţeide gegeben ḩar. 
Benn aber biefer (Begeno nod) ber alte, unrüf̨m. lidie Name bleibt, fo folget nidte, dak fie ganj un. frud)tbar (eny. PHanzen, weld)e trocfnen Bonen lieben, wacthen fiaufig darauf; ungeţeure Flecte fino von Difcutella didyma gelb, bie von bem fabrairzlid)ten

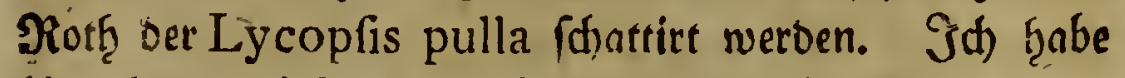
Gier egeobem Aftragalus Onobrychis, Thefium linophyllum, uno Teucrium montanum hicufin angetrof fen ; uno man ḩat mid verfichert, oaß̧ and Teucrium Chamaepythys, Teucrium Botrys, uno Sideritis romana ḩier wadhfe. Şin uno wieber wiro vie Şaibe yon fleinen $\mathfrak{W a ̈ l b e r n}$ unterbrod)en, bie fogar Saubf̧ol tragen, unter beffen Sthus bie verfd)iebenen Zfrten ber Conballarien, das Lilium Martagon, uno andere faftreidfe Pflanzen forffommen. - 2lber id) hrabe ood) nidt) im Sinne, J̧̧nen eine vollftanoige flora ber $\mathfrak{B e l f e r b a i b e ~ z u ~ f a j r e i b e n , ~ u n o ~ f i e ~ m u ̛ r b e n ~ i n ~}$ ber That für einen Brief viel zu lang ausfallen. Der Flleiß ber Menfdien f̧at fithon groffe Bsegenden bollfommen urbar gemad)e, uno an andern befámpfet er nod) immer mit zweifelf̧aftem Siege bie Unfrudtt. barkeit bes Eroreidfs.

Einen Ţ̧eil biefer (J)egend mufite id surd). faf̧ren, als id) ben vierzeţnten biefes Monatţes in (S)efellfdafft bes Şerrn Rathes Sd)iffermúller's, ber midj bis Sambad) zu begleiten bie Ģüte bৃatte, ḩief̧er zuruicf reifete. 


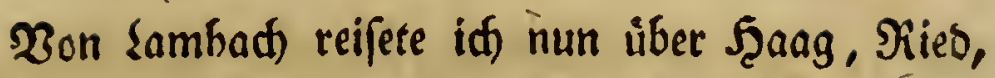
Edjarbing nad Paffau. Die Begeno niro um Sam. bact) ftion bergidft, gleidfwob̨l berbeffert fict) bas Ero, reich alle Stunden, und man fömmt grififten f̧aag

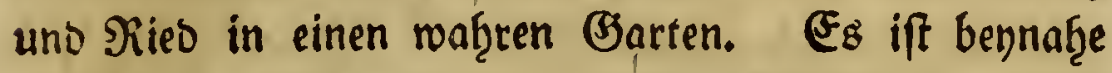
unmóglid, baß̧ es eine frudtbarere Bsegeno geben fóne, als bas Bebiet um Rieb. Sites felbft ift ein ganj f̧übfdher Martfflecfen, ber mir immer fo lieb ware als bie Stabt Efferoing. Die groje fruaft.

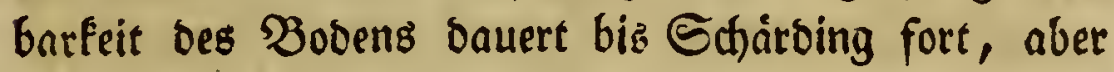
von Sdjaroing bis Paffau nimmt fie wieber frark $a b$.

Id) ţabe auf biefer Reife eben nidfts merE. murroiges gefunben, auffer daßß id jwifhen f̧aag uno Sites Das Geranium phaeum an ber Straffe rild. mad)fend angetroffen ţabe. Sine X!penplange auf einer zwar bergid)ten, aber nitdst gebirgigten (S)egent? merben Sie fagen. Dennod) ift es 10; wiffen Gie Dodf fdon aus einer andern Edfrift your mir, onß id Alchemilla alpina auf einem fleinen sanoberge bey

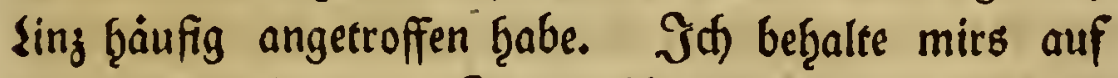

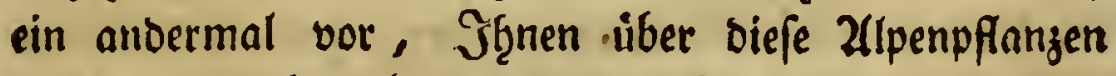
auf bem fraten lanbe meine $\mathfrak{M e n n u n g}$ ju fagen. Sis baf̧in u. F. f.

Gdrant.

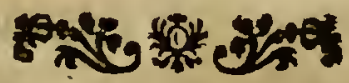




\section{Dritter Brief.}

Surgbaufen, ben 25. Suniuß 1783.

Mabreporiten von 23 offfecin. Giviner Mrarnor.

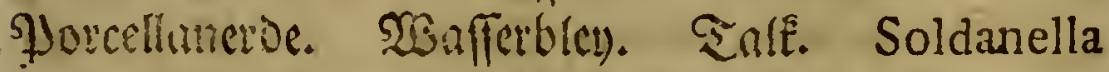
alpina. Jjerfenmuldetm. Stive. Lleber bie stipens

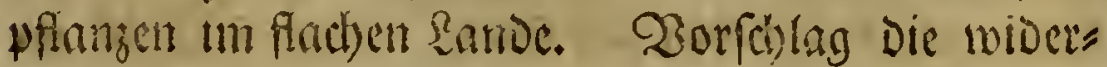

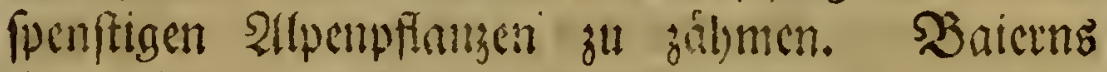
Frudstbaréeit. Sivitie einer Stelle in Den Biviefen cines Tranzofen. Sftindider Derbaum. Sinnfender Eteributud).

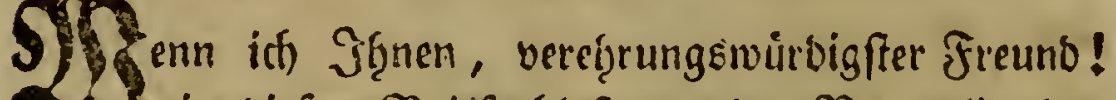
in diefem $\mathfrak{B}$ riefe blö von den Naturalien des

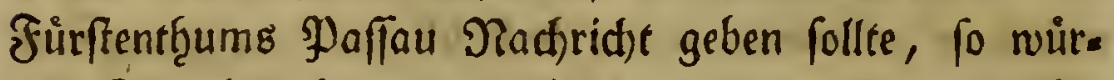
Den Eie eben feine lange Unterf̧altung ḩaben; nict)t, als wenn es ein sano wäre, bas bem Naturforfd)er nidits aufzumeifen haăte, was feiner Zufmerffamteit wưrtoig wäre : fontern meil es mir allemal bely meinem Zlufentf̧alse in demfelben entweder an Miufe, ober an

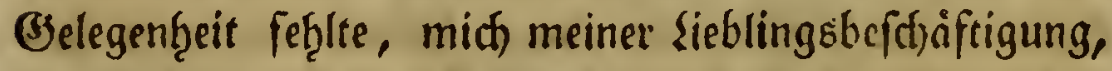
Der-Naturgef(d)id)te Der Begento meines Zufenthalts, zu ủberiaffen; mein $\Re$ nabenalter aber, bas int ganz in Yyaffau zubractfe, war bie Beit nicte, zu ber man es mir zumuthen fónnte, etroas in biefem ̧radje geteiffet วu ถูaben.

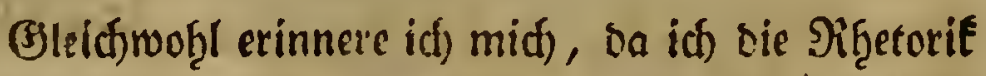
frubitte, einen Stein geféfen zu ḩaben, ber unter bie

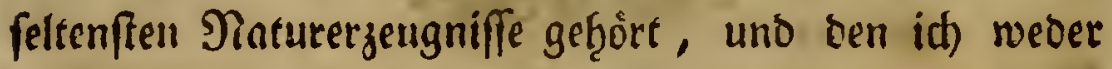
feitţer in irgend einer Maturalienfammlung angetrofs, fon, nod) in Yaffau firboft meģr exfragen Eonnte. 


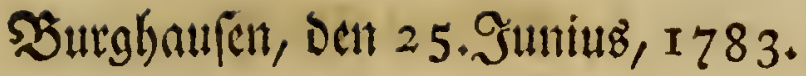

Es war ein Mabreporite von blaß̧rother Farbe, uns fo geringer feftigfeit, baßs man ib̧n mit bem Ragel fhaben fonnte; utbrigens hatte er feine beftimmte Sigur, fonbern glid einem Stůcfe, bas man irgeno bon einem Felfen modhse abgébrod)en ţaben; feine rȩ̣e beutliden Belld)en, wegen weldyer man if̧n einen Eternftein geneunt f̧atte, waren ungefäḩr von ber Groffe eines Gaamenforns vom gelben Eommers veild)en (Cheiranthus Cheiri Lin.) Er foll im

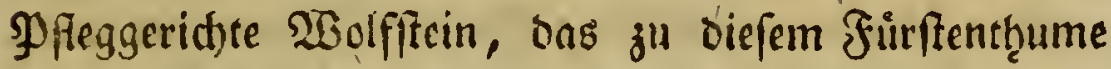
getzoirt, gebrod)en worben feyn. - Seine von benen Figuren, meld)e Fougt in feiner 2(bfrandung de Coralliis balticis Edit. Biwald. geliefert hat, ffellt bies fen Mabreporiten genau vor; am nàd)/fen fómmt if̧m fig. 24 aber bießs ift eine Millepora, und ver Stein von $\mathfrak{B}$ olfftein hiat beutlid)e Sterndien ; gleidywoht vermuthe id, daß es einerley (Eorallioliţ̧e fen, meil if̧n Fougt befdyreibt : Millepora fubrotunda poris minimis confertis (biefe fonnten mir Damal nidgt auffallen, ober waren im verfteinerten Stůcfe wof̧l gar ni(f)t Da), majoribusque crenatis remotis. nunc cinerea - nunc in rubedinem aliquantum propendet. 2(ber bie Etralen waren bod) etwas mę̧r als Einferbungen (Crenae).

Man f̧at midh nath ber f̧and aud verfichern wollen, es breche in biefem jurtftentḩume cine 2 frt grumen Marmors. Da id) fein Probefticf bavon zu

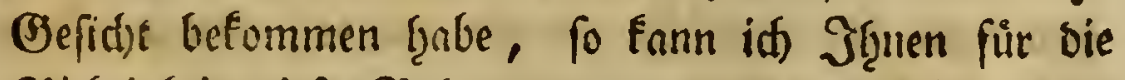
Dirfftigkeit biefer Sebtauptung nid)t ftę̧en. Org̨ne auf 


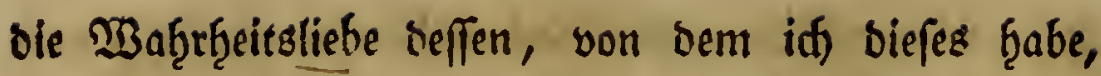

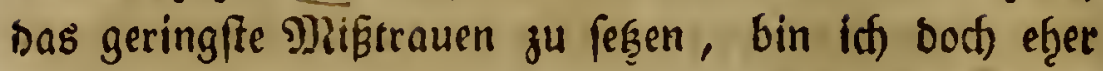
geneigt ju glauben, baß er einen Eerpentinftein módfte gefehen, uns für grünen Marmor geţnlten baben: benn Marmor vermutf̧e id) recoer im Paffauiften, nod) in ser ganzen Walorevier von Ef̧am, an ber oberpfälziften (3)ránze, bis Brieß̈bach), bas an ber Ġranze von Depitreidg liegt, uno von Den Paffauern zum Unterforieie von bem baierfden Pfleggeridte

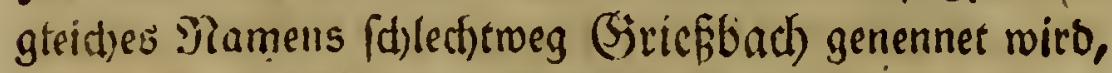
ba fie, hingegen das andere batrif(h) (jrickbach ju nennen pflegen.

Defto berifhmter ift hingegen biefes f̧ürftentf̧um megen feiner idjönen miltsweiffen Porcellanerbe uno bes Wafferhtenes, die man bense in bem $\mathfrak{B}$ ejirtée bes pafe fauifdhen (Brieß̧bact)s gräbs. Sie miffen es ox̧neţin

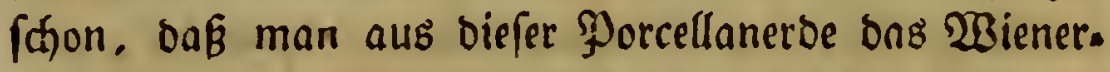
und Münd)ner Porcellan verjertige, uno snß leģtere Erbart bortrefflid)es Ethmargefdjirr, befonders fef̧r ḩaltbare Ed)melzztiegel gebe. Der Şandel bamit ift to betráct)tlic), baß ber Marftflecten Dbernzell ber vielen ₹opper wegen, von benen bie meiften biefes พBafferbley) verarbeiten, und ganz nob̨lf̧abende teute fino, gemeiniglich bie Shafnergell, uno bas Edwarggefdirr Sanfuerzellergef d)irr heiffen. Weniger bürfte

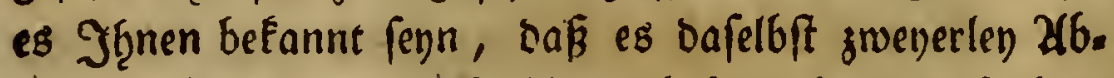
arten gebe; bie eine ift fhattentraunlidft fitberfarben,

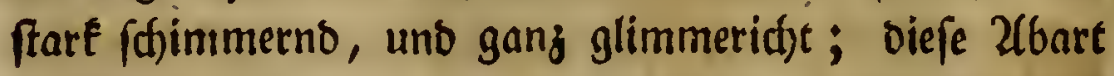
b̧ălt man für foblect)ter als bie anbere, viel bunflere und feinförnichtere. 


\section{Burghaufen,ocn 25. Juniuz I 783.}

If) muß fier im Borbengef̧en einen geograpḩi. fifien Jrtţum rügen, ber fith) in bie gmelinfthe Ueber. feşung von Simme’s Maturfyftem des Mineralreichs cin.

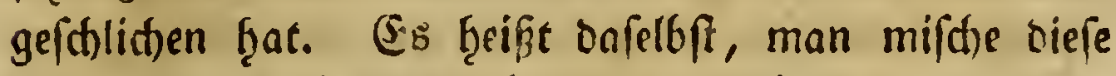
Eroe zu Jafueizill bey Siegensburg unter ben ร̧̧̧on, um Tiegel 11. bergl. zu mact)en. Şafnerzell, ober, wie es eigentlidf) beist, Sbernzell liegt aber nidft ber) Piegensburg, fondern an ber Donau nod einige Stunoen unter Yalfau.

Zufferbem bridst unweit bes Porcellaner.

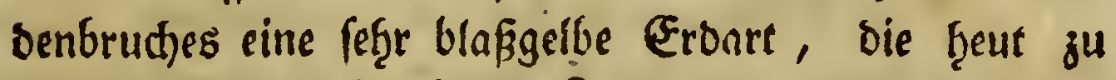
Tage von einem Sopfer. zu Jlg, einet von ben oreyen Etäbten, alts reld)en Paffau beftef̧t, mit Ton vers mifht, zu fef̧r guten Befáffen verarbeitet miro. Diefe Eroe bridjt in Eleine Rlöfe zufammengebacten, melche aus byalb ourdffdneidenben Eduppen beftef̧en, bie immer auf einer Geite etwas eingebogen, auf ber andern aber etwas ausgebogen fino. Eie ift fett anjufüḩ̨len, uno fărbet ein mentig $a b$; im $\mathfrak{B a} a$ fer zerfállt fie, audf lópet fich) mandmal etwas weniges auf (vermuţ̧lid) weil fie nidft gang rein ifit), uno madtst ons $\mathfrak{B}$ affer trübe, aber allemal riect) fie nad) oem Benajen, wie Rreibe, weldtes fie audh im trodtnen Buftnnoe, aber fd)waicther, unb etwas füßlicht, tf̧ut, befonders wenn inan fie in einem meffingenen Mörfer zu gan, feinem Mef̧l reibt, in weldhem Falle fie aber thre Farbe nid)e merélid verlieret, fonisern ein weiffer Etaub miro, der nur etras mę̧r graulictit ift, nls bie zufammengebactenen $\Re(o ̈ \beta(j) e n$, weldje $\mathfrak{B}$ einfuppens 
furben find mit untermengten filberfärbigten $\mathfrak{B}$ lättchen. Das (S)ervid)t ift gering, uns in Echeioerwaffer branfet fie nicht, macht aber fowobl biefes als tas gemeine Sioffer efwas fómierig, wenn man fie barinn herum, treibr. - \a) hृatte mit einem' Morre fagen finnen, biefe Erbe fer gemeiner Salf (Mica talcofa Lin.); aber Eie baàtten mir bann ood) auf mein $\mathfrak{B o r t}$ glaus

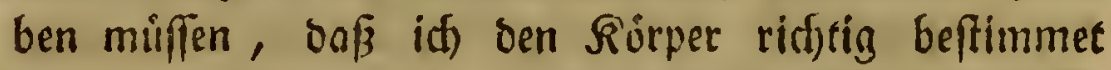
habe; nun aber hrabe id) Eie in oen Etano geferget, felber ju urtheilen: $\mathfrak{j a}$, dab ift $\mathfrak{c r}$.

Das Pflanjenteid) ţat in ber (Scgeno biefer

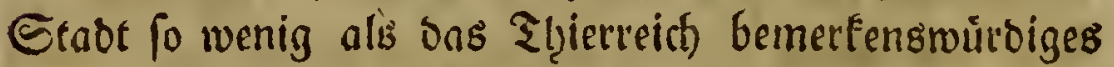

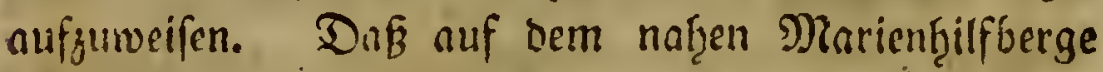
tic Soldanella alpina, eime waḩre Zlipenpflanje, Ģaufig

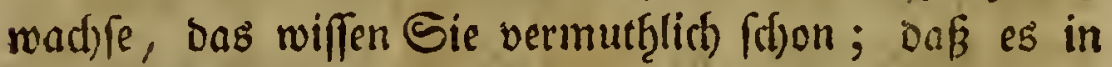
Dem Iljfluffe Perlen gebe, weld)e fich in ben Ed)aalen ber groß̧en frluß̧muffjeln (Mya arenaria Lin.) bes firion, thaben Sie vielleid)t auch gelefen, aber ich $\mathrm{mu}$; J̧̧̦nen baju fagen, baß̧ Der Perlenfang Fef̧r unbe.

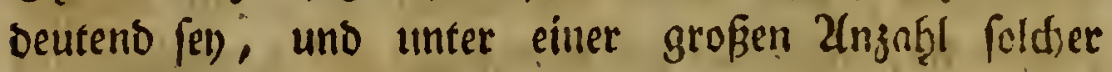
Mufdieln Eaum eine ober zwo eine Perle bely fid)

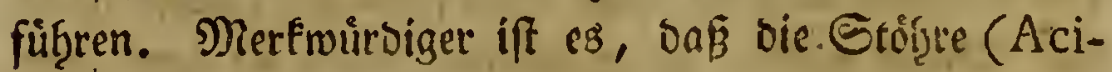
penfes Sturio Lini) auf ber Donau bis ilber diefe Erabt heraufaieţen; da man fie aber zu, fdionen $\mathfrak{U t}_{\text {t. }}$ rache hat, uns ig̨r frang ziemlich foftbar ift, unters nef̨men $\mathfrak{c s}$ bie fifider nur bey aufferordentlicten Belegenf̧eiten, Darauf antşugę̧en.

In ber 9ladjbarfdaft ter Stabt am reffent ufer Der Donau, in Ber Begeno ber verfdiebenen Şuen, 


\section{3urghaufar, ben 25. Эunius 1783.}

Flucil, ober Donauinfeln, waid)fit ber Edfnittlaud (Allium Schaenoprafum Lin.) wilt. Sd) grabe Siefe Pflanze, bie id) Gier nid)t vermuthete, nid)t unt genau unterfuctet, fondern nuth) mit eben blifenoen (Bartenfकuiftlaud) zufammengeb̧alten, uno nid)t ben geringften Unterfdied gefunden, ben (berud) ausge= nommen, der im wilben wibriger iff.

Şiet woire nun das Bischen paffaniffiter গlatur: gefdidte beifammen; aber id) bin Jģnen nod) meine Mennung ủber bie 2llpenpfanjen idulbig, was idf

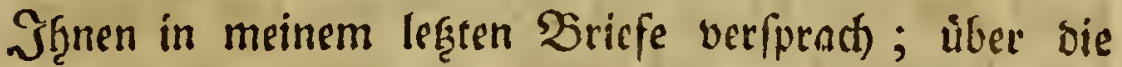
alipenpflanjen, bie man auf Bbegenten finder, bie nidfts weniger als zllpen fino.

Jat) bqabe in meiner Senturie botanildyer 2 n. merfungen zu Sinnés Species plantàrum mel̨rere Zlipenpfangen angemerfet ; 子. $\mathfrak{V}$. Galium rotundifolium bun bem $\mathfrak{P}$ óflingberge bey Sinz, Alchemilla alpina vom freubenberge ben Sinz, Primula farinofa von ber (Sirgens um Cfiemfee und Miunchen, Phyteuma fpicata von Sing, Paffau, Surgfinufen uno (Jiern, Gentiana verna von ben fladyfen Gegenden Sberoffreichs uno SBaierns, Potentilla alba vom Freubegberge bey ling, Hyoferis foetida oon bee Gegend um Surghaufen, uno dem fę̣r måfígen Berge ben Marf́t, breb Erunben von bier, uno,

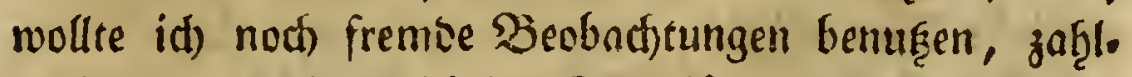
reidse andere fino ridftige Beweife, baßs viele allpen. pflanzen audf) auf nieorigen (Gegenden, auf Dem fläd)s.

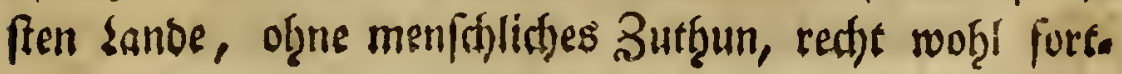

fommen, 
fommen. Nun frigt fid) aber, wie fie bon biefen iḩren freimifhen (B)egenden auf bie iţnen, wie man glault, unnntürliden Niebrigungen gefommen fenen; wabrifteinlid) ift eE nidft., baßz fie von Menfden gefáet worten, ba fie meiftens unanfergnlidise, und übers ḩaupt eben uidft die braudjbarften Pflanjen fino. (b):meiniglid, ruft man, biefe Erftheinung zu erflären, bie Etrôme und Flliffe zu J̧ilfe, bie bie Saamen von

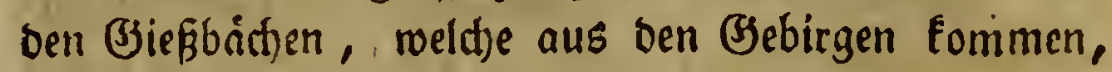
uno fid) mit if̧nen vereinigen, erf̧alten, waifrendem Saufe gier uno ba an bie Ufer abferzen, uno Folonien von 2lipenpflanizen tief unten im sande anlegen. Betradite id bie Stellen, an benen ids vorgeobatie Pfanzen gefunden f̧abe, fo läßjt fich) bie Endie meineto wegen bey einigen ber obengenannten Derter mutfy. maffen: aber andere benefimen ber Endje wieber alle

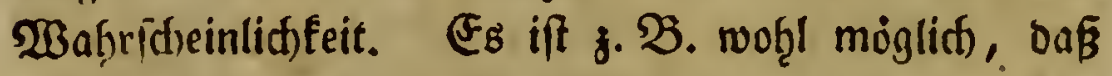
bie Saamen von Hyoferis foetida, welde man be Burģ̧aufen antrifft, von ber Salza mògen f̧ertier). gefüḩret worden fenn; aber biejenigen Pflanzen diefer 2(rt, weld)e am Martitlerberge, wad) fen, fonnten io wenig bem Innftrome if̧r Dafelyn, als Alchemilla alpina, Potentilla alba uno Galium rotundifolium oas if̧rige ber Donau zu banfen b̧aben.

Iđd) glaube vielmeg̨r, es gebe gar feine eis

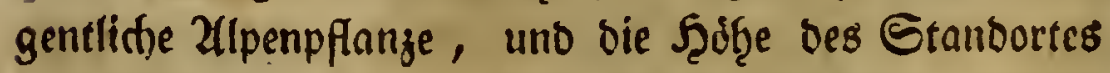
(er) bloke Bufälligfeit. 2lber andere Umftinbe, bie fita

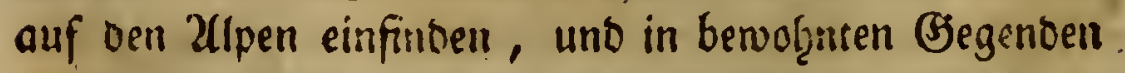
feltner anzutreffen finb, mogen die Urfadje fenn, 


\section{Büghaufen, Den 26. Suniuв I 783.}

warum bie wenigften fogenannten 2llpenpflanjen unter uns mof̧nen, bie man aber ef̧edem gewißs an ben Etellen unjerer Tieberlafingen angetroffen hat, ba fie nod) in ber lage maren, in weld)er sas J̦orígeburrs

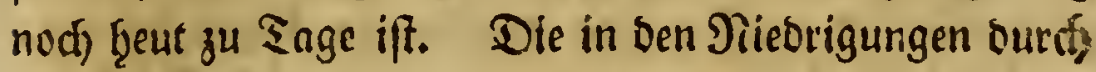
Zufammenwirfung ber Natur uno ber Menfichen une gentein ftarf vernef̧rte Dammerbe muß̈te PFangen,

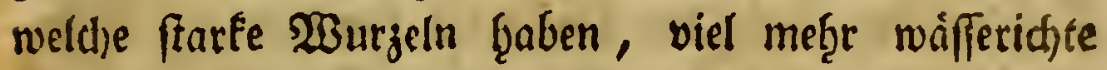

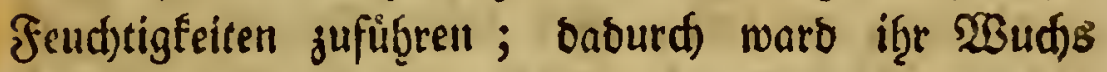
allmáą̧lig geiler, aber eben bnourd bie ganje \$flange (d) wóadflidher, fie fonnte baf̧er bem nicht weniger als auf bem f̧adigebirg: ftrengen frofte fowob̨l, als ber

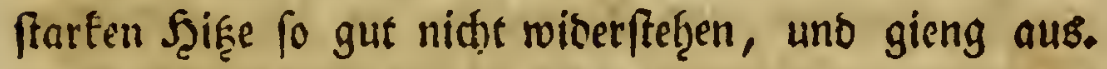
Daßß aber bas aromatifte, Das ift, dlige, Der Rälte fonvogl als ber Bertroctnung wiberfétgente $\mathfrak{B} e$ fen ourd) das faftreichers Eroreich) ber 9iebrigungen ges

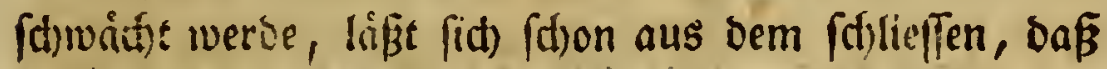
bie $2 \mathfrak{a g n e s p f l a n z e n , ~ b i e ~ m a n ~ v o n ~ b e n ~ a l i p e n ~ i n ~ b i e ~}$

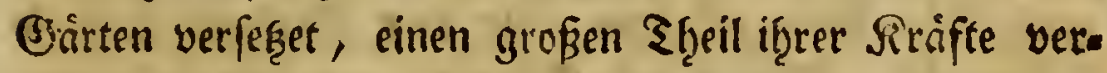
lieren. WPangen mit fagrachen $\mathfrak{B}$ urzeln wuroen bon

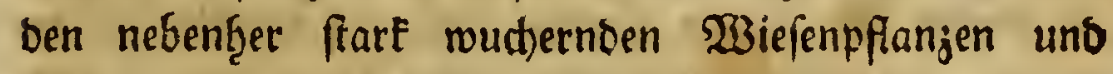
(Brasarten verrrieben, bie wof̧l mand)mal zugleid) an ver ju groffen Frudfibarfeit bes Eroreid)s eine neue Duclle i̧̨res Unterganges fanuen.

Seģen Sie nun, was idf inmer vermutfe, baß

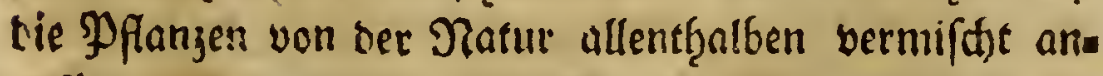
gefáet worben, Dod) fo, Dnß jeber afrt ein befonoerer Boten, eine befonvere Ervart, fogar eire befonbere Etellung gegen bie Eonne, als notb̨wendige Besing. Faturbif stiefe, Loband. 
niffe ifres Fortfonmens nngerwiefen wurse, to muste

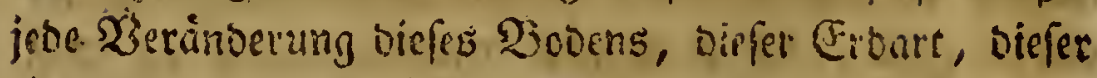

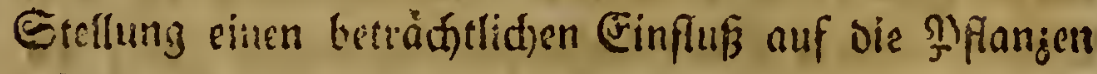
Eqaton; fie muigen ba ganiz ausgetren, too es für fie fa nadththilig waro, uns musten oa frefen blciben, wo feine Şerånoerung vorgieng, ober biefe Berände.

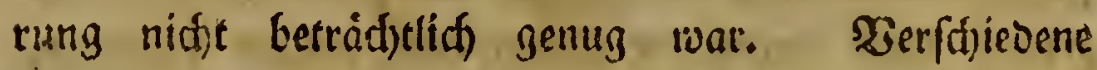
Sledtenarten uno ber F̧uflattici) fommen auf Ş̧on. grumb gang gut fort; aber laffen Gie ien ?fien zu

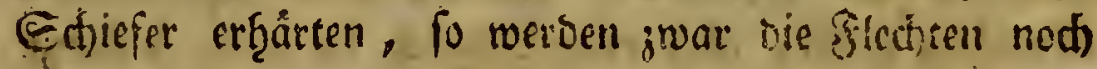
fortwadien, aber ber Feuf(attid) nit' beriftivinden.

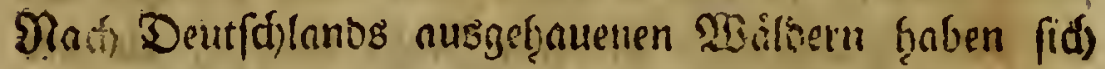

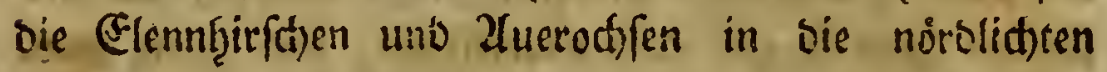
(J)egenden zurutrigezogen, wo fie bie giantur noth jiems lic) fo, wie fie ju Cápars Beiren in Bermaniens Mitte mar, antreffen.

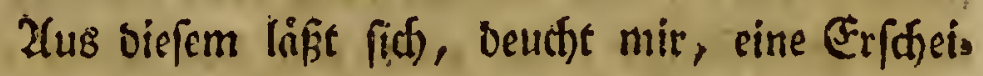
sung fehre leidfte erflären, bie bem 21nfdjein unch fo fortfan ift. Wis braben Pfanzen aus viel wairmern (Jegenten mit gutem (Erfolge bel) uns einfreimifd) ges mad)t, interferi es uns bey vielen âlpenpfinonjen noct) nitite gelungen tyat, fie in unfern Bsitton fortjuptan. jen. Unteroeffen rá!nlid') jene in unferm viel (d)lect)term Soten, als ser if̧res Baterlandes ift, jwar fleiner blicher, uno magerer, aber Dauerf̧after, und gegen unfere froofte abgegénirteter f̧eranwutd) fen : fanben biefe rin befferes Eroreid), wurben gróser und faftiger, uno ftarben, weun ith mith to ausbruicten barf, an Den Jolgen ser Bollblutigfeit. 


\section{Surghaulen, Den 25. Stunius $1783 . \quad 35$}

Sabat hat an verfdjiedenen Pflanjen und SBàu. men, bie von Franfreiff) nad Martinif́e gefommen

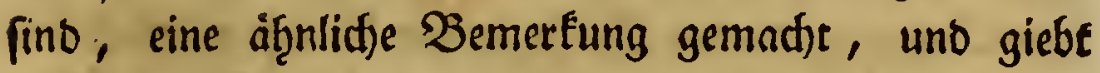
von if̧rem $\mathfrak{B a u e}$ eine Regel, bie bie Erfaf̧rung be. nóf̧ret ḩat, uno bie wir ganz leidft auf unfere allpen. pflangell anmenden Eoonnten. Er tritḩ, man follte fie aus bem nådften Sanbe, weldhes mit bemjenigen, wo fie ḩinfommen, an meiften übereinfömmt, ḩerneţ.

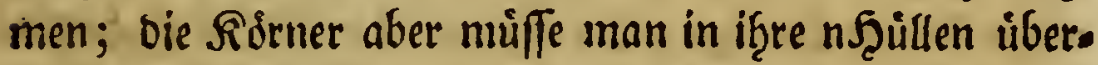

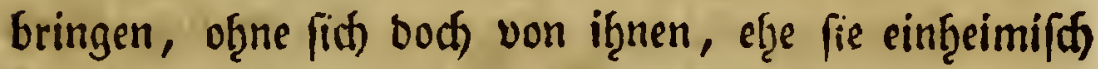
gemorben, eine meḩr als fef̧r mittelmåsige frudttbar. feit zu verfprect)ell. Man follte, Dácite id), von ber. gleichen miberfpenftigen 2llpenpflonzeu eine groffe Menge Saamen anbauen, nadjoem man für fie zubor, fo viel mogglid), einen Det im (Barten ausgefuchet hat, ber if̧rem natưrlithen Gtanoorte åţnlid) ift. $\mathfrak{B}$ on ben wenigen Pflarizen, bie man vielleid)t erf( nad) meţrern Berfud)en gerettet ḩat, follte man bie Eaamen zum frifchen Ilnbane, uno bie Gaamen biefer leģtern zur britter Enat wähtllen, weldje nun einţeimifa) genug geworben, um nad) und nad) in befferes Eroreid) vers pflanjet, aber viefleidft freglici) auch zu ganz anbern SPlanzen zu werben.

Enolidi reifete id sen I I. Bractimonattęes nad Şaufe. It) naf̧m meinen $\mathfrak{W}$ eg über (Briesbact),

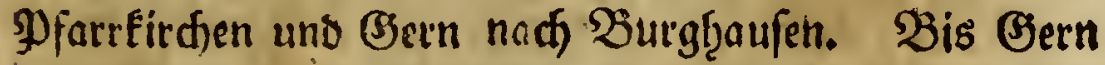
hatte id feine gemadte Etruffe, und bie Bieife mürbe felze unangenetim gemefen feinn, wenn mid) nid)t allentf̧alben ber Znulicf ber faoinften uno frutbitbarften 


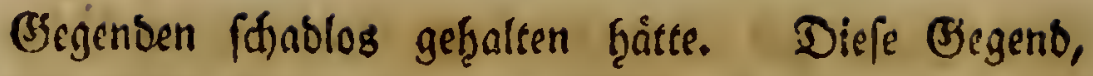
gleidmoly! nod) weber bie fhionfte, nod) bie fruchtharfte von Baiern, Ģătte ber Berfafer ber Briefe einez Jrans zofen bereifen follen: gewifi, er múrbe feinen lefern Baiern als einen (Sarten, nitht als eine Miffte ge. fohilbert graben. Tan mußs auf Sicifen von cinem

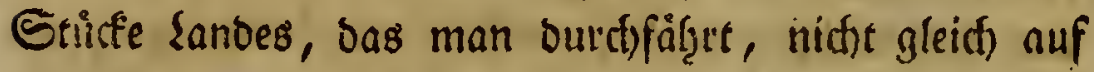

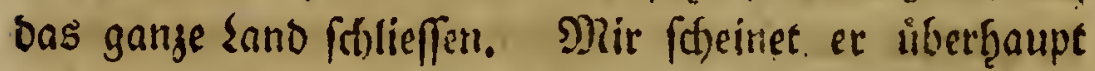
einen Eleinen Brollen wioer Baiein im Sufen zu hegen, fonft bichte id), würben wit aud) bie brollige (Eari. Eatur eines Baier's bet) if̨m nitbt !ejen, bic er irgeno von einem Bierbrauer ober Jiteifd)er módte entleñnet ந̧aben. Es giebt feine Probing in Deutfdtlano, no man nid)t eine MRenge wob̨lgemåftete Sjenfdien fänoe,

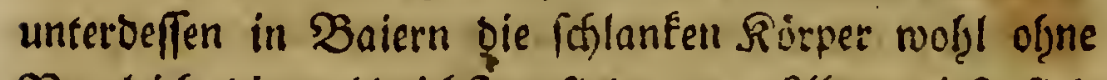
Bergleid) oie zahtreid)fen fint. - Zuer sirß̧ fino nicf)t oie einzigen Unrid)tigféten in siefem $\mathfrak{B} u d\} e$, bie id) Ju riigen ḩâtte.

Untermege traf idf bey Birnbadi, uno mieşer bey) Pfartfircten bie rotfie (Sartenrofe (Rofa gallica Lin.) f̧ăufig wilomact)fend an. In biefem Suftande bleibt fie niebrig, uns breitet Etamm uno alefre allente

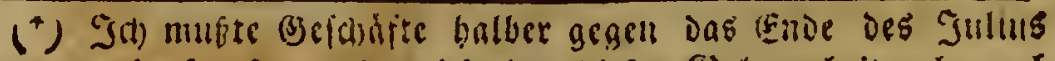

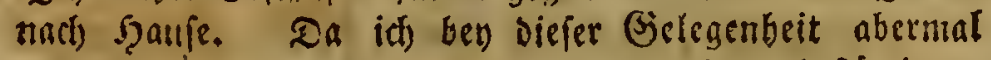

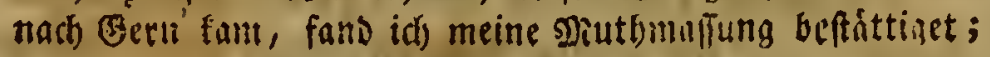
Der Delsaum trug nut fdjon feine Schoten, uno war nichts weiter als cint Pettids, Der nur barinne vont ge: meinen sisinterrettids verfobjeden mar, DaB feime

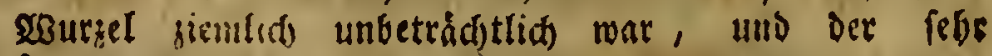
daftige Stengei einc feht grofie 2anjabl Enfoten trug. Der wsohar mar von Şirfen, Den wir auf unjern gelderti

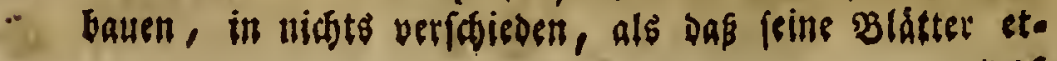




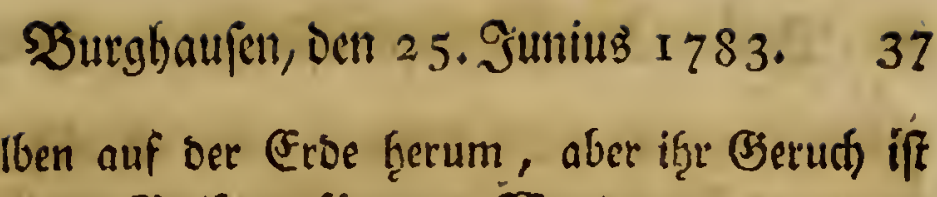
nid)t minoer $\mathfrak{B a l f a m}$ für ben $\mathfrak{W} a n d e r e r$.

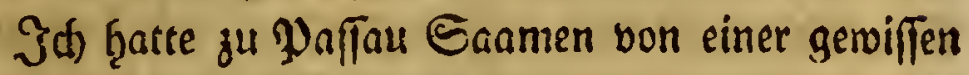
(Jraknt, bie von ben J̧lingain Mothar genannt wirt, befommen; man fagte mir, fie wadhle wie Sdjilf, (el) aber fif 3 , nno werbe als feeu von Den Bsroffen in

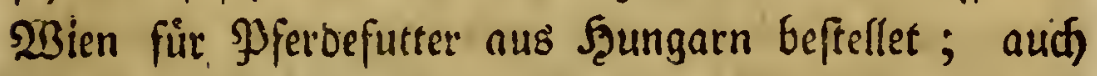
Ganmen einer amcrifanifden Gierfte, uns eines in= bianifhen Delbaumes ergielt ich eben bafelbft. alles biefes f(hictte id) im Fruithiag̨re nebft nod einigen be.

fannten Eaamenarten vou Seerrn P. Chriftian Bauts namm, ben Eie aus feinen ófonomifoen Edjriften vielleitit forgon Eennen, nact) (Bern, wo die vortrefflidje Befigerinn Diefes fdjonnen Siitterguts, sie Frenfrau von Singenlyeim, bie Bjüte ḩatte, bie $\mathfrak{B a r t u n g}$ ber: felben ferbft zu beforgen. Die Pflinghen waren alle bey meine Znfunft (d)on jiemlid) herangewad)fen; und id) fah es sem vermegntliden Delbaume fidon an, Daß er bon bem befannten Delrettig nifft verfdieden feyn bưrfte. ( $\left.{ }^{*}\right)$ Bu eben Der Beit-ftanden meine SPlangen von Saxifraga farmentofa, bavon mir

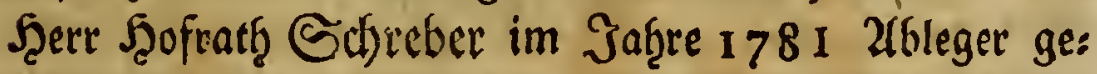

$$
\text { C } 3 \text { Pentet }
$$

was raub, und dic Staubbeutel purpurfarben waren. Die amerifantiche Gerfite ift Holcus Sorghum, Lin. Der 2teldh) ift einblumight, zwey Bálglein, gróffer als Die

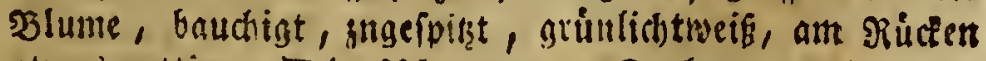
etras zottig. Die blume zwo Eversen, weir, am Ranve fottigt gefranget, ungleid) : Die gró|jere fiviz̨ig, an Den Geiten eingebogen; Die Eletnere fofhe flein, an

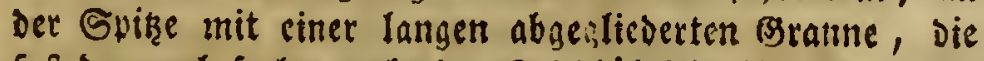
faft brenmal fo lang als bas Seldabáglglein iff; fie if bis an Das Gelent fámujig getlo, Dann mildsweís. Die 


\section{Dritter ßrief. Burghbafen, Den 25. Sun. I 783.}

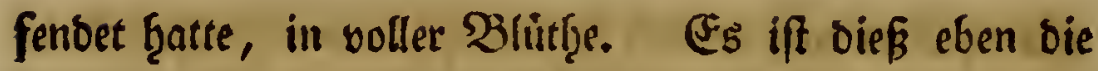
Pfanje, bie bel) Şerrn Bergrath Jacquin Saxifraga ftolonifera Mifcell. auft. vol. 2.p;327.) Ģeist, nno

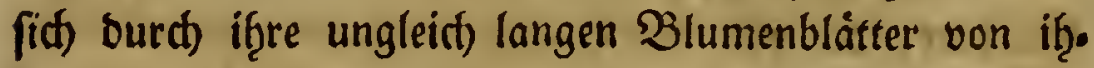
ren Baattungsgenoffen fo betrád)tticf) ausgeidnnet. Der Ritter Murral) zu Bosttingen ḩat fie in ben Nov. Comment. Gottingens. und norb) vor if̧m . গ̧err Jृofrath Sd)reber in ber legten Zlusgabe feiner Ueberregung Der ellififden Sefdutreibung Der Dionaea Mufcipula bef(t)ricben; audi) Der zu frith verforbene

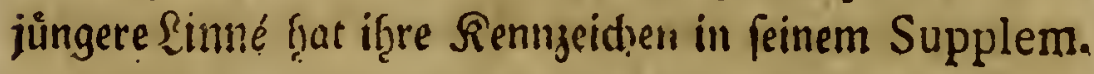
plant, p. 240. vortterfflith auseinanber gefegetet.

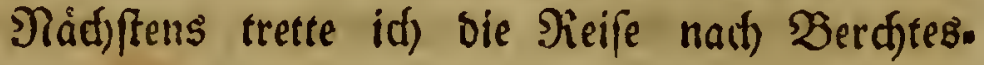
gaben an. Zutú) yon biefer forlen Eic umftánolidie शaffridt) befommen, Ђis daģin u. f. f.

\section{Edrant.}

Tnges

Glaubfisen zrveen, ober oren, fo lang als die groffere Spelge; die Strubbcutel lanzetfórmig, gelb. Stertgel; Der fructinoten enförmia, grunlidst gelb; zmecn Briffel, meis; Die garben feberartig, weif. Die jlattanfäge, unter ben sieldbulglein senóbntion zueen, febr furs, faft linienrodrmig, meiblidst, mit cinem Purpurpunfte. Die

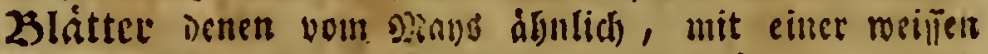

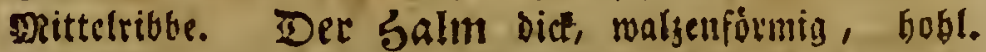
Die Kifpe abrenformig, fulbenábnlid). - Mian bat fie zum ófonomifoton (3etraucise angeratbell; id glaube aber.

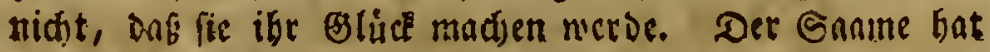
cine dice Soulle, die in ber siuble gebrodsen merden nuibte, uns ba tourbe. Dann cin ziemlicf fleines. forn überbleiten. 21 cine artige Pfange nódite fie fich in Bårten cher empfeblen. - Belegenbeitlid) merle id) nod) an, Dab Der Reta Dicfer Pflanje, renn fie im Soden. fteft, jwesblumigt fer. Gortentópfe balen ocn sotaniferu

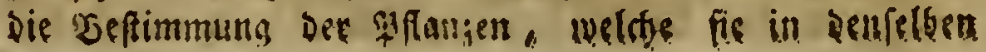
zoben, foban ifter efchmeret. 
Bierter Drief. Sell im Eitlenthall Den 28.24ug. 39

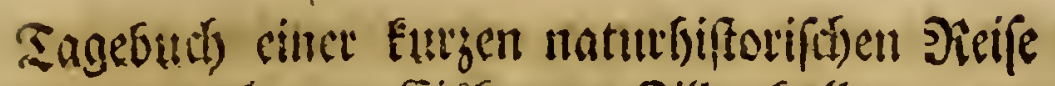
nach Den Eišbergen Billettgalls.

\section{Rierter Brief.}

Bell im 3illertínall ben 28 . 21ugulfts monber, 1783 .

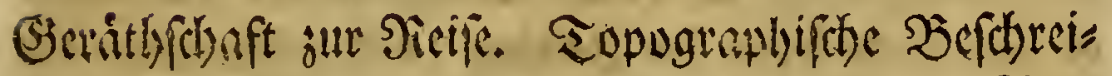
bung Dez Billertlyales. Fernete Sicife. Ders

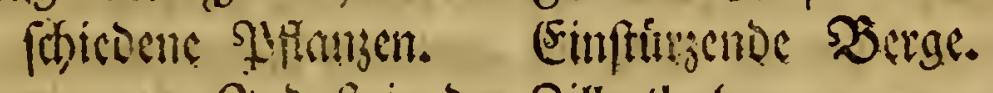
Sindufte Der Billerthaler.

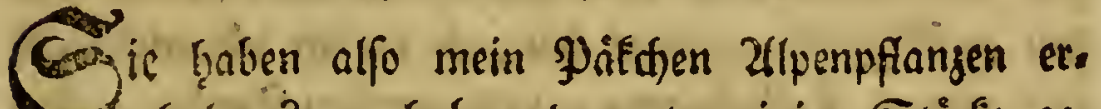
braiten? - Graben barunter einige Strude ge-

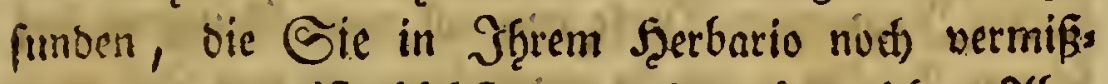
ten? - das ift bood)ft ongenergm für mid): Z(ber

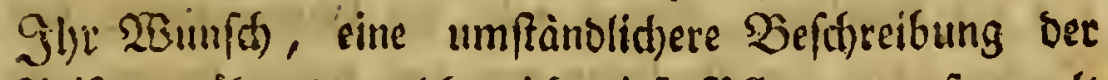
Sicife, woiffreno welder ich) biefe Pflanjen gefammelt

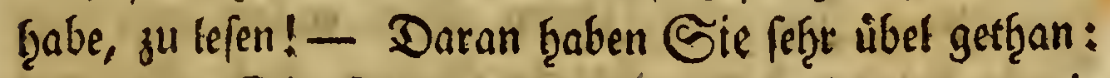
benn wic Eic feţen, fiz' iaj mirflid fiton an mei, nem Edfreibtiffise, um mit einem langen Briefe an

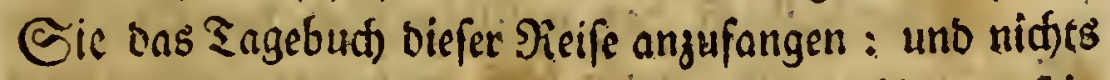
in ber $\mathfrak{B}$ elt foll midh irre mathen, es bis an fein Eno fortjufegen: Macjen Sic Sidj alfo menigftens auf ein halb Duzeno foldser Riefenbriefe gefapit, uno

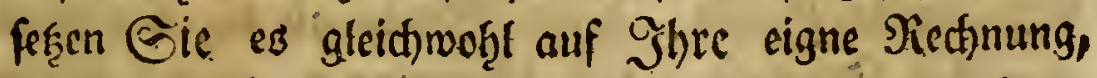
wenn fie nidfs fo ganj in ser afrs finb, wie es einem M)

Det 22 te Jृeumondes war's, als id ungeadtet

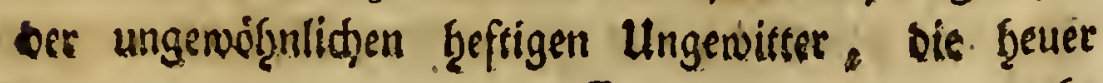

$$
\text { C } 4 \text { meģr }
$$


mef̧r als anbere Jaf̧re in unfern Begenden muitheten,

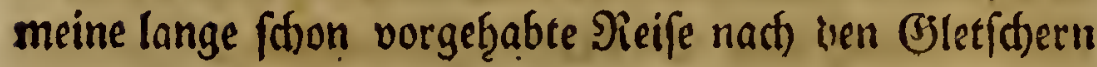
Billertl)alls ontrat. Mein gang Gepridfe beftand in Sieufens Compend. Botanices; in ben erften Sain: Den ber Fabrizius'jthen Spec. Infeet: ber Sorjis fichen Bentrage - uno bes allgemeinen fiffematija)en Berzeidnifes ber (3iewaid)e Deutfdslanis. ferners in einigen Sánadteln für Jnfeften, unb Söfthpapier für

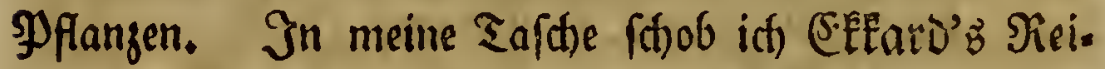
fenden: uno ba man auf áf̨nlidjen SReifen mand)mal fef̧r ubel daran iff, menn man nirgt nud) feinen Mia: gen ju (Semúthe geführet ţat, fo verfạ ich) mid) aud) mit etwas Salbsbraten, uno meiffem Brone. Fin ftarfer Junge vom Dorfe trug all mein Sieifezeug auf feinem Fiùcfen; und fo wanbelten wir von Bell weg, um bie Bletffier, Davon wir im f̧iubergrunbe bes Thales fafon bie Borboten vor uns liegen hatten, zu

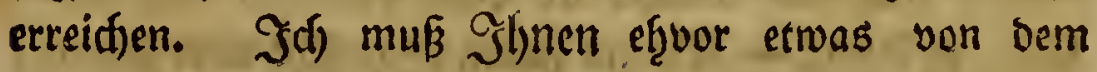
Zhale fagen, beffen füblidje Gránge biefe Eistherge umidulieffen.

Billerthall ift ons weftlinfe Eno bes Ergftiftes Salzburg. Sie foinnen dief in unjers Doilo Giuts rathers Rarte von Galjburg fef̧en. Es gränjt

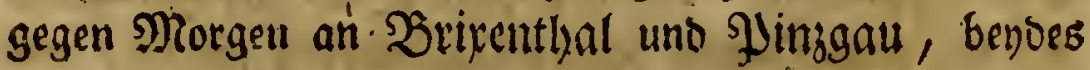
falzburgif(h) (Janen, gegen Mittag an bie tirolifd)en Beriffere Taufers, uno Gterjing : gegen albeno liegen Steinach, stettenberg, uno Freundsberg. (Begen Mitternad) Siattenberg, uno ber Innftrom. . Nur von ber erften Geite ḩängt es mit Dem übrigen Exaftifte zufamm; won ben anoern fiófit es cunoum 
Bell im Billerthall Den 28 . 24ugufmondes I 783.4 I

an Tirol. Das J̧auptţ̧al bef̧âlt nicht in feiner ganzen Sänge oie nemlidye Jiid)tung. Der nòrolid)e Theil bef: felben liegt von Norb gegen $C_{i d}$; eine fleine $\frac{I}{2}$ Etunbe unter Sell fpringt ber, an ber weftlidyen Geite zelegene 3ellberg, mit einer Ecfe oftroints für, uno bas Thal beugt fid) stwaz fỉbreftwairts; ungefáger $\frac{x}{2}$ Etunbe liber-Scll maht ber an ber óftlithen Eeite liegenbe

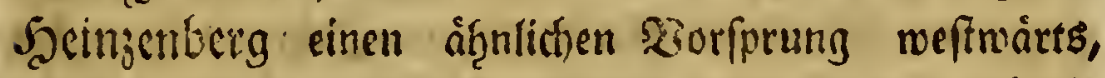
uno sas Shal lenft mieder mef̧i füowärts cin. Mieţ. rere Eestenţ̧aler ftetien in redsen uns fojicfen SWinfeln auf bas Şauptthal von 2 beft gegen Sft ; fo wie fid Der nốrulide Theil Des leşten in einem red)ten ßBinfer

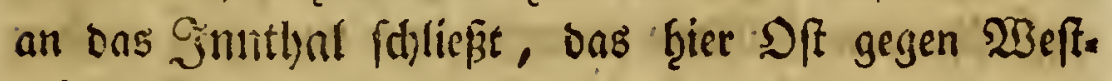
wôrts liegt.

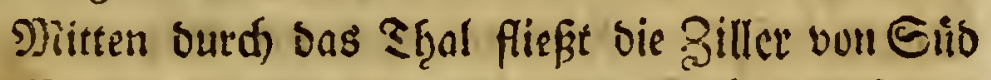
gegen গord: fie if̂t ein mittelmáfigiger, aber reiffenber Etrom, mit bem fiú) bie sisáffer von. I 3 máfigen Eeitenbådjen vereinigen, bie felbft fijon 26 Eleinere

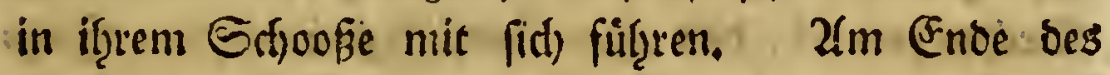

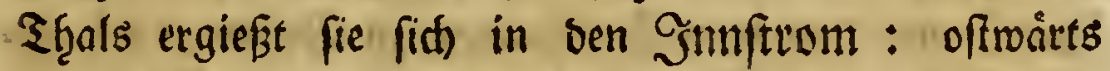
am 2lusfuffe liegt ouf einem fexigel bas Ed)loß Sropfiperg : eine alte $\mathfrak{B u r g}$ mit bidsten Mauern und

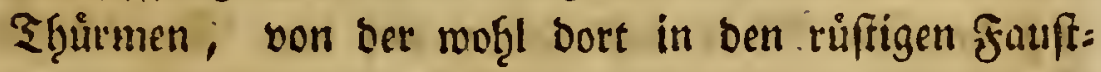
friegsjeiten mancher fdinne Rnappen, und reifigen Sincé)ten feinen blanten Säbel umfonft gefdhroungen bृaten burfte. Num find bie Mauern berfelben gutentheils eingeftùrat; bod) iff nod) cine Edylogftapelle, uns mef̧rere bernog̨ubare Simmer für dic Erzfiftlidsen Beamten, einen fomman.

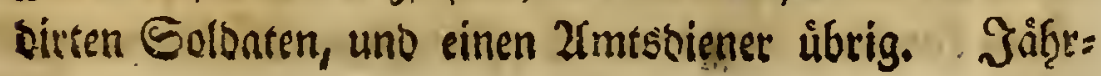

$$
\text { Cs lich }
$$




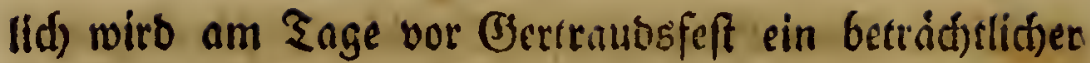

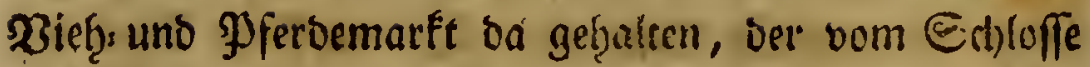

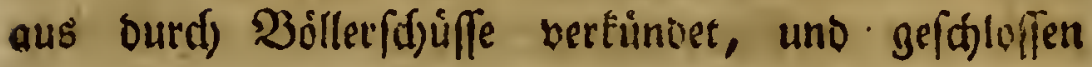
wiro.

Bon Siropfaberg fübrairts gen Bell fino bie betråd)tlidfften Börfer im ₹̧̧ale: Gchlitters, wo peit

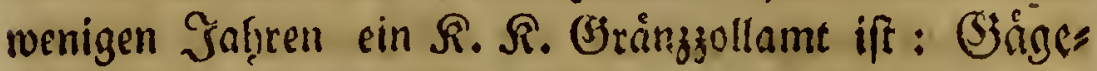

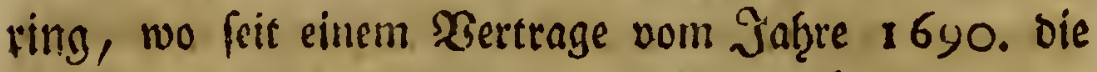

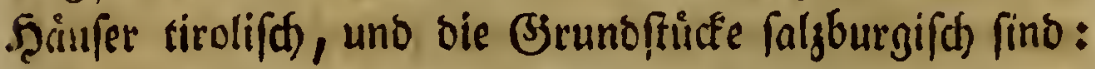
Shigen mit einem Salzburgfhen Pflegamte, einem R. A. Eifenţandel, ber vormels ber Gráf. Sruegeri. fojen Jamilie getriorte, und bazu bie Şammerwerfe ungefähre eine $\frac{x}{4}$ Meile fübmeftroàts von bier liegen; benn einem artigen Sandfraufe, das ift ein Bronf von Tario befigt. Feier ift nud ber Sig eines sand.

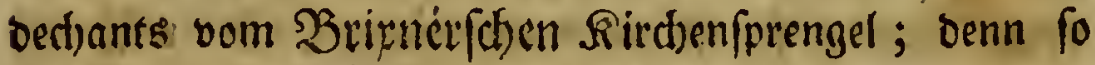
wie bie Biller ons Seauptţ̧al in tas offtlid)e und weft. lidge thieilt, fo ift fie aud) bie (braingf(d)eite ber beiben Rirctenfprengel, bas vom SJürferrbifd) of von Ealzo burg, unb bas vom Sirfthifón of zu Bripen : bie ơft. lidje Geite bes ₹b̧als ftejyt unter tem erfern; die weftlide unter sem zweysen. Bon Jingen ift nod

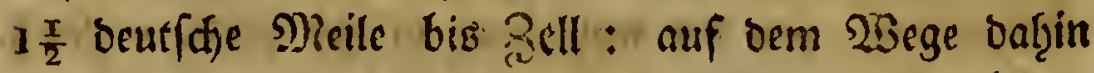
fommen vor : Sapfing : Dic Bripild. Sanmenbergs foren Dorfer, ficfing, llderns, uno Sitco : ferners Saltenbad), if̨m gegeniber jenfcito ber Biller bie (Gråflic) = Siffingidse, vormals Sdjibenbofiche Sherr.

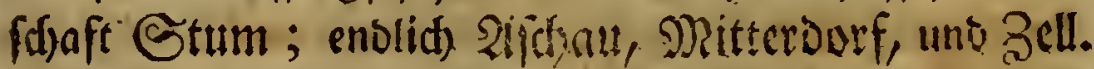

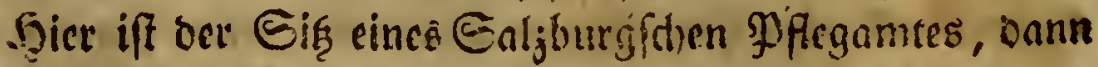

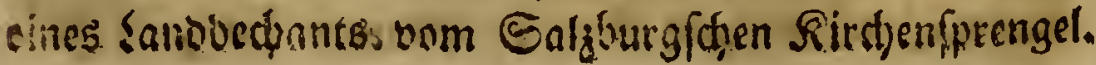


Bell im Billertlall, Den 28.21uguftmondez 1783.43

Die belsen getadjten Borfpringe, bie ver Bellberg uno Saimzenberg madien, bilben um Bell eine $2 \mathfrak{d r t}$ von Seffel : ber erffe verthindert bie 2 (usfic)t in bas nórolide Thal - : Ginter bem zwenten verbirgt fids

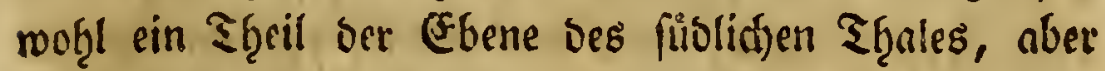
nidht bie ḩoben (3ebirge an Şintergrunbe beffelben, bie mit aller ihreer Majeftät ủber biefe nieorige Erte ţins wegfef̧en. Hebrigens hat Bcll aud) eine artigere Rirche, als mtan f̧ier vermuthen follte, aufjuweifen: ber ver. Diente tirolfche Baumeifter 2 fnure. Scueber, in Sibgicht,

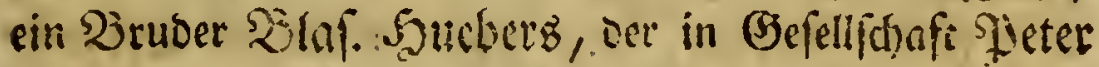

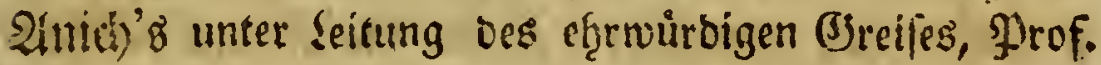

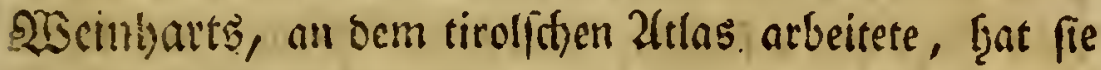
im Jafze 177 I ju bauen angefangen, I 782 wart fie vollendet. Das $3 \mathrm{rmen}=$ ober fogenannte $\mathfrak{S c i l i g e n}$ buth, vormals Hofpitale S. Joannis, ḩat bisferer nur ein biơljernes (B)baiube getrabe, bas aber 1780 abge. branut ift : mir ḩoffen nun balo ein anfeģnlid)eres Ges

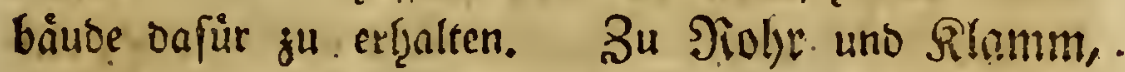
jebes eine fleine $\frac{\pi}{2}$ Etunde im Diten bes Dorfes, fino

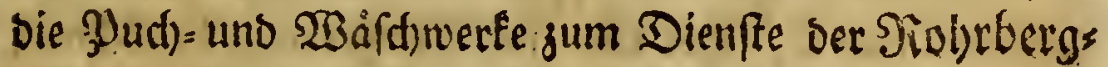

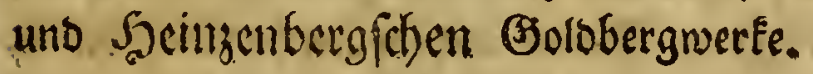

Billertlyall ift cin fojones, fruchtbares కุ̧al. Blumigte $\mathfrak{W i e f e n}$ - frud)tbare 2 (ecfer - und freund. lictse Errenauen theilen fid) in bas Evoreid) Deffelben. Slut im nörolidien Sgeile liejt eine groffe moofigte sisicfe, bie von ben Beffifern Derfelben, den Bewohnern. Des Dorfes lloernis, Das an ifrem nơrolidien Enbe.

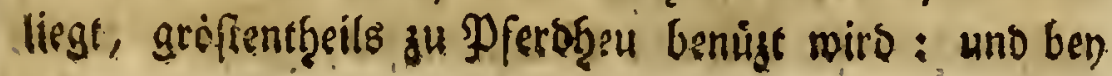


Den Dórfern Dittreroorf, Ctum und Edjitters milften fids fpiegelnoe Seiche in bas (strün ber anlic:

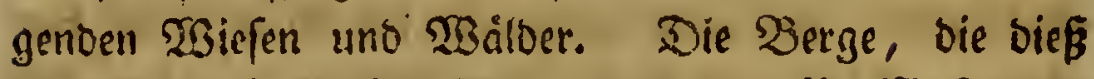

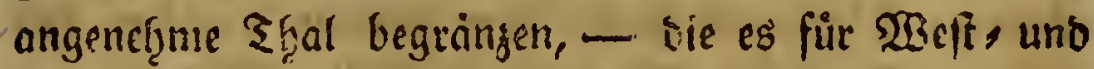
Oftruinten idvişen, aber ben falten, regenbringenben Siorbminben ofnen, - bie ven wob̧lthäätigen Etrablen Der Sonne burd) wiebericholtes Burvictprallen, uno Beus gen bie wirkfamere $23 a$ àme gében, - fino im nơro.

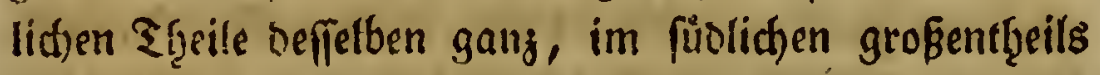
grin, uno frudysbar : nidf) fthaubernbez Fellengemainbe, nid)t haartes (Beftein, bas jebam nieorigen Etrauche

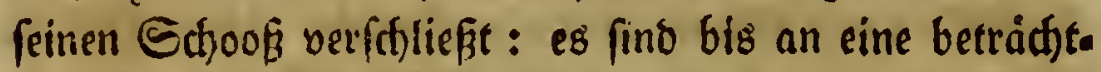

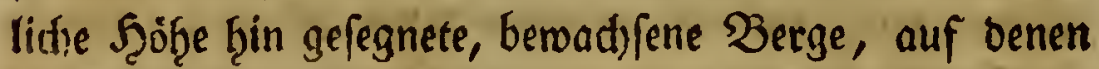

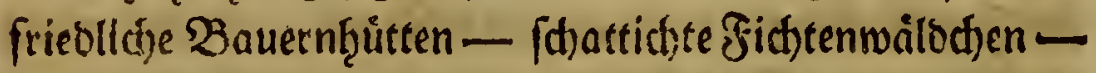

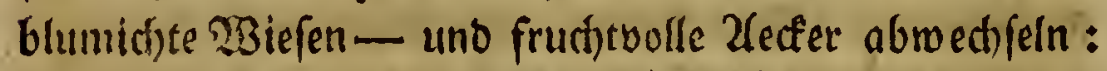
on fie formiegen fich lachenbe $\mathfrak{B b e n b e n , ~ u n o ~ B o r a l p e n ~ : ~}$

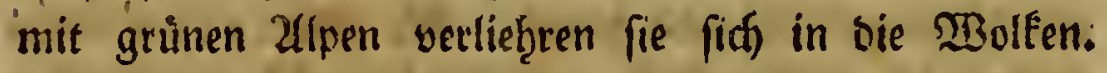
Meţr nod b̧äten fie Reis für ben empfinsfamen Jreund ber Siatur, - mürben nid)t oofters milbe, reif=

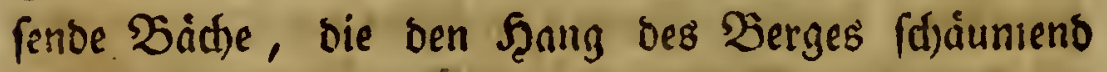

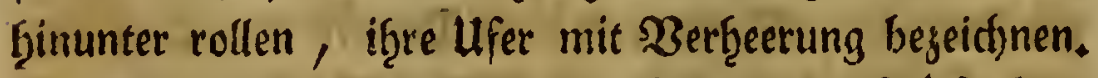

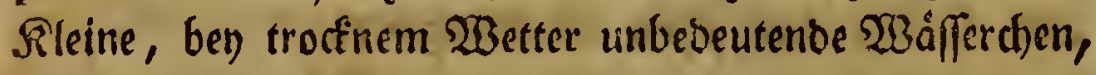
bie izt faum if̧re Diinnfaule füllen - ein slagregen uns man fennt fie nid)t mcḩr - fie fino fürtfterlidje,

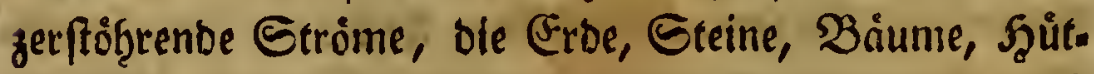
ten, $\mathfrak{B}$ tef̧ uno Menfd)en mit fid) f(t)leppeli, uno groffe grünenbe $\mathfrak{B i e f e n}$ in menigen 2 ugenblicten tief unter Roth uno Eteingeldiebe begraben. Dafür finb bie Berge, die bas fiblidje Ţal gegen Tirol falleêen,

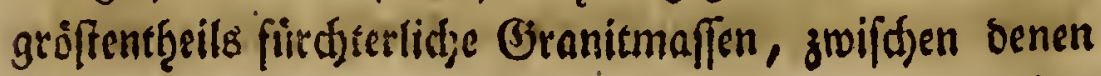




\section{Bell im Billertlyall, Den 28 . 2hugufimondę̉ I 783.45}

froftige Eistf̨aler innen liegen - uns Eisgebirge, bie mit if̧ren weiffen, bepubertem Siủcten die $\mathfrak{B o l f e n}$ Durd)oringen.

Sic ḩaben fyier, mein sieber! eine Sifiz̧e bes

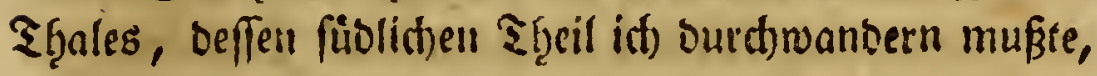
um die (J)letfcher, bie if̧n begrinzen, zu erreichen.

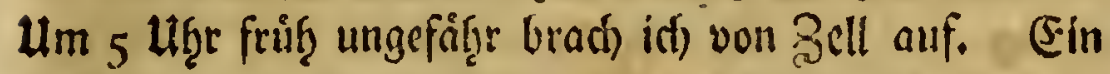
beitcter, angeneţmer Niorgen wars: und bie liebe Eonte hatte fthon bie im Eliboft liegende Jelswant, Gierlosfiteit, mit einer rofenförbigten Binbe gegurtet. Der fuffreig läuft zwifa)en Hectern, sibiefen, uno (d)attid)en (Erbenauen finsweftwirts, Durd) bie Mitte

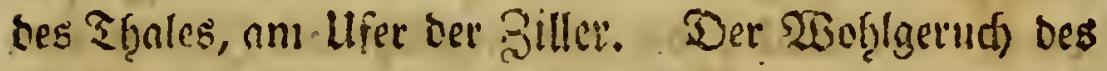
Byffus-Jolithus L., Der an Beffrabe berfelben auf Den (Stranitgefdieben wadd)st, if waf̧re $\mathfrak{B}$ olluft für Den SBantherer. Dief ift b̧aufig auf sen Etcinen, bie an beiben Ufern ber

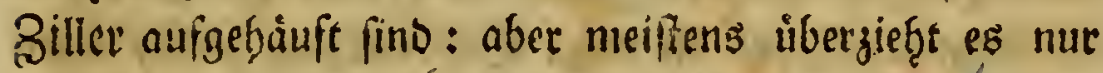
bie (Branitgefdiebe, bie aud) ben gruffen Theib biefer Eteinţaufen cultgmađten. Biele Gtunben, bie Gie im Thyale an biefan Befraden Ģinmandern, begleitet Eic ber fiffelelBeild)enouft bavon. Eeine Jarke iff

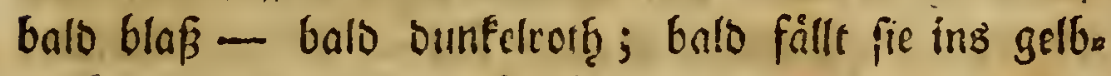
lidjte: je bunfler bie Sarbe, befto vortrefidjer der Beru氏5.

Siectits jenfeits ber Biller lag mir ber (S)uggel=

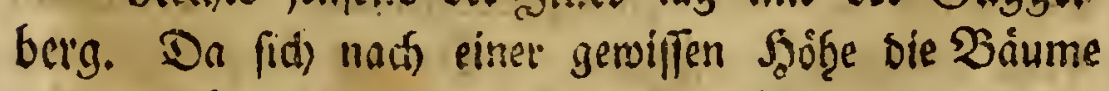

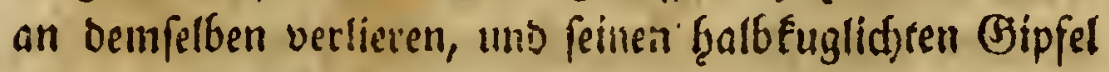

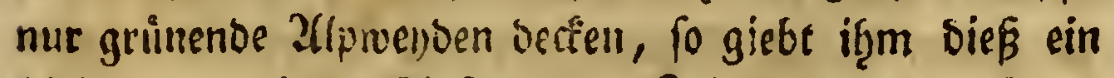

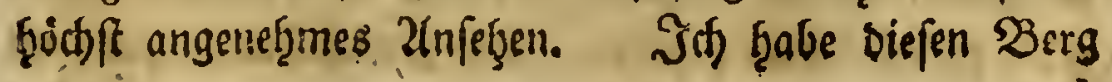
noti 
neck nie beftiegen : woĥl aber pflúfte mein Bruber in ben erften - Jrưfilingstagen an ber Szofhe teffelben Azalea procumbens L. geftrecter Felfenftraud).

Primula farinofa L. Miebligte Exluffitblume.

Gentiana acaulis L. Étammloje Engiane.

Soldanella alpina L. 2llpenoraftel6lume.

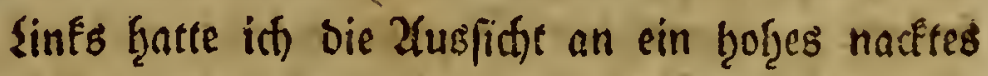

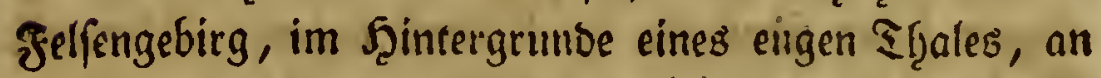
oas fich von der গlotsfeite der Gierfosterg, won ber

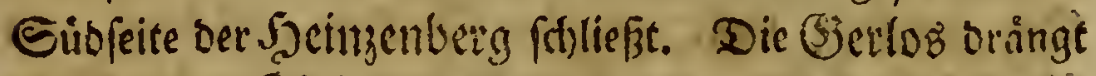
fid) an ben finffen biefer Berge ourd) tie Enge binis burd) : ein $\mathfrak{B a d}$, ber vor ungefáber 20 Jaf̧ren bem Dorfe Bell ben voolligen Linfergang brob̨te, uno bie (Segeno runb um fith einige Sus tief mit Enno uno Eleinen bebectete. Sie bereinigt fid eine halbe Etunte untenferer Sell mit ber Biller. 2(m Fußze bes gebarfien Jellengebirges, bas bie bobe Schwarzach freife, lirgen

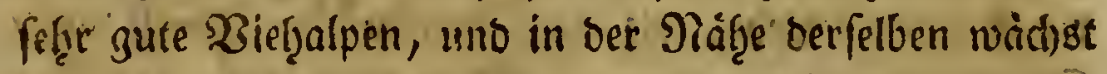
am Suffferige fef̧e viele Niondoraute (Osmunda lunariaL.). 2(m (jorlosberge ḩab idf), ben einer anbern

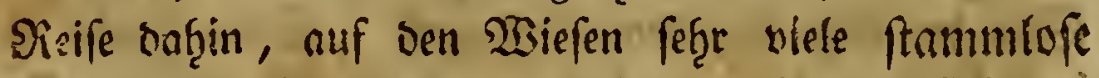
Cberwurt (Carlina acaulis L.) - in ven wálberh haufing sie Ofmunda fpicat. L. unb cine benumiderns: muirbige Menge von Scidelbeceftraud) (Vaccinium myrtillus L.) uns Jjecufelbcerftrouth) (Vaccinium vitis idaea $L_{\text {. }}$ ) gefunben. $D a$ id) mef̧r als ène

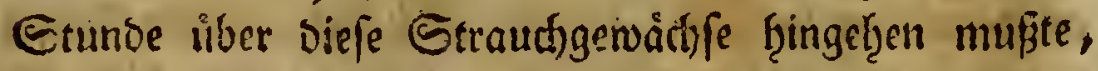
fo waren meine Edjufze von ben Beeren ses erffen

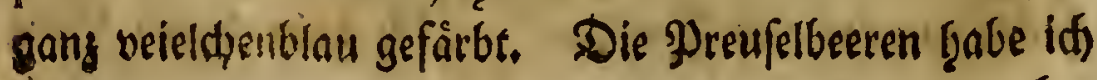




\section{Fill im Billerthall,oen 28.21 guftunonoes I 783.47}

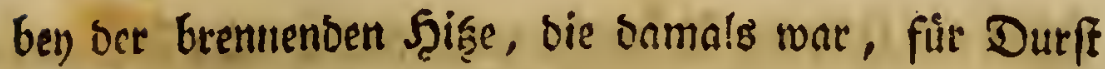

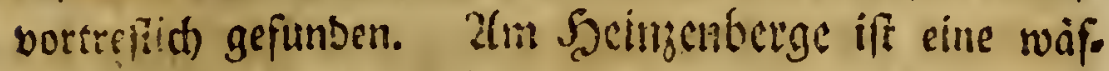

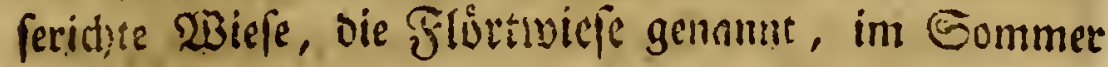
ganz mit Carlina acaulis $L$, und Trollius euro-

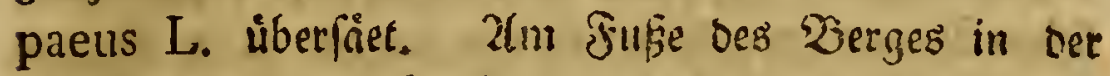

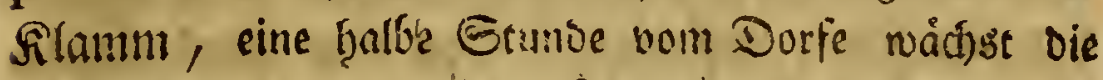
Alproje (Rhododendron ferrugineum L.), von ber unter ben Icon. plant. medicin. Centur.II. tab.200. eine fefre gute. Zlbbiloung ift, fêtr Ģăufig. Şerr 3oller, ber ijt ?ffabemifer an ber $\Re$. $\Omega$. Zeidjnungs , uno Rupforfted)er 2ffabemie in SWBienn ifr, hat von biefent grofpefte eine 3eidnung entworfen, sie Sie, mein lieber freunb, wenn Sie meine sibinifise eben to gerne erfüllten, nls id bie J̧̧rigen, - bolb in Scll felof betradten, uno mit ber 9iacur vergleid)en wirben.

Shit warmem (B)efüb̨le fưr bie mannicffáltigen Edjoinfieiten ber Tatur burnwwanterte idi) bie Juffteige, Die das Beftade ber 3illev verfolgen. Joblone 5930 gen bon Roggen - Niseizen - uno Bierfté frrơmten an meiner linfen Eeite für mid) vorủber: balb lad)e mir ein mit ber lieblicsen $\mathfrak{B l u t t h e ~ b e s ~} \mathfrak{B u c t h w e i z e n s}$ (Polygonum Fagopyrum L.) bentalter $\mathcal{H e c f e r}$ entgegen : balo fufflite mich ber Echatten freunblic)er (Eroen:izt ward min (Gefutbl bur(t) eine Safaare munteren

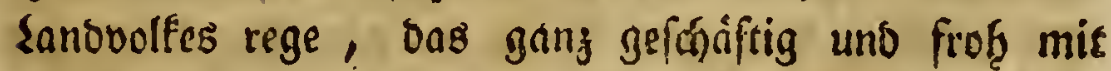
ber Sid)el ben bantbaren 23oven feiner teidfen Berfien. frucht entbuirbete. Fiant einer fleinen Stunbe roar id) sem Dorfe Şippach gegenúber, bas jenfeits ber

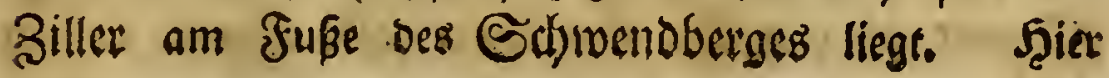


frehenen am Ufer bie Felberftauben, (Salix alba L.), auf

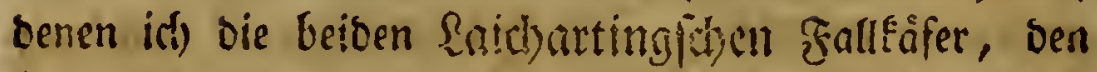
hicuvglywliforen (Cryptoc. hieroglyphicus), uno Den trautigen (Cryptoc. triftis) in eivier nufferorbents lichen Sslenge antraf; to fie' mir fonft in biefer Begeno nur Fête felten vorfamen. Bon einer Senbadtung; Die id baben gemadyt f̧abe - zufolge ber beite Talléffer nur eine Snjeft fint, - werbe id) in ber brittent Siez

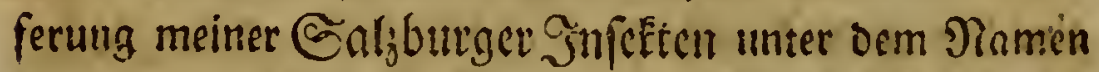
Chryfomela Laichartingii meţr fagen. Eine fleine ḩalbe Erunve von Gier fanz id) an einen fdomalen Eteg, Der mid uiber bie Billier nath) bem fogenannten Gfftan

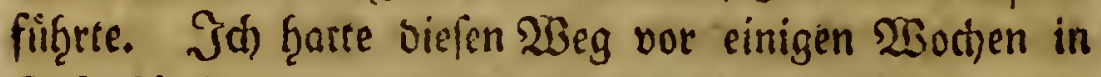

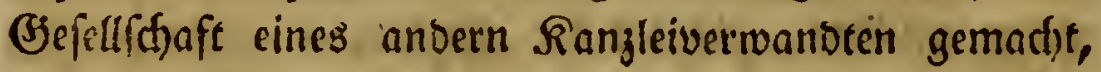

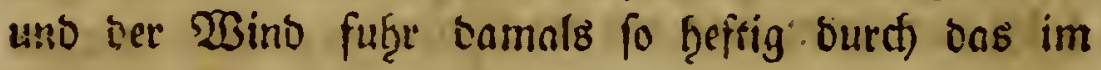

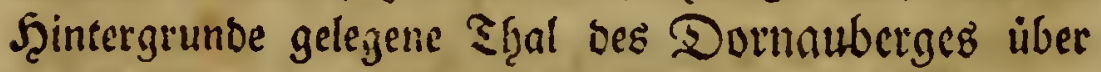

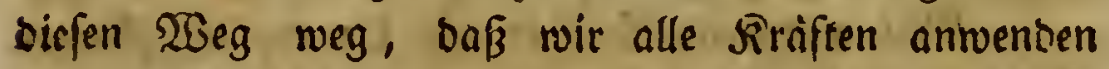
mufften, um nidjt in ben Strom f̧inabgeffoleubert zu merben.

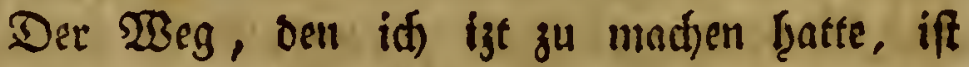

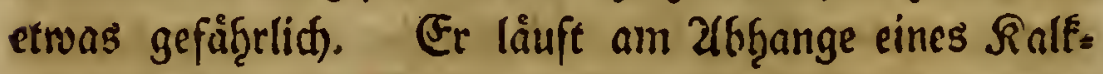

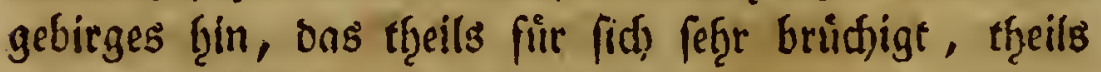

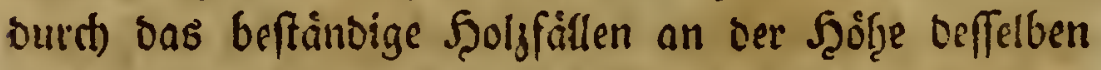

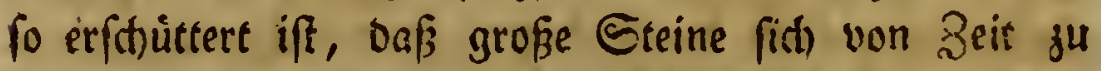

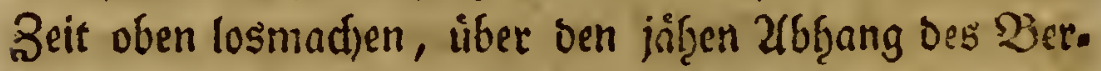
ges hetab, und zum Ţ̧eile in ons Etronkeft ber

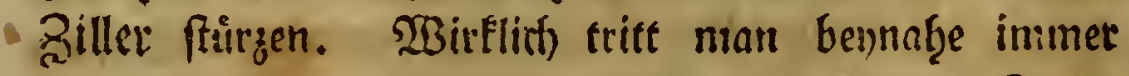

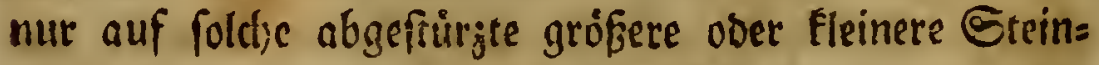
trümmer ; uns baf̧er vermutģfid ber Tiame biefer

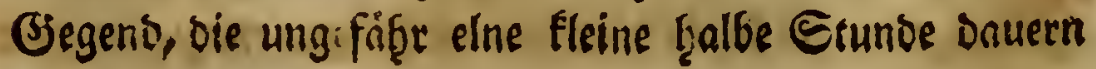


Bill im Billerthall,ben 2 8.21uguftmondes I 78 3.49

mag, (Siftan (Gefteine). Man Fann biefen $\mathfrak{B e g}$ bermeiden, wenn man ourd) bas Dorf Mairbofen, meld)es int Dften Der Biller liegt, nact) Şethftegen geb̧en will; aber ber $\mathfrak{W e g}$ ùber bas (Sjtan ift birt

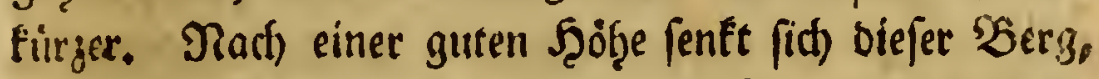
noromeftmarts in ein formales aber angeneffimes,

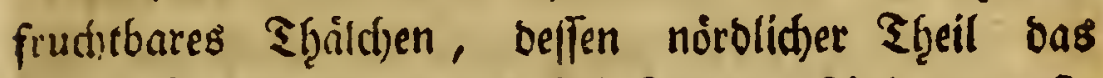
Eattlek, ber füblide bas affete freigrt. Zui bas erfte

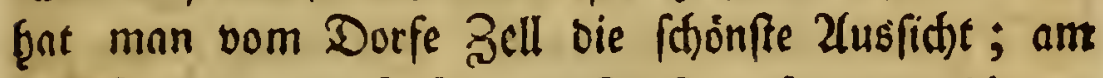
2:fteke frabe id Şafer und Berfte gefunden, bie an Wud)s, uno Nenge Der Rorner jete anbere in J̧augthale weit ubertrafen. Zus biefer Begend erbielt ich), einen neuen Fugenfáfer (Ciftela): er ift bey weitem oer groffte unter ben befannten, uno übers

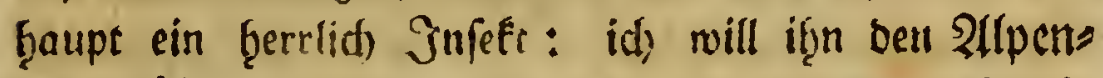
fugenkiffer: Ciftela alpina, nigra, nitens, elytris fufco-caftaneis, rugofutis, futura nigra, nenner.

afm (G)frane ónete fid meinem \&uge wieber ein betråd)tlid): Geitenthal, im Dften bes Şauptḩales, eine fleine Strecfe ủber sem Dorfe Mairlyofen: es

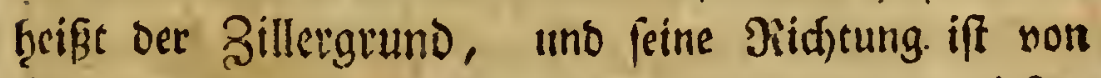
Dif gegen THelt. Die Biller entfpringt in biefem

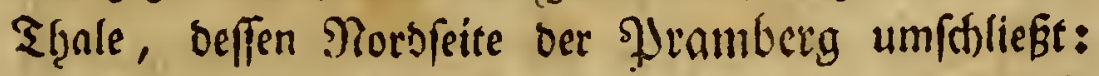

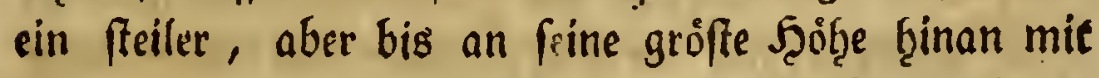
ミanuernfiutten, Eantfeloern, und Wisiefen sejietfer

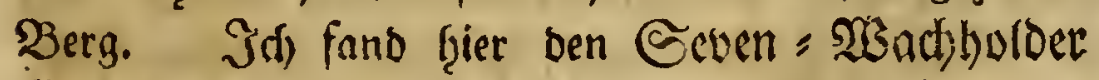
(Iuniperus Sabina L.) in grofer Nenge, ba er entgegen in bem librigen Thale g̨ơhff felten iff. Jath mag ifgn nidft (Gevenbaum nennen : benn er erreid)t,

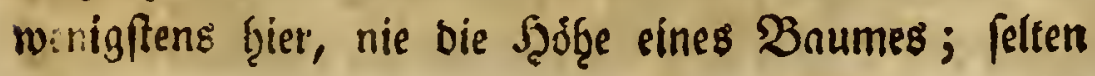

Eiaturbift. Sriefe I. Zant. 


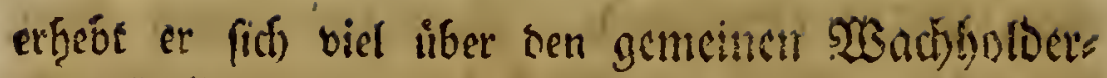
ftrauth (Juniperus communis L.). Ith ḩabe über

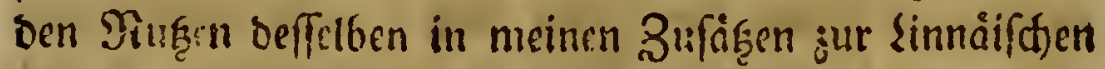
Noxa infectorum efrons gefagt. Sjert von Faber,

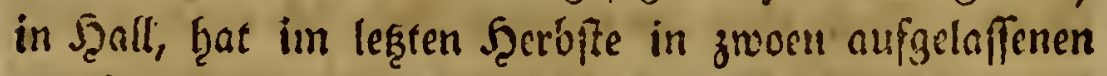
Ornben auf Blen zu baun angefangen: Die eine ift am Sochfteime, bie andere nod) ung(sid) bioferer, nuf Der Slich): aber er bat forjon in biefem Sommer feinen Bau wieder aufgegeben. Die SBerge, die fid an ber Mitagfeite bes Thyales erigeben, fint theils mit Walbunigen, theils mit grunnenben alfiwiten bebecft,

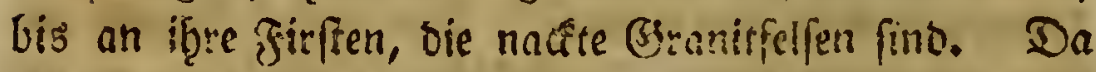

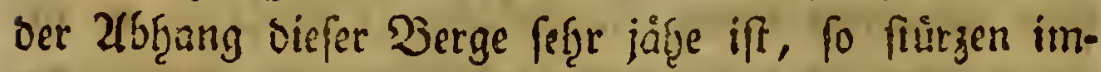

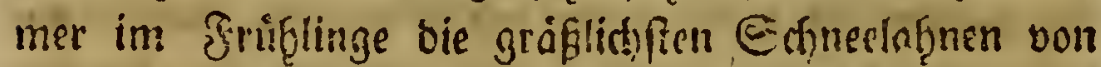
semfelben ins Tfal berab. Bor wenigen Jaf̧ten ḩat tine Salne bie Bauernbitte zu firitus, nid)t neit

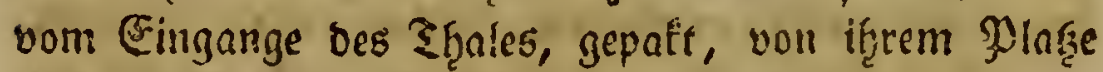
vegzeriffen, uno fo über uno unter gefestren, onf fie

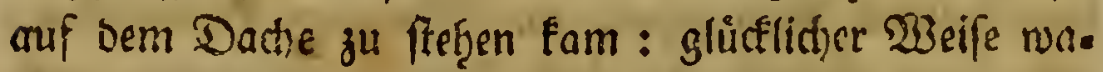
ren bie Bewnthner berfelben gernde zur Sirthe, bis an bie alte Mutter, oie im Şaufe zuritfgeblicben war,

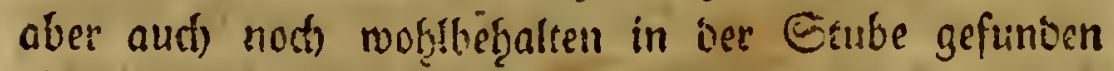

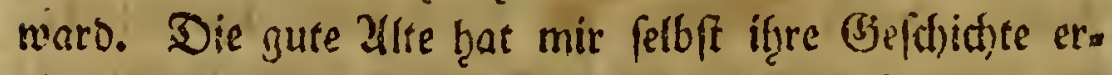

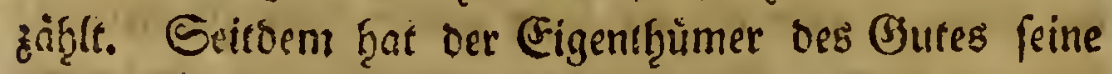
Şutte mit mifre Borficht an eine Etelle verforgt, wo

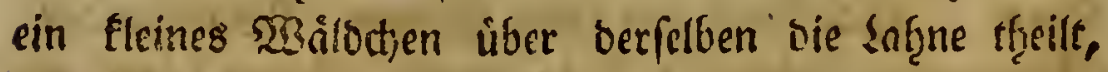

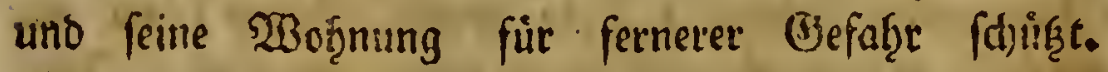

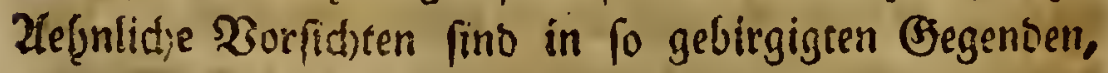
wie bie unfrigen, ḩơdft notf̧wendig. 'Im Şinter-

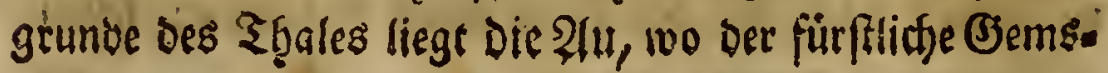


Bell im Billerthall, Den 28. Afuguftmondeb I 783.5 r

jäger woß̨nt. Şier ôfnen fid wieber zwen Seitenţ̧ăler :

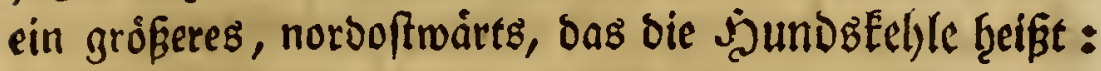
füownits bie Goldder, bie wiener in jmen fleinere

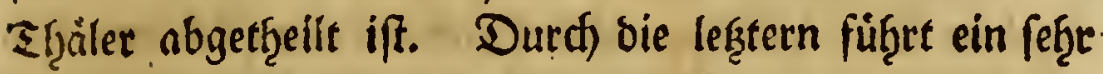

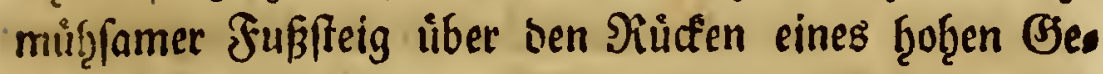

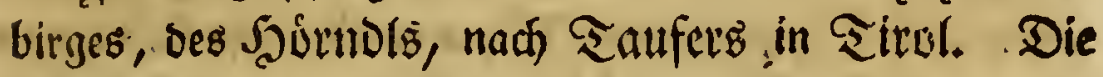
Berf̧eerungen, bie bas unnuff̧orrlide Zloffürzen ber gröften Steintrümmer von ben ḩohূen Şirften ber um. liegenden Berge, in ben am Fuße derfelben liegenden 2(ipen verurfad)t, fins wirflid) traurig. Nur in bies fem Salize murben 4.2 Sagmeiten, oder nach einer

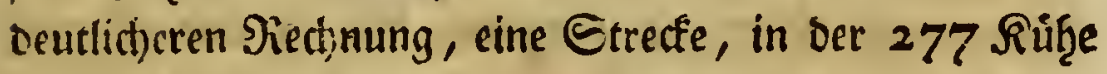

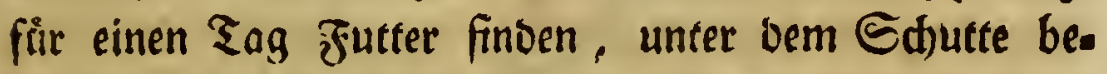

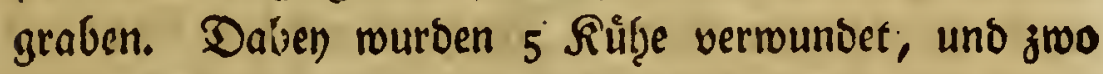

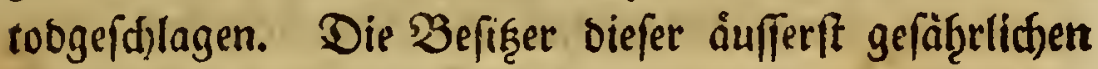
alpen fint burdaus siroler, aus bem jenfeits bes J̧ưrnd:B gelegenen 2riern. Sie mûffen bafür eine

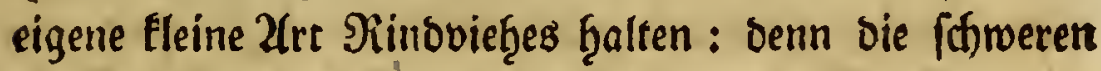

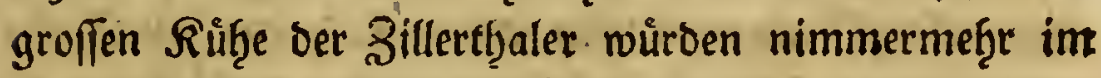
Etanbe fenn, auf biefen Felstrummern ḩerumzuellim. mern, uno das bisctien (Gras, oas noch) Ģie uno ba

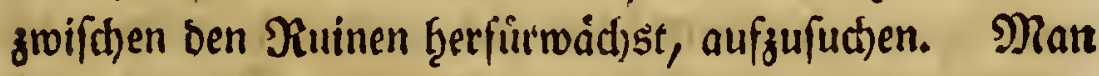
fann fidh) feine fonberbarere Ecene benfen, als bie, meld)e bie auf ben Firften biefer wilben Berge ftef̧envent Brud)ftutufe von Jelfen bilden. Bange $\mathfrak{B u r g e n}$ mit Tghürmen glaubt man ba zu fef̧en, Die jeben 2lugen= blicf auf bie fthon untenliegenten Eteinţaufen natg.

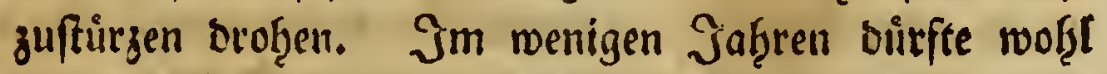

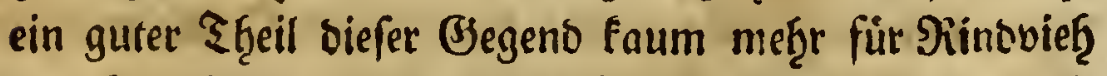
gangbar reyn. Sergeben Sie, mein lieber, biefe 
52 Bierter Brief. Zell im Billerthall, Den 28. 21ng.

etmas lange Digreffion, und laffen Sie mid) wieber zu meinem Fupfteige om Gjftan zurñdfeffren.

Şier fant id nict)ts um mid) als (Ellern - ger meinen $\mathfrak{B a c t i f j o l}$ er - uno Gafwargborn (Prunus fpinofa L.). Eparfam entrecte id ein Pflángchen von einer 2lrt Silpenquend (Thymus Acinos L.), uns eine fpanifobe fritge (Meloe veficatorius L.), bie

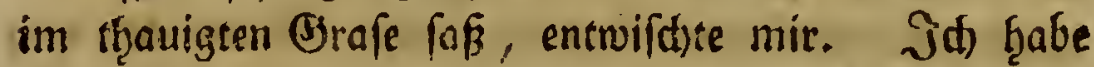
Diefen $\mathfrak{Z}$ eg feit einem Jaftee ofter gentad)t, aber nie war id in ber geringften (befalgr : auth biefimal fam id) ganz mob̧lbeţalten an ber Siob̨lftâtte, uno bem Bauerngute Irrcuzlau voriber, bis an eine fd)male, aber bequeme ḩilzerne Bruice, bie mid) über bie Billcr

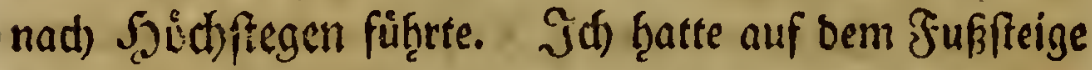
bief̧er manct)mal Bjelegenfieit bie Jnouffrie bes Biller. ţalers zu berounbern. Jeber fleine filecten Eroe, ber nur irgeno einer Rultur făḩig war, war entwebers mit Flact) (Linum ufitatifimum L.) ober mit Sartofeln (Solanum tuberofum L) ober mit $\mathfrak{b o l}$ )= nen (Vicia faba L.) bebaut: alles bief̧ ftand in bem mef̧ligten Booen am Geftave ber Siller, ber von if̧ren 2(ustrettungen ba entfteḩt : wenn aud) zmifchen Dem Fuffteige uno bem Etrome nue

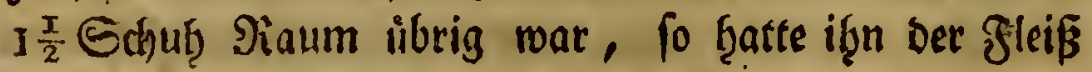
bes Sanomanns ju benifęen gemußt.

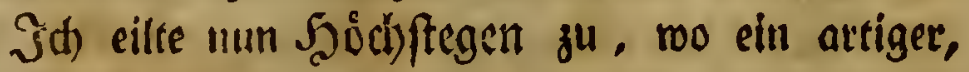
rediff)afner snnemann, Snfob SIroffer, ber ouf ciefer

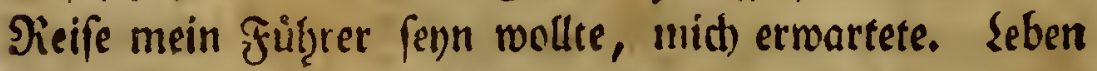
Gie mir wohtl, red)t moţl : im nåchffen Briefe will id Gie in bie alpe Gduwemm füferen. 
Simfter Brief. Bell im Billerthall, Den 7. Sactb/tm. 53

$$
\begin{aligned}
& \text { Funfter פrricf. } \\
& \text { Ball im Gilletthall, ben } \\
& \text { 7. STerbfrm. } 1783 \text {. }
\end{aligned}
$$

Fortfesung Der Sieife. Wech zum Eauen. Tabats Eauen Der Billertlyaler. Zerichicoene Zoralwen.

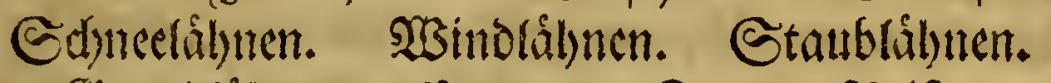
Girundiklynen. Nurren. Fernere Sicife. Rafertar. Breitliner. Eillwencht.

Sun resten Briefe habe id meine Pieifege(d)idte $N$ in bem 2ungenbliffe abgebroden, ia id zu Şidb)fregen anfam. Wir wollen fier nidst lange weiben, mein Freund; benn mein Frihtrer hat fọon

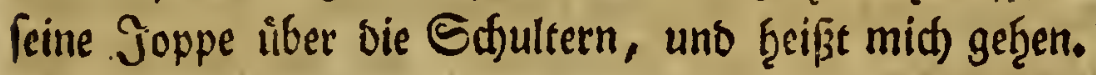

Tur nod) ein Felb $\mathfrak{B}$ eges Ebene war uns tibrig: un b benn giengs rund um an bie $\mathfrak{B e r g e . ~ W B i r ~ h ̧ a t t e n ~}$ Ģier zwel) క̧̧ailer vor uns liegen, broon fich bas eine weftwairts, ons andere fübroarts jiefit. Das erfte füf̨rt ourd) bas Dörffien Finfenberg nad) bem Sure,

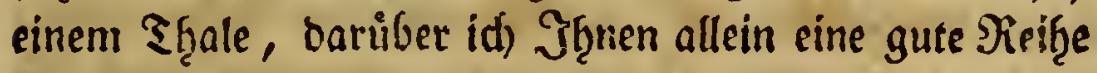
Briefe fobreiben fönute, fo gar fonterbare, gang von Den in angrånzenden Billertḩale, verfđjiebene Sitten,

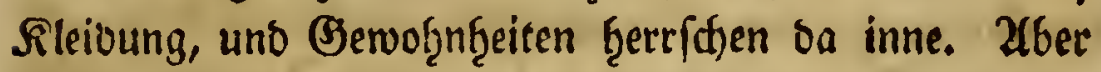
wenn wirben wir in unfere Eisberge lommen?

WBir beftiegen sie 2fnţōbe, bie in bas füblid)e

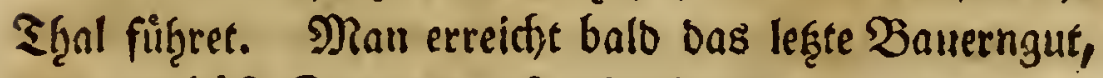
ons bie bofe Dornau, fo wie biefe Begend liberigaupt ber Dornatberg f̧eißr. Nun warb es allmåb̨lig en:

$$
\text { D } 3 \text { ger: }
$$


ger: nidhts meţr als eine fhmale, tiefe Epalte zwiffen zroeen f̧of̧en Sergen, Die Faum nod) einem fid) burdbrångenden Sact)e Siaum genug übrig laf: fen: nad) bem 2 (btgange bes Serges, ber fid) an bie

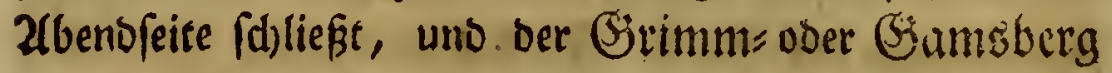

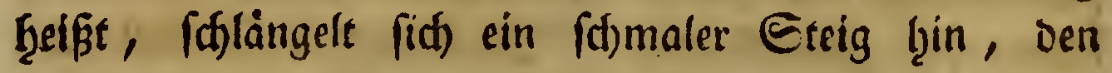

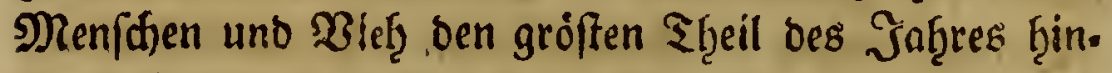
burd). beroandern, um burd) biefe Enge in ein etmas

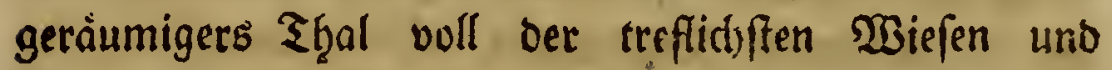
Bief̧wenden zu fommen. Der Sriftenpipie, ein ḩoḩes Jiebirg, bas fich in einen abgeftuzten Rigel enoigt, uno auf allen Eeiten feb̧r fteil abgeld)nitten ift, be. gränzt die Offreite biefer Epalte, uno raubt if̧r bie

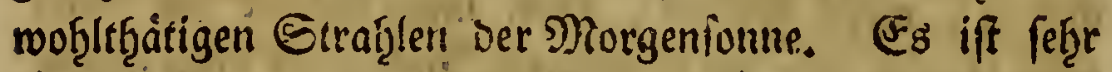
überrnjheno für ben $2 B$ anberer, ber ein Freuno bet lieben Natur. in jeber iggrer Bjeftalten ift - fie mag

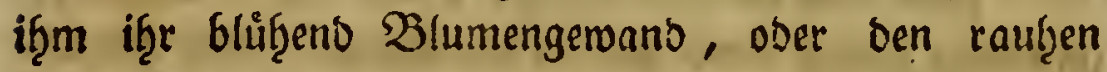
Felfenridfen zumenden - mandjmal mefrere Edjritte wie unfer einem Felfenoadje wegzumanoeln : Denn groffe fürtsterlidfe Steinmaffen ragen oft über bie J̧álfte bes $\mathfrak{B}$ eges, unt ebent fo oft ưber deffen ganze,

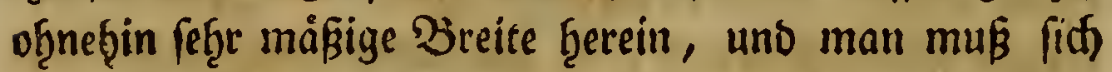

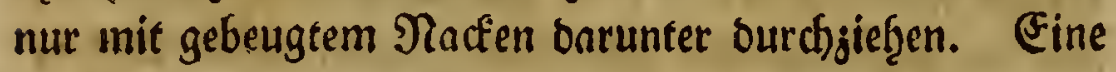
ziemlich geráumige J̧öle zrwifthen groffen Selfen, bie bidht am $\mathfrak{W}$ ege ftano, zog izt meine Zlufmerffamfeit an fid, und id war liffern genug bas Innere berfelben su befţauen. Mebrere ins Rreus gelegte Bjipfeln bon Gichsen, über bie ein Best aus weid)em Mioofe

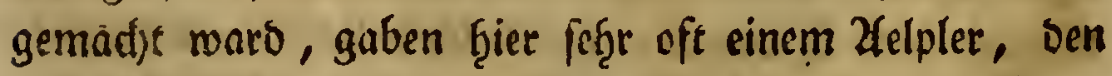
fifrectlid)e Ungemitter ober bie $\mathfrak{N a d )}$ auf bem $\mathfrak{2 B e g e}$ ùber: 
Bcll im Billerthall, oen 7. Scrbffm. I 783. 55

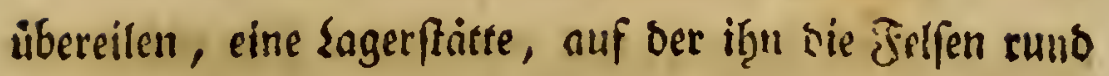

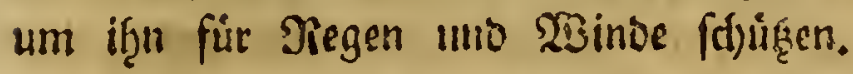

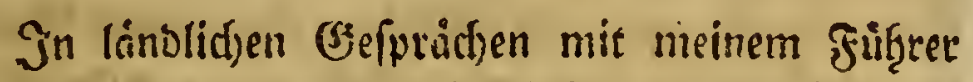

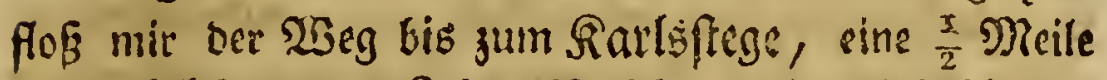

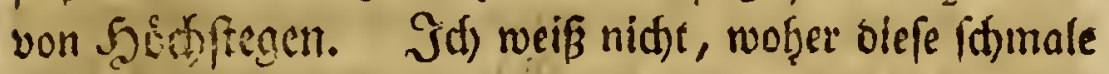

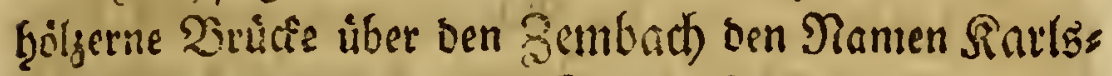

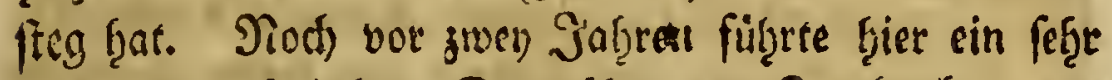
nievriger, folledter Eieg úber orn Zembach): Das

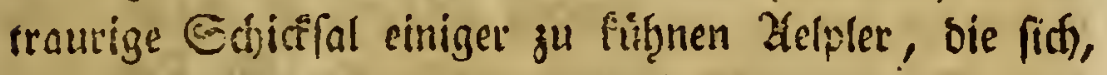

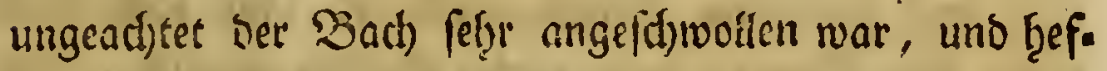

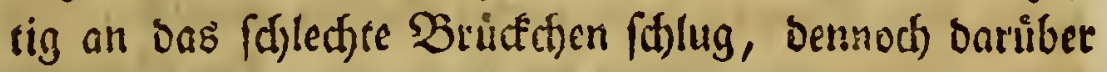
wagten -, aber aud) unglüeflid) genug waren, if̧re

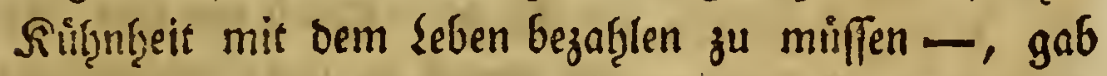

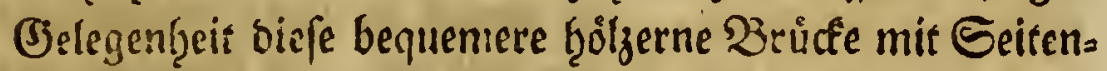
wisnoen anz̧utegen. Gie verbinder den Giamsberg

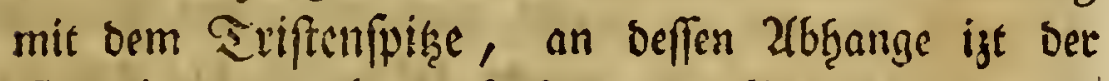

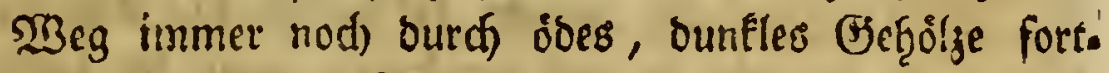

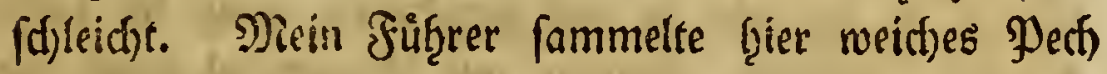

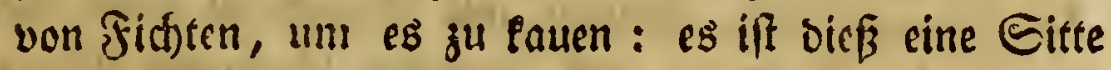

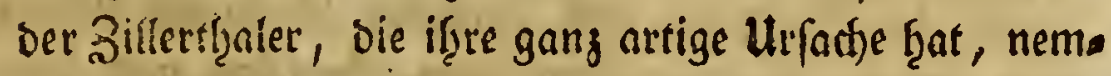
lid) oie blenbense 2 Beiffe bor Zånhne, bie man baburds

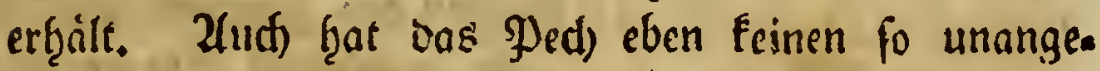

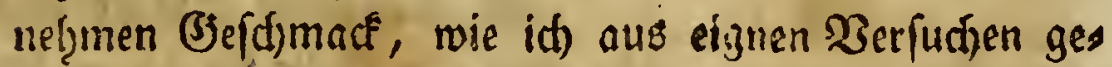

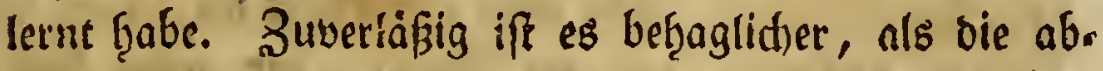

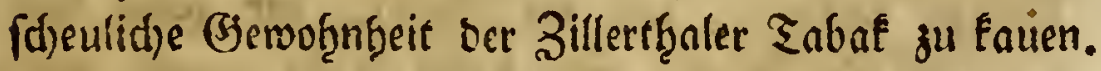

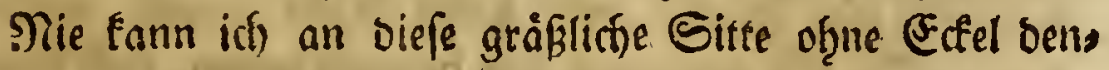

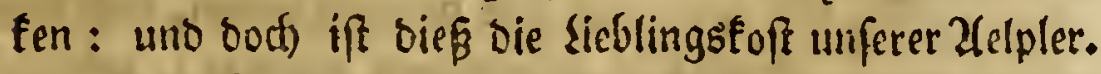

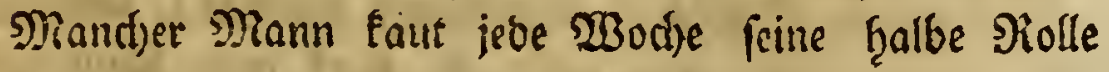
₹abaf : uno mef̧reere $\mathcal{B}$ auernfnedffe verfplittern samit

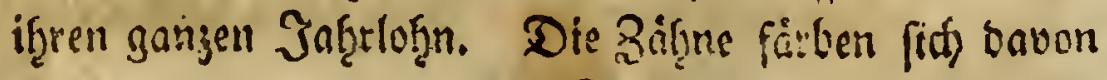

$$
D_{4}
$$


rechet ecfelfaft braun, uno man fann fiif) nictits ab. fdheulidhers benfen, als ben Epeirbel biefer Seute, uno if̧ren (S)erud) aus bem Runbe. 2lugfpurg fdricff un. ferm Sanomanne biefe artige Roft : $u-$., und Billerthall allein nimmt bem Raufmann Delafont, und einem genvifen Rind, ber im Bürgeripitale Daferbft lebt,

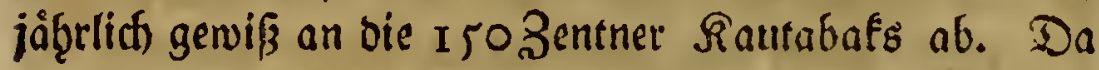
bie hirfigen Rramer ben Bentner von diefen ફૃands!s. leuten für 10 - I 2 Bulben ablófen, fo mad)t bief;, nach bem geringften preife gerectnet, für Billertģall allein eine Summe von 1500 Gutben jeigrtidid), fe!gr elend verfplittersen Bjelbes ins Zlusiano. Die Bewof̨n.

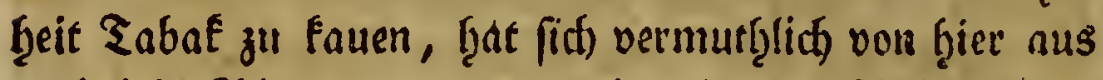
aud) in's Jimbgau, uno meiter in oas s3ripenthal,

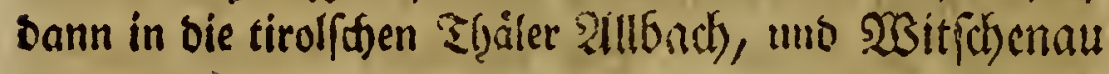
verbreitet - und fie liteint aud) unfer ben Bauern im Unter "Suntlyale immer befounter z"l rerien.

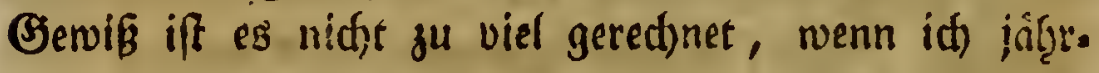
lid) für Billertrzal, Pinggau ıno Brixentf̧al (betm ber Bauer im Эangaue, uns \&ungaue (d)maud)t lieber) 400 Bentner Rautabal annefimen : weld)es, nad) bem

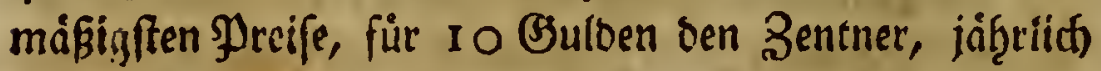
4000 (Sulben für eine red)t armfeelige $\mathfrak{W}_{\text {Baare }}$ nus bem Erzftifte zief̧r. Şier wiro eine Folle für 12 - I 3 Rreuzer verfauft, uno ein Mann, ber gerne fout,

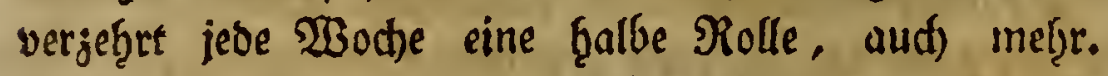
Unteroeffen will man bod) Gier bemerft f̧aúen, oaßs biefe

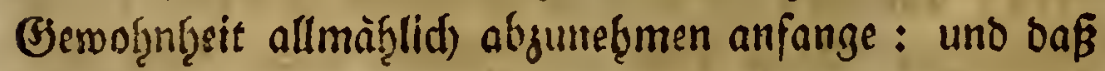
izt jüngere seute nidft meter fo ftare nad) Rautobaf fragen, ba bor bem jeber fleine Bube frton feine Roiatll, wie fie fagen, fraben mughte.

Ein 
Bell im Billerthall, Den 7. Serbftm. I 783. 57

Ein fürd)terlid)es f̧ochwetter ḩatte in ber leşten

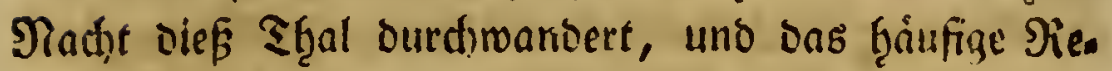
genwaffer, ons von ber Jedghe bes Sriftenfpişes abs

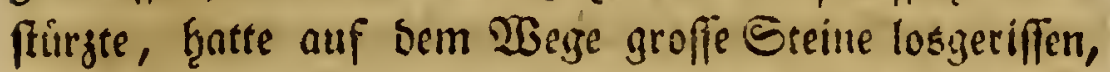
bie, in Falle auf biefen jukiteig, an einizen Orten fo groffe (didjer ourd)/(h)lugen, oa fortfommen Eonnten. 2ather ballo fieng es an freuns, licjer unt unż herum ju werben; uno wir famen aus

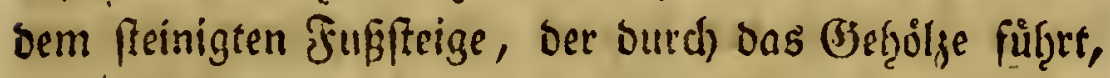
in bie Moralpen, Die Mutter Satur mit Der fdyónften grinen Sapete überbecft haatte. Dieje frud)tbaren (Se: genoen, Die das trefflioffe Futter für Das $\mathfrak{B}$ ieh geben, fennt man hier unter bem allgemeinen Siamen Aleften. Jd) weroe J̧̧nen in ver Jolge meţr von ber altr fie zu benúgen zu fagen (selegenţeit ந̧aben. Rechts jenfeits bes Bartes ift bie erfe Gruffecin : ber Befişer bringt bie herbften $\mathfrak{B i n t e r}$ fo gut als bie freunolidffen Eommertåge in biefer 2 oralpe ju : fonberbar ift es, onß̧ Das Bief̧ Ģier fo allgemein vom $\mathfrak{B l u t h a r n e ~ b e f a l l e n ~}$ wiro ; vermutb̧lich ift bie Urfacte biefer Rranflyeit, ents webers irgeno cine fdjablide Pllanze, bie ba wád)st ober ein ŢBäperchen, bas burd) biefe Weiden hinriefelt,

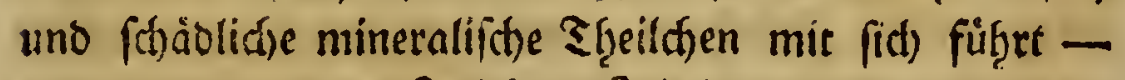
ober woḩl gar ein Jinfeft. Jath hatte noth nid)t (5eles genţeit nàhęe Unterfucfungen bartiber zu madjen.

Mein $\mathfrak{B e g}$ füfrte midh burd) bie Boralpen Fanmebe unb Edjlifitein. Bon ḩier aus false ta dos arfemal die befondern $\mathfrak{W}$ irfungen ber Ednee. läg̨nen. Ein tiefer (Jraben burd)fdneibet bie $\mathfrak{B o r a l p e}$ 
E(f)ranbad), bie jenfeit des 3embuat)cs, am Jufe

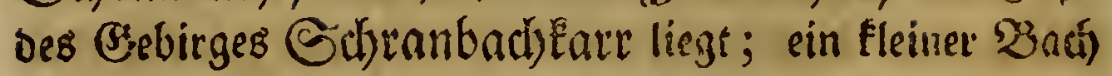

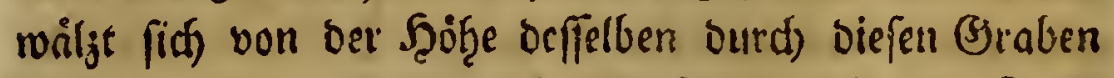

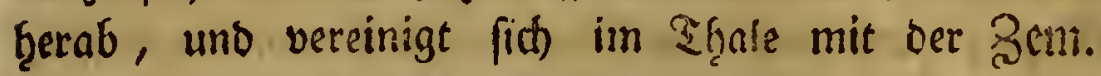
WBenn nun bie warme, aufthanemee $\mathfrak{W B i t t e r u n g}$, oper

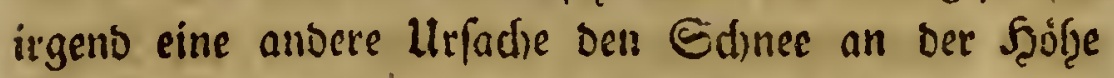

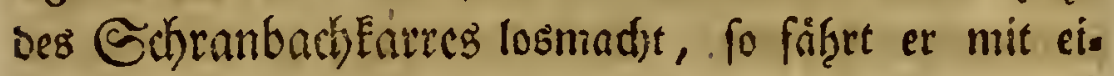

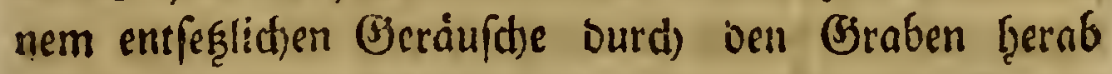

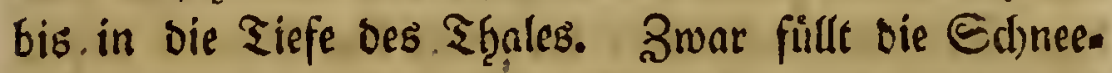
maffe nun ben ganzen Giraben: aber sas 2 Báfferdsen bogret fid) unten feine Etraffe Durd), fisfeidt) unter ber Deffe bie Jjöhe Gerunter, unt formmt in Thăle unter Dem Şaufen, wie eine Suelle, mieber hieraus. So

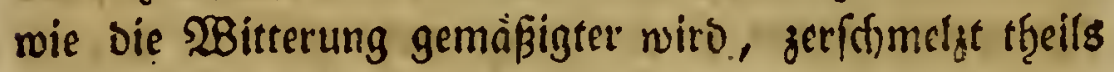

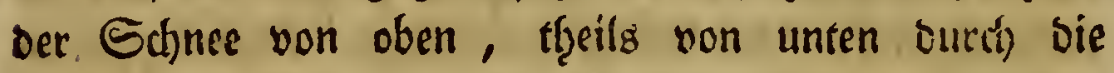
Warme ber unter ber Esthneemaffe eingefperten Dinfte : Das unter ig̨r flieffente bådggen wiro auds

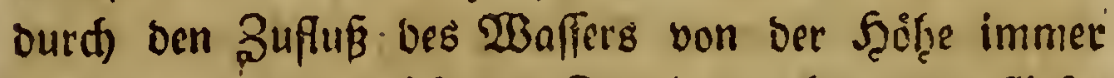

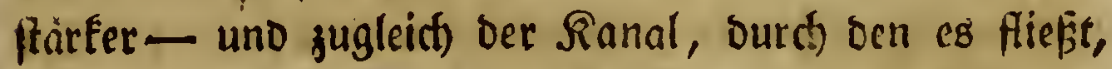

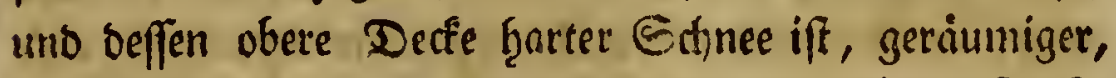

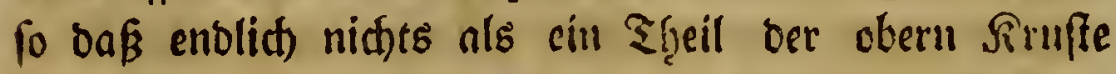

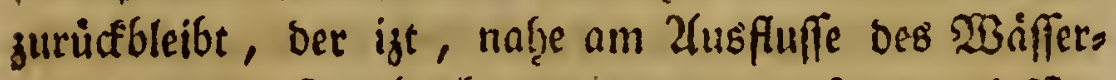
chens in ben Benchatid), no ber Şaufe am tieffiten war, eine orbentlidje $\mathfrak{B r u d f e}$ úber Den (Jraben bilbete.

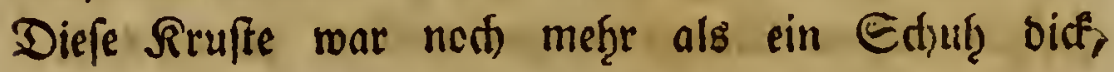

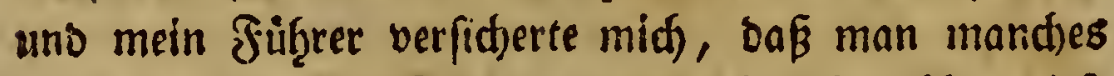
Jaa̧r ben gangen Eommer ḩinourd) fidher úber biefe Edfneebridfe geb̧en dơrfte.

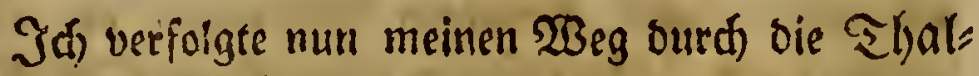

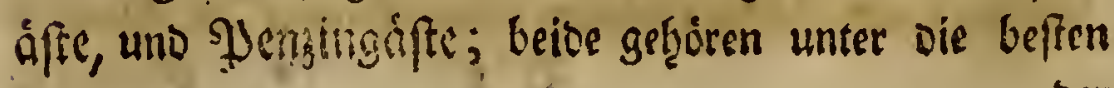


3all im Billerthall, oen 7. Saerbftm. I 783. 59

Dee hiefige $n 23$ oralpen; man mar eben in ber erften mit Mähęen ju Enbe, und id) wunberte mich) Der Menge Erbober, auf bie man bas Gras aufgefgáft ḩatte. Jenfeits oer Zacun lagen bie Boralpen semgnu uno Sippenifit. Enolid erreidse id) oen Gimfling, eine ref̧r fructibare Boralpe. Sm Sffen serfetben of net fich füooftroirts ein Thal, Das bie filutte beeift: id habe es auf ber ?Riutfeife von ben Eisbergen befud)t, und will Jhnen in ber Folge etwas bavon foreiben. Wir lagerten ung an einer bृơzernen Scutte, uno ent. folloffen uns hier Mittag zu inadien. Eitwas Bwet. fdyengeift - Ealter Ralbsbraten - Srob - uns \$affer vom Bembact)e waren alle unfere (Beridste, uno wir bact)ten uns babely fo gut, als jeber franjơfifde Beneralpåd)ter ben feiner nodüffigen ₹afel.

Saffen Gie mid) Shnen bis jum Enoe unfers Gaftmals etwas von den Ectneeląhrnen ergähilen, weil wir boch gerabe zubor eine $\mathfrak{B}$ irfung berferfen gefeb̧en ḩaben.

Nidits, mein lieber freund, madte in ben (Jee. birgen ben $\mathfrak{B}$ inter, oder vielmeţr ben Frrūfling fürdftet. lidfer, als bie Sdgneelähnen. Iđ habe nicht nơtţig,

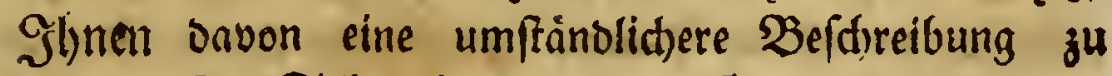
geben. Da Scheuth;er, oc Suc, Gruner, Altmann, Şald)er, Sacquet, und andere die trefentifen $\mathfrak{B i l b e r}$

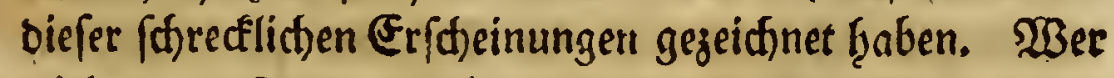
bódjte, Daß̧ Die, auch) nur leife Etimme eines Men. foten - Der Flang eines (Slockdjens - Das Selupfen eines Eleinen frof̧en Bögeldjens an einer Etnute die 
Utfache des 2lbfaf̧rens einér unbefifreiblidgen Maffe Sdjnee's werben feunnte. Id) bin im গiovember. monare 1780 . von STsublam liber ben Shaftatters tauen nact) Galyburg gereist, lange hatte ta f(t)on hioher Sdince gelegen, uno oa nun eben Regenwetter eingafallen war, fo batte ith ben meiner Durthreife wobl Urfadje für ben Sdjneelaingnen beforgt zu feyn. Diể gab meinem Fufrrmanne (S)elegentieit mir eine lange \$ieif̧e non Unfállen, bie in biefen Begenden ourd) bie Ectincelåf̨nen entfanden wåren, ḩerguergäb̨len.

Die geringfte Erffultterung in ber suft madte ein Sd)neeflümpchen von einem $\mathfrak{B a u m e ~ l o g ~ : ~ e s ~ f a ̈ l l t ; ~}$ nit if̧m vereinigt fid) ein anders, im f̧allen wirbs ein Sెnll, ber enticlly ju einer namenlofen Maffe Gájnee's armåd) bie Tiefe Des. Thales nicberfturgt. Die Bemalt, bie reiffenve Brefhwinbigfeit, mit Der fie abftürzt, und

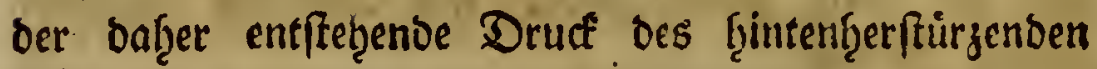
Ed)nees auf bem vorbern Theil ber láb̨ne, mactjen, Dổ fie oft nod) an ber andern Seite Des Trgals eine gute Strecte meit allfwairts getrieben wirb. Daju foimmt noch an ber (Gegenfeite ein fo gemaltiger $\mathfrak{3} B$ ind. ftó, Daß mand)mal Båume uno f̧ưtten bavon nieber. geriffen werben. Man ift baf̧er genơţ̧igt, mandje

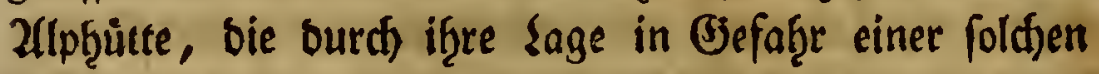
Bindląhne ift, baburd) für Schaben ju bervaf̧ren, oaßß man für bie Sţitte hiin, an ber Geite, wo bie Winbląngne anzufallen pflegt, bis an bas Dach thinan, eine Steinmaust, gerabe in ber త̇eftalt eines $\frac{x}{4}$ Bogens 
Bell im Billertlall, Den 7. Seerbfim. I 783. o.

eines Birfels; auffrînet, ser 2 Binoffor preflt an berm

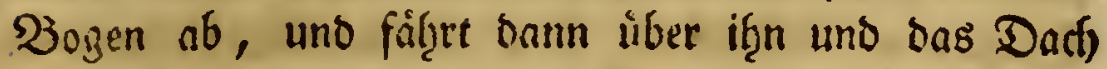

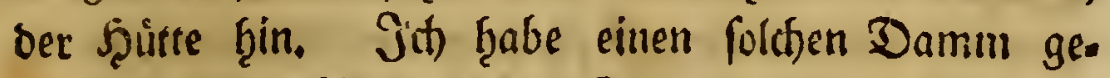

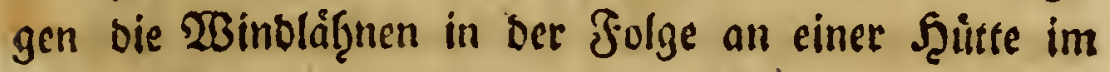
Floitentlbale geferenen.

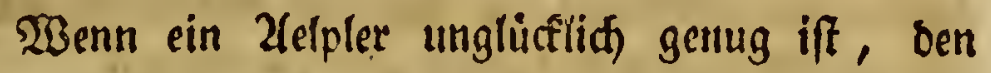

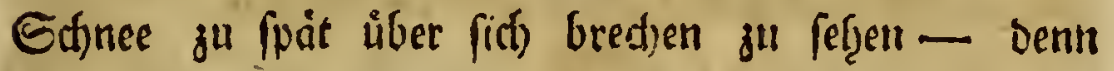

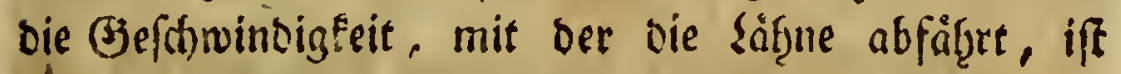
aufferorventidid) : man fief̧t oben frauben, Ģơrt ein Dumpfes (Betófe mit furrdserliden Edylàgen - und ba

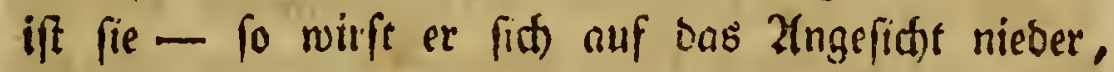
etwa unter einen fellfen, wernn gerate einer on iff,

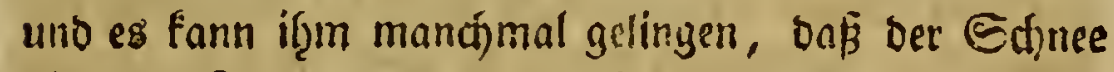

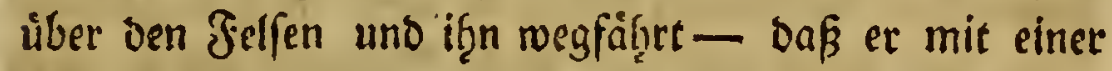

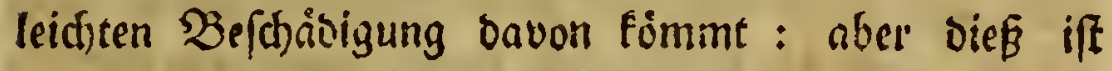

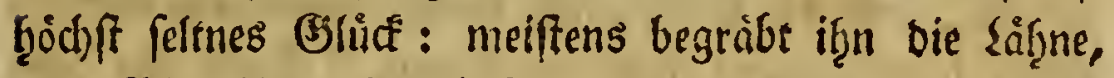

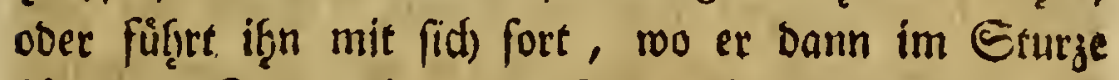

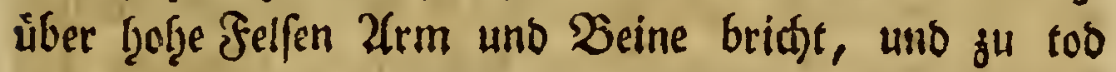
gequetfdet wirb.

Gemeiniglich), befonders, wenn ble Săg̨ne großß ift, if Edmee - Etaub - uno $\mathfrak{B}$ inolähne vercint:

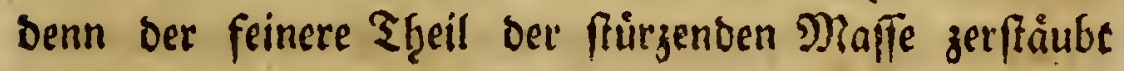
im Salle, uno Der zante Estnneeffaub breitet fids oft niber weite Etrecfen aus : zugleidh entffereft auf ber gegenüberlitgenben Ecite bes Thals ser fîrdfterlidge

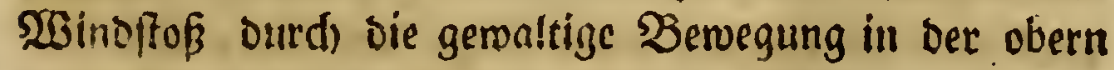
Suft, uns ourd) Den Druck, ven ber abffirizende Ednnee auf bie untere Suft madft, treldefe gany natürlidy auf bie Esite weilfet, wo fie feinen 2 Biberfans

fintot. 
findr. In biefem $\mathfrak{X e r f t a n d e}$ werben bie $\mathfrak{B}$ orte Sinub - SBind - uno Sefneefibne hier gen nommen - uno fo fino fie aud ben 23 ald her in fei. ner 2 fbrinnolung uber bie tirolffen Eisberge, uno ben Gdienthyer in peiner Naturgef(d)id)te bes Sd)weizer= lanoes zu verftef̧en. Giruner verbinoet mit ben Staublifynen ganz einen anbern Begrif.

Die ŞBirfungen der Săg̨nen fino wirflid) ferger traurig. Sie füferen oft in ber ganjen Etrede iģres Saufes bie groffen Steine une felstrummer mit fich fort : reiffen bie bióch)

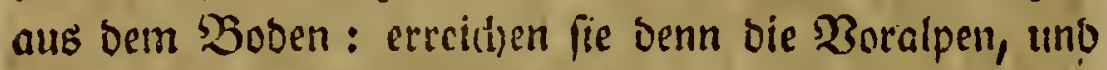
rollen riber bie $\mathfrak{B B}_{\text {iffen }}$ serfelben weg, fo bezeidynen fie ifgren (auf ourd) tiefe uno breite (Srriben, bie fie auss werfen, woburch ber Sefigerer biefer verwüfteten Grund.

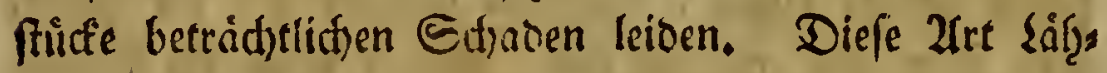
nen werben aud) jum Unferfdiede von andern weniger verf̧eeremben Girundifilmen genannt. Giruner ḩat fie int oritten Theile Der Eisberge Des Ect)meigerlandes,

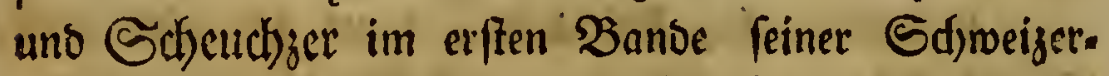

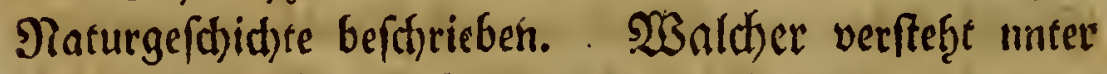
Dem $\mathfrak{B}$ orte Giundliflynen bie in Sirol fogenanten Nurren. Diefe heiffen Ģier Brifhe; uno cine (Be: geno, bie aus to leimigtem, wàfferictitem, nich)t ţalt=

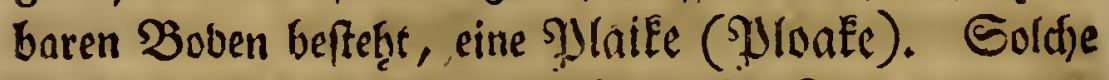
Plaifen trift man allf bem $\mathfrak{W}$ ege ins Tux, uno vorn zùglich am Sierloğ uno Şaimzenterge, ferge viele an. Eie berraţ̧en fid) auch in ter Jerne bem 2luge bes. Reifenden; benn man jief̧t großje Strecfen bon weislid)t 
ober graulifficer Jarke, von oenen ser fidoone grủne Siafen weggeffinenmt ift. Der eole, menfdent.

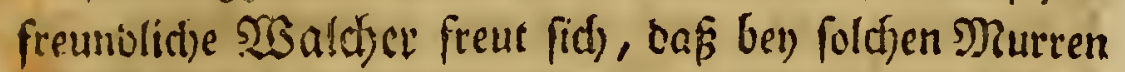

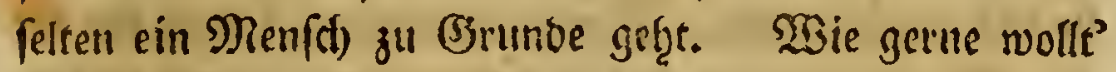
id) mid) mit if̧m Daruber freuen, menn id) nitht in

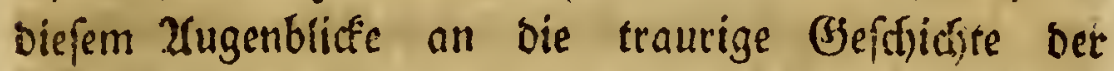
Nurre, bie im Jaḩr 1764 im tirclfdsen Billertb̨ale naţe an Dorfe Gtum abfuņr, erinnert mürbe: Der Allumbad), ber fid) vom Ctmmerberge terab= wålgt, war entfâslich angefdiwollen : bie $\mathfrak{B}$ auern eilten on ben $\mathfrak{B a h}$, Dem 2lusreiffen seffelben zu meţren: aber einer von ingnen, ber zufsulligerweife

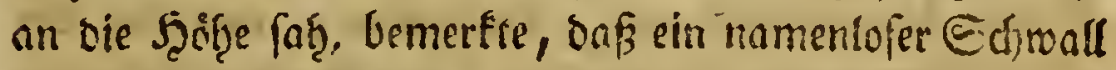

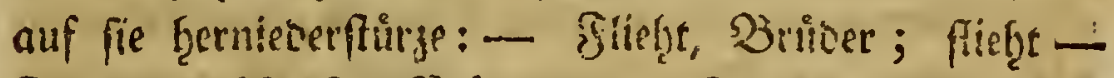

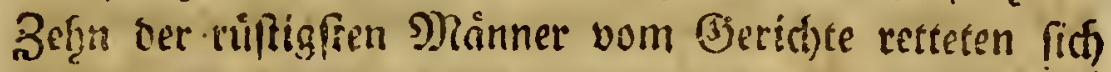
an ben 5 girgel, an beffen Jufe faton ber $\mathfrak{B a d}$ weglief Die Ungluicflidsen! - Der Siafen brad) unter iferen Jưffien weg - ftürzte mit if̧nen in die Tiefe bimabund bic (Seroalt oer nieberfaḩrenten Murre rifí fie mit bem $\mathfrak{B}$ actie fort - fie waren alle im Ectlamme bes graben. Seffer Frenns! ein Mann, ber diefe fürdter. lia)e Szene mit anfah - in beffen 2 (rmen joeen biefer Unglucflid)en nod) vollends verf(d)madteten - zeidfnete mir bas Wilo berfelben auf oem şlaşe, wo fie fié)

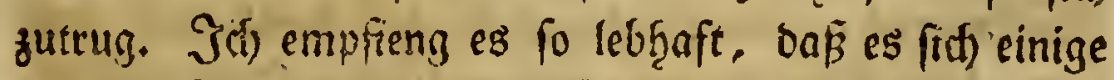
folgente ?age in alle meine 5 challoingen mifd)te. Die

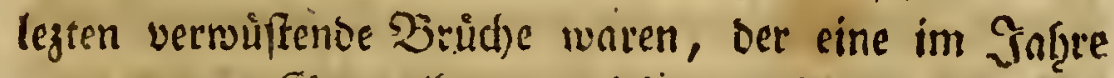
1767 vom Ginggelberge : iff) war 2lugenzenge ber Bertieerung, uno, obwobfl idf Damals nur erft ben fiebenten Sommer wiederfommen fáf, to babe iff 
Doch bas andenfen berfelben nod) bis ijt lebraft be. balten. Die Murre rif non einem f̧aufe bie jween Eeitentf̧eile weg, uno ließs den mittlern ftehen, ben fie tief einfdylammte. Tiod) nad) einigen Sagen frectite ein aites Mitterchen if̧re 21tme aus oen Siuinen ţerfür, uno bat mit gefalteten Şånden um Scilfe : man roagte

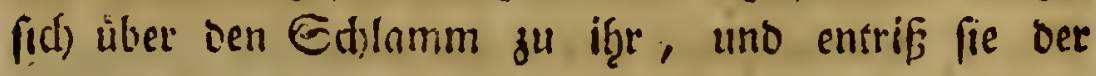
Gefnlgr für Scunger zu fterben. Unterweilen mit ver. einten Sräften grarbeitet warb, um bie noch weitere Z(usbreitung bes Sirud)es ju f̧inbern, oer gerabe einem natzegelegenen Baurenfiufe Drofite, mufite ein rafher

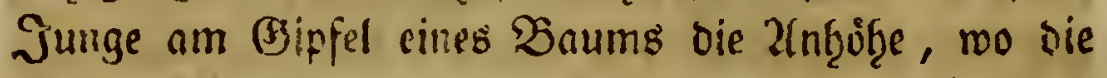
Miurre loegegangen war, beobadten, uno bey ber erften $\mathfrak{B e r w e g u n g}$, bie er oben bemerfte, ben arbeitern Das Beiden ğur fludt) geber. Denten Gie einmal: ber Booben, Der eçebor mit allen úbrigen an Frudtes barfeit um bie Wefte frritt, war in einigen Minuten mehrece Slafter tief unter Ethlamm uno Steine be= graben: ber $\mathfrak{B a d}$, an Den fich elgevor biumigte $\mathfrak{B i e f e n}$ f(t)niegten, láuft nun mitten ourch) einen freinigten Boben, Den eine junge Ellernaue becfi, die feit bem

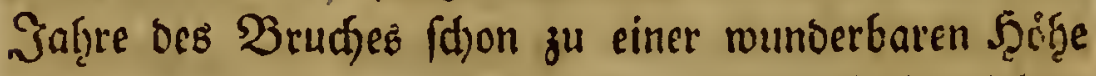
angewad)fen ift. Der lef̧e ungleid fürdterlichere Brud) war I 781 nad) einem befrigen Ungemitter uno

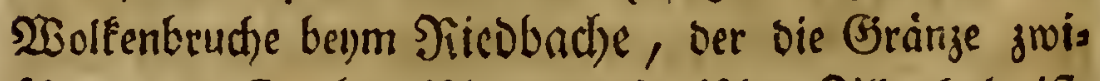
fhen bem Ealzburgif(t) "uno tirolifden Billerthale iff. Bende Theile fübiten die traurigen şirfungen oeffel. ben, bie nod) in bieferm alugenblicfe feţr fidhtbar fint. Die. Siller wart babey niele Rlafter weit von sem jenfeitigen Beftade weg an bas bieffeitige geworfen. 
Sell im Billertball, den 7. Şerbftm. 1783 . 65

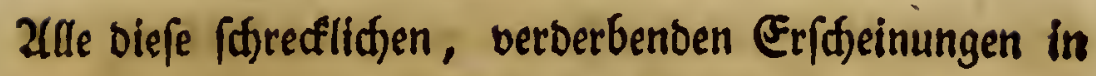

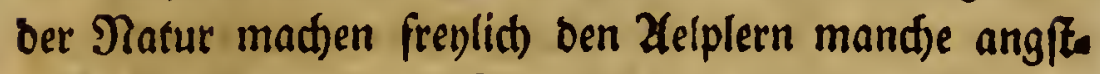
bolle Etunbe. Saffen Eie mid ben traurigen Bilbern ein Ent maden; Sic fino zu menffienfreunolid, als

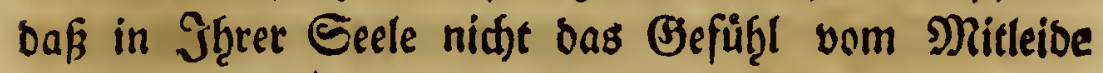
oarüber rege würbe, bas in biefem Zfugenblidé begm Znoenfen berferlben in mir entfteţt.

sange fáon war unfer Dinér vorüber, als míf)

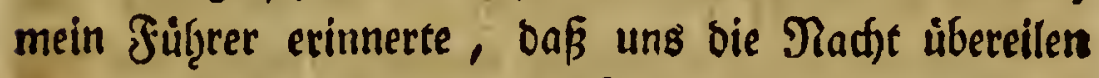

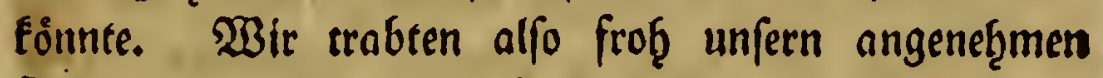

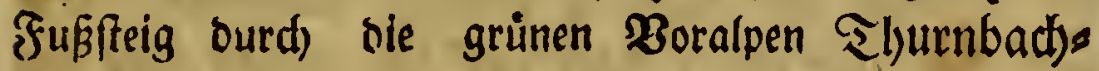
Sinsling, uno Dender fort, ,ro wir auf einem ferer fdfmalen, fdimanfenben Etege über ben Bembady

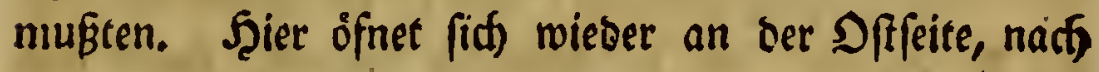

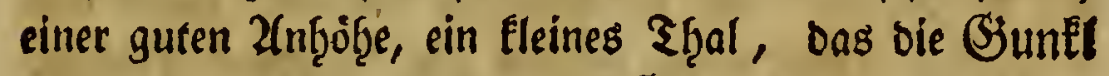

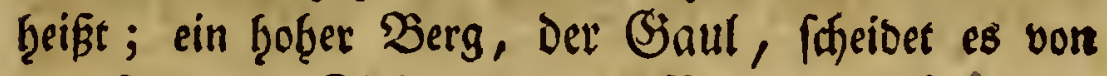
oem Thale ber Floiten, das im Norben beffeltben liegt.

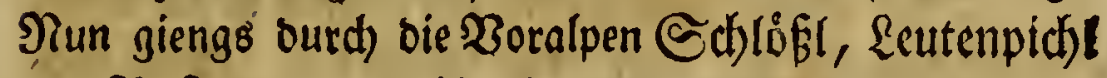
uno Siofllang : fie ift bie legete unter ben Buralpen.

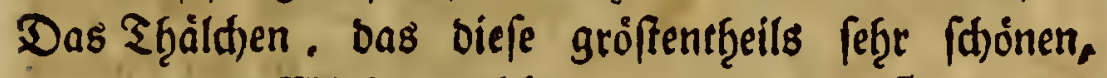

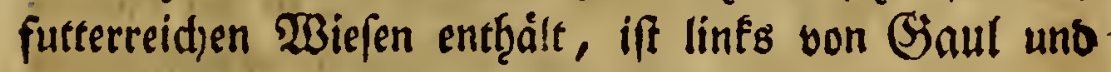

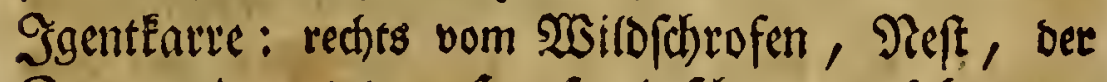
Gaunwand, und bem Schaujergletfher, ber fidi, wenn

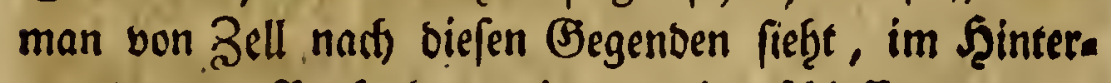
grunbe bes grofpeftes geigt - eingefdiloffen.

Der $\mathfrak{B}$ eg waro nun immer feeinigter - und bie

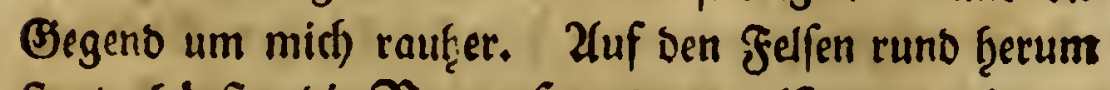

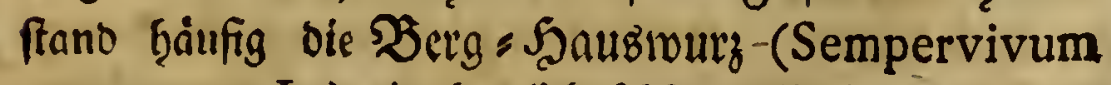

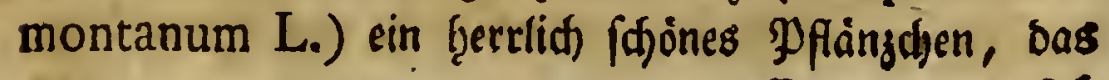
ETaturbifi briefe, I.Band. 
fid ourd bie Ģod)rothe Farbe ber SBlumenblätter, bie freulifí) im Şerbarium blaßßpeild)enfarbid)t wiro, -

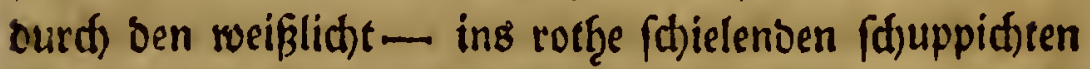
Etengel, an bem bicke, fleine rotfic Blätfer, fo wie an Thuffilago farfara L. gereihet, figen - uno ourd) bie golbfảrbigten Etaubfäben ungemein gut ausnimmt. Eonft waren bie $\mathfrak{B}$ Beiogange Gier voll Euphrafia officinalis L. unb Dianthus carthufianorum L. Man ift bier beftandig ben fogenanuten Eteinläb̨nen bon Der Jृof̧e eines feţr bruidtigten Branitgebirges, an Defs

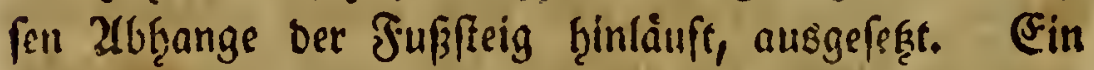
felge muiffamer Etrig führte von ţier gerabe uiber ben

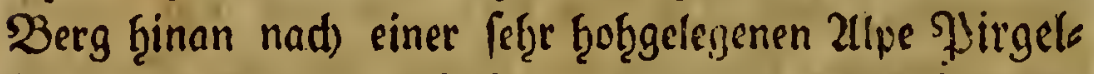
berg uno Jirgelbergheffel: uno es ift nidfts jeltenes, Daß̧ bie Zlelpler, bie fie befteigen molten, Durdf bas beftänbige 2abrollen ber Eteine zurúctigefdjeut wurben.

Salb giengs nun wieber cin biegen in bie Tiefe, uno id fam in bie erfte zllpe Sififerlar. Die lage

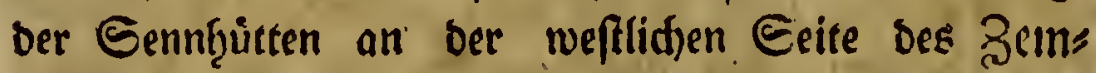
baches ift gqöt)ft romantifoj. Berftreut in ein fleines SBálod)en geniefen fie ben Ed)atten wohtltḩatiger

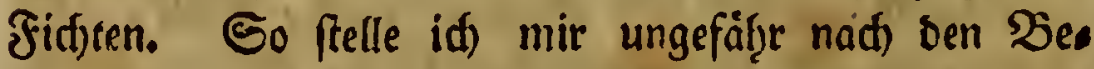
fdreibungen Der Eeefaḩrer, bie fleinen Dörfer ber In. bianer vor. Ein fanftes, fủbles arbenolufftchen fpielte izt mit ben 2reften biefer Baiume, uno baburd) mif(d)te

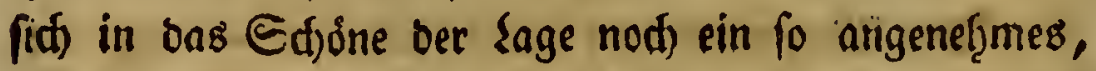
tiefes (seråufd)e, bas einen hiod) fet feb̧aften Einoruce auf mid) madife. (Sefellfd)aftlid) zmar - ein fleines Dơrfdien - und bodf fo einfam - po eine ę̧rwúrbige, 
Bell im Billerthall, Den 7. Sherbftm. 1783.67

in ţoţem Brate ḩeilige Erille b̧ier. Unmòglid) fann

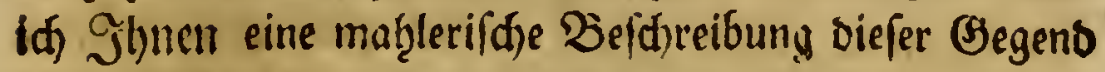

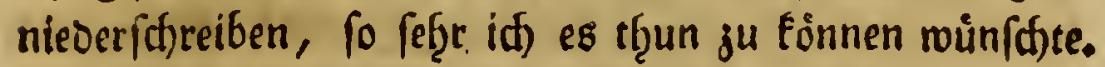
aber lange ftano id) unberogglid) Da, uno betrad)etegieng einige E(t)rifte vormárts - uno fef̧rte wieber um - unno fonnte mid) nie fatt fệen.

Es ift eine artige Sitte ber Giefigen Xepler, baß

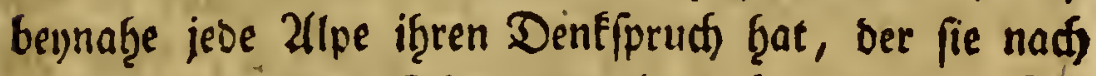

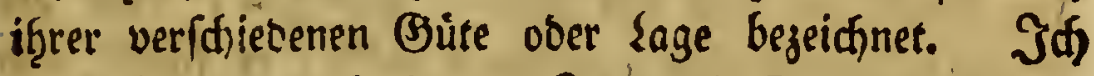
mußs Slynen bod) in ber Jolge bie Denffpridife ber 2lipen, fo wie it) fie nadeinamber burdfreifet ḩabe,

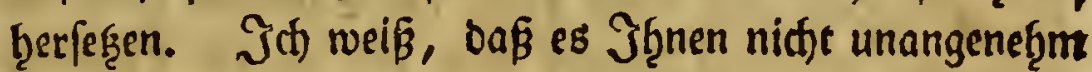
feun Éann, fie ju lefen : id) fano es wenigftens für

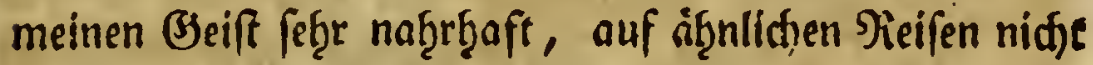
nur bie Matur in ben Tfieren, Pflanjen unb Minera lien - fonbern aud) in bem Menfdien, uno feinen

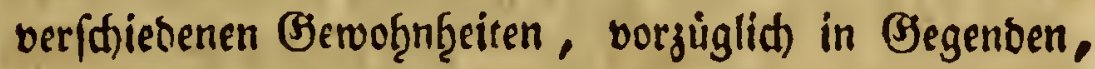
wo er im naruirlidffen Buftande zu fenn fideint, auf

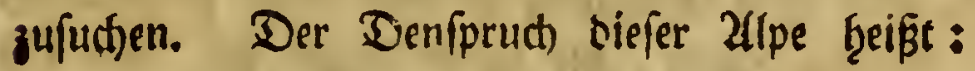

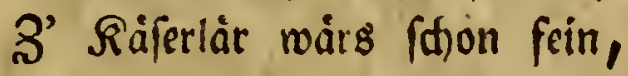

Benn man nicht milft tragen

Das Echmaly von auffen Ginein:

Der Borberfás biefer Debife beftattrigt Das, was ián Şbnen viber bie sage der biefigen Eernthütten gefagt habe, aus bem, was int Shlnen biefen 2(ugenblicf fagen werbe, fönnen Sie fid ben J̧interfás erfláren.

Nad) einem furgen $\mathfrak{B}$ ege erreidfe ind $\mathfrak{B}$ reito

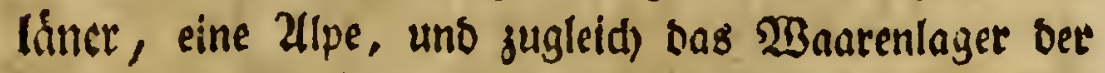

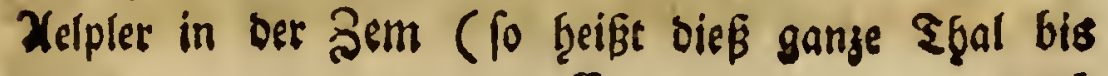


nach) ber innerften alpe Gd)marzenftein). Won als len Zlipen in biefem Şgale - es fino if̧rer act)t - fleffen hier alle Erzeugnifie an Butter, Fiajen unb Edhotten zufamm, uno werben in Eleinen f̧ólzernen Sctitten, bie Siffiften beiffen, aufberwahret. Die Mels fer ober Şirten tragen gewoóţnlid) jeben zrognten ober Dritten ₹ng bie Eržzugniffen Der zween leģten Táge

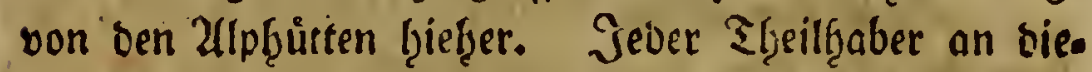
fen 2lpen ḩat ḩier fein eigenes f̧uttthen, tem ber gange Saftiginsnukgen anvertraut nito; er fdjicft bon feinem Sute im ₹̧̧ale gelegentlich ₹rigger ḩię̧er, bie

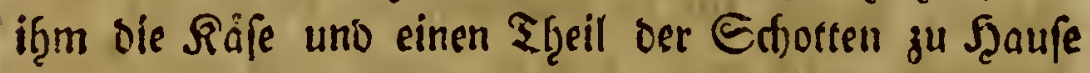
bringen; Denn bie $\mathfrak{B u t t e r}$ und ein groffer Theil ber Edjotten werben unmittelbar von bier aus in eas nalje Tirol verfauft. Sur Eitherheit il̨rer Magajine balten fie Gier einen Mann, ber ben ganzen Eommer über ba wof̧nt, uno gegen nádjtlidje Einbridje in bie

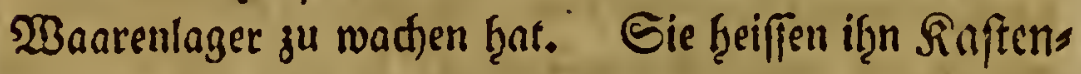
butter, und feine $\mathfrak{B}$ ob̧nung ift eine eben fo (d)led)te,

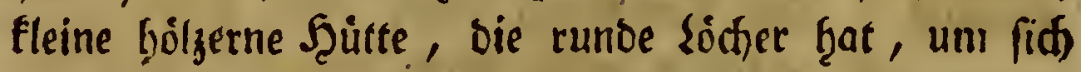
mit Sd)ieß̧gervehr baraus zu vertheioigen. Hebrigens ift Breitlinner felbft eine 2llpe - mo aber bie $2 B e i b e$

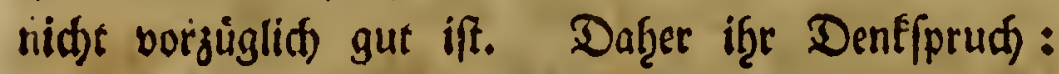

\section{Braitlanår, \\ Edjottenfämár.}

Denn wo sie $\mathfrak{B}$ eibe niddt gut ift, erzeugt man wenige

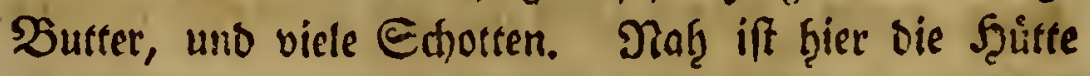
eines fürftliç)en (jemfenjägets. Im $\mathfrak{B}$ effen ber allpe

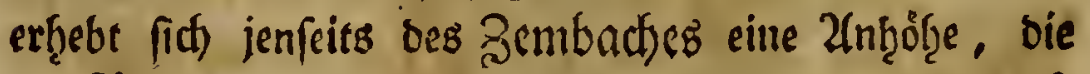

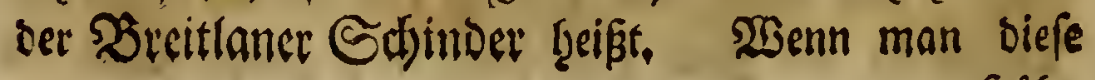


Ball im Billerthall, Den 7.5erbftnt. I 783. 69

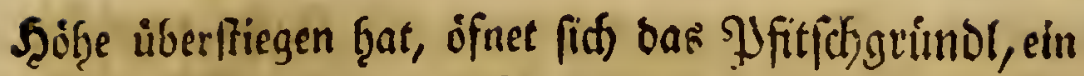

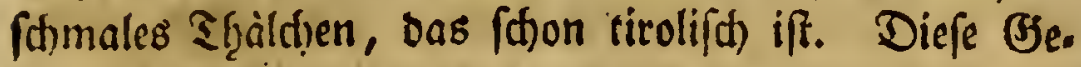
geno ift reid) an verffoiebenen Steinarten, vorjugglid

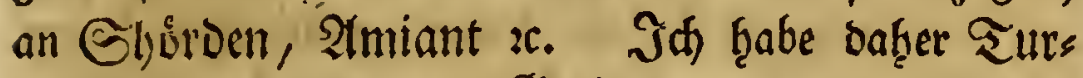
matine, wie bie vom Girciner, und cinen fdjonen biauen Ef̧ờ in einer amiantartigen Mutter erf̧alten, Der fict) ungemein gut ausnimmt. Man finbet alles

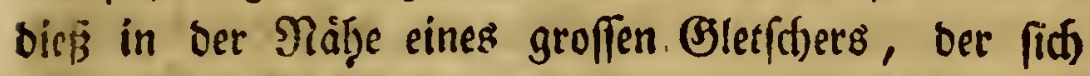

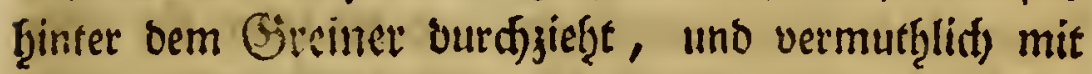
Der Rette von Eisbergen; bie id Şlynen im nádfften Briefe befthreiben will, jufammenf̧ängt. Belegents lid) will id) Ģier bemerten, daß bas $\mathfrak{B}$ ort (Siruno, Gruitnol bier überḩaupt ungefáf̨r fo viel als That,

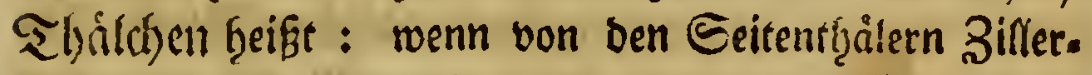

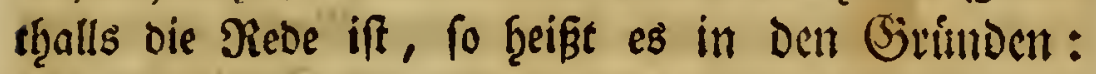
baher Bittergrutti, Bemgrund, und eben fo aud Jfitficgrimbl. Im Sungaue beifie es unter bem

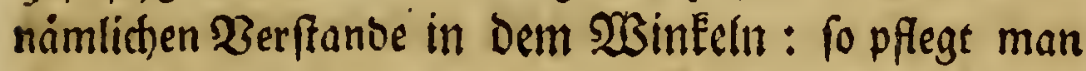
bort bie Geitentf̧äler zu nennen : 子. B. Nurrtwintel.

WBit fanben f̧ier niemanb zu Şaufe; benn bas Bief mar izt in bet alpe Sdfwarzenftein : bod)

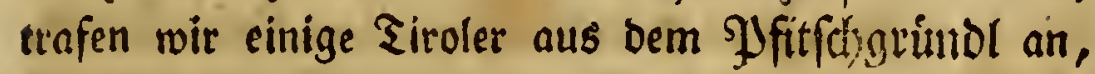
uitr bie ganze Raravane lagerte fict) auf einer $\mathfrak{B a n f}$,

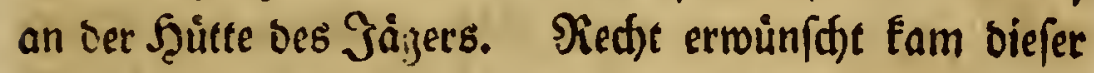
gerabe vom $\mathfrak{B}$ erge heer, uno trug einen gemidjtigen Gemsbof auf oem Siucfen. . Der gute 2llte ófnete uns bie ₹̧̧ưre feiner Şưtte, uno bot mir bie leber bon sem gefällten Thiere. Da mir múbe waren: fo

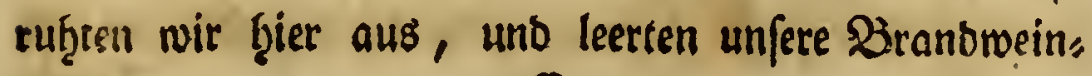




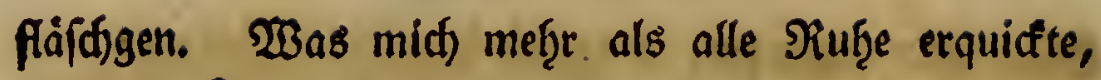
war bie Freube, bie id) batte, bier ichon einen Blumenftranß Satyrium nigram L. - Lilium martagon L. After alpinus L. - uno Aretia alpina L. zu feģen: er frano auf Dem Tifche, um ben wir uns gelagert

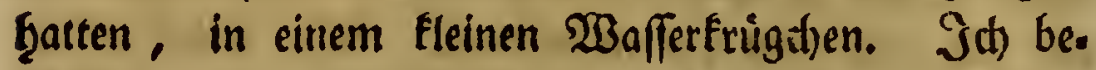

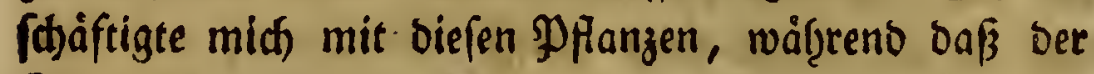
Jager feinen Bsemsborf ofnete, uno bie leber für mid) Ģerausnaţm, bie mir ḩơt)ff willfommen war. Der gute alte erbot fich aud) mich auf bie (Eisberge zu bes gleiten, - uno nachoem idf) if̧n auf ben finftigen Morgen zu uns auf bie 2llpe Sthwemm beidjeoen

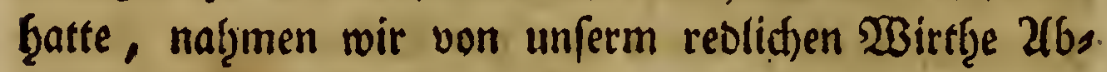
fajies, uno wanderten mit neuem $\mathfrak{R u t b e}$ über eine fleine 2lnţöb̧e, uno denn immer am Zembathe Ģin

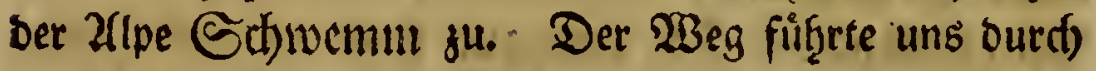
bie 2lipe Silaujen: vermintf̧lid hat fie biejen Namen: won einer alten f̧olzflaufe erf̧alten, bon ber man noth

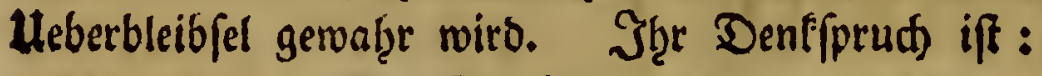

\section{in Der Rlaufen}

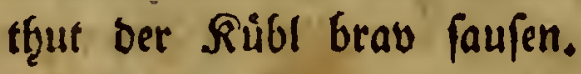

Sie feţen fation oḩne mein Erinnern aus biefer Devife, baß gier treffirte $\mathfrak{B e i n e ~ i f t , ~ w e i l ~ v i e l e ~ B u t t e r ~ g e m a c h t ~}$

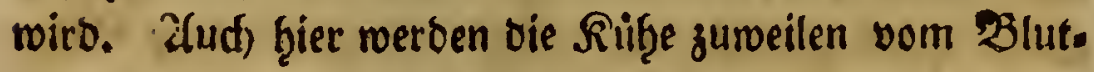
tharne gequailt, aber nid)t fo beftanoig, wie in ber Boralpe Gauftein. Naat) einer Biertelffunde ets reidten mir bie alpe Gd)wemm. Die Berge, gni-

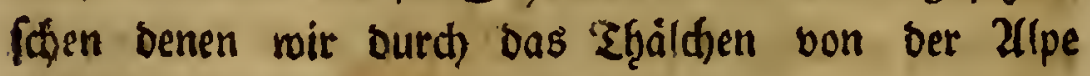
Sieffertár bis giefget tumen, find redfts, ober an ber abenos 
Bell im Billerthall, Den 7. Saerbftm. I 783. 7 1

Z(benbfeite ber Sifffler uno Epiegel, an ber Dffeeite bie Ephtentarre, Das Edhlinofiart, uno Der Braitenterg : alles feģr bobze, jum Ţgeil fab̧le Be. birije, bie muntere Siemfen beḩerbergen.

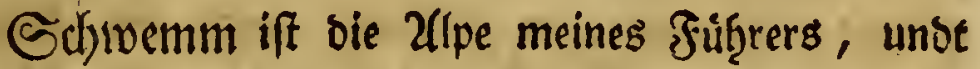

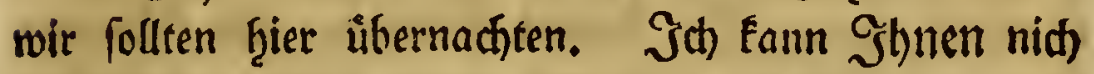
befdreiben, wie zufrieben id) rar, als wir fie etreich. ten; benn bie Begend ift Gier angenebm, - ber Şoris zont ofnete fid) wieber, unt bas Thąild)en marb etroas geràumiget, als es auf meiner Reife ḩief̧er immerḩin gemefen war. Die Eennfyitten auj biefer alpe liegt

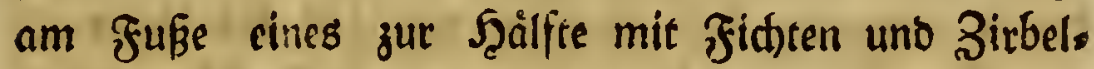
báumeil (Pinus cembra L.) berwad)fnen, jur f̧älfte fablen (Branitgebirges, bas, fo wie alle übrigen Berge

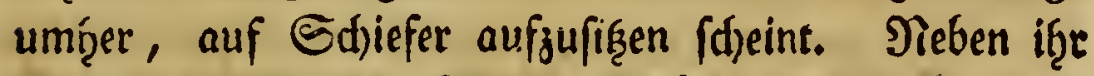

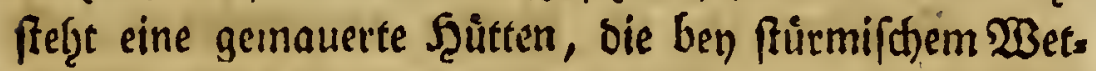
ter bie Ziengen, uno ben Sanneegeftobber bie Rüb̨e bef̧er.

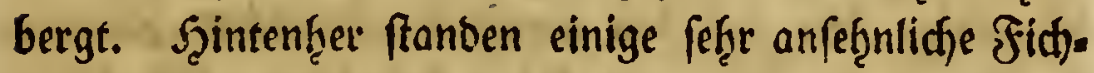
ten, uno eine fleine Errecte bavon quoll ein filberflaree Brunn aus bem $\mathfrak{B}$ erge, ber trefliches $\mathfrak{W a f f e r}-$ 2Baffer, bas idf in biefem 2lugenblicfe mit feinem

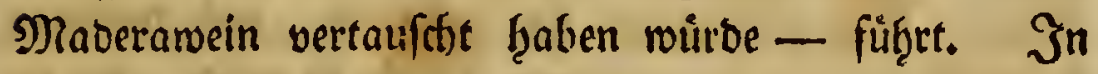
bas Riefeln oer Suelle mifdte fich ber traurige Ton

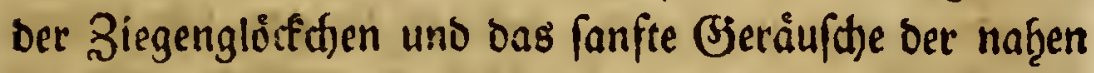
Jiditen. 'SBeld) ein fenerlides Toinen burd) bie $\mathfrak{N a}$. tur ! Gerabe vor ber Şuttte lag ein geráumiger $\mathfrak{B}$ eibe= plaz, den hie uno oa fleine 2(ermaten bes Sembathes ourd)/thitten : uno bie $\mathfrak{B}$ erge runo umber bilbeten eine Zrt won Seffel, Denn norbwairts, wo wir ḩergefommen 


\section{$\eta$ \\ Sủnfter $\mathfrak{B r i e f . ~}$}

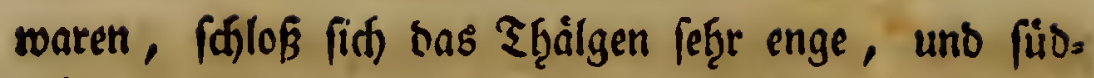

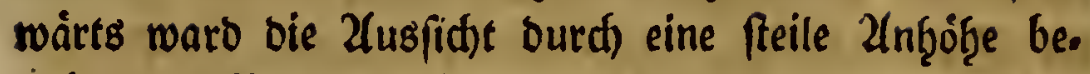
graingt, bafür midj aber eine fffóne Rabfabe, bie ber Bembach da madte, vollenos entichäbigte. Sonft

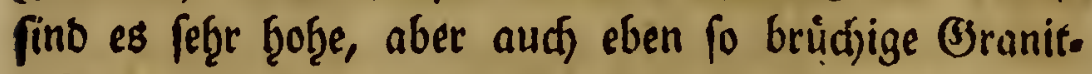
gebirge, Darunter in Subweft auds ber befantte Greiner war. Die fleinen (Slet(d)er, bie fith) juis fhen ben boit)ften Etyatten biefer Berge einfenfen,

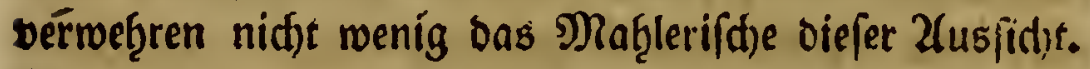
Jd) fand fie ungemein fffion, und wurbe fie untefilbar ijt nod) linger betradbet haben, wenn id) nid)s in meinen Blievern fo etwas gefübtt haitte, Das mid) Daran erinnerte, baß id) beut von Bell bis . b̧ieģer 7 gemeffene Stunden über einen jum tḩeile feg̨r raub̧en \$eg ju Juke gegangen war.

Nadjoem iff ein bischen ausgeruf̣et - unb meine Buidter uns Sdjact)eln auzgepact ḩatte, fihergte id) einige Beit unit ben menfdenfreunblid)en . Biegen,

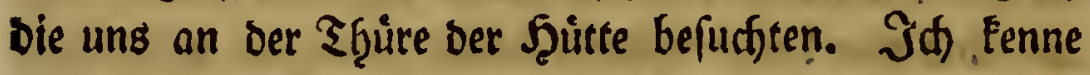

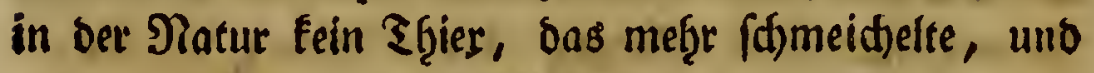
meţe bie Befellifhaft bes Menffhen liebse, als eine Biege. Sie fifmiegten fid) an mid), uno brangen Durch) Bemegungen mit bem Ropfe in mid), fie zu ftreiddeln, uno zu traşen, weld)es fie gar feḩi liefien. Die Melfer eilten um i็̨r $\mathfrak{B i e f}$ zu melfen, uno iđ gieng mit J̧roflern eine tleine Etrecte für bie f̧uttte Ginfpaziaren. Das Ufer Der $3 \mathrm{~cm}$, bie fich in einem felgr freinigten Bette oem Thale zunálzt, ift mit ver. fümmerten Ellernftauben berwadjfen, unter benen hice 
Scll im Billerthall, Den 7. Sccrbftın. I 7 83. 73

und ba ein Erigeron acre L. ftand. Ungemein vers gnůgt war it) über ein fdjonnes Exemplar von Aflpens Dorant (antirrhinum alpinum L.), oas id) Ģier fand.

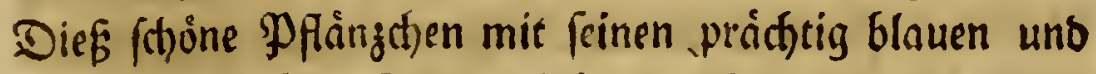
orangenfärbigben Blumenblättern frano einfam am

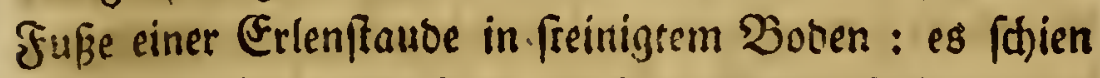
mir nur zufälligerweife ba gu feyn, uno id) fonnte in Der Bjegeno umb̨er, ungead)tet ber b̧eftigen Begierde,

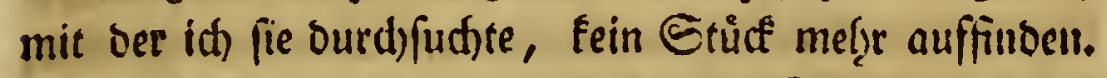

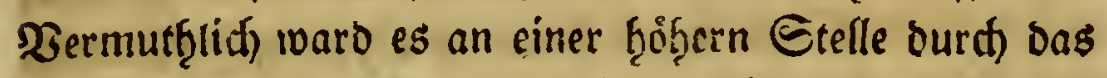
$2 \mathfrak{B}$ affer abgefd)wemmt, uno fier fanft niedergefegt, no es Denn nieder $\mathfrak{Z}$ urjel gefaß̧t ḩaben mag. Zuf einer Urtica dioica L. fano id) einen Blattéfifer, ben ids

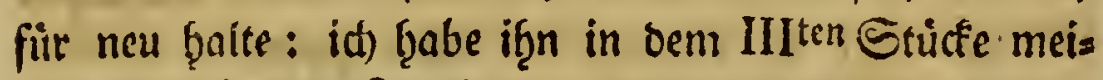
mer Enlyburger Snleften, bavon ith unferm lieben

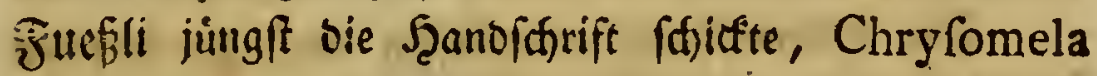
fubalpina genannt. Seine Beftinimung itt : Chryfomela nigra, capite, thoraceque rufis, clytris obfcure viridibus, ftriate punctatis, antennis longioribus, bafi rufis. It) finde unter allen be. fifriebenen Blattéffern feinen, ber if̧n gleid) wäre, bis auf Chryl. ruficollis Fabric., Der, wenn id bie Jựffe ausnef̧me, to jièmlid) juttâfe. Mit aller ber Raltblütigfeit, bie jedem Entomologen fiơdfft noţ̧wen. big ift, wenn er anderft Entbecfungen in biefem unter. baltenten fadje madien will - unb ganz bes Gecfs fioi'falden Zusfprudies : une feule de ces bouzes (des vaches) devient une efpece de trefor pour un Naturalifte curieux, et qui n'eft pas trop degouté : ingebenf, unterjuch)e iđ einige vor mir lie. 
genbe Ruf̧waben : aber id fant auffer ben getwơthn. lidfen gemeinen Dungfafern Scar. fimetarius, confpurcatus, uno nuchicornis nici)to als cin Ettiff bes Girabers (Scar. forfor L.) leler viel lánglid)te (Scar. oblongus Scopoli), narunter cinige vorzùg(iit) bicfe Unterleibe hatten, uno vermuth(id) befrud)tete 23 eibden waren : und das fidjone nelle, fleine Entuffäferden, bas id) Sltnen unter sem Siamen Hifter fulcatus, minutus. niger, nitidus, antennarum apicibus grifeis, capitis foveo:a frontali, thorace elytrisque fulcatis, illo fulcis fubquinis, laevibus, his fulcis fex vel feptem ordinate punctitis, alternis profundioribus, alarum macula antica teftacea, tibiis tarfisque ferrugineis: ju fiticfen bie Eŗre ḩabe.

Die Sonne batte unterdeffen nud) bie Ģöd)fren Gipfel der berge verlaffen, uito bie lejten Epuren yon Dámmerung erinnerten mid, uni meinen Fuif̧rer, baß man uns in ber Şitte erruarten wirrbe. Wrir fef̧rten froh nad biefer gliudliden, patriardyalifiden

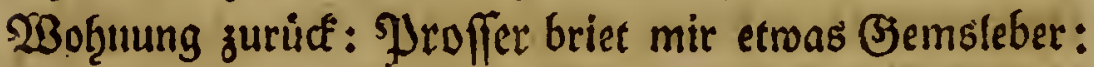
ich) Ins nod) eine 2 Beile am Rodffeuer : und bie Melfer waren am Enoe gefällig genug mir cinige Zllpenlieter z" fingen. Bollen Sie, mein Freunt !' eill Bitb unfers länolid)ẹt Rlubs ţaben, fo nef̧men Sie bie

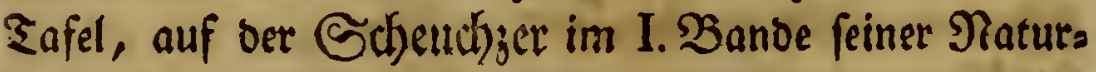
geidhidjte des Edhwrizerlanbes bie Zeidhnung einer Senn. biutte' gegeben hat, fü fict); reiben fie ben Reffel vom Feuer $a b$ - fesenen Sie mich, Juroffern, feinen Bruber, feine beiben Sôţme, und meinen Trioger in einem ḩalo 
Bell im Billerthall, Den 7. Sherbftm. $1783 . \quad 75$

ben Birfel um felbes, - Denfen Sie Gich auf allen (5)efichsern bie Büge. ber innern Seelenrufge, uno bes Bergnúgens - uno bas Billo ift fertig. Waare if De Suc, ber tiefferzenbe Forifher jeber Falte bes nrenffy. lid)en Jెergens - b̧ătte id Den fanften, gefüb̨loolien

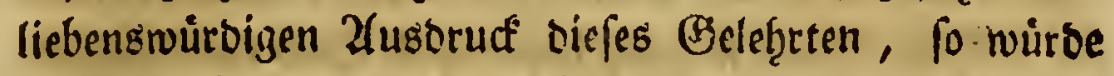
id) Diefen $\mathfrak{B}$ rief mit einem $\mathfrak{B l i d f e}$ in bie Befeidichte tes Menfdien foflieffen. 2lber id) würoc zu viel raagen, wenn id) es ůbernåb̧me, bas (jservebe von (Empfinoungen zu entwidfeln, Die mir in bem noiferem Umgange mit ben glùcflidgen allpenbewothnern biefe. Abenoffunden

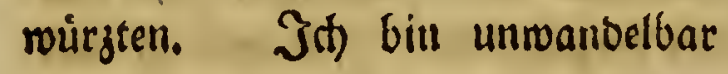

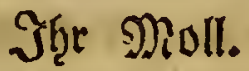

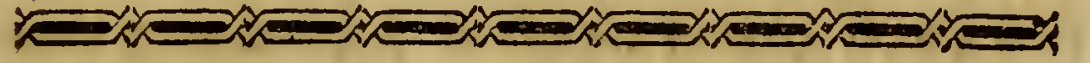 \\ Ged)iter Srief. \\ Sell, ben 13. Jeerbftm. $x_{783}$}

Eine Sllpenfene am Morgen. Eine Rašcade. 2rlpenpflamzer. Sirawander Sdjinder. Gonders barer ßergweg. SIlpenpflanzen. Sieife nad Dem IGd)warzentein. Edwwarzenfein. Şorngletfder.

S ant fen bem Şimmel! 's ift mieber ein Ģeiterer; angentef̧mer Morgen. Die rofenrothen Epişen

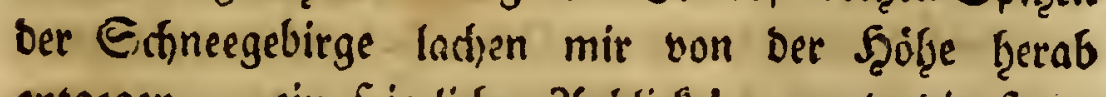
entgegen - ein feierlicher alnblife ! - uno bie fetten Rühe fetren faton mit Seb̧rfud)t nad, bem Melfer, ber įgren vollen Eiter ber Maild entladen foll. . 2lber es 


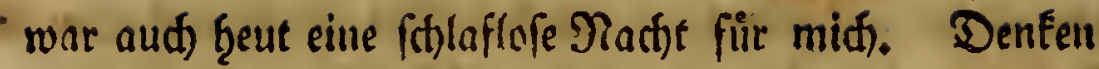
Sic einmal, mein freuno! oer. obere boben einer ofenen Szutte, oie aus Báumen fo nad)läblg gejimmert ift, Daß jwifthen ienem Paare serfelben ein ofner Raum von meḩr als einem Bolle bleibt, woourti) mir

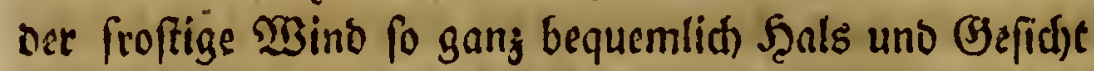
beblafen fonnte - bieß war bie Ed)lafftube - ein míffig Buntel f̧eu mein $\mathfrak{B e t t .}$ Daju rar bie Nad)e -

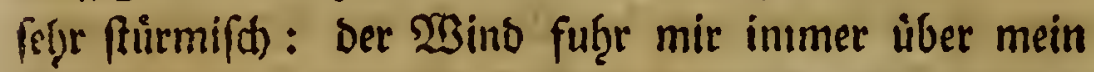
3(ngefid)t, uno ber ftarte Regen mad)te mid) an ber Mo̊glimfeit naturḩiftorifher Unterfudungen für ben finfrigen Sag vollenos bergmeifelin. Id) war fo voll

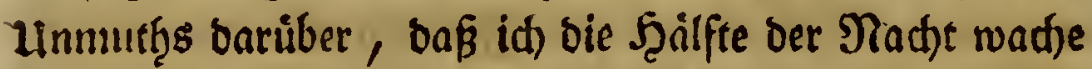
blieb: aber in ben wenigen Stunben, bie id) verfdlief,

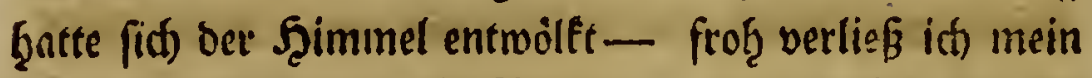
Sager - blicfte in bie Natur - uno bact)te im in.

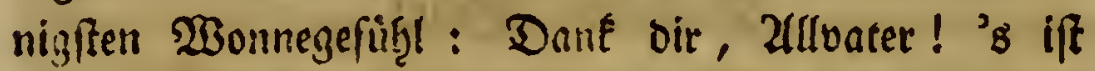
wieder ein ḩeiterer, angener̨mer Miurgen.

Die Gonne wat nod nifft bुoch genug ain Şori. zonke, Daß̧ wir ihre belebende Etralen unmittelbat entpfangen fonnten. Die bohen Berge, an ber Dft, feite, verbargen uns bieß woblithaitige Bieftitn - nber fie waren nid)t hyod) genug, um fein Sblicfen auf bie

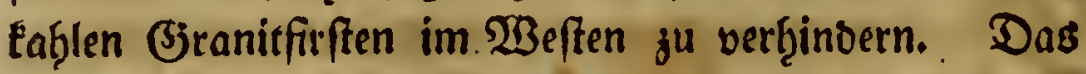
unbefdreiblich fidone Bemifhe von Echatten uns Sidft,

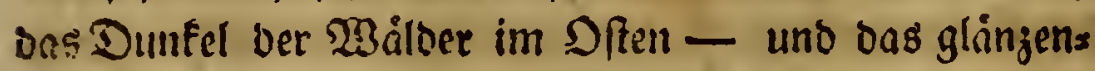

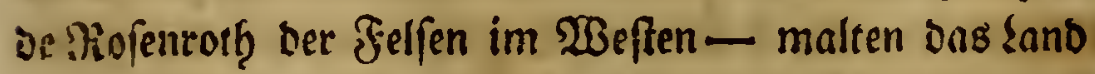
um midt) fo herrlid), Dofs id) es nid)t gerne îbernefgunen midiste, Gibnen alle bie Farben mit igreen zabillofen. 
Nuancen zu nennen, sie biefe 2 usfitht red)t in bie WBette verfduonerten. Daju bilbete die um bie Şuttte

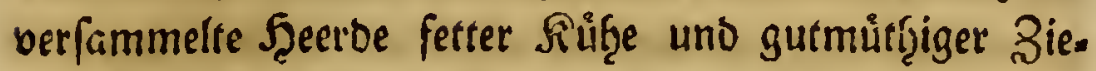
gen fo mannid)fältige (3ruppen in biefe sanofd) aft, tap id) mir in oem zugenblicfe Yloufing uns Sivós'ens Dinfel münfdre, um mit bem erfien ber פlatur bie ḩerrlid)e (anofd)aft abjuborgen, und mit biefem bas ३ief? tarein zu malen.

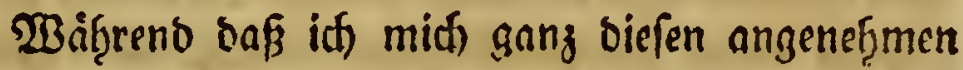

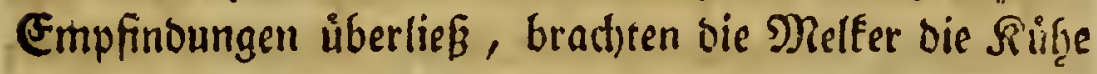

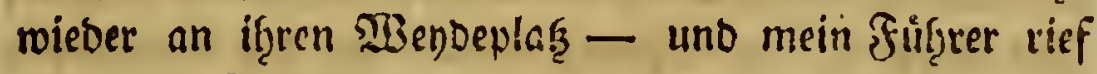

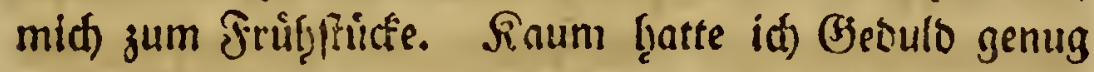
meine Mild)fuppe einzuithlürfen, fo febre eilte idf), biefen angeneţmen Tag zur Jorrfegiung meiner Reife nad) ben Eiêbergen zu benůg̨en. Wir hir ţatten nur eine furze Grrecte vor uns, um einen $\mathfrak{B} \mathrm{berg}$ zu erreidjen, ber ber (Sranander Gdjinder heißt : ein fonverbaree গame, ben id) J̧̧nen balb erfláren will. Heber bem Inblicte einer fdoinen Rasfabe, bie ber ßenibad) ùbex

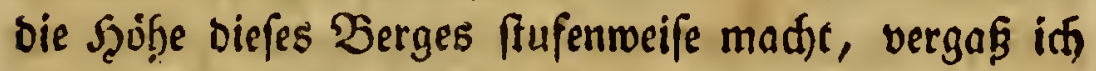

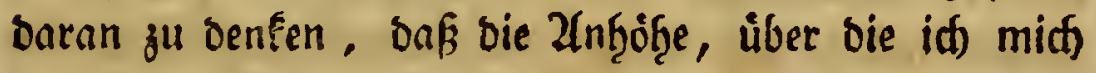
Derfelben någerse, jientlich ftell war. 'S raar gerabe mieber eine Szene für midx), uno id) erinnerte mid babey nidjt of̧ne frohen Empfinoungen ber herrtidten Rasfade, oie bie $\mathfrak{A}(d)$ en in ber Rrimml im Sber pinsgaue mad)t, uno bie firt) mir ist um fo leb frafter

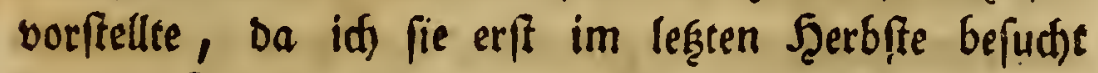
bुatte. Erenlid) ift bort die Maffe von $\mathfrak{2 B a f f e r}$ une gleiđ gróffer. Das zGftüryen Deffelben verutfadte eis 


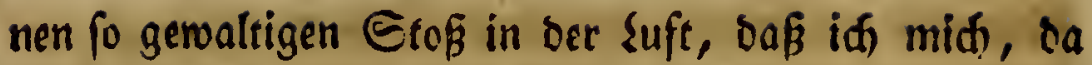

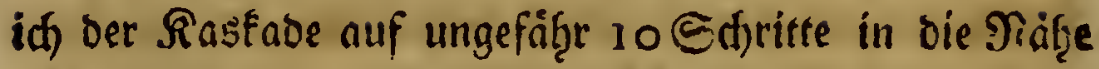
getommen war, geff̧rwind umfefren mußste, um nidht erftict ju merben. Mieine Sileiber waren von oben bis unten naßs von bem jarten Etaube, Der fich vom

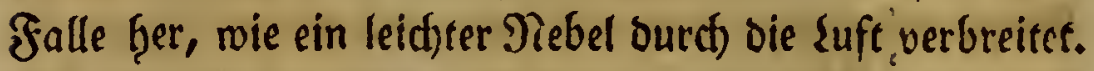
Moidten Gie bier, mein frreuno! eiren Slict in be Suc's fünften Srief unter feinen plyyfifaliff) = moralifften

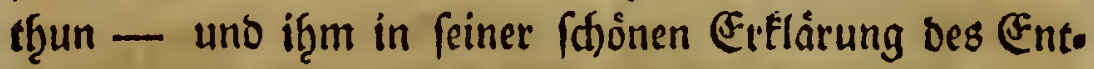
ftę̧ens Diefes zarten Etaubes folgen. - J̦ier fino

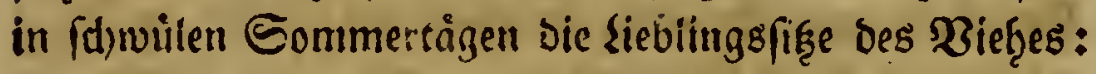

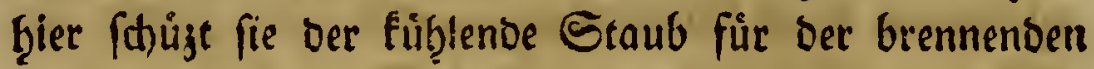

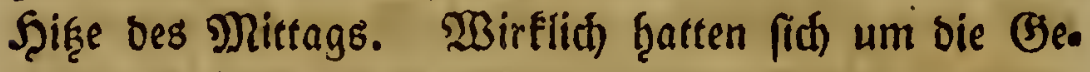
geno Des zalls viele నůţe gelagert, bie izt ruf̧ig ingr Sutter wieberfáuten.

Die Rasfabe, sie in biefem 2lugenblife vor mir liegt, (jatte freylid) fo viele Reize für mid) nidst, wie bie Srimmlifohe : aber id fano fie immer fojón genug : um mid, einige Minuten von iḩr beftáuben ju laffen. Wir ftiegeri nun ben $\mathfrak{B}$ erg weiter Ginan, ber

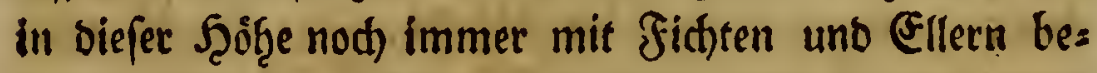
rad) fen war: aber fo wie wir bother famen, bers fhrwanden bie Erlen, uno bie angenef̧me Jaarbe dee Birbelbáume (Pinus cembra L.) mifote fia) In ons

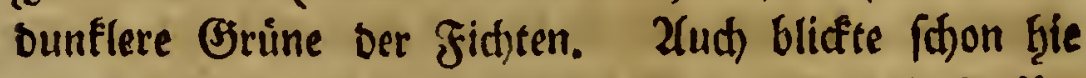
uno sa eine Bawergficl)te (Pinafter pumilio) Geerfür.

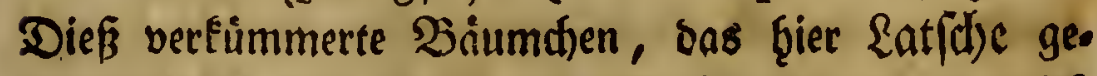
nannt miro, ift Jlbuch of̧nebem befannt genug: id barf es Şlunen nicht năbzer befdyreiben. Die Pinus 


\section{Zell, Den I 3. Scrbfm. I 783 .}

montana, Rrummliolibaum, Eleine SIlpenfiefer,

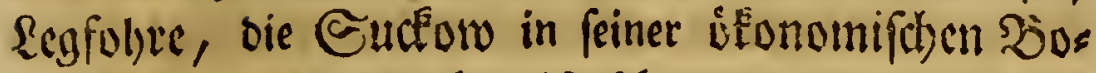
tanif S.22. befdreibe, ift fofmerlid) etwas unders, als unfere 3wergfid)te. Z(u(f) sunct mid) ber Namen Segfol)re mit Dem Proninjialnamen Secferfinude, un. rer bem bieß $\mathfrak{B}$ siumcten in ben oberófterreidfifd)en (Fis. gebirgen befannt ift, einige Berwanofchaft ju haben. Eonft fano ich bier and) bie bärtigte Giloctenblume, (Campanula barbata L.): unb cine 2 (bainderung ter rmbblátterigten (Campanula rotundifolia L.) mit einblumigtem Siengel, bie icl) für bie cinblumigte (Campanula uniflora L) geţalten ḩaben wúrbe,

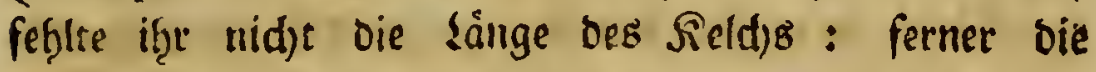
Allpen Johannisberftande (Ribes alpinum L.), bie

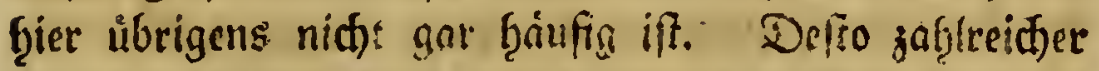
maren bie 2flprofen (Rhododendron ferrugineum

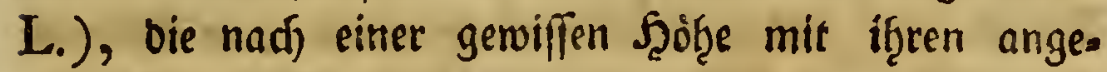
nef̧men rotţen Slüthen Spalieren an Dem $\mathfrak{B}$ ege zu

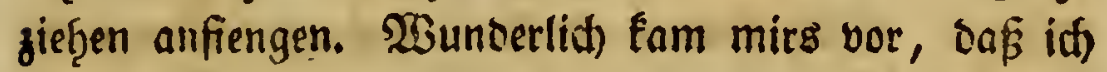
bey ber Menge des Rhodod. ferrugineum 's, bie id) J̧ier und allenthalben auf bem Şege nach bor zallye 2Haregg fanb, nidte einen Etraudy von Rhododend. hirfutum L. entoecfen fonnte.

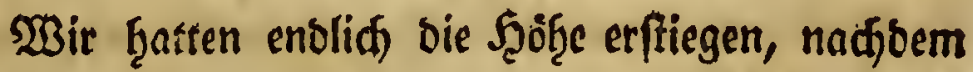
mir ehevor bey einer Quelle vortreflid) füfes uno faltes $\mathfrak{B a f f e r}$ getrunfen f̧atten. Şier fanben wir einen bausbáfigten, blutrothenen Zelpler, ber ßutter uno Säfe cus ber zalpe Schwarjenftein nad SBreittáner

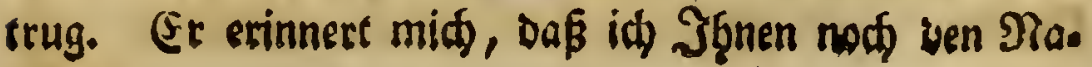




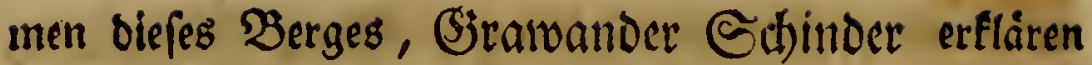
muß. Denten Sie, mein greuno! an ons zurúc, was id) Shnen im legten Briefe ben Breitliner von dem bortigen Sufammenfuffe Der Ergeugniffe aus allen $2 U_{0}$ pen diefer (Segend gefagt ḩabe. Die Melfer von

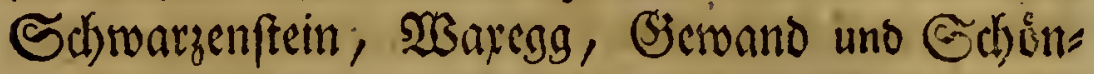
gidfl múfien jeben gweiten Sag, fo lang fie auf biejen Weijoeplázen fino, alle Eirzeugniffe úber biefen berg

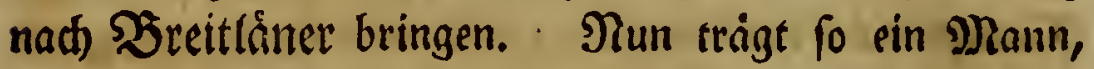
wenn ex eine J̧eerbe von ungefáf̧r 30 Rủben zu bes

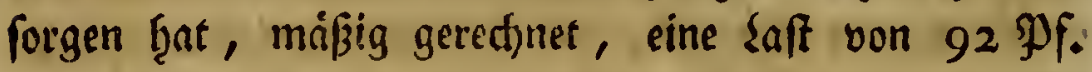
auf reinem Siucfen : id) neţme bie Butterfugel, von

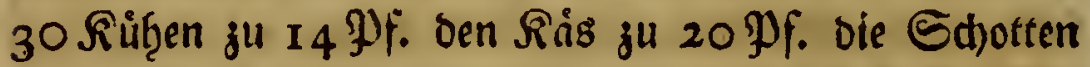
zu I 2 Pf. : von jedem trägt er jwey Stủcfe - weil es allzeit oie Erzeugniffe von jwen Sagen fino- bie Eumme gezugen giebt $92 \mathfrak{D F}$. Diefe laft muई ber Zelpler auf feinem Fiuffen úber einen bren Biertel. ffunben langen, fteilen, fehre freinigten $\mathfrak{B}$ ege abwairts tragen. Sie fönnen oenfen, Daß̧ bießs eben nidjt

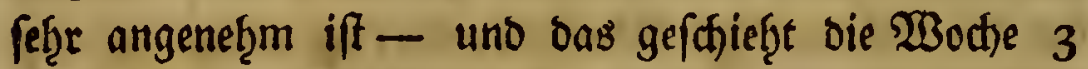
bis 4 mal. Dazu frabe idh noch oie fdomere Maake gering angenommen : in ben beften 2 Bodjen - fie find um Jafobstag - miegt bie Butterfugel von ungefäfifr

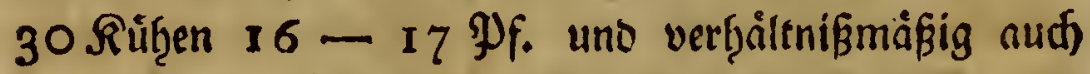
bie Ráfe uno Stjotten. Gie begreifen nun, warum bie Zelpler diefen für fie fiodt)f läftigen $\mathfrak{B}$ erge den $\mathfrak{N a}$. men (Srawander Siljinder gefren. Nur muß̧ ids

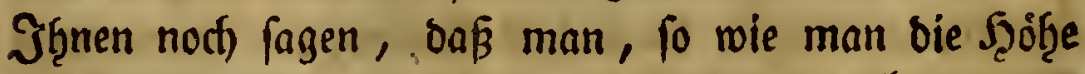
biefes Berges erteid)t Gat, in vie 2l(pe Girawanto tỏmmr. 
3cll, Den I3. Serbftm. 1783.81

Diefe allpe gef̧ơrt ben $\mathfrak{B} e f i k e r n$ ber alpe B̉reito laner, uns liegt fef̧r angenef̧m. J̧̧ৃr Denffprud) ift: 3' Grawano

ift ber Sdinder an ber Şanb.

Bon Pflangen fand id) Ģier nid)ts merfwúroiges, als

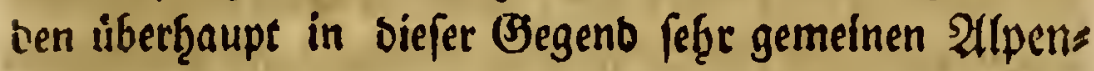
anwpfer (Rumex alpinus L.). Diefe $\mathfrak{P f l a n z e ~ m a ́ d s t ~}$

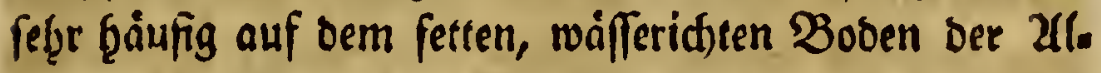
pen, vorzugglid runo um bie Eennḩutten, welther Pla Gier bas Dród (vermutţli id) trett von tretten,) genennt wirs. Da bas Bief fier am offten jur Melf zeit verfammlet wiro; fo wirb biefe Stelle inmerfort burd) bie Rüf̧waben gedüngt. Unterbeffen find bie allpen ampfer nidfts weniger als ein gutes fFuttertraut, auffer soenn fie nod) feģr jung fino. Eben ber gute Bober, in bem fie fteţen, ift bie Uurfact)e, baß fie gar zu fónell aufroadfen, uno gar zu ftarfe Stengel uno Blätter erḩalten, bie das Bief̧ nidjt meţı freffen will.

Der $\mathfrak{B e g}$ warb nun immer fteinigter : aber roas follte mir bieß für ein Şinberníß fenn? izt, ¿a

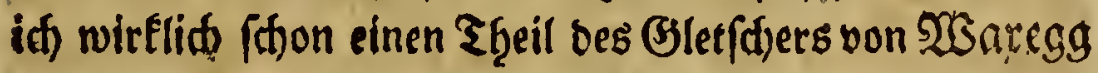
vor mir liegen b̧abe? - Lieber Freuno! id meiß nidt, ob Gie fiton Eisberge bereifet ḩaben: aber was idf ben oiefem eben fo fdionen uno prächtigen, als

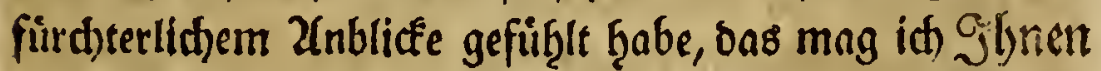

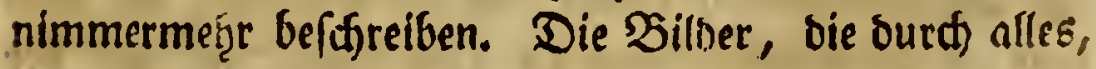
mas ich) von biefen groffen Eridbeinungen in ber Tatue

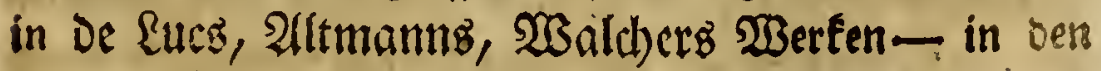

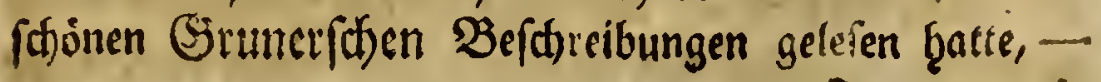
Faturbift.25riefe 1. 25and. 
in meine Geele gegraben waren, veiffirwanten, als id) Die Natur felbft faţ. Eo fdtoon, fo unnad)af̧mlid) zum Theile bie Befdreibungen ber Eisberge, von fo groffen Naturforfahern entworfen - fo meifferb̧aft bie Beid)nungen ben Giruner, uns in ber franzöfifden Encyclopedie fint - aber bie Natur, mein fret1!o!um wie viel fdoner - unnadjalfmlidjer id fie! Slods tyabe id)'s nie fo ftare gefingle, wie wenig 2 borte bas ausorưden, was man empfinoct, als in tieferm 2lugens blicfe. Mein 2lug war zu fibre an ben Bjletfher ges beftet, als baßs id) nid)t dariber mand)e (d)onne PDfanze überfeben bzaben follte. Unteroeffen erreicten wir bie Waregger Sergmäber, ober freilen $\mathfrak{B e r g w i e f e n , ~ m e n n ~}$ Eie mollen. 'S war ein fonderbares Pfat, das uns Daf̧in füfirte. Denten Gie Gid) einmal über ben 216 . ḩang eines betrådtliden Şgeiles bes Berges, an bem wir hinmanderten, eine fürdstetlidse Decfe von binnfen Eteinen- eine eingige, weitfd)id)tige Eteinblatte, bie ijt vom nádjtlichen Regen wie Spiegel glånzte : in ei= ner fdiefen sinie über biefe Decfe hin befertigen Gie

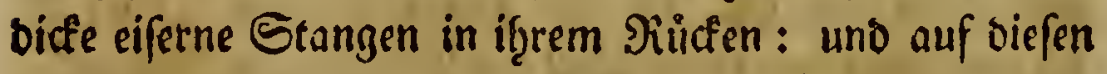
fonderbaren Grund bauen Gic Gith eine lange,

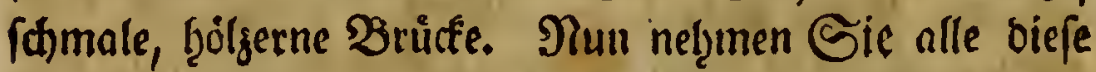

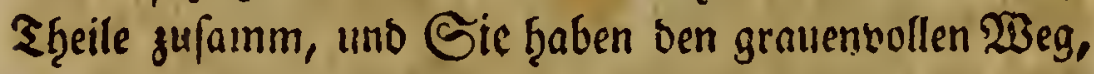
ber uns nad) ben Bergniabern füf̨rte. Was if̨n nod am unangeneismften madte, ift bie 2usfidite, bie man bon ba in eine abfdjeulidje Tiefe ḩat, in ber bee Bembach) fliefst: man fofjwebt gleid)fam über berfelben ouf biefer Brride - uno fief̨e fie nur gar zu oft burd) bie Sugen zwifhen ben Báumen, auz detten bie $\mathfrak{B r u d f e}$ 
gezimmert ift. Da biefe gefährtlitfe Straßge füt jebes Stúf $\mathfrak{B i e f ̧ e s , ~ b a s ~ i n ~ b i e ~ 2 ( l p e n ~} 23$ aregg, Sdhwarzenftein uno Sdjenpid)t gef̧en, ober von Dafier jurúcffommen foll, unumgainglid) iff, fo liegen auf ben Báumen immer feg̨r viele Ruţroaben, bie Durch ben Diegen anfgemeid)t, oie Uriacte maren, oaß i(t) beynaḩe mit jebem E(t)ritte ausgleittetete. Frey. lid) iff ein (Bselänber angebrad)t, weld)es für Menfd)en unb Bief̧ ḩud)ft notf̧wendig ift; aber ben allem bem bleibt diefem 2 Bege nod) vieles, bafür ber Stndrmann

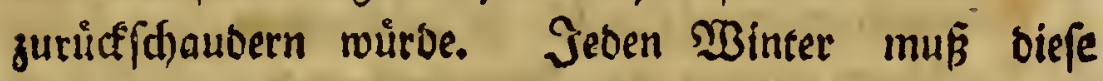
Bructe abgetragen, uno erff, menn aller Edjnee in Diefer S'́egeno abgefalgren iff, mieber angelegt werben: meil fonft ber Sdinee, ber fich auf biefem blanfen Eteingrunbe nid)t b̧alten fann, immer alles mit lï in bie Tiefe füb̨ren wưrbe.

Sheidflid) warb id) bafuir burd bie (Enbte, bie (d) auf ben Bergmàbern für bie Botanif madte, ents (t)åoigr. Sith flimmte an biefer freilen WBiefe, to un. angentę̧m es ben ber Nâffe roar, ganj begierig ḩerum, und wenn es Eteflen gab, an bie id mich) bey aller meiner Mann(eit niche magre, fo warf Jjtoffer bie

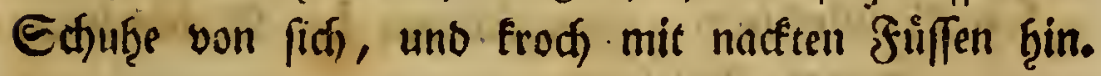
f̧ier fammelte idf bie (Srasart, bie id) Şlynen unter Nro. 2. zu fenten bie Ëbre ḩatte, uno bie Sie fir nidjts meiters als Juncus campeftris L. Galten : eine anter $\mathfrak{B}$ infenart, bie Rinné inid)t verzeidfinet : wob̨l aber SEupuli in feiner Flora carniolica. Edit. $I$. p. 225. unter Dem Namen Juncus foliis planis 
culmo unifloro befdrieben bat, Da fie vielleidt nods feinen Trivialnamen ţat, fo erlauben Gie mir felbe Juncus Schrankius ju neunen. Ferner maren b̧ier

Primula minima L. Eleinfte Sdblüfiétblume.

Geum montanum L. Bergmergourg.

Atragene alpina Xipenatragene.

After alpinus L. a alpenfternbfume.

Silene acaulis L. Etammlofe Eilene.

Achillaea atrata L. oas gefdenarzte Zddillenfrauf.

Gentiana lutea L. oie getbe (smgiane : (d) on verblüḩt.

Convallaria majalis L: Maablůmaden.

Tuffilago alpina L. a(tpenţuflattic).

Imperatoria oftrutium L groffere Meifferwurz.

Orchis pyramidalis L. piramibenförmiges Rnas benfraut.

Lilium martagon L, bie Berglilie.

Thymus Acinos L. ber alipenquentel.

Satyrium nigrum L. fdimarzer Etendel.

Fìligo Leontopodium L. Bergrug̣rértaut.

Hieracium alpinum L. Zflpenf̧abidtftrauf.

Osmunda lunaria L. Monosraute.

Valeriuna officinalis L. gemeiner Balorian.

Hypochneris maculata L. geflectes Gaufraut. Chryfofplenium alternifolium L. Weidffelbläto teriges Miljfraut.

Globularia cordifolia L.Şetzblätteridter Mlaffüffel. Aretia alpina L. Zlpenaretien.

Leontodon aureum Lo golofärbigter sómenzafin. (Enolid) - Inula provincialis L. - Aftragalus Cicer Lo - Polygonum viviparum L. uno Hedy- 
Sell, Den $13.5 \mathrm{serbftm} .1783 . \quad 85$

farum obfcurum L. wie Gie mid belef̧ren, nidfe Hedyfarum alpinum L. fuir das id es b̧iclt. Bromiß nußs ser unterf́rsies zwifthen biefen zwoen PPlanjen fef̧r gering fevnn. Şert vọn Şaller b̧ielte fie mirelid nur für eine 2(rt, wole id aus einer Xlumerfung in

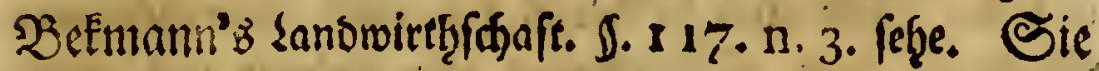
werden mid Shtunen allo fef̧r verbinden, menn Sie, mid) ourd) oie ver(prodgene Uteberfenoung eines Hedyfari alpini L. Diefen Unterfájies fenuen lef̧ren. $\mathfrak{D a}$ id) zu feftr oen (Eisbergen zueilte, fo fammelte id) fiir

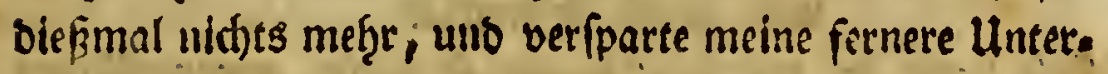
futhungen bis zu meiner Rilidfunft b̧ief̧er. Ifl bem. feinigten $\mathfrak{B}_{\mathrm{ege}}$, Dem Bembade ju, ftanben bee Scetfftecintect) (Saxifraga autumnalis L.), Der moosartige Stcinbrech (Saxifraga bryoides L.) ferner oie Saxifraga mutata L - aber mit weiffen Blumen, uno oer fajone Steinbred), in bem Sie bie Saxifraga cuneifolia $L$ : zu finben glauben : auds wảdsst úberall hier bie Ziwergbirfe (Betula nana L.)

Während daß i id biefe Pflangen fammelte, fam id) unmertlid immer nảber an bie Eisberge: war

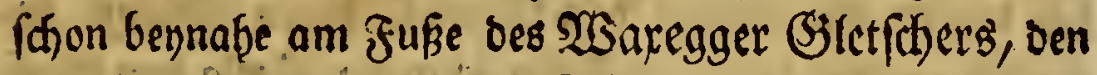
alpḩutten, vie nut menige Sd)ritte vom Bletfiter jen. feits bem Senibaches liegen, gegenủber. Zugfeid) Fâ̧

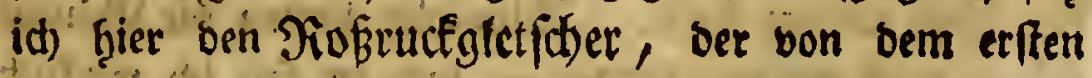
nur biuld eillen fhmalen felsterg, Riofrute, abge. fd)nitten ift. Uebrigens parallell mit ibm läuff, uns fict) von Noro gegen Siro allmáf̧lig in bie $\mathfrak{B}$ ollen erţebe. Эd) blieb f̧ier lange Reęn, une ließ mir von 


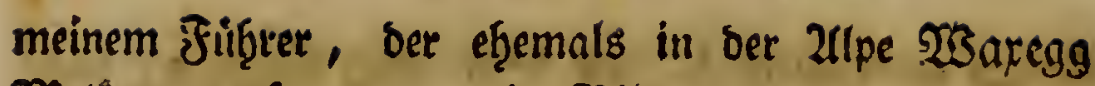
Melfer gervefen war, ein 2 illo von den Eisbergen unb ifgren Eigenfdjaften mathen, barunter freylid) aud) mandfes Jabelwert war, wie 3.3 bas fiebell-

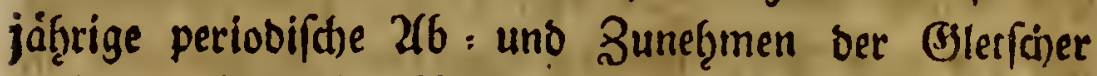

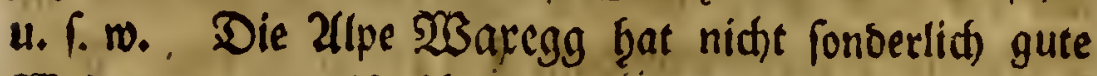
Weibe - und ift uberḩaupt fef̧r fteinigt uno rauf̧. Dab̧er iḩr Denffprut) :

$$
\begin{aligned}
& \text { j' Waregg } \\
& \text { gibts Fleine Butter, } \\
& \text { uno groffe Sd)ottfát. }
\end{aligned}
$$

Эd) Ģafte in oer Tadjbarfdoaft biefer unermeglidjen Eismaffen nidfts anders als eine fürchterlicte Rälte

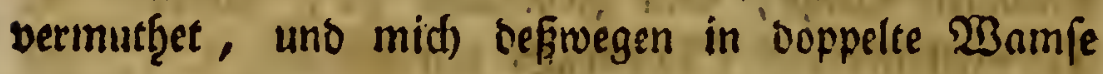
geftecft: aber zu meiner Bermunderung lifpelte hier ein angeneţmes, laues sůftgen um unfere Şaare. Mein Bergnügen barúber. Dauerte nid)t lang - Denn id er. innerte mich) balo irgentro gelejen zu halien, bás laue Winde aus ben (Eisborgen fier Siegen verfünden, uns fieng an fef̧r unzufrieben mit oiefent, Bephigr zu werben.

WBir fegeten unn unfere Эeeife weiter fort. Bis. heer racen wir immer von Norboft gegen Süboft ge. gangen - nun fübrte mich ein f(t)maler Steig von $\mathfrak{B}$ eft gegen Dft ber 2(lpe Ed)warzenftein zu. Dicfe war bas Biel meiner গieife. Zulumäḩlig ḩatten bie Bäume fidi) zu verlieren angefangen - uno ift fah id mirfiich auf eine Strecte un midh ḩer nid)t einen eingigen, nidts als f̧ie uns ba verfüummerte Fiefern (Pinafter pumilio), bie fin freundlich ineinander fdlungen. 
Bunveilen blicte aus bem Grafe bie gologelbe Blütf̧e bes Geum montanum L. : fonft war hier feine meb̧r von ben SBlumen, bie id) auf ben $\mathfrak{B}$ ergmábern fano, zu fę̣en : von Grasarten war bie b̧åufigfte ig̨r Juncus monanthos - er gef̧ơtt unter bie fauren Gsaáfer, unb iff fein gutes Jutterf́raut fưr bas Şornvieh : für श) ferbe burfte er etwa beţaglider fenn. Id ḩatte Gier wipoer einen fehtr unangenef̨men $\mathfrak{W e g}$, um in bie allpe Sidwarjenftein zu fommen. Ein Pfab, ber faum

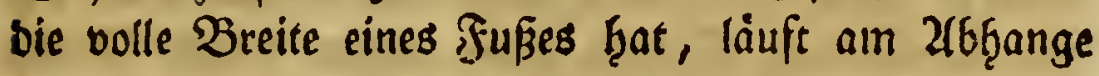
einer wáfferid)ten Bergwenbe b̧in , tief unter ibm rollt fhaumeno ber Zembad) : onju ift oiefer f(f)male $S_{t e f g}$ nod) fo ziemlid) fdief ber Siefe zu, uno sa er fid in beftánsigen Sirummungen an einem fef̧e moofigten

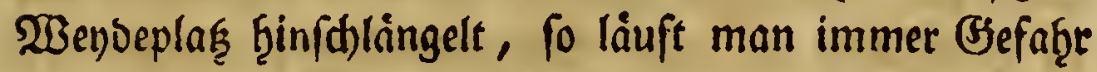
ausjugleiten. Man würbe bey bem geringiten fsef̨l.

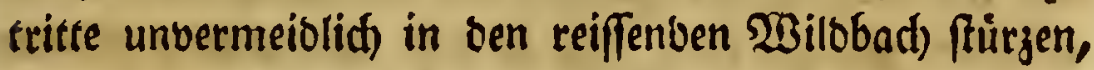

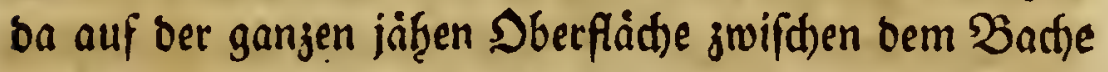
uno bem Steige nidt) eine Staube ift, bie if̧re woḩ. tģàtigen Zefte jur Rettung leib̨en fóntute. Man wage es nid)t, bas $\mathfrak{B i e f ̧ ~ d i e f e n ~} \mathfrak{B}$ eg geţen zu laffen, fondern es wirb unten in ber Tiefe ůber eine fleine Bride an bie (Jegenfeite gefüh̨rt. (B)lücflid) gieng's barúver weg, uno nun war id in ber alpe Gd)warzenftrill. Meine

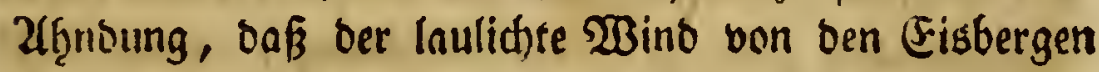
ber ein Siegenbote fern wurbe, fieng mir nun an bee ftåttigt zu werben; Denn immer mef̧e waro uns ber 2(nblicf bes fajoinen lafurblauen Şimmels burd) trübes Bemoilfe entzogen, oas fid) nun fetge fannell über unfere Edheiteln verbreitete.

$$
84 \text { Stwars }
$$


Sdimarzenftein ift bie biodffe Zllpe in biejer

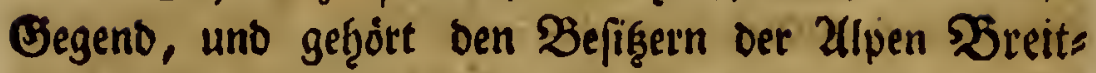
laner, Rlaufen und Grawand : eigentlid) find biefe 4 Zlpen nut bie Theile, ober verfffiebenen Raiger einer einzigen. Diés wirb Stbnen in einem folgenten Briefe flar werben, wo idf meg̨r über bie Defonomie auf ben ZIlpen fagen werbe. Dic Genngürten fint hier fệt nadbläßig ans Steinen, Die mit feinen Mörtel verbunben find, aufgefübrte , weil man auf eine betrádftlidje Strecfe runb fierum Peinen $\mathfrak{B a u m}$

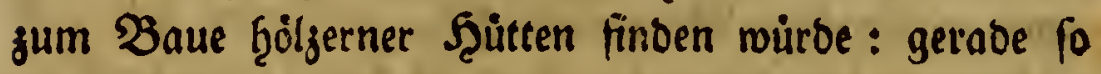

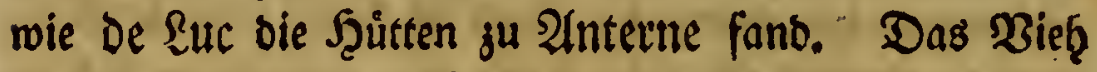

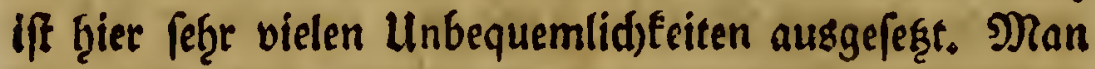
Eennt hier taum ben গiegen: benn er verwanbelt fii zu gefdininbe in faltes Safneegeftober : ba nun, fo roie auf ben meiften Zllpen, fúr bas $\mathfrak{B i e f}$ mit feinen Stállen geforgt if , wo es ben fuutrmifferem 2 Better Sdugs finben fönnte : fo muß es nidft felten megreere Tage im neueı Sd)nee unfer frevem Şimmel jubrin. gen, weldjes frenlidh nadjtţeiligen Esinflußs ouf ben

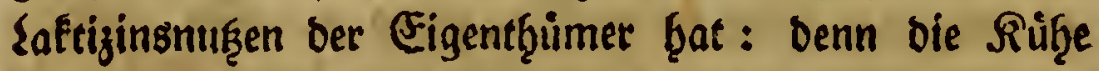
fónnen an folden sagen nur feģe wenig millden.

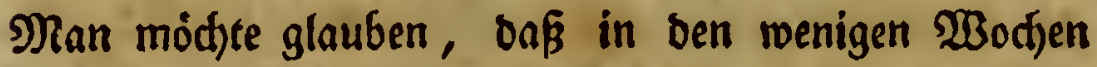
bes ţödfften Gommers, bie bas Bief̧ auf diefen ţoḩen Wenbeplägen zubringt, Die $\mathfrak{B B i t t e r u n g , ~ m e n n ~ n i f f i t ~}$ immer feger gemásigt, bod) niemals fo raub fenn fónnte, um biefe Gegenten mit frifdem Edjnee gu becen. Zaber man würte firf) babely irren: wirflidg

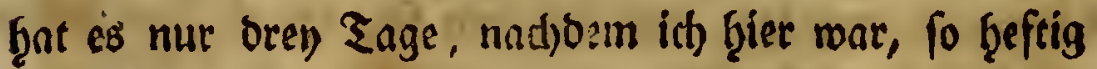

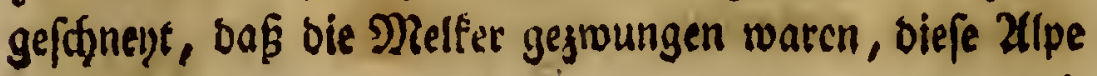




\section{Bell, Den 13. Serbftm. $1783 . \quad 89$}

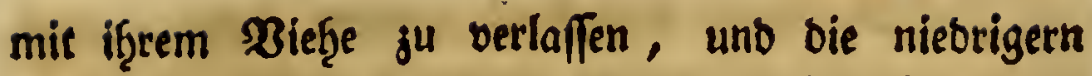
כBenden ju Giramand ju bejief̧en. Şier bleibt ber WBinter immer Şerr im Şaufe - nie verorängt lign ein Srüb̨ling ober f̧erbft baraus, uno man fönnte fagen, daßß ex in ben wenigen, fdoonen, heeitern Tagen, bie man bier geniebt, ben Sommer nur als (5aft bey fid bemirtye: Der Denffpruch) biefer 2lipe ift:

3' Schwarzenftein,

fleine Watel (Waben) groffe Bein, uno Enfel wie bie Sentnerftein.

Proffer finf̧rte mid) in eine Zlpḩưtte, in ber in) einen lebįaften, roţ̧bàrtigen, mof̧lgewadffenen Melter antrnf, Der feb̧r leutfeelig uno gefällig mar. Эळ) ruf̧te ḩier uur wenige Zugenblide aus - benn, Da es immer trúber am ફ̧orizonte zu merben anfieng, uno id) vermutffete, bie $\mathfrak{B}$ itterung moidste fid) gang in Ernfte in Ednneegeftöber um[daffen, to eilte id) ben Sdywarzenfteiner, oder Sorngleticher zu be. firdtigen. Die Jaútten liegen fef̧r naf̧e an biefem

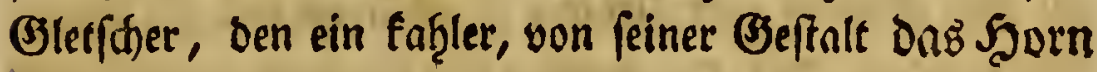
genannter, Granitberg vom Siokrufgletidter fdeibet. Woir mußsten, um ib̨n ju befteigen, úber ben Bembad)

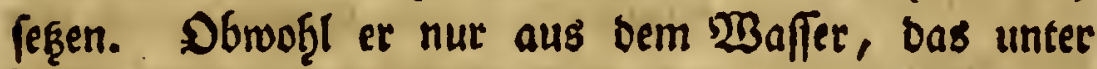

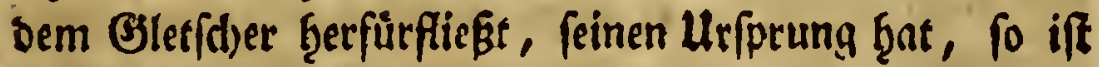
er bod) Ģier fdoon ziemlid) breit uns tief : und ba in Diefer Obegend nirgentes eine Brücfe Darúber gejajlagen ift, fo fiel id,, ungead)tet mir mein f̧üḩrer bie Şand bot, zugleid) tief in bas $\mathfrak{W B}_{\text {affer: }}$ aber nod foflimmer gieng es meinem Srìger, ber fidi) enolid), nadfbem 


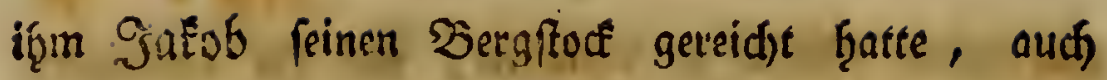

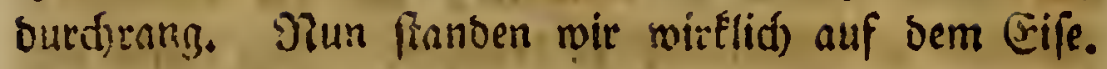
Der erffe Edjritt, ben id) auf einen (bjtetf(d)er thgat! Da er firts bom funfe- weg eben nidsi fo gar freil, fonbern allmálshig ertgebt, fo warb es mir gang leidfte

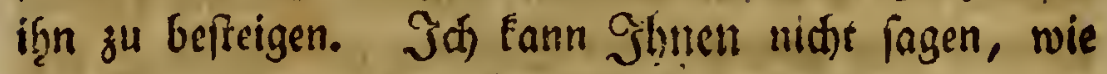

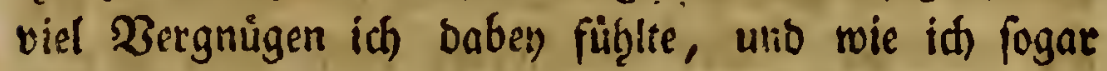

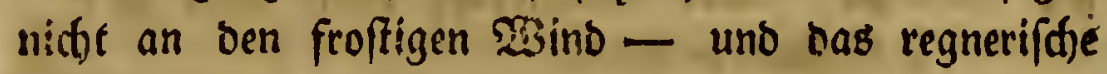
2 Better badise, bis mid) meine erfiartre Şänbe Daran erinnerten.

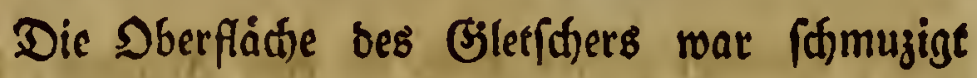

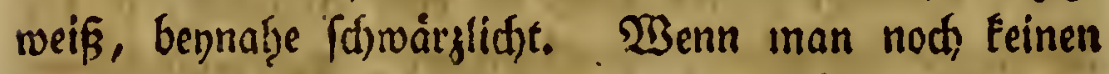
anbern, als ben gewớçnlidgen 23 inter fdrnee ouf fladem Sanoe, ber fein Zlter erreidste, gefetęen Ģat, - fo fann

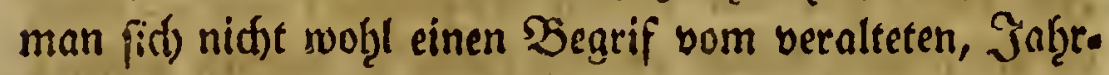

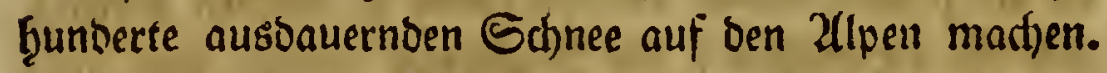
Eie war ferners ḩófridft, an vielen Stellen, wie mit Sano ủberfizeut - nirgenns fo geglättet, wie Eis. baf̧nen auf bem sanbe - aber audif nirgenos naţ̧m

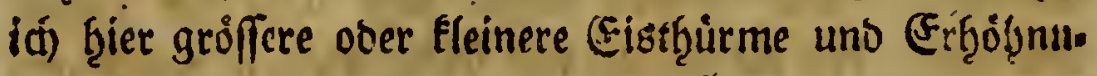
gen gerwafir, wie bie meiffen ben) Struncr gezeidineten

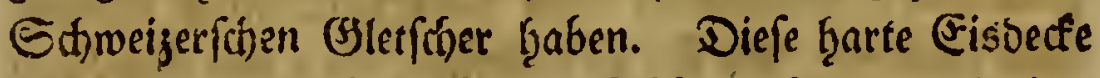
wour übera!l burd) unzåălige Slüfte gefpaltet, bie im. mer tiefer wurben, je Ģóber mir ben BJletf(d)er Ģinan flimmten. Zllle bon mir bemerften Sluffse ftanoen redtetwinflidft auf ber Bafis bes Bletfiders, fuir bie id) feinen zlusgang unten am Zembad)e annaf̧me:

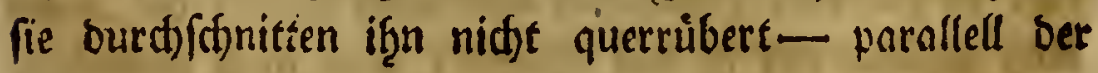
Bajis- fonbern, wie ing fagte, parallell ben Bergen, zwifden 


\section{Bell, Den $13.5 \mathrm{Serb} f \mathrm{fm} .1783 . \quad 9 \mathrm{I}$}

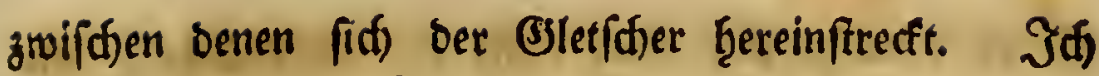

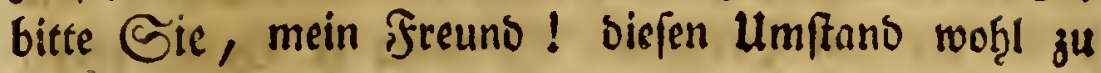
bermerfen - Denn id werbe im folgenden 'Briefe, wo id) Slgnen einige zufällige Bebanten ůber bie Eigens

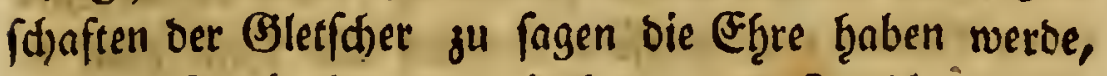

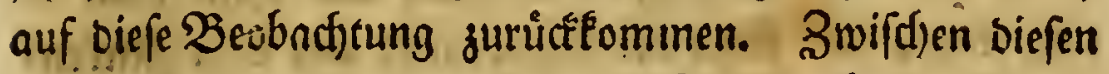
Silüften, Deren einige meţrere Ectuhge breit waren,

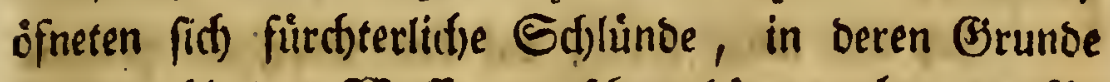
man woogl bas 23 affer rauffden Ģorte, aber es für

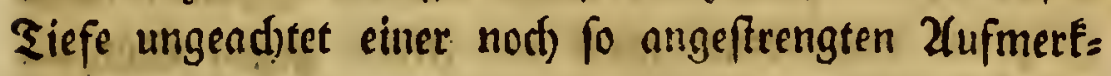

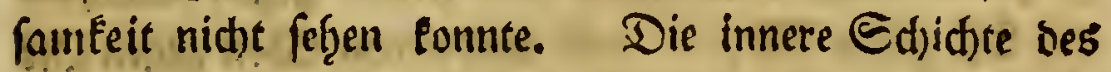
Eifes jeigte firt) in biefen Siluften, fo weit id bie J̧arbe woofl unterf(d)eiben fonnte, meergrin, unt dos Dunfel ber Farbe ftieg in bem nàmlithen $\mathfrak{B}$ erf̧äalts niffe, wie fie fict) meģr bem Brtunbe năḩerte. Der

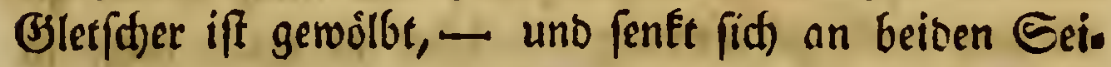

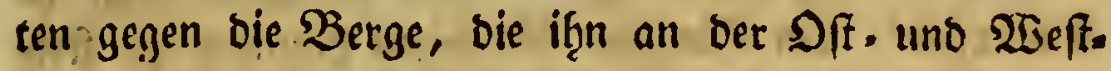

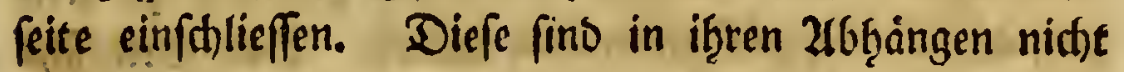
mit Eis beoeft, fonbern naftes Jelfengemánte. Gie

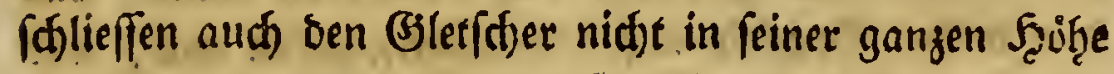
ein, fondern fie verlieren fidf nads und nart), uno Der

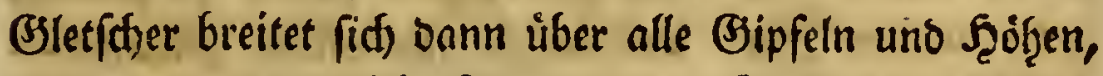
wie ein unerméflid) MReer, aus. Snvifden if̧m uno biefen Eeitenwaínben liegen máfige Giråbden, ourd) weldje in Eleinen Biunften Edgneeroaffer làuft: aber

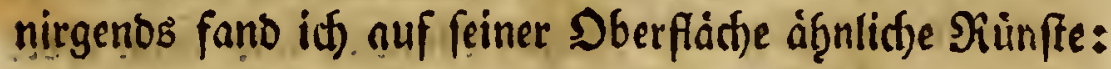
wogl afer an metgreren Stellen gróffere uno fleinere

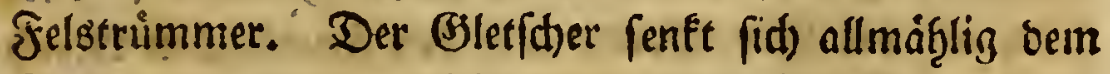
Boven ju, er wirb nidjt ourdh eine jăḩe Eismano ab.

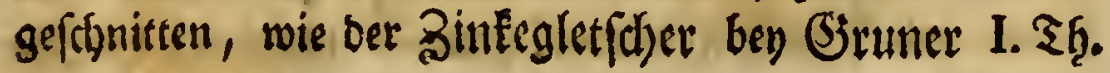


5. 48.: enbigt fid) aud) nidft in Eisfdruntoe, roie

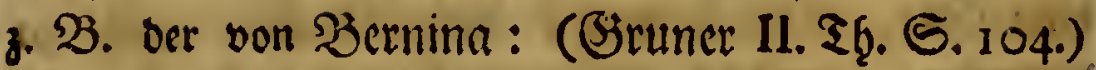
fondern verliert fict) fanft in ben 3 embach), ber if̧n nach feiner gangen $\mathfrak{B} a$ fis hiin benezt, uno in berfátec benen fleinen uno gröfern Suellen aus ifim eben fo entfpringt, wie etra bie Snuteraare aus bem Binfens gletfiter.

Jd) rar lange genug allf bem (Bletffher b̧erum. geflettert, - Gatte iber cinige fleinere Spalten über: gefezt - taufenomal mid) vori Noro gegen Suid, von Dff gegen Weft - und wieber von $\mathfrak{B e f t}_{\text {gegen }}$ Oft getef̧rth, und alles rund um midh betrahtet und bes nunbert, als mich meine farte Şande uno ourdigea nejten Silciber erinnerten, baß̧ es nun aud) Seit wàre;

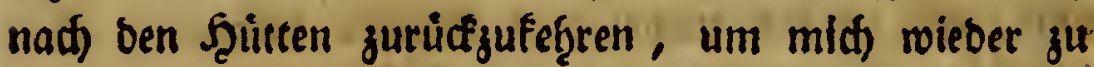
wärmen und zu troffnen. 2(ber es marb nid)t fo

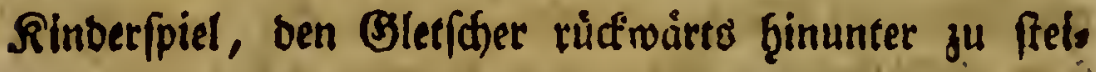
gen, als wir bergan gefommen waren. Das Bemirdhe von Regen uno Sdfnee, das einsweilen immer aus bem truben Bewoilfe auf uns niebergefallen wair, uino an bas wir biz izt nicht Zeit gețabt lqatten zu benfen; hatte unfern $\mathfrak{P f a b}$ abfd)eulid) fallipfrigt gemadit uno id) gleitete anfangs bey jesem Sdyitte aus. Da uns ber $\mathfrak{B}$ eg mandfmal zwifden zwo Spalten burdjo fúţrte, fo gaätte id fo ganz bequemlia ben einem Zuso gleiten in ben adbgruns fturzen fónnen: uno denn Gott befoģlen, Sie unb alle meine lieben Sreunbe ! Nie haatte id bie liebe Gonne uno ben freundidjen $\mathfrak{M}_{\text {onb }}$ wieber gefegen - Gắtte nimmermę̧r zu meinen Jno

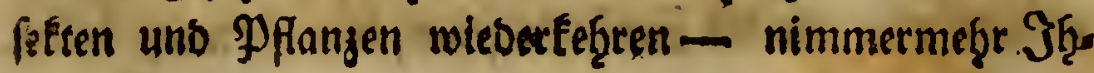


nen bon nflem belli, ras id) Ģier fạ̧ und faunte, eine Sylbe fagen fönnen. Xlber 's wat bod fo arg nech nidt. Mein fdarf gepipister Etocf - meine ge,

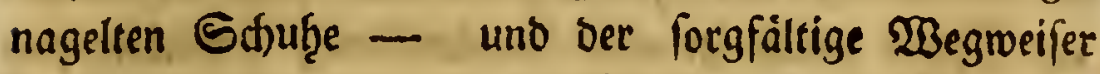
bradten mid) gut unb wob̧lbeţalten, wie Sic mid)

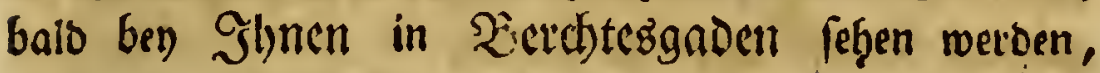

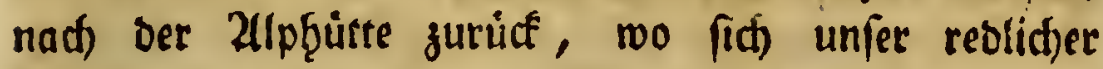
Wirtţ eben bereitete, Mittagfuppe für uns zu Podjen.

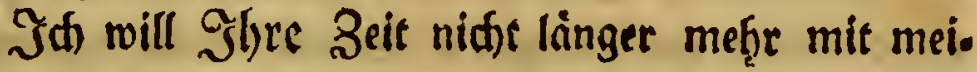

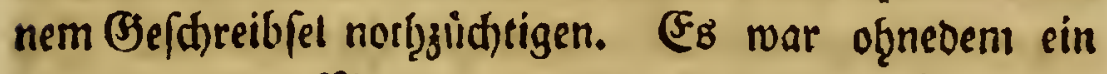
ḩerglida) langer Morgen um ben ḩeutigen. Im nàd), ften Briefe will id) Sie jum Gee am rotl)en Siopfe fübzren. Jab bin unwandelbar

J̧̧ז Moll.

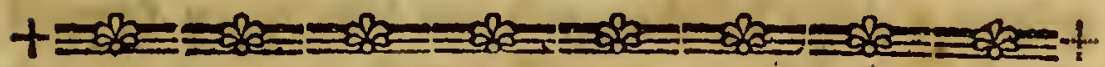

\section{Siebenter $\mathfrak{B r i e f}$.}

Bell im Billertlyall, ber 10. Ditob. 1783 .

Stumbenred)nung Der alpenbewolner. 2(ud) etwas von ibrer Stcrnfuntide. Sicife nach cinem Eisfee. Werfatedene atpenpflanzen. Ein neuer Gdumetterling. Einige Rifer. Eisberge. Ueter.

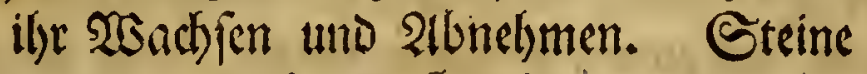
auf Den (Sietfdern.

9) ber. fẹgen Sic bocí) einmal, was es für ein art (iget $\mathfrak{R o d}$ ift um einen Melfer : eben bereires er mir ein Ruß , ober, wenn Sic bas Ding lieber 
fahmeizerifof genannt ḩaben wollen, eine Stunfenwerne. So angenef̧m mir's in bieferm 2lugenblicfe ift, an peine Manipulation baben jurùct zu denten, fo gerne moidte

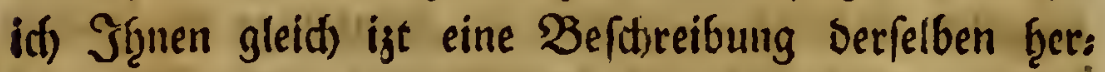
fegenen : aber id) b̧offe noth beffere Oelegenţeit in fol: genoem siriefe saju zu finten.

Woif̧rent bem mir in unferm Baftģnufe eine

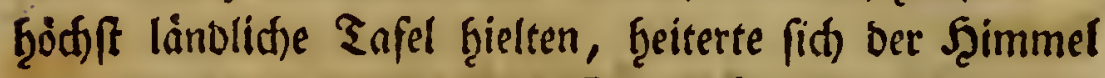
fanft mieser auf, uno bie Sonne blidfe mit berbop.

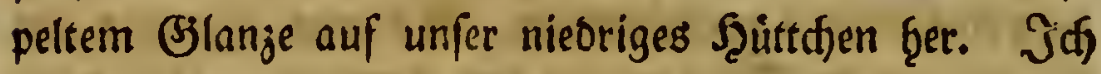

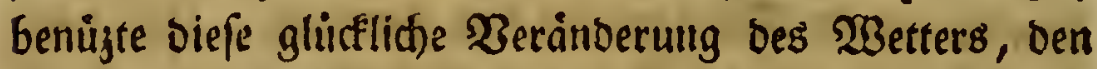
Melfer unt bie Etunbe bes Tages zu fragen. Unge.

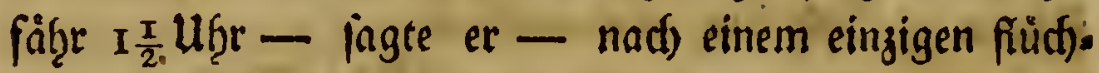
tigen $\mathfrak{B}$ licfe an bie Sonne, gerabe, wie wenn an ber golonen Sdeibe da ohen, und am lnfurblauten ફ̧immel um ibre bie Biffer fo beutlich gezeid)net wairen, als on

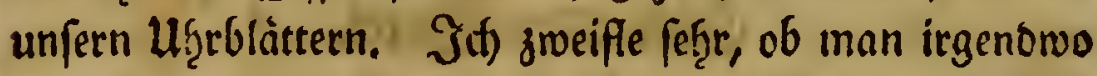
fo genau uno to ḩurtig bie Etunoen bes Tages blok aus bem 2 Unfdachen ver Sonne anzugeben weißs, als es bie alipenberwof̨ner tḩun. Gie werben felten eine ḩalbe - ifters feine Biertelftunde fef̧len. Die Entz fernung von Dơrfern, noo ifgnen ons Bifferblatt am Blocfentf̧urme bie Etunbe fagt, und der Rlang set Ǵlodfe ben Mittag varfindet, f̧at es if̧nen notḩwendig gemad)t, auf anbere Mittel zu benfen, surd) vie fie bie Stunben bes Tages moglidift genau ju beftimmen, in Stano geferest wuirben: weln fie anderft die fo nơtţige Dronung in iţren lanbrirtthi(d)aftlid)en (B)ef(b)aften ţa: ben wollten. Das erfte, was ben 2(elplern baben in 
Sell im Blllertlyall, Den r 0. DEtot. 1 783.95

bie 2(ugen fiel, war zanz natürlić) das 2(ufgeţ̧n ber Eonne, uno iḩr Untergeḩn. Es war eben nidft vicie Beurtheilungsfraft Daju nơthig, um den Edfluß zu macten, Daß̧ man um fo viel weniger im $\mathfrak{Y}$ Sormitraye

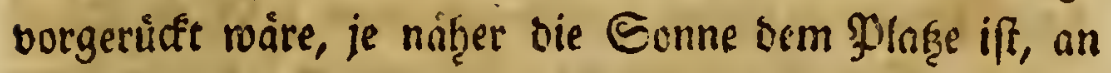
bem fic aufgieng, - uno daß fict) Der Nachmittag eben to gegen ben af(eno fenfte, wie bie Conne fid dem Plaze des Untergchens näbert. Daraus gab fich nun

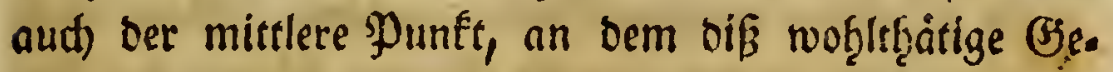

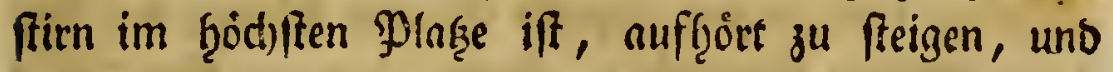
gegen ben গachmittag zu finfen anfängt. Dif gab

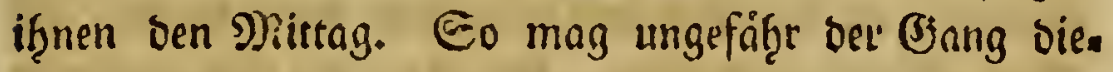

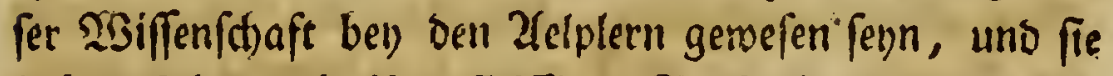
Ģaben fid) Durd) if̧re feeifligen $\mathfrak{B}$ eobatt)tungen in Den Etand geféğt, aus ten verfbjiesenen Etellungen der

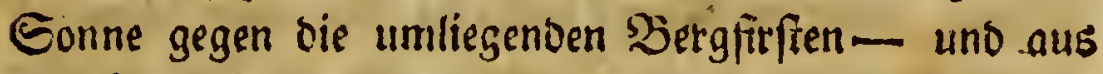
Dem Sortfdreiten bes Scthattens und Sidftes in Dffen

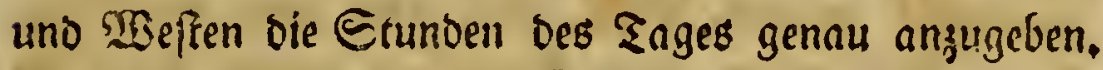
Der 2elpler meiß in jeser Gafrobeit - in jecem $\Re_{0}$ nate - bon dem 2fufgehnn ber Eonne zu fagen: F̧eut

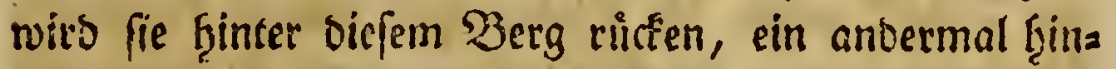

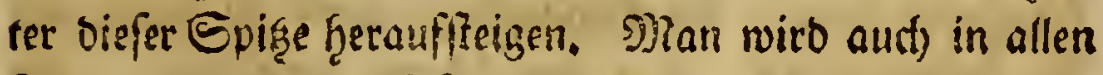
Serggegenten genviffe 2 erge finben, bie man befonters

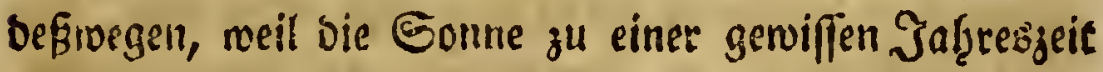

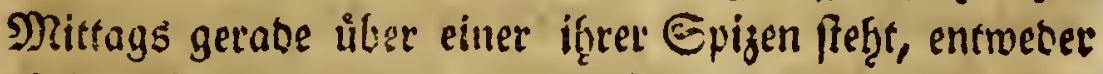
Mittngipişe - over Mittaghorn - ober Mittags

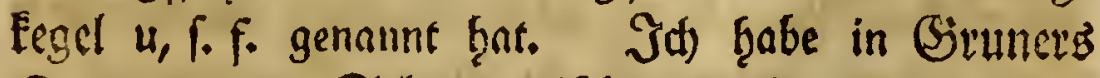

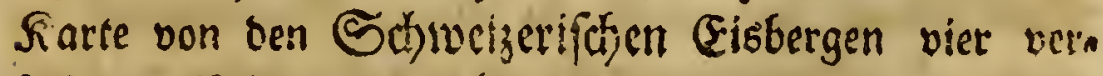
ftieoene sinittuglyorne bemerft : wie viel werben derent nod) unter ben Edjyeizerfden sBergen fenn, bie nid)s 
Cisberge find? Nod) erinnere id midh bon bem Rremsmumfterfden Sanogute Sdjárnftein in Sbers

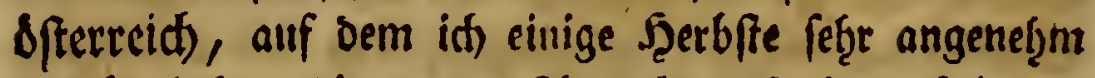
berlebt Ģabe, bie gerabe 2(usfidtet auf einen Mrittags fegel geţabt gu bुaben. Eo ḩat man 3. 20. in Tilol auf ber 2tlpe Struben im Yyfeggeridte Dirgen einen Nittaglopf in Befidte u.f.r. Die Sammlung ber

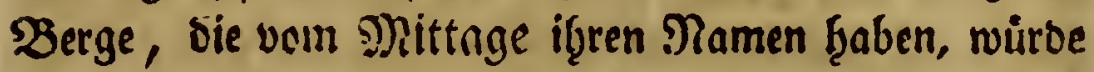
allein ju einem máfigenßante madfen. $\mathfrak{B}$ enn aud

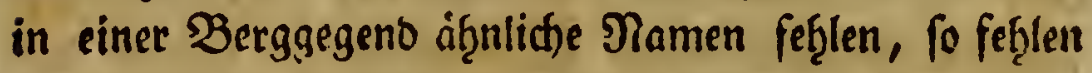
bod) niemals Spigen - Bergrücfen - ober Jels. (h)atten, bie ben Zlelplern vorzuglid) baju bienen, bie. 5) Rittagftunde anjuzeigen. $U_{m}$ nur ein Benfpiel von Der Begeno, in ber id) Shonen bieß (dreibe, ju geben, to fraben in ben Sommermonaten bie S3erlesberger Mittag, wenn bie Conne fentredte ùber einer fámalen Epalte zroiffien ţongen $\mathfrak{B}$ ergen, bie fie Das bumtle Rlúmmel uennen, ftefit, uno felbe gans erleudtet, sa fie fonft wegen ifger Enge ben ganzen Sag über

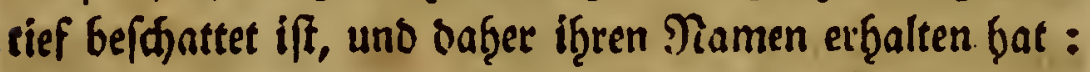
bie Zeller, menn oie Eonne ùber bem Gierlositein= Eugeldhen fref̧t: bie Şiggacher, wenn fie gerabe auf bie Triftenfpige, ober ben Jaun ḩerabfieg̨t. : (Fs fdjeint biefe Beitfunbe ber 2felpler fef̧r alt ju fenn, beiss

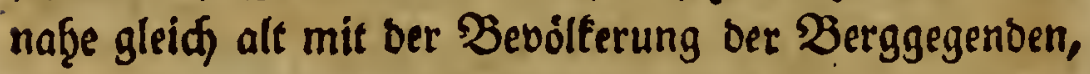
und bem baraus entftefenden Bebirfnife derfelben.

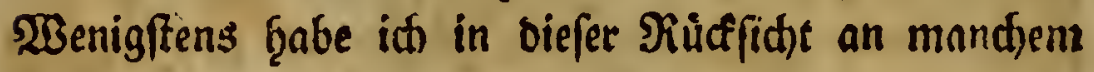
Drte gefragt, wie etwa ein Berg, Den man mir ift Mittagfogel nannte, vor altem geḩeiffen f̧aben möd)te? und immer erb̧ielt i 
Bell im Sillerthall, ben 10. Ottob. 1783. 97

eines andern Namens erinnere, baß man ig̨n bon jes ḩer unter biefem geffonnt fabe : Bum flaren $\mathfrak{B}$ emeife,

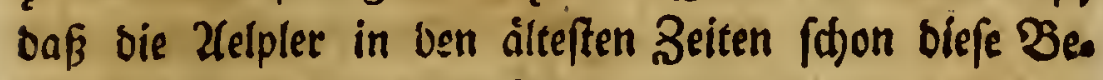
obadjungen gemadjt ḩaben.

Nimmermegre will id eines Unterridtes vers gefifen, Den mir bor menigen $\mathfrak{B}$ odjen ein alter Sanbo maun gab. Er ţante mids am Gserloz̧berge auf einen

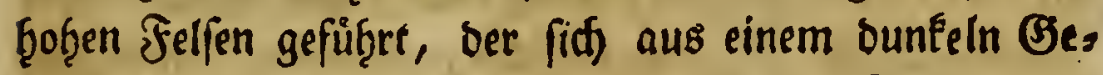

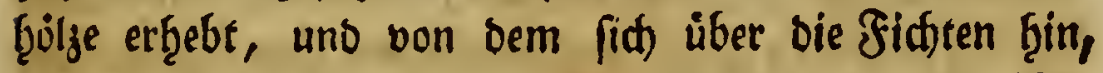

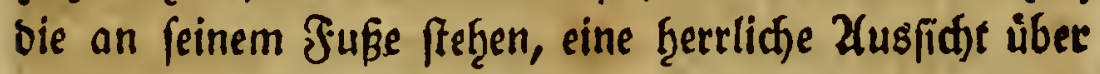

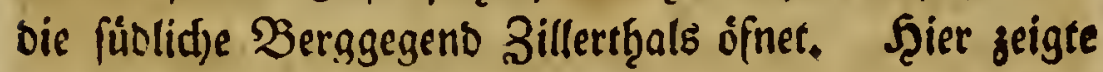

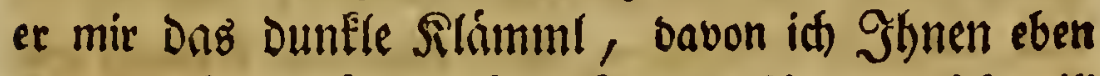

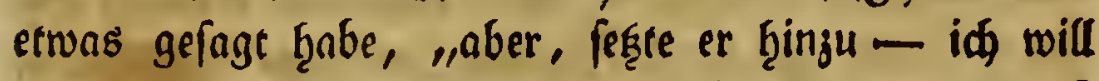

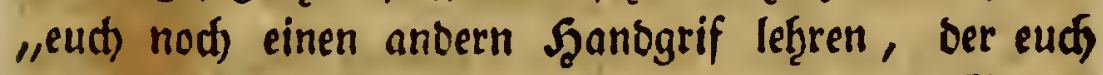

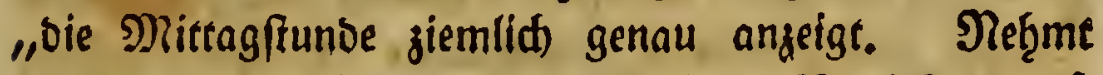

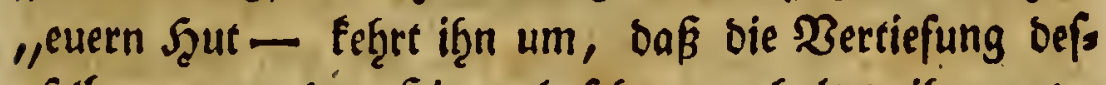
"feltben gegen ben Şimmel fef̧e - Ģalfet ifhn unter

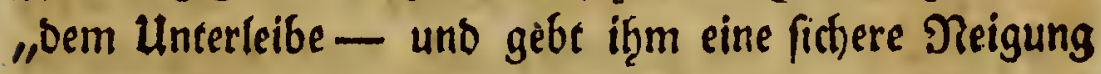
„gegen ben f̧orizont : nun fiefllt eudf fo gegen bie

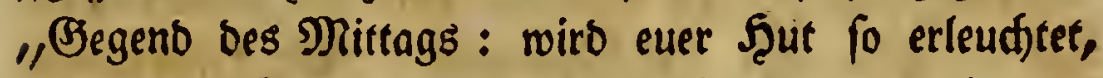

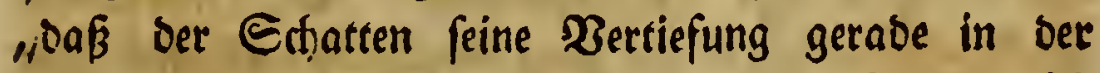

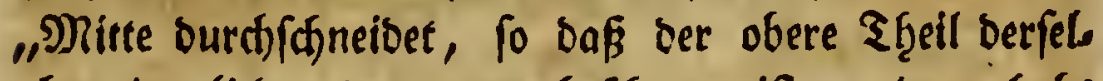
„ben im sidft, ser untere beffoattet iff, - Daunn ḩabt ,ifbr - glaubt mirs - fo giemlid) genau bie. "Mititagftunde. "

Diefe Bemerfung über bie Beitfunbe ber Zeeipler führze mid, auf eine antere, bie mit biefer in $\mathfrak{B e r b i n a}$ Dung. freht : fie betrift bie Eternfunde biefer \&eute. 2lud bie Eternbillber beobaditet ber zalpenberwog̨ner in Fiaturbjif Briefe I. Bamb. 
mandyer Pilicfiffy, unb benennt fie theils nact) äf̨n

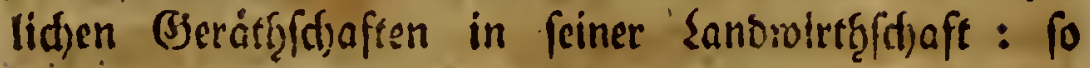
nennt er z. B. geniffe Esernbilber bic Sidud, bie Genfe, Den rifid), die Gtube n.f.f. theils mit iffen nllgemeinern Namsn, als Den Jjecrungen, bie

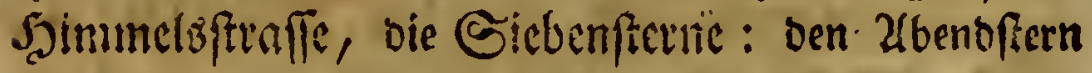
nenne er mit einem 2lusbrucfe, bet ifgn fef̧r gut bes zeid)net, Den Detiger, aud) Den Şágerftern. Ber Natraier ḩat für bas Geftirn, Das ber Billerthaler

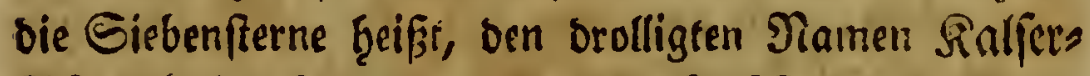
Yjfantholz erfunben, weil es im J̧erbfte binter einem Berge, ber Mnatrai von Salz foneiset, herauffigeigt,

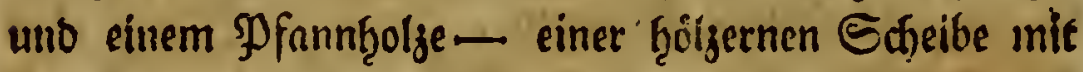
einem Etiele, ouf ber bie Pfannen mit ben Megllfpeifen

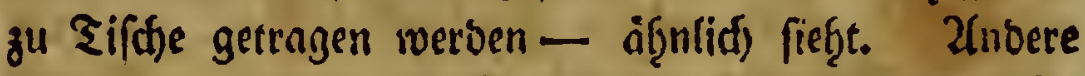
Grerne fino bey oen Billerthalern ocr Juetersftab,

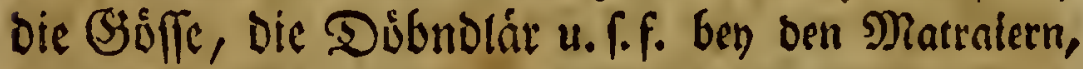
Die Barbcrin, Das heilige Sireu u.f.f. Das $\mathfrak{Z u f}$ uno Untergeßgn ber Sterne, uno if̧re verffiebenen Etellungen gegen sie Berge, tḩun ben Zfelplern bie Dienfte einer H(gre bey Nad)tzeir. Die Jigiger unb Wilobiebe verffeţen fid gang vorguglid) auf biefe arte bon Beitrectynung.

Bergeben Gie, theuerfter Jreuns! baf id to lange ben biefen $\mathfrak{B}$ emerfungen fref̧en blieb: uno laffen Sic uns mieber nad) unferer alipḩustte feģen. Die

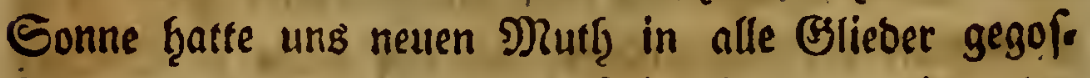
fen - uno ein fertre laaftiges Mánncten aus einer bee b̧iefigen 2llpḩütten muß̣te mein Frobgenn ju feinem Bor: 
Bell im Billerthall, Den 10. Detob. I 783. 99.

Borb̨aben zu benúzen. Er madtse mir ben Boridhlag, mid) zu einem Eisfee ju fự̆ten, oer, nur eine fleine fralbe Stunde, wie er fagte, entfernt waire. WBie fonnt' id fo etrons ausfdlagen? 3roar ftanb bie Eonne fhon hुod über unfern Echeiteln, und wir follten heut nod) in unfere allpe Schiremm zurute. 2fber gृeiterer Şimmel, und nur eine ţalbe Etunbe-: uno damit wairs in bie Şano gefdlagen. Meine Begleiter waren anfangs nid)t fef̧r geneigt, ben $\mathfrak{B e g}$ mit zu madjen - aber als fie fab̧en, baßs id) aud) oḅne J̧̧nen bem Ģurtigen Jungen zu folgen entfollofien

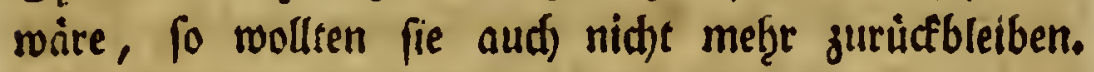

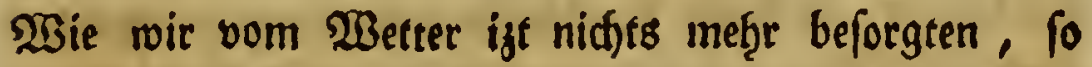
lieffen wir, um gefdrwinber wegzutommen, unfere

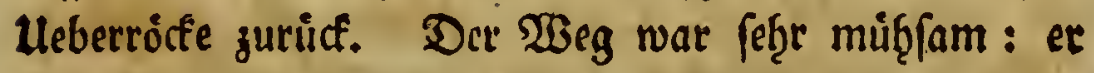
gieng gerabe über bie Zntyobhe, die im Norben ber

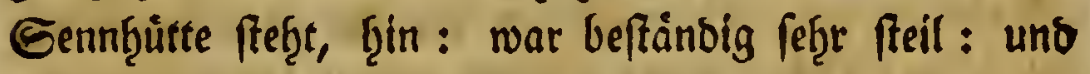
von einer fo unzàbligen Menge fleiner (S)räbden ourd): fánitten, Daß id) bes endlofen నigzags enolia) mübe

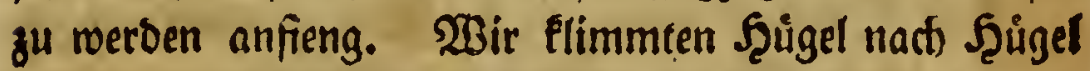
Ginan, - Durdfreuzten Bräbchen auf Bräbdhen -

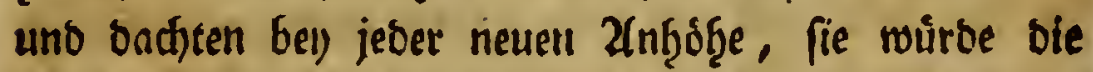
legte feyn : aber ḩatte fid) wobl mit unferm Şofen : ba war \$fonen alljeit wieber eine Eleine Tiefe, und jens

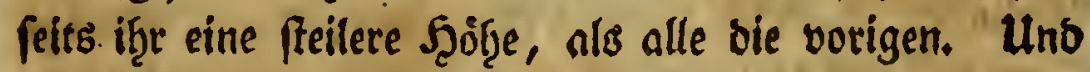
bie fleine Galbe Etunbe bis zum Eisfee — Das war mir eine allerliebfte :Recínung. Id mơdite jebem Naturforfder, ber altpenteifen zu madien anfängt; ratben, ja nidjt auf bie Entfernungen, bie į్m bie Zeipler angeben, zu zåf̧len. Die guten keute bringen 
taben immer iţre muntere, bes Eteigents genofinten Juiffe - und ifgre leid)te Rleioung in 2(nfd)lag - : und ba mag ber Mann bon flad)em Salte, ber bren Biertfieile feitner Erunde auf feiner Erubierfube ver-

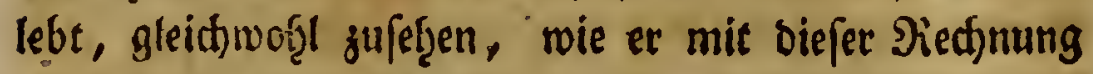
zurechre fommt.

Iक fano fier bas fo allgemein gepriefne Sutter. traut Phellandrium mutellina L. ziemlid) Ģaufig. Wherr Diof bant es in feinem Berzeidjuife berjenigen Edjweizerpflanzen, weld)e vorzủglid) zur Nab̨rung Des Biefes sienen, unter sem Nomen Sefeli, foliis multifidis, lobulis lanceolatis acutis feftr gut bes

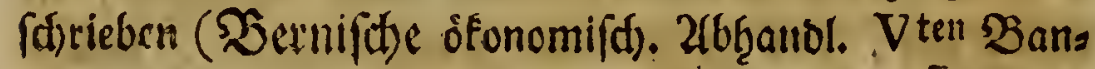
bes. 2tes Enưf. E. I 48.): ferner bie Fëlobinfe (Juncus campertris L.), oie Ģier Miartel ḩeißjt, uno von einigen gepriffen, von ben meiften aber unter bie

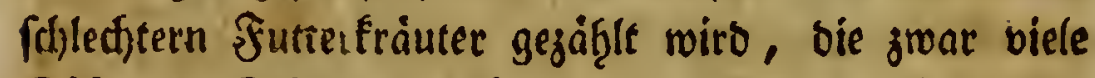
Rápe uno Ectotten, nber menige $2 u t t e r$ geben : bie inthligte Gdyluffetblume (Primula farinofa L.)

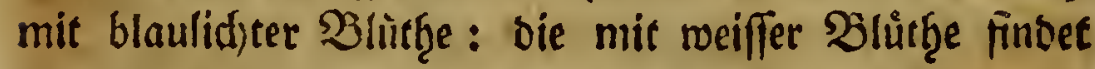
fich) Ģier auch), wie mir mein Füḩrer fagte, aber etroas feltner, enolich die 2llpenarctie, (Aretia alpina L.); bie Der mürbige Sacquet im I. B. Der Edrift. Dee

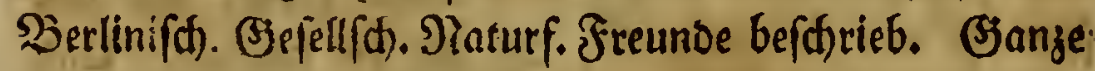
Bcyenden fino blau gefärbt, bon biefer wof̧lriectenden SPfanze, bie Gier unter bem allgemeinen Samen ber Edlüffiblumen - blater Epcie, - audh mit bee griffen ift. Eie ift in Berbinoung mit ben Primulen. ein vortreflid, Jutferfraut : Die Butter etf̧ält Davon sine 
Bchl im Billertlyall, Den 10. Oftob. 1783 . 101

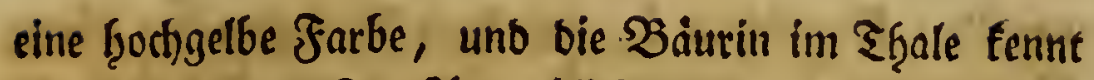
es mit Dem erfen 2ugenblidé aus der Sanrbe, und

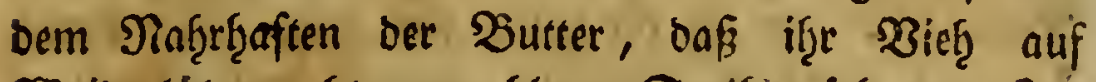

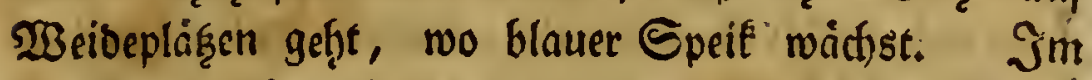
Rodhen vertgált fich bie (Ergiebigfeit Der Butter, bie auf

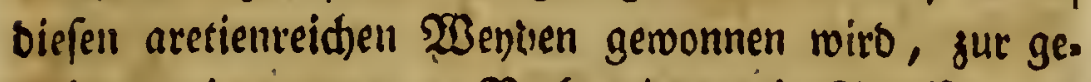
meinen wie $2: I \frac{x}{2}$. Nod zeligte mir \$leoffer eine

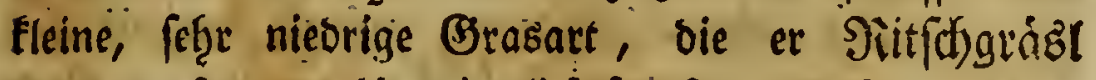
nanute, fie war Ģier ziemlith) fparfam: beffo gemeiner foll fie aber in einigen benadbbarten 2 Seideplatgen foyn.

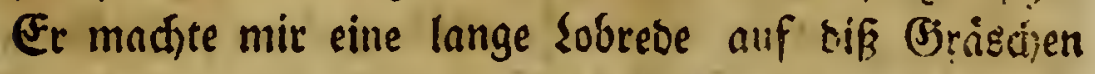

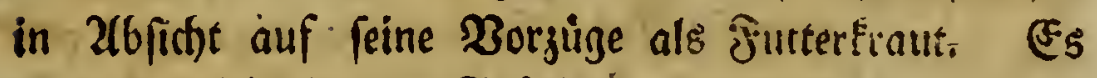
war izt nicfte in ber Blüthe : id ḩielt es für bie Poa alpina $\mathrm{L}$.

Unffer $\mathfrak{B}$ egweifer verfolgte, und fieng mir eills, weillen mit einer bemunbernstwürbigen Beḩanbigfeit einige Extmetterlinge: iff erffaunte barüber, wie ber Junge es wagte an bern freilen abţángigen $\mathcal{B} e r g e$ fo fdinell queer siber zu laufen. Zaber frenlidi war aud Der grơfite Theil ber gef̧afdeten Eremplare giemlidh be.

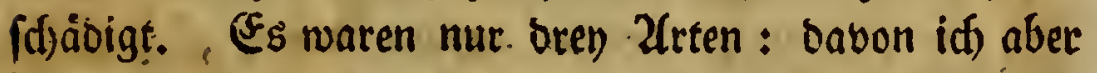
eine, weldfe unter bie gologlanzenben Falfer (Papil.

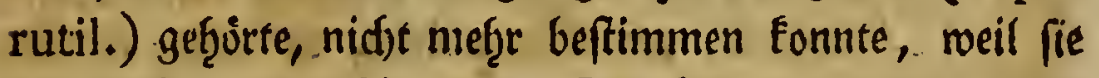
gar zu feght verwifdot war. Die Geiden andern waren eine fartfe: ZfGånoerung bes Papil. Daplidice L. wie mich aus ben feltnen $\nwarrow$ Rupferfafeln zu SElopoli’s. Entom. Carniol., bie id surth bie Juite, unfers lieben von Saidjarting's bor mir liegen ḩatte, bünft, fo

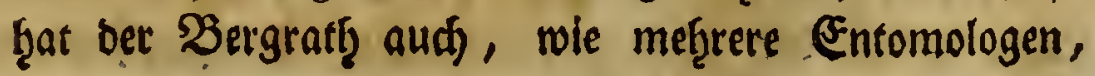


ben Pap. Daplidice L. mit bem $\mathfrak{B}$ Bibden bes Papil. Cardamine L. vermengr. Die oritte $\mathfrak{X} \mathfrak{r t}$ war ein ₹aguogel, ben id für ganz neu ţalte, uno Papil. Danans feftivus glacialis nennen will, weil er ber ben Esisbergen gefunben warb. Scier ift bie Befdreibung.

\section{Papilio Danans feftivus glacialis:}

alis primoribus fupra fufcis; maculis fex vel feptem fulvis; ocellis duobus nigris, pupilla alba, unoverfus apicem, altero verfus angulum pofticum; margine anteriori albo nigroque variegato; fubtus pallidioribus; ocellis ut fupra; verfus apicem nigro albo. que variegatis,

pofticis fupra maculis fulvis, uti in primoribus; ocello unico, nigro, papilla alba ; infuper punctis tribus albis; ad marginem interiorem grifeis, nigro obfolete variegatis : fubtus totis nigro - albo - luteoque nebulofis; ocello unico nigro, pupilla alba, ad angulum pofticum.

Antennis nigrefcentibus, apicem verfus fulvis.

Pedibus pallidis; femoribus nigris.

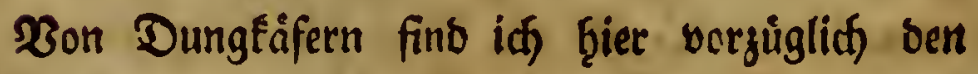
Scar. haemorrhoidalis L. - nidft ben, in meinem Berzeidnniffe ber Ealyburgifften Infeften befdriebe. 
Sell int Billerthall, Den 10. Dftub. 1783,103

nen - fonbern ben waf̧ren, bisţer nod) immer ver. fannten Scar. haemorrhoidalis L, navon id) SG)

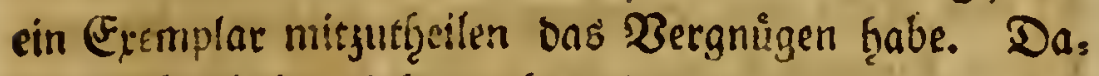
burd) beffuttrigt fid sudu meine $\mathfrak{B e r m u u t h u n g ~ , ~ D a ß ̧ ~}$

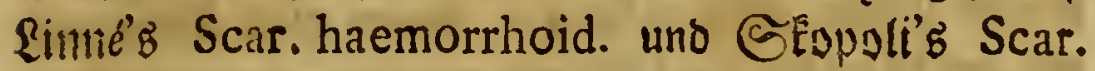
alpinus cinte, und die nemlidge Folbentáferart fenen, bie ber $\mathfrak{B}$ Bergraty nie auf Dem Sanbe, nur einmal auf einer Zlipe in Sbertirain gefunben frat, obwofil fie

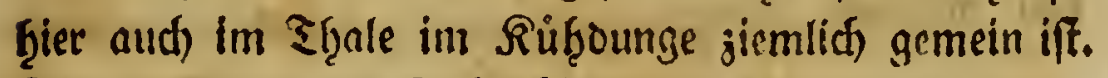
Jerners cine uelle Rolbenfäferart : Scarab. arcuatus, niger, nitidus, capite antice rufo transverfim trituberculato; elytis ftriato - punetatis; futura, macula bafeos marginali, vittaque abbreviata rubra, futuram verfus inflexa; pectore obfcuro; abdomine flavefcente; pedibus, antennis, palpisque

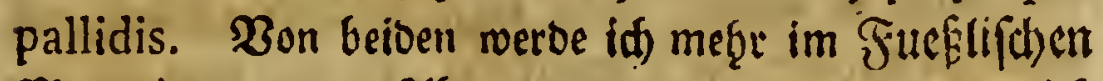
Mngajine reben. Zlber bas roar nudf alles, was idf

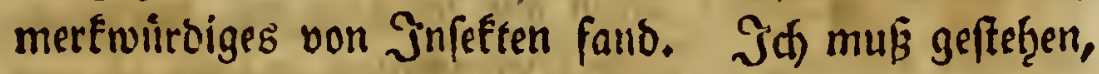

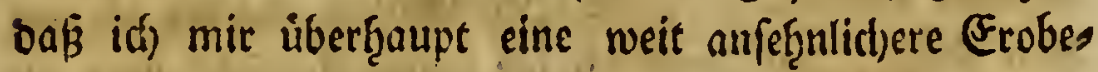
rung für meine infeetenfammlung auf biefer zllpenteife biofte, uno idc fann, wenigftens bier, be Suc's Meys.

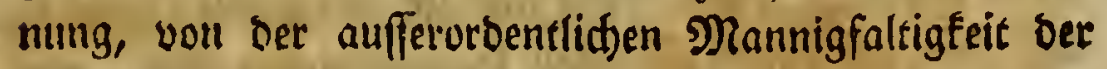
Jnfêtten, auf Dem Gebirge, ourd meine Erfaģrung nidst) beftättigen.

BBir famen unterbeffen immer boobser, ben Eis. bergert gerabe gegeniber : und bie 2fusfidht auf biefe war izt prähfiger, als ie an einem anbern Drte. Şier

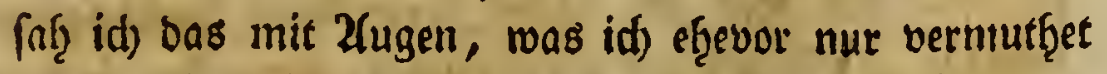

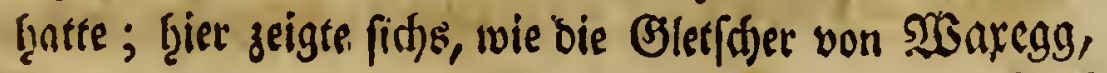




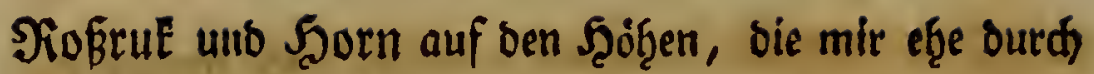
bie Berge verbectit wurben, zufummenfliçen - uno ba eine Rette bon Sthneegebirgen madjen, bie man feinen 2lugenbliaf ofgne ber tieffen $\mathfrak{B}$ ewounberung bet

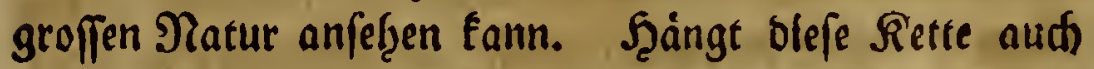
mit den (Sletfhern im Sुfitid)grunde - unb mit benen von ber Floite und Stillupe jufammen, wie ę mir immer waḩrydjeinlidjer waro, fo ift biek ein Eisjelo von mę̧rern Meilen.

WBir murren oft úber Ereigniffe, bie begm erften 2(nblicfe viel unangenef̧mes zu ḩaben fd)einen, obrob̧ fie im Srrunbe $\mathfrak{B}$ ob̨ltḩ̧at fĭr uns fino. Zufóllige Uum. fánbe madfen uns mandfymal unfern Jrrtf̧um einfę̧en: aber mandjunal Fommen fir zu fpát; bie erften fino

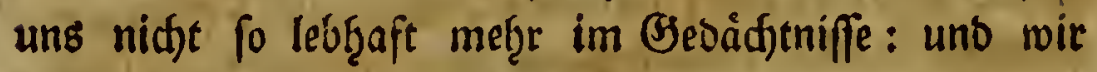
vertieten barüber bie Bemerfung, zu ber uns bie lez. ten fự̂ren würben, wenn wir fie nod) mit jenen ver. gleichen fơnnten. Diefe Betratitung mindtete ids auf

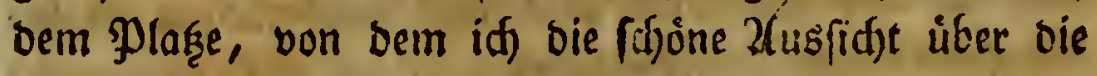
Bjletfder genoß - benn id) befano mich izt gerabe im

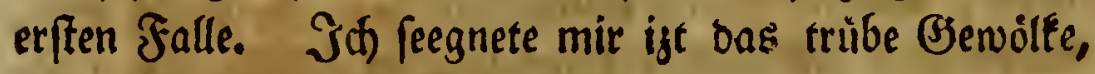
mit bem idf) einige Stunden fo unzufrieben mar, weil es mir bamals úber ben fhjonen Şimmel einen Salleier

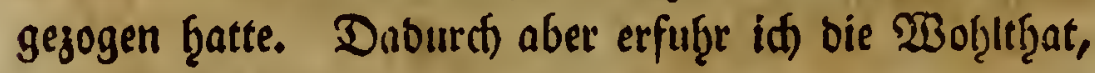
Daß id in Seit bon wenigen Stunben bie Bleerforer in zwo gang verfdiebenen lagen, in $2(b / i d f)$ auf bas mab̧leriffbe, das fie ser Begeno unņer gaben, zu foţen befam. Es finb zwo hiojdjt verfófiebene sand/d)aften um bie 2lusfititen auf bie Bletfder, wenn if̨re Jirften 
Sell im Billerthall, Den 10.0ktob. 1 783. 105

an tribes Gewolfe grainjen, und wenn fid bie firbers weiffen f̧ob̧̨en in ben lafurblauen Şimmel verlieren.

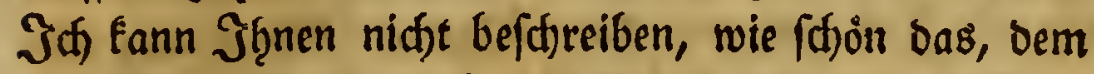
Ed)eine nach) unmittelbare, 2(ngrainzen bes fdjonnften $\mathfrak{B a u e s}$ an bie blendenbe $\mathfrak{B} e$ iffe ber Edjneeberge iff. Je ḩöbler wir ben $\mathfrak{B e r g}$ ţinauffitiegen, uno je reiner Der Dunftetreis wurbe, befto reizenter marb aud) bie (antofd)aft : benn im nàmlidjen Berf̧áltniffe murbe auḑ bas Sllau bunfler. De Suc ḩat liber biefe Erftheinung in ber Natur mand)e fdjoine Ifnmertung gemad)t.

Zange fdjon war bie ḩalbe Etunbe voriber, oa wir unterbeffen immer bennahe gelaufen ḩatten, um unfern $\mathfrak{3}$ egrweifer nidft aus bem Befidite ju verlieren : wir ḩatten úber Sdnnee uno Eis weggefergt - als wir uns auf einmal am Beftade bes vorgeblidjen (Eis. fees befanben. Nimmermeţr Eann man angeneţmer úberrafdet merben, als id es in biefer 2lugenblide. ward. Ith ferzte mint) am Geftabe nieber, und bes tradtetere ben Gee, ber nod bennahe gang mit (Eis bes beffe war; und nur erft runb am Strande berum et. was aufgeeist ḩatte. Er ift bon mittelmápiger Brôffe, bennaḩe Birfeltund, unb your ben Eeiten mit ţoţen Felfen uingeben, bie faft fenfredfit abgejahnitten find, uno ein pråd)tiges 2 (mp̧̧itţenter bilben, bas nur ge. gen Gíb offen ift. Um wie viel fhoiner wúrbe biefe Z(usfid)t nod) fenn, - weenn bie Eistinbe, die bes Sees Dberflärhe becft, das Epiegeln ber Jelfen, uno Des blauen J̦inmels im $\mathfrak{B a f f e r}$ beffelben nidft Ginderte. Uleber bie Firften ber Jelsflumpen, bie im Şinters 


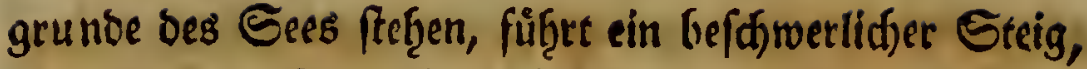
Den nur oie Eemsjäger fennen, in bas gegenúber

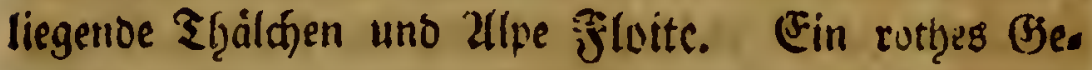
ránbe, bas am linfen $\mathfrak{u}_{\text {fer }}$ bes Gees ftelte, heifist Det ruthe Sipp. WBeftwarts am Jufie deffeloen

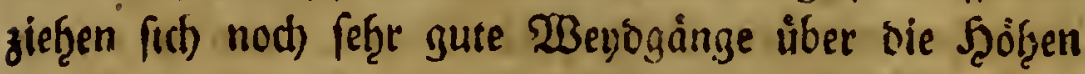
ber Berge bin, bie zur allpe Gabwarjenftein gefercen, uno Der Ddfenfare genannt weroen. Wenn bas

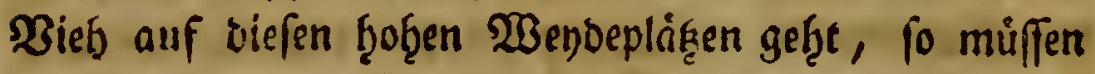
bie Melfer ieben 2lbent uno Morgen den müf̧famen Beg von ber Gennţuttte bis tabin ntadien, um oa if̧re Rủbe gu melfen. Eie fónnen fid) vorffellen, wie

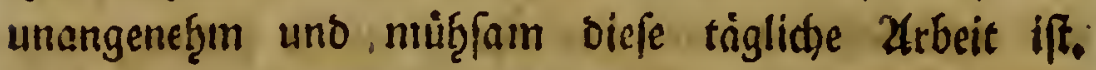
Daher der Denfipruch) viefer $\mathfrak{B}$ eibgänge:-

Wenn's melfen im Ddffenfarr,

fo fajinot's ber Melfer naheno gar. (Getnahe)

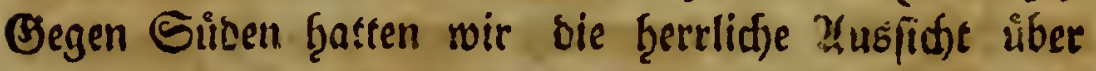
bie Eisberge - uno bie baran grånzenben faflen Bstranito felfen, unter bie fich im Sủboft grünetibe Edfaafwely)en mijciten. Bom Bipfel eines Berges im Siboft blicfe ein Steinmånndien auf uns b̧er; fo ḩeiffen bie alelpler freinerne Saiulen, bie bie Siirten auf ben hiort)fen Bergrưdén für if̧r Bergnigen aufbauen, wenn fie

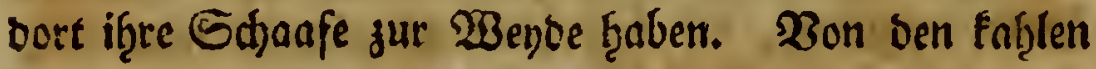
Jelswainden, bie fid an bas fotmale (seftabe bes Eees faflieffen, rollen bie săf̧nen gerabe in ben 2lbgruns Deffelben. Brofie Ţaufen Stjnees lagen, befonders am

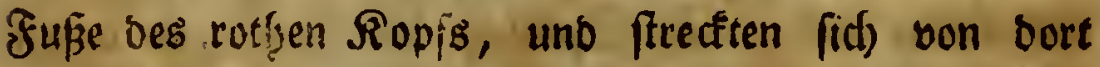
an ben Gee ḩin, fo baß eine f̧allfte bavon wirflid

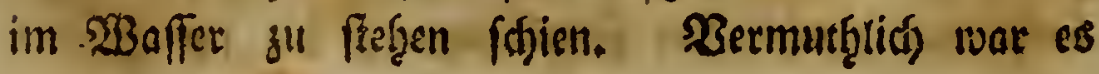


Bell im aillertlyall, Den 10. Oltob. I $783.10 \%$

weralteter Edfinee, ber von bem jäbrlid)en 2lbftürzen B̧ier jum Theile zurůcfbleibt. Nad) Ginuners Eyftem bou ben Eisbergen mifife man biere Edqneelagen unter feine Eislagen jåf̧len.

Ltnfer গ⿱龴 diefen Sommer nut erft yor wenigen Sagen auf einen Jelfen, der einige Ed)uf̧e vom Ufer weg fid) aus sem Eee heerfürftrectit, gewagt, uno bon ba cinen fleinen Etein, ben er an bas eine (Eno eines brtrádtlidjen Sineuts faben befeftiget ḩatte, bimuntergelaffen ķabe : Der Fraben wàre aber gand ju Ende gerwefen, nls ber Etein nod) immer gejogen f̧åtte. Jd) glaube, ber

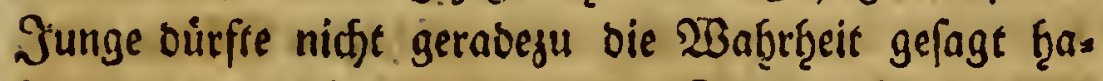

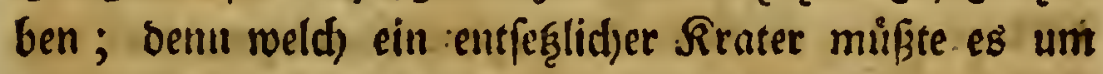

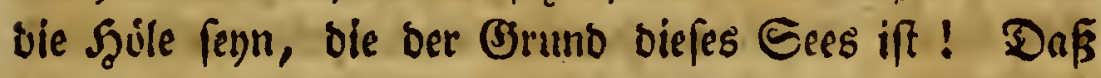
unterbeffen ber See von einer nist)e geringen Tiefe fern

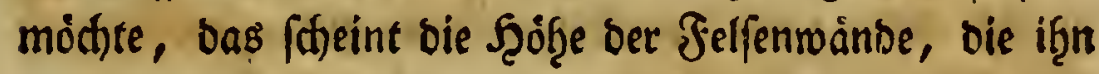
umgeben, ju berveifen, wenu boch biefe nidht ungerwoifgn.

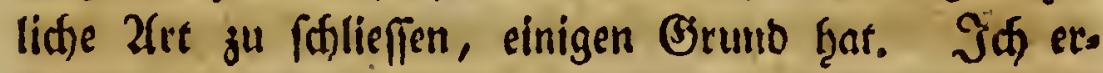

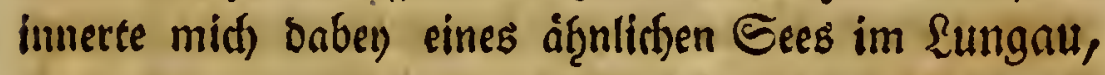
ber auf einem fef̧r ḩohen Bergrưden liegt : id) weiß nidht, ob mir mein (Şedadjtnis getreu iff, wenn id

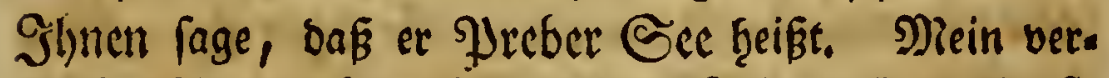

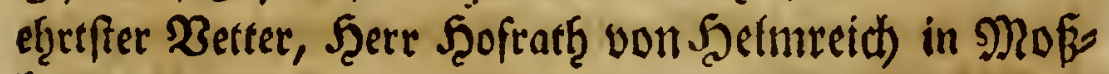
hant ţat in biefem Gee bie treflidffen Eälblinge (Salmo alpinus L.) frefen. Ith thabe biefen in

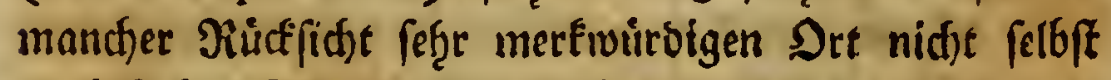
zu befudjen Belegenţeit geţabt - aber ber 2effdreibung nadf, bie mir von bemfelben bey einem langen 
unvergeflid)en 2(ufentţalte in Mofblyam gemadjt warb,

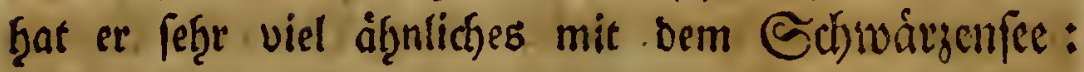
nur. mit bem Unterífhiebe, Daßs. es Ģier woh̨l niḑt moglid fenn murde, fifde ju erfyalten, ba ber. See begnafere nie ganj aufcist, unto bie Berwalt, mit ber bie Edjnectgaufen von ben umliegenben Felşpişen fiá)

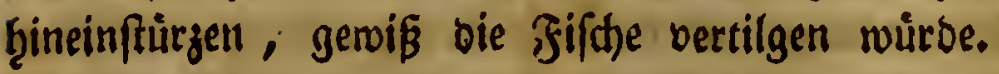

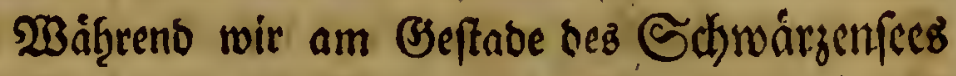
fajen, Elimmte unfer 2 Begweifer an ben benndbarten

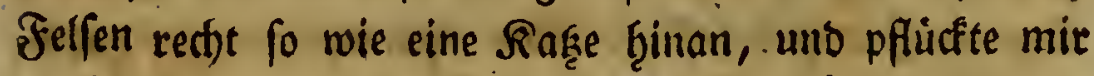

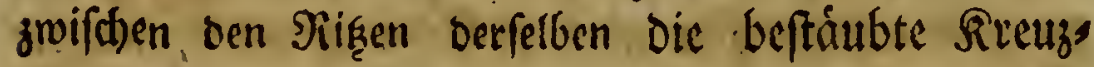
pilanze (Senecio incanus L.). .. So oft er ein Eremplar biefer hier ungemein geftrajten פPlanje fant, vertúnoigte er es uns mit frohem. Jaudgen, ons von ben Jelfen umf̧er taufenontal wieberf̧olt maro: uno balo fam ex mit einem STute boll EDefraute ju uns zurưce. So beisist ber Zleipler biefe Pflanze. Es ift ein vielbebeutetiver $\mathfrak{B o r}$ ug für einen Melfer, Der einen fojonen Strauß oon Foeltaute auf feinem Şure triggt, wenn er an einem Rirchfefte ben Tanjboben im Shale befudft. Die guten (eute machen auch)

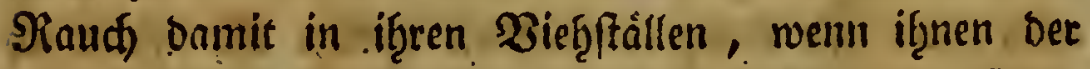
elenoe alberglauben, oen cein groffer şgeil if̧rer Eeels

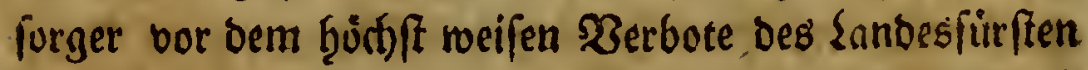

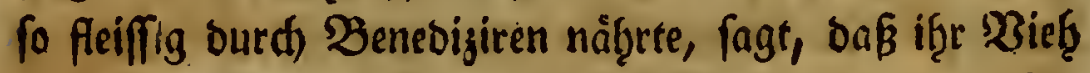
verţert, oder, nad ibrem 2lustrucfe - vermennt fev.

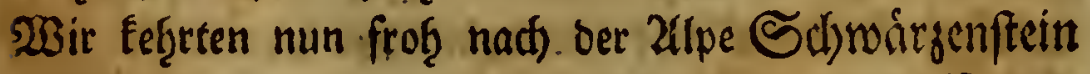
zurtick, wo ber Me!fer, mein $\mathfrak{B}$ irth, mir eine Menge voll orn obengenannten Aretien, uno bnju eine Planze 
Sall im Sillerthall, ten ro.Dftob. I 783 . 109

bract)e, bie er 2 (ltthec nanute, uno mid' verfidferte; es woire fier ber feltenffen eine, und fie whidjfe gang

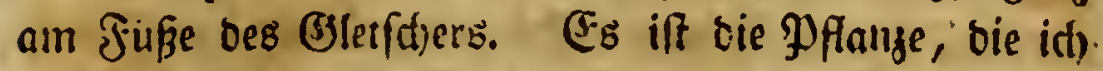

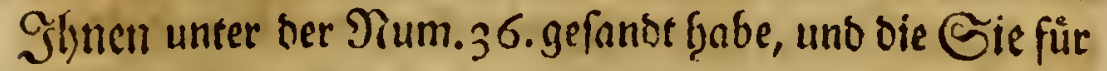
nid)re mef̧r als Doronicum pardalianches L. f̧altert.

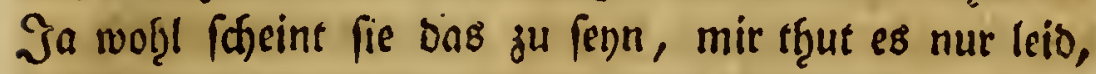
baßs oer Melfer feine $\mathfrak{B}$ urgeln an feinen Exemplaren mitbrad)te, und baß mic) bie Beit zu feģr an bie

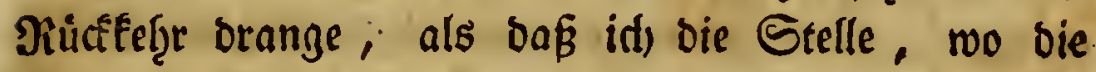
DPflangen gepflucft wurben, fetbft haatte befucfen foonnen.

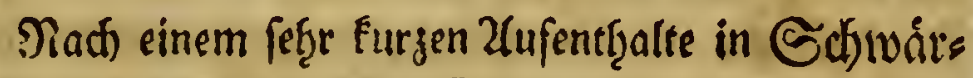

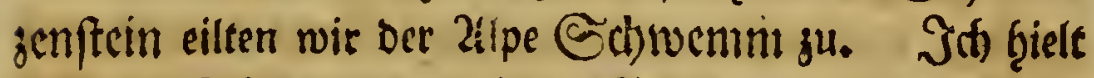

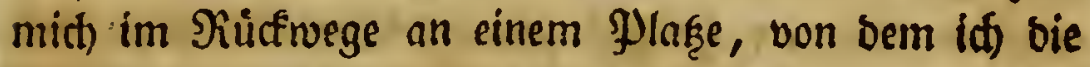
(d)oinfte 2lusficht auf sie bren (s)letfdier ḩatte, nod) lange ailf, uno fonnte mid faum von biefer merts. nuiroigen (Begent fheiben. S(d) will es verfud)en, Slynen einen Begrif von biefer 2(usfidt) zu getien. Der Siùcten bes Serges, von beffen füblidjem $2 b_{b}$ bुange id) meine Lanb/f)aft entwerfe, f̧eifft Der Rámpel: an ber redten Seite jenfeits bes Bembadies, blicfen bie Sirften bes গiifflers uno Epiegels heerfür; zmeen

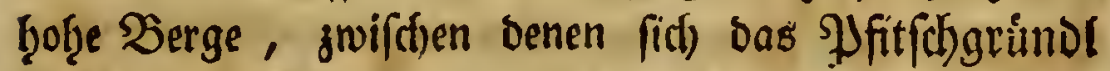

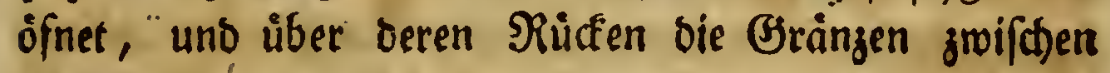
Salzburg uno Sirol laufen. গiad) if̧nen folgt ber Gireiner, ber burd) bie Müllerít) albḩanolung über ben Turmalin to befannte $\mathfrak{B e r g}$, den id ben funf: tigen Tag befteigen mollte. WBeiter fübroairts ftef̧t

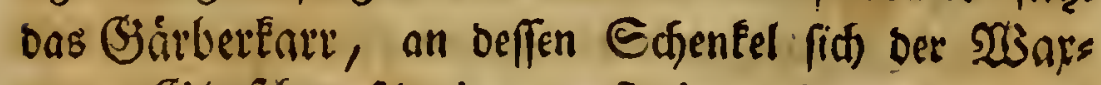
egger (3)letfid)er fajmiegt : oftroàrts, î̧m gegenúber, 


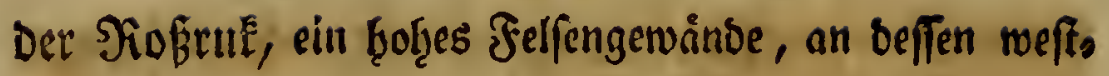

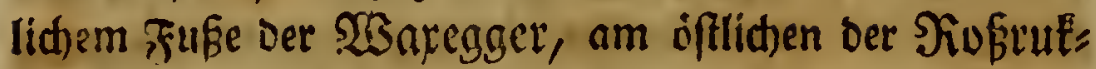
glet/fhet liegt, Diefen begrángt im Dften Dab J̧orn, ein fineufflicher, thoher Bjranitfelfen, ver ben Scornce:

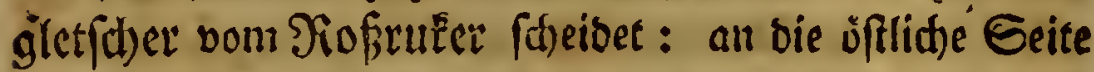

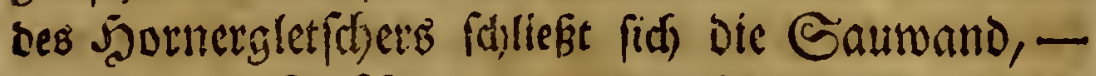

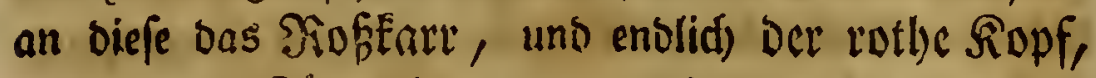
ber an ben Siámpl, von too wir ausgegangen fint, gränzet. Şier b̧aben fie einen beträdstlichen Rreiß̧, Deffen झeripherie aus lauter ungeţeuern Felfengebir.

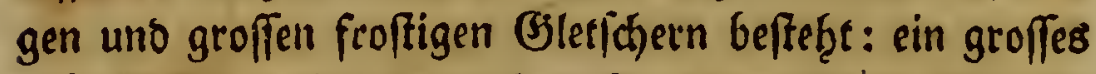
Gtücf Eroe, in bas fict) nafte, grauenoolle Eranit= maffen, unt ewige, unerfdjöpflithe berge von Sdnee

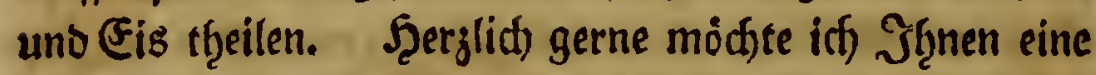
Beid)nung ber 2(usfics)t auf biefe (Bletfder von biefem Etanopunfte entwerfen. : 2lber Inffen Sie midi)'s in biefem 2fugenblicfe wieber von ganger Seele bereuen,

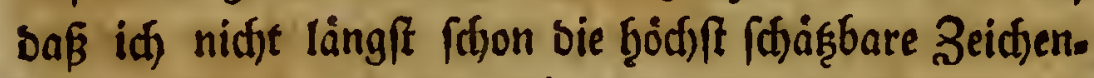
Eunft mir eigen gemad)t ḩabe, fo wie idss viele ḩunberts mal bet) imeinen meteorologifften Unterfuct)ungen bes reuet ḩabe, wo fie mit fo vielen, fo gar vielen $\mathfrak{B}$ or. theilen verfnipft ift. S(t) will Shlinen bod) einsweilen eine ibeale Beidjnung ber Ejegeno bes Ectwwángen= fteitterglet fh)ers nact) einer åg̨nlidjen im (Struner geben.

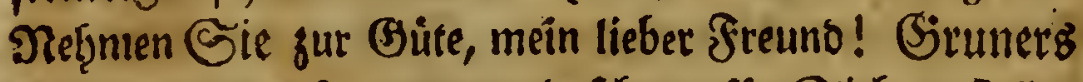
2(bbilbung bes Snuteraarglet/chers fur Sid) : frellen Sie Sith vor, Daß ber Şaufe Felsfteine, ober ber Guffer (lit. g.) bie waifferid)te $\mathfrak{B}$ ergwente fen, an bea

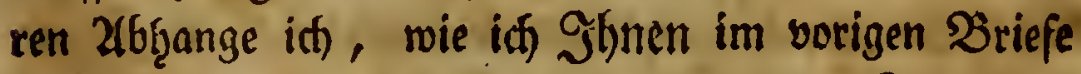
fagte, einen feģr unangenę̧men fdjmalen Etrig hiin. 
Bell in Billerthall, ben ro. Oftob. I 783. I 1

wantelte. Swifden biefer, uno dem ßirandidmmers borne (lit. f.) Denfen (Sic Gidh einen tiefen Bras ben, oen oer Bembed) Durdffliefit : Den Eisfhrunb am Jufe diefes berges laffen Sic weg, uns nemnen Sic das Brandifimmerlyo:n, und das herborragende

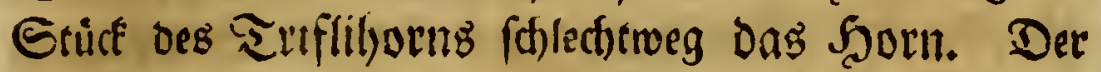
(Bletfder (lit. k) bleibe, mie er liegt; das Finfteraars houn (lit, 1.) দৃeiffe Snumand; bas sauteraathorn

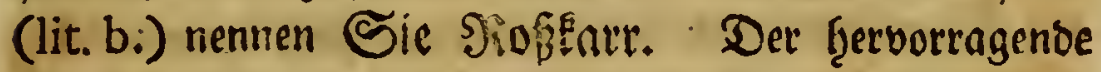
Jeigel zwiffjen biefem und bem Sberginle (lit. a.)

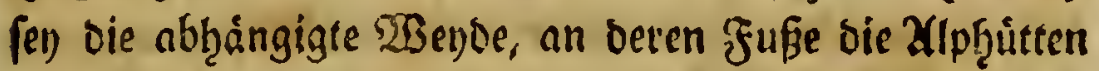
oon Shywarzenftern liegen, uno viber bie id nach dem. Sdinuizzerjec gieng. Eo fzaten Sic genau die 2lusficht uno bie Esegens des Sdywázzenfteiner= ober Saornergletfhers, bavon bie wáfferidjte Sergmiefe am פIInģe (lit. g.) Der Gtanopunft måre. Denfen Sie Sich ferner, Daß bas (Jebirg (b) fict) burch ein naftes Steffengewände an ben Sberzinfe anfolieffe, uno alfo mit if̧nen einell Şalbzirfel ausmadten, fo fraben Sie ben rothen Ropf, an beffen fupe Der Schmarizemfee liegt : es toàre ber meftlide ?tgeil bes \$Nittelgebirges.

Die Rette von Eisbergen, bie id bier vor mir

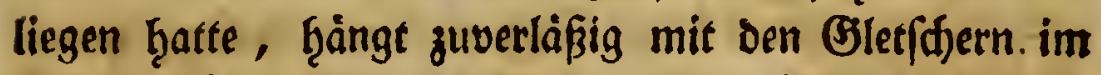
SIIfitfatgrimbl, und benen bon ber Floite, uno ber Stilluppe zufammen, uno mef̧rere sanbleute, bie biefe Begenden genaul fennen wollen, fagten mir, Daß audh bie (Bleeticher ven ber wilden Gerlob, und von Der Sd) innad), Die 5 bis 6 Stunben oftwarts von Sell liegen, Glieber biefer Rette feyen. 2lber nod 
Eenne id) grwifden biefen uno ben erften sie eigentlidje Berbinbung nicf)t. Der Rrimlertauern,' ber zmifden Oberpinggau uno Taufers liegt, foseint biefe Rette von einer antern fefgr groffen zu fdeiben, bie aus ben Bletfdern yon $\mathfrak{B i r g e n}$ in Tirol, aus bem Sdblofer:

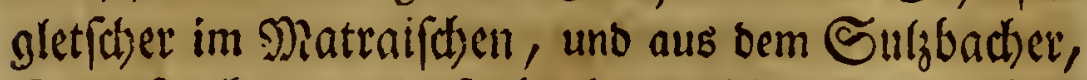
Şollerfwacher, uno Sçabadhergletfdyern im Dbers pinzgau befteḩt. Bon ben hiefigen (Bletfdjern fino ber $\mathfrak{W a r e g g e l}$ und ber Sarnerglet focher wanbelbar : bell Siofrufter aber madjen feine fajrectlidje Eteile, uno bie abfdeuliffen Slüfte jebent Menfdjen ungang. bar. 2(ud) ber $\mathfrak{2 B a r e g g e r g l e t ( d ) e r ~ w i r o ~ n i d ) t ~ f e h ̧ r ~ b e s ~}$ wanbert; aber über ben J̧orngletf d)er wandern meţrere ber jenfeits wofgnenden siroler in ben Eommermonaten Geruber, obrob̧hl es aud) ba felten of̧ne Befaf̧r ge. fhieg̨t. Mian jăblt eine gemeffene Stunbe bis an bie J̨ơhe bes Sdfwarjenffeinergletfd)ers, unb von bort zwo in bas ₹rgal ḩinab bis nact) 2 seiffenbach im 2 (l)ete. Der Junge, ber midh ju bem Eisfee gefüḩrt ḩatte, fagte mir, baßß er diefen $\mathfrak{B}$ eg einmal mit vielen (Ses

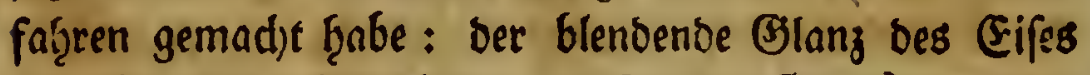

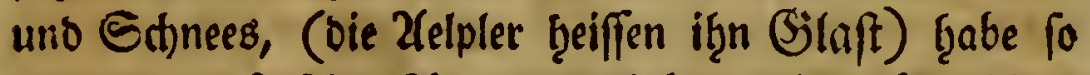
gemaltig auf feine 2lugen gemirft, oaß er ben feiner

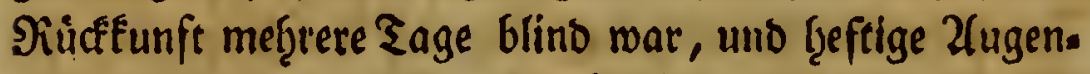
fahmerzen ḩatte. Der Bembach entipringt aus bem Şornergletf(her, und ein tleines $\mathfrak{W}$ äferchen, bas vom

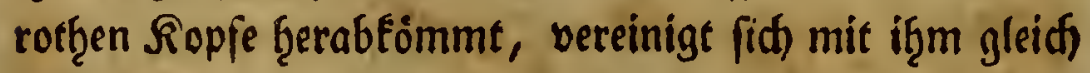
bey) feinem Urfprunge. Stion von ber \&uelle reg

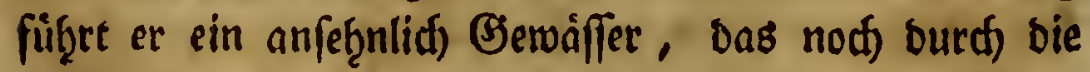
Zlusfüffe aus. Den benden andern (s)etfdern, bie ex bentegt, 


\section{Zell im Billitthall, ben 10. Dltob. 1783.113}

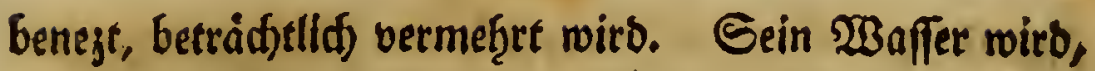
fo wie bas Bleteffinerroaffer überţaupt für feģr gefuno

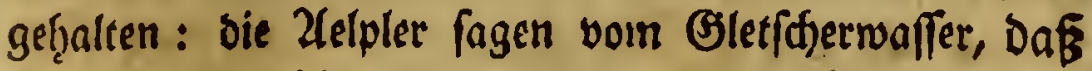

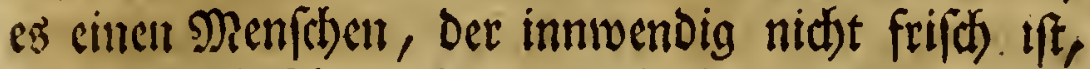
entwederes frifot madse, doer leerbuje (tóbte). Die Melfer auf Der Zlipe Edgwarzenffein bedienen fich befs fetben, wern fie buttern, bie afbfönberung ber Butterthetlle von ber פild ju befortbern, mit ungleidf ges fofjwinberm Erfolge, als Des gemeinen $\mathfrak{W a f f e r s . ~}^{2}$

Die Eisberge werben ţier Ráfé gerrannt, 3. B:

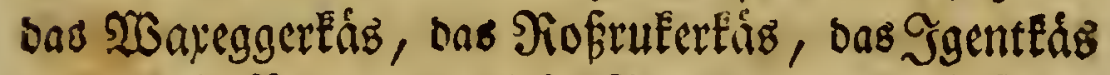

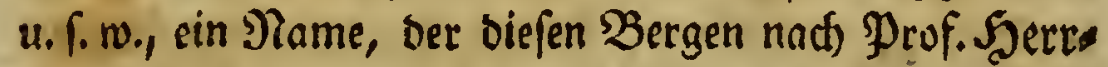

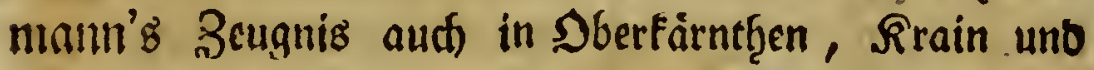
Dberfener gegeben miro. (afbrif der phyfizatia

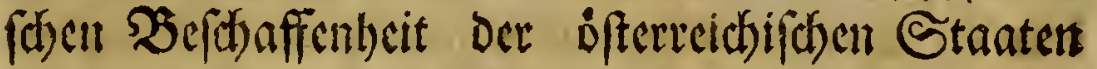
(..53.) $\left(^{*}\right)$. Die Râfe, bie in thier bereiste, finb

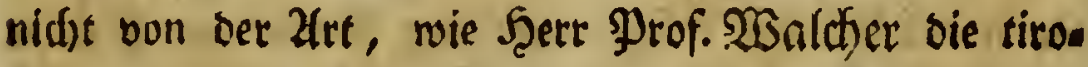
lif(ten Eisberge befdtreibt, bie nad) S. 3. Feiner Pad)ridten von Den Eisbergen in Sirol nur aus

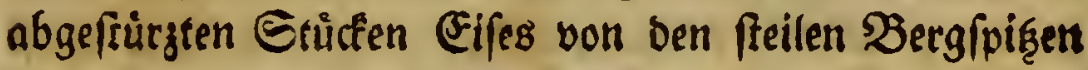

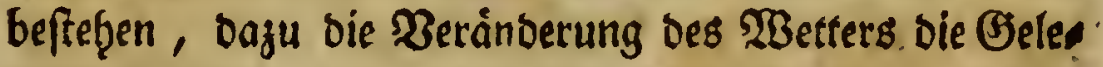
genheit gåbe. Sie find allmäbllig freigenbe, ourch gráslide Epalten zerriffeme Berge von Eis, beren fros

pige

(*) Es máre zu febr auffer meinem Befidstowunfte, bier eto was ùber bie Etimologie biefes sBortes zu lagen: id will dies in meinen Beytrågen ju einem Saljburgifhent

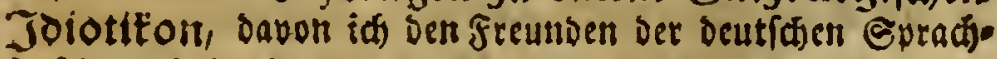
furichung bald eitte Proge vorgulegen, Die Eghe baben werder thuit. 
frige Gipfel uiber alle benachbarten Jellengebirge hiin.

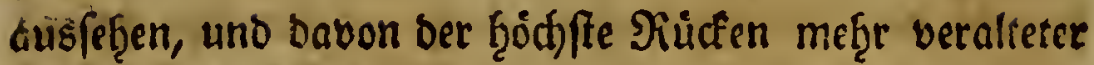
Edjnce als (5is zu fenn fitheint. Nimmermeţr fann

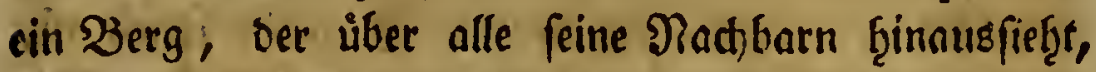
aus bem abftürzen bes Eafnees von bemfelben entftef̧en.

Jd) bitte Gic, mein freuno! mit meinen 2 sorten Eisberg, Gjletfder u.f.r. nidt bie Begrife zu ver.

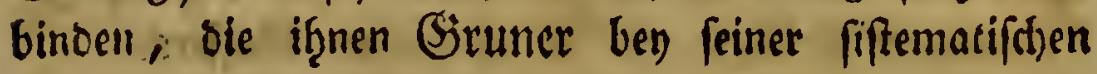
Eintf̧eilung ber (Eisberge giebt. Id) ḩabe biefe beys ben Worter bisţer ganz im weitumfaffenteften $\mathfrak{B e r}$ frante genommen. 2(ber it) will fie um jeben $\mathfrak{M i ß \beta}$ verftono gu verfindern, in ber folge befiimmter ges braucten, menn Gic mir erlauben roollen, biefen Bes trad)tungen liber bie hiiefigen Eisberge einige anoere

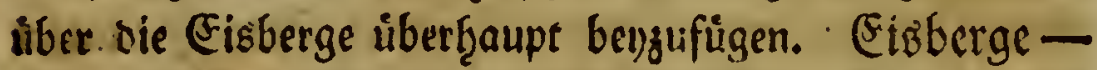

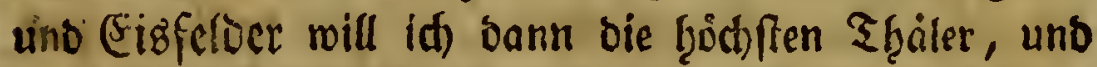
the in bie 23 diten fid verlierenden (bipfel von Ed)nee

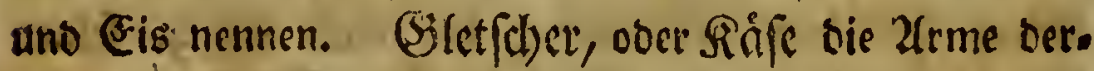
felben, bie fid) gwifduen ben nieorigen Ţ̧âlern ḩernug.

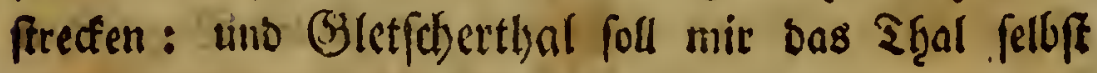
beiffen, in oem ber Bletfcher liegt.

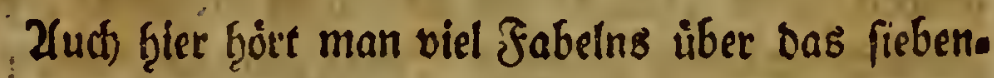

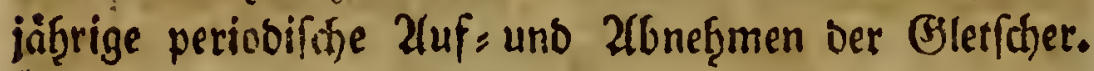
Die \{cute nollen uumiberfpred)lid)e (Erfahrangen Dar. ùter ḩaben: man zeigte mir, ols ich) am Jufe bes 53areggerf́ấcs vorúbergieng, baß biefer (Jileffd)er eben

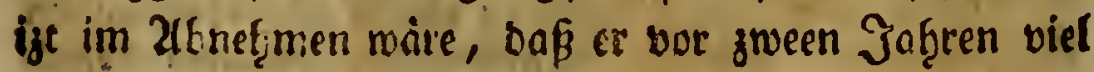
reiter an sas bieffeitige ufer bez Bemíad)es gereicht

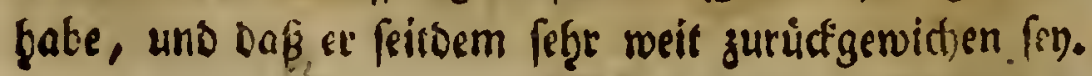


3ell im 3illenthall, Den 10. Dttob. I 783, 1 15

Wirtelid) hatte id) and) bie übergeugenditen Spuren bavon, oấ ber (5iletfd)er fich) viel weiter herausgeftrecte hatte, unb's ift eine betrådatlidje Etrecte, um bie ir feit ben paar Jab̨ren fleiner geroorben maire. Unter.

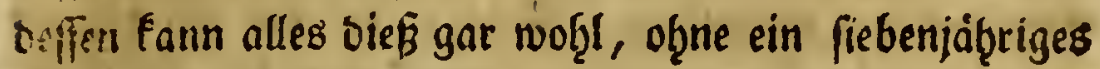

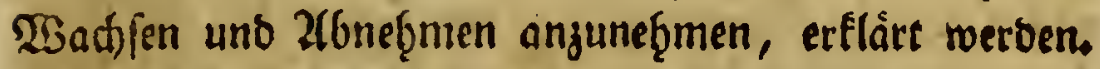
Eonderbar ift es inmer, daß ber Stimeizer, unb bee Firoler, unb der Ealjburger fo eirmürţig auf oie Zaf̧l Sicben verfielen: ein Beweis bon ber enthufiaftifhen Neigung, weld)e bie guten leute für biefe myftifde 3aţ̧ yon jef̧er ţatten.

Die Gdriffifeller, bie bas $\mathfrak{B e d}$ feln unb 2 (6.

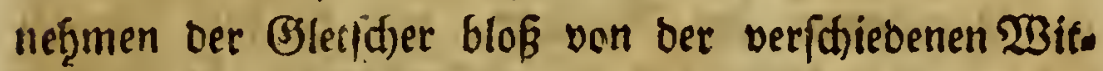
terung, von ben verfffiebenen (Sraben von Rålte unb 23 arme in verfafiesenen Jaḩren ģerleiten, fommen frenlid) am furrzelten uno am bequemfren baben burd)aber fie f̧aben aud, bie Sache baburd nidfs meniget als Flar gemad)t. Zroanjigmal habe id) de Suc's

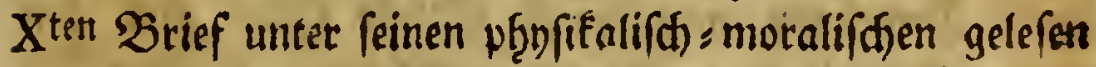

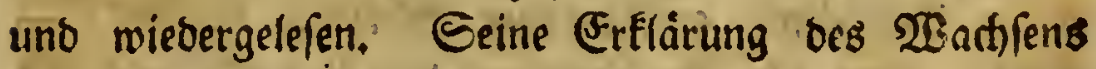
und abnef̧mens ber Bjletficher lft ungemein treffeno

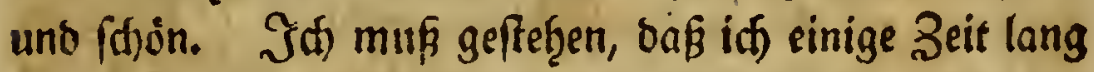
mit Grunbe batan jweifeln ju fonnen glaubte, weil id) mich nidyt erinnern fonnte, anbere Epalten auf

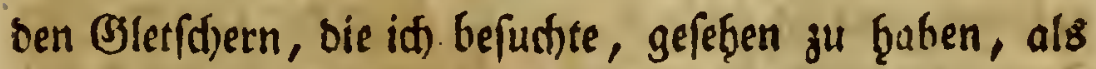

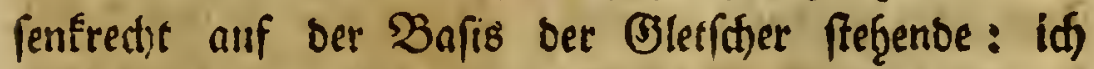
batee fie oefirwegen in meinem lezten Sriefe gebetters, biefen limftans zu bemerfen. Zber feitbem mith seute,

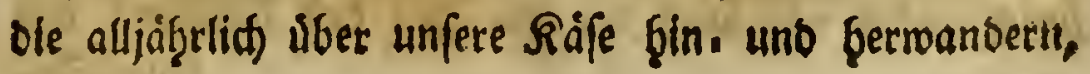

$$
\text { गู } 2 \text { ver: }
$$


verfintert baben, baß die fenfredten Spalten nur in ber (B)egeno bes Juffes ber (Jlet/d)er fo zab̧/reid) màren-

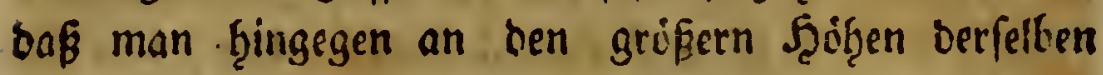
mebgr quecriberlaufende, uno inrunter biele, bie hen

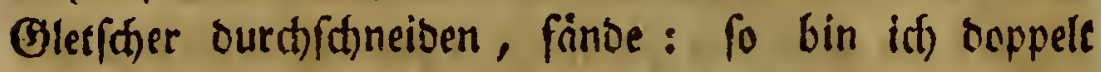
von De Suc's Dielynung úber das fortrúcfen ber Blet. fher úberzengt. Ji) erwarte mit lingebulo ben nád)ft. fommenben Eonmer, um ben einem efwas lángern Xufentḩalte an oen Ģiefigen Eisbergen biefe Queerpalten felbft aufzufuchen.

Zlltmann unb Giruner f̧aben mit menig Bufaß̧ und Berinterung, bie nemlide Urfacte bes $\mathfrak{B a d}$ fens uno Zanef̧mens ber Bbler/d)er, wie De suc angege. ben - aber biefer bat fie einleudjenber zu madjen gerouft. Wirflid bin id) nidst fo gans mit (Srunern

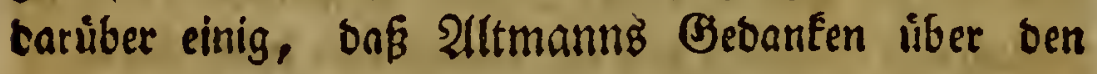
Urfprung ber eigentlidjen (B)tetfher fo gar wiberfinnig fenen. Sieffe fid) benn nidjt ein burd) eben fo gefdee. benes Fortrúdén meitläufiger Etride Eifes aus ben obern (Eistbailern entftunbener Bler/d)er benfen?Miiffen benn bie Zualeerungen des Ueberfuffes diefer Eisfelber gerabe in Eisfdjollen gefdetgen? Sointen nict)t aud) bie Eisttfäler ganze meitlaufige Etùcte Eifes burd) bie Defnung bes (Blet(d)erthales eben fo herab.

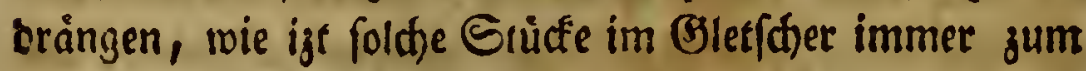
Fortrüfen gebrad)t merben? Snffen wir einmal burds fo einen Eisfopo den erften Bruno zum Btletfder gelegt fenn : er miro burd) bie nachoringenden Stúde immer fortgefducben - wirb mit ber నeit ben 2fusgang oes 


\section{Scll im Sillertffall, ben ro. OHtob. 1 783. I 17}

Bletfherthales erreichen - uno fo fann nadi vielen Jaḩren oaraus ein ganjer Bletffier entftanben feyn : beffen unuuterbrodfines Fortrúcfen erft benn dufhorrt, wenn oie zuerft ausgeftoffene sage eine Siefe erreid)t, in ber fie gefówintor zu fotmeljen anfängt - wo

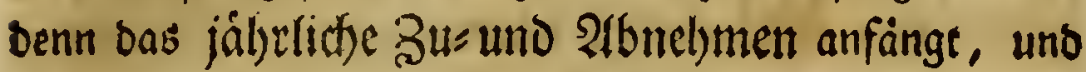
burd) bas beftänbige Rad)rúcten bon obenţer unters falten wirb. Th verftebe bas unumterbrodine ?forto rúden hier nidft in a(bfit)t auf oie Beit: fonivern in Zlbficht auf ben Sort. WBenn bie erfte lage ausgeftofo fen ift, fo folgt ing nad) einiger aeit bie zroote : bie roieber nach einigen Jaf̧ren ourd) cine britte fortge. fitsoben wirb u. f. w. Thun aber erreic)t oic erffe lage oen Dunte im Dunftetreife, no bas Eis ge/d) winder fafmilgt - und wirb zugleid) burd) bie Nenge bes

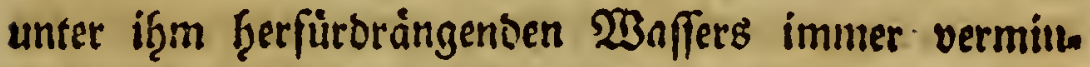
bert. Scier wirb bas fortrúcten unterbrocten : es ift จณE Non plus ultra bes Bletfdhers : bie lage Eis,

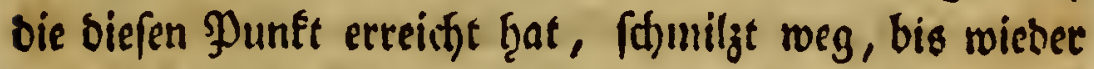
ein neuer E(f)ritt vom (Bletfder gemadit wiro : unb wenn bann bie sage f̧inter î̧r forttưdt, fo findet fie nid)ts mef̧r zum vor fid fdjieben : fie füllt alfo nur ben Plaş wieber aus, ien bie roeggefdamolizene lage. offen lákt, uns ber Bsletfder färeitet benn im eigent. lid)en Berftanbe nidjt meḩr noeiter - rúft nidjt meţr

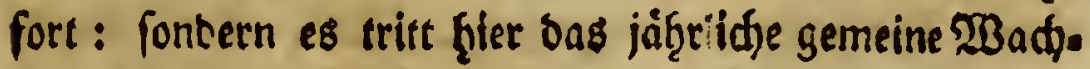
fen unt Xoneţmen ein. So ftelle id) mir bie Eade sor. WBenn in einigen Begenben bie Bletfder immer

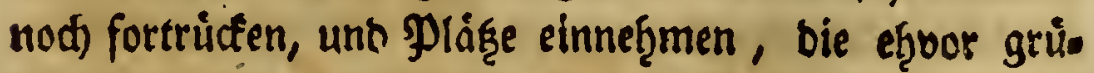
nenbe, frudjtbare ßief̨rwenten roaren, fo gef̨e bieß gan

$$
\text { รุ } 3
$$

natủr. 
natưrlid 3u. Der (Bletffier ţat frin Non plus ultra ben Purift, indem bieß fsortruidfen unterbratien mirt, nod) nid)t erreidyt. Unfere Niachfommen werven diefe Beit erleben - uno werden nid)t melir bie: Silage fübren, bnß if̧nen ber Eletffher, ber izt not) fors. rùdt, if̧re Bief̧menden raube.

Nan weißß Bletffyer an Drten, bie noch Innge nid)t ḩod) genuig liegen, Daß̧ fid) bas $\mathfrak{B}$ affer , bort

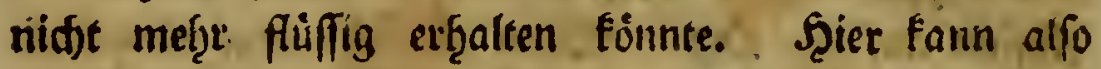

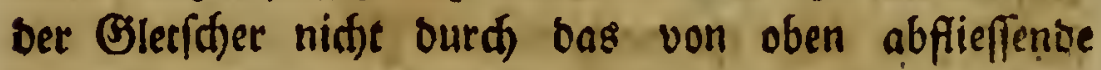
\$aaffer, bas hiter zu Eis geworben wàre, entfanden:

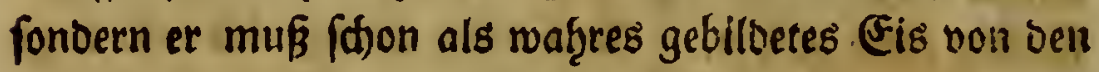
obern Eisthálern ausgeftoffen morben feun. Unto wenn

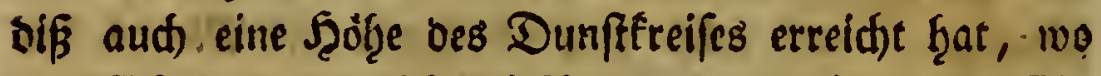
bie Warme orbentlic) wirffam genug raire, bas (sis zu fatmelyen, fo miro bod) felbft bas Dafenn ors fdion bidén Eifes, uno oas beftànbige Rad)rủcéen oeffelben in ber suft, bie unmittelbar auf bem Bletfder liegt, fo viel boul ber naturrlid)en 2 Barme auff̧eben, Daß̧ Ģier (Giletfd)er ausbauern, wo ein fleiners, einige Edufge tiefes uno breites einjelnes Stüf Eis nid)t ausbauern murtbe. Bubem ift ber Brao von 23 ärme, ber gemeines (Eis famelzt; nod) lange nidft wirffam genug, um Bjlet/f)ereis zu fa)melzen. Darúber ḩat man bie ar. 'tigften Beobaditungen gentad)t. 2(us nllem biefem folgt, ons bie Edjid)t im Dunftereife, in ber bie Wairme bas gemeine (E) zs zu fajmelgen anfaingt, nod) nictit bie Sinie bes Non plus ultra ber (J)erfd)er ift,baß folglid, Bletfder an Sorten fino, wo bas 23. ffer 
3ell im Sillertlfail, Den ro. Dettob. I 783. 319

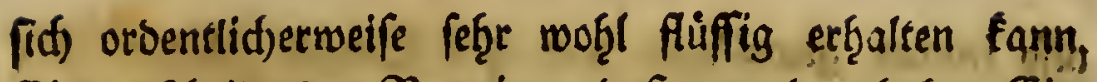

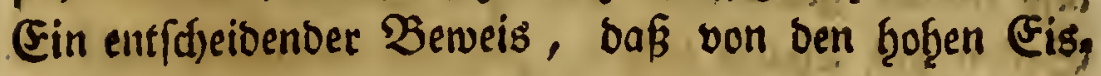
tháalern ber Ueberflü nidjt allein in Sdyneemaffer, fonbern mirflidi in gebildetem Eife ausgeffoffen miro,

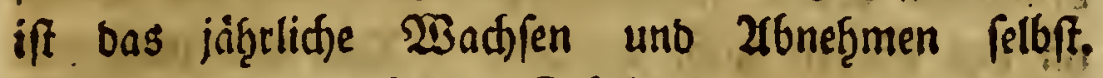
$23 e n n$ bie vorgerúdten Eticfe immer fo, wie ber Gileffder mit einem neuen Jalle votwarts tritt, wege

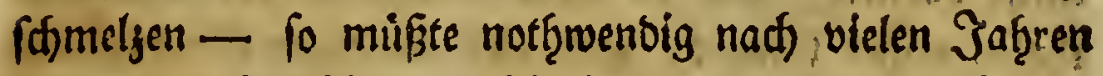

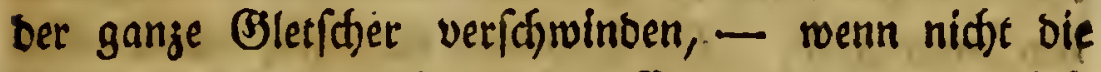
Eisfcloer bas meggefdomoljene (íz mieber burd) wirt.

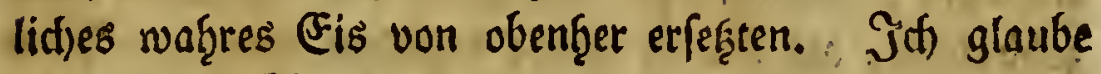
nicht, baß Sic bießs láugnen werben, mein Freunb !

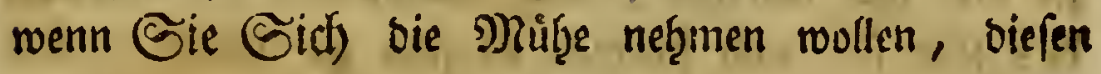
wunberlidjen Errfdeinungen in ber Siatur nachsubenfen.

Saffen Sic midt biefeen allem eine $\mathfrak{B} e m e r f u n g$ über ben Uriprung ber fenfredten Spalten auf oen

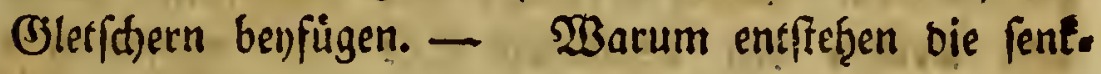

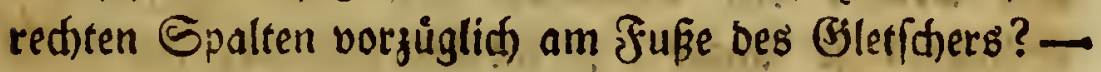

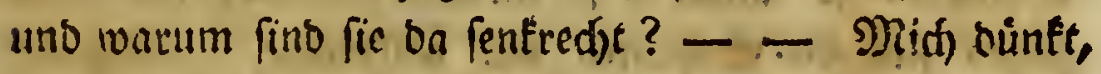

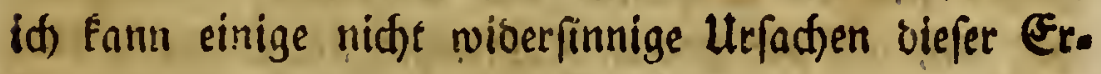
fdeeinung angeben. Das Eis wirb von oben immer in bie Tiefe bes Gleetffitertf̧ales niebergejdoben, uns nùct fo bis an ben Punft bes geffiminbern Edjmeliens

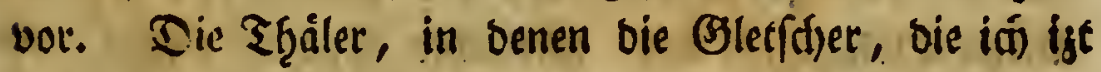
befud)t ḩabe, liegen, merben gegen bie Şổ̧e Ginan geraiumiger, uno verengen fiid gegen ben Juß̈ bes Gletffers. So wie alfo ein Stúcf Eis vorgefdoben wiro, fủmmt es aus bem geráumigern Theille bes (B)etffdertf̧ales immer in einen engern, - uns muß 
alfo immer gewaltiger von ben Bergen, bie an ben Seiten bes క̧̧ales ftef̧en, gebrưft reerben, weil es. bodf burd bie eigene Sdimere, uno burdy bas nad,s bringenbe Stủd fortgeorangt wiro, uno bie Berge an ben Eeiten nidjt weidjen. Das Eis mußs alfo notb̧mentig borften, uno in eben bem Berb̧ältnißge meg̨r borften, wie es bem 2lusgange bes Ģletfcher= thales nảher fómmt, weil bieß in biefer Bsegeno in. mer enger wirb. Die Spalten, bie butrd oas $\mathfrak{B}$ orften entfteber, muffen alfo am Suke bes (Bletidjers viel zaf̧ireither, als an ber şojge beffelben fenn. - 2lber fie fino hier fenfrecht, nidt) queerúbar. - Bemis, bas fino fie, uno fheirten es feyn zu miffen. Die vorberften Sagen Eifes, bie. ben Suß bes Bjieffdpers bilben, Egalte id) für bie ailffen, weil fie frưber vom Eistḩale ausgeftoffen wurben, als bie b̧inter if̧nen bergebenoen. Nact) Struners uno Siltmanns Betrgs nife merben bey beraltetem (Eife bie rongerechten Estifften je lainger, je minder fitfibar, umb bafur zeigen fid ole Sdididten alle fenfrect). Ilttmann hat biés auf ber zrooten Rupfertafel lit. L. vorgeffellt. Das Eis mirb alfo bier mef̧r fenfred)t berften, fo mie feine Sdjicteen fino, als wageredif : uno folglid

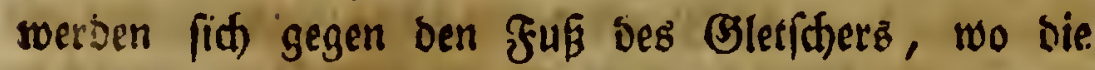
áltefte Eislage ift, mef̧r fenlredte Epalten zeigen, als

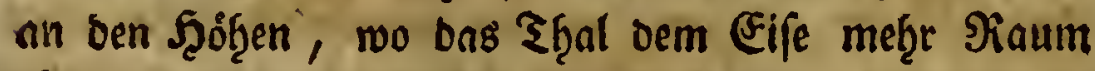
lípt, uno bie jüngern Sddidten liegen. 2aber genug über Das Wadjeen - 2tbnef̧nen - uno Entftȩ̂en

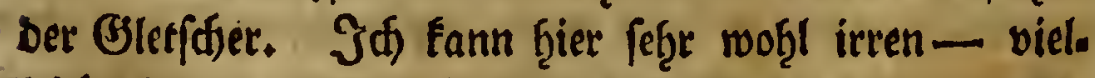
teidft bat irgenb ein Sdjriftfteller Nadjridten von ben 


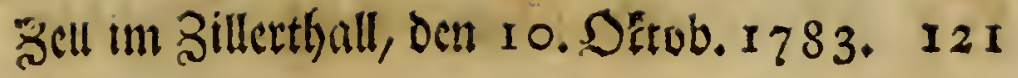

Eisbergen gegeben, bie meine (Jesanfen mirflic) roiberlegen. Zaber aus benen, Die id) vor mir liegen f̧abe, ftheinan fie immer mef̧̧e befáttigt, als entéráftet werben zu fönnen. Dr. Sattingerz Erfafrung liber bie magered)ten Extildten bes (Eifes, bie (STruner felbft

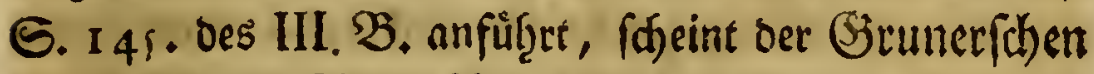
uno altmannfichen úber bie fenfrediten ju wibers fpredjen : und mid) wunbert, baf (Sinner bieß nidje

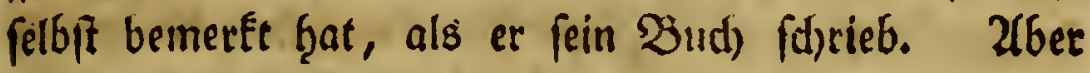
man imúpte nuch erft viffen, wo Dr. Sottinger feine Erfaḩrung gemad)t bat : ob am Fußje eines (Jlet. fithers, ober an ber Syodze beffelben, weldies nadi meinen Geoanten allerbings einen groffen Unterfdgieo geben wirbe.

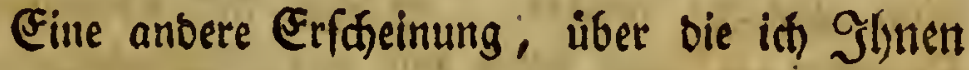
auch) narif bem, ras id hier bemertt ḩabe, meine Bjedanfen fagen muß, fino bie Strine, bie mands, mal auf ber Mitte, ober auf irgeno einem Sheile ber Pberfladje bes (jletichers erfodyeinen, oa man auf oies fem פlaz̧e rénige Tage zubor feine Gpure bavon be.

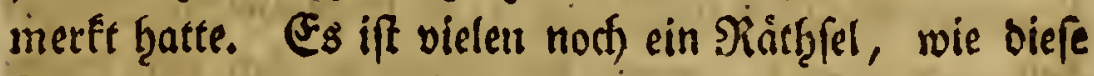
Jelstrummet saf̧in fommen. Der gemeine ग)ann läpt fie burdjgebenos aus ber Siefe bes Bletffters Durd) bie Epalten berauffommen. - Die Rälte reípt bie Steine in bie J̧ôţe, fagen sie Zelplet, - uns wirft fie mit Gewalt bey ben Sefnungen ber Spalten

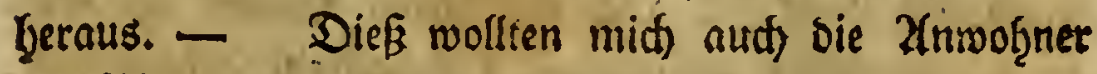
Der Pinzgauer. (\$letfher verfitfern. MRein Füürerer, Jafub Jroffer, war einige Zalt Meffer im 2 Jaregge, uno er betḩeurte mir, cinemals 2fugenzenge bavon ges

$$
\mathfrak{H} 5 \text { wefen }
$$


wefen zu felyn, wie unter einem gewaltigen Rrndfen nus einer EFalte des bortigen Räfes ein geofer Etein herfür, unt hyol in bie suft gefitfleutert waro, ber

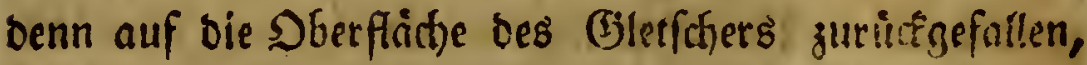
uno ba liegen geblieben wäre. Dic Bielebzten halten

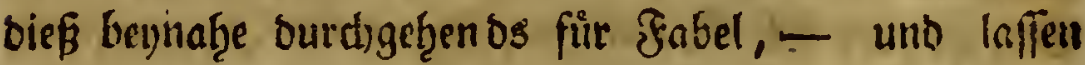
bie Eteine, bie auf ber Sherfåăthe bes. Bletichers erfdeinen, von ben Geiten ber Berge, bie î̧n bes gränzen, abfturrzen. Diefer Mgennung fino oer größte Thyeil ber Edrifrfteller über bie Erisberge. Eie

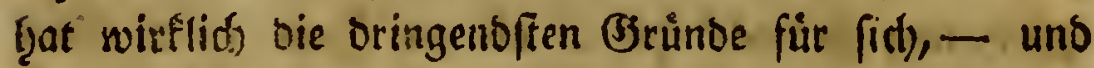

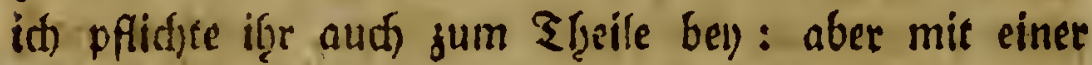
geviffen (Finfdrånfung bunft mid) auch) bie Meinnung bes gemeinen Mannes nidyt fo gar wiberfinnig, als man glauben mód)te. Eogar mit philofopheifden Brunben - uno felbft mit ben Beobachtungen ser

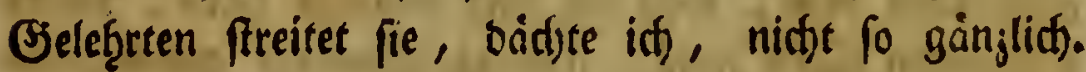

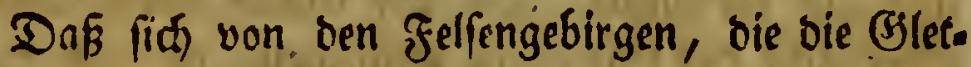
(d)er begränzen, Steine lostreiffen, uns auf bie unten.

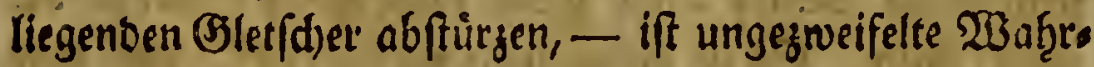
Geit: aber wie, wenn bie (s)let(d)er fid bon biefen Bergen entfernen? wie, wenn fie, fo wie bie beffories benen, nad) einer groffen Jृdfge turd) fein Felfengebirg mefre befdränft, ein umunterbrodjenes, weites Eigjels, bas fid) über bie (Bipfeln ber Berge hin verliert, aus. madjen? roenn fid) aud auf biefen Theilen ber (Fiz. berge, bie ḩobher als alle umliegende Jelfengebirge finb, Steine finden, - wo fommen fie benn frer? bon meldjen $\mathfrak{B a}$ anben futrzen fie $a b$ ? - Seier ift biefe 
Bell im Sillerthall, Den 10. Oftob.1 783. 123

Meynung gerabezu unanmentiar. Daßj finf aber auf ben Giod)ften Eteflen ber Eisberge Steine finben;

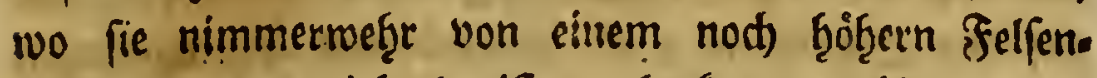
sebirg, - bas nid)t oa ift, - Jycrfommen Éonuen, ift unläugbare Erfaf̧rung. S(d) muf Şbticn b̧ier auso fdrreiben, was ber vortreflide De suc in feimen Briefen an oie Róniginn von Broß̧britfannien über biefen (Sea genftand fagt: es bient gar ju gut, meinen Breifel ju beftarfen. Nadjoem er bie Ltrfad)e bes 23 adfens uno Zanef̧mns ber Bletfder angegeben ḩat, fo fitigrt er fort:

i Indeffen mus man bed befennen, baß cB

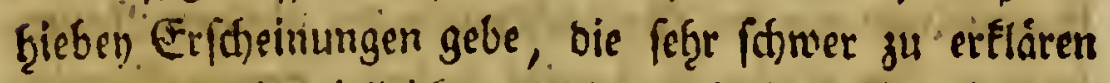
fino, uno rie vielleid)t aus jener eingigen turfadje gne nid)t jil erflairen ftef̧en : vorneţmlid) bas Z(uswerfen von Strinen, ferbft bon zientich) gropen Feleftucten,

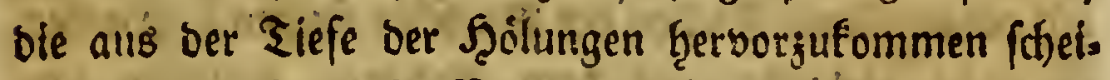
ren; fo aud) grope Mengen von Ereingruno, uno von Eteinen, bie man nitten auf Der Breite Der (Eistgalden firtset, bie, allem Infheine nach, gar nid)t von ben felfen umber fich herfdretben fönnen: uno es verwumbrrt mids baf̧er feineswegs, baß bie Einmofinner ber (Sebirge ben biefem allen etroas Zaubes rifçes zu fę̧en vermennen. Ith habe mir felbft nie. mals einen Brunb bavon angeven formen, ber mir (Genugge geleiftet ḩătte, uno id) habe aud von Znoeen nie etros geţ̧ort, oas es beffer erelárte. Jith glaube,

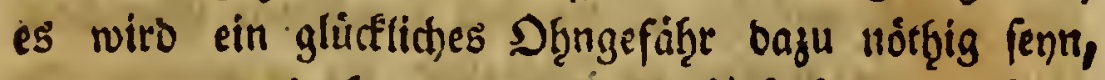
um es ju entbrafen, ba man die Urjadge in ber Ş̧at iberrafds! " 
So meit de Suc. Jdh fann hier mieine Be. imerfung über bie \$lennung oerjenigen, bie alie Steine auf oen BJletfacicn von benad)barten Felfengehirgen berleiten, nodh nidft faflieffen. Sogar ben ben Zlrmen, mit oenen bie eigentfidjen (b) letfd)er fiós zroiftjen ben Felfengebirgen burd) gegen bie Tiefezu verliefgren, hat

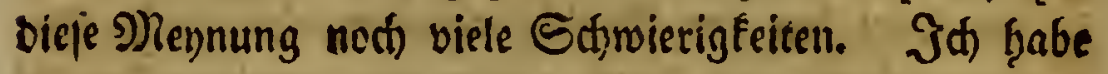
in meinem leg̨ten $\mathfrak{B r i e f e}$ gefagt; onß bie Dberflädje berielben in ber Mitte geroòlbt, uno gegen bie Echenfel

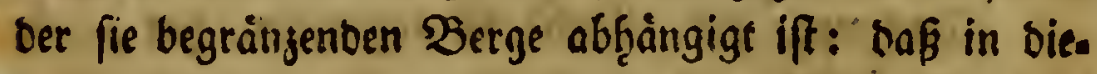
fer Tiefe immer fleine Bräbcfen liegen, ourdf bie in verfchiebenen Runften das Eistraffer abläuft. Dißs frimme auct) mit Den $\mathfrak{B}$ cobactstungen der übrigen $\mathfrak{T a t u t s}$ forfder, bie Eisberge bereist b̧aben, genau überein. saffen wir mun vort biefen Felfengebiegen Steine abs fiùrzen. Werten fie wob̧l liber bas Gemólbe bergan auf bie mittleren Stellen ber (Bletfder freigen? Wierden fie nicht bielmę̧r auf tem $\mathbf{2}(b$ ḩange berfelben den Dergen zu entwebers liegen bleiben, ober über benfelben in bie untenliegenben (Bräbdjen rollen? - Esçen wir

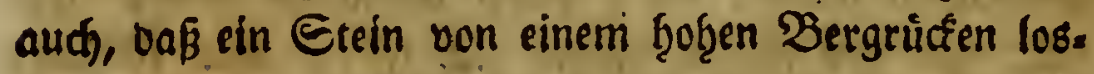
reiße, - uno in Sturje auf herborragente zelsftrufe

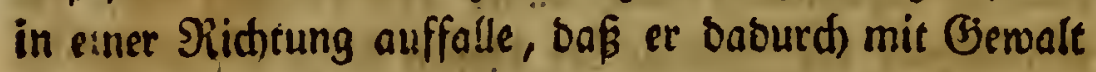
einwairts gefकleubert merte, - wie biß aud) ganj juveriajpig feber oft gefdeţen mag, - fo wirb man fids Dod) Diefe nie to beftig borftellen fönnen, bak fie ben

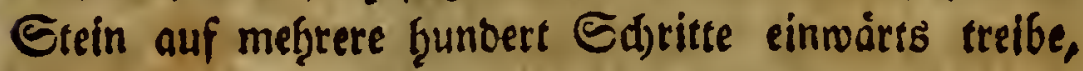
uno if̧n to auf bie Mitte bes gerwolbsen Bletfders

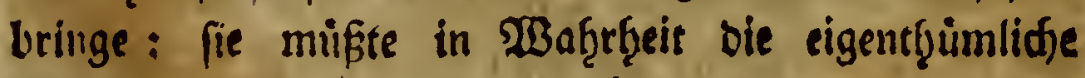
Edjwere beffelben gan aufgeben, mit ber er, nach 
Bell im Billerthall, Den 10. DEtub. I 783, I25

Diefem 2lufpreflen, balo mieber fenfred)e nach Dem Boben zueilen mirb. Das mag de Suc ungefäbr bey hen $\mathfrak{B}$ orten gedadit ḩaben, bie id unterzeidgnet bुabe. Biele biaben biefen gerviß̧ beträdstlidjen Einturr uno bie Edfwierigfeiten, iffnlidse sagen ber Eteine burd) bas adbrollen bon ben benadfbarten Felfen zu et. lautern, eingefegen. Gie mollten fid bamit beraus= miffelii, Dás fie glaubten, bie Etcine, bie nuf ber

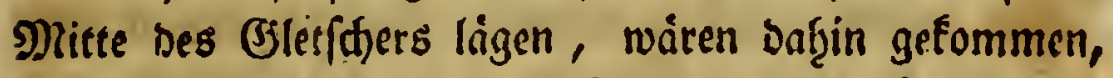
als er nod) nieorig war : fo wie er fid) aber erbebt,

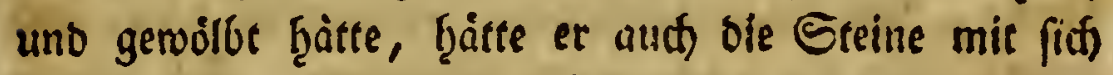
nufgetroben. 2(ber dißz grürtbet fid) in sem eben fo falfthen Waf̧ne, daf bie (Jletfffer bon unten mathfen, uno bie Sberfládje oerfelóen immer unveråndert bleibe: eine Berfauptung, bie lange ffton buth) bie triftigften Brünbe wiberlegt ift. Co wie vie (Jletfdjer zuberläßig bon obenterer bur(f) den all;äfrlictien Shinee, ben bie Eonne nur jum Ţ̧eile iveguufdimelzen vermag, bes tràdtlid) zune(zmen; fo würoen birfe Steine aud) mit jebem Jabre unter bem nenen Sunadjfe begraben wer. ben: nimmermetcr wourben fie fid) alle Jabre auf ber

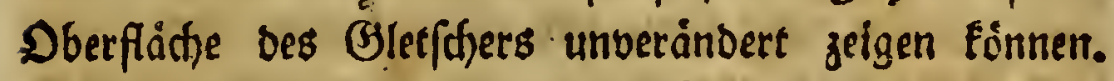
Suberm fief̧e man ja Eteine an Etellen, wo man nue wenige Tage jubor feinen bemerft ந̧atte.

Dem gufolge ift es wob̨l ridtig, baß cin Theil ber Steine, bie auf ben Bletfidern liegen, bon ben bee nadfbarten Felfengebirgen Ģerabgefturgt: - aber gewiß even fo rirjtig, baß nicfis weniger, als alle biefe Eteine bab̧er gef́ommen find. Wollen feģen, of es 
benn fogar wiberfinnig if, onk audd ourd bie Epalen Gteine terreufgefdsleubert werben.

Daßß sie (Siletfaber effers toote Rörper bon Men. (d)en uno $\mathfrak{B i e f}$ ourd) sie Epalten auswerfen, - ift bei) Siruner fribf S. 158 . Des IIIten Sianbes anges merft: Daf dieß aud) mit Steinen - J̧olz - uno

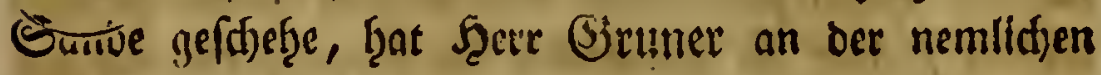
Gtelle bemerft: bafi bie unter ber Eisbecfe verfammelte Suft oft in befigigen Sivinien burd) bie Fisbecfe aus. breche, uns fie jerborfte, ţat Giruner S. 1 41 . und meitf(t)id)tig 5. 136. 137. beţauptet. Jä glaube nid)e meţr als diefe Єảz̧e nơtb̧ig zu f̧aben, um einige

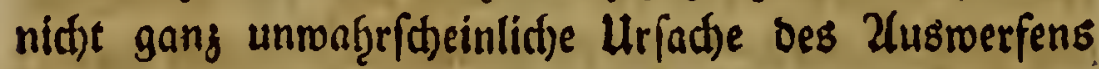
ber Eteine ju finben. Nur benn Eann bie von Girus nern angegebne Urfadje bes 2luswerfens tooter Rör. per bie wirflidge fenn, wenn fie gang unten am 2fus. gange bes (Jletffiers, wo biefe गaffe bes Eifes ges wớg̨nlich nur von ciner máäigen Decfe ift, ausge= worfen worben. Dort wiro bas viele unten verfam. melte $\mathfrak{B a f f e r}$ bie Eisbecfe aufzuheben im Stanoe fenn. aber nimmermef̧r fann bas $\mathfrak{B} a f f e r$ unter bem Bles. fher fo entefeflich anwadten, uno fo gewaltig wirken,

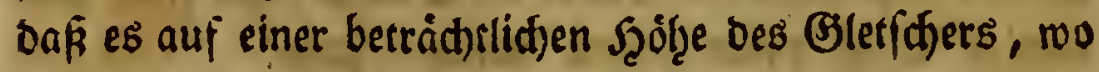
bie graislidyen Epalten oft eine leinem 2luge erreid). bare Tiefe bes Eiles zeigen, - audh nod bie Decte Waben follte. Das $\mathfrak{B a f f e r}$, Das an fo einer Etelle in Der Tiefe bes Sletfhers flief̂t, fann wobfl einige Edfutge fod) in bie Spalte Geraufgeonuctit werben, wenn es ju fehre anwòid), : aber fo eine Eismaffe, wie oa liber

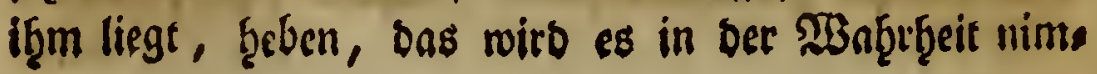


Bell im Ballerthall, ten 10. Dftob. I $783 . \quad 12$ ?

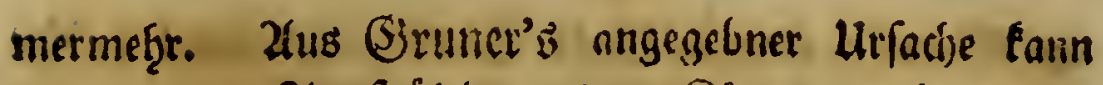
id) mir oas 2(tr\&fpilylen tobter Rórper, aber nid)t

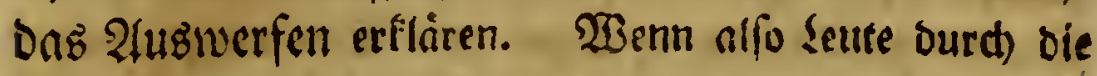
Epalten ausgenorfen nurben, burdh bie fie Ginein: gefallen, uno umigefommen find, fo fann bies nur fef̧e felten am Zfusgange bes (H)effchers gefdethen fenn, weil

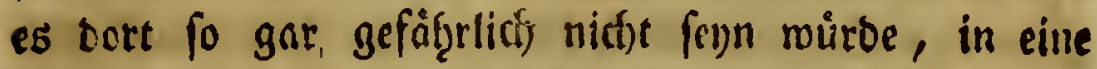
Epalte zu fallen, ba cort die (Fistede von feiner bee

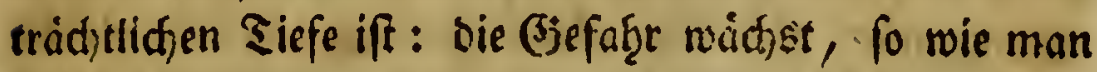
ben Blet/d)er ţơther binanfteigt, Denn in eben bem ßer. baältmiffe nin:mt nud) tie Eisbecte an Didfe zu. Stz

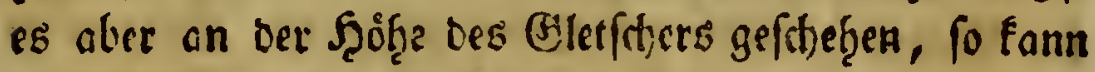
bas Zuff̧eben Der Decte nur feţr felten bie Urfactje babon groefen fern. 2luch bie Erflarung, roie bas J̧olz bas Eis gerpaltet, und zlusgang fud)t, thut mit nidit genug, fo menig als bie folgente von Steinen

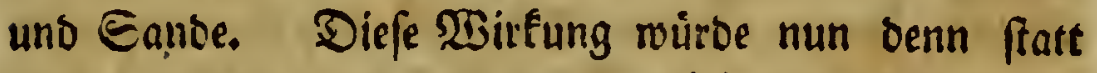
haben, renn alles bieß nuf ber Dberfache bes. Gilet, fthers láge, fo Dak die Etraf̧len ber Gonne unmittele. bar baranfahluggen: Denn wie founten fie fonft to wirffame $\mathfrak{B}$ ärme ben Steinen unb Sande mittheilen,

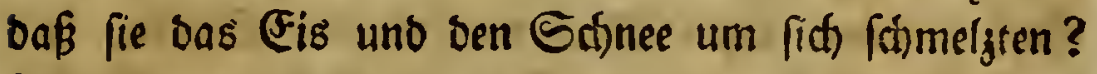
Wie follte dießs gefdetgen, menn fie einmal im Eife begraben fino? Llno fino fie auf ber Dberfläche bes Bletfders eingefroren, fo Eommen wir wieber an bie Srage zurưf, wie fino fie bafíngefommen?

Es muiffen alfo nodf andere Utracten ba fenn,

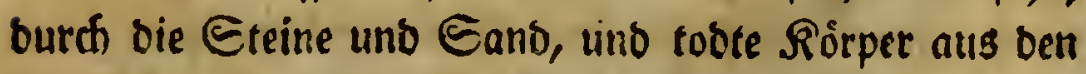
Epalten ausgeworfen werben. Daṕ burch bie unter Der 
ber Eisteçe eingefhlognen Dünfte mandimal befrige

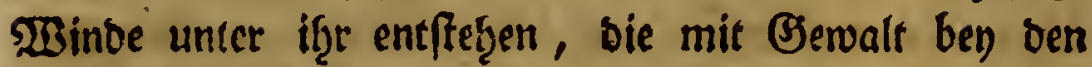
Spalten ausbrectien, - fogar neue Epalten ju werfen im Stanbe fino, wo fie nicft fhon gebaf̧nte Etraken finben, das ģat Siruner gezeigt. Seg̨en roir, es oringe fo ein $\mathfrak{B}$ ino unter ber Decfe fyeraus: - er finbe eine Spaite, burd) bie er ofine SBitoerftano aug. brechen fónte : es ftȩ̂e if̧ı aber ein Etein, ein Sndraufe, oner ein tobter Rórper, Der burd) bie Spaite ḩineingefallen, uno wob̨l efma gat in berfelben frecten geblieben, of̧ne bis an ben Sorund ju fommen, im 2 ege. Wenn ber Winto fáb̨ig ift, Epalten ju werfen, wie itgn Giruner felbft für bie grófte Uurfadje berfelfen angiebt, fo wiro er noch) leidteter bie Steine, Sanof̧aufen, uno toote Rörper, bie if̧m im $\mathfrak{B}$ ege liegen, um frey bey ber Spalte ausjubred)en, loss maden, uno auf feinem $\mathfrak{B e g e ~ v o r ~ f i d ~ h i n ~ a u s w e r f e n ~}$

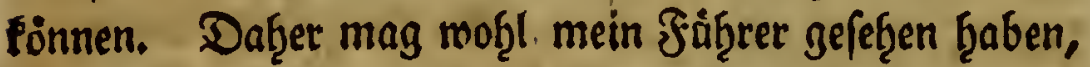
baß̧ ber Etein einige Rlafter fyod) in bie Suft flog, und benn erft auf ben Sleticher zuruidfiel. Jat) finbe gar nichts wiberfinniges in biefem Zuswerfen ber Eteine. Entffef̧t burd) einen $\mathfrak{B}$ ino wirflid) eine Spalte, uno es ift in ber Bjegeno verfelben ein Stein eingefroren, ber izt tief im Eife liegt, fo wiro itgn ber $\mathfrak{3}$ Bino auf bie nemlide 2(nt losteiffen, uno zur Spalte hinaus: faleubern fónnen. Man wiro fid Saruber um fo weniger wuntern, wenn man fief̨e, weldfe Menge 10. fer Steine mandimal unter bem (Stetfdfer liegr. Jo warb bavon am Fufe bes ŞBareggerfäfes ủberzeugt,

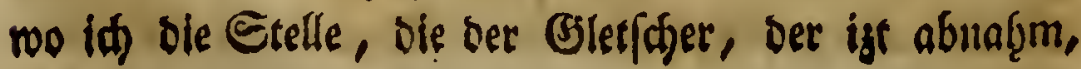


Bell im Billertball, Den 10.Dltob. I 783. 129

eingenommell hratte, mit Steinen ůberbedt fant. Das, was in Erobeben, uno bey Bulfanen unter. iroifthes Jeuer, uno bie baburdf mit Beroalt ausge. bef̧nte Suft tḩun, bas wirb Ģier ein J̧aufe ber burds anbere Llfacten unter bem (Slet/d)er in Berwegung gee bradten Duinfte berwirfen. Man reiß boch, daß̧ bie Banberer auf ben Biletffern ofters ein gerwaltiges Beben Der Eisbecfe erfaḩren ḩaben.

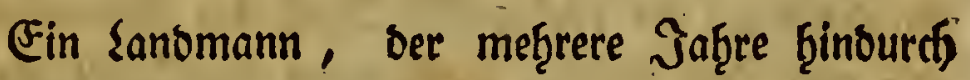
über unfere Bletfther gereifer ift, fagte mir : Das Rás leibet feimen Stein unter fich, wenn es wád) st. Ja) will hier ben 2(usorucf des Mannes eben nict)t gutheiken : aber in biefen Saz̧ umyefthafen: Der Gjleticher wirft melbr Gteine aus̉, wenn cr wád)Bt: Eo̊nnte feine Beţauptung wirflid gegrủnbet fenn. SEenn wir De Suc's Erflärung Des $\mathfrak{B a d}$ )ens füe

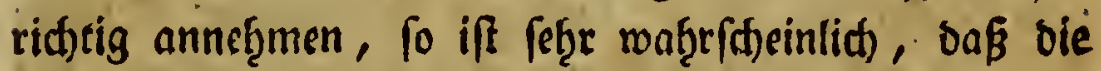
unter ber Eisbecfe verfaloffene suft niemals meţe in Bewegung gefegrt werbe, als benm Fortruicfen bes Bletfithers. Er fann feinen Ed)ritt vorwàrts mađjen,

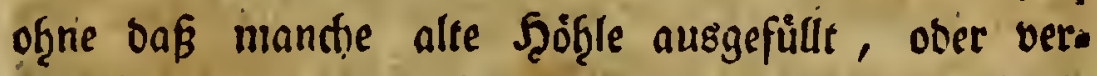
किloffen, - und mandie neue geòfnet wirb : auch) bie Wirfung ber auffern suft auf bie unter bem Eife einges fperrten Dünfte wiro ourd Das $S d$ )lieffen alter Epale ten, unto ourd) bas Entfetęen neuer fegre mannotffaltig geänbert. Aflles bieß muß benm Jorffhreiten bes (3) let/chers notf̧rendig eine beftige \$eroegung in ber unter iţm eingefdloffenen Suft Gerborbringen, Die aud) fu mandbem Eteinnurfe bie Urfadje merben fann. 


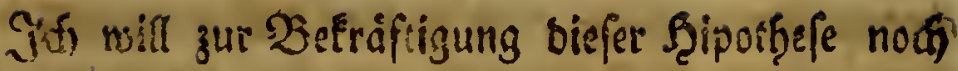
einige Besfpiele nnführen, bie, wern idf) nid)e irre,

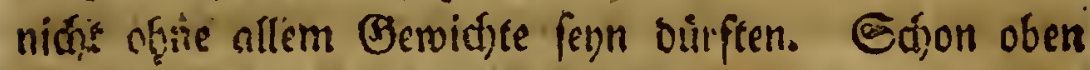

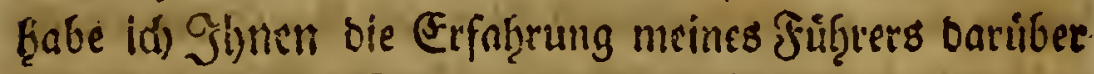

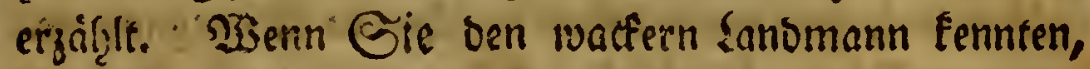

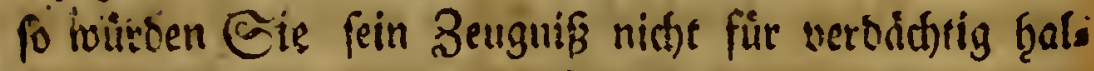
teir. Selute von biefer Rlaffe ţaben meg̨r Gelegenţeit über äg̨nlidje Erfftheinungen in Der Natur Erfab̨rung

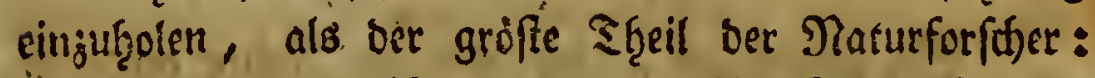
unt man irrt genvis, wenn man if̧re Beugniffe of̧ne Unterfajiés, ais Bergniffe von keuten, bie zu reenig Bestrtgeilungstroft heben, verbåd)tig madjt. Ein groffer Theil berfelben fyat meģr als gemeinen afletags

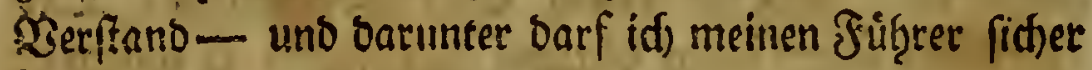
such) jäflen. Zlber eine andere Erfaf̧rung, vie nid)t meniger flir meine Mieynung fprid)e. Ein Freusto, der von Galibutrg cus vor meţreren Jof̧ren úber Mitter= fiil, uno sen Felfortrucrn nać) Sp?atrai reiste, frat

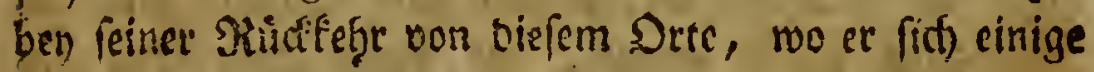
Wood)en aufgielt, folgende Bemertung ain (Sd)loffee Silife gemad)t. Diefer weillaufige Bjetfther liegr ben Sieifenden, roenn fie den findliden fuß bes Felbertauerns erreidt) Haben, an ber rectiten Seite: am Junfe bes Biatifhers liegen bie Stbloffer Alpen. Mnein Freuns

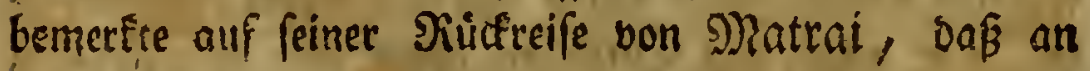
tem Niande meţrer Epalfen viele Steine und gange Saufen Gantes uno Eroe lagen: ba ond einige 33effen vorber ben feiner Durdreife von allem bies fem nidjte zu feţen war. Daju verficferte er mith), Daß̧ alle biefe Steine uno Sanbḩaufen nimmermę̧r 
3ell im Sillertfall, Den 10. Detob. 1783. I3

burd) einen Sturg von einem Seitenberge fiâten an if̧re Etelle gebradjt merben fömten. Uno warum wairen fie gernde am Fiande ber Spalten liegen ges bliebẹn, wenn fie burds einen fall von obenţer baf̧in gefommen måren? - Dißs bünft mid) ein unumftò lidjer 3 erweis zu fenn, baß alle bieje Steine uno Ganbbraufen burd) eine 2(rt Erbbebens bey ben Spals ten tzeraufgetworfen murben. Es bürfte alfo mof̧ bie Befdrelbung, bie Şerr Ģregoriuz von ben Eisbergen mad)t, nid)t in allen if̧ren ₹̧̧eilen fo feltfam fenn, als fie Girunern vorgefommen ift.

Es wàre nun einmal genug über bie Eisberge; uno bie Erftheinungen auf Demfelben vernunftelt.

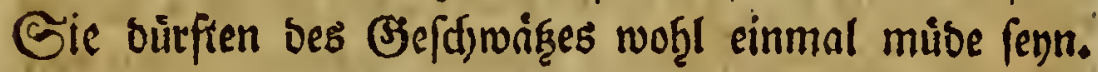
Darf idf Ojhe Mennung Dariber - uns sie Eins murre, bie Silnen etwa ben Durdslefung biefer $\mathfrak{B}$ e. tradtungen beryfallen, in einem Sllecr antwortfdreiben

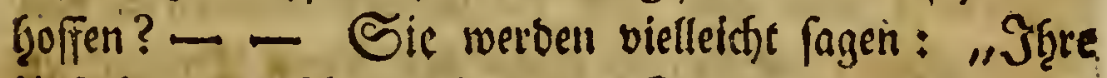
utratien bes 2luswerfens ber Eteine, ber fenfredfen Epalten, u. f. w. thun mir eben fo wenig genug als

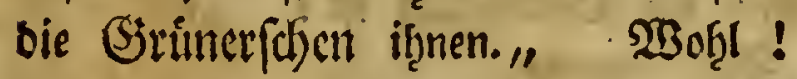

- - Si cui videor non juftus, inulto Dicere, quod fentit, permitto. Horat.

Iक) (d)äme mid nidjt bey Gegenftänoen zu irren;

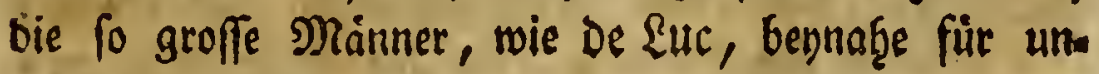
etlläbar ḩielten.

Jat bradgre auf ber Stelle, von ber id Shynen

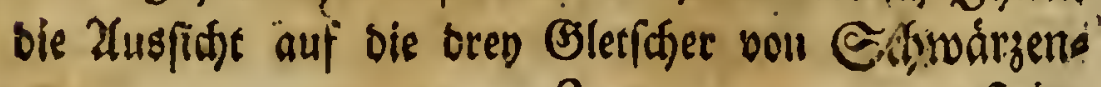

$$
\text { I } 2
$$

ftein, 
ftein, Niof́ruf und SBaresg entworfen habe, nog̣t meţr als eine Etunbe zu. Viclleid) múrten mir

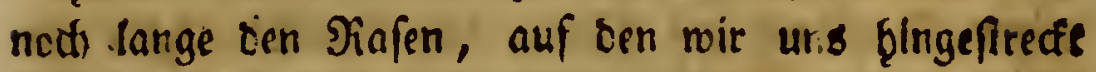

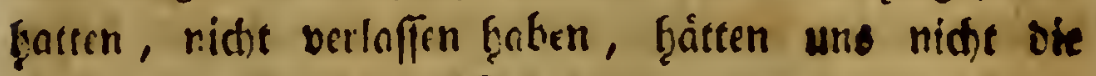

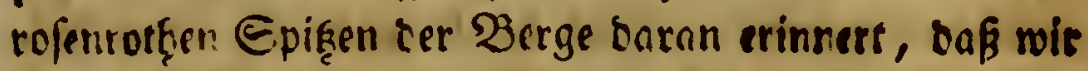

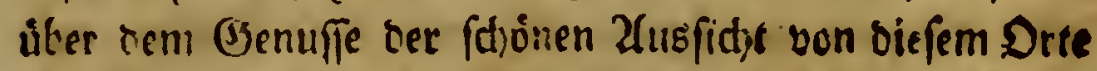

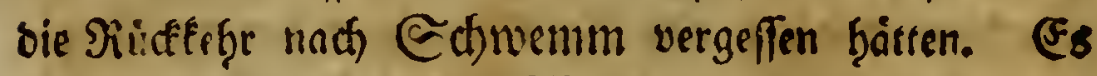
war ein herrlich fdiorecr $2(b \mathrm{cno}$ - uno id) badte bas bey an eine E:elle in $\mathfrak{S}$ siclanos beutffiem Merfure:

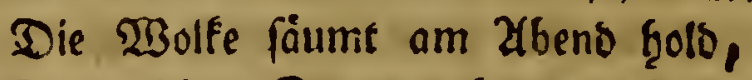

Der reicten Eonne geborgtes Bolb.

Zuf cem Siucfwege fammolte id aus Miargel ber Beit nichts meģr als ein paar (Exemplare des Ranunculus platanifolius L., der unter oem $\mathfrak{B}$ ege zmi/den $\mathfrak{W}$ are egg uno (Siramand fȩ̣r fparfam mád)st: Dann ben Rumex fcutatus L. - uno sie Rofa alpina L., eine Bilt:me bon einer unnactaf̨mlid cunteelrotben farbe. Man fiff̧t von bier auf bie fdjonen Zlipweisen bon Echỉnpichl, bie ben Befizern ber alpen $\mathfrak{B}$ rcitlänes uno $\mathfrak{W}$ aregg geb̧oren. Eie liegen jenfoits bes Beme

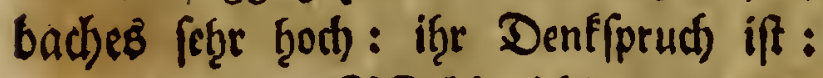

\section{Z’edjonpidsl,}

if 's's ben Rúben wobl,

und sen Melfern übel.

Dem Bleţe bef̧agen biefe Weiben fefre wob̨l; benn to

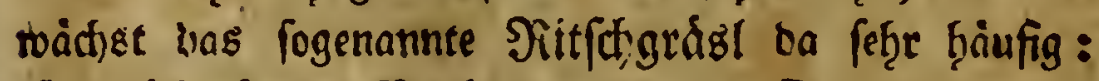
aber nidjt fo ben Melfern, bie bie Erzeugniffe son

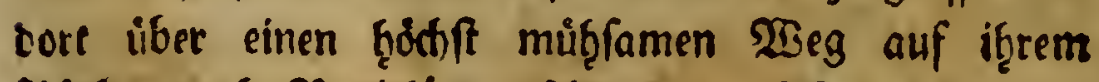

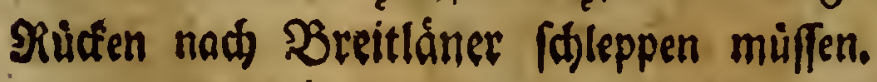


Bell im.3illerthall, Den ro. Dftob. I $783 . \quad 133$

Nun fam auch ber graue Gemejaảger von ber

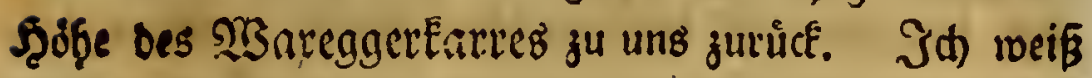

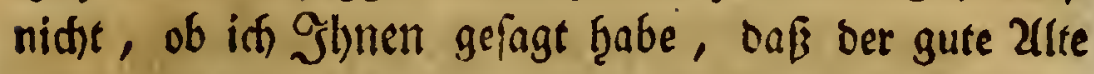
genau bie Etunbe bielt, bis meldher id) ifgn geftern nach ber 2lipe Edfwemm beictieoen hatte. Zluf ofm

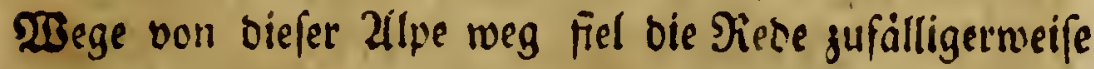
auf ben Epeif. WBie id Shlunen fagte, heiffen die biefigen allpenbenofinner bie Ed)lüffelblimat)en und âte. tien úberhaupt ben Epeif. Im sungau fennt man unter biefem :amen ganz eine antere Pflanze: ncmlid ben Seltiften Balorian (Valeriana celtica L). Ja hratte offers nadgefragt: ob auf ben biefitsen Zllpen biefe glflange nid)t gefunben muirbe: aber allgeit warb nir alle frofnung, fie bier su erţalten, benom!nen. Nun forfdite id bey bem alten (Semsiáger barnad), und er erbot fid) mir, biefe Pllanze auf ber Sçobe bes Trarceggerfareres für midh zu pflùcfen, wo fie, roie el fagte, nid)t felten wáre. Sie fönnen fidt) vorftellen,

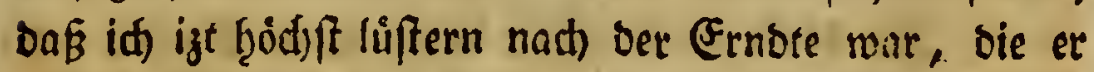
meinem $\mathfrak{B e r m u t h e n ~ n a d ) ~ v o n ~ b e m ~ g e l b e n ~ E p e i f e ~ g e . ~}$ madit baben follte: aber id fand, baß feine Pflanze nichts meniger als Valeriana celtica $L$. ; fonoern Primula minima L. war. Untelbeffen war id

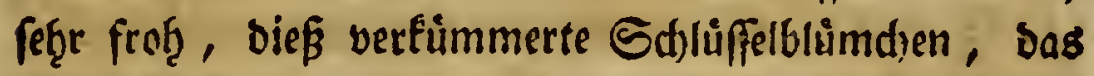
feiner 2uffage nad) auf ben Ģodfften Sirffen biefer Berge wåd)st, zu erf̧alten.

2Bir eilten nun, ba es allmàtflig ountel $\mathfrak{j u}$ merben anfieng, ber Zllpe @d)wentm zu: wo id) beym Sodffeuer uod einige meiner PPflanjen uno Infeften

$$
\Im_{3}
$$


unterfuct) ; uns nad) einem lönoliden Xbenomable bon ber ḩeutigen befdmerlictjen Sieife austuḩte.

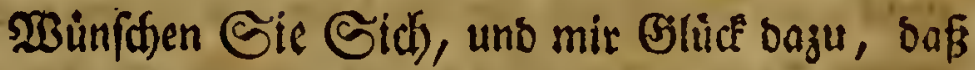
id) enblid) von biefem unbanbig lnngen Briefe ents bunben bin. Mir (d)anberts belym Durchblattern ceffelben. Dennudf habe inf Slinen vergeffen zu fragen,

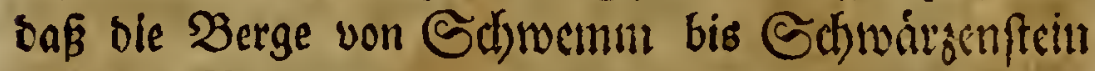
linfs Der Bieitenberg, an beffen Juffe bie Eetrif̨utte von Sdyemm liegt, denn Das Gtcinkeirl, Die Scenns

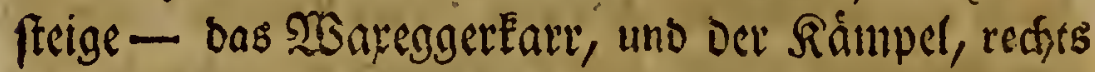
Der Sireiner uno bie oben fifion genannten Biletfdiet uno inge Swiftgenberge fino. Die Scentlfteige ift ber sieblingsfif ber Gemf : fie ift gerabe über ber Eenn:

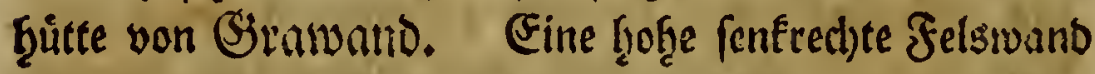
unterftủjt ein fleines angenef̧mes sisalbden von Fid). sen uno Birbelbäumen, Ginter bem wieder faf̧lere Je?. fen Stufe auf Etufe Şimmelnn freigen.

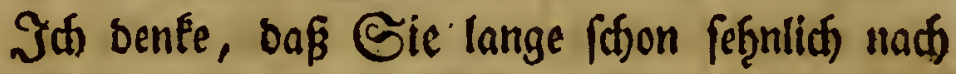
Der Berfidjerung von unwanbelbaret Freunb/djaft ḩar. ren, mit ber if bin

\section{J̧̧ Snoll.}

\section{2(d)ter Srief.}

Bell im Sillerthall, sen 15. Dec. $x 783$.

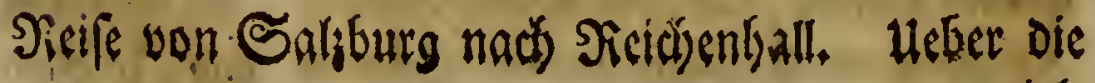
vicls 
Bell im Sillerthall, Den 15. Det. I 783. 135

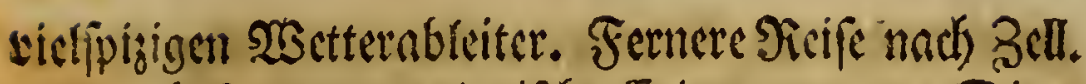
Sorliufige entomologifche Erimnerungen. Dic falzburgiffjen Sothriterenten.

5 ier fig' id wieber in meinem Embrio von 刃us feum - mitten zwifdsen meinen Infeêten uno Pfanjen inne - und benfe mid in bie glüculliden,

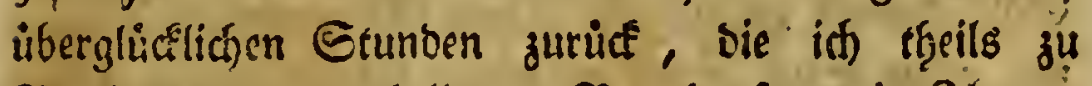
Berd́tolsgaben, thells zu Burgḩaufen mit Shlnnen,

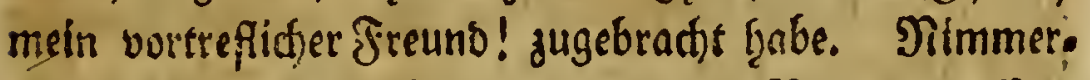

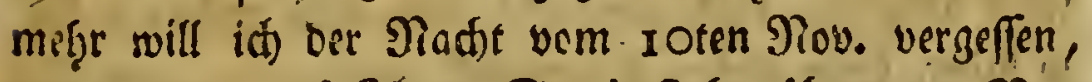
in ber wir auf Shber Etubierfube itber bem Ber: gnủgen, bos uns entomologiffde und botanifde unter.

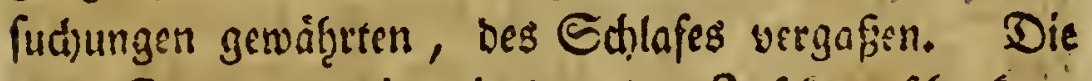
paar Stumben, bie wir bort ben Infetten frisenffen,

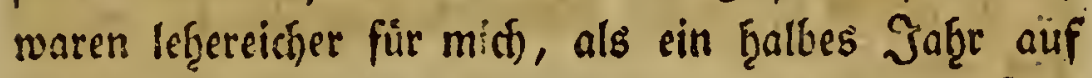
meiner Erubierffube. Unio wie waren fie's? - Das werbe inf Sģnen gleid) fagen, mein sieber!

Daf in wisber in Befl bin, bas wiffen Sic nutt:

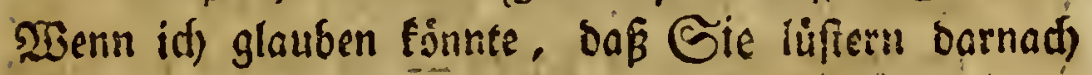
wären, zu erfahren, wie mir's auf ber shidd héfe gieng,

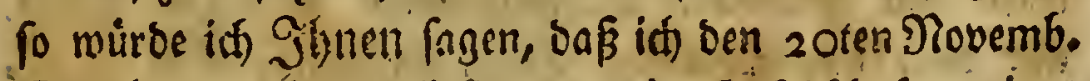

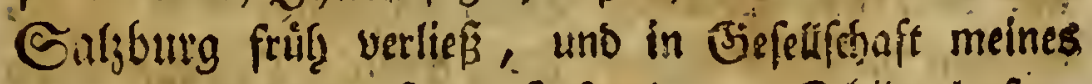

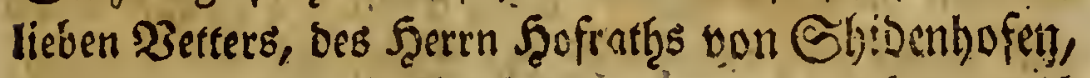

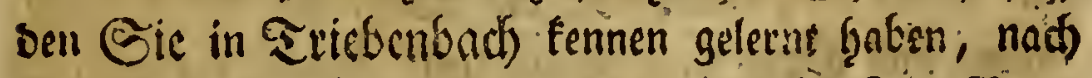

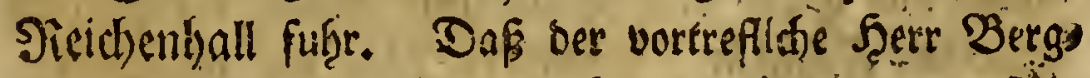

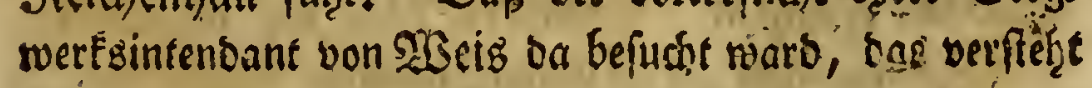
fid) : und baßs wir von if̧m mit viefer Befálligferit ems pfangen wurben, Das barf idf Shlmen and) nidfit er(t) fagen; bens Eic fennen ben liebenswürtoigen Cḩarafee

$$
34
$$

biefes 
biefes berbienten Mannes. Eine ber erften fragen war nach bem Woḩlbefinben unfers Fucfili - ber Jृerren Sling uno Biegler. Denn id) muß̧ J̧̧̧nen

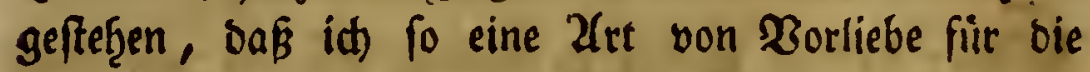
Ed)weizer frabe. Şerr von Slats war bann fo gúrig, uns an ber Şano ourd) bie neuen von iţm angelegten

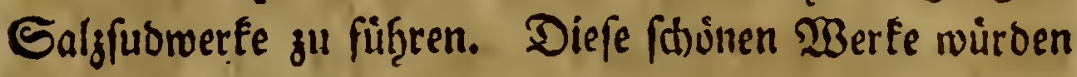
mir allein Stof genug zu einem langen Briefe geben, aber Sic ḩaben fellbe unfeb̧lbar von Berd)tesgaben aus feloft befud)r. Llno nuenn @ic bas nid)t geţ̧an f̧aben, fo laffen Gie biefen Zlugenbliaf anfpannen, uno fahts ren gerabenwegs nad) Sicid)enball. Beit uno Reife.

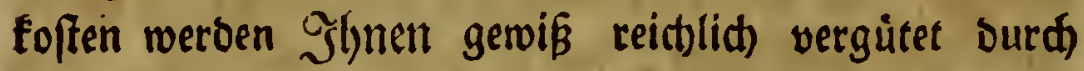
Das $\mathfrak{B e r g n u g e n , ~ D a s ~ S i e ~ b e n ~ B e t r a d t u n g ~ o i e f e r ~}$ in fo vielem 2 etrad)fe fetgenswurbiger $\mathfrak{3 e r f e}$ empfins ten werben.

Wàre id) Anaftrcon- ober, wenn Sie wollen, aud nur einer unferer toaben Didsterlinge, bie irgento

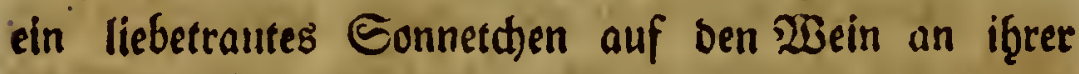
seier b̧erunter geflingert b̧aben - To wuirbe id lange fhoon ben herrlidjen Ed)weizermein befungen ţaben, mit bem uns f̧err von Rlais am Enoe nod) zu bes wirtţen bie Bhite hatte. Dieß steflicte Dejeuner warb mit einem Befprådje ùber bie ß̉etternbleiter gemuirzt, inbem uns Şeur von $\mathfrak{R}$ lais mit peines vortteflict)en

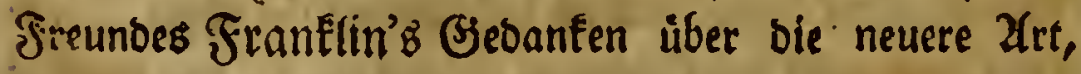
Di: Wetterableiter zu erriditen, befannt mad)te, bie er exft vor furzem aus bes groffen Zmerifaners Runde gef̧ört ḩatte. 
3ell im Billertlyall, oen 15. Dec. 1783.137

Frantlin ift nidts weniger als einverftanbek mit ben vervielfad)ten Epig̨en uno Bufammenführungen ben einem groffen \$geile Der neuern ßetterableiter: er ginubt, Daßs ourch bas Bieţen an fo verfthiebenen Ecfen und Enoen bie eleftrif(d)e Materie - wenn iid) fo fagen barf, meb̧r vermirrt, als langfam abgeleitet mirbe, uno viefleidft bürften noblit bie Ed)läge baburd mand)mal mef̧r heförbert, ols abgezogen werben. Eeine 2 (rt $\mathfrak{W e t t e r a b l e i t e r , ~ b i e ~ e r ~ a u d ) ~ a n ~ f e i n e m ~ ( a n d , ~}$ gute Jjaffé angebradit ḩat, ift hiơffit einfad). J̧err von Elniz war gưtig genug, mit einem Blciftifte eine Jeidgnung bavon ju madjen, und er niro eine äḩnlidje auf tem Ealzpfonnengebatioe in Sicidjenlyall errictsten. Eine cinjige Etange, dabon bie Epige ein paar Boll breit gut vergolbet ift, uno bie benn fo ganj einfach mit etra fingerdicfell Eifenftangen, - wie man fie ben ben (Eifengittern für ben Jenftern frat, - nart) bem

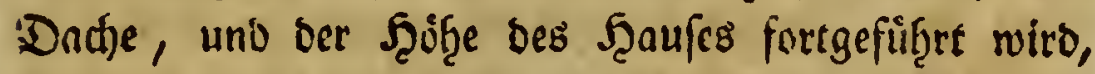
Darinn beftef̧t bie ganze 3uriffung. Sie hat nad) Franklin's Mennung $\mathfrak{B}$ Birfung genug auf eine gute Etrecfe, - id babe bie benláufige Sabl ber Rlafs ter vergeffen, - und ift babey ungleid) reniger foft. fpielig, als bie meiften zufammengefescten Wetter: ableiter. Die abzief̧ende Stange fann in einen fiölzers

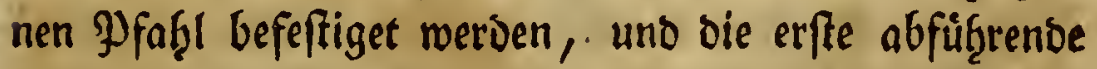
wirb in ber abjief̧enden einge/dhreift, bie folgenben aber forgfältig miteinander verbunben. $2 \mathrm{fm}$ Enbe fann man fie aflenfalls in einigen Éleineren Strabjlen in bie Erbe füţren, wenn fein $\mathfrak{B a f f e r}$ in ber शią̧e ifr. 


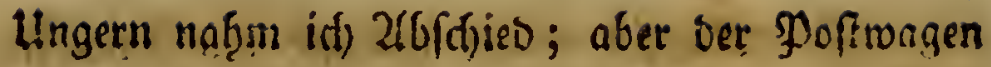
fanto vor berm haufe, uno ber Eduute von Sulfillon fliffd)Elatfd)te uns bie Ofreen boll. Ba war alfo meines $\mathfrak{B}$ ergmigens sin Eno, uno ics) mufite mids

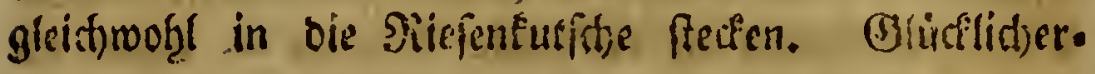
weife war meine (jefellffinafit ta gerabe fo flein, als

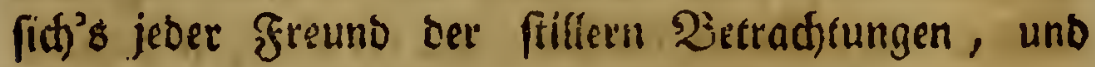

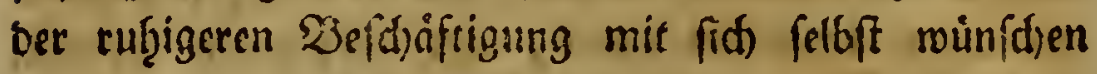
wiro, wenn ar je einmal sas Linangenergme ber ge= moif̧nliden Befellfdaften auf bem Yoftwagen zu ers faģren Belegenņeit geţabt bat. Der Sonbufteur uno idf - uno cenn waren wir alle. Jith noţm mir

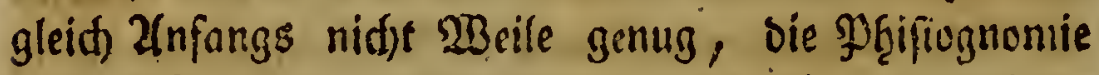
meines Jüuzrers zu betrndjten, benn id war nort) zu

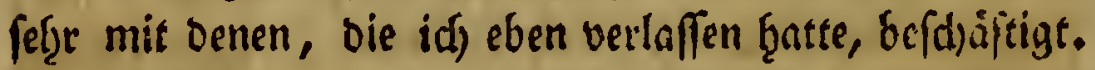
Zlber ber Mann, ber mir an ber Eeite \{aß̧, unterbrnd) oie Etille, uud fthmurrte mid) aus ber Mafe gar lictes

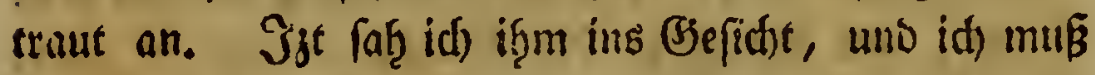
nody ladfen, wenn id) an bie SBirfung jurictentenfe, bie feine fonderbare gebilbete Scabida)tinnafe - fein bon gapern in bie Rreuj uno in bie Durere burdiwirftes angefid) - fein fónmugig grůner mit fotwarger Ed)aaf.

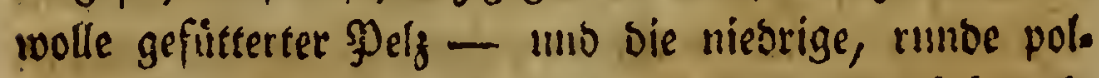

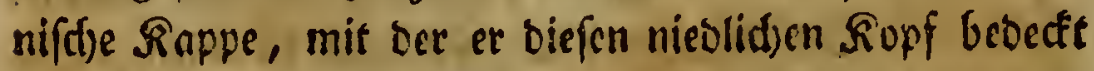
batte, - auf mid mad)ten. Saare Sauater in sie. fem 2lugenblicfe bey mir gefeffen, fo bàitte er ficher mit allem feinem phrnfiognomifden Entb̧ufiasm ausgerufen : Welcje Nafe !

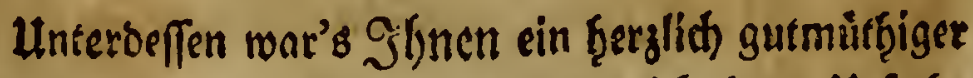

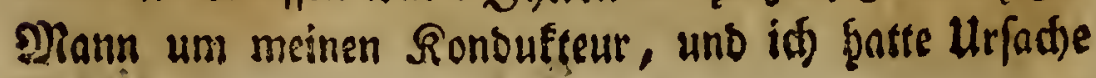


Bell im Billertlyall, Den I 5. Dec. 1783. I 39

zufriesener mit if̧m ju fenn, als mit ber $\mathfrak{3}$ sitterung,

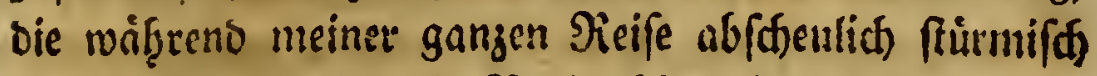

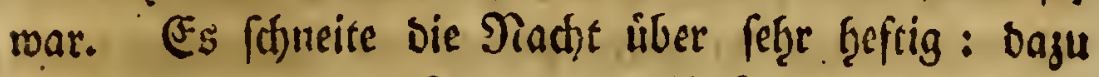

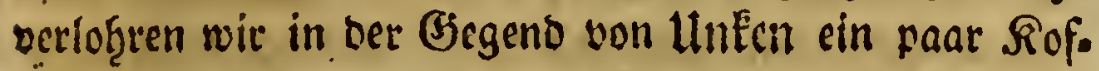
fers, unter benen auts bas meine war. Blicelidjers

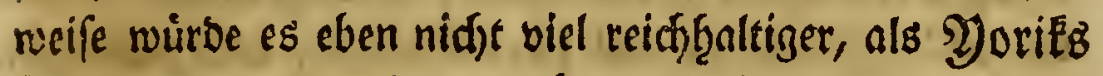
Fefleifen gewe en renn: aber es rove nad) langem Fludjen uns Eshimpfen bes Ronoufteurs auf unfern Softillon wieber gefunden. Ş6 verfútzte mir bie Brit, fo viel es bie gar fanften Bewegungen unferer

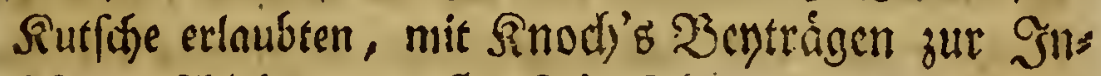

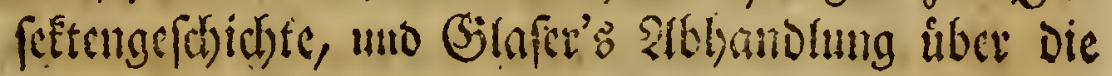

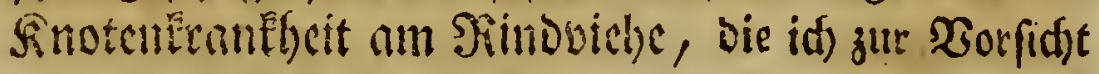

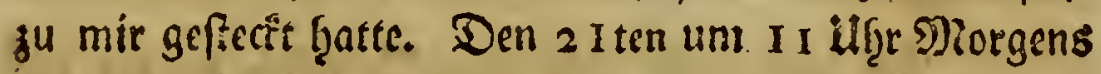
bielt ber $\mathfrak{Z}$ agen an bet Sonne, einem Gaftţofe in hem fleinen unbetråd)tictsen Gtádedjen Siattenberg. Ein Jguidff trauriget Drt, am Ufer bes Innfluffes, ber bie

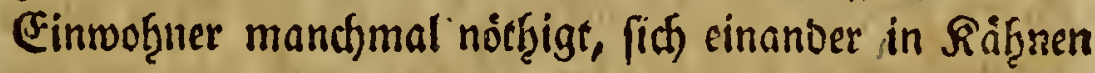

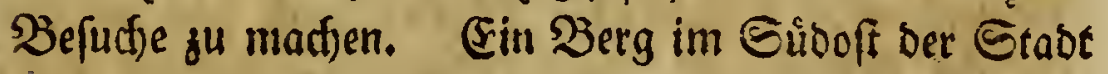

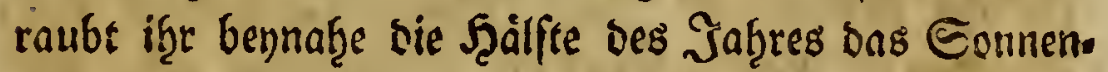
lidft. STur vor furgem war Ģier nod) eine Beftung, und es lag einige $\mathfrak{B} e$ fázung ba; aber man fiat es wirthfidaftlider gefunden, bie $\mathfrak{B e f t u n g s g e b a ́ u b e ~ a n ~}$ Partifularen ju verf́aufen, uno bie $\mathfrak{B}$ elaģung aus bem Etábtchen zu zief̧en, bas izt ganj offen ift. Die Zluguftiner ḩaben f̧ier ein Rlofter. Nad) einems

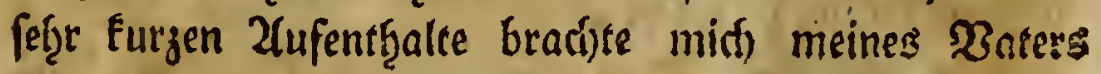
Pirutfe in wenigen Gtunden in's Billerthall zurute.

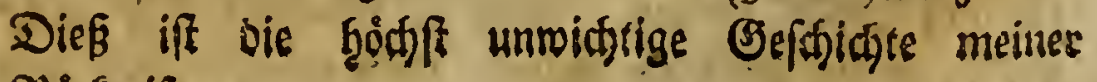
Siưfkeife. 
Cine meiner erften Sefdafftigungen war's, bie Bemerfungen, bie id mir bei unfern entomologiffen Unterfudgungen in mein Portefeuille gefdrieben hatte, mit meinen Snjeften, uno Dem, was id) in THefli's Mingajine darůber gefagt f̧alle, ju vergleidsen. Zaber ba zab's Shlnen fo viel zu verbeffern, und juzufezen,

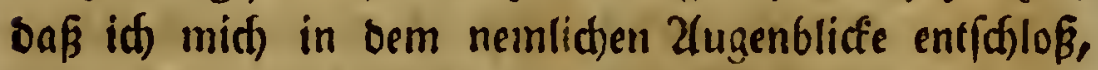

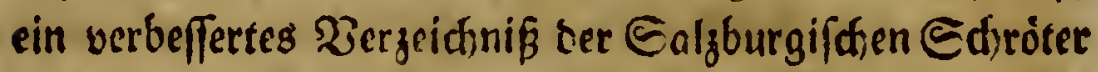
nno Rolbenfäfer (Scarabaeus L.) in Briefen an Gie zu ridten. Woitflid) bin id im Segrife, bieß zu thun, - uno nill nur nod) einige allgemeine $\mathfrak{B e m e r .}$ fungen tiber bie $2(r t$, mein $2 e r g e i d f n i \beta$ elnjurid)ten vorausfdicten.

Id) will nidit bey nllen Injeften fo gar furr fenn, als id) es, vorgugglid in ber I ten sieferung meines Berzeidniffes im Fuefflifden गagajine war.

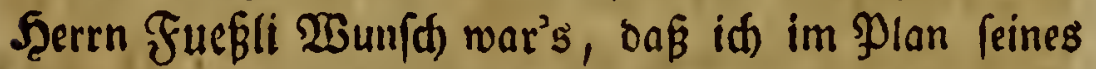
Berzeidyniffes Ed)weizer. Snfeften aud bas meinige für if̧n bearbeitete. J̧err Siúmer ḩat feitbem einen

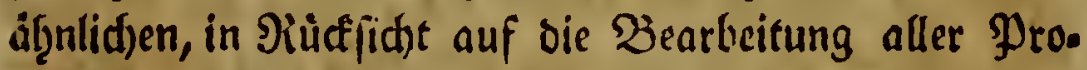
vinzialgaunen ủberf̧aupt, im Miagagine geáuffert. Das that id) nun treulid), aber ict) mus befennen, saß id ju feģr, von unfers wúroigen Fuefli's groffen ento. mologifden Renntniffen überzeugr bin, um ben $\mathfrak{B} u n j$ (d)

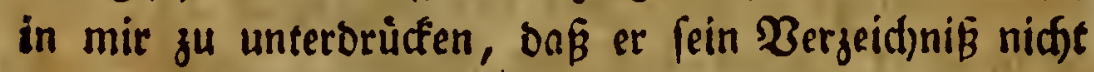

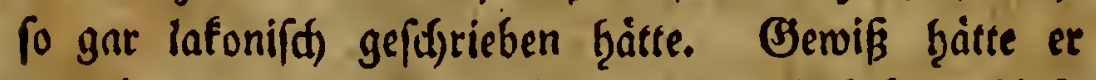

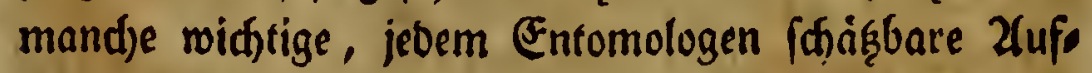
tlärung geben fönnen, wenn er feinem Plane nidbe gar zu getreu geblieben wäre. Mir gieng's aud) zum Sheile fo: bavon will id Sie in biefem, und einigen 
Ball im Billerthall, Den 15. Da. 1783. 141

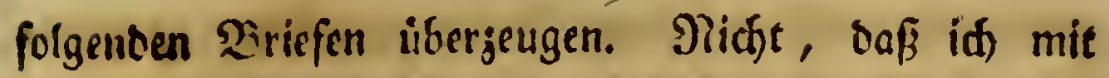
taufenomal fifon gefagtern, uno wiebergefngtem Bic. zeuge Shye Beit not!guidtrigen wollte, ober jebem In. fefie sinen langen Edyweif von వెitaten anjub̧aingen gुe.

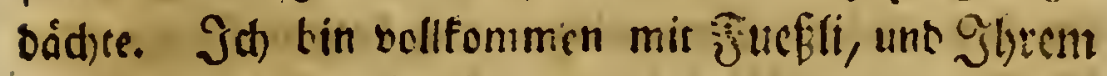
trefliden Breunie Scerbft bariunn einverftansen, iaf bießß, wenn es nicht oer eigeneliacie Begenftano des WBerfes ift, wie in ben mưlylamen Shentrigen tes Şerrn Paftor Gionic - ober wenn es nidht die $\mathfrak{B e}$. ridtrigung ber ben einem Infefte auffollenden $\mathfrak{B}$ erwir. rungen in ber Einonimie nơrthig mađt, - eitel sruse Ift, bie blos oaju targt, bas Papier ju fülten, unb bie entomologifityen Sivider unfinnig anjufdnellen. 2ther sas, was id von feinem, mir befannten, Enfo. mologen bemerft finde, - und was hennod) bemerfeng.

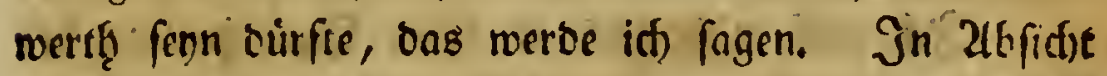
ouf tie Bisutare, , fo will id miáj genau nad) Jeerbft's fthonem, uno midtrigem, fritifden $\mathfrak{V e r z e i d j n i f f e ~ G a l t e n ~ : ~}$

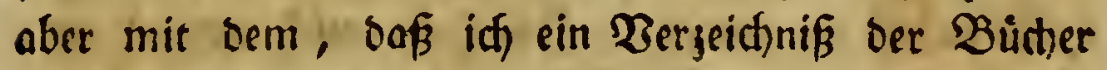
mit adbGilbungen, bie id) baben gebraucht hanbe, vorause fajicfe, und mid baten erflare, tap idf jebe in biefen Bưdiern enthaltene albbilsung bey ben Infeften", mo fie von Sionze, Fabrightus zc. 2c. angefühtgrt werten, für rictfrig angsfüb̧rt ţalse, fo lang id) nidjt bie (Srunbe, Die mid) zum (Begenţ̧eile beffimmen, nambeft mache. Id) finbe biek aus folgenber Urrfacte für

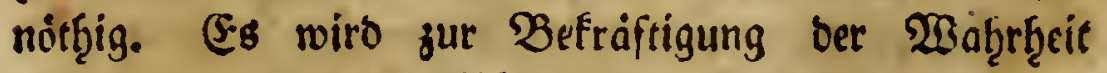

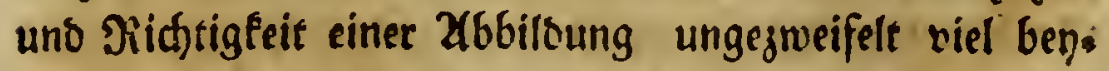
tragen, wenn ber ausorúcfliche Benfalt ber meelffen Entomologen bafürpridte, tak fie ádit fen, ober 
wenigftens bieß ooer jenes Infeft zuberläizig vorffelfer foll: fo wie es aud) fidfer Brweifel erregt, wenn cin Sditufetfeller, ber aus einem gewiffen Suuthe bie $2(6$.

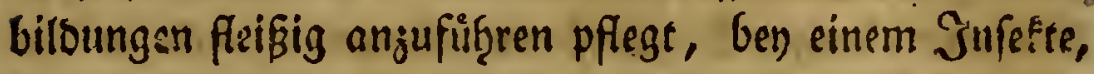
bey bem anbere Entomologen aus biefem $\mathfrak{B u d f e}$ eine abbiloung angefüţrt ḩaben, feine anfüf̧rt. Jeoer Naturforffher, ber ben móglidiffen (Jrab von Benanigi feit und Sidjerbeit in feiner $\mathfrak{B i f f e n f d j u f t}$ muinftht, mus es bedauern, renn fich ber Edrriftefller über bas W3eglaffen fo eines Bitates nidht erflart ḩat. Nun biefe (Semißgheit fann nid)t anders erreidft werben, als wenn ber Sdfriftfteller alle abbilbungen eines Infeftes, bie er vor fid hat, zitirt, ober eime Ertlátung, wie bie meinige ift, voraus/dicft.

S(d) will midf) b̧ier in Eeine Beurfb̧eilung bet Sifteme einluffen; - fie müroe midj zu meit füf̧ren, unto idf bin nicbt zuverfisftlid) genug, um meinem Ut. theile babey zu trauen. Joh bebe nidi) einmal ents fabloffen, ben sinme's Gifteme in ber Şauptfatbe zu bleiben. Da aber bie vielfältigen neuen Entbeckungen von InfeEten feit ber Zeit, in ber uns sinné mit feinen: Eiffeme befdenft bुat; fef̧e viele Berwandidhaften uno Unterf(hiebe ben Etof ju vialen neuen (Jefhlect)tern gaben, bie soir ben Rinné unlángbar zu "Fefgr unter. eirnnber-gemorfen finben, fo werbe ifí) biefe neuen

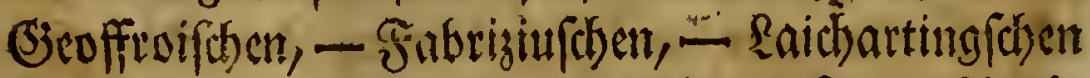

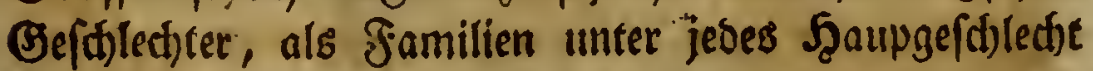
sinne's, mittheilen : iffi werbe alfo fagen Scarabaeus (Melol.) folftitialis; Scarabaeus (Trich.) fafciatus. 
Bell int Billertfall, oen 15. Dec. I 783 . I 43

Siur bann, weenn bie Şauptgattung Simne's mit ber

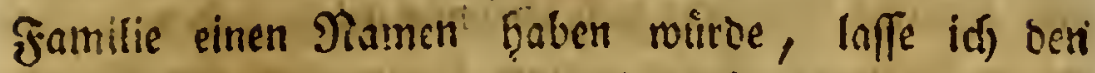
Familiennamen ganı weß : fo 3 . $\mathfrak{B}$. werbe iff für Scarabaeus (Scar.) naficornis gerabegu fagen Sca. rabaeus naficornis L.: Denn bie erffe Familie ber Rolbenf́áfer wáre ber eiggentlitife, yon allen Entontologen rogennnnte Sinrabelis, Dunghiffer. Die Natur.

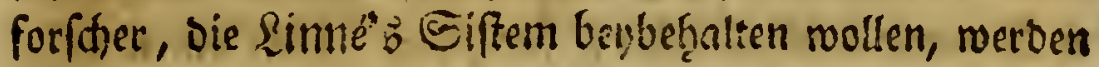
ben siefer Metrizode mandjer fonft fetwer zu vermeis Denben Werwirrung ausweichen Fơnnen : Gie werben

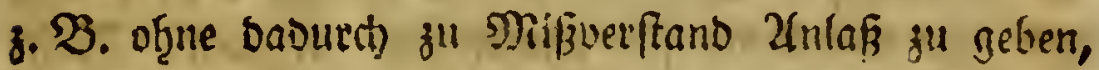
fagen fónnen- (Scar. oblongus - uno Sçar. (Melol.) oblongus. Jebermann wiro verffef̧en,

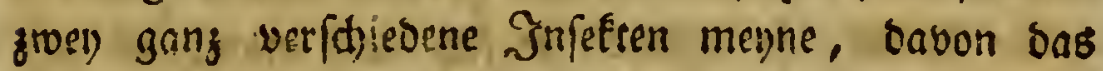
erffe, jur erften 'zanillie ber eigennlidfen SEarnbeen -

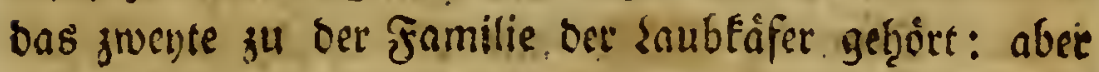
nidj) fo, wenn man gerabegu jwenmal frggte: Scar. oth-

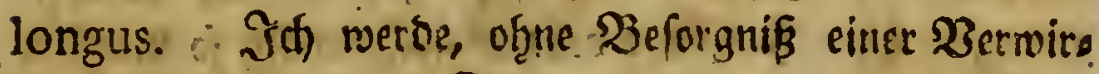
runiz fagen fônnen: Chrys. vittata - und Cliryfo (Cryptoc.) vittata : uno Chryf. (Crioc.) vittata.

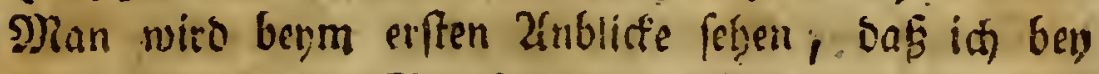
Der erften an Die Chryf. vittata. Fabric. Spec. inf. p. I 16. n.3.- ben ter znoten an Crypt. vittatus Fabric. p. I 44. n. 36. - ben ber britten an Crioc.

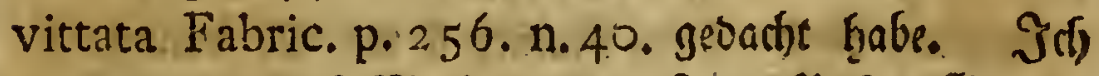

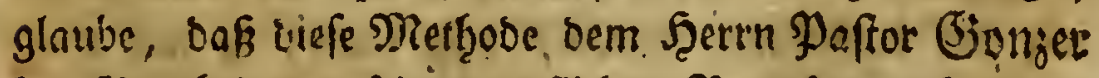

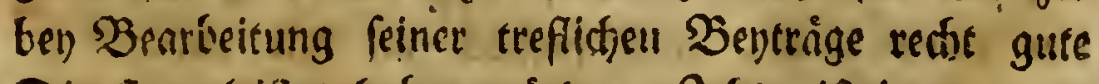

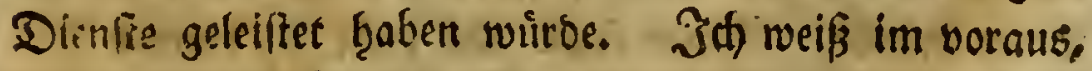

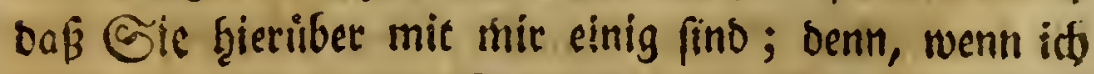

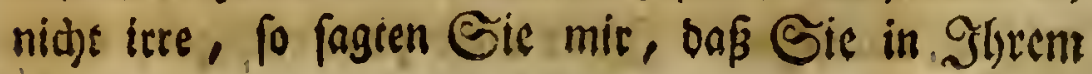
शిans 


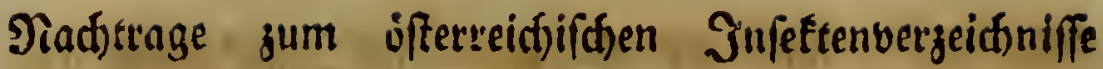

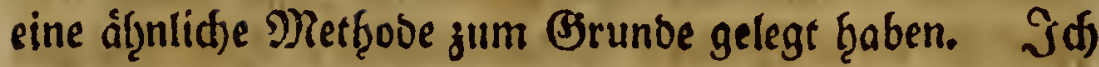
werbe fie in ber folge meiner sieferungen ins Maga. jin gebraudjen.

Enolid glaube idf) nod) eine Bemerfung in $2(6$. fidst auf bie beutfd)en Benennungen ber Infeften beys. fügen zu múffen. Jđd) weiß nid)t, warum man nidjt aud) Daran benfen follte, für jebes Infeft einen beuts

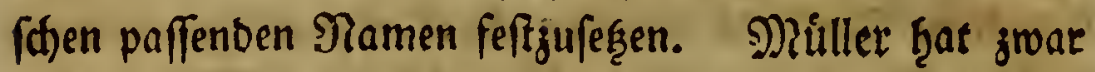
alle simneifichen Infeften beuríh getauft : aber was fino das mandjymal firt rounderlide Namen! Nidje genug., bak fie oft to unbezeidineno fino, baß man

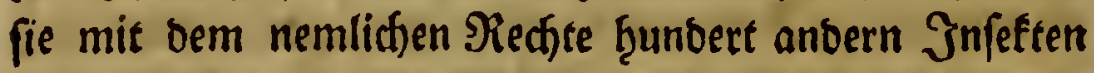
geben foinnte, fo begief̧en fie fids nodf mandsmal auf Eigenfdaften, bie man am Infefte felbit umfonft fuct)t. Denn da Miller alle bie unzăb̨ligen Fef̧ler Scouttuy'ns in feiner Ueberfergung getreulid) herriber= getragen hiat, fo muste baraus notbriventig fo mandjer

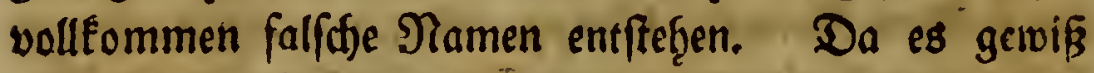
zur Bolltommenţeit jeoer' 'Biffenfd)aft, uno zur (Er. leidferung bes Etubiums berfelben nidte wenig bet). tràgt, wenn alles batinn eine gemiffe $\mathfrak{B}$ ejief̧ung auf. einanber ḩat, fo wutroe es aud) in ber Entontologie zuverlåßig von groffen Borţ̧eile fưr bie Stubirenden fenn, wenn bie beutffen Namen fo viel $\mathfrak{B}$ erwanorfdhaft mit ben lateinifthen ḩătten, báß man, fo ivie man ben einen fiórte, aud) fogleid) an ben anbern er: innert wưroe. Man follte alfo erftenz, immer nut bie lateiniftsen Tamen Simne's, unb anderer, fo viel móglid, ưberfergen. Sft es nidjt zmedtmápigiger, wenn 
Bell im Billerthall, ben 15. Dec. 1 783. 145

man Cicindela campeftris, butd) Feld:Sandláufer, unb Cicindela hybrida, ourd) gefleciter Sandonifer

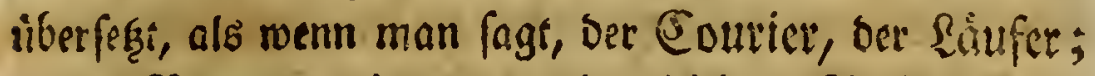

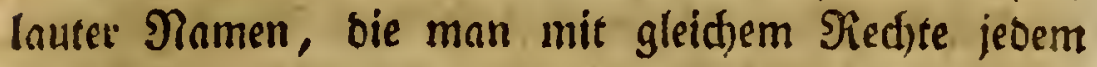
Candrkufer (Cicindela) uno cinem grofien Tgeile ser \&aufeåfer (Carab. L.) benlegen Eann, - benn alle diefe faufen fegre ḩurtig. Bweutens. WBirbe

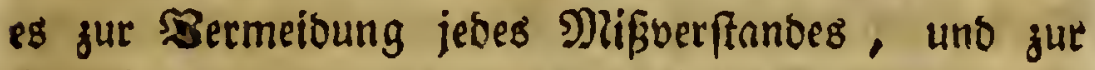
Erleid)terung bes beutlicten 2usbrucfes ungemein jurriglid) fenn, wern jeberzeit ber (Befd)lectes: namen mit ben Sriblalnamen im Deutfhen eben fo verbunsen wurrte, wie tieß im satein gefd)lef̧t. $G_{0}$ múrbe id) 8. B. fagen: Der gellffifigte Gambliufer, Cicindela flavipes L. in Sisnlirheit beftimmiter, als wenn id) fage: Dei (Selbfuff: : Man wiro Dabey nidfe wiffen, of ith Sicufriol's Dermeltes fulvipes, ver bie Chryf, flavipes L. - oter Gieuffroi's Chryf. flavipes - ober Mitlier's Curc. flavipes - ober Fấrigius Elater flavipes - ober ben Staphylinus flavipes L menne. - Doer lafien Sie mid mie Shnen vom Siotifufere reben: was werben Sie baben Denfen? - Wile merben Sie beftimmen, an meld ein Jnfett id snbey benfe? - on id Slynen nur unter ben J̧artfdaligten gefdrwinbe 20 nennen mill, bie ben Trivialnamen Rufipes füf̧ren. Diefer Mangel iff ge* boben, fo balo man fagen miro: Der rothfufígite

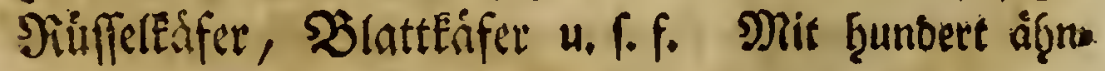

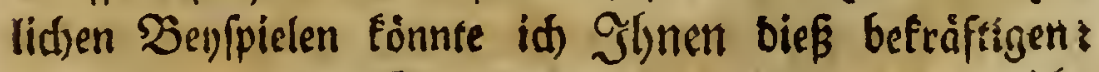
aber id glaube, Sle burften eben fo ungeneige midits penn, in biefem Etuicfe meiner Rennung benjutteteth. 


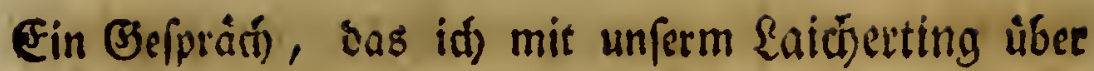
biefen Begenenfant ḩatte, war es, bas midf juerft ouf Diefe (Jetanfen bradjee. Saicharting bat fich aud) wirflich in feinem Rerzzeidnniffe an biefe Điegel gethalten,

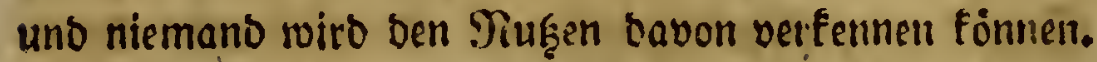

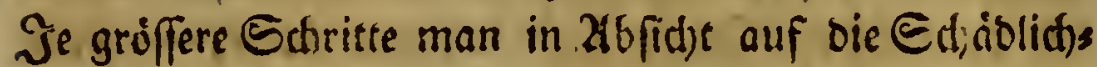
feit ber Infefteri- uno. Die Mittel bagegen; uno in

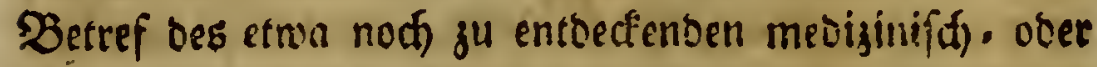

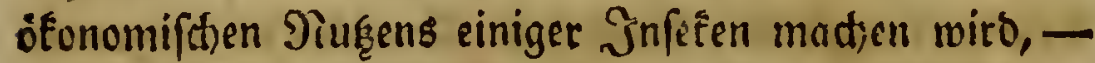

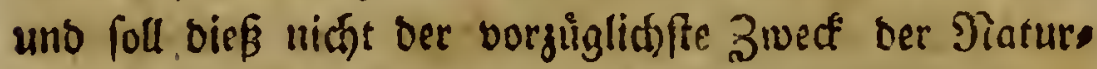
forfder fenn? - Defto meģr wiro man bas Bedúrfníß guter beurffder Tamen fíflen: Drnn man wirb bie Infeften mit beutf(d)en Vaamen nennen müfen, um fid) badurd) ben unlateinifden Deforuomen verftänolid)

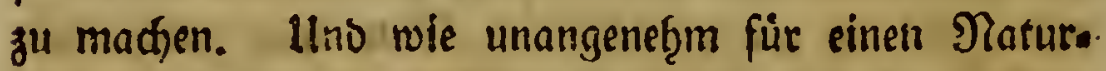

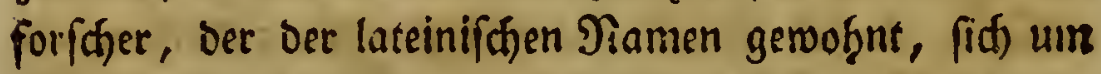

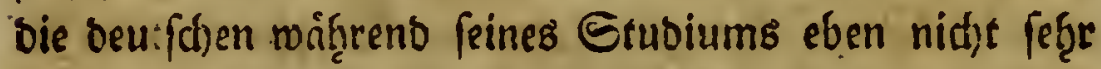
befümmert Gat, wenn er fo ein Buth liegt, uno bey

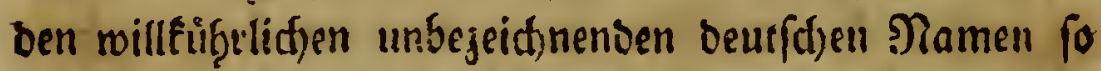

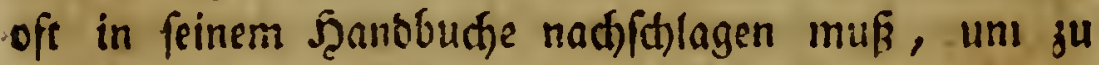
wiffen, bon weldsem Snfefte eigentlid bie Ficbe iff? Denn bas wiro Dodf feiner, ber bie unbändige Mienge

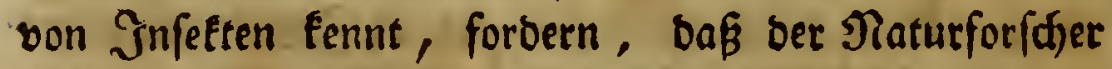
alle Tateiniffden und beurfiden Namen berfelben wie ein Edjulfnabe aus bem Gebaditnißje herfagen foll. - Zfber

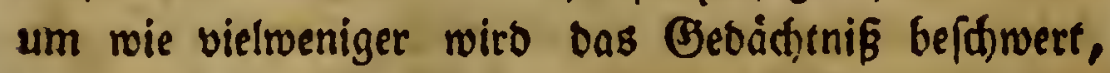
menn bie beuffifen Namen allgeit Ueberfegęung ber las teinifidten fino? Man ḩat bann nut einen bon benben zu ḩơren nờţ̧ig, um benoe gu wiffen, - aber man muß

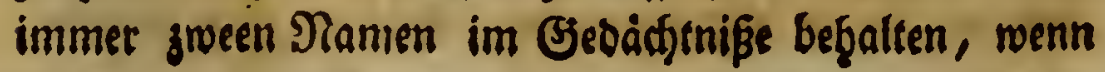


Bell im Billerthall, Den 15. Dec. $1783, \quad 147$

fie iim Zusbructe fo entfernt von einander find. $\mathfrak{l}_{n}$

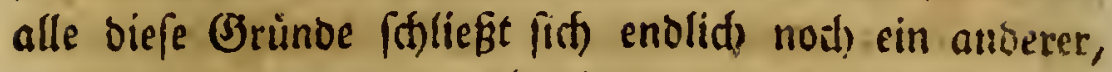
ben feerr grof. SBfumenbad angab, uno auf sen hiff,

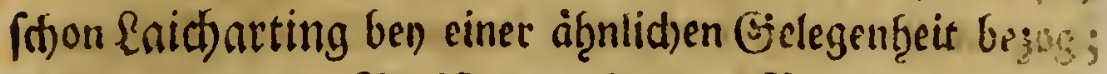

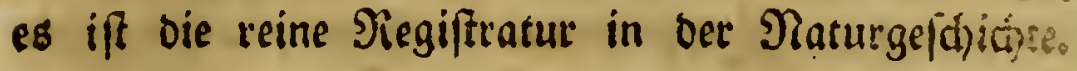

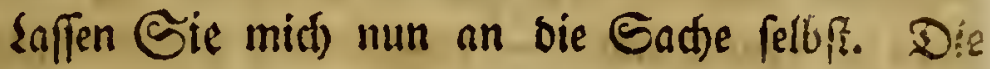

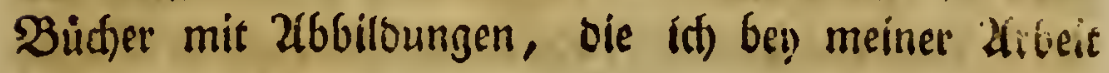
allzeit vergliden ḩabe, fino

\section{a. gemtablte.}

Riufetz Tuferten.

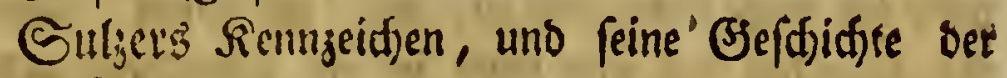
Jnfeften.

Suesti's 2urdio. Rinuct)'s 3 entråge.

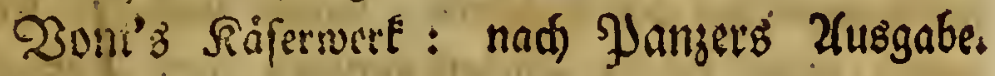

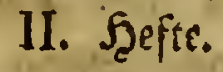

\section{B. Supferfitide.}

Geoffroi Infectes aux environs de Paris. Dicßs if in meinem 2luge immer nod) eines der befen, braudjbarfen enromologifthen $\mathfrak{B}$ erfe. Eeine $\mathfrak{B} e$.

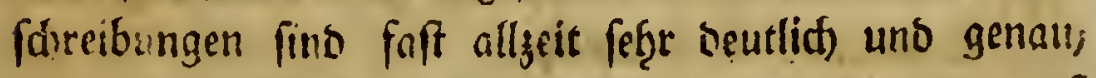

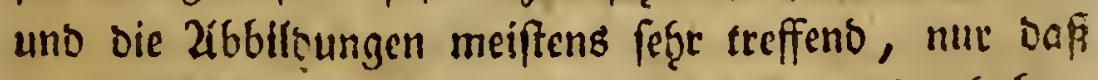

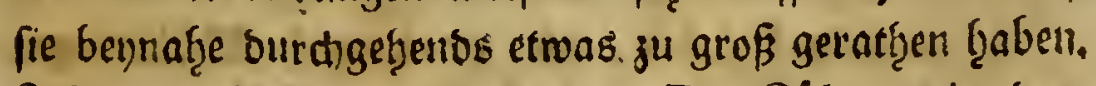
Sd) begreife onger nid)t, wie Dr. Sillhn, in bem

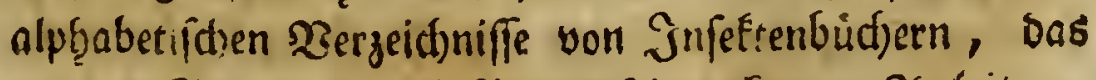

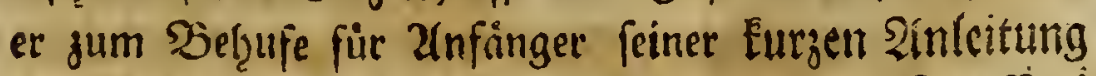

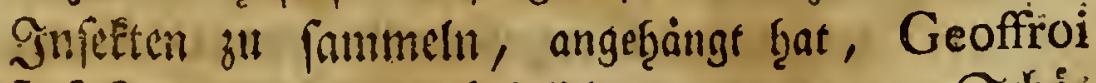

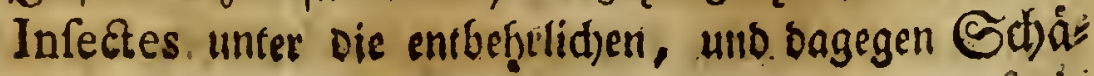

$$
\Re_{2}
$$


fer's Icones unb Elementa unter bie borgugglid) ju empfehtenden fegęen tonnse. उめ) bitte Gie, mein Jreuno! was foll ein Znfänger, aus einer über allem

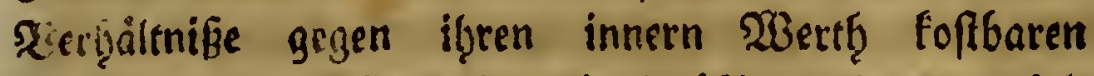
Camnilung, gröftentf̧eils mittelmábiger, in ber ecfele Fafteften, oder vielmefgr gar feiner Dronung, - of̧ne Ş:an - oţne Namm - aufgeftelter Zlbbiloungen ler. nen? Def finb abre tic Icones infectorum in 20 afgr. Geit, - und man lainzunet baben bie ansermeitigen groffen Berbienfte bes Saern Paft. Cebificre gne nicht, menn man fagf, Daß̧ fie é fins. Die Elementa Wabe ith ned nicht gefeben, aber ein Brief cines gue ten Freundes fat mir felbe in ein úbel sidit geftellt. Weldi)er 2(bftand zwifthen (S)eoffroi's Infectes, unb CWhafer's Icones! Dak man Gienffroi mobel ifters

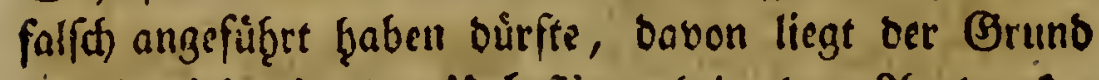

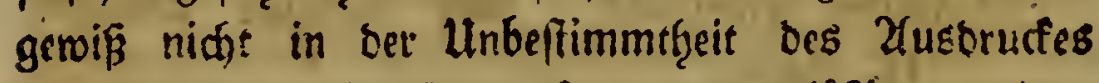
biefes wuirbigen Gelef̧rten, fondern zuberlábiger Darinn,

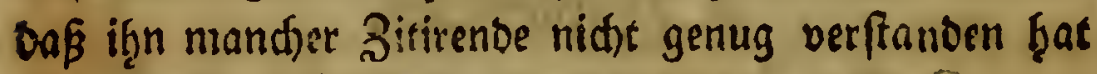

Frifich, Jureetten Deutfalanos.

Schluga primae lineae.

Goldartius cum notis et tab. mut. Lifteri.

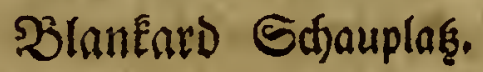

Zinbere Bücher, bie zwar aud 266 bilbungen bon Infeften entḩalten, aber nidft von folknen, bie in meinem $\mathfrak{B}$ erzeiffniffe fethen : $3 . \mathfrak{B}$. Fllue Enumerat.

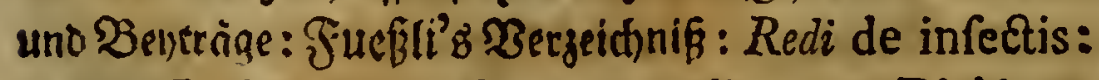
bie groffe franzofifine Encyclopedie von D'Alembert, uno Diderot, von Deren entomologifthen Theille 


\section{Sell im Billetethall, Den I 5.'Dec. $1783 . \quad 149$}

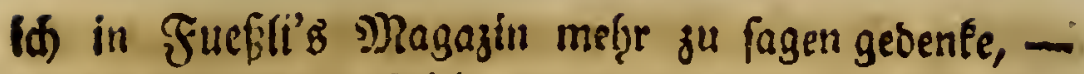

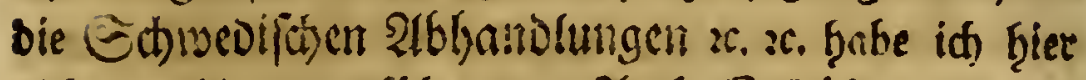

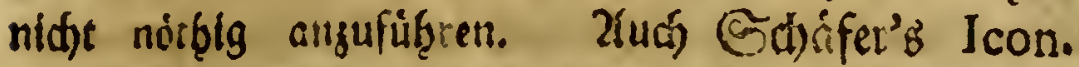
uno Scopoli's Icon. ad Entom. Carniol. babe id

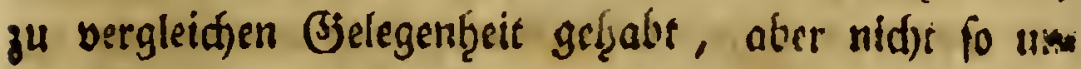
unterbrodjen, dás idf fie kgier in mein Berjeidjuis aufneţmen fónnte.

\section{Lucamus. Sthriter.}

1. Lucanus servus. Der Shiffafforitter.

Magna, I. B. P. 377. n. 25. Encyclopedic Vol.II.

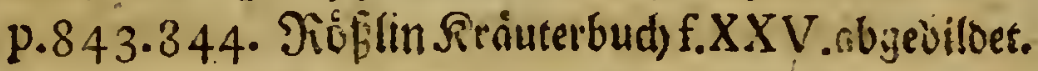

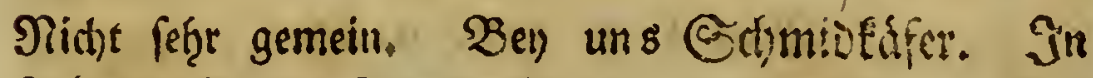

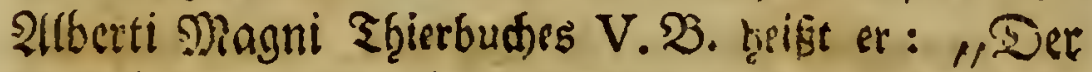

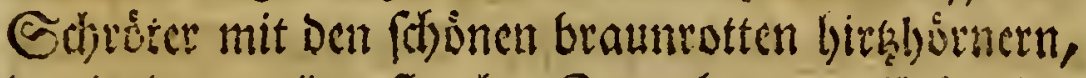

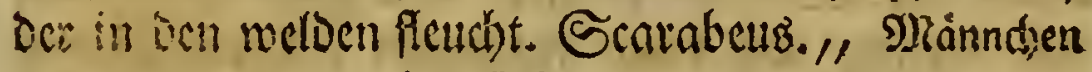
und Siscibchen find bey Elopoli gut abgebilbet. Er ànoert ungemein ab in Der Broffe. İ berifge ein Exemplar, bas iç lange für Den Capreolus L gefgalo

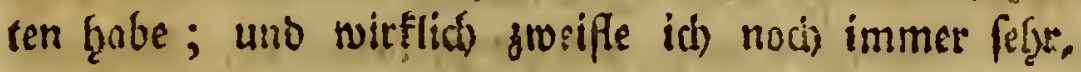

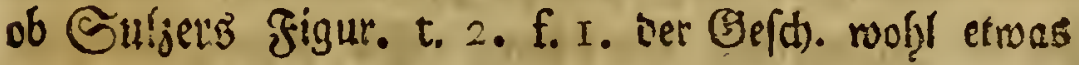
anbers, als eine fieine 2fbainberung bes Luc. Cervus ift. Uteberthaupt if beym Luc. Caproolus nod) viele Berwirrung. Paftor von Slecven verbiniet then mit. Geoffroi'z Chevrette brune. (Gounze fondert fie ab,

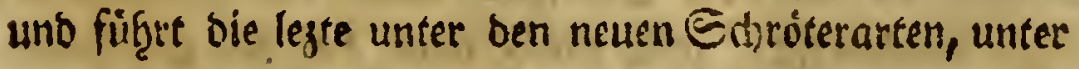
bem গiamen, Lucanus fufcus, auf. Simie fagt: maxillis apice tantum furcatis: ber Rammertherr (Degrer bagegen: apice fimplicibus. Ben Yleof. Fabriflus ḩeiffes : Dens mandibulorum lateralis omnino

$$
\Re 3 \text { deef: }
$$


deeft: Dieß wollte vermuţ̧lid sinne ourd, fein apice tantum furcatis, bagegen lefe ich bey Degner: latere unidentatis : Gulzer uno Fuefli ragen nofl gar, ber 3 af̧n in ber Mitte f̧abe verfdbiebene Epig̨en. Id) wage es nidtt, biefe $\mathfrak{B e r w i r r u n g}$ auseinander gu feģen. Prof. Fabriziuz begief̨t fich beum Capreolus

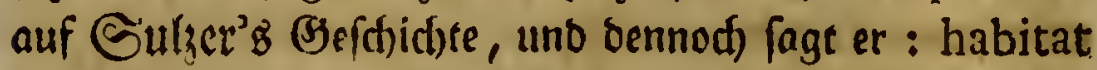
in America boreali. Entwebers ift Sulzer's 2 lb. bilbung niát fein capreolus, ober biefer ift nidjt aus/(h)lię̧lid) in Torbamerifa ju J̧aufe.

2. Lucanus parallelipipedus. Ser $\mathfrak{B a f E e n f f f r i ́ t e r . ~}$

$$
\text { Ming. I. p. } 378 \text {. n. } 26 .
$$

Bên uns etroas felten - in 2 sârbern. Zlucti bieje 2 (rt ánbert feģr ab in ber Broffle. Die zween Punfte auf bem $\Re o p f e$, von benen Fabrizius, Ģeoffioi uno Degnet reben, fabe ich nad) langem Euchen an mei: nen fleinern Exemplaren gefunden. Sic b̧aben voll, fommen red), wenn Gie fagen, baß $\bar{\beta}$ man ben $\nwarrow$ Ropf genou betradjen muß̧, um fie zu bemerfen. Bielleidt

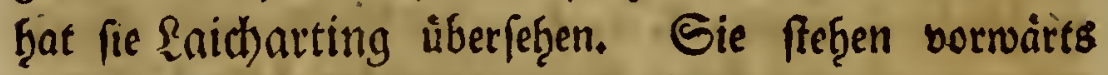
ouf bem $\Omega_{0}$ opfe, queeruber, naf̧e aneinanber, uno man wiro fie am eheften gewaḩr, wenn man beu $\nwarrow$ Ropf nad) ber Seite mit einem Suct)glafe betratitet. Der Rórper ift auff ber Sberfeite unter ben Fluigelbecten fajoin bunfelroth, aud) finbet man ben ben (Fingliebe. rungen der Fứffe glänzenb bunfelrothe Flecten, bie tḩeils ferbft auf ben Einglieoerurigen ber Edjenfel, theils über benfelben freferen. Borgủglid) werbe id) über ber

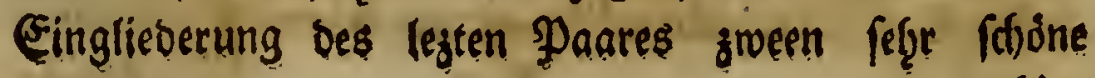




\section{Bell im Billertlyall, Den 15. Der. 1783. I5 I}

rubinartige Punften getwahgr. Die Fubshlatter fint mit

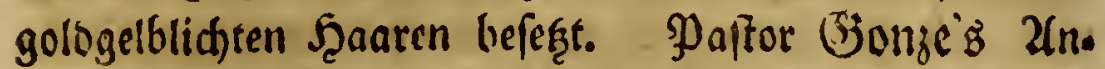
merfung im I. B. Der $\mathfrak{B}$ cutraige, S.122. (*") bey JJontoppio:ms punctatus iff etwas ounfel. DRan

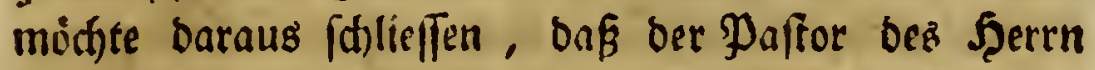
\$rof. Fabrizius- $\mathfrak{S}_{\text {Borte }}$ : fexus alter minor \&c.\&c.

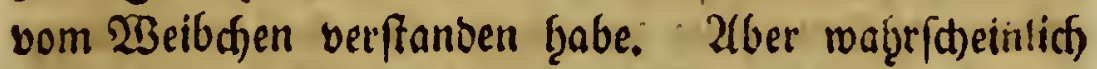

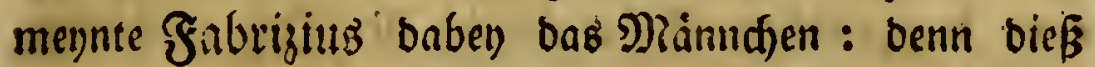
foll ja nach nad) (J)evffroi uno Glynen fleiner fenn, uno

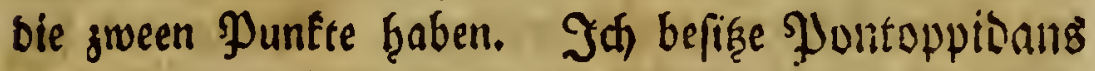

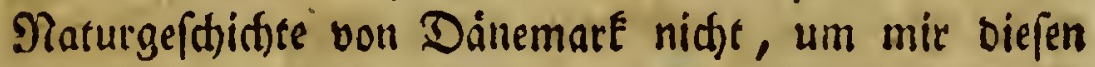
3rweifel zu erflaren.

3. Lucanus caraboides. Der $\mathfrak{E a u f f a ́ f e r ~ = ~ a r t i g e r ~}$ Sditster.

Nur einmal im Billertl)ale gefunden. Brün, oban und unten; etwas ins blatlichte giefentent. Die 2 lbbilbung ben GFopoli grat nidht aufs befte gerathen. Der ganje Sáfer, oben unb unten, ftarf gepunfitet: ant 2 ffer lange graulidfe Shaare : audh 3 Siand bes

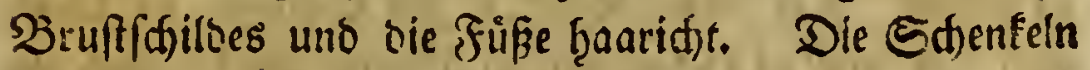

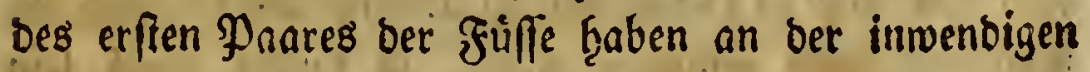
Eeite naf̧e bey oer 'Einglieberung einen goldogelbers

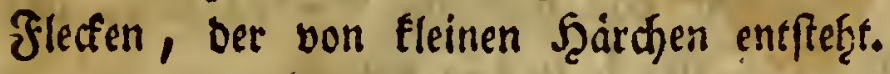

4. Lucanus cylindricus. Der walgenfsernige Sd)roter.

Magaz. I. p. 378. n. 27. Sul . Renut. t. I. f. 2 . Sueferi n. 3 an?

Rỏmmt felten auf faulem , Sholge vor : In ber Gegeno won Saligburg. Mein Mànnden ḩat an ben vordern 


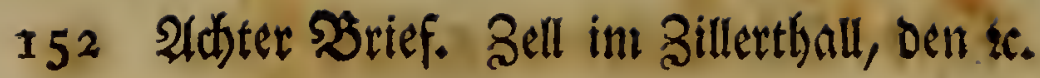

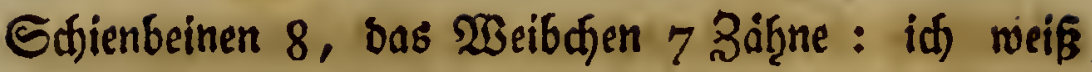
nidft, ob sis immer fo iff. So hyat aud bas $2 B e i b$. den am Srte bes Bruffidfilbes, mo Seich)arting bie

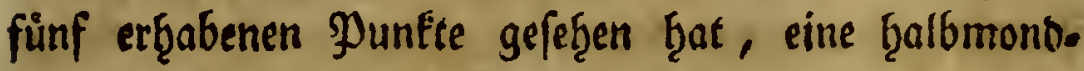

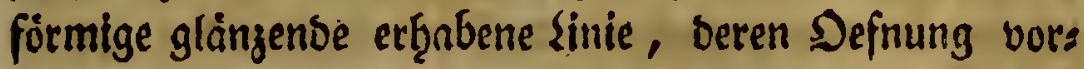

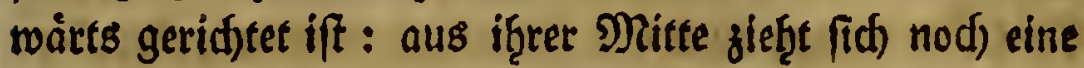

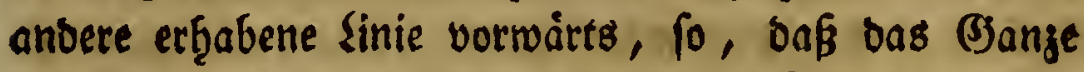

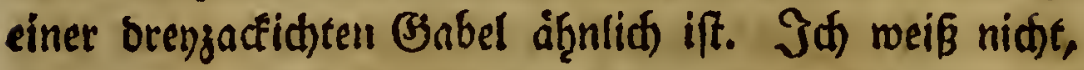
foll id Gier aus Suevigit ben Scar. cylindricus, ober Den Scar. fubterraneus anfứrren. WBenigftens ḩat er bie @ulferfiche a lbbilsung biefes Ráfers, ble @utlzer für ben fubterraneus L, ausgiebt, bey bent legtern ans

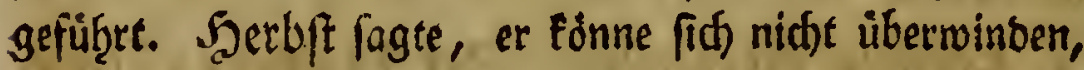
îgn mit Saicharting und SElopeli unter bic Edirster zu feşen, weil er bie meifen Renngeidjen mit ben

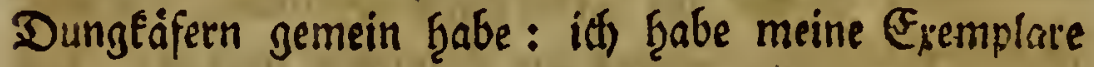
biefes Răfers von neuem für nida) genommen ; aber

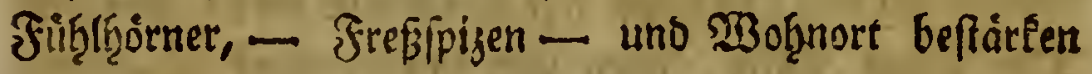

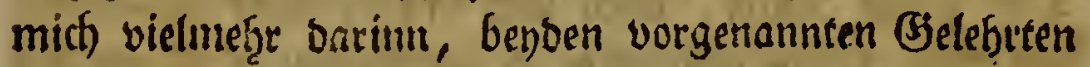
zu folgen. Da biefer Rafifer in ber Edjweis, in Tirol, utno von mir in Defferreif ju Siremsmunffer, uns im Salzburgifden gefundent warb, fo buirfte woofl fein Woognort niast nur bas nórsficse Europa Penn, roie Sabrigiug Spec. infr, I. p. I2. glaubt.

Seben Sie wothl, mein lievéer Jreunb! im nädfften sBriefe will id) über bie Ealgburgf(den Rolbens tojfer (Scarab. L.) zu tommentiren anfangen.

Ş̧ূ̆ Noll. 
Neunter ßrief. Zell, Den 2. Jänn. I 784. I 53

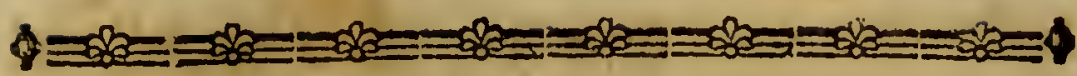

\title{
গcunter Brief.
}

\author{
Sell, , Den 2. Sinnt. I784.
}

Zorláufige Erimnerung.

Saliburgifate RolbenEifer.

5 ic Gaben in meinem lejten Briefe bie Salyburgs fden Edfrusel gefunben. In bieferm will if bie Rolbenkéfect (Scarab. L.) eben fo befranteln : will fie nad) bem entworfenen פlane in Jamilien ţ̧eilen: nemlid) ber eigentlidse Scarabaeus : oer Trox : Die Melolontha : oie Cetonia : uno oer Trichius. Jür bas Befdidecist hyabe idh im Deutifiten feinen $\mathfrak{N a m e n}$ eigentlider sefurben, als Sivblber Eiffer. Sie bahen in Ghrer Enumerat. sie Scarab, ourdaus Shart: kaffer genannt, aber miá) beud)t biefe Benennumg auf bie Melol : Ceton : uno Trich. nidft anmenobar zu

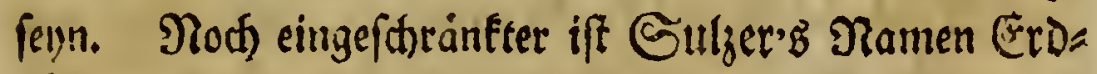
Eiffer : tenn man wirrbe iģn in engerm Berfanbe nur für ble Troc. paffent finbera. Degner ḩat bie Dung. fâfer (Scarab.F.) Scarabées de terre genannt. Im weitern $\mathfrak{B e t f f t a n b e}$ fónnte man wohgl alle Rifferarten (Scaraboides Lorich.) fo nennen. Die wenigen, bie Das $\mathfrak{W a}$ affer bewolinnen, ausgenommen. Mid bünft alfo

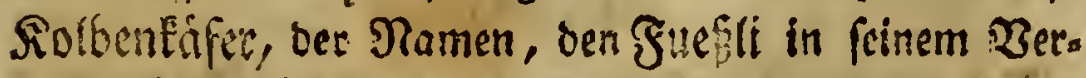

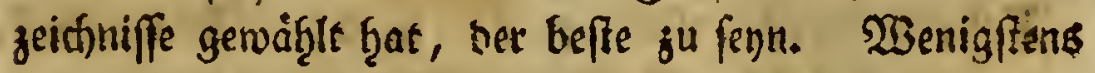

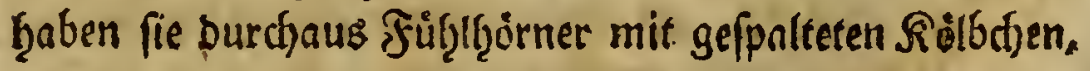
Qoer Rnötden, wenn Sic woollen. Dleßs wäre nun einmal genug über ben Namen. 


\section{Erfte Samilic.}

Dung = Siolbenfaffer. (Scarab. F.)

A. mit Edjilbden.

a. geţơrnte.

I. Scarabaeus naficornis. Der Pagkljorn Rollicnéf́ficr.

Mag. I. p. 370. n. I.

गiod) fam mir ber Roufer felbft nict) zu Befirfte; wohl aber f̧abe id bie Sarva beffelben in bes Şerrn Dr. Ernft von Sectmreide'b Barten, zu Salzburg, an oer füblicten Eeite Des Rapujinerberges, im Dunge gefunden.

2. Scarabaeus foffor. Der Giriters Rolbenkiffer.

Mag. I. p. 37 I. n. 4 .

3. Scarabacus fubterraneus. Der unters iibifube Solbenéafer.

Fabric. Spec. infect. p. I $5 \cdot$ n. 58 .

4. Scarabaeus terreftris, Der Eroes Rolbenfáfer.

Fabric. Spec. infect. p. 16. 1. 6I. Mngajin I. p. 372 . n. 5. fubterraneus. p. 374 , n. 12 . piceus. Enumerat, inf. auf. p. 5. n. 7 . fubterranęus. 
alle bie $\mathfrak{B e r w i r r u n g e n , ~ b i e ~ m i t ~ b i e f e n ~ \Re a ̈ f e r n , ~ v o r - ~}$ zuiglid) mit ber oritten uno vierten $\mathfrak{A}$ rtt baf̧er ent, fantion fins, weil fie imnter fo unbeftimmt bef(h)ries ben wurben; weil bie gar zul furze Cbarafteriftif, niger, capite tuberculis tribus transverfim pofitis, fo vielen fleinern uno gróßeren Siafern zu. funmt: alle bie Schwierigfeiten, bie bie Entomolo. gen bisţer fanben, aus biejen Ungewişgeiten etwas ridftiges ju entgiffern, f̧abe idf felbft lange genug

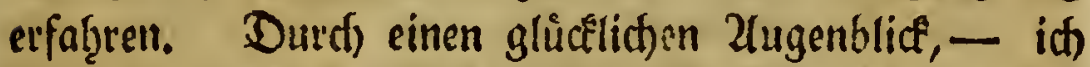
habe lange feinen fo froben gefrabt, - glaubte idi) mid) ill Etand gefezt zu ref̧ell, mir bieß (Ş̧⿻上丨, bel oem id) felfft im Magnzine geftolpert habe, zu ent. ruideln. Jal fano endlid) ben Rafer, ber bie ge. Eerbten Streifen : ftriae crenatae - vereint mit bes Szerrn Prof. Sabrizilus : Follori affinis, at multo minor; fenntlict) genug madjen. Eine ge. naue Parallefle biefer orey Rolbenf́afer wiro mir's am leidteften mad)en, mich Darübei żu erElären. Die Maaß̧e bृabe id auf bem Maaßifabe, den Gcopoli feiner Entom. Carniol. vorausgefę̧t şat, genommen. 
Scarab. foffor. Scar. fubterraneus. Scar, terreftris.

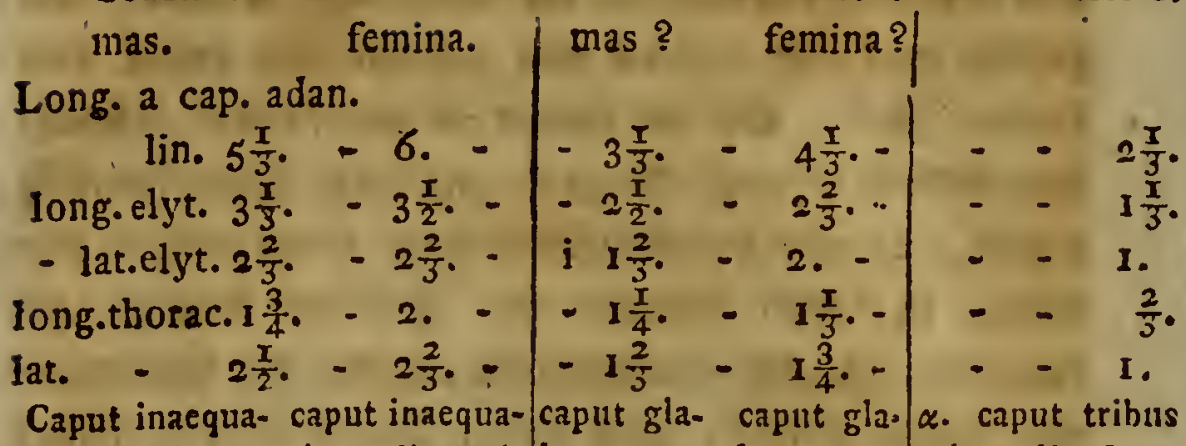
le,tuberculis tri- le rudimentis brum, tü- brum, tul- tuberculis. $\beta$.cabus, transverfis, tribus tuber- herculis tri- berculo un-put: rugis duaper lineas ele- culorum per li- bus, exili- nico in bus, elevatis, vatas connexio, nças elevatas bus, trans- medio ca, transverfis, pamediofubcornuto. eonnexis. verfim po- pitis vix rallellis; poftica fitis vifibili. trituberculata.

antennis. ferrngineis, capi-anternnis nigris, artitulo fufco.

culo tertio rufefcente, antennis ut in capitulo grifeo - nigro, foffore.

a. thorax maginus, convexis, parce punstatus, foveola antica $\beta$. thorax rotundior, puncatior, thorax nitidus, pluri-thorax nitidus, defectu foveolae. mis punetis fparfis. punetatus.

elytris ftriis punelytra ftriis crenatis, ctatis, intervallis elytra glabra, vix puncata, intervallis numerofe vix punctatis, Striis parce punctatis. punctatis. margine exteriore et apice fubinde rufefcente.

pedibus nigris, turlis rufefcen-pedibus ut in foffore. pedibus rufestibus.

centibus, femoribus magis, tibiis minus.

oorpus cylindricum, con-corpus ovatum, de-corpus, ovatum, vexum. prefsciufculum. convesum.

feutellum cordatum, magnum fcutellum fubcorda- \{cutellum parfoveolatum. B. planum. $\left.\right|^{\text {tum, parvam, }}$ vun, rotunda. tum. 


\section{Bell, Den 2. ginnn. I 784 .}

Shier ift eine genaue Jurulletle biefer säfer,

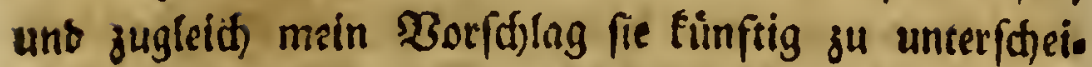

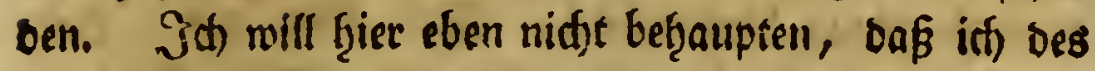

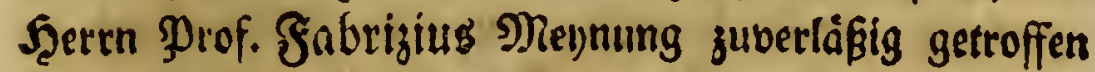
habe : aber feftr waf̧rfdeinlich) iff es mir, bafi er bie Râfer, bie izt vor mir liegell, gemennt b̧abe. Jots will nod) jebem insbefonbere cinige. Bemerfungen benfügen.

Scar. follor. 2luf bem Ģaifberge ben Ealjburg babe ich mefre als zeţen unter einem alten Soljfocte in (b)efellffiaft gefunden. Eonft etwas felten im Dunge. Die 2 lbarten, bavon if bie eine für bas

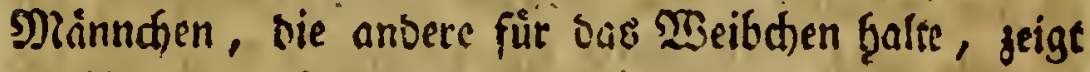
bie Parallefle bei) ber (Brö́c - Ropfe - und Bruftz

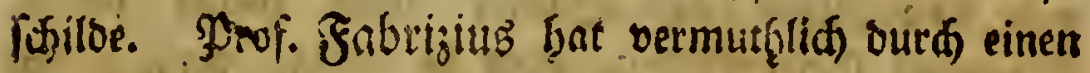
Drudffegler ben feinem foffor ein fonberbares Sitac aus Schaffern: es geipit Icon. t. 144. f. 78. Nun fino auf ser angefüţrten Fabelle weber 78 Siguten, nod) unter ben ouf berfelben abgebilbeten Infeften aud nur ein einziges, bas Dem foffor áf̧ntid wäre. Ueber saidarartings Bitat aus Stopoli bुabe id im Migajine etwas gefagt.

Scar. fubterraneus. Diefer Sáfer if ţiet ziemlid) felten : id babe sie znoeen Exemplare, bie id vor mir babe, im Billerthale, auf ben Siuggelberget Soralpen, in Rubmift gefunben. (Sleid benm eriten Znblicfe zeigt er viele Zleb̧nlichfeit mit bem folfor $\mathrm{L}$.,

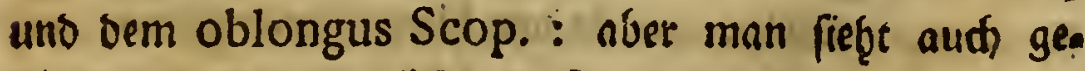
ffroinoe gan; oeutlid), dak et eigentlid feiner von 
benben iff, bebor man fid) nod) felteft bie naddfte Uto fad)e babon angeben fann. Er ift in orr Parallelle beutlid) genug bef(d)rieben. Nur ůber bas : ftriis crenatis : muई idf mich, um allen Miß̧uerftano zu vermeioen, nod) erflaren. Die Streifen fino eigentlich) einfad), aber bie Flgeile ber Fllugelbecten, bie zwifhen ben Etreifen lirgen, . finb geferbt : gerabe fo, wie ein geferbtes $\mathfrak{B l a t t}$ einer Pflange. Dadurd) er/cheinen aber aud sie Etreifen felbift geferbt. Man mus ben Rífer unter cin gutes Euấglas bringen, um dieß red)t gevau zu fetenen. Şlly fubterraneus if nidjt

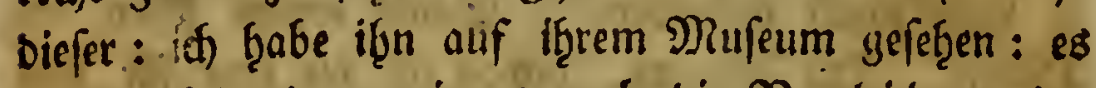
ift ber folgenbe, wie es aud) bie Bergleidung ber Manaje zeigt. Fueßti'z fubterraneus fann id we. gen bem Zitate aus Sulzer nid)t ţię̧er jăf̨len. Degners fubterraneus eben fo menig, benn er frat fein Sdiltoden, aud) Scerbft'B fubterraneus mage id) nidjt angufưf̧ren, Denn er foll vollfommen Grooffe, unb Seffalt bes fimetarius Kaben, meldjes ben mei. nem säfer nidje zutrift. Es bleibt mir alfo fein Einonim vibrig, als Prof. Sabrijius.

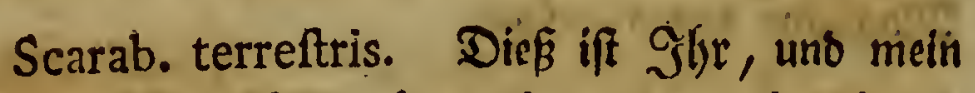
fubterraneus. Ming. loc. cit, : uno mein piceus foheint nur eine Eleinere 2 (bart bavon ju feun. Die

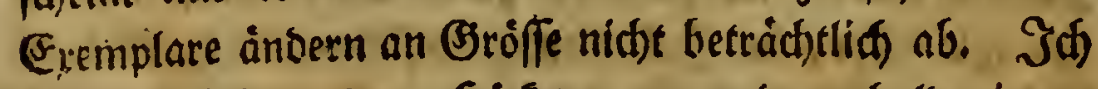
fetze on einigen oren feocfer : an anbern balb einen,

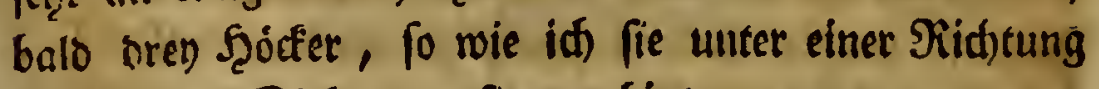
betrachte. Sief̧t man fie von ḩinten gegen vornen an,

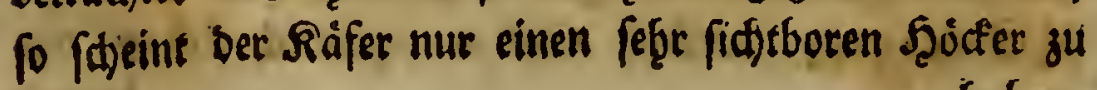
ţaben, 


\section{3ell, Den 2. Jinnt. 1784 .}

Ģaben, ber mitten auf einer erf̧abenen Sueerlinie ffețt: vor biefer Sueerlinie liegt nods eine aribere, etwas

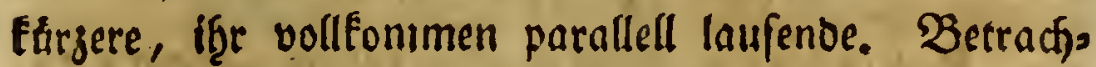
tet man bagegen ben Ropf. von vorne, fo fietet man beutlid) 3 Sgoffer auf ber tgintern Dineerlinie. Eie incroen es mun reid)t entfaleiden, ob meine $\mathfrak{B e r}$.

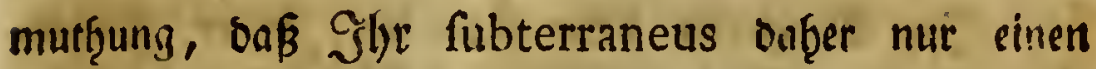

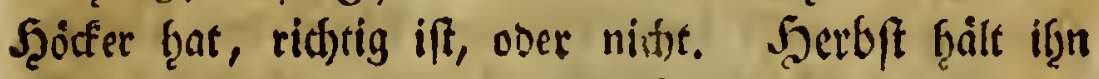
bep̈regen fir einen von feinem fubterraneus verffices Denen Räfer. Díß if aud) molį móglich; aber mein terreftris ift er zuverlåipig. Eollte nohl Gieoffroi feinen forfor mil biefem vermengt baben? die gar fleinen abailberungen beffelben laffen fo was bermuthen.

Was úberf̧aupt in biefer Deduftion waf̧r obet falfhi ift, ons wiro Prof. Sabriǵtus. an zuverlápigigften entfheiben Eannen. Wollen Gie bennod) meinen ter-

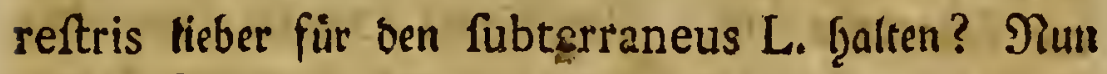
mob̨l! Jat) bin's audb) zufrleden. Zaber benn merben Sie meinem fubterraneus einen netuen Damen geben mulfen, benn er wirbe unter bie noff) nidfe befdriebenen geţơren.

5. Scarabaeus fimetarius: Der Mifte Solbentéfer.

$$
\text { Magas. p. } 372 \text {. n. } 7 \text {. }
$$

In jebem Rothe ferge gemein. Er if mit bem confpurcatus L. Der erffe, ber fid im frúb̆linge in Dungliaufen jeigt. Mit ben Bitaten aus Śdjáfcr' Icon. find die Entomologen bey diefent Rolbenéfifer nod) nid)t einig. 
6. Scarabaeus rufus. Der braunrothe Solbenéáfer. Magaz. p. $37^{2}$. n. 8 .

Rufus totus, folis oculis nigris, capite tuberculis tribus, clytris ftriatis, punctatis, tibiis anticis unidentatis, deficiente foveola thoracis.

Im Magazine vermuţ̧ete id), Diefer. Rolben. fáfer fónnte robgl etwa nur eine 2 fbart bes borigen,

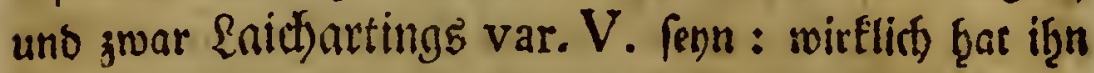
mir aud) biefer (S)eleşrte für eine 2(bart bes Mliftelbens fäfers erflatrt. Zabei, wenn mid) nid)t idjon S5erbft's Zleufferung in feinemt ftitifd)en $\mathfrak{B e r z e i d y n i f f e ~ u ̈ b e r ~ b i e ~}$

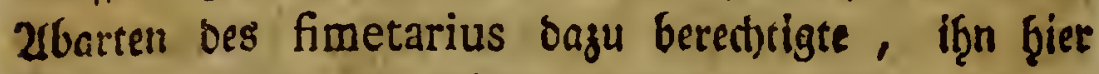
fefegen zu laffen, fo wuirbe id es bod) tḩun, weil id

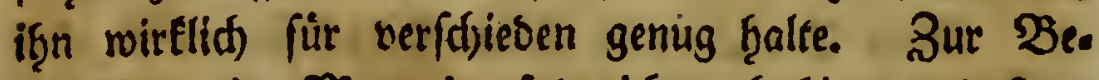

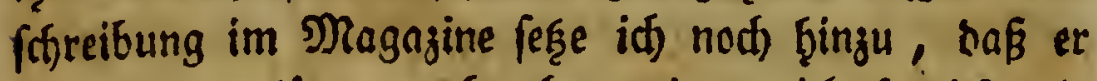
wob̨l etwas langer, aber ber) weitem nid)t fo bick, als oer fimetarius iff. Brufffdild, ounfel, ofgne bas (Brưb(t)en, bas ber fimetarius mit fo vielen Dung. fáfern gemein ḩat. Er fómmt felten bor.

7. Scarabaeus arcuatus. Der bogigte Rolbentáfer. Scarabaetis niger, nitidus, capitis clypeo antice rufo , elytris ftriato - punctatis, futura, puncto marginali bafeós, vittaque abbreviata, futuram verfus inflexa rubefcentibus, pectore obfcuro, abdomine flavefcente, palpis, antennis, pedibusque ferrugineis.

Sie wiffen, im voraus, baß̧ id biefen Rolben. fâfer auf ver alipe Edjwárzenftein gefunden Gabe. 


\section{Bell, Den 2. Jọnn. I 784 . 16 I}

Er Gat fo ziemlid) Brơffe uno Beftalt bes folgenben, aber Jarbe und Beidjnung fino ganj auffalleno bers fhieben. Ropfffilto, vorne rotb̧braun, Ginten forwar: bren J̧ódferdfen, bavon bas mittlere etroas meiter vor=

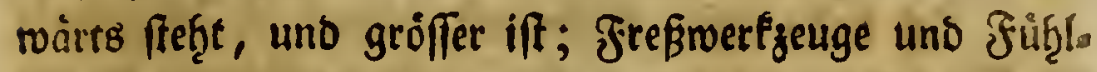

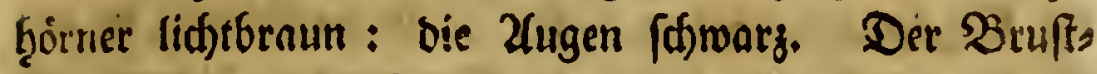
fíllo, fdiwarz, glänjeno, zart gepunftet : wie benm fimetarius, vorne ber braunrothe flecten. Die Sluigels

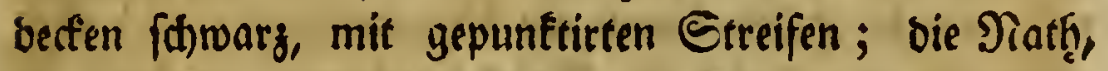
ser Suctel am Grunbe ber Decfen find ròtţlidit. Zuffer biejem aber fängt etwons unter bem borbern

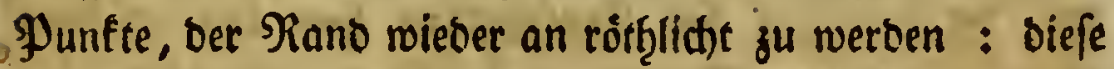
Jarbe zief̧e fid bis unter bie Mitte bet Decten, wo fie fint) in einem $\mathfrak{B}$ ogen gegen bie $\mathfrak{R a t h}$ zu feģrt, aber fie nidft gang erreidte. Die Bruft bunfel. Die Füffe liditbraun : oer Unterleib gelblid)t. Er änbert ab mit bráunlidfem Brufffrilloe, Jluigelbecten uno Unterleibe, Sumeilen ift aud) bie Spişe ber Decten rơtţlidft.

8. Scarabaeus confpurcatus. Der fifmuzige
Rolbenfifer. Mag. p. $373 \cdot$ n. 9 .

In jebem Dungḩaufen feţre gemein. If beo fiģe einige Exemplare; benen bie Dorne fef̧len. Dieß̉ fotheint Shber Bermutf̧ung in ber fritifthen Sievifion, Mag. I. p. I 36 ., baß ber merdarius Fabr. uno confpurcatus L. ein, uno berfelben sáfer fenen, neue Buverlásigfeit zu geben: benn ber erfte ift unbewafnet. zather aud Şerbft haalt benoe für gang verfajiebene Siafer.

Daturbif.25riefe 1.25ant.

(. $9 . \mathrm{Sca}-$ 
9. Scarabaeus teffulatus. Der gemuirfelte RolbenEáfer.

$$
\text { Mag. II. p. 33. n. } 70 \text {. }
$$

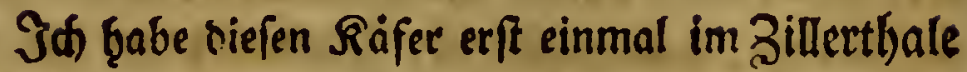
im Ribgforf̧e gefunben. Er iff fo verfticeden in ber (B)rơfle, Daß̧ man babey in Berlegenheit fömmt, ob man iţn für biefen balten foll. Der meinige iff beye nałye um bie J̧älfte fleiner als ber confpurcatus: aber menn idh if̧n Stüd für Stúd ourdggebe, fo fann ida ingn für feinen anbern, als oen teffulatus bzolten. Die Mafeln auf ben Dedfdilben fino bey meinen Exemplaren bem Brunde ju bgăufiger, als gegen bie Epize, oie jiemlich unbemafelt ift. SBeoffiroi fiteint biefen Rafer unter ben fleinern abarten feines Rolben. fáfers n. 19. mit zu begreifen. Plof. FabrijiuB jiebt zmar biefen $\Re$ äfer zum confpurcatus, abee Giesff:oi bat feine völlige frauptbeftinnmung bon sinne's fimetarius grifeus genonmen.

10. Scarabaeus futuralis. Der St)warznatfy Siolbenéáfer.

Scarabaeus niger, nitidus, capitis tuberculofolitario minuto, elytris fordide flavis, friatis, minute punctatis, fcutello, margine exteriori ad bafin, futuraque nigris.

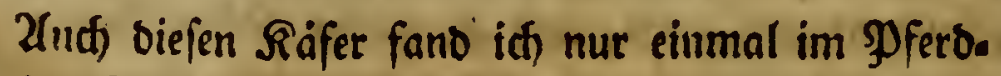
Fothe im Billerţ̧all. Er fỏmmt zmift)en bem Scar. lordidus, Fab. uno foetidus Serbft'

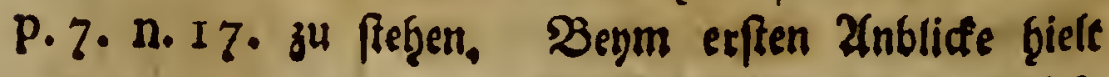




\section{Bell, Den 2. Jiknn. 1784, 163}

id) Şin für ben merdarius : bepiwegen fagte id in Der III. Sief. meiner Salzb. Jnf. gegen Jlbre Zeufferung im Magaj. P. I 36. n. $5 \cdot$, inith hiielte sen merdarius nid)e fîr einen mit bem confpurcatus, weil id erft feit wenigen Tagen ben waḩren merdarius ju ḩaben glaubte." aber er iffs nidft. Unter bie befannten vermag idf if̧n einmal nicht ju bringen. Ropf, Bruft. fíilo, Unterleib und fruffe fofwarg: auf' bem erfen

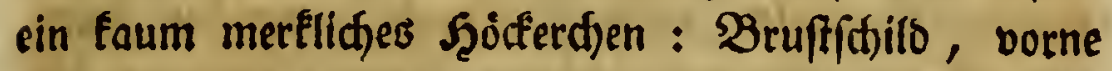
rotgbraun eingefaßst, fef̧r zart gepunftet, ziemlith groß unb, runb. Die Decfen famujig gel6, jart gepunftet, nur bie leztern Etreifen gegen Den ánffern Miandfangen unter ber $\mathfrak{M i t t e}$ ber Decfen an tiefer gepunftet zu wer. Den, $\int_{0}, \mathrm{Da} \hat{\beta}$ in einer Dronung, bie von-bem Ranbe ber Nath zugeţt, jebe biefer befonver's gejeidfneten Streifen etwas lánger iff, als bie anoere. Nath,

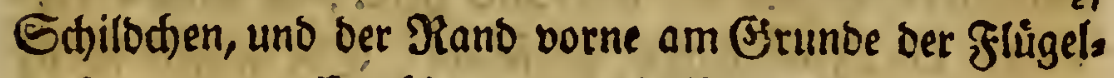
becfen, unt auffen bis gegen bie Mitte Ģerab fintrars. Der Siano bes Ropfs, bes Srufifdilbes, aud ber

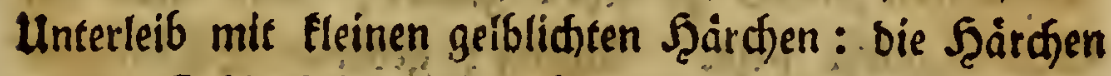
an ben Edjienbeinen róthlidf) : an ben vorbern Edfiena beinen zween Badf̧ne : 10 groß , als ber fimetarius. Şier ift er genau beidgrieben : id) múroe if̧n für ben merdarius F. halten, weltn nid) biefer unberonfnet

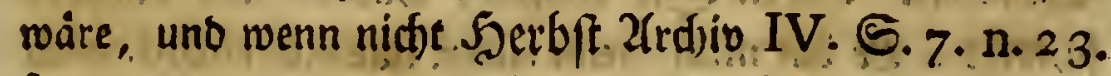
fagte, ber quisquilius fêt dem merdarius fo âfznlid). SBie weit er an ben foetidus uno fordidus graizze, bas werben Sic aus ber Bergleidung oer Bejdrei? bungen biefer zrween Ráfer felbft feb̧en. 
I 1. Scarabaeus haemorrhoidalis. Der গiothar fof Rolbenkififer.

Fucfiti n I I. Scopoli Scar. alpinus p.9. n. 2 r.

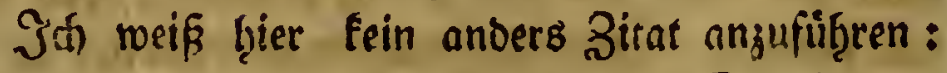
benn oer Scar, haemorrhoidalis, Den Gie in oer Enumerat. n. 8. unt ict im Jiagajine p. 373.n. Ir. befdy:ieben bauen, ift fidter nidts ander's als Sphaerid. haemorrhoidale. Soffen Gic nith Stuncn hier fagen, wie id) Ju, und von meinem feģler gefommen bin.

Iक . hatte mef̧rere fo fleine Dinger von Rotḩarfa) vor mir. Da biefe Ráferdien feb̨r flein waren,Oa überbem einige bavon bie früblģorner unter bem Ropffatib eingejogen hatten, uno folglid noth fafwerer zu unterfuntien waren, fo glaubre ith an einigen berferben antennas, crpitatas, capitulo fiffili, an anbern clavatas, clava oblonga, ju feḩen. Sie wiffen felbff, wie oft man bey foldten Dingerdien, uno in folden Umftänoen etraas zu fefen glaubt, was man im Brunte nidjt fiefte. Bening : getornte Edjienbeine batten alle. $\Im_{f j}$ nafim alfo jene fur Scar. haemorrhoidal. - biefe für Sphaerid. haemorrhoid. Bon ben erften fofictite id) unjerm lieben Dr. \$anzer ein Exemplar! Storen Ste einmal, was er mir Darúber (d)reibt : Er bat midi meine Scar: haemorrhoid. nod) einmial genau zu unterfuchen; oenn er batte an bem auf ber Pieife gebrodinen Eremplare bes úberfdicften Siafers étwas bor cinem Dermertes zu finben geglaubt.

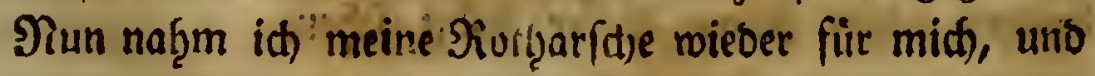
je ôfter uno lánger iđ unterfuctee, befto meģrere famen 


\section{Sell, Den 2. Sănn. $1784 . \quad 165$}

nun bem Sphaerid. haemorrhoid. - zu ftef̧en, big enolid) fein Scar. haemorrh. methr übrig war. Já

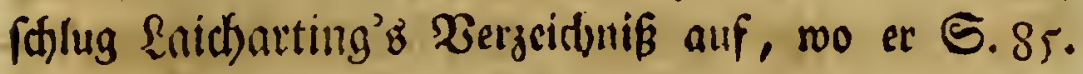
fein Sphaerid. haemorıh, beidfreibt; uno rerglid) bamit J̧ren Scar.haemorrhoidal.: Gic fagen : ad lentem elytra ftriata. Seichauting : oie übrigens fnum mertid) geftreift finb. Gie fagen: tuberculum capitis in meo fpecimine deeft: tibiae multidentatae, denticulis exiliffimis. Zlher alles bien ift aud) Şauptfennzeicten des Sphaeridium's. গiun glaubte id), Saidfarting ḩabe witeflid) ganz ridftig vermuthet, es birfte Spaerid. haemorrh. - und Scar. haemorrh. L bisweilen verned) felt worten frun. Nod) meţe woro id beftáret, als id bier, in Dunge,

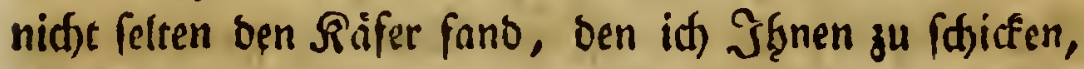
bas Bergnugen ţatte, und ben aud) Sic, wie Sic Sidi) ausbricten, für ben lange berfannten Scar. haemorrh. L. erflarten. Sie wiffen nod, mein

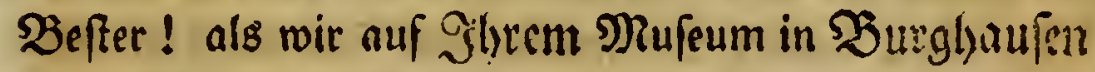
Shye Roleopsern burd)giengen, wie id ba Jjeren Scar. haemorrh. gerabe fo fano, mie ber, ien id felbit fâl(c)lid) bafür geţalten ḩabe, - wie Gic, ouf meine Bitte, if̧n gennu zu unterfud)en, if̧n feltêt für Sphaerid. erflärten. Wir Ģàtten alfo beube ben Scar. haemorrh., fo wie er izt ftef̨t, aus unfern Berzeictiniffen ausjuftreidjen, uno onfuir bas artige Rolbenfáferden

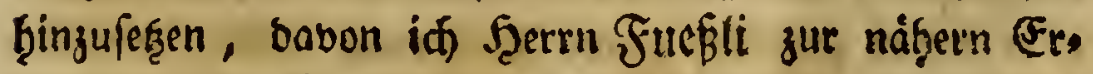
láuterung ein Eremplar fenden werbe, mit ber Bitte, cs abbiloen zu laffen. 
Da b̧aben Sie, mein Jrennb! eine genaure

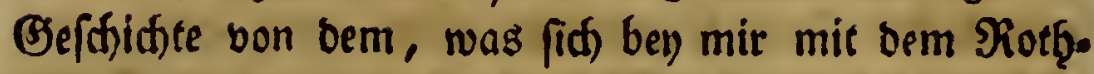
arfă Rolbenfáfer zutrug. . Ein bisden langmeilig?Frenlid) wobel! - Je nu! Was merben Gie ants

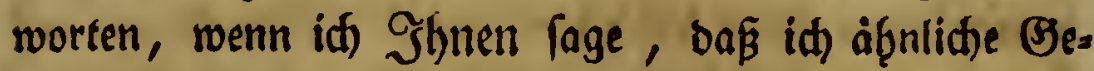
(d)tdten, wenn fie fef̧e fparfam fino, nid)t für fo gans

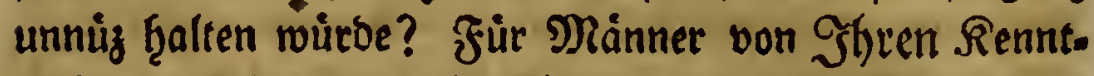
niffen frenlid) nicht; aber 2 (nfänger in ber Entomologie wirben baburd) bef̧utfamer im Beftimmen merben, wenn fie åf̧nlide Benfpiele von Bermirrungen ju lefen befámen.

Felix, quem faciunt aliena pericula cautum.

Diefer Råfer ḩat ganz ben f̧abitus bes গnifts Solbentáfers, aber er if viel fleiner - ganz fálwarg, Die Fluggeldecfen geftreift, gepunftet, bie Epizen bunfet-

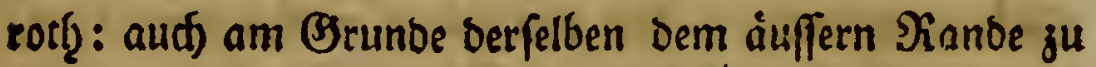
ein rother Flecten. Dieß trift aber nidft allzeit zu. Bey allen meinen Exemplaren fino nur bie Fuß̧blátter rotḩbraiunlidit : bieś if bas einzige, in bem er von Sinne's Beffreibung in ber Fauna fuecica. 358. abgeht : aber niagt bon ber fürzern im Siffeme, wo

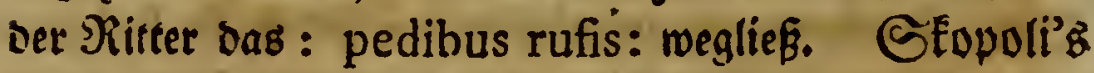

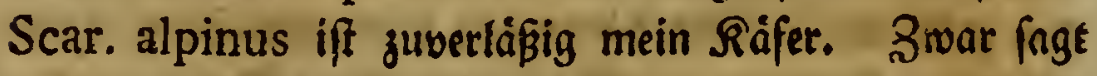
et, bas Rólbden fen zmengetheilt : id finbe es immet orengetḩeilt, uno ftatura coccinellae I opunctatae: aber bubon zeigs fich in ter 2(6biloung nid)ts, bie mids

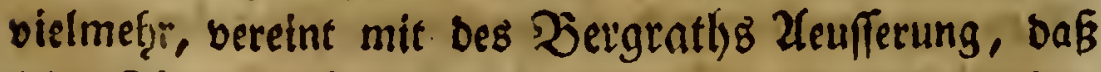
fein Rafer faum vom fimetarius berfáienen fenn

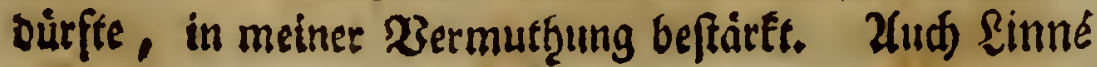




\section{Sell, Den 2. Jänn. 1784 .}

bat feinen haemorrhoid. gleid an ben fimetarius angeidiolofen.

In ter Enumerat. p. I 8 . finbe id aud sinen Scar. alpinus, uno Gie berufen fid) baben auf GFos poli's angefübrte Etelle. Tagegen haben Sie in

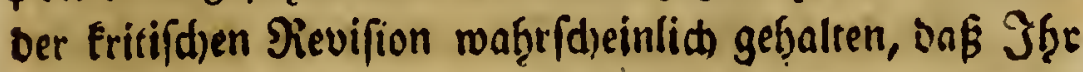

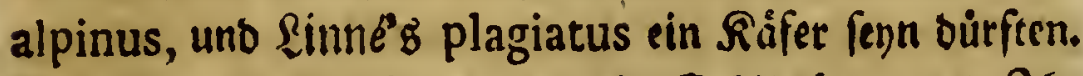

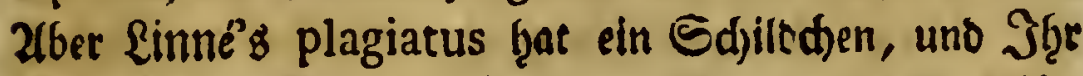

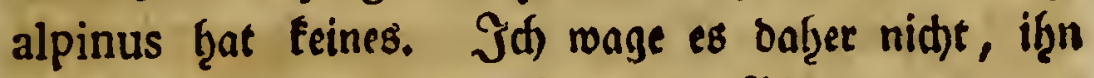
ben meinem haemorrhoidalis anjufưţren.

12. Scarabaeus granarius. Då $\mathfrak{B a i z e n t o r n}$ Siolbentáfect chen.

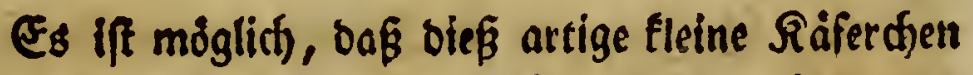
bas fich) audh f̧ier im Dunge offers fȩ̣en läprt, von been borigen nidte wefentlid) unterf(dieben ift : aber es iff viel fleiner, glänjenber, unb ḩat groar nur einen woḩl

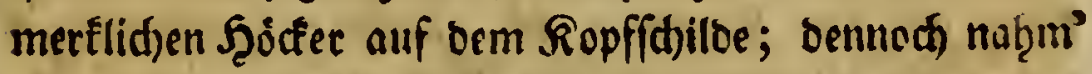
id) an allen meinen Eremplaren bie Epuren, von zirten antern an ben Geiten getraḩr. Der rothe Jleden ift

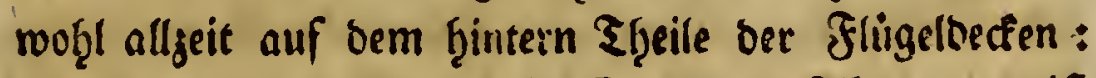
aber erreid)t nid)t alljeit bie Spisen berfetben, uns ift mandjmal feḩr Elein; vielleidye iff er bann Scar. pla . giatus L.? Borausgefeget, bák biefer Ráfer ridtig Sinne's granarius ift, fo bürfte Prof. Fabrigiub wobll nid)t unred)t getţan ḩnben : va er if̧i mit bem haemorrhoid. unter einem Namen Jufammenzeg: aber id) wollte bann lieber sen Mamen haemorrhoid. als ben ungleid) meniger begeidenenden granarius Dafúr bef̧alten.

$$
\{4 \text { I3. Sca- }
$$


13. Scarabaeus ictericius. Der gelffiutrtige Rulbenḱáfer.

Enumerat. inf. auft. p. 5. n. 6. copris. Nag. p. 136. n. 6 .

Diefer Ráfer ift im Billerthale im Dungḩaufen gar nidft felten. Id befige Eremplare nit ben bren Jुócferden, aber aud eines, oģne biefelben. Diês ift ein Umftano, ben Sic, mein lieher Freuno! moght bemerfen muiffen: benn er erregt folgende $\mathfrak{B}$ ermutf̧un. gen in mir. (Bleid, als id) Jibren Copris fâ, ers fläte id) if̧n für ben nemlidben Râfer mit meinem ictericus, id) f(tyicfe Şbnen meinen ictericus, und Sic farreiben mir in Shrem langen Briefe vom 18. Dec. "iEtericus : gerabe mein Scar. copris." 2lber $\mathfrak{J l y}$ copris ift muticus - uno Saicharting' 8 ictericus ḩat orel Şodcferd)en. 'Meine obige Bemer. fung lost ben Rinoten : id) glaube : Snid)arting's uno mein ictericus ift bas פianndhen - uno J̧ge copris, uno bes ḩerrn Prof. Fabriziu's merdarius bas Weibrtien. (Secoffrui's Ruricola if aufferbem, was fie fhon im Magngine bemerft ţaben, aud viel żu großs. Was $\mathfrak{F u c f i l i ' s ~ m a r g i n a t u s ~ e i g e n t l i d ) ~ i f t , ~}$ uns riberf̧aupt, was in allen diefen Bermurf̧ungen

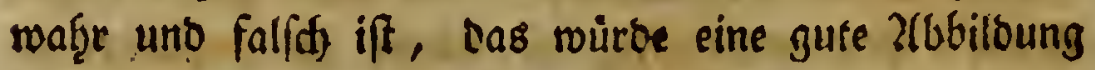
Des ictericus bey oen Erlaiuterungstafeln ju Snichar ting's Bergeidniffen beftimmen. Da werben bie En. tomologen famt und fonders fagen fornnen, of oer aba

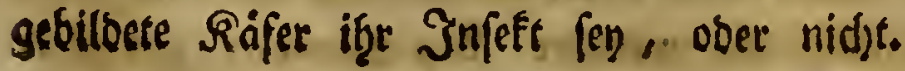




\section{Bell, Den 2. Jånn. I 784. $\quad 169$}

Zflles bießs ftheint fid) gang wojt benfen zu taffen: aber gernoe finbe idt) nod) eine Edfwierigfeit. She copris hat fein Sdjitodjen, - uns ber ictericus ift

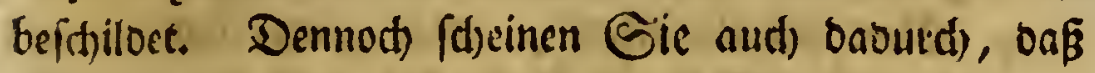
Sie meinen ictericus für einell Ráfer mit $\mathfrak{J}$ hrem copris erflaren, - und daß Gic Eseoffroi's Ruricol. nur negen bem punftirten $R$ opf und Sruftidjilbe nicht mit Э̧̧rem copris vereinigen wollen, zu fagen, bón Şror Ráfer ein Edjillod)en habe : Denn ber ietericus und ruricola finb fcutellati.

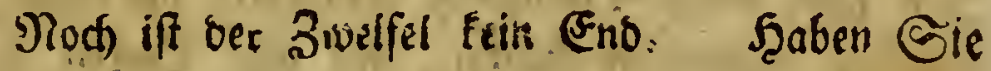
woģ Gibien copris unter einem guten Sudiglafe be. trad)tet? WBenn id) meinen ictericus unter bas Branderfde Mifrostop bringe, fo fef̧e id gar beutlidf bie Puntten in ben Streifen ber Decffajilde.

\section{B. Itnbewafncte.}

14. Scarabaeus ftercorarius. Der Drects. Rolbentífifer.

$$
\text { Mag. I. p. } 370 . \text { n. } 2 .
$$

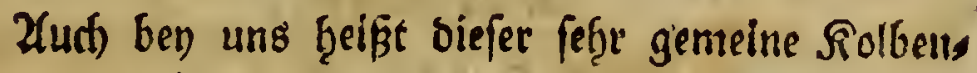

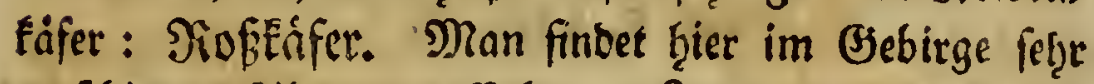
verffiebene albarten beffelben. In Jjinzgau biabe id

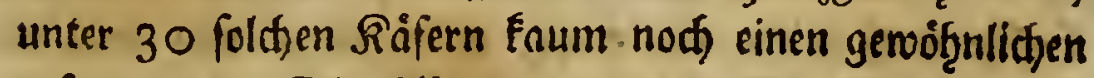
gefunter. Die Zllonrten waren :

a. Oben fatwar $z_{3}$ init einem blaffen Rupferglanze, unfen gruin mit Bbolbylanje: nur ber Theil bes Unterleibs, swifden bem grweyten uns britten Puare ber Füfe blau.

$$
\text { \& } 5
$$

B. Oben 
B. Dben fupferfärbigt, unten buntelblau, ber ₹̧̧ell Des Unterleibes jwififen bem 2 ten und $3^{\text {ten }}$ Paare ber J̧úfe ròtţlid) mit Bologlange.

y. Dben metallglänzent, unten gang Dunfelblau: ber $\mathfrak{B}$ ruffifitilo fpielt ftare' ins Borune.

d. Dunfelgrün, unten fojơn ftab̨lblau.

Im Magasine f̧eißst bas Bitat aus Frif() burd) einen

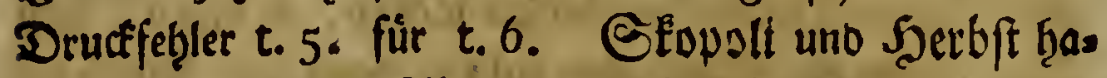

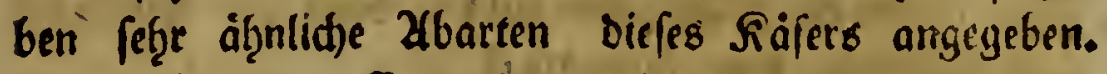
Id) bqabe einige Eremplare gefuncen, bie rotbe, mit Tarben bejeid)nete Jlecten, auf ben Decfen ḩatten. Järben fith etwa bie ju ber Beit, ba bie Dectén nod) biel Eaft b̧aben, verleģten Theile berfelben, eben fo ròttho lid)t, wie einige Pflanjenblatter von ben Etid)en ber

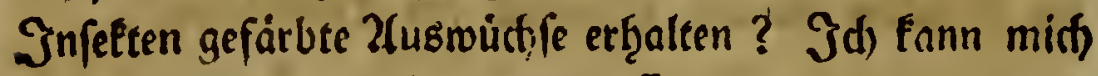
soofl erinnern, aud) mehrere Cocc. 7 punctatas ge. feb̨en zu fraben, bie äf̧nlid)e, fatworge, ober ounfelbraune, benarbte Etellen auf ben Decten batten.

15. Scarabaeus vernalis. Der Friblinggs Siolbentáfer.

Mag. p. 370. n. 3 .

Nidit fo gemein, als ber borţergeţenbe. I befite noth nid) ein einzig Exemplar, von bem id im ridbtigften Berftanbe fagen fónnte, baß̧ es unges freifte Jlügeldecten ḩabe. Zulueit zeigen fich menigftens bie Spuren von Streifen, wenn fie (for on mandimal fo feidt fino, oak man fie benm erfen Anblide faum bemertt. Stopoli hat bon bieferm uno bem vorḩer.

getgenben 


\section{בెell, Den 2. Эănn. I 784 .}

gef̧enten gute $\mathfrak{Z}$ tbbilbungen gegeben. Dns Bitat aus Raj. p. 90. n. 7. elytris laevibus, bas Gicoffroi ben bem. ftercorarius anfüţrt, fdyeint ḩief̧er zu gei̧ơren.

\section{Scarabaeus oblongus. Der linglidite Rolbentifer.} Mag. p. 37 I. n. 4 .

Diefer Råfer ift bier eben fo ḩäufig im Dunge, als ber Bröber: Rolbenfífer felten ift: Gingegen fỏmmt et in ber Begent bon Ealghurg um fo feltener vor. Id) habe in Magajine 1. c. Die Rennzeichen biejer beyden Siafer in eine Parallelle gefergt, Die ich hier nidht wiederbolen will. Shrnedem laffen SEopoli's uno Ş)re Befdreibungen feinen Zweifel mę̧r น̊brig. Der móglid)ften (jenauigfeit willen feģe id) nod) Gingu.

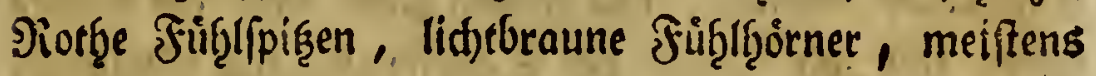
bunfelbranne Jüffe. Die Beibden merben feģr bicf; wenn fie befrudfet fino. Die albbilbung SFopoli's iff, glaublid), von fo einem $\mathfrak{W e i b}$ hen genommen.

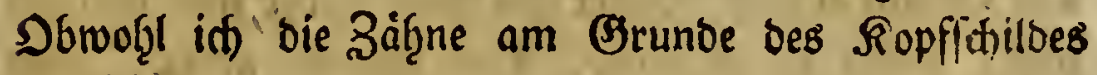
nidft finbe, wenn nid)t ber Bergraţ oie fpişigen Edéen, bie er gerabe vor ben 2lugen madft, für Zåd̨ne gee nommen ḩat, fo bin id) bod), aud) ourd) bie Zlbbiloung,

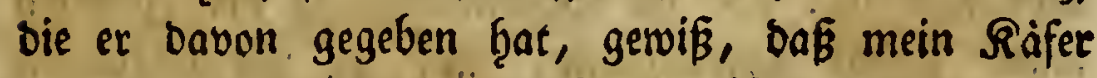
voll bem reinigen nidft unter (d)ieben ift. 2(ut) on ber

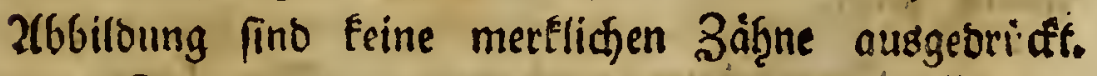
Daß $\mathcal{S} b t$ oblongus mit bem meinigen ein Räfet fey, bavon fraben Sic mids nut erft in einem Briffe vom I8. Dec, verfidert. 
17. Scarabaeus gagates. Der Şagat, Rolbentufer.

Scerbff. 2(rdio. IV. p. 9. n. 30. arator. Gieoffroi I. p. 83. n. 2 I. Scar. jaget. Nitilicr Scar. gagates.

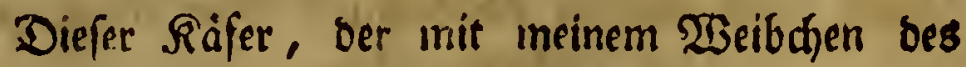
fubterraneus fefgr viele alef̧nlid)feit ḩat, ḩabe id immer fừ die 2lbart bes Scar. rufipes : pedibus nigris geţalten: vielleid)t ift er es aud). Sctubft's arator fơmmt vollfornmen oamit likerein : bie groffe Zlegnlidléeit, bie er mit Scar. rufipes heat, beftartft mid) aud, Darinn, taß id) oen fubterraneus L. rid)tig beftimmt habe, benn sinné fags boum rufipes : firnillimus fubterraneo, fed elytra ftriis minime crenatis, et thorax minus gibbus. Mein siáfer ift gang fowwar: : nur fino bei) cinigen Ercmplaren bie

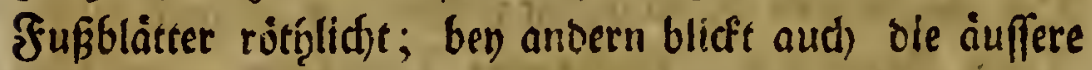
Seite unb oie Dorne oer Sobjenbeine ing rơtblidjte.

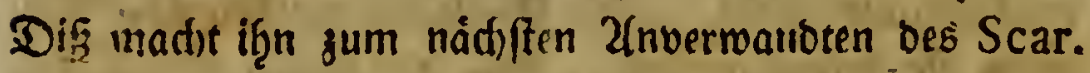
rufipes L. Ja nethme fein Bedenfen Die Bitate aus Geoffrot uno Miller, bie Paft. Gjüze jum rufipes zieb̨t, ḩieber zu neh̨men. Den Namen arator, ben

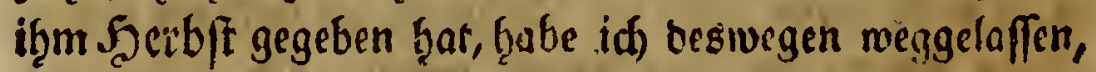
weil Prof. Fabrizilus aud) einen arator bzat, ber aus

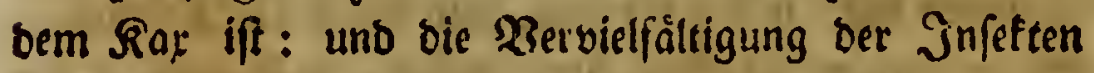
unter einem ₹rivialnamen nur zu Betwirrungen Zulaß giebr.

18. Scarabaeus variegatus. Der bunte SolbenEáfer.

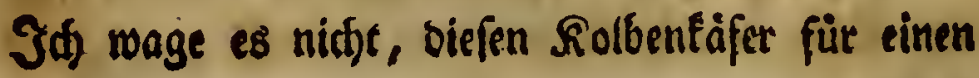




\section{3ell, Den 2. J\&!n. I 784 .}

neuen ausjugeben, obmolel et mit 5̧erb/t's Scar. variegatus nid)t vollfonmen libereintsmme. Bon ber Grôffe bes vorhergehentell, ganj glångend fajwarg,

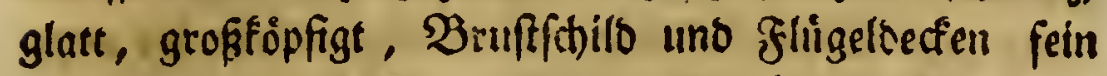

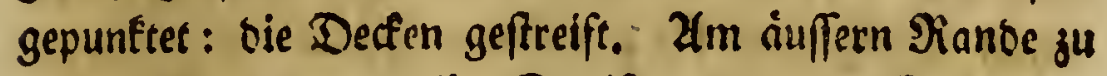
laufen einige mattgelbe Grreifen von ber Spige ber

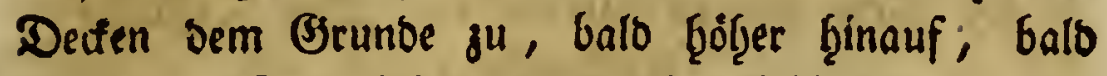
meniger. Sie fajeinen aus gelben ડ̧åård)en zu ento ftệen, unb finto fehre matt, fo daf fie nid)t benm erfen Zublict ins Befict) fallen : audf find fie nur in ber Bjegeno bes äuffern Siandes ber Decfen, gegen bie Naţ ju wiro man feine geraal̨r. Şerr J̧erbft wiro entifbeiben, ob bieß eine Zfbänberung feines variegatus ift, wie id) feţr geneigt bin zu glauben.

19. Scarabaeus foveolatus. Der gegrúbelte Rolbentedfer.

Im Billertb̧ale gefan:7uelt. Eine genaue $\mathfrak{B}$ e. fofreibung beffelber wiro in meiner II. Sief. jum $\mathfrak{N a}$, gajine vorfommen, worauf id mich) bicr berufe. D6.

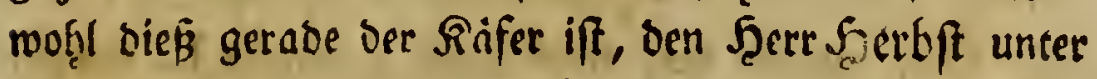
Dem Namen porcatus ouf ber. 19. Tafel. I 9. fig.

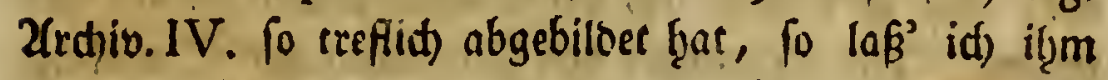
bod ben Namen, ben ich) iffm im Shagazine gegeven

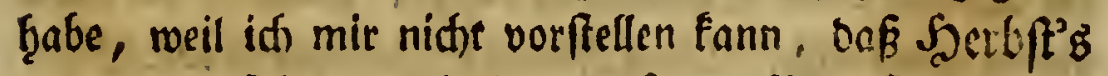
uno mein Rafer mirflich des Şerrn P̧rof. Sabrigiu's porcatus pri. deren elytra porcata nennt, diefer. groffe Naturforider in feiner idcinen Philofophia entomologica p. 33. fulcata, fulcis punetis ob. longis elevatis adfperfis. 26er oié if gerave 
bas Bjegentḩeil meines Râfers, betun er F̧at vertiefte, runbe Srübsen in ben Furchen, - nicht erţabene, länglidyte Punfte. Dieß bejeugt and) Scerbft's Zlbbil. bung uno $\mathfrak{B}$ efdreibung. Soer id verftef̧e bie ange. fuif̧rte Etelle ber Phil. entom. falfab.

20. Scarabaeus quisquilius. Der Refrict Rolbcnfáfcr.

\section{Efopoli. n. 29.}

Nur einmal f̧abe ich bießs wingige Rulbenläferdjen zu Enlzburg im Dunge gefehten, - aher auds nur ge.

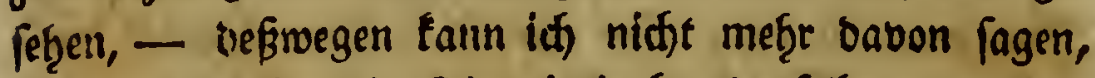
obroobl id úber sie Sinonimie bey benfelben aus bem Grunbe zroeifle, meil einige $\mathfrak{B l u m e n , ~ a n b e r e ~ D u n g ~}$ zum $\mathfrak{2}$ Bof̧norte angeben; und weil bey $\mathfrak{P a f t}$. Sonze

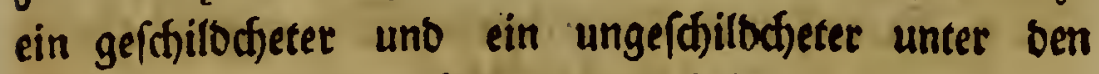
Einonimen gleid) nebeneinander frȩ̧en.

\section{B. olme Gdildaen. \\ a. gelyarnte.}

Sie merben bier einen Scar. Lunaris L. ermars ren; aber nod) frabe id im Saljburgifden feinen gee funben. Die Exemplare von Mănnden uno $\mathfrak{B}$ Beibd)en bie id in meiner Sammlung befigęe, habe id) ber (Gute unfers gemeinfdjaftlicfen Freundes, bes Seerrn Dr. JJan = zers' zu banfen. SEëlegentzeitlid merfe id frier an,

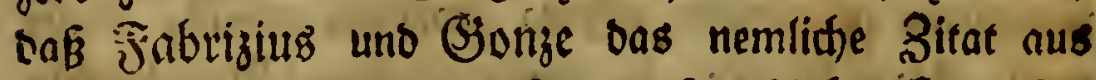
Schdfer's Icon. t. 63. f. 2. 3. formobl ben Scar. bilobus, - als ben Scar.lunaris anfütheren, - uno den. nod bat ber erfte ein Edilbden, ber zwebte feines. 


\section{ลell, Det 2. J゙ล̊nn. I 784 .}

2r. Scarabaeus quadrituberculatus. Der bier bóctrichte Rolbenéafer.

Erft einmal im Pferbefort gefunben. - Sillerthall. Raid)arting ḩat iţ̧ ref̧r gut befdrrieben : nur fino

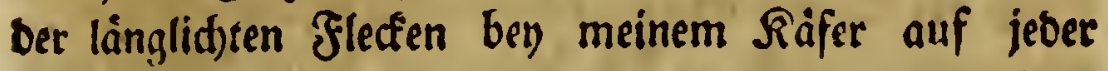
Decfe vier, wovon bie lezfen zmeen, bie ber হath zu ftệen, einanber nàther fins, als bie übrigen. Man mußs roobl adjt ḩaben, daß man biefen Räfer nidbt für einen Nactenţorn , Sáfer bält, unb unbemerfe feine $\mathfrak{B}$ ege geţen läpt. $\left({ }^{*}\right)$

22. Scarabaeus Taurus. Der Stiers Rolbentaffer. SEopoli n. 23. rugofus. Eaidjart. p. I9. n. I2. var. $\beta$.

Diefer Ráfer fơmmt im Billertllale im Ruḅift oft vor: aber mas fefte fonderbar ift, fo trabe id than immer nod) pünttidi) fo, wie Sfopoli reinen rugofus bef(h)reibt, gefunden; folglich lauter 2 Beibden vom Taurus, uno nod fein einzig Näunchen. Unter.

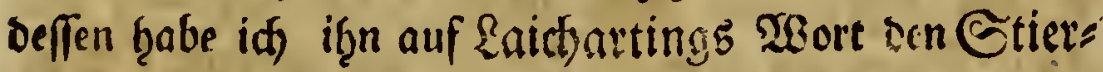
Rolbentíffer gennnnt. Die länglidte Errhabenţeit auf oem Bruftidgiloe finte id) nidi, wof̧l aber merte id) am Grunce deffelben g̈egen ben äuffern Rano auf jeder Seite cin runbes Snopistien gewohis, das in einer Bertiefung fteḩt. Die Farbe ift ben meinen $\Re$ safern gang

(*) Sherr Gdjoller frat Diefen Solbentifier im I. 3. Det

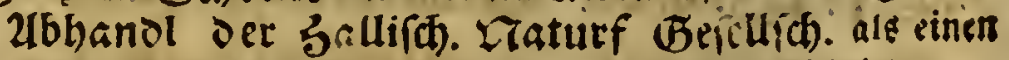
neuen unter bem Mamen Scar. ropunetatus beídricbern.

Doll. 
ganz formarg: nur ein Exemplar befige id, bas eine merfroüroige abart fenn buirfte. Ropf, Flligeldecten uno Unterleib mit einem ounfeln Rupferglanze, vorzuggs lich) ber Unterleib; ber Brufffdsilo glángeno grun, bie Raţ̧ Der Decten erţaben, Galbfupferfärbigt, glänzent, insgemein fpielent. Die albbilsung biefes Ráfers ben Gfopoli ift mittelmàfig : aber das Månndhen beffel. ben würoe man aus ber 2 fbbilbung, bie ber $\mathfrak{B}$ ergrath bavon gegeben ḩat, woh̨l fdjweilid erfennen, denn fie bat ganz ben Umriß bes thoracocircularis Eaictarcings.

23. Scarabaeus nuchicornis. Der $\mathfrak{R a f t e n l y o r n}$ Siolbentáfát.

Mag. p. $372:$ n. 6 .

Eefre gemein in jebem Rothe. Xlle meine Exemplare ḩaben gerave fo gefärbte Brufffailoe, wie bie saichartingldsen, nicht eines einen fotwarzen, mie Scerbft'b feine. Bel ben Bitaten aus Shdufern giebt es hier nod) Dermirrung. Paft. (Sonje unb Fuefili führren aus bem Icon. t. 73. f. $2-5$ an. Prof. Fulbrizius oagegen f. 2,3 . bei taurus, f. 4.5 . ben vacca. Saicharting fúf̧rt meber bey taurus, nod) bey nuchicornis eine 2(bbiltoung aus bem Icon an.

\section{B. Unberwaffinete.}

24. Scarabaeus ovatus. Det eltunde Rolbentéfer.

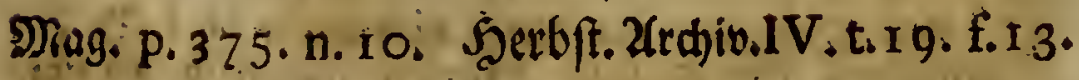

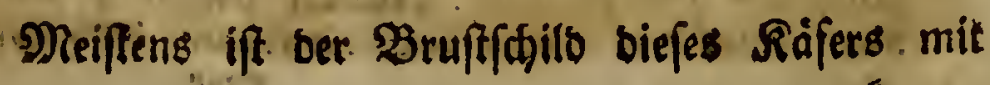




\section{Bell, ben 2. J̣ånn. 1784 .}

Eurzen Şąärájer befę̧t, aber eines meiner Eremplare if am Bruff(t)ilbe, Unterleibe, und vorzúglted) an ben Frifien, ungleid) pelzigter, als bie tibrigen.

Şier ţáben Gie alle mir befannten Ealzburg. fóch Solbenfáfer, bie jur Familie ber Dungeâfer ge. Fiơren : aber auch babey Debuftionsmáffige Bemer. fungen. Sie bürften both etra, wie id mir fhmeid)s le, oen grơften Ţbel berfelben nid)t für ganz unnư f̧als ten; und jubem barf man in in einem freunofd)aftlititen Briefwed) fel úber fo Begenftánde etwas weitláuiger felyn, als in einem $\mathfrak{B}$ erzeid)niffe, oas man gerabewegs

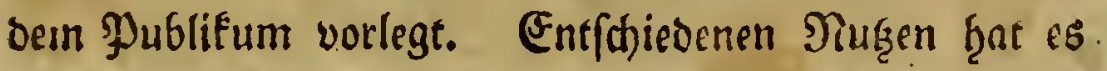

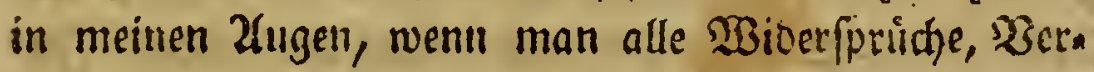
mirrungen uno Sinoten, aud) bie fleineren, ber jebem Sufefte, in fo weit man fie noch in feinem andern

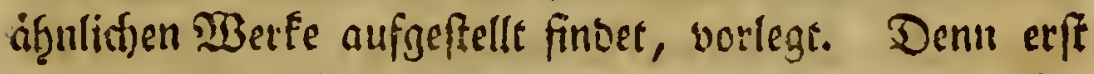

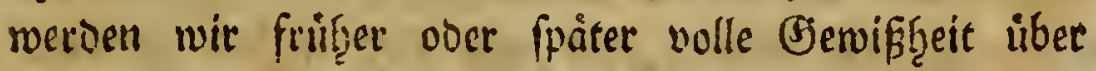
jebem einzelnen Saturfórper erf̧alten. Jd) glaube,

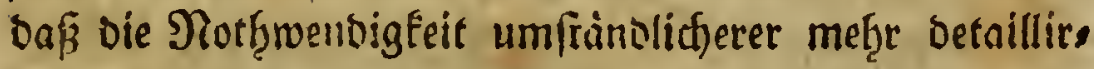
ter $\mathfrak{B e f d x h e i b u n g e n ~ D e r ~ I n f e f t e n ~ i m m e r ~ b e f t o ~ m e f ̧ r ~ i n ~}$ bie 2lugen fallen mirs, je meţrere Nnturforfher bie Entomologie bearbeiten, uno je faf̨lreidger folgliat) bie neuen Entsecturigen in biefem weiten, bennab̧e uner. foipflicten Sieidje Der Sintur werben. Denn man wiro 4,5 , uno meţrere Infeften finden, auf bie, bie mandimnal to furg gefapten Rerinjeidgen eines Snfeftes geriau paffen werben.

Mit Sefznfunthe erwarte id jeoe Glyrer anntwor. ten auf biefe birlefe; uno bie Şeleg̨rungen, bie Gie Daturbift.25iefe 1. Band.

M

mir 
mir ůber einige ber geniuferten 3meifel zu geben bie (S)น̆te ţaben werben. Joh bin unmanbelbar

$$
\text { 3ฺุ̧e Mioll. }
$$

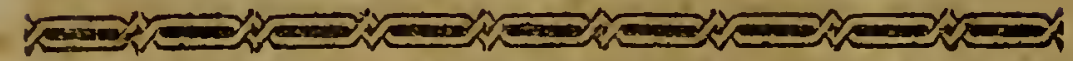

Zebnte Şrief.

3ell, ten r. รånn. $x 784^{\circ}$

Gortfegung Der RolbenEfifer.

Co ift bod) cin gar ju hinteiffendes Stectempfero um sie Tiaturgefádid)te: man mód)te ben gans jen Eag saralli herunibacfeln - und man wiro bes

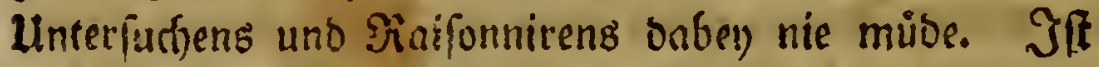
faum mein gar weitlid) langer Brief iber bie Dung:

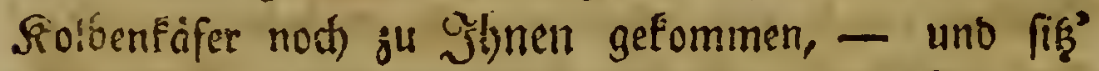
fajon micter an meinem Edreibtifhe, um Shinen eis nen nicht biel flirgern viber ben Sieft meiner Rolben. fáfer z" fthreiben : 2(m: Ento miffen Sic bod) alle

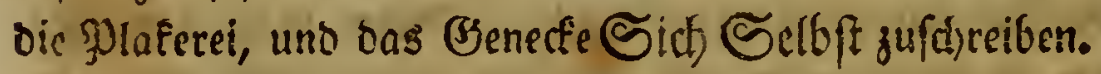
\$2arum bzaben Sic mid) Shltien fo gang eigen geo mact), - uno warum wiro man auth nie fertig, wenn man an Sie fofreibt.

Dle Reibe trafe nun bie zinote Familie: bie Staubsoldbentáfer (Trox. Fab.): aber id mus

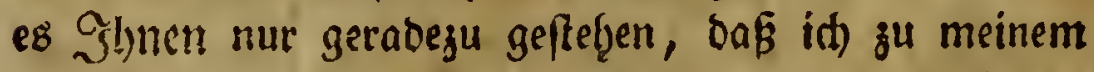
grofien Z(erger bisher nod) nidht einen, nuth) feinen Scar. fabulofus, im Enlzburgfden nuffinden fonnte. Der Bienius ber Entomologie miß̧ßte mir in $\mathfrak{B a h ̧ r t ̧ e i t ~}$ abfdjeulid) gram fenn, wenn er mir aud) biefen frrúţ̧. 
ling, an eitrem $\mathfrak{B}$ of̧norfe, ber mich zum nơdtyften Nad)bar eines Lanbes madit, in bem nad sald ar: tings Zeugniß biefe Rajer ziemlid) gemein fino, feinen berdicrte. $\left({ }^{*}\right)$

Szt babe id) bie \&nub = Solbenkfifer (Melol.) vor mir ftef̧en. Saffen Sie mich fie muftern, uno ragen Sie mir benn, ob idf jebem berfelben feinen Şlą̧ rictitig angenviefen ந̧abe.

\section{Dritte Familic.}

Saub= FolbenÉfifer. (Melolontha Fab.)

25. Scarabaeus (Melol.) maialis. Jer $\mathfrak{R a}$ Rulbenkafer.

Scar. Melolontha L. Mag. p. 374. n. I4\% Gendart. Lifteri, t. I I I. Folled)t.

Bely une leiber auch ferte gemein unter bem Namen Simnent : erft lezten Sommer haben bie Karven unfere (Sterfffart ziemlid mitgenommen, uno wir fürden heuer fur unfere Sorume eine gervaltige

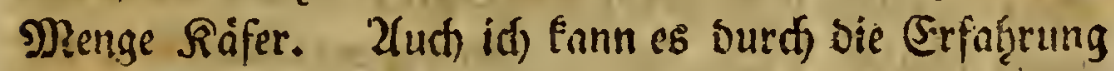
beftáttigen, Doß siafer mit braunrotberl - uno ounfel. violeten Srufffdilden in einem Jathre erfd)einen.

$$
\mathbb{2} 2 \quad \therefore \quad 26 . \mathrm{Sca}-
$$

(") 'S'ifî mir eit gar gutmùtbiger (Ssenius um den entomolo.

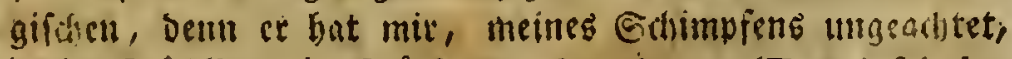

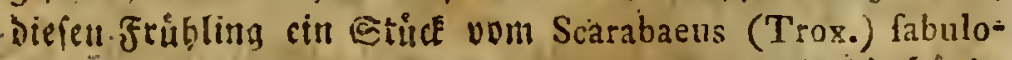
fus - unt cines vom Scarab. (Trox.) hifpiatus in Die fgánge gefpielt.

piol. 
26. Scarabaeus (Melol.) folftitialis. Det Dradimonid = Folbentâfer. Mag. p. 374. n. I 5 .

Nidjt to genietin, wie vorf̧ergef̧enter. Die

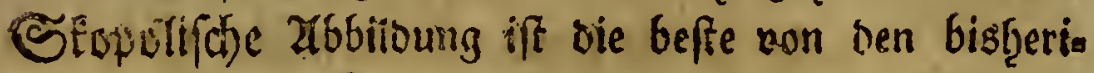
gen. 2Suf siffer's tab. mut. fteht biefer. Ráfer gleid neben orm vorigen, aber er ift riber uno riber fo pelo zidht gemadyt, oaßs man iḩn faum dafü erf́enuen follte.

27. Scabaeus (Melol) dubius. Dir zweifer bafte Rolbenkfer. 5iag. n. $308 \cdot$ p. 377. n. 22 .

(So felten id biefen Rafer in Defterteich) uns um

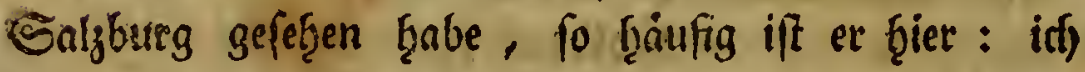
wriroe in einet Bierteffunbe gar teidft einige f̧unbert gefammelt . Jgaben.

SSict giebt es (mmer noti) fef̧r viele Berwiltum. gen. Einige Entomologen, wie z. 23. \$orof. Fatrizitus,

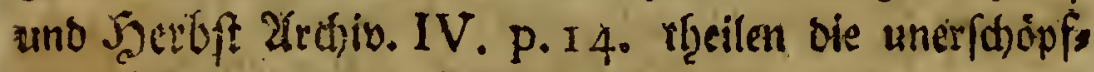

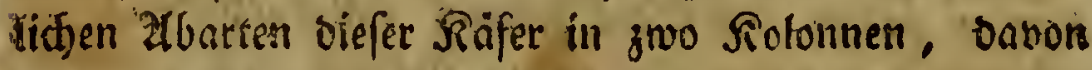
bie cine unter bem গnomen Melol. Frifchii, bie anbere unter Melol. Vitis freget: 2(nocre verbinben fie, wie Siopoli, Saicharting, uno ith mit if̧nen unter bem Damen bes żeifellaften Rolbentáfers. WBietlid

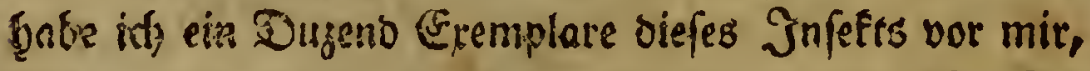
bavon nid)t eizes gerabe fo, wie Das andere iff. $D a$

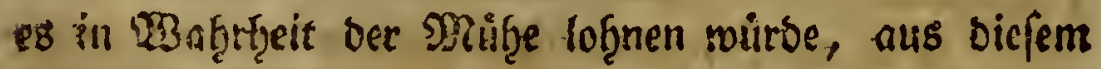
Chyaos zu fommen, fo mill kf Slbnen erft meine Zibarten kutz befolyreiben, uno benn meine Bemer. Eungen benfugen. 
3. Viridis tota, elytris teftaceis, thoracis lateribus flivis.

2. Capite viridi - aeneo, thorace teftaceo, vitta duplici viridi (gerabe fo, mie var.2 in ber GFopolifhan (2ibbiloung) elytris anoque viridi teftaceis, corpore fubtus obfcure viridi, teftaceo maculato, pedibus lividis:

3. obfcure caerulea, thoracis lateribus, macula media futurae pofterioris, elytris, anoque teftaceis, pedibus obfcure caeruleis, femoribus flavo-marginatis.

4. obfcure viridis, thoracis lateribus, vittaque media flavo-rubris, eiytris, anoque teftaceis, caerulefcentibus, pedibus viridibus, femoribus flavo-marginatis, maculis abdominis flavis.

5. tota viridii aenea.

6, eiusdem coloris, at faturatior priori.

7. viridis, capite et thorace nitore aureo, fubtus faturatior,pectus cum pedibus aeneum. 8. tota obfcure viridis.

9. capite, thoraceque viridi, elytris caeruleis, fubtus aeneo nigra.

io. eiusdem coloris, et canut es thorax viridis carulefcunt.

I x. caerulea tota.

12. atrocaerulea tota.

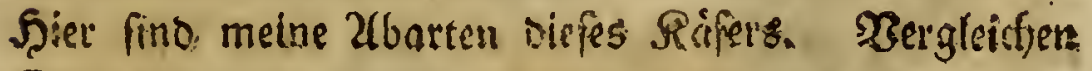
Sic felbe mit benen von ?cichatuting befdyriebenem. nuo Sie werben fie einanter fegre sifnlis finten.

$$
\text { श } 3 \text { Durtens }
$$


Danfen will id Şbnetr, mein Beffer, wenn Sic mir afle biefe Epielwerfe unter bie zno Rolonnen Mel. Frifchii, uno Mel. Vitis fo mittheilen, oaß jebes jus

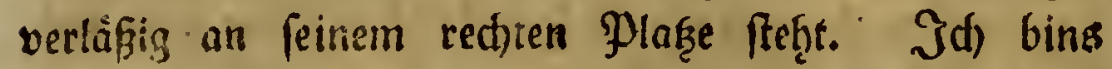
nidt) im Stande. Jad will bennahe aus allen J̈rifdens

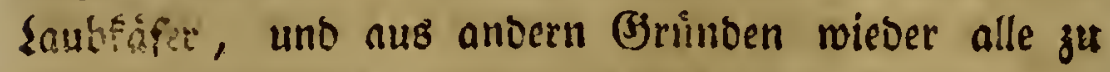

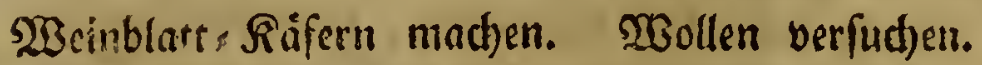

3.2. Die $2 \mathfrak{l b a r t}^{\mathrm{I}}$; bie wâre nun ene $\mathfrak{Z}$ bart ber Mel. Vitis, elytris teftaceis, nang $\mathfrak{F a b r i j}$ in ; bie folgenven 2. 3. 4. fint's mit eben Dem ßiedre, benn bey allen fino latera thotacis flava: nur iff biefe zar be meg̨r ober weniger breit, fo baj man balb Das gruin, balb bas geltb jur Graundfarbe ans nȩ̣men mởdte. SGollen Eic bas nicht? Ie nun!

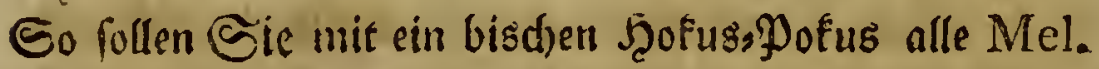
Frifchii fenn, Dem sas nigro-aenea, elytris teftaceis, trift aurfin in feiner afrt ben allen zu.

Die 2 buarten $;-12$ wáren, bådtse if, alle Mel. Vitis ; wenigftens fagt 5 cerbffit, biefe fenen alls zeit golbglånjender und Gellgrunner als Mel. Frifchii : abee n. 8. wirb audh Mel. Frrfchii fenn: bie Zlbart, elytris thorace concoloribus. $\mathfrak{Z B O}_{0}$ wollen wir n. 9 . binifeşen; biefe 2 bart ift aud nigroaenea, aber fie Vat Glaue Decten: alfo wirb fie Mel. Vitis fenn? Das wird fie frenlids, weil bort Scar. dubius bes Bergantrys anaefuigert wirt, uno n. 9. ift gerane bie

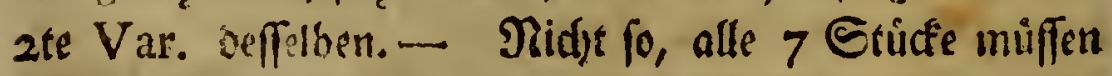
notfgendig Mel. Frifchii fenn, weil Mel. Vitis gelbe

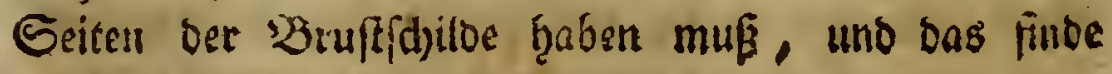
id bon $S$ bis $I 2$ an feinem. 
Saffen Sie nich) auffeoren; i id wứros midh felbft

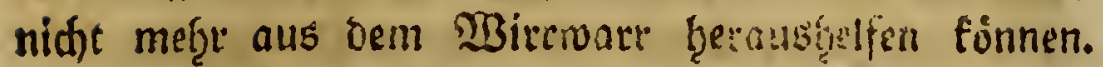
Zlles dieß, buntt midh) wenigftens fo vial zu beweifen, ans es faum móglid iff fur oie Melol. Vitis, und Mel. Frifchii zuverlópige Lnterftheioungejgeingen ju nennen, uno oaf GEopoli's Scar. dubius nidft nue bey oer erften, fondern aud) ben ber lejten angefiugert werben múbte; osnn bie Variat. 2. offfeiben woltte in noc) alljeit liéer zu Mel. Frifchii, als z" Mel.Vitis zieģen, um to meţr, da bieje nad) Sacrbft'z

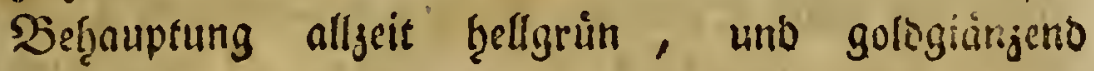
renn foll.

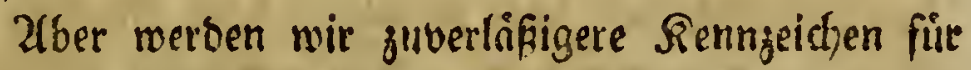
ben Scarabaeus dubius ḩaben? - - Eben fo fdower zu benntworten. Die Farbe miro fdyerlidy ein Renn=

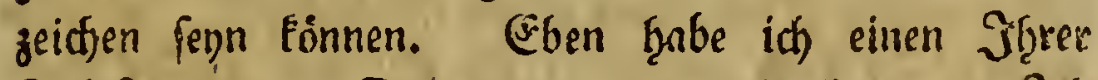
Briefe vom 2. Dec. I 782, bur mir lizgen. Sth fajicte Shlnen bamals eine Befdreibung meines Sca: dubius sils einen Bufaf zum ofterceidfichen Infetten

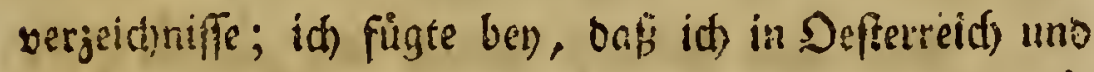
un Ealzburg nur biefe abore gefuncen (es war var.9.) - Dap aud Das Exemplar, bas ich in ber fleinen Jin fettenfammlung auf ber Sternwarte gu Sremsminfter fano, gerabe biefe 2lbart jey:- mo Eis antworten mir barauf: "Die farbe follte moḅt f̧ier unter ben Sennzeiden nid)t flefen, weil fie fo verftsieten iff.

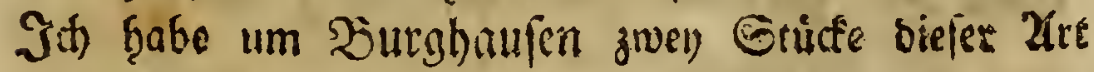
gefunben, bavon bas eine gerabe fo war, wie Sie Shleen Rafer beffifreiben; bas antere war burdiaus fell tupferfarben (meine vas. 5.) ors gnfrigius Mel. 
Frifchii uno bielleidt aud) Vitis geţơren f̧ief̧er. ") allo bie farbe fann fein fideres Rennjcid)en fenn.

Satcharting glaubt, baß ber unbedectie Tbeil bes 2ffters immer mit oen Decfen gleidfärbig fen: Daran ḩat aber Şerb/t mit Brunbe gejmeifelt, roie mid) aud meine var. I. vom (Jiegentheile ùberzeugt. Dennod) trift Jjerbft's $\mathfrak{B e f h a u p t i n g , ~ O a ß \beta ~ e E ~ b e i f f e n ~}$ múffe, biefer Theil fen allzeit mit bem $\mathfrak{B}$ ruffechiloe gleidffärbigt, bei) meinen Rifecrn nod) weniger ju: benn alle meine Exemplare mit gelbbràunlidten Jlugels. becten fraben auch) folche Zffter, obrobgl bie Brundfarbe bes $\mathfrak{B}$ rufffdilbes, balo grưnliajt, balb bläulidyt ift, bie

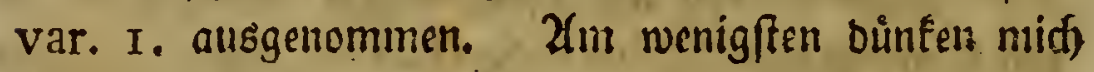

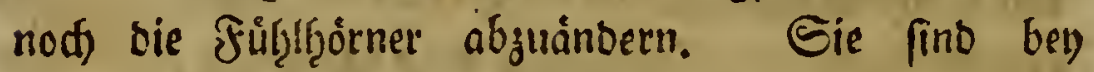
allen meinen Exemplaten allyeit lidtbriunlids, zuweilcn etrase mit grün gemiifd)t: Die Rolbdjen' oabon allgeit bunfelbraun, wenn bie Decfen lid)tbraun fins, - uno Dunfelgrün, remu die Decfen grü oder blau find.

Bey aller Muxḩe, bie id mir gegeben b̧ave, war's mir bisher nod) nidft móglid) cin eingiges juber.

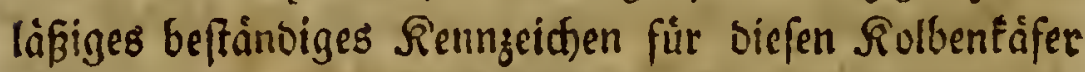
zu finton. Bermutblid weroen Gic aud in Grorcm Nat)trage jum Deftert. InfeEtenverj. von biefent Sna fefte frredjen. Danfen wirb ३̧̧̧nen jeber Entomologe, wenn Sic fierinnen glúctitid)er fino, nls idf.

2fber horen Sic einmal, was wirb erft aus ben Mel. Frifchii werben, menn Sie auf Jģrer Bef̧nup:

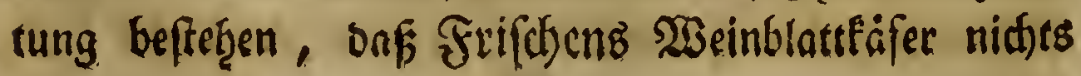




\section{Bcli, Den 15. gånn. I 784 . I 85}

uno wieber nidfts als ein Scar. horticola L. fen. Das f̧aben Sic in Sţrer Entom. p. I2. gețan, unb in einem Sriefe an mid), als id Slbnen meinen

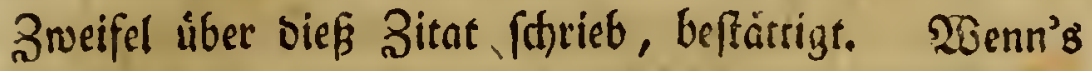
fo wáre, fo muspre die Mel. Frifchii ans bem Sifteme

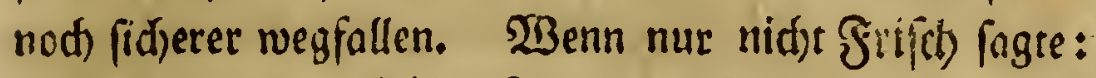
sie Dectén feiner $\Re$ âfer feneu mit Brun untermengt: eine Eigenfdaft, bie idy an feinem Scar. horticola L. woḩl aber an vielen Exemplaren bes Scar. dubius bes

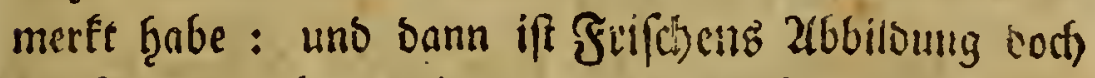

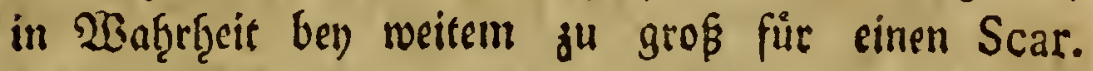
horticola L.

Sd) weißr gar nidft, wie Paft. Siocze in bem

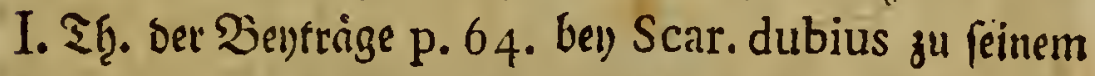
fonberbaren fseb̧ler getommen ift. Er führte eine Etelle aus ber Ent. carniol. an, Davoun bie leģte

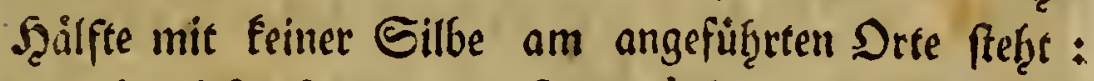
vermóg biefer fefeft er ben Scar. dubius unter bie ger

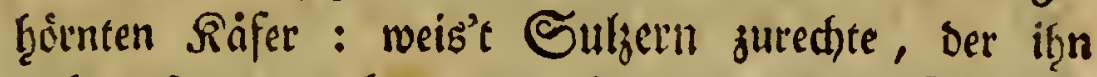
unberwafnet angab: und nimmt bennod) Sulzer's Sigur - und in ben Beridtigungen des 1. Bandes p. I 8. Der $\mathfrak{B u r r e b e}$ zum II. $\mathfrak{B}$. - ben $\mathfrak{Z}$ ontît Foenifex americanus als Sinonimon an.

28. Scarabaeus (Melol.) horticola, Der Garten תolbenlefifer.

Mag. p.377. n. 23. p. I 37. Seckft. tab. I 9. f.23.

Eefrr gemein auf allen Báumen uno Pfanzen.

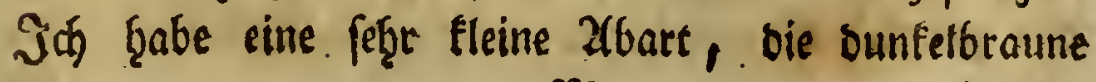
$\mathfrak{M} 5$

Decfen 
Decten hat, und üteraus pelgidft iff. Stepoli's adiaphorus gef̧̧ort rit)sig bieb̨er: id bin ourd) oie aboiloung Deffelben ùbergengt.

29.Scarabaeus (Melol.) argenteus. Der fifbertic

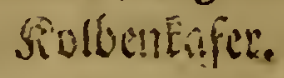

Enumerat n. I3. farinofus. Saichatt. p. 42 . n. 7 . Magaj. p. 370 . n.20. farinofus. p. 263. p. 309 . feq.

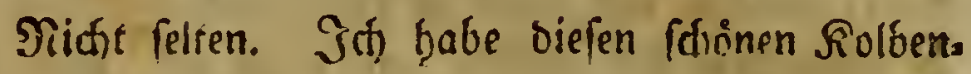
Eáfer im Nagagine unter som Namen farinofus, fo wie Sic in oer Enumerat. aufgefüfrzt, aber, ndjoem,

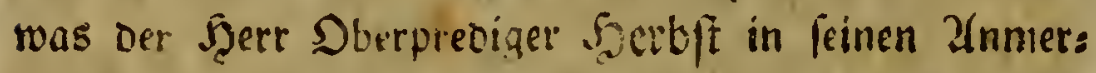

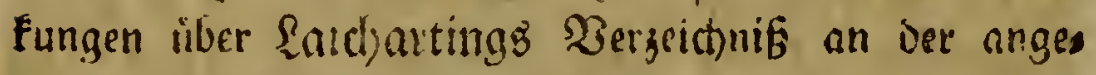
fribrten Stelle bes Magajins lagt, gloube id) nirtlict) felbit, Daßz wir ben maţren Scar. farinofus L. vera

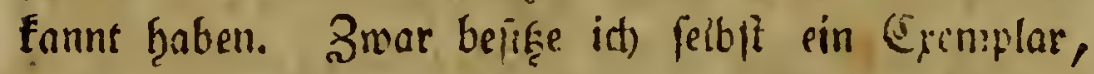
Daz auf foiner ganzen Döerffict)e ben Eraub, bis suf etroelche Punfre, verlolicen ḩat, und am ganizen Leibe, auch bie Juiffe niche aufgenommen, fowars iff; ein

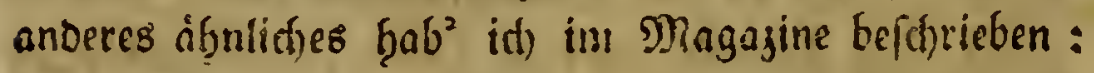
alber vermutblid fint beefs nur fegr feltme 2iborten bes argenteus, Da im Begeritgeil sicerbft foinen farinofus

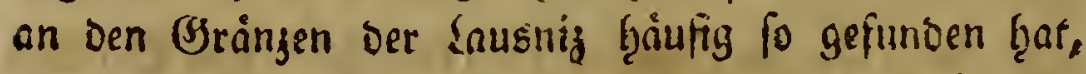
wie er if̧n im Magajine befdreibt. Ţक ţabe alle meine Ráfer von biefer $2(\mathfrak{r t}$ unter ein $\mathfrak{B}$ rinberfd)es Miftroftop gebradt); - uno ḩabe auf allen perlmutter. artige Ethuippdsen gefunden : auf einem Exemplare, bas jiemlid abgewifdst iff, zeigte fid Etaub, - aber 
idf bemerfte gena!ı, Daß̉ es nur bie $\mathfrak{B r u d f f f i c f e}$ von

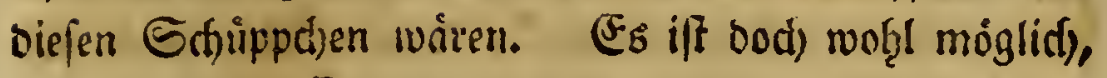

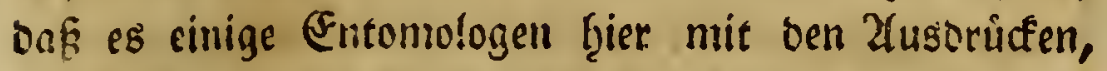
Staub uno Sclsippditen to gar genau nid)t genom. men ţaben. 2ulle meine Ráfer ţaben nur eine Rlaue an oen Șinterfiffen.

\section{Scarabaeus (Melol.) Fuefslini. Fuc $\mathfrak{F}$ li'z Rolbenéffer.} Mag. p. 376. n. 2 r.

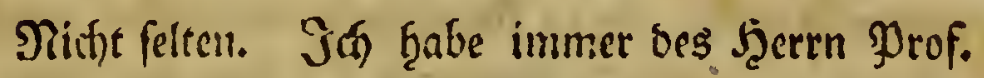
Finbrizins Melol. pulverulenta um fo melir fur bie Fen Raffer gef̧alten, weil biefer verbiente Encumologe felbft bie Sulgerfit)e Sigur beffelben anfübst, aber

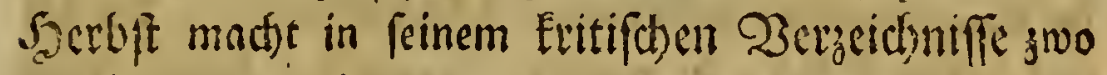
abgefonderte, obwof̧l feţr berwande Zaten baraus. Es ift fo leidjt nitst, für biefen Rolbenfififer gang ges wiffe Linterfochoungzzeichen in Bergleichung mit bem vorigen zul géen. Zlber. er ift alljeit ein gutes flei. nes, folmaler, glatter, uno nie fo ftarf gefduppt, wie Der filberne, wie dieß auá bie Eulzerfite Finur zeigt. Don bie eingeine lange Flaue an ben f̧interfüffen mud Der firberne Rolbenfäfer Gabe, gabe id) eben gefagt: alfo Earafterifirt if̧n Dont's গిamen unungulus frey. lid) nidst, wie midf \anjer ganz ridttig erinnert. Dennect) fann ids mids nod) nidse enffchlieffen, biefen Rollentiffer, wie mir mein lieber freund in Dem nemlidion Briefe ju berveifen fudjt, blop für eine. Ilbart bes Scar. argenteus z̧u ḩalten. Unter fo vies len Rilipern biefer 2 drt , bie iff sefangen ţabe, fam 


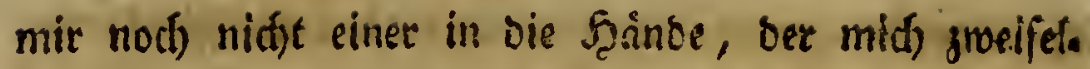

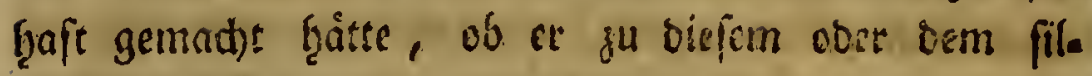
bernen geţơre.

Uebrigens ḩafe id) Sitticers Beḩauptung p. I 8. ber (J)eftid)te : "Daf bie einzelne Slaue an ben Jinter. firfen ben meiften Eroféfern, bie auf Slumen wohnen, gemein $(E)$, , an meinen Rolbentäfern biefer uno ber folgenben Jamilien nithe beprittigt gefunten.

Run wären auds meine Rolbenf́fifer von ber britten framilie alle. Piod) frabe ich weber ben Scarabaeus (Melol.) agricola L. ben id) aud) mit Shluen fứr Seped)inz crucifer Galte, noch ben 20 alter $=$ Solbenfáfer Scarab. (Melol.) Fulla L. in Galzburgithen gefunden. Die Exemplate, bie id von biejen $\Re a j e r n$ in meiner Sammlung babe, babe id) meines lieben Betters von Saichartimg's Bjüte zu DanÉen.

\section{Bierte Samilie.}

Sdhirmblumen = Rottentiffer. (Trichius F.) 3I. Scarabaeus (Trich.) Eremita. Der Einficolers SRolbenÉáfer.

Mag. p. 374. n. I 3 .

Diefer anfegrnlid)e Rolbenfäfer - ber Şerfules unter ben unberwafneten, if bey uns feltner, als in Defterreinh, wo ith iţn ofter gefunben f̧abe. Sith. folge fier mit waferer Ueberzeugung bem J̧errn von

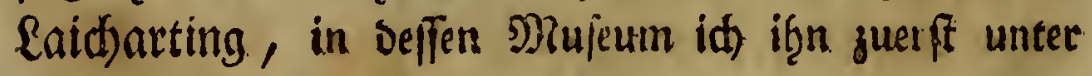
biefe familie geotonet fand, in Der er meines Biea 
bünfens, Feinem Şabitus nad), fo wie ber folgente, una gleid) naturtidfer ftef̧t, als unfer ben Mietallétáfern (Cetonia F.)

Zan (jiroffe ànbert er siemlich $a b:$ id) befige ein Eremplar, das etwas mefir als bloße 2fbart feyn

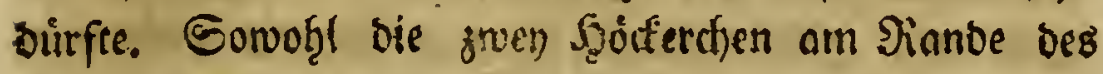

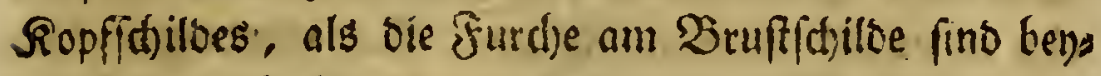
naţe unmerflich baran: nur gmo Errfioflungen weroe - idf an ber Grefle geroafe, wo aud) bie Jurde an ben anbern etwas erfiohter ift, wie Stopoli fręr genau

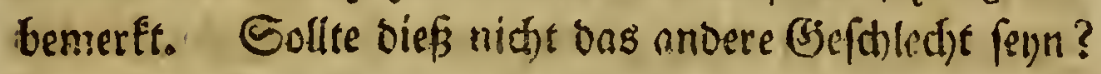
Daju glaube id) nod) ju bemerfen, on ob ber affer etwas hyalbfegelförmig verlángert ifr, ba er bel) ben nnoern

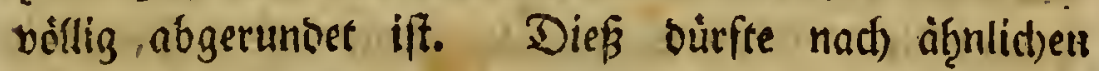
Bevipieten an viclen andern Jinfetten, jum Bef̧ufe bes Eierlegens feinn.

Brưnglänzeno fino id if̨n beym Eeitwerts: anfegen:nie, nie $230 n t$ - and nie zanj fdrwar, wie Sfopdi - inmer etwas feeller ober sunfler fupfer. fárbidt. Die Etopolifate abbiloung ift gut.

32. Scarabaeus (Trich.) nobilis. Der cole Rolbenkifer.

Enumerati n. 14, auratus. Mag. p. 375. n. 18。

Diejer fdjóne Rolbenfáfer ift um Galjburg fel. ten, um Nattfec uno Saufen gemeiner - aud ţabe id fgier sinige nuf Sambucus ebulùs L. gefunden.

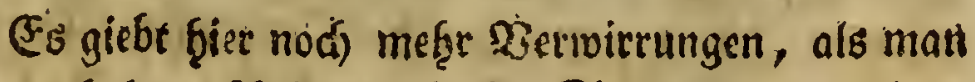
bafür ju bुalten fajeint. saffen Sic mid) verfuct)en, 


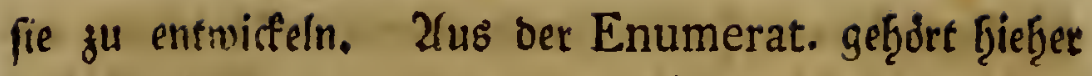
nic)t nobilis. n. I 5. - Der miro in ber folgenten Familie jeinen $\mathfrak{P l n}$ cr erbalten, - fondern auratus. n. 14.- Eonberbar, Duirfte irgend ein Entomologe fagen, hat nid)t Jeerr Sdirank felbft an \anjern gen fdrieben, ,fein auratus fen mit $\mathfrak{S}$ ont's anthocantharo viridi - corufco (aurato L.) aflersings einer. lev)." Lefen Sic cinmal 6.20. bie Slote F̧errn \ુan= zer's zu OJontż igneus. - - Darat: fiaben Sie gang red)t, mein lieber Freuns ! - alver nun will id Sl)nen nudh zu lefen geben, was f̧err Sdyrant ben 2 Dec. 1782. an mid) fdrieb. "Mein Scarabaeus auratus if weber Scar. auratus L. noct) fein Scar.

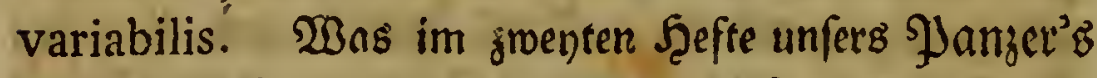
ferbt, fdrieb id) if̧m vom sanbe; entfernt von meiner Naturalien: Sammlung : es betrog mich abẹt mein (Beodicftnif : - mein Scar. auratus gef̧orrt in bie Battung Trichius \&aithart. - Diefer Belef̧rte ḩat

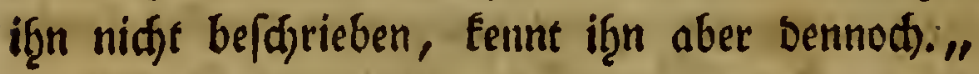

Diefe Etelle aus Grbrem Briefe if mit ref̧e widłtig. Eie bilft alle meine ミermutf̧ungen beftảrfen.

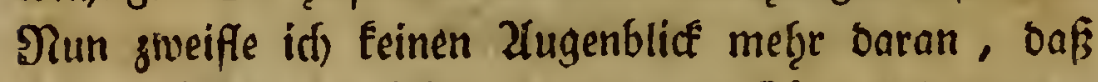
mein nobilis, uno $\mathcal{S}() \mathrm{r}$ auratus ein Råfer fino; bern auth mein nobilis ift ein Trichius: er hat Umrißß unv alle Rennzeid)en, bie Şerr von said)arting zu einem SdjirmblumenEafer forbert. Bennabe follte it glaúen, Janizet ḩabe den wafren Nobil. L: niche gefannt, ba er fagt, bey der Sontichen Jigur bef-

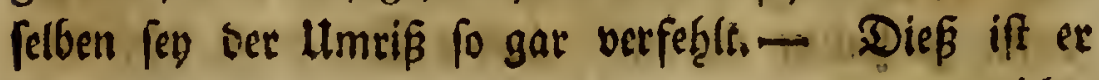
nichr. 
nidft. - Die Zontfche Sigur ift zu ffimal, uno zu linglid)t - aber fie ḩat den Habitus eines Trichius; -

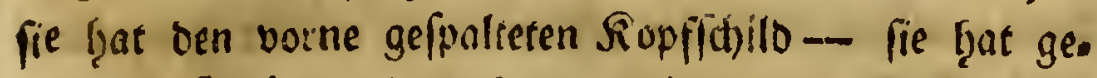
rate bie Sarbe, bie metgrere meiner eblen Rolbenfåfer traben; oenn fie tommen fefre oft unbemafelt yor.

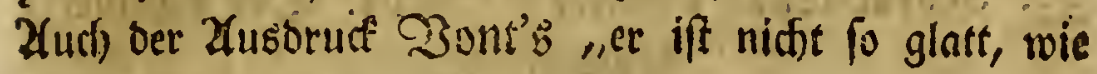
Die meiften anvern Blumentéfer - ift gang bezeid). nent. 23ont wollte baourch Fingen: or ift runglicteer, nif(t) fo abgefdiffen, wie Scar. variabilis, auratus fticticus \&c. \&c. Fribrizius une Fueffit diriten midf) aljo mit gutem (Srunte, Sont's igneum an.

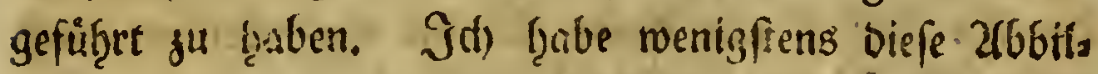
oung, of̣ne nofi) Den Tert getefen zu baben, benm er. ften Ifrblicfe für nobil. L. erfannt.

Tiidtig Eennt Saichnating biefen Rifer, wie Gie fagen : aud er fonnt ig̨n als einen Trichius - fint ingn weder unter Cet. aurata, nod) unter Cet. ftiEtiça begriffen, - uno oarinn binft mid), b̧aben Sie

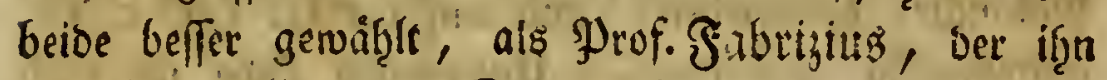
zur Ceton. 'matst. So viel hiver das Bitat aus ber Enum. - Die Bửe ber Sortichan 26bilkung uno bie "Etellung biefes Solbentäfers im Eiftente.

SEopoli'z nobilis geffort ber Befdreifung nach ungezmeifelt hithber; aber bie abbilbung if fafled) - fie bat volltommen ben lumtis bes unbe. fantinigen Scarab. (Ceto.) variabilis L. Die నífet fommen ţier balo bemntelt, balo unbemafelt - unb Dann fenerrotg ober grinlichte vor. Die Mafeln fino gelbgraulidt, to mie Soffitein. \Janzer ţat b̧ierủber 
eine artige Xnmerfung gemiad)t: id) befif̧e ein Elem: plar mit einem Slümpdhen foldher Erbe am after. Das nemlidje beobad)te id) an einem meiner (Einfieblers Rutbentéáer.

33. Scarabaeus (Trich.) fafciatus. Der bandierte Solbenéáfer.

$$
\text { Miag. p. 375. n. I } 9 \text {. }
$$

Die Stopoliface abbilbung gut: bie ßontifhe fệr mittelmáfig. Eie ḩat anftutt eines gefpalteten Ropfichilbes waf̧re Zangen (maxillas). aber warum

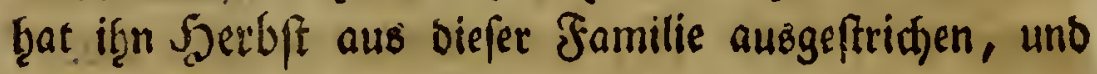
unter bie Melol. gefeget?

34. Scarabaeus (Trichius) hemipterus. Der Sanlboecten = Roltenentifer:

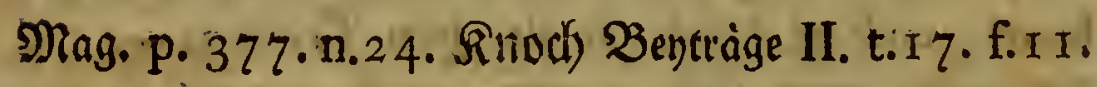
Nicht gemein. Sfopolf ḩat gror nur sas Mänudjen befdirieben, uns auf ber, II. Taf. f. 28 . mittelmápig abgetilloer. Unterbeffen finbe id) bod) nuf ber I. Taf. zwifhen f. 7 . unb 8. bie albbilloung

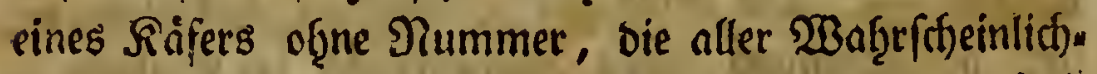
feit nad) nidfte anbers als bas $\mathfrak{W}$ eibdjen gegenmártigen Rolbenteáfers vorftellen fann. Der $\mathfrak{L} m \mathrm{mi}$, bie

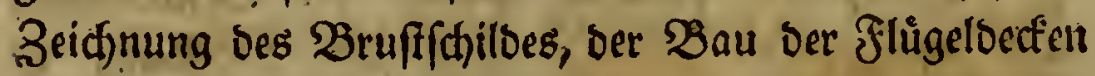
unb ber Etadjel am 2(frer madjen mid es vermutţen. Warum biefe Figur Eeine Numer frat - und wie fie in bie Safein fam - Da SEapoli gar nidtes bavon fagt, ons merben bicjenigen, entfdeiben, bie ùber sas Edjidffal biefer. Tafeln náţer unterridjtet fino. 
Simfte Familie.

\section{Metall=Rolbenkáfer. (Cetonia F.)}

35. Scarabaeus (Ceto.) variabilis. Der unbes ftándige Siolbentáfer.

Mag. p. 3 I I. 375. n. I6.398. Enum. Inf. Auft. n. $15 \cdot$ nobilis.

Eef̧r gemein. In ظetref ber Bitate berufe id mid) ganz auf bie angefuibrten Etellen Des Magazines. İd, glaube, wir find Şerrn Fucfili Dante, fefor vielen Daint safür fdulbig, baß er bie Nierwirrungen, ben viefem, und dem folgenoen siäfer fo treffeno auses

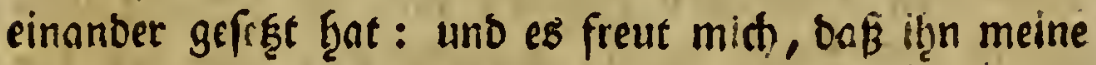
2limeifurigen ju unfers \anzer's 2lusgabe Dont'B saju veran!abt ḩabell.

Duß $\mathfrak{g h r}$ nobilis Gieg̨er geţơre, bezengt mir

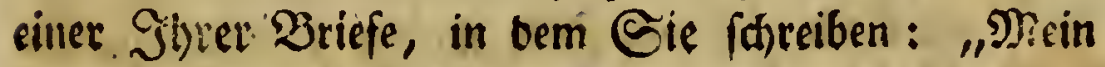
Scar. nobilis ift Cet. aurata Saicharting - " anthocant. Viridicor. \Jont. Scar. variabilis \anzer, wohin ficher oud) anthoc. metallicus aeneus geţơrf, Der aber von. Scar. Aticticus fimmelmeit unteridjieben

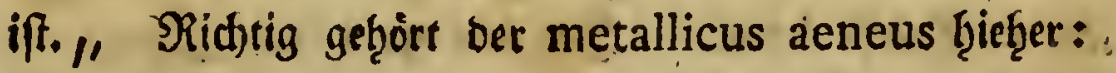
man vergleide if̧n nur mit ben befllyrünen, unten fupferrotben fleinern 2 Irfen, urio man miro fternum porrectum, uns fegmentum fecundum abdominis mobile, utrinque dente terminatum, benbes nad \&aich)arting Şauptfennzeidjen diefes Sivibentäfers geo nau findert. Wollte man bie Adbrtzelluug nash Şerbit P. 3 I I - 3 I 5. Der Mag. matjen, fo múrbe ber me- 
tallicus aeneus ju feiner leften 3 frt Cet. metallica

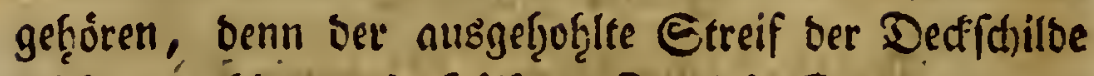
reidst nú bis an bie Shalffe. - Zu biefer Cet. metallica

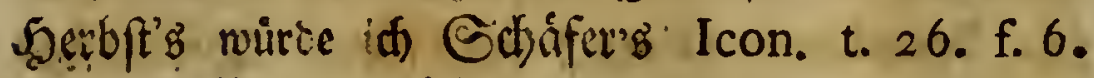

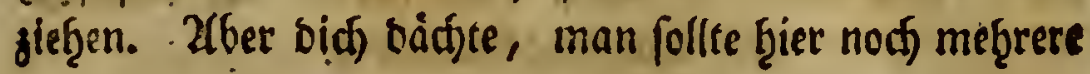
Unterarten madjen.

36. Scarabaeus (Cet.) fticticus. Der weife flectigte Rutbenkiffer.

Mag. p. 375 . n. 17. p. 39 I. 400.

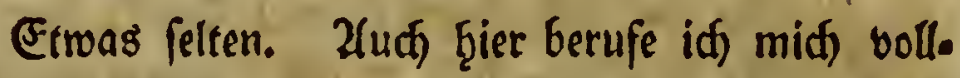
formmen auf bie angefüftrten Ereflen int Magagine. Ilus allen bisherigen Siguren find bie $\mathfrak{B}$ rufffafillog. punfte auf feiner fo gut auggebruxidit, wie auf ber

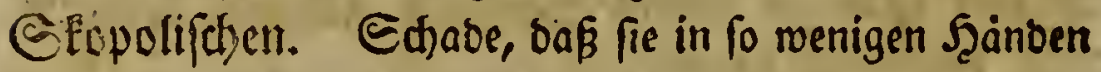

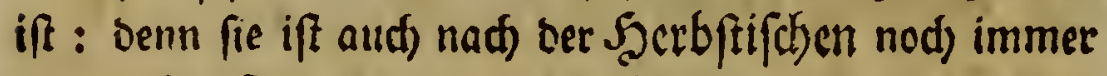
bie trefentife.

J̧ier bin id am Biele meiner entomologifften Briefe - aber nur ber entomologilften. Denn, traun: id) habe Silmen fdon mieber ein \$äfdicn Edjriften von gar fonderbarem Infalte aus meinem Sdireib. pulfe zugedactit - Die follen Gie in einem פaare fols

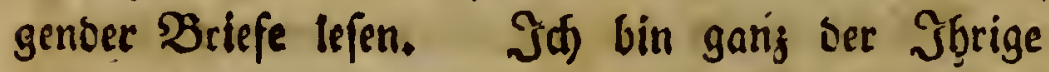

\section{Moll.}

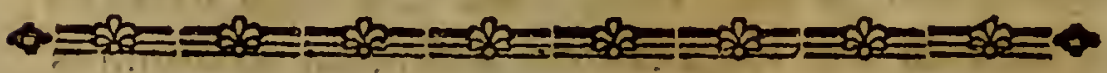 \\ Bilfter Brief. \\ Burghaulfun, ben 5. Sanun: r784.}

Ficife nad) Safjburg. Papilio Lucilla. (Jeffílts 


\section{Şurghaufen, Den 5. Jånn. I784. I95}

lițe Bicbinde in Sarburg. Dentwirbigleiten in Entghurg. Der Sapuinerberg. Sicife von Saliss

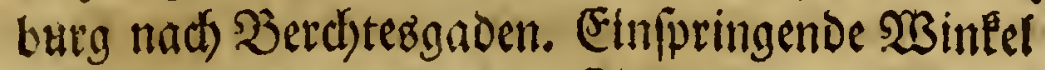
Der Berge. Heber die Syjfeme von Ent: fethung der jegigen $\mathfrak{W}$ clt.

(Gitwas fpat erfiflle id) mein Berfprecten, Dos ids Glynen im norigen November getf̧an bzatte, ba Eie mir die Ețre ermiefen, mid) bon Ealjburg naw Burghaufen ju begleiten, umi on ber Befidtigung meiner Tiafuralienfammilung einige Erunben zu fiten. Een. 2(ber wiffen Cie wob̨l, baß̧ id mid) an bem

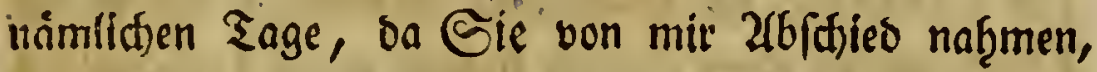
ter Pafete und Briefe ungead)et, bie wir auf meinem כ̄immer antrafen, eine fleine Sieife vornaf̧m? So

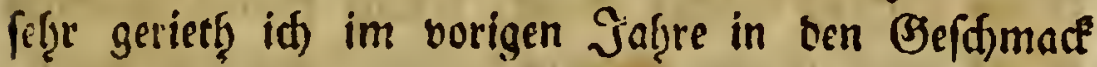

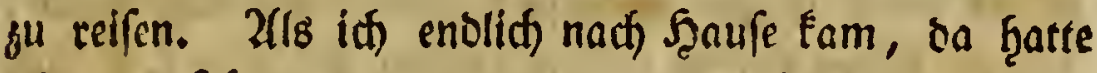

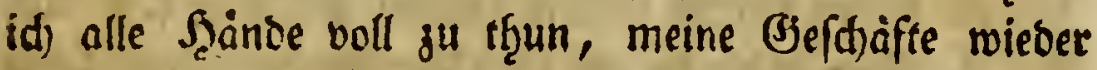
in Dronung zu bringen, und alle bie Briefe ju be. antworten, bie faton fo lange da lagen. Nun aber enoliti) follen Sie alles lefen, was id) auf meiner Reife nac) Berctresgaben beobadtet ţabe.

It) reifete ben 27. Brad)mon, vorigen Jaf̨rs von $\mathfrak{B u r g h a u f e n ~} a b$. Nein $\mathfrak{B}$ eg gieng. úber Titt: manning, Salfen und Ealzburg, oḩne daßs id bis caf̧in erwas feţre merfmúroiges gefuncen haătte. Die PHanjen, die an ber Etraffe mudten, waren alle gang

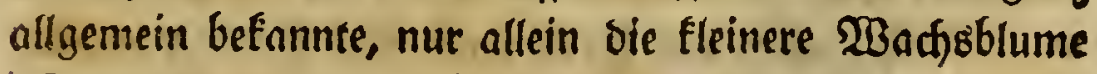
(Cerinthe minor L.) ḩatte ist) um meine Biegeno bुetum nod) nidft gefunden, bie aber úber Plattenberg

Ginaus 


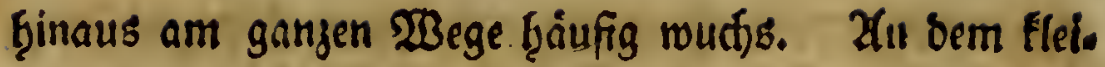
nen Fluffe, ungefáb̨r elne. Etunte vor Ealzburg, ben man mir bie Gaale nannte, glanbe ich ben Papilio Lucilla bes $\mathfrak{B}$ ienerverzeictniffes gefefenen zu ḩaben. (E) flog bid)t am 23agen vorbey, uno ungendetet id alfobalb ausftieg, iḩn zu b̧afden, fo war er bennods fdon ganz aus meinem (Sifidite. Diefer Ed)metter: ling ift eben ber, weldyen Gulzer in feiner Befdidfte auf oer $18 \mathrm{ken}$. Faiel fig. 2. 3. unter Dem Namen Papilio Nymph. phalerat. Leucothoë nbgebiltet bat.

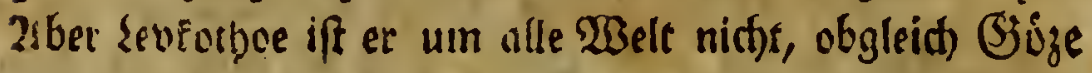
uñ Fabrigins oie Guljerifds abbilouniz ben Papil. Leucothöe, oser, wie if̧n lezterer nennt, Leucothea, angrführet baben. Engt bođ) Simné beutlid): alisfupra fufcis, fubtus luteis, fafciis tribus macularibus albis nigro-notatis; Fabriziub fagt fogar: alis - fupra fufcis fafciis tribus albis u. f. f. roie \&inne. 230 fino nun auf oer Dberfeite ber Eulzerfotyen Edymatserfinge bie bren weiffen Binden ? 2(ud) auf ber Unterfeite, no fino fie? man müpte nur ben fthmalen reiffen Dueerftreif, ber grwifchen ser exfen uno jwenten Bittoe auf ber Unterfeite ber Şinterflügel Gjizláuft, eine Binde geiffen; aber bann fino gar vier Binden sa : benn bie monoformigen Mafeln ùber bem Sinterranbe beroienen wohl eben fo gut biefen Namen. Llno wo bleibt in Der angefinfrten abbilbung Das nigro notatis? Die ffiattenbraunen 21bern bes flugels laufen mojl ourd), aber bas beibt fonft bei) Rimté venofis, Fier wưroe es nigrovenofis beiffen; ober bzătte sinné nuf ole fdfmárzlid)te Einfaffung oer flecten geferten, fo 
wuirbe er nigro marginatis gefagt babern.- 2lber

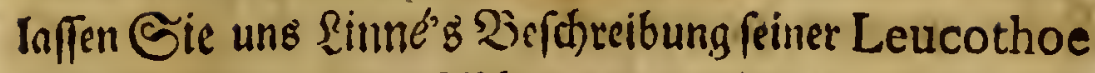
mit ber Sulgerifthen 2(b6illoung jufammen f̧alten: Gier

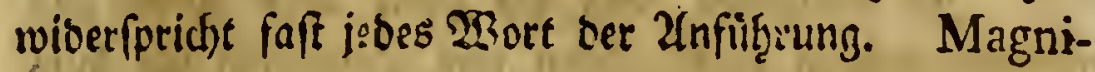
tudo Papilionis urticae : cas modte hingabert. Alac utrinque fafciis tribus transverfis : nun bas finoe id ben Sulzer waḩríd nidjt. Coëuntibus verfus angulum exteriorem primorum : id) musite nid)t Satein verftefgen, wenn ith dieß benm Gufjer. finoen follte. Fafcia prima in primoribus nigro interrupta : ia, ber länglidte Streif, ber aus bom Srrunbe bes fluigels fómmt, aber bas ift feine Fafcia, fo byat fid) Sinné fonft nirgenos ausgeorúcft; zwar

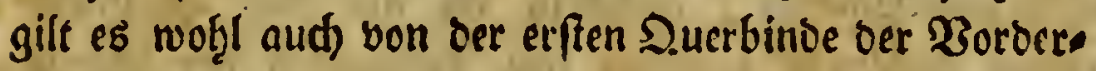
fligel, aber was iff bann fafcia fecunda uno tertia? Secunda per pofticas et primores utrinque linea nigra notata: bas finbe id nun abermal ouf oer er. ften, nidft jmenten, Duerbinde ber Dherfluggel, unb gwar nicht utrinque, fondern nur auf ber Sberfeite. Tertia in pofticis ex maculis fe tem albis : biefe fieben Mafeln fino mof̧l ba, aber mact,eu nidit bie britte, fondern bie grente und lezte $\mathfrak{B}$ inde aus. Puncto nigro notatis, int bilbe mir nuf mein gutes Erfid)t was redfers ein, aber biefe fdiwarzen PJunfte (bie burd)laufenden abern mogen Stridpe, Lineolae, Ve-

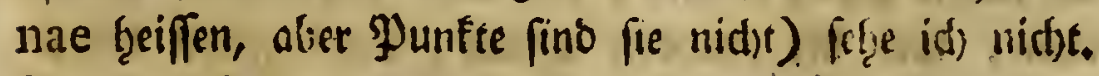
Dagegen ftellet ber Eulzerfde Ed)metterling bie Lucilla, welde id in sinz bey Şerrn Sinth E differs miller gefehen fabe, uno weldse id) jejt surd bie

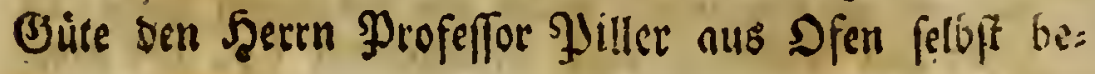


fige, fo volfeommen vor, buß es unmogglid iff, bie. Natur getreuer zu Eopiren.

In Ealgburg, das iđ grwar eben nidjt bns exffemal gefêten ḩatte, fielen mir gleid)roofil bie lotha. redten Bergmainbe auf, neben weldsen man sleid, wenn man in bie Etadt frineinfómmt, Gerfáf̧rt, unb. bie anbern, welthe man liber ber Salza am Rapusiner. berge fief̧t. Id) fann es niemal begreifen, wie man

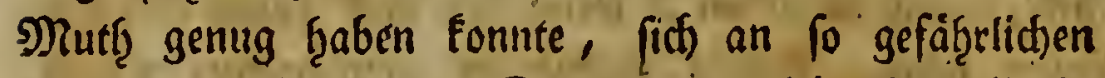
Etellen anjubauen, an Etellen, wo nid)t bie geringfte Böfdung bem ruthimentig betorftebenoen (sinfturze bes Berges wiberftebc. Mieine Beforgniß hatte nut nod) jugenommen, nactioem id auf ben Berchtes. gasner Bebirgen zmildyen oen Srimmern ber Berge bon biel fefterm Beftein heerungeftiegen ıar. "MBirflid Soraz hat red)t; wir fino

Audax Japeti genus; Audax omnia perpeti. (*)

2lls in) vor einigen Jaf̧ren zu Ente bes', Wein. monatḩs eine fleine Jieife b̧ieģer getḩan ḩatte, befah ich bas ftoone Mirabell, uno oie fitơnen Bàrten ḩerum, fạ bie groffen prädtrigen Dranienbáume in ib̧rem Winterquartiere, aber bod) in ben Boben gefeset, ber von unten gef̧eijet miro; futh das angentef̧me f̧elles brunn, uno bie, fremben Tģiere, bie bafelbft unter: f̧alten

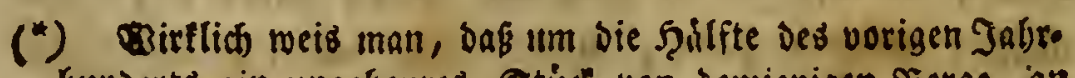

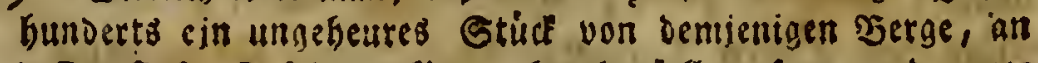
Deffen. Fus Enfjburg liegt, berabgejallen fen, unto ganze Gafien be sraberl Gabe. Le grand thẹatre hiltorique Tom.5. 


\section{Şurghaufen, Den 5. Jănn. I 784 .}

Dalten werben, fah bie Bögelfammilung bes f̧errm Grafen von Sirmian in ser secporbsfrotie, fah etien Dafelbft bie foftuare Pottrátenfanming beriftemter Minler, bie bielleid)t eine ter bollftantiften in bieferm

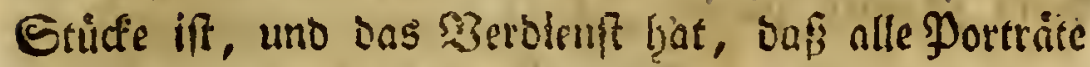
von ben Riuftlern, sie fie vurfitellen, felbfit berfertiget worben. 2lbet bie Jahzrsjeit, uns oie Umfrànoe, uniter weld)en idf biés alles fals, erlanbten mir nidfte einen

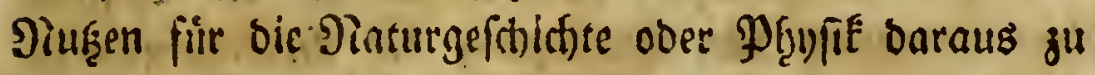
giegen. Nut auf Dem Niunnberge fanmelte id) einige PFlanzen, bie aber nidjt unter bie feltmen geţơren, felb/t ber noch) blütenente Thymus acinos, ber bafelbft auf altem (Bemaiuer f̧áufig wåkst, war mir nid)t ul. stwartet; id) hatte if̨n um Bobaun in Unterófterreich) in viel grófern Entfernungen vom eigentliden (B). birge fajon gefunben.

Эđ) weis aber bennod), bap bie (Gegeno um Enljburg, befonters ber Sapuginerberg, an feltenen 2(lpenpflanjen reid) (el). Jd) wollte onf̧̧er, als id gegen bie Mitte bes Eeptembers jum juentenmale. vad Serd)tesgaben reifete, und Frühgereitig genug in Ealgfurg anfam, bafelbft heerborifiren. Zllein man wies mich an ben Sterrn Commentanten an, bon bem id) ein Billet haben múfte, wenn ich in siciem Sibalo

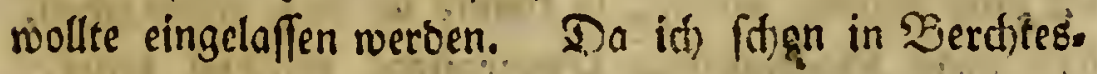
gaben bie Ȩ̣re gef̧abt f̧atte, biefem Fecrrn befaint ju werben, fo waire es mir allerbings ein leidtetes geweeren, siefe Beoingniß zn erfuillen, wenn nsir ber fafjon fin: fenbe Tag Den Gebraudf babon nidje felbft unnüg ges

$$
\mathfrak{N}_{4}
$$

mad)t 


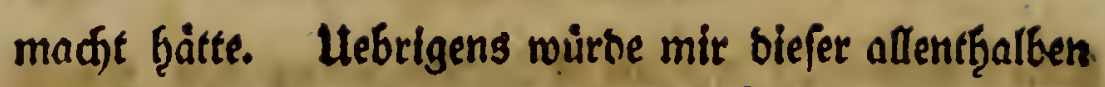
fren bafteģente, eben nidft b̧ohe $\mathfrak{B e r g}$ mit feinen Zlpenpflangen einen neuen Semeis für meine Şypos thefe über bie Zllpenpflangen im flactjen sande an bie Şand gegeben baben.

Bon Ealjburg aus reifete id faft beftanbig am Unterfperge b̧in, einer ungef̧euren Marmormaffe, bie allein finreid)end ware, bas Romantifd)e ber Bufffon. iden Mennung von Entfteḩung ber Marmorgebirge. Darguthum, wenn wir nid)e iezt nod) toeit überzengenDere Ėrübe b̨ătten, fie als gemiß oer Erfab̨rung zumiberlaufeno zu erflàren. Baiffon felbft froeint jegt ganz anders zu benfen. Wenigftens meil iff) es

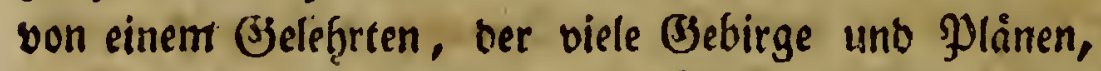
auch in De Suc'B (Befellfd)aft, bereifet, und bem Brafen

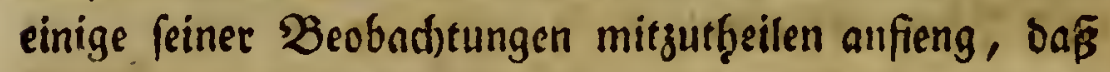
Búffion biefelben nid)t hioren wellte, weil er, wise ex fagte, zu vieles gefdrrieben b̧abe, bas er mun gans abánbern múfte, moju er aber nidjt meģr Riâfte. genug ḩâtte.

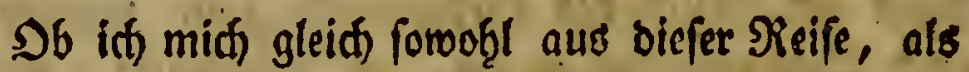
aud) auf allen meinen folgenben Reifen surd) bie ₹f̧äler uno (Stebirge Berdtresgaben forgfältig nach) bek einfpringenten $\mathfrak{B i n f e l n}$ ber Bebirge, zmifhen reldjen id mid befano, umfarg, fo fornte id bod) nidts fefgen,

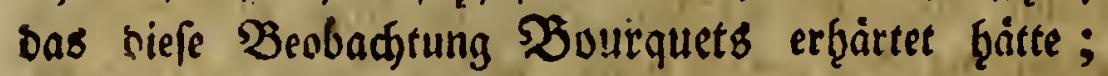

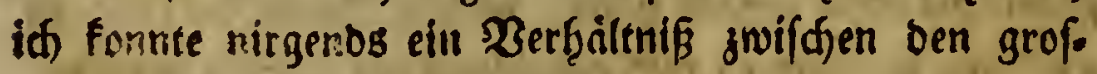
fen Thâalern, uno den Borfprüngen ber gegenüber. liegenden USeburge entoecfen. , Wenn man einmal 


\section{SBurgbaufen, den 5. Jänn. 1784. 201}

ùber bie Ecfe bes Unterfperges herum ift; fo fiat

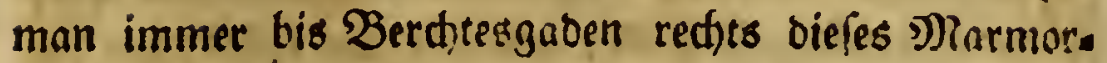
gebirg : linfs die Berditesgatenfthen Relfffeingebirge. Je weiter man fómmt, beftomef̧r entfernt fidf erfiteres, und beftomeģr náţern fich lejsere, weld)e enolid) am Sönigfee nud) auff̧óren, um jenfeits befferben auf ein, mal miever fortzufegen, babey aber immer fortfof̧rent, fid) bon bem Unterfperge zu entfernen, ser feirle Branze an ber Erraffe fat, ourd) bie man von Berch.

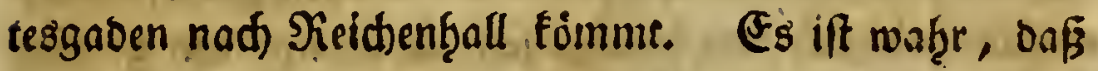
biefes legte Gobirg nur einen 266 fprung ju madjen fojeine, um in einer etros einwairts geféę̧rten Shich. tung, aber mefir jurùctetetenten flàd)e, uno unter anberm Namen wiever fortzufegen, uno fid enslich mit bem linfs; fortgefgenden Gebirge zu vereinigen. Daourd) entiftehen alleroings verfobieoene anfeţnlid)e, oft mit niebrigen Bergen burd)zogene Ţ̧áler (jurưffs tretende $\mathfrak{W i n f e l )}$ ), of̧ne baß man bod) an bem (Jebirge, bas biefe Thăler vorwárts uno rưfwairts fofliefit, ers

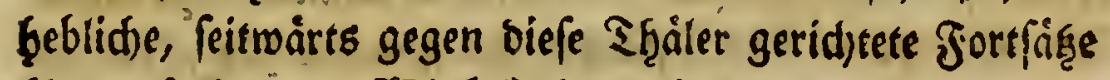
(6̧ervor(pringende $\mathfrak{B}$ infel) bemerfete.

Da id meine Reife nad) Berditesgaten bas zroentemal madte, feine anbere befellfdaft alo bies meines Mietḩutffhers hatte; uno auf bem mir fhon befannten $\mathfrak{B}$ ege nidts neues anjutreffen hofte, ver. fdilog id) mid) in mid, felbft, ftellte $\mathfrak{B e t r a d f i t u n g e n ~ a n ~}$ uher bas, was id bon ben verfidiedenen Enftemen über bie Eneftef̧ung ber jezigen $\mathfrak{W B e l t}_{\text {gelefen, uns }}$

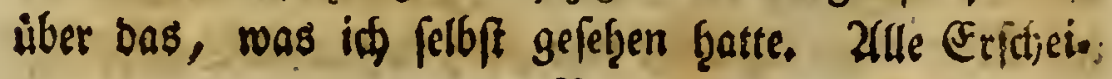

$$
\mathfrak{N} \text {. }
$$


nungen, bavon idf jum Theile felbft 2fugengeuge wat, liverwlejen midj, baß ba, wo wir jegt feftes sand has

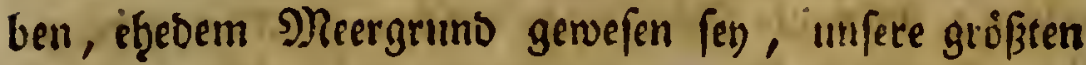
(5ebirge músten biefer Erfd)einungen zufolge unter

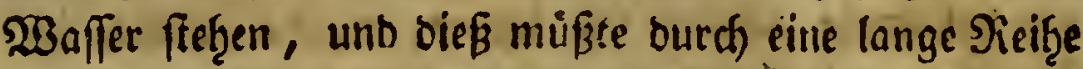
von Jaf̧ren fo fern. Bulfane müsten ba; wo rir jezt unjere 2 Beinberge Ginpflanzen, genuitţet balien, Berge aus bem $\mathfrak{Z}$ affer gef̧oben ḩaben, bie Infeln bilbeten, und oft mieder unter die $\mathfrak{B}$ ellen fimabjanten; eadict) frob fid) foas beutige fefte lanb mit ben groffen Infeln fo ziemlidh auf einmal empor, ober, roeldies eben Daf̧inauslåuft, enolid fant das Meer fo ziennlid) auf einmal in bie jezige Siefe hinab, uns alles gerterth in einem bleibenben Buftand.' Bor biefer Pievolution muiste fdion sano da getwefen fern, uns lanotbiere, Derer Rnochen man in bet Eingeweiben ber $\mathfrak{B}$ erge finbet, músten es berwof̧net ḩaben; ba ber $\mathfrak{B a u}$ ber Zähne an ben gefunberien Gerippen bie 2rênnlid)feit; felbft bie BIleithf̧eit mit unfern befannten şierarten beweifet, fo mingten Pflangen oa gerejen fenn, die fie genoffen : fo mufsten Siatte ta gewefen fenn, aus tenen

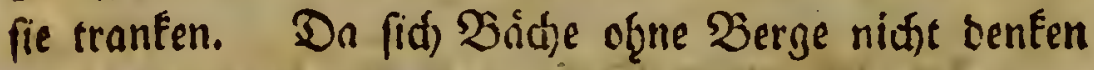
Inffen, fo muffen in ser niten $\mathfrak{B}$ elt fdon Berge ges

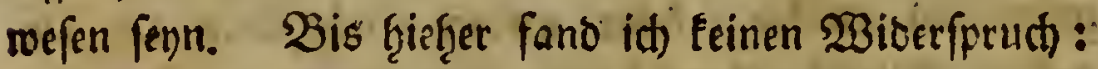
alles fdien mir ridjtige Folge; aber f̧ier fano ia aud) mein Non plus ultra, wenn i(d) mid, nid)t in Şupothefen vertiefen wollte, bie erwig J̧ypothefen blein

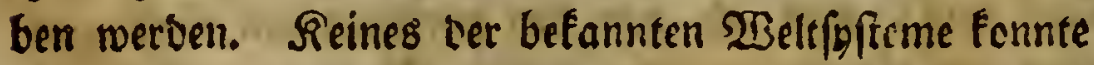
mir Sefriesigung veridaaffer. It) fano in meiner al= ten $\mathfrak{X}$ elt, menn id nidte eine ganz nagelneue Edjopfung 


\section{Burghaufin, Den 5. Jălt. 1784.203}

annef̧men wollte, eben biefelben Ş̧iere, eben biefelten

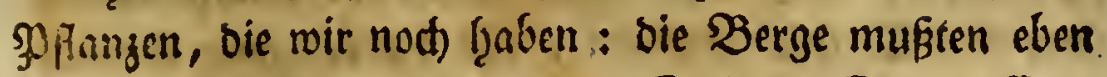
fo gut, als bie unfrigen, aus Eteinen, Eano, Eroe oajteten, bas, was wir Metalle u.f.f. nenuen, mòd)te. wobll in einer andern Bernifd)ung uno (Seftalt ba fenn, als wir es beue ju Zage hraben, aber ba muste es boch immer fenn, in ber ftarfen Ulebergeugung, Die id bon ber gẹnauen Berfettung aller. Weefen ந̧abe, mußte

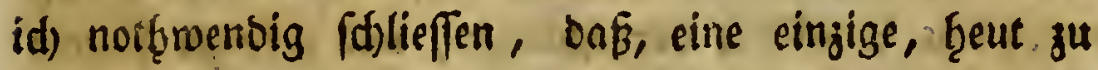
Tage befannte Ş̧ierart vorausgefaget, alle übrigent

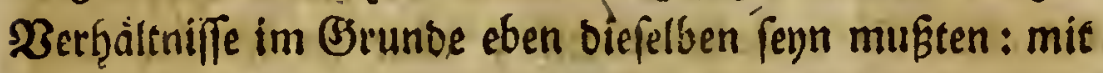

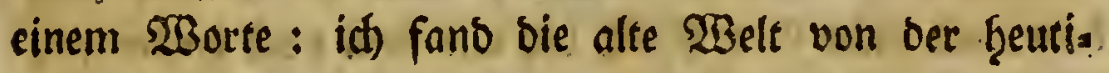
gen in feinem betradjtlidien Etirfe berfohicoen.

Mir faneinet bie Encte, aus biefem (B)efidhts. punfte betrad)tet, ridftig; und biefip vorausgejejet, was verlieren wir bann, wenn wir eingrfteţen, die $\mathfrak{B}$ elt fen. gleld) beh if̧rer Erfftifffung fo gemefen, wie fie Ģeute. ift? Warum ḩứcen wir uns benn fo angftig bom Sdjopfer ju reiven, menn bon (5efdjöpfen bie Sicbe iff? Jmmer mujesten bie Eteine, bie (Frof(d)idten eine gen wiffe Sage baben, uno warum zerbrectien mir uns Denn bie Ropfe buruber, Daß̧ fie gerabe diefe lage haben, uno bauen in oer erfigiten Einbiloung BBelten aus Siartenblättern, die jecer f̧aud) tiber ben f̧aufen witft? Sidutig ift es allerbings, baf unfere

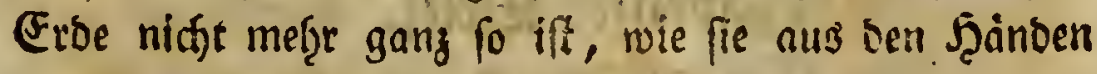
bes Edjopfers fam; allerbings girng mit ifir unter Baaffer eilue entegglidge Fevo!ution vor, und raģro fajeinlidf fältt die Epodje derjelben mit ber Eunofitut 
zuffammen; obeßs woire nun gmar eine Mutf̧maffing ilber bas 2 Sann verfében, fúr bie iff) gute Bründe beybringen fónute, aber ids merbe mid wolly ghuten,

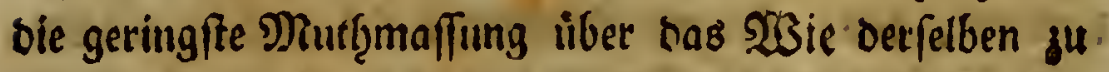
wongen, weil alle Mutḩıaffungen ùber biefen Bsegen. frand envig Sanothefén bleiben werben. Swar fofeint es ridteig, Daß́ die Eúnsflut von irgenb einer andern, nud) weit mirffamern Urfadbe begleitet worben, baF enffeğlicte Einftürze vorgegangen, und gefolget fenen ;

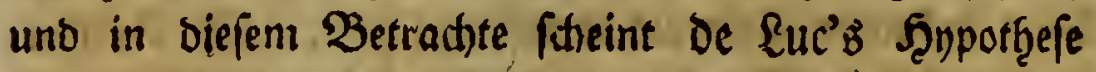
aus allen bie wabgrfdeinflidffe; aber fie iff beg alle bem nod) niddt (Serwi Die zwar alle Errfiteinungen fȩ̣r finnteid) erfläref, aber eine Urfadse vorausfegzet, bie nidft ermiefen iff. Geftegen wir es nur aufridttig, daß es in allen Dingen ein Maximum gebe, über weldhes unfere Siråfre nidfe

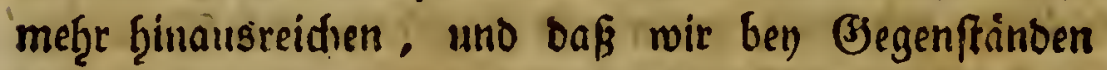
Des bloffen Borniges siefes Maximum feg̨r balb erreididen.

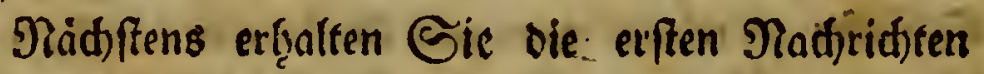

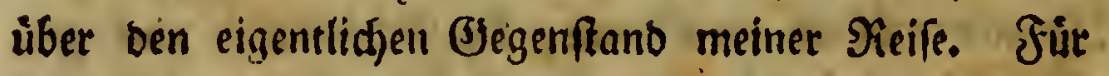

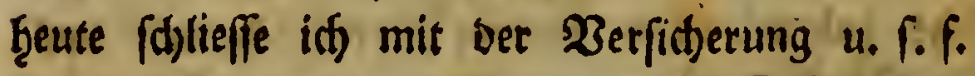

\section{Schrane.}

\section{Şwilfter ßrief.

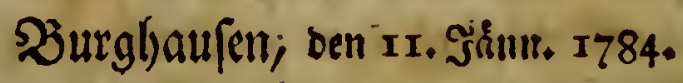

Gruno und $\mathfrak{B}$ oden von $\mathfrak{B} e r d$ teşgaden. $\quad \mathfrak{B} e r(f)=$ tesgaben. Sddofllenberg. Siamfau. Singeniclume 


\section{Şurghaufen, den I r. Jín. I784., 205}

Segenden. Eanowirth/d)aft Der Berdfteb: gaoner. \&uft. Э̧nfeEten.

$5 \int$ enn man Ealgburg eine Etrecfe weit Ģinter fich getaffen f̧at, merft man es ref̧r beutlid), Daß bie Oregend gebirgig merbe. Zfllenţ̧alben ţat man bie. forwarge Niefrmurz an ber Etraffe neben fidf, uno balo fängt das fleifd)farbene Szeibefraut an, bie Eei. ten ber Straffe zu beberfen. Die Felfentrimmer, zroifthen benen man hinfährt, find von Marmor voer Salfftein, unb non eben diefer Eteinart in fleime Sloffe

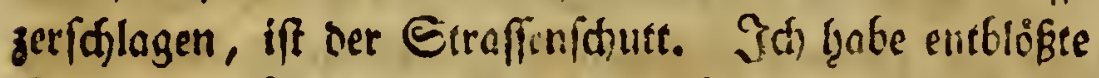
Etellen am Suffe bor 2rnfiogten neten ter Erraffe geo

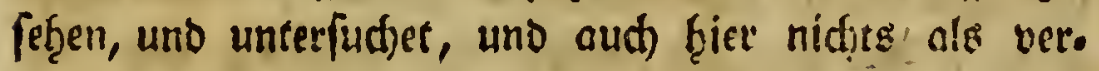
witterten fleinbrdaflictsen $\mathscr{R}$ alffein gefunien; on andern Oreen begegneten mir nafie Felfen, die ben $\mathcal{F}_{\mathrm{B}} \dot{\mathrm{B}}$ von Bergen ausmaditen, weldfe ben uns mitten im sanbe

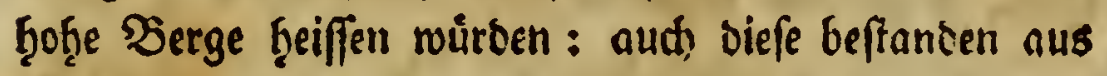
Ralffein, ber gleidfam in bidfe 2 làtter getheilet war, bie fid in einer fobiefen Siidtung offwairts neigten,

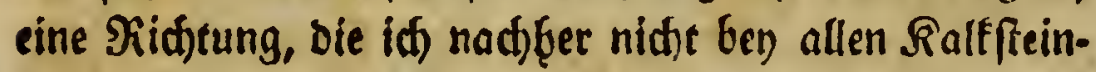
gebirgen angetroffen batte, bely denelt biefe शeigungen oft aud) meftwairts, und immer mit bem 2ibḩange Der Bergţ̧áler gleid)laufeno ftreid)en. Id mod)te Den Boben ba, wo er blok lag, unterfudien, wo idf wollte, fo fanb id) faft allentbalten Falffein ober Bsips, ober Mergel. Den Eanteftein forno id nur in ben Bàden, aber f̧ier fo fdpwere Stüte, daß̧ biefe

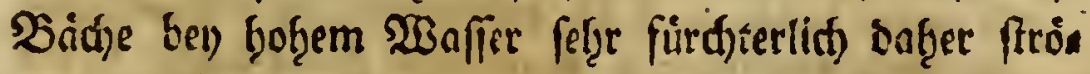
men muiffen, um fo fd)were. Ereine mit fid fortzun reiffen. Diefe Steine zeigen allerbings an, Dak ser 
Raleftein, weldter ber Şauptftoff ber bafigen (Sebirge ift, blok anfgeferget fen, uno in feine betród)tlide Tiefe fortefge. In ber Namfau, einer Pyarre, ble

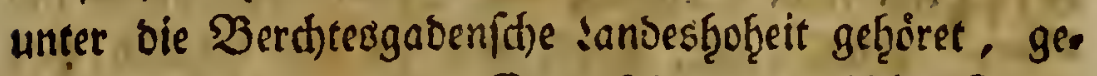
- winnet man aus ber Erte fef̧r vortreflide Eand. freine, bie mirflit) eine Zurt von (Branit fino; man berfertiget Muiflfteine baraus, bise fef̧r gefd)diget merben. Der Bsips ift meiftentf̧eils roth, und miro ref̧r gelobt.

Sef̧r fonberbar war mir ber Fall, baß man

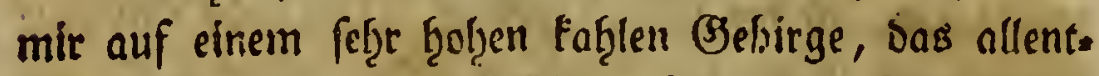
balben lebighidh aus Ralffitein beftand, Eteinfoblen zeigte, bie von ber beften 2 trt waren, uns fein anbers Salband als Silffiein ţatten, wenn man eine bunne Edfidit setten ausnimmt, bie fit) in ifrem siegenden befant.

Da alfo der unmiftelbare Boben meiftentţeils

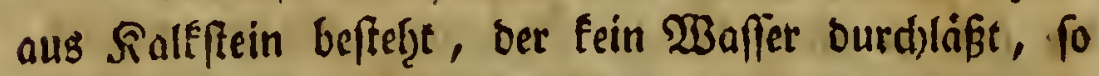
iff die Dammerbe meiffentheils feudst, und wirb allents frafben von fleinen Bàd)lein ourd)fdnitten. WBeil es

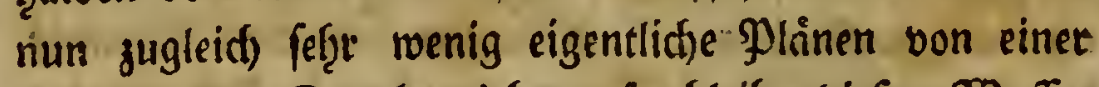
beträdtflidien Strecfe giebt, fo bleibe diefes $\mathfrak{B}$ affer nidat lange ftefgen, um faulen ju Pơnnen, uno bie luft ou verberben. (*) Das Ȩinbert aber gleichnof̧l nicft,

(*) Daher tommt aud) dic Eridjeinung, bie mir anfänglido

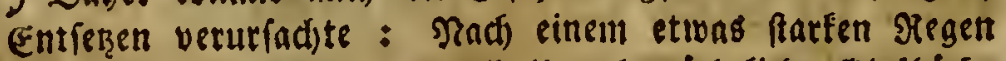
fommen vom Bebirge allenthalben betråchtlidje Biegsische berbor., Da id fie an meinem benfer aus Begenden foms 


\section{Şurghaufen, Den I 1. Jłnn. I 784. 207}

Dak, es nidft fier uno bort betraidtlidfe Sümpfe geben follte, sic auf Torf weifen.

Эक fenne im ganjen Lante eigentlid nur fedfs Eeen, ben ben ben Bellern, ben Sberfee, ben Mittlerfee, ben F̧interfee, den Briunfee, und ben f̧unbenfee, bet auf einem lef̧r b̧of̧en uno wilben B̈ebirge liegt, unt

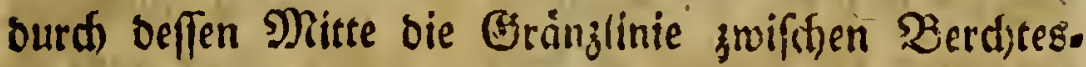
gaben und Enlzburg gețt. In biefem leztern findet fid) feine andere Sifdtart, als PFriflen, nidts, als ob nidft aud bie fdimactfyaften Ealmilinge barinn borfreff: lid) fortfommen wirben, fonbern weil bende ?hgeile

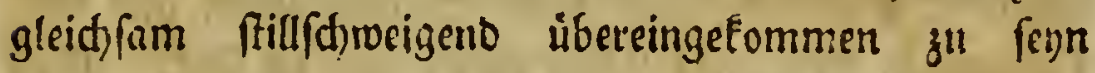

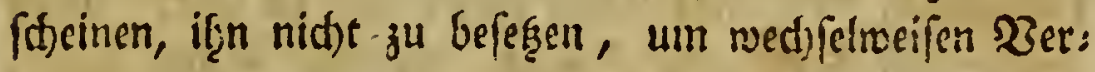

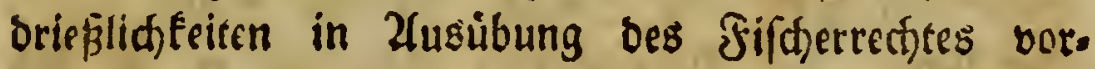
jubeugen.

Der erfte Drt, an ben man in Berdtesgaben foommt, renn man von Ealzburg bahin reifet, iff Ed)öllenberg, ein Marfiflecten, bey oem fid eine Ealzo paanne befindet. Der Srt liegt am vorbenflieffenden Şod)waffer, thas aus bem Rönigsfee tommt, ift aber nidt) fonderlich) großs. Er ḩat ein eigenes Pfleggeridgt, und eine eigene Ifarte.

Berd)tesgaben feloft, bas im allgemeinen f̧ifforiThen Sexifon eine Etadt genennet wirb, ift nid)ts weiter als ein guter offrer Marftfleden. Eeine áge

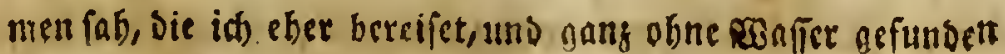
batte, fo gemarn dic ericheinung. Dajurch sin Defto fürdj-

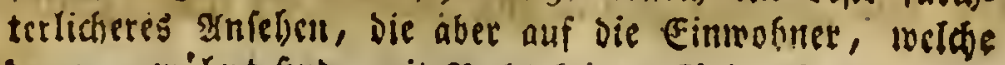

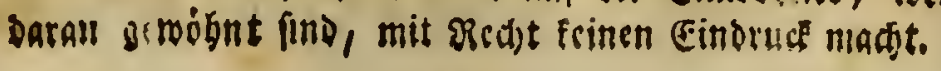


if etwas erf̧aben, wenn man fie gegen bie $\mathfrak{B} a f f e r$. flache ser Roinigsftadt uno bes baraus abflieffenden

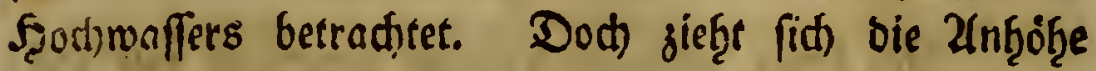
gleid) Ginter bem Marfte hinauf, uno läuft, nadbem fis einigemale verf(fiebentlich unterbrochen worden, bis an ben Unteriperg fort. Dos reidfsfunftlidfe Eḩorftift, tem ber Marft, uno vielleidit alle baherum angebaute Jiegenten iø̨r Dafeun ju Danfen b̧aben, liegr ganj in Der Mitte. Es roaro zu 2lnfanz des orensef̧nitu Jah̨r.

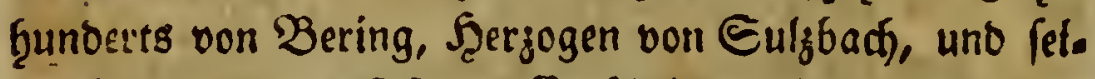
ner (s)emahlinn gefliftet. Es fd)eint onfger ber ḩeutige

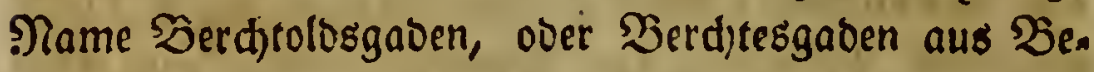
ringsgaben (berings (Sut) entitanden ju fern. Man nennet nod) Ģeute eine (brgeno, Die bem Erifte gegen.

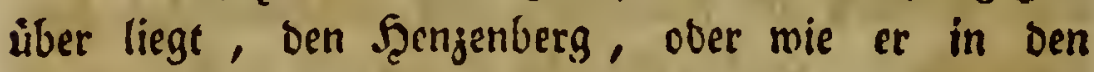
(Jerictitsbichern beißst, J̧erzogenberg, uns id) wat felbft auf oiefem $\mathfrak{B} e r g e$ in einem $\mathfrak{B} a$ aurenţaufe, meld)es vormals bie Sisog̨nung eines J̧erjogs von Sulgbad) in biefer (Gsegeno gerwefen fen foll.

Man ḩat mir erzäb̨let, bie erften Ȩ̧orh̨erren, weldie fid) ḩier niebergelaffen ţaben, ந̧aben es un. móslid) Gier ausbauren fónnen, uno feyen balo wies ber nad) if̧rem vorigen 2(ufenţ̧alte jurŭcf gereifet. Nir fam bie Ead)e gar nidte unglaublid) vor, menn ici) ben Fall feşe, ber ridtig zu fern idheinet, daßj fie.

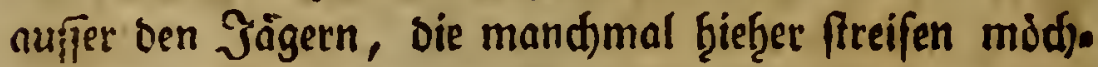
ten, wahref(heinlid) hier bie erften Dienfahen geroejen fino. alles das nun neggetedsnet, was SBetriebfam. feit uno গiotḩourft ber fí einmal ba niebergelaffenen 


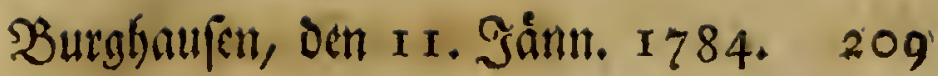

Menf(j)en veränbert, uno fogar verfotonert bat, muß̧te

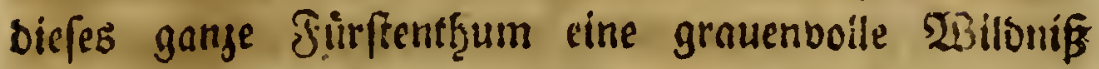

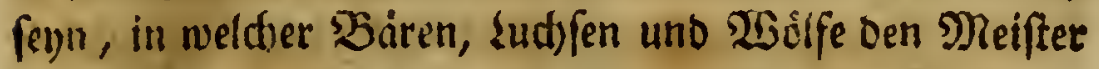
fpielten. Nod) beut zu Sage leben alte Minner, weldie fict) erinnern, baf man an einem gemiffen Drte bes hiefigen Stiftgebaittes in ig̨rer Jugeno nod) Z(uers ḩứfner fohieffen fonnte, Die man jist in einer Entfer. nung von vielen Einnoen auffud)en -mukr. Sonnte ein einziges Menfid)enalter eine foldhe Berånderung. hervorbringen, mas müffen niadt alle bie Menfffen. alter bis ins orenseg̨nte Jab̧rf̧undert zurùcf benoirfet

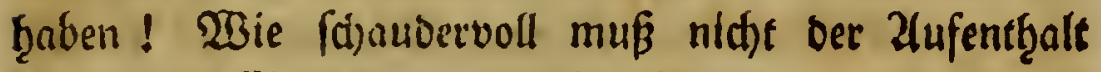
ber' crften Einwog̨ner gemefen fenn.

Der britte Snt biefes Lanoes ift die Ramfau, ein Dorf, ju vem aber nody ein ziemliches Fievier ge. f̧öret, bas unter biefem Namen begriffen wirt. Die Dafige Safarre gef̧ort jur Decaney von SBerchtesgaben, bie sinen Dinconus barauf baalt, eben fo wie auf ber PJfarre von $\mathfrak{B}$ erditesgaten felbft.

2luffer biefen brciyen Derfern giebt es feinen mef̧r, in welthem bie Menfthen in grêffern (j)efello fhaften benfammen lebeten; unterbeffen ift Das ganze Sano bis an bie Ralfgebirge hin mit einffhidtrigen

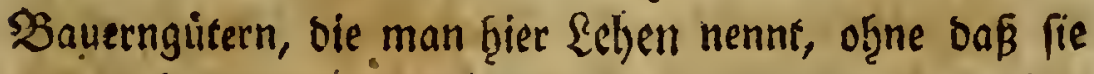
Darum Seģen im eigentlichen $\mathfrak{B} e$ ftanbe máten, gleid): fam überfäet. Es ift fefr angenefgm, mann mon, unterieffen man auf oen Bergen tererumfteigt, uno fich ganz bon Den Mienfthen cutfernet f̧àlt, allentţalben wieber auf woh̆lgebaute seg̨en fümm, fobalo man els

Liaturbif. ¿riefe 1. zand. 
nen STingel überftiegen, ober einen $\mathfrak{B a l b}$ jurücfgeleget bृar. Mian erftaunt úber bie Menge $\mathfrak{B o l f e s}$, meld)es an den Senertagen zum (Sottesoicnfte nach) Berctess gaben fómmt; gleictwob̧ if bleß nur ungefäb̨r ber oritte Sheil, weil eben to viele Menfihen in bie benden antern Sirdfficle eingepfartet fino. $\fallingdotseq$ d) meis es zmar nidft, woḩer ber $\mathfrak{B e r f a f f e r}$ ber Briefe eines F: ungofen bie Siactridtt habe, Daß̧ fich die Bolfemenge Diefes lanbes etwa auf 3000 . Rüpfe belaufen moidte. Epin Zufentf̧alt faceint biel ju turg gerwefen gu renu, als oafi er felbft einigen Ueberfdalag f̧ätte madien fónnen, uno bon juverláâigen Perfonen f̧at er bie

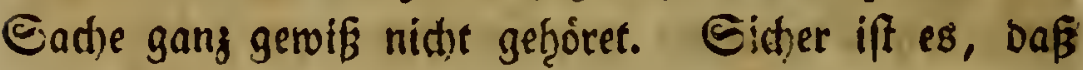

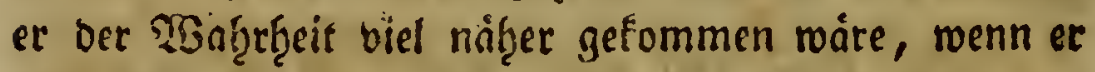

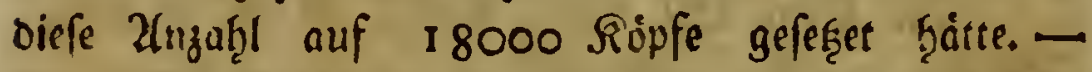
Was für einen Glauben verdient ein Edjrifffeller, Der fict) mit feinen gefammelten Tindstidsten fo breif macts, uno daben to viele Untidfigfeiten in bie WBelt Ģinaus/dfreibt?

Der farecflidje Buftanb, in weldem fid) Das

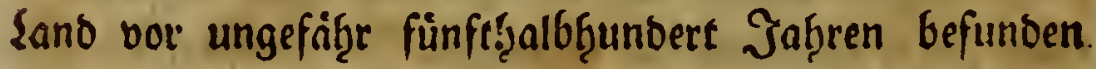
bat, ift jezt gainglid) berfdimunden. WBeit entfernet,

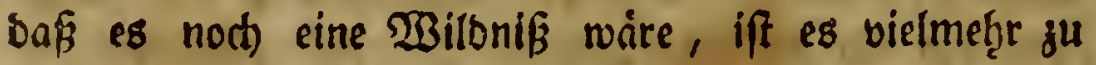
einem Sanve geworben, bas für einen Manu, ber nicb) gleid) liber Eteintten binftolpert, vorguglitise Nieize befizet. Man fonnte frenlid) reber bie Berge ab.

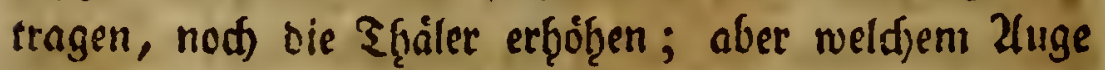
fo̊nnen noḩ unerme Blidide Ebenen in sie singe gefallen? Der Zublide einer groffen Pläne ḩat etruns groffes, aber 


\section{Şurghaufen, Den Ir. Jánn. I784. 2 II}

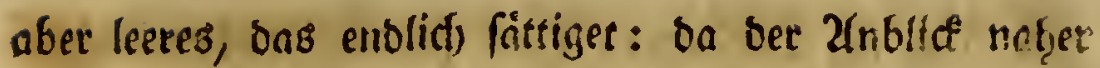

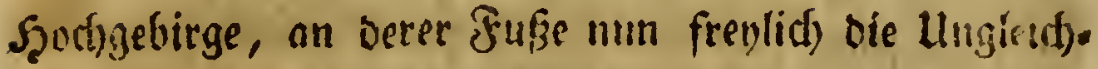
Geiten betråd)tlid) felyn müffen, großß uno erf̧aben iff. jd) fann in ber volfreid)ften und lórmentfen $\mathcal{E}_{\text {:abr }}$ tagelang am Jenfter figen, oggne einmal ouf oie (5iaffe zu feñen: aber in Bertiseggaben frano id) funbelwatig am Jenfer, von meldsem idf Den Edfmarzore, ten

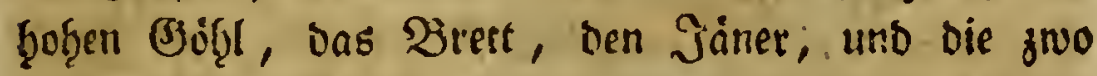
majefrátif(hen Epizen bes $\mathfrak{B}$ ngmanns feţen fonnte, uno weryvete mid) an biefem feelenţebenten Zunbiicf, Der mir badurch, Daj id) fie fdon vorḩer felbft bereifet fonte, nur nod) feftlid)er warb.

2(ber bief fint groffe, bielleitht nithet für alle Menidjen fübzlibarc Edhintrciten. Es mangett baben feinesibegs an fanftern Ecenen, nithts Eann reigenoer feun, als bie Zlusficht, bie man an ben Renftern bes Ediloffes su Jrietnsberg geniept. Das Ed)lok liegt felbit anf einem fefgr másigen Sanbocrge, Der fich

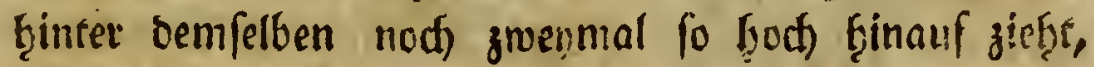
uno oann ift man am fuffe bes Luterfperges. Zuif biefer Eeite find aber bie vorzuglidffen Sinmer nidit

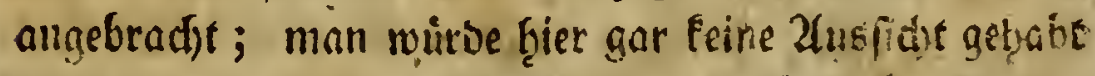
bzaben, bic ouf ber arbern Eeite fo begnuterno if :

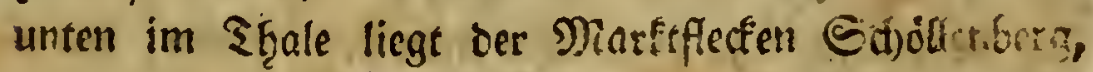

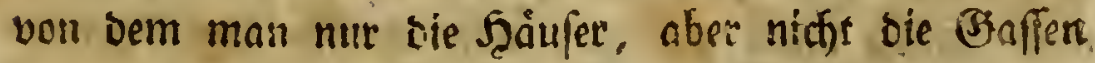

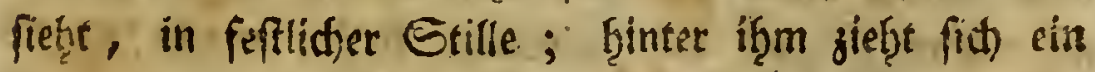
Foterer, bem 2(nfid)ein nach jiemlid) freiler, aber beiwarty ferer, uno eben barum benofenter Betg aufwairs: benn bier find alle Berge von Menfaen berooghat, 


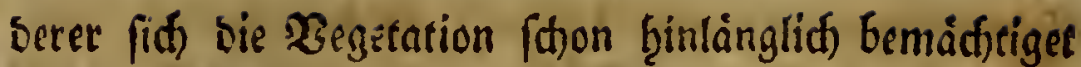

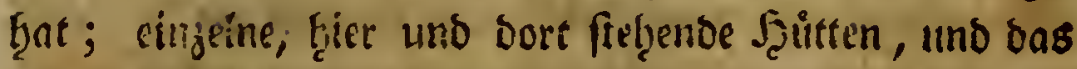
Iebignfte Brun ber Wiefen, sas von ber 2 lidffarbe ils ternser Biune unterbrod)en wiro, unb einjelne gefdjä:

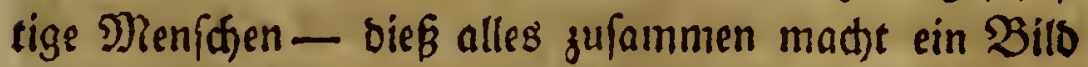

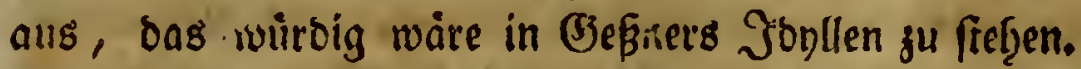
(5.s ift baher fein 2 Sunder, wenn bie philofophifate

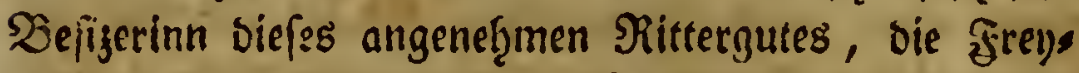
frau von ङ3rußsoorf, eine gebotzrne Brráfinn be la Tour, Sen Commer himourch biefe länolidje, aber wonnevolle Einfamreit den glänjendern, aber lange nidje fo innigen Bergnügungen Enlzburgs vorzief̧e.

Joth werte nod) ofter sie Eģre f̧aben, Sie mit Dirgleidien Biefiten, die der Einbiliung fo viel didste. rifiaen zuffinumg geben, ju unterbialten. Entbft (Jisgensen, bie gang unferm sanbe gleichen, baten fier

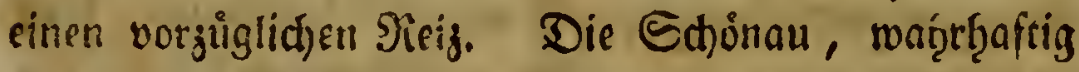
eine foino Zlue, fief̨t fo vollfommen einer flad)liegen=

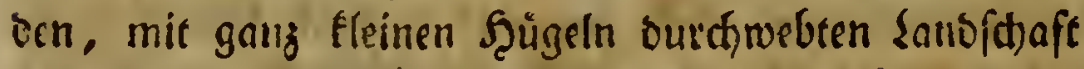
von Saciern gleid, onßs man fid) auf einmal mitten in Gaiern ver $\left[e_{g} \mathrm{et}\right.$ glaubt, uno auf bie ungeţeuren Jerge vergist, jwifdain welden fie liegt, und bie

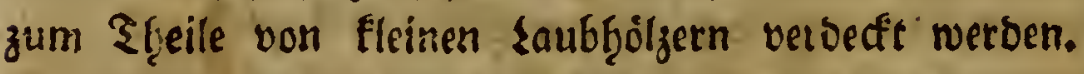
Die lleberrafdung, in meldjer man fid) befindet, wenn man mitten utiter groffon, aber ernften Eduónf̧eiten urvermuthet auf eine nieolidje ftöft, madt, baß man ganz von bem Gingeriffen miro, bas man ba foum fir bemerÉenswurbig Gielt, wo man es aúltaglia fieģt. 


\section{Şurghaufen, Den I I. Jånn. I784. 2 I $_{3}$}

Die Sanorvirtşid)aft ber Einwofiner beftefet, wie allentigalben in oem gelindern Europa, in Bief̧zucht

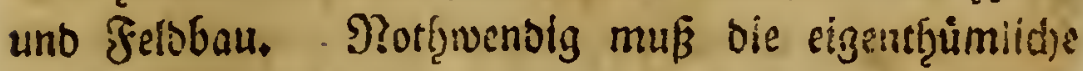
Befdaffenteit eines jeden lanbes, ooer, wenn bas Sano groffer ift, ciner jeden Syrovinj, betráchtlicile Berfdiebenfieiten in birjen benden Bweigen ber Lanb. wirttgf(t)aft veraslafen. In Baierns Walorevier um

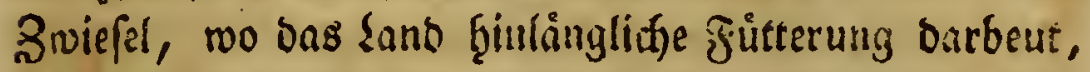
baben juvar ḩod) liegt, aber niche geburkig ift, wiro alle Feloarbeit mit Od)fen verridjtet: man beţailt

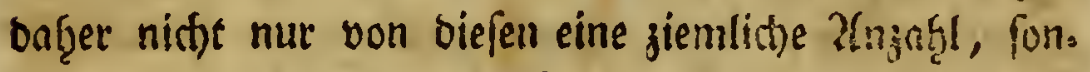
Dern aud) alle Rúb̨e ju Şaufe, uno tethit nur bie jingern Sillber und bie überflüfigen Daffen auf ben Radfel ober 2frbo; blof bem Şirten wito für faine

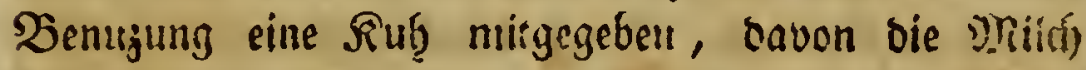

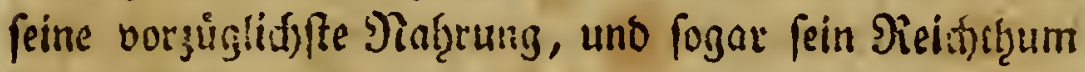
ift. Shas wirben aber bie Berditesgatener mit lang. famen, fótwerfálligen Ddjpen austictsen? Şier wirto. bie Felbarbeit mit Pferben getrieben, bie if̨ren Bigens

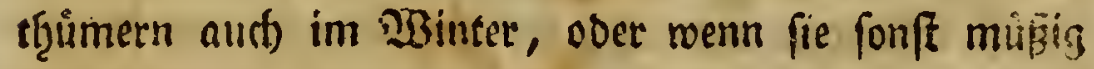

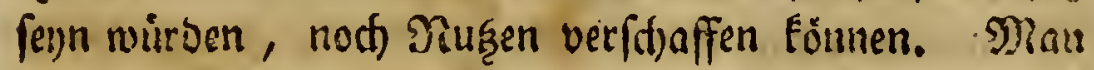
findet bofzer wenig Dafeen, uno oen Sommer Ginsurd)

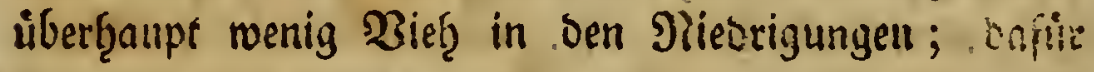
fino bie grasteid)en (bebirgthâler mit Şeerion von

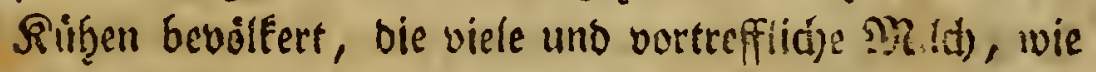
man fiof rof̧l benfen fann, geben. Daraus vev; fertigen bie Gentreximum (fo geifist mon Gier dis

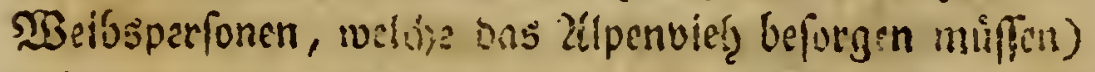

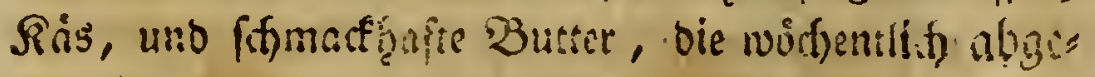
trageit, bas if, you ftarten Bauerferten, Die fich 
Damit auf Sopf und Riucfen fdfmer belaben, nach ben Sejen fierabgethadit merden. Man nennt aus oiefer Urfoche aud) bie Sennthutten fiffer, ober nach ter

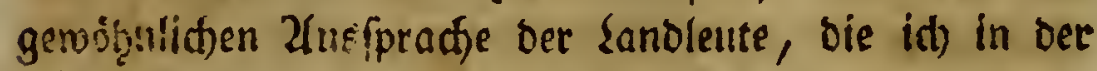

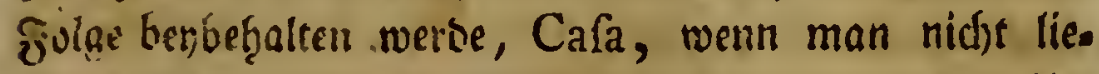

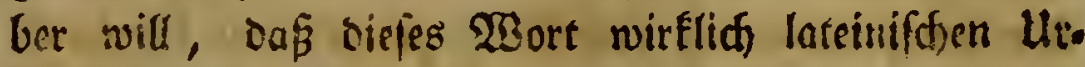
fpruriges ift, weil vermutgelich) bie 2llpenfahtet exft cus Jiclien nady bem Tribentiniften, und bann weiter nait Inrol uno Deurftilano gefommen iff. Die Wenseplaje auf oen alfen fino übrigens gemiffen kefeen

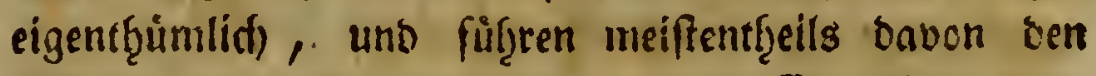

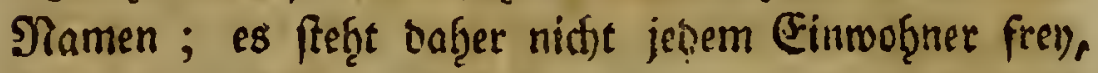
fie zu benuzen, er fann aber mit ben Efigentf̧umern eineti Bertrag mathen, sabi fie fein Bief unter ger

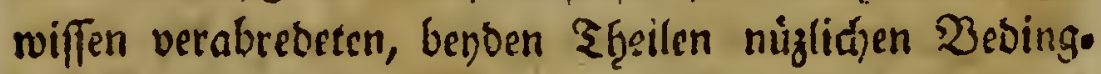
niffen unter bem if̧rigen werjoen leffen. 'Säglich) mirb bas Bief. -zweenmal gemolfer, meldes allyeit in ber

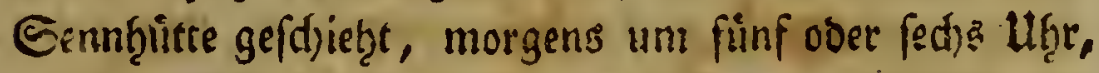
uno um bie gleiche Zeit গind)mittags, miţ̧in alle gmòlf. Etunben einmal; bie Swifhenjeit roirs von Eennecinnen mit ber abflårung ber \$Milć, mit Berfertigung ber Butter, Dé হájes, und des Gdhotenß, uno was nod) ùbrig if:, mit Stricfen zugebrad)t.

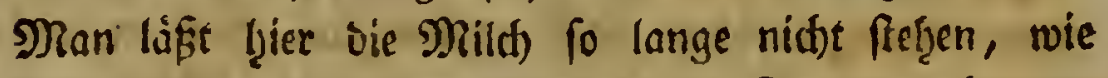
in Baicrn, unt fauren Siaum z̧um Buttermat)en รู"

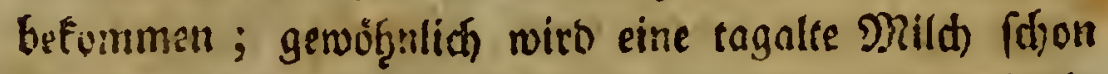

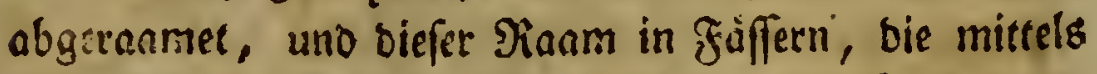

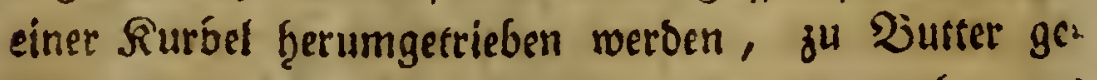
fdjlagen, Dafere man auch) hier bas, mas man ben uns faure ober geftócfette Mild') nannt, nicojt befómmt. 


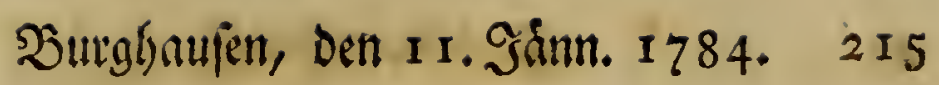

2fus bem Uebrigen, bas notfimenbig noch viele Butters.

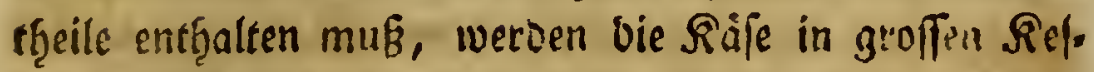
feln berfertiget. Die faute und wáprigte Mitid, wèlthe von biefer atrbeit uibrig bleibi, wirt unter Jein frifdeen Nolfen gegoffen, in roaldient basurd) alle nod) vor:

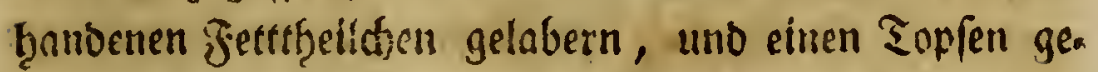
ben, ben fie Sthoten nennen, uno feţr fómacthaft finoen; bas lezte ineinfuppenfarbene $\mathfrak{B}$ anfer, weld)es

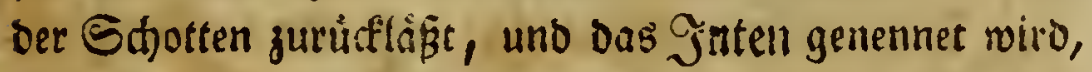
wiro in Gooljerne zroige gegoffen, uno ben Rálbern uno Rủhęen zur ₹rånf́e gereidfet.

Seber Bauer, welther eine Ilpengeredtitigfeit befizet, ift menigitens von zween, letridtitid) entferns ten, Wendepläzen Befizer. So balo es die Jaţrézeit zulábt, wito auf oen untern, gelintern, aufgetrieben, we!d)es fie auf die If(penfalyren ḩeiffen. Sladjoem

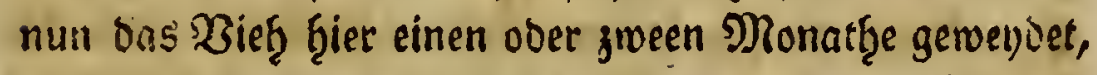
treibt man es auf bie bojhere Begent, uno läft ben Plangen ber untern 3eit wieber nad)zumad) (en, um fie im Şerbfte abermal zur $\mathfrak{Z B e n b e ~ j u ~ b e n u z e n . ~ T B e s n ~}$

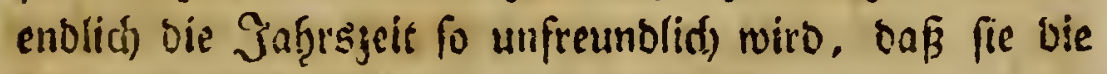

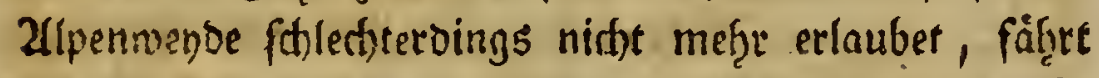
man heras, das ift, man treibt bas $33 i e f$ nad) Ş̧aule.

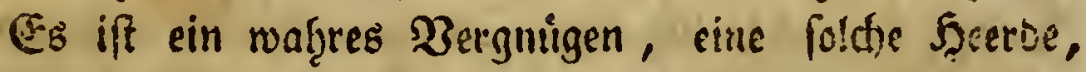

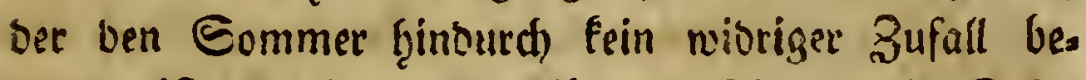

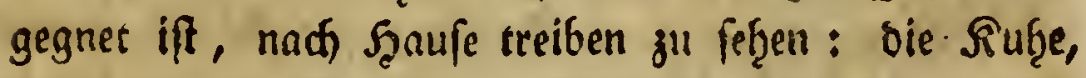
weld)e bie Blocfe traigt, befoimmt ben gnnzen Rupf mit Rränjen umwunben, unb not einen 3 weig bon 2(lpraufini) (Rhododendron hirfutum); ober funfs 
irgenb einen Zl(penffraudf), oben orein; aud, bie Rällber wersen mit Rränzen umffodtsen; oie ůbrigen Etricfe befommen nur einen, ober zwenn foldher Siringe um bie Stirne geflodten; ţintenorein fömmt bie Eesunerinn

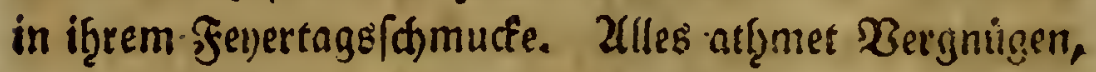

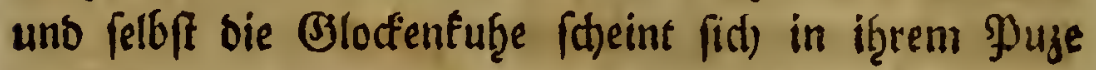
zu gefallen, uno die fesener zu fübilen.

Der Felobau iff gerabe fo, mie i̧̧n bie Utmpánbe

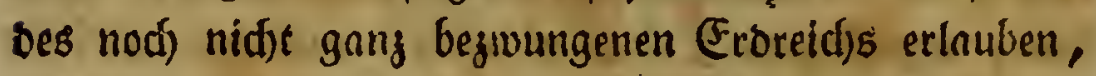
uno of̧ne unfere ótonomifchen Biutter gelefen zu ţaben, ift oer $\mathfrak{B e r d f t e s g a v e n f d y e} \mathfrak{B a n e r}$ ein vortrefflidjer

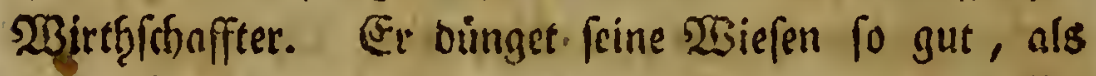

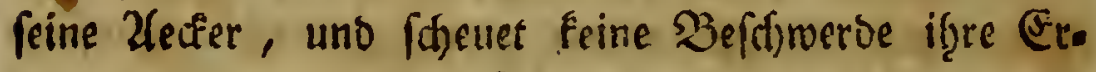

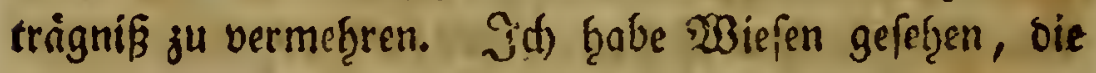

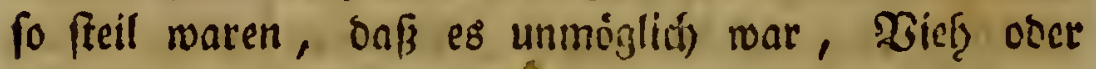

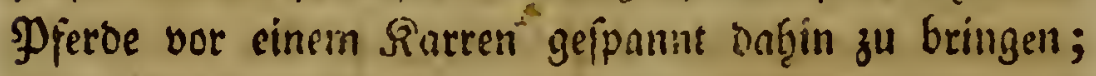
fie murben nidbs beffoweniger geobinget, und ber Dünger ward von Menffien, die fidf Juspeifen an bie Edjughe gebumben hattén, in Edjiebfarren zuges fübret. Diejenigen $\mathfrak{W}$ Biefen, we!che eine bequeme saze

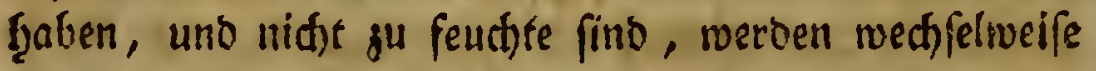

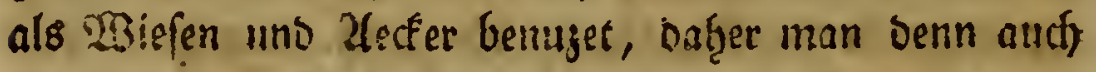
bon ven wittowadfenten Yglanzen forvolil bie, bie ben

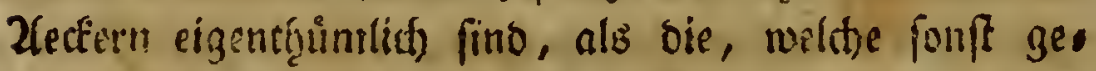

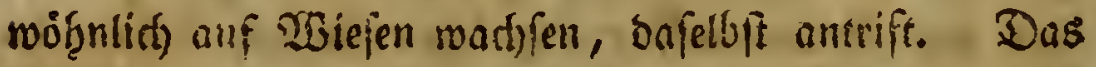
ift aber eigrntlid) feine Bradse; Die $\mathfrak{Z B i e f e , ~ w e l d e ~}$

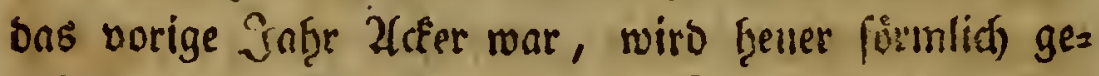
màbet, uno bleibt offter meţrerere Jaghre Ginteceinanber

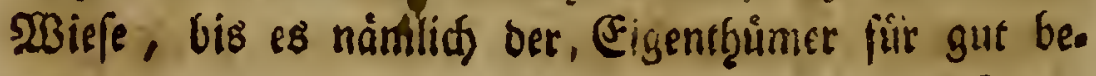




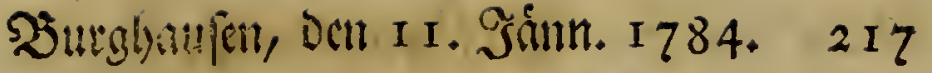

finbet, fie abermal zum $\mathfrak{d}$ cfer zu madjen, um einen acter zur Wiefe meroen zu laffen. Jan pfligt eine

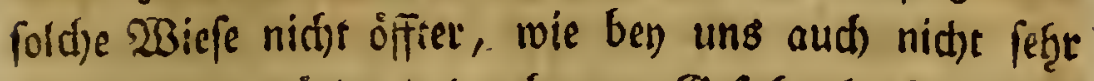
tief : man nưrde daburd) nur (G) faf̧e laufen, ben

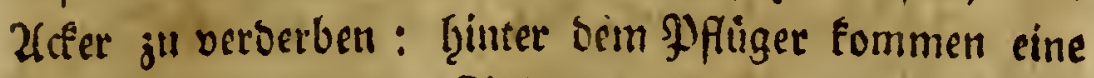
Menge Weiber unb Riluber, bie bie anfgeworienen

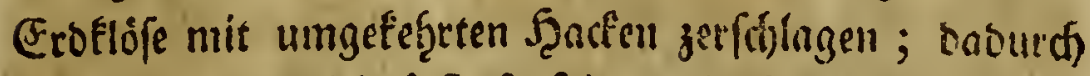
wiro das (Eroveich faft fo fein, wie Barsenland, und Da bie Dammerbe mit Syan wanig vermifdtt ift, baher aud) fo leidst nidfte jufammenbidd), fo erbialten bie Burjeln alles Das, was fie noch ber sullifiten Me,

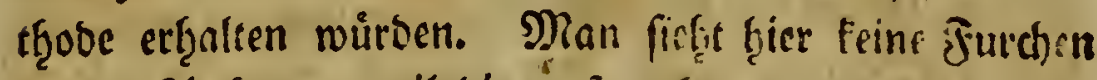
in ben 2lectern, weil bie aufgeacieste uno zafdolagene Eroe wiever geebnet wirb. Diés erpart nirtlich ber Begletation bielan Plaz, weil in ben Jutrdien megen

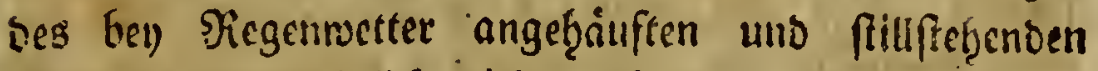

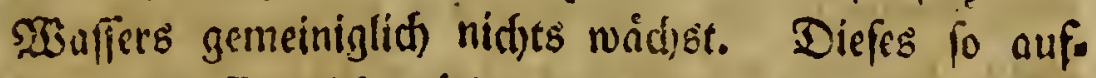
gelodjerte (Eroreid) wirb bann oigme weitere Ulmftande befäer, undo zuzeegget.

Piachoem bas Betreibe, won weldier $2(\mathfrak{r t}$ es auch) fernn mag, $\mathfrak{B}$ eizen, Sỉorn, ober Şaber (BSerffe

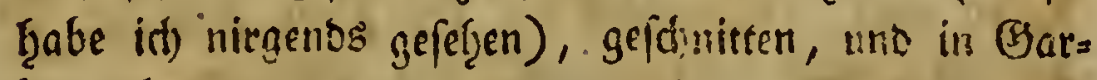
ben gebunden worsen, wits es nidit in Mandeln nufs geftellt, wie in $\mathfrak{B}_{\text {aiern uno }}$ Deftreid, fontern geb)iffelt, Das ift, man befeftiget cine groffe 2luznģ Etangen, baran bie Zeffe nur grob weggeidtonitten fint, cusf bem Etoppelfeloe, uno maitht an benfelben ungerifir is bis 16 Barben fefte, die oben, mie bie Mhandin bey) uns, ein Etrofgoad) besommen, uno beylåı,frig

$$
0.5^{\circ} \text { sine }
$$


eine Epanne fort) von bet (5rbe entfernet bleiben. Fine folthe mit Barben beţangene Etange ḩeiğt ein Şifel.

Die suft ift f̧ier, wie in allen gebirgigten sins bern, aufferlt rein uits gefund, daf̧er auci) bie Ein. woģner von Sranflgeiten wenig wiffen, uno 23 ruft. beflemmungen aufferordentlich felten fino. Seure bon einem vorginglid) betenen alleer firto unter oen Einges bof̧enen nidgrs weniger als cine Eeltenlyeit, und fino in itgren graulten Gaḩren meiftens nod) gute JuBs

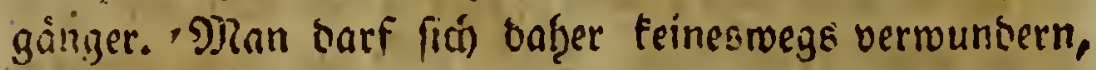
baki Bieņfeucien fogar dem Namen nad) unbefannt fenell; aber bießs iff nod) lange fein Beweis wiber bie Brunte, aus benen man bie Shutivenben auf bem flasiten, oser nur menig hergidsen lande verwirft: man bringe einmal zlpenluft auf unfere glănen, und

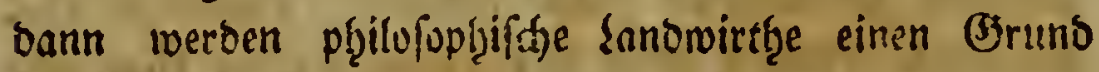
meniger haben, bie ફ̧utweysen zu beftreiten. Sth menigitens habe auf oiefer Reife feine Ulyadre gefunden eine Sylthe in meiter abhianolung ùber bie Etallo futterung zu anteern, ausgnnommen, oaß idf einge. ffechen muş, es mad)ien auf ben alpen meiftens nur niebrige, aber befto vortrefflithere Dflanjen.

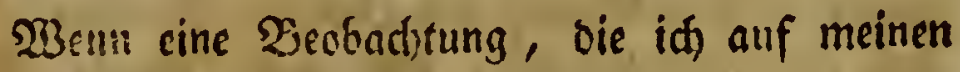

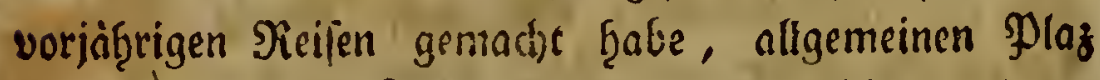

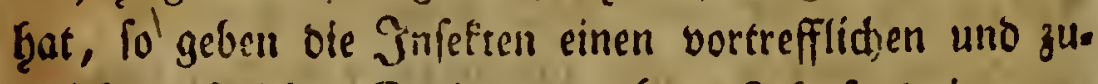
gleid natirliden Eubiometer ab. Ia fano im ver" floffenen Fruif̧linge und Eommer in Deftrei Paffau, uno an allen ben Sertern Baierris, Durds 


\section{Şurghutufen, Den I1. Jainn. I 784.219}

melde if gereifet war, bie Dbftbaume und Şeidfels bisume bon Shaupen gang ungemein zerfreffen, und sin gleid;es hat man mir bon ber fladen Begeno um Ealigburg erjốlet, uno war etroas leid)tes ju wiffen, weidje Sinupen an biefen Berfirungen vorzúglith Ediulto hatton; allentrfalben fath id) nuf ben Etraffen,

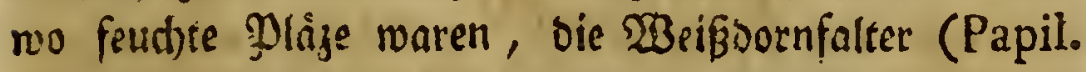
Crataegi) jı গ̧aufen berfanmelt, uno auf allen Zlrten bes Prunus sic leeren Berpimmfe ifrer Pianpen; sie Rinupe des Rirfd)enfalters (Papil. polychloros) Frods um sie Mitte des Junius an allen Siganoen auf, in

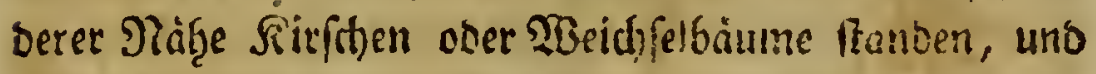
folten fonnte man unter einem folden Sarme wega

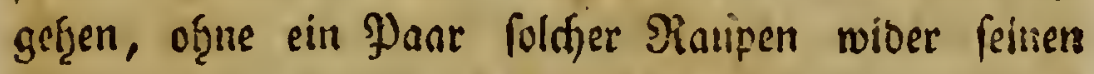

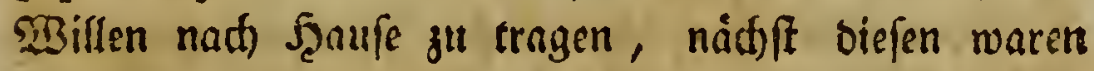
Phal. Bomb. Neuftria, Phal. Bomb. difpar, uno Phal. Geom. brumata fef̧e zaf̧lesid); iberf̧a:apt wat Das vorige Jabre für bie fortpflangung ber Infeften siberaus vorff̧eilf̧aft: aber biefe Frud)ebarfeit war in Werchersgaben, verglicisen mit jener ber benactbarten Sånoer, feģr geringe : vom $23 e i$ Foornfolter Eamen mir

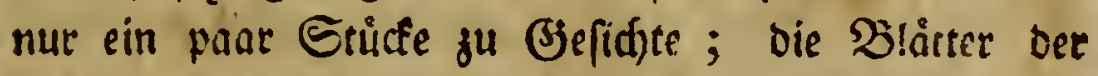
Bảume, unb ber nieorigen Pfangern waren fegre wentig angegriffen, und ob idf gleid) offiers, blof uim In: feften ju fangen, ausgieng, fo fonnte id ood) nut

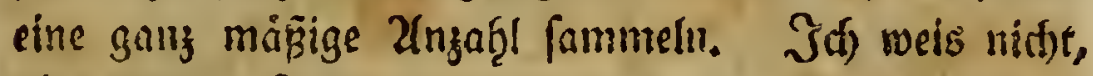
ob sies alle Gafire fo fen, uno ob siefer Mangel an

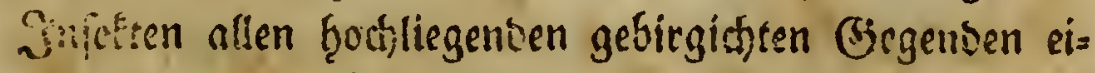
genthiuntich jutomme: aber faft follte inf vermutizen, Don es fo fey, 'uno sap man bie lurfadje tavon in 
ber sufiart ju fudyen habe, bie auf ḩofien Begenden

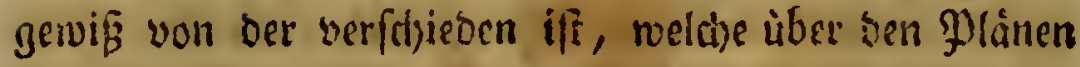

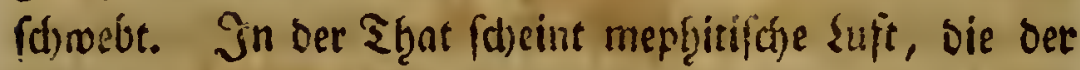
Befundbeit ber Enugthiere fo fdabiid) iff, gerabe biejenige zu fenn, weldise ten Jufetten in einem ţof̧en Brabe robl beformmt : leben nidte bie meiften Siffer mitten in Dem gifrigften (Bas? uno haben wir nid)t in ben Nadjrid)en ber erfen weftindifden Maif onnrien und Sieifenden bie bitferften Rlagen ủber bie unges

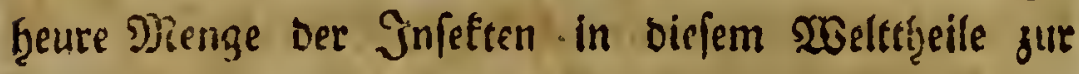
(5emige gelejen? gerabe in bem 3 elttheile, Dem Şerr von $\mathfrak{3}$ aw bie allerungefundefte Suft von ber $\mathfrak{\text { Welt }}$ zus

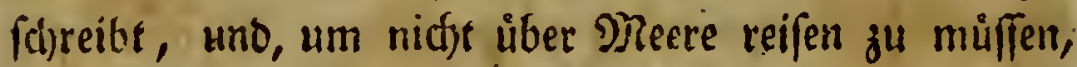
fino nidht bel) uns felbft eben bie Derter, aus welden Die Beinlidffeit berbannt zu feun foreinet, gerabe ber vorgiglidffe Sammelplaj Der Snfeften?

Zlber id) befinne mid, ' Dap id) Slynen einen Brief, uno fein Bud) f(breiben wollte. Эoth verfpare inir Dab̧er bie ferneren I?adjricteen auf bie folgenoen Briefe, und bin unabänoerlid' $11 . \uparrow . f$.

\section{Edrant.}

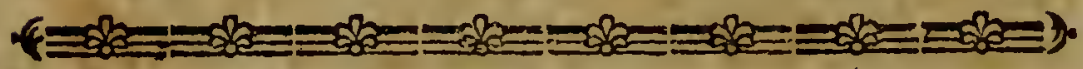

\section{Drengebnter SBrief.}

Büghaufen, den 15 . Jänner 1784 .

Sd)af̧utut. SBicnenzut)t. Şutzarbeiten. Salz= fabrten. Gieninthourt Der SBerdytergadner. sob ik jejigen Furiten. 


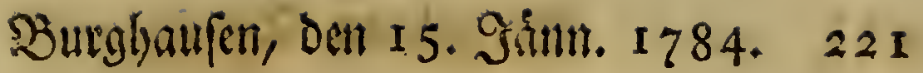

Sd) babe Glinen in meinem lezten Briefe eine

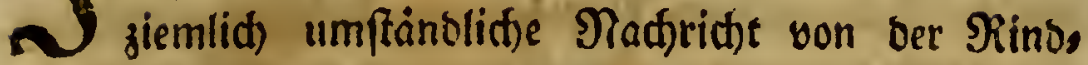

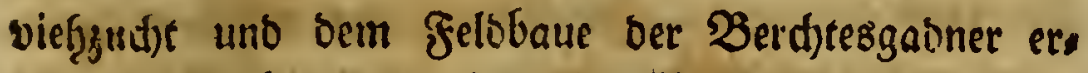
theilet, ben bensen wefentliden Nab̨rungswegen eines Sonomannes : idf werbe in bem gegenruartigen bas Hebrige nadtglen.

Die Sdjafzuctit würbe vortrefflich geraftenen, aber fie miro aus mir unbefannten lirfadjen nidf

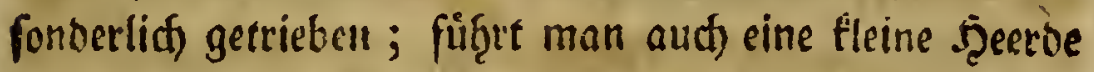
auf sie allpen, fo if man ifrentwegen fo forglos, Daf fie viele Etunben meit megjiehen formen, of́ne Daßs man fidis einfallen läpt, fie aufzuifuchen. - Unterberfien ift man ood verfictert, baß̄ fie firk) niemal in bie Nie. brigungen ferab verianfen werben : fie gief̧en inmer bergan, und inf) fant Dergieidien herumftreifente Şeet. ben meiftens an foldien Zllpengegenten, wo nidits meģr

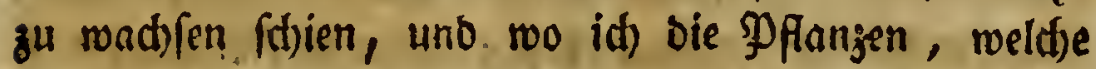
id) fammeln mollte, guvifthen ben Steinen f̧ervorfud)en mufte. - Die Biegen merben nur bel) ben Şärifern in geringer alnzablerzogen, weil es verboten ift, fie

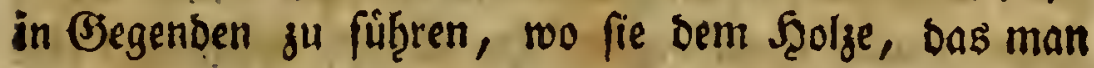

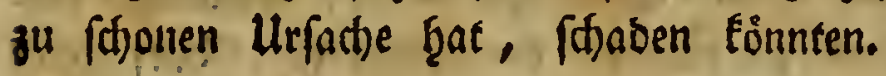

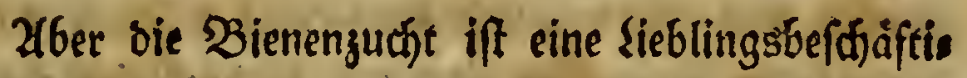
gung ber Berdtesggabner. E\& ift bennabze Eein Kef̧en,

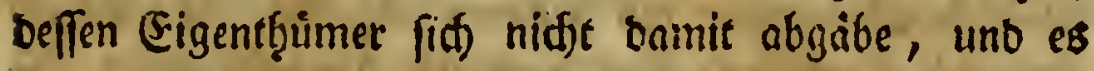
ift gar nidtz feltenes, boß uno now mefitern Etơften iff. Eie betienen fid ger

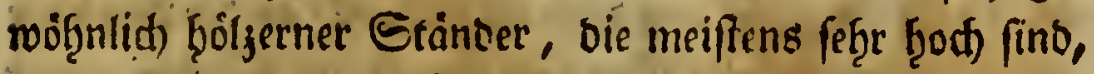
uno woḩl ofters im Eommer von ben Befigetn in die 
(3ebirge getrngen werden, bamit fid) bie Bienen ba bon ben balfamifhen alpenfraiutern befiffigen moidten. f̧ier uno bort weis man fdjon etrons von bem Unter.

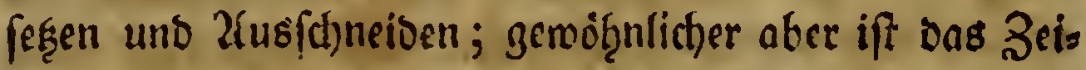
beln, in weldfem Falle man bod bie Eadje niemal

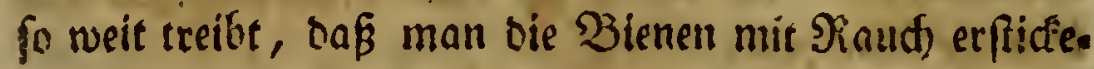
te, of man fich gleid) bes Siaud)es fu finer eigenen Sidjerb̧eit, um wáb̨rentoer Zarbeit nidjt geftodjen zu werben, bedienet.

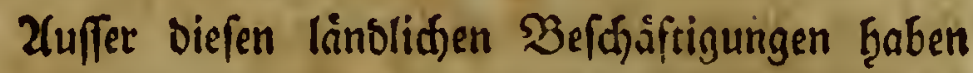
fie nodh f̧àusliche, baburch fie fich etwas verbienen; baģin geţoren bie fogenannten $\mathfrak{B}$ crcktesgabrerarbeis ten, bie auffer ben Edjaditeln meiftens" in Epielmerf, zeugen fur Rinber befteţen; faft jeber Sanomann, auch viele, bie blof in ser J̧erberge finto, bearbeiten einen Zweig biefer Inbuftrialprooucte, weldhe unter biefe $3(\mathrm{r}$. beiter orbentlith vertfeilet fino: Denn es fömmt Eeiness

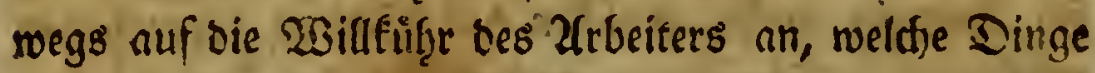
er bearbeiten wolle: Daburd) ivirben Bermirring, Fila. gen, uno mannigfaltiges $\mathfrak{M i} i$ bergnigen unter biefen guten Senten entffehten. Derjenige, meldjer Eleine Edaadteld)en ju madjen berectstiget ift, barf feine groffen verfertigen, uno umgefeffrt; aud wiro fich niemal ein Figurenfdineiber nit Echadtelmadien, ober ein Edfact)telmadjer mit Jiguren abgeben. Fs ift er: ftaunlid, wie offt bas geringfie Probuct biefer ?fre bem $\mathfrak{B e t a r b e i t e r ~ i n ~ b i e ~ S ̧ a ̈ n b e ~ f o ̈ m m t , ~ u n o ~ w i e ~ g e r i n g ~}$ Der Preis iff, für ben biefe Erzeugniffe an bie $\mathfrak{B}$ erleget

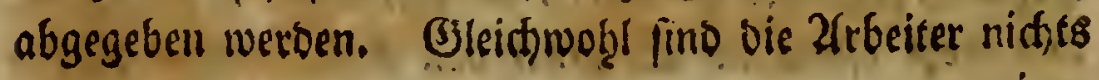
reniget 


\section{B̧urgljaufen, Den 15. Sun. I 784. 223}

sweniger als Seute, bie finf mit nichts befferm zu bes fthâftigen roúften; fie roiebmen ben ganjen Sommer. tag ibrer Felbarbeit, uns nur am albente, ober wenn fie fonft Muffe braben, ruhen fie ben siefer if̧rer Tebenarbeit aus, bie fie offt tief in bie Mad)t fortfegeen. Die 2 arbeiter fino übrigens feineswegs bie gewòthnlitisen Berfáufer ifgrer Errgeugniffe; fie liefern biefelben an bie Berleger gegen einen-feftgeferzen preis ab, bee ingnen aber nidht gang ausbezahlet miro, indem ber Berleger von jebem Bulden einen ebenfollz feltgefefcten Z(bzug mad)t, für weldhen er Dem Z2rbeiter Farben. materialien, Seim, Pinfel uno dergleit)en Notbzendig. feiten feines (sewerbes abreidt.

Sab habe unter biefen Seuten einige fef̧r gefdritfe gefunden, bie allerbings im Stande wàren, if̧re Er. zeugniffe of̧ne $\mathfrak{B}$ ergleid) gefd)mactvoller zu madien; aber bann mufieten frenlich aud bie Berleger sie Zúbeit,

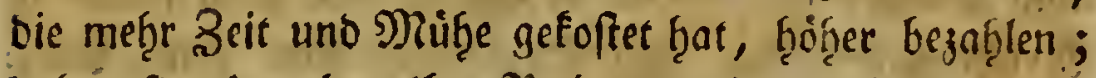
babein fie aber eben ibre siectumung nictit ju finsen glau. ben. Finige, oie an feinen Berleger gebunten fino, maden in ber Ţat foldhe Dinge, Die man ibnen mit

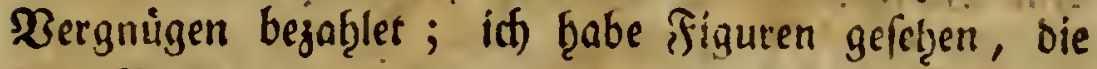
eine Jlinte abricheffen, uno volfommen guif gebilbet waren; bie Becter, berer 50 bis 100 incinanoer. feefen, uno fo oinne als Papier frito, fino Shgnen fd)on befannt, aber Eie miffen viffleidst nid)t, enß man aud) bas menfdilide ?lug mit aflen feinen innern

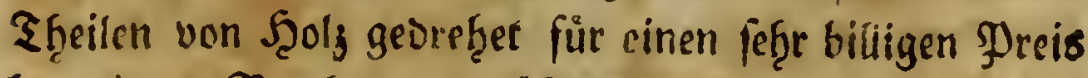
bey einem Berdjesgaden/djen Zurbeiter ḩaben fönne. 
Die $\mathfrak{B}$ eiber bringen alle if̧re Beit, zu Der fie nictes aniers zu thun ḩaben, mit Giricfen $3 u$, in weldser Z2trbeit fie benors, fo emfig uno sefdjictit fint, dâß man fie fogar auf ben (Soffen beftannig bamit be. fthaiftiget finbet; felsff wenn fie Eleinere saften ju tra. gen baben, wiffen fie siefalben fo gefthicft auf bem

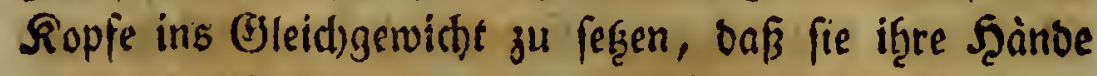

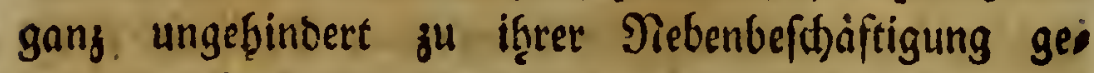
braudjen fónnen.

Die Snljfą̧rten fino ein anberer Zmeig bes Erroerbniffes. (5in groffer ₹ģeil der Einwoḩner ift

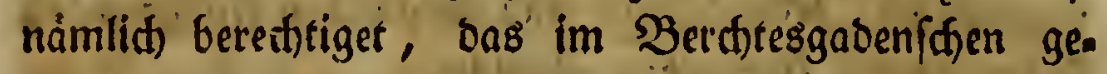
wonnene Salg gegen eine geroiffe (Jebriţr nad Sieid)en. b̧all in bie furfürftlidjen Magajine zu fügren; fie ver. rid)ten diefe Ealgfof̧rten meiftentf̧eils in ber falten Jaf̧rsjeit, ober ju fold)en Beiten, wenn fie if̧re Pferbe zur Beffellung if̧er Felber nid)t vonnothen fraben, uno genieffen Dabel ben Portteil, baß man ifgnen in Sicidjenţall gegen iţre $\mathfrak{B}$ ezab̧lung eine neue Ealz: lasung abgiebt, weld)e fie weiter bersin firitren, ba abfergen, uno dafur Getreibe abjolen, das fie in in. rem Batetlande mit Wortheil abfeçen.

Das find nun oie vorgiglidfften Naņrungss

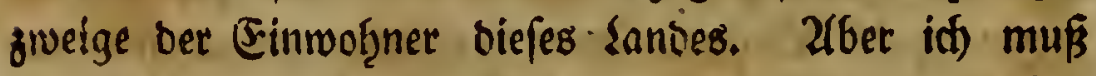
Sie not) mit einem anbern; aber einfad)en, unb eben barum für einen Philofopţen merfmüroigen Producte befonnt madten, bas jwar auth im Saljburgifachen, bejonbers zu Grebing, verfertiget wiro, wo id aber bie $\mathfrak{B e r f e r t i g u n g ~ n i d d ~ f e l b f t ~ m i t n u g e ~ l e f ̧ e n ~ f q a b e ; ~ e s ~}$ fino 
B̧urghaufen, ben I 5. Jૈ?n!. 1784: 225

fino bieß bie Silucter, bas ift, Eleine Rugeln boin unnùķen Trùnımern Marmor ober Ralffetein, bie un. gefäḩr einen ḩalben Boll im Durdsmeffer ţaben mơd). ten. Id) ḩatte mir eine feb̧r zufammengefę̧te $\mathfrak{M a}$ fdjine eingebilbet, menigftens eine Múf̧le glaubte id baju notḩwendig, uno jȩ̣en Sic, mie oie Einmoţner bie Eacje anfdjicfen. - Eie mäblen eine ziemlid)

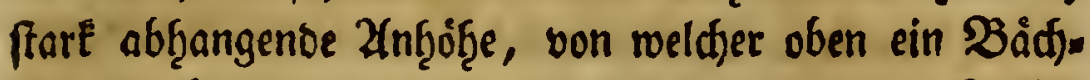
lein herabfromt, bas man etwa mit einer ftarten

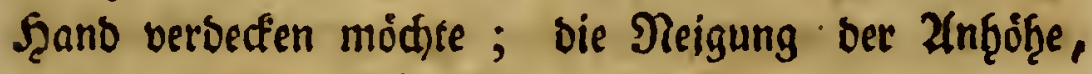
meld)e idf fah, fajajze id to ungefäb̨r auf $45^{\circ}$; ber

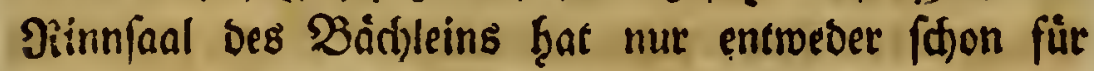
fid) felbfft fleine albfólle, ober fie graben if̧n in verz fápiebenen Entfernungen $a b$, um Eleine $\mathfrak{Z a} a f$ ferfälle zu erf̧alfen; unter dem Drte biefer $\mathfrak{B a f f e r f a ̈ l l e ~ v e r f e n t e n ~}$ fie in bie Eroe eime Ennofteinglatte, in bie fie vorţer verfficedene freisformige Sinnen gearbeitet, uno in bie Mitte ein Sod, bas aber ben Stein nidtt gang

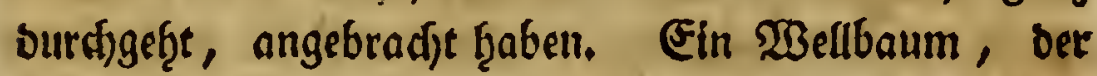
etwa etrons mef̧r als cinen fuß ḩod) ift, unten gleid). falls eine Platte von Gansftein, uno um feine Mitte.

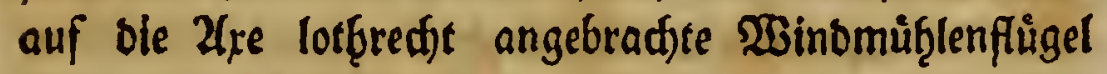
நat, wirb in biefes loch eingezapfet, unb von bem auf feine Flügel ḩerabfliefferden $\mathfrak{W}$ affer ḩerum getrie. ben. Die Eteine, weld)e zu Siluctern beftimmt find, werben vorf̧er in unförmlida Wirfel gebroctien, alle Ecten abgeftumpfet, uno baun in sie rinnen geleget, roo fie von dem herumtreibenden $\mathfrak{B e l l b a u m}$ of̧ne

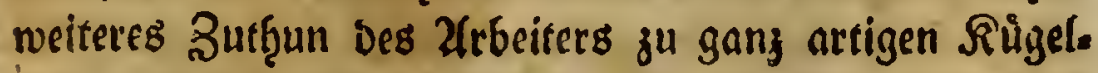
d)en gebrefeet werben. Der (Bebraud) bavon nuß 
mannigfaltig fernn, weil fid einige fleine Samillen

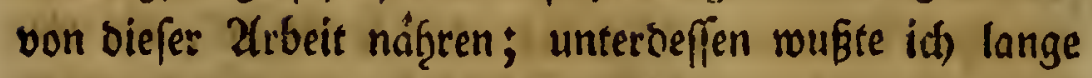
feinen antern, als gewiffe Spiele unferer Rnaben,

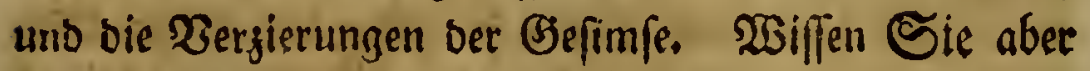
rooger, oaßs Bibraltar - -

2loer in siefen Srief

Rrade fein Zlammenffifuno,

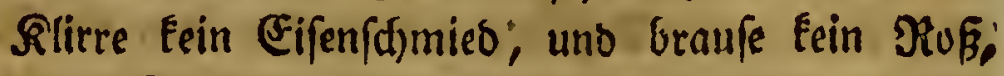
Evrlaffen Sic mir biefe 2 neffbote, wern alle Baiern

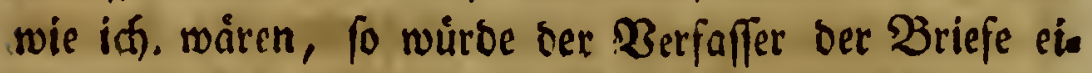

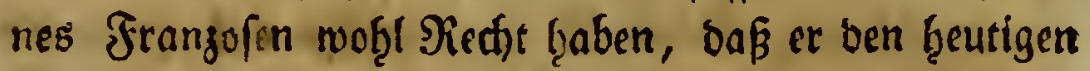

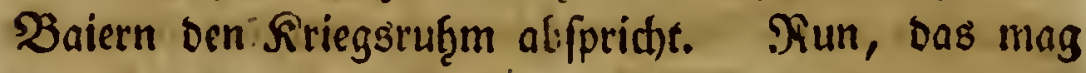
er meinetwegen; aber miffen mơdt)e id es bod), wo und wann er biefe widftige Entbeckung gemacht ţabe.

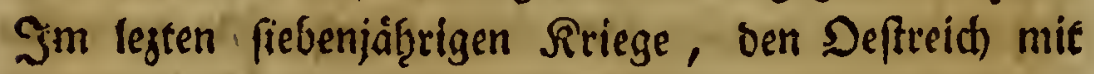

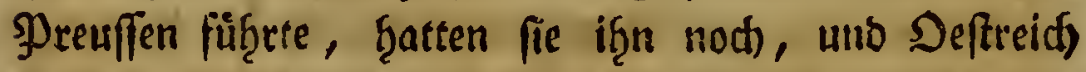

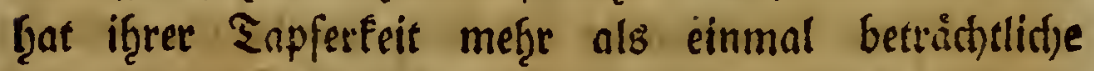

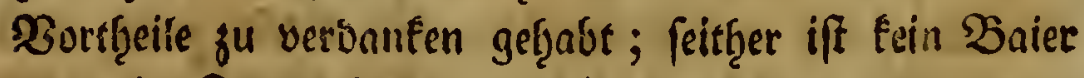
meģr im Jefo erfonienen. Doer will er, Daß fie im SaĢre 1778 cen bỉmmften aller Grreidje b̧âtten an. fangen follen, Dof fie in einem fladen kande, ofgne

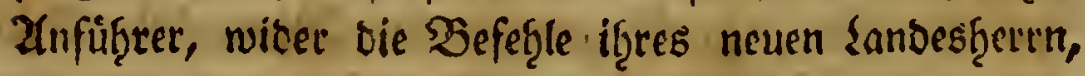
sie bie $\mathfrak{B o r}$ (id) ten im Jelbe erfffeinen folfen? - Zlber sie Müttae

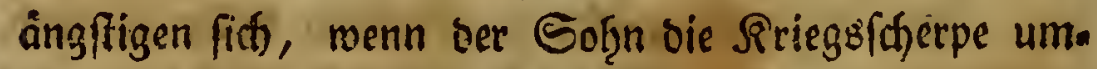
giirtet, uno bie Soffene woolten feleft nicsit gerne baran.-

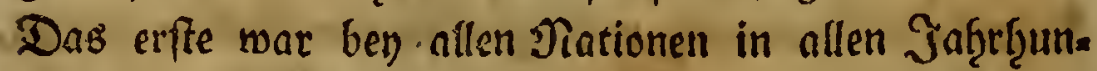
Derten eben fo, nur vielleid)t (bielleid)!!) Die Epartaner

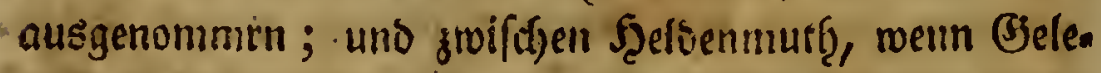
gentheit baju iff, und geizenbem Diang nach einem 


\section{Burglyaufen, Den 15. Jinn. I 784, 227}

färglidsen Lunterf̧alt, wie ber bes Rriegers in aflen sandern ift, finoe id ju viel Uuterffoies, als has id beyse biefe Dinge fo gröblid) verwedffelu foùnte.

Der Eģarafter ber Einwof̧ner biefes sanoes, benn it) Eeḩre mieber zu ben Berdftesgabnern jurúcf, ift ber glücflisfffe von ber $\mathfrak{Z}$ elt. Whre $\mathfrak{B}$ orte fino mir lieber, alb anderwairtig (Eibe, Eibe, bie id), waire id) ein (5) Fefęgeber, immer mit Folter uno Feuerptobe in eine Rlaffe forsen wirbe. Zllentb̧alben, 10o id ḩin. form, fano id) fie offentergig, uno uneigenmúgig, zmo ₹ugenoen, bie mit ien 2flpenpflanzen aus volfreid)en Santoern verovinget morben. Jlgre Sieligion if wie ib̧re Berge, einfăltig uno grof : if̧e Bottegfurd)t ift onģar ungef̧euthelt, und fómunt aus Ueberzeugung; gicid) weit von Zfherglauben uns Starfgeifreren ent fernet fef̧en fie feltme Dlaturerfiteinungen mit ltner,

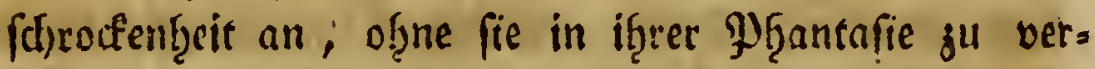

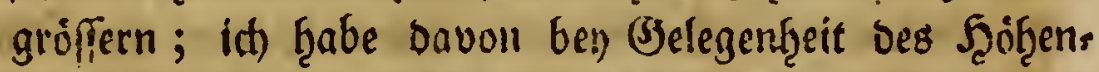
rnudics, ber im vorigen Eomnier allentf̧alben fo viel Sårmen verurfad)te, ein merfmůrbiges $\mathfrak{B e v}$ (piel felbit etfofgren. It) fam auf einer 2llpenreife mit meinem Sróger, ver nichte weiter als ein Styadfelmadter war, in eine Eennḩitte, wo wir unjer Mittagmab̨l einzu. Hef̧men Dachten ; die Senneriun unterhielt uns, wie ps

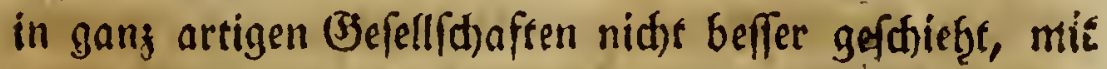

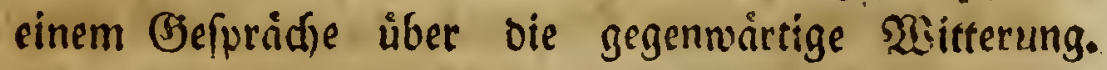
ISotzer mag wof̧l biefer f̧ibhenrauch) fommen? fragre fie enslid. Jd) wollte if̧r eben antworten, als mir mein Tràger bevortam; laffer uns um bie Lirfadje 
nidft fragen, fprad) er; er mag herffonmen, aus weldjer Ulifadse mall wolle, fo fef̧en wir, baß er in feinen Wirfungen feilfam fene: wir fraben feltnere und fofwidere Donnerwetter als ansere Gafire, uno bie Suaten fetegen fo freubig, als fie lange nicht ftanbert. Sie Sennerinn war mit biefer 2fntwort iufrieben, uns id) F̧ielt es für ìberflüffig if̧r meine Mennung ùbee bie Urfacte zu fagen, bie bamal of̣ne bieß nur noct) Mutf̧maffung war, uno aud) jejt nidjt viel meţr iff, ob fie gleich) eben biefelbe iff, mit weld)en de In Sande feine garifer getroffet haat. Iक werbe bey einer an.

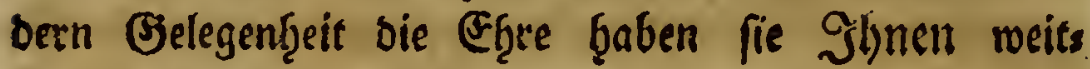
läuftig genug zu erflàren.

Einen vorjuglidfen 3 ung im Eḩarafter biefes

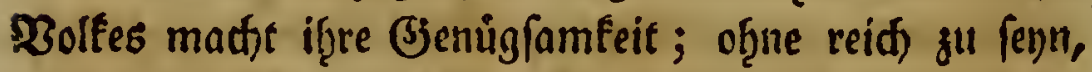
ia mand)mal bey wirtliçer 2 (rmoutb fins fie nit if̧rem soofe zufriedan. Ein paar $\mathfrak{B e n f p i e l e ~ m o ̀ g e n ~ G i c ~ b a v o n ~}$ u̇bergengen. - Es brannte einem Bauer zur Beit meiner 2 (nwefenf̧eit fein Scaus $a b$; ber J̧err Dechant, ein Siraf von Dictrid)ftein, gab bem erarmten Manne ein Befditenf, bas er mit sem rairmften Danfe all. naḩm; als er if̧n aber zu fidf befdrieo, if̧m bie fdsiftlidse Erlaubnis zu ertheilen, für feinen neuen BSau zu fammeln, verbath biefer bie Binabe, weil et fo mof̧i burd) bie gnábigfte. Unterftîzung bes f̧irften,

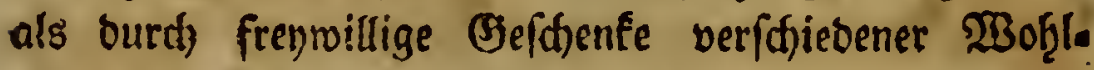
thiter im Etanbe ju fenn glaubite ben $\mathfrak{B a u}$ zu unfern nef̧men, uno feinen (J)ewififen zu naţe ju treten bes fürdtete, wenn er butch) feine Sammlung Bellegenţeit 
geben follte, baß sielteicift Dürffigern, als er, etwas

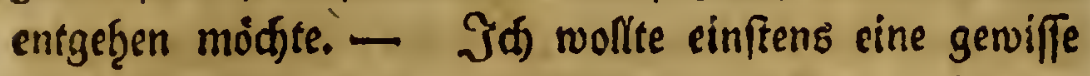

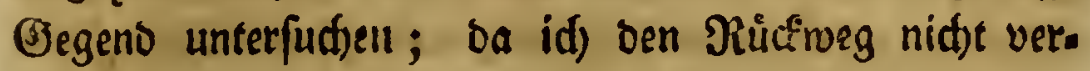
felfyen fonnte, fo gieng idf an einem Sunntage mit einem Manne, ber dafeltbft anfáffig war, nad) sem Bootesbienfte dab̧in; aber Der Drt war weiter ents fernet, als id vermutfeete, uno id fano midf ges

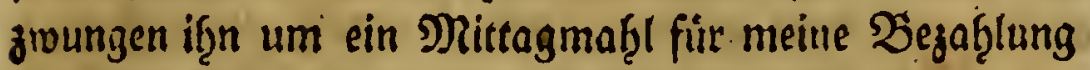
3u bitten; Der Mann entf(d)uloigte fid Zlnfangs, Daß er fein Sleif(d) batte; ; Dns will idf oud) nifft, ants mortete id, idf effe mit euff; wir baben nidf)s als Edfoten, gab bie Srau zuruide, ber ift aber fir ben

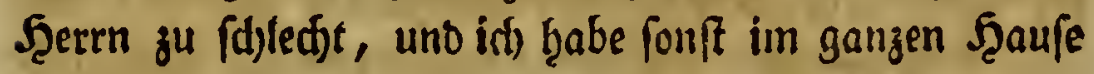
nidfts, als ein wenig Milds. Gut genug, war meine

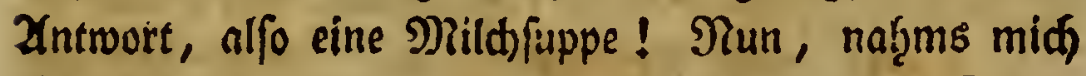

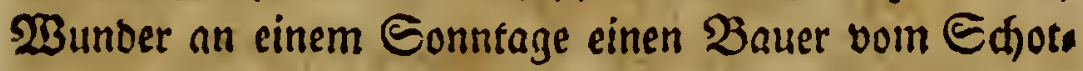

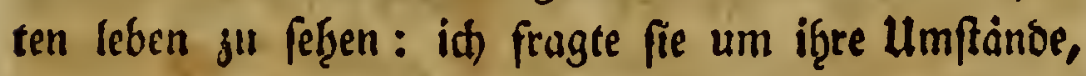
uno erfught, fie máren nidfts meniger als $\mathfrak{B}$ autersleute,

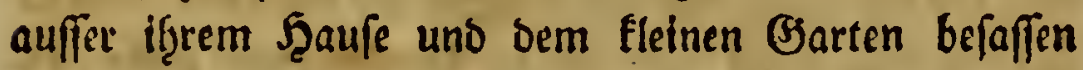

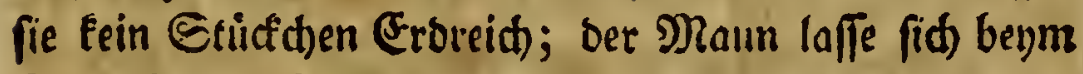

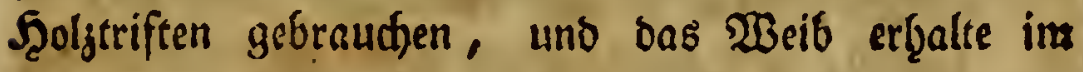

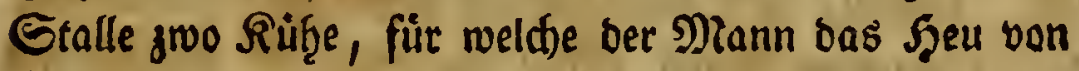
naben (jebirge aus unbenujten Gegenben Ģerborbrätte. uno bas iff alles, moobon ig̨r lebet? fragte idf. Zflles, gab mir meine $\mathfrak{B B i t t h i n n}$ gur Zntroort, ausgenommen, ba备 mein Mann fid) mandimal fo etruas bey einem Bauer veroienet, ber ilfm Ztbeit giebt; immer zwor wenig, aber bod, , Daß̧ wir leben fónnen, unb się if genug, zu Grunbe gefgen wir gewiis wiemal : Ģabcn

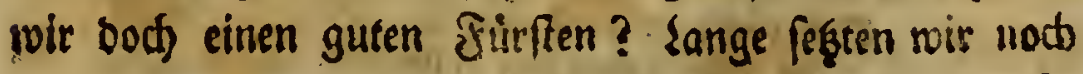


unfer (Befpräch) über biefen Begenftand fort, und iđ fofied voll ßenuunberung von iḩnen, gab if̧nen benm Ed)eiben ein fleines Bjefhent, und ḩatte Miủhe, fie ju überreien, es ganz anjuneţmen.

It) ergähle gerne sergleidfen Begebenbeitelt, weil id) mid) gerne an bie Sugent erinnere, bie in

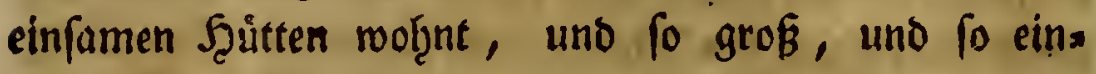
fach) iff, oa hingegen bie ₹ugeno in Palajten meiffens viel (B)othif d)es ḩat.

Der Gebanfe an ifgren f̧urften fheint übrigens Der lieblingsgetanfe ber :erchtcegaoner zu fenn; bey jeber Belegenţeit erzăhilen fie uen Fremben, nuie glüd. lid) fie feyen, dafi fie feine Unterthanen find, und oft fagen fie es unter Freudenţ̧tånen. Gott hat uns meb̧r geaeben, als wir verbienten, als mir jemals verdienen fonnten, fagten fie oft zil mir, uno fanien

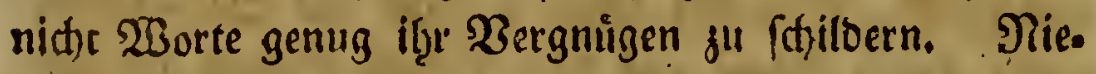
mal ift ein Jürift von feinen Uuntertḩanen mef̧r ge. liebt morben, aber menige verbienten biefe siebe fo feģr; leutfelig uno Gerablaffeno gegen jedermann, für

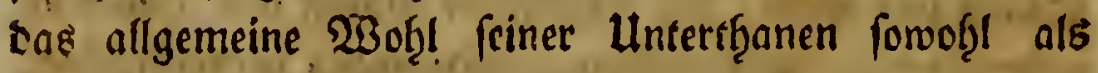
fúr vas fonberţeitlicfe unabläfig beforgt, wirb er gwar nicht

(*) Sofeph Sontad aus Dem frevberrlichen Shaufe yon Edjrofenberg ward ben 3. Sorn. im 9. 1743. aebohren, legte in Berditegganen bie fenerlecthen Droengeclibbe ab Den 28. Noremb. i770; mard full allgemeiner Freude zum

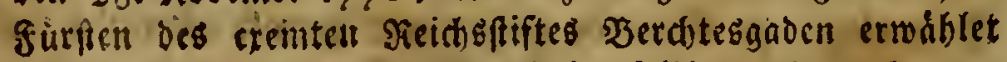
Den 8: Mado 1780 , morauf noch im felbigen Nonathe, am 2 sften, - feine untertbanen Dett Şuldigungseio ablegten, und 
Sourghaufen, Den 15. Jănn. I $784.23 \mathrm{E}$

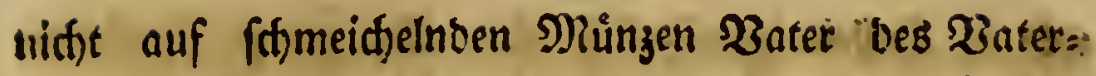
lantes genennet; aber if es, uno tief in tie Şerzen.

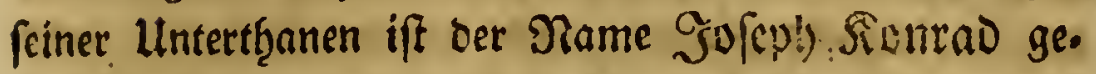

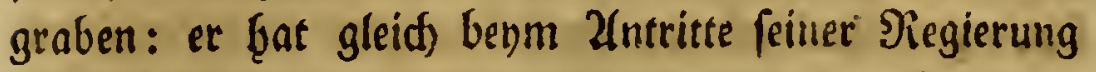
23 unber geţ̧an, und wie meife und gerectit feine

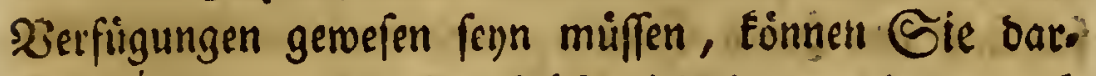

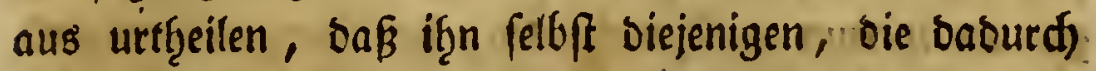

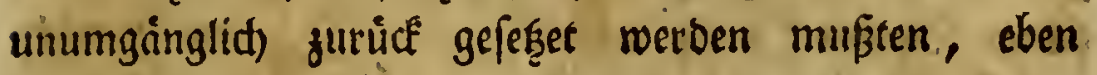
barum beloben. (*)

Noidffens, mein ₹̧̧eurefter! folten Sic etroas

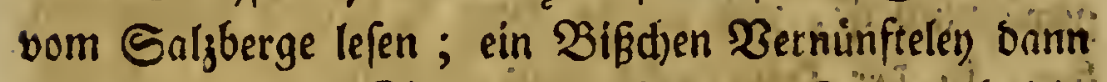
oben brein, bie Sic mir gefâllig ju Bütem haltên

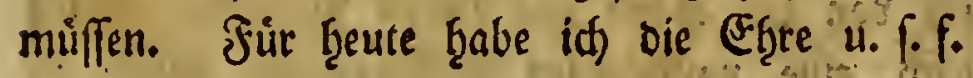

Edrane.

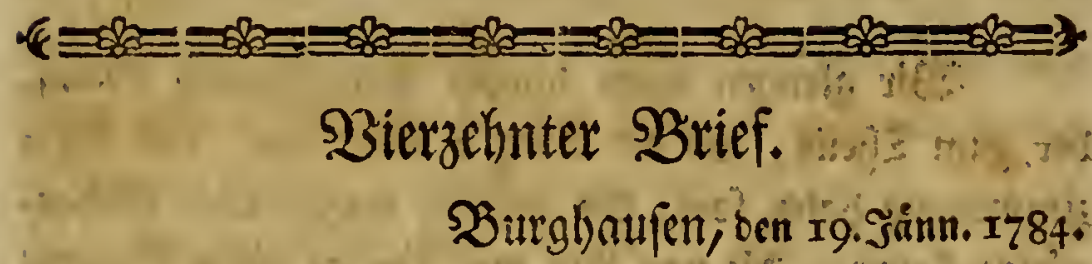

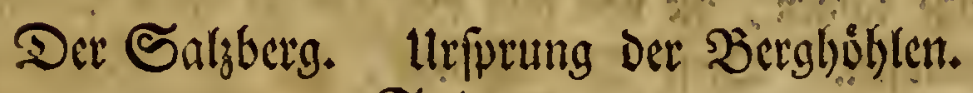
Salzquelle.

(S) en 30 Sunius befungr id das Galjwere, of̧ne babey) fo viele Umffainbe miadjen zu múffen,

$$
\text { शु } 4 \text { mie }
$$

am 24. 2uguft eben Deffelben Safres nard ar jum \robften: eingefegnet. Der allaemeine $\mathfrak{B}$ unifio aller Untertballen ift: Serus in coelum redeas.

Saben meine acachwartiae \$rriefe einigen 2 Berth, fo fallt aller Berbienft cavon auf dicfen crbabenen firtiten, gurủc,

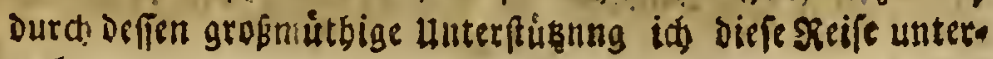
nabni. 
wie in Saallein gerwosthnlid iff. Die ganze Borberels tung beffant sarinn, oaß id meinen ?iod mit einem weiffen leinenen Rüttel, und meinen Şut mit einer fdiwarzen Grubenfappe bertaufdhte, uno das Bergleber um bie Senben gurrtete. So gefleiteet gieng id in (Ges

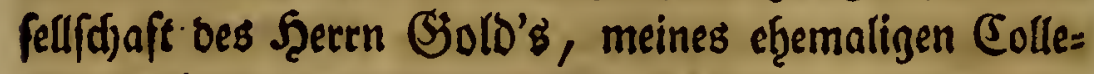
gen am \{nceum zu Burgḩaufen, bes Bergmeifters uno feines Brubers nad) bem $\mathfrak{B}$ erge. It werbe mid ben $\mathfrak{B}$ efffreibung biefes $\mathfrak{B}$ erges furz faffen fönnen,

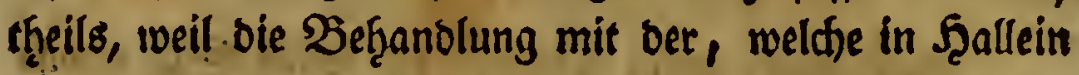

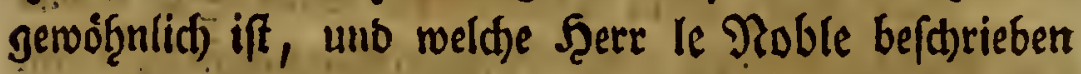

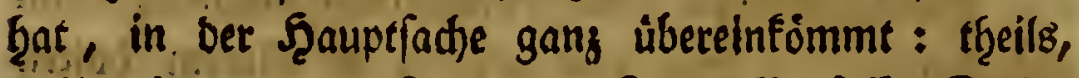

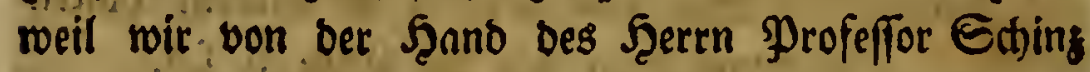

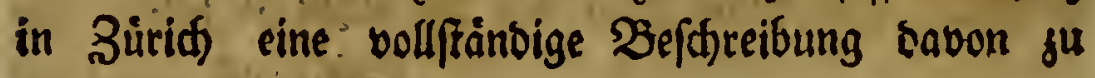
erwarten Ţaben. -

Wir giengen einen langen unteritbifheu Gang, ber zum ₹̧̧eile, wo der Bèrg brüchig war, eine Bero zimmerung Gatte, fort, bis mir un verfdjiebene Neben. gänge famen, bie von ingm wie Zweige eines Boums auslaufen. Diefe Nebengånge, oie fie Sdaftrite (Edjadftridte in Şallein) nennen, werben jeber burd bas Einfwert begrainzet, uno fino evenfalls an ges fág̨rlicten Dertern vergimmert. 2luf sem Bimmerwerfe fan' id Gier und bort eine groffe weiffe Sdjimmelart (Mucor) feffifigen, bie mir feḩi auffalleno war ; id

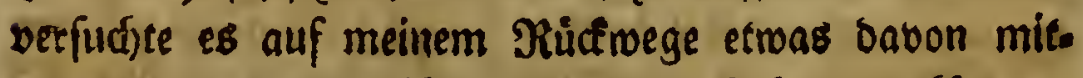

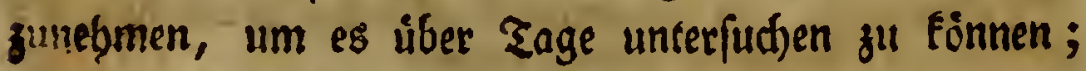
allein als ids barnad) langte, befam idi) nidfes als

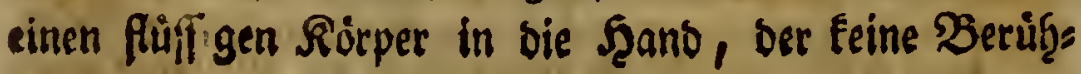




\section{Burgliaufen, ben 19. Sămn. I 784. 233}

rung ausfieett. Da, roo Das Geffein ber Strfadftridft oģne Bimmerung, aber bodt mit weidfern $\mathfrak{B}$ ergatten

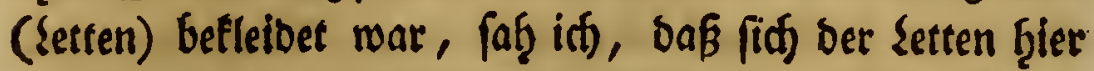
uno bort vom feffen Geffein ablófere, ba bann alles mal Ginter bemfelben bas reinfte, weiffefte Sals. auswitterte.

Enolid famen mir in bas neue Rapiter; to ḩeíft das Sinfwert, aus meldhem die leęte Surr war

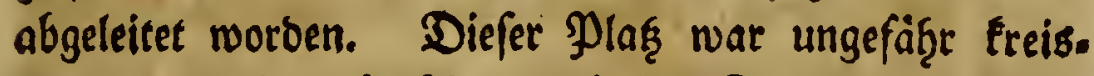
runb, und b̧atte in feinem mittlern Durdfffdnitte ettiva

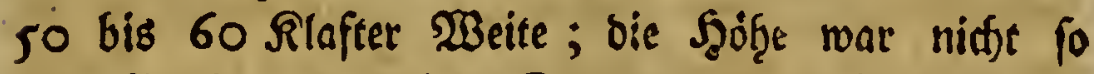
gleidformig: an einigen Etellen war fie benuahe von 7 Sdifuber uno nod) groffer, an anbern mod)te fie

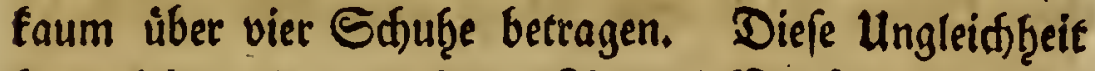
fam nidft są̧er, Daßß ber Şimmel (J̧irfî) nidft follte bollfommien wageredtit gemeferen fenn, fondern theils weit

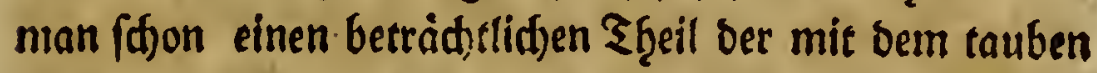
Ealje zu Boben geftürzten tauben Bergarten, bie als Edfutt zurưçgeblieben waren, ausgefübret ḩatte, tḩeils von ber waâtrfdeinlid) ungleiden Madattigfeit Des Saliffocfes felbff. $\mathfrak{u}_{\mathfrak{m}}$ mid, Gier verffanblich genug ausjubrùcfen, muß idi bie Bearbeitung Des Ealigftoctes in Rürze erzáglen.

Nadfoem man bie Sdjaftrit (ben Stollen) fo weit getrieben ḩat, als es notḩwendig war, bas iff, bis man in einen mád)tigen Theril bes Saljffectes eingebrungen, baut man ju banden Єeiten einige furge

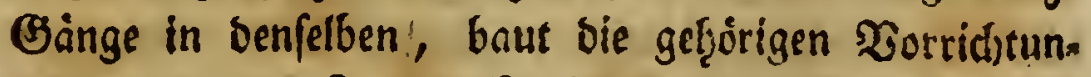
gen, um das Tagewaffer in biefen Drt einzulaftu, 
einen Damm, damit es nid)t wieder ablaufe, uns

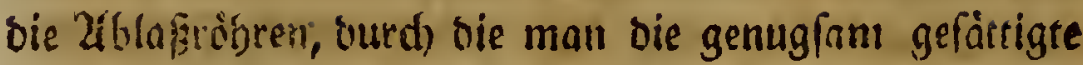
Sully nad) Beslieben ablaffen fann. Siad)iem bieß alles fertig gemorven, und bas sagewalfer nad) ben nun j̧ugemauerten (veroámmten) Ţ̧eil ber $\mathcal{E}_{d j a f t r i t}$ zu flieffen angefanigen hat, berliert biefe ben biskgeri-

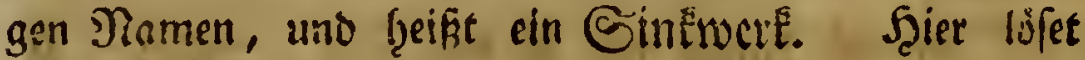
nun ens Baffer, bas bis an ben Scimmel (firft) berauf gef̧en muß̧, allmåģlig ben Snljftein auf, uno färtiget lid) mit feinem Ealge, unterbeffen bie tauben Sergarten ju $3 o$ en fturrjen, bie nothiwenoig oa in grôffern f̧aufen liegen mulffen, wo fie von dem Ealje weniger ourdorungen waren, bas iff, wo bas Salz einen Eleinern Ţ̧eil if̧ret Maffe ausgemad)t ţatte.

Die Rebensart ber $\mathfrak{B}$ ergleute, daß Daz Salz bom Scimmel fonme, ift nitht fo zu berffergen, als

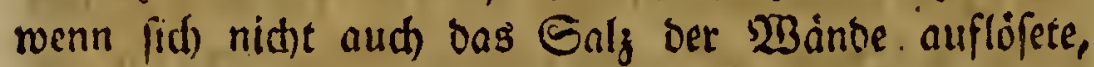

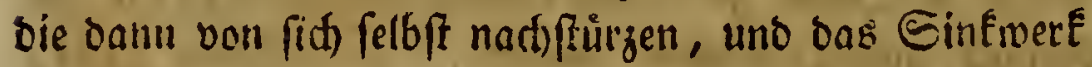
erweitern. Da man nun von Beit zu Zeit wieber neues $\mathfrak{B}$ nffer in bas von ber leztern Sulze gereinigte

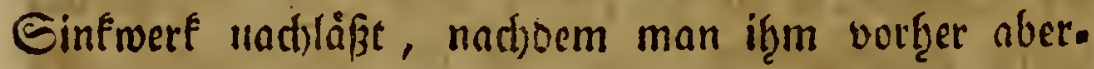
$\mathrm{mal}$ in sie $\mathfrak{B}$ inde Gincin vorgearbeitet ḩat, fo ift es ganz natürlid), oaf bas Sinfwere nad) uno nad) eine ganj erftaunlidje siseite befommen múffe. Es iff in oer SGgat unmơglid einen fo meiten Faum of̧ne Woólbung uno Stüz̧e zu ref̧en, uno oariber fiir fein

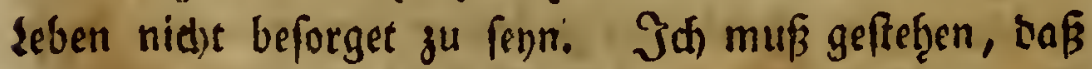
id) ben aller meiner Llebergeugung, baß sergleiden Sintwerfe Jaf̧rḩunberte ftę̧en, unọ fogar benufgt 


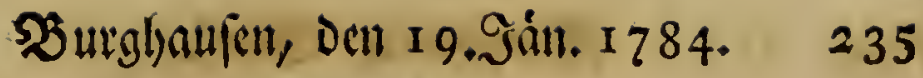

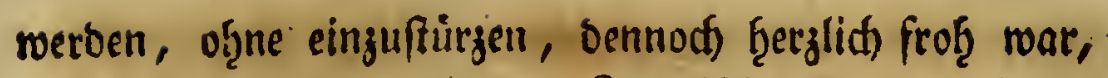
als id wieber ţeransfom. Ingwirfthen fielt mid ther

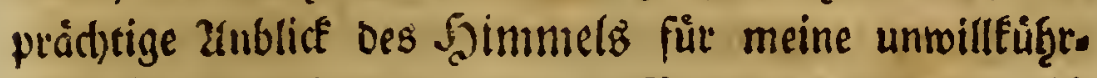
lid)e Furd)t fadablos genug. 230 maan şinfieftet, ift

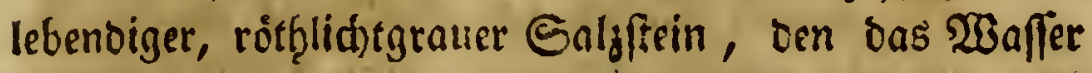
bienenzellig burdsfreffen bat, an beffen (Eden fia) bie (s)tubenlidter, rwie an Diamanten, fpiegeln.

Der 2fnbficf biefes Einfwerfes, wobey, wie Sie

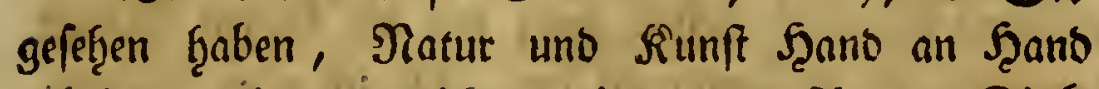
arbeiten, erinnerte mid) an eine unoere art von EinEs werfen, bie blok 23 erfe ber Natur fino, ić menne,

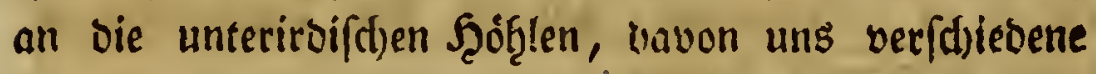
Belef̧rte fo vortrefflid)e $\mathfrak{B}$ efdireibungen geliefert ţaben.

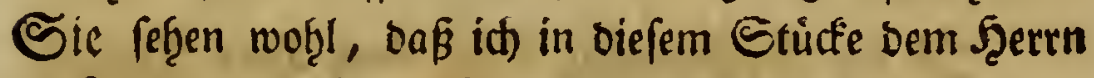
be suc nadbonfe; aber id) fobere jeben auf, ber feine SBriefe úber bie (befdid)te ber Ertoe uno bes Menffiten gelefen, und bann einen Salyberg befaf̧ren ḩat, ob if̧m mỏglidf fen, fid) Des Belffalls ju er. meţren, ben bie fdjarffinnige Ţ̧̧eorie yon ben unter:

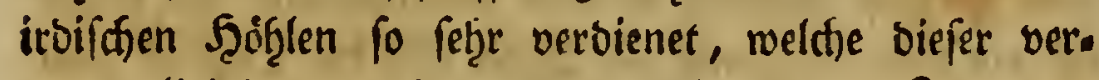
traute liebling ber Natur entroorfen frat. Sil) waro nad) fer auf meiner Seife nad) ben Serdtensgadenf(d)en Ralfgebirgen in meiner erften Soee nod) mef̧r beftäto tiget, bort fah̆ idjs mit eigenen 2(ugen, wis bas $\mathfrak{B a f}$. fer ben Ralfftein auflofet, fah bie lecther, bie es f̧ier uno bort tief in ben Bruno gefreffen hatte, bitte an einigen Eteflen, wo er feid)ter log, fein unteriroifdjes Eprubeln, uno mun ftano id feinen Zugenblice meţr an, mir bie \$egriffe über biefe Eadfe, bavon biefes 
feltere Benie nur einige fluddtige Büge bingenoorfen, ganz zu entmideln. Gef̧en Sie, wis int mir bie Endje vorftelle.

Die groffen Maffen ber Ralffteine muifen fo gut, als jebe anbere Steinart, iḩre Siluffre ḩaben; bieß ift eine notf̧wenbige Folge ber Berwanblung ber Erben in Steine, bie burd) ble bloffe Zattraction ber ₹geild)en ganz gewiß bewirfet wirb. Diefe unter. irbifchen Spalten ftellen ganz gut bie furzen. JSänge am Enve ber Sdjadtredjt bor, bie id) oben befdrie. ben ḩabe. Wir b̧aben uns nun blö um einen 2 Abgang: fdhurf zu befümmern, baburd bas Tagewaffer in biefes Ginfwert fomme.

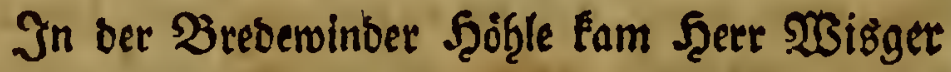
bis an bas Ende berfelben, no fid ons Bebirge bis ein enges Sod) abfdynitt, bas cine ftarfe Bugluft ver. rieth, baben aber fo enge war, baß faum ein fieben.

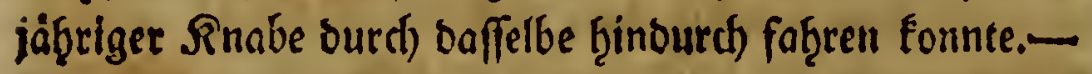
Eine Bugluft verrâth offenbar (B) luft vor bem Eingange ber א̧ōb̨le mit ber Tageluft b̧in, ret biefem Sodhe. (*) Gollte man nidjt biefes lodf für Den Stollen balten, meld)e ehebeffen bas Sagervaffer in biefes Sinfwerf leitete? Es mag renn, baß etheo oeffen úber biefem lodge ein Gee geroefen; aber wir

ḩaben

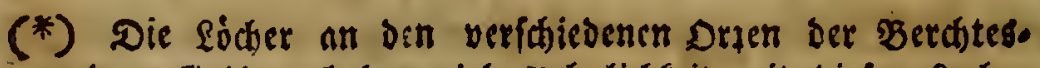
gabner Bebirge baben viele alfinlichfeit mit biefem Soche. DSen einigen bơrt man fogar, wenn man sinen Stein binabuirft, tief unten cin 2tufwlaksen, wie, ween bet Etcin in soafler gefallen wáre. 
Burghaufen, Den 19. Jun. 1784. 237

bzaben biefe Rorausfeģung nidit vonnơtḩen. Das langfain burd bie Spalten burchfinfende, uno biefe Epulten felbft erroeiternoe $\mathfrak{B n f f e r}$ bon Regen uno fanmeljenbem Edinee ift mäctitig genug, bergleidjen f̧bुglen zu bilten, befonbers, ba es nicfte gerabe Durch) eine, fonoern ourd) unjäḩlige Rlúfte juges füf̧rtet mirb.

Dos $23 a f f e r$, bas num einmal an ben unter. irsiften Epalten nagt, wiro freylid wofgl bie auf. gelöpeten Eteintf̧ailition irgendroo abfoģen müffen, weil

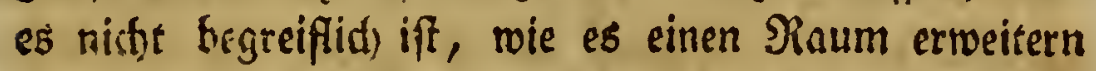

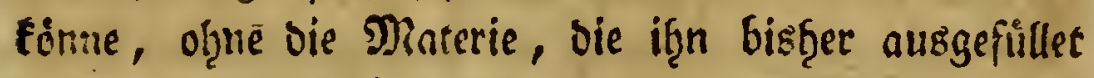
batte, ous bemfolben fortjuld)affen; nidfts fann if̨m aber leíter fenn, als biejes: ba bas (bebing nad) allen Siidtungen, mitf̧in audh in bie Tiefe, Epalten Ģat, fo wiro es die aufgelópeten Theile mit fich in oie Tiefe fortführen, fid) balo biefe Deffnungen mit ben unternegs abgeferten Sheilen felbft verftopfen, balb

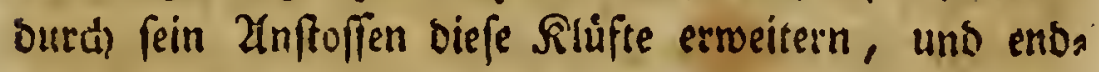

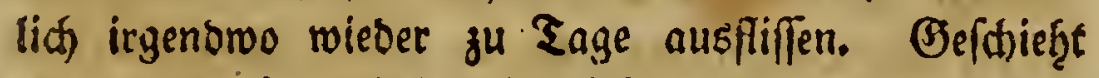
bas lef̧te, to entfeţen in tiefern (S)egenden \$uellen, ober woffl gar Eeen; gefdiefft aber bas erftere, fo firto misder meţrere Fálle möglid): entweber berftopfen fich bie mit Eteintz̧eilen nngefdiwängerten $\mathfrak{B a}$ âfer nach) und nach felbft alle 2(usgange, uno in biefem

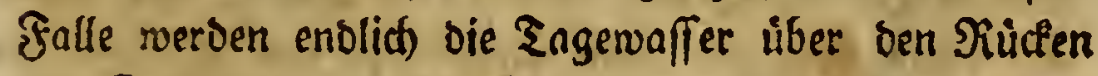
Des Bebirges in Eleinen Baitien baf̧inniefeln, uno gleich nnbern Bebirgmaffern bem ermübeten Săger, ober bem eblern 2 Banderer, ber bie Natur auf biefer 
ந̧oţen Edgule ftubiren mill, lange gemuinfdite Erfrio fatung reidjen. Dringen fie aber nach und nadf burd) bie abgefeģte, aber nod) nidft feftgemordene Materie, bnoon fie måḩrendem Durd/flieffen immer wieber etwas nbreiffen, fo lange fort in bie Tiefe, bis fie enolid) einen 2lusgang finben, fo haben wir aber.

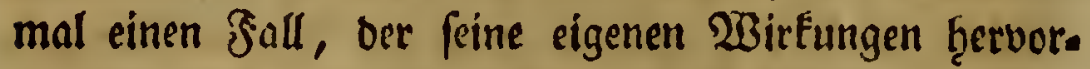
bringen mus. Je länger fie on biefenn Bafteine lecfen, uno bas aufgelöpete forffuifiren, oefto groffer wiro ber Shaum, oen fie fid) f(haffen, befto groffer bie Dberfládhe bes $\mathfrak{B a f f e r s ,}$ bie an ben $\mathfrak{B}$ inden nagt, befto groffer ber Drang nach oen $\mathfrak{B}_{3}$ antoell, bis fie enolich) irgenowo gar einffurrzen, uno bem zerftorrenden $23 a f f e r$

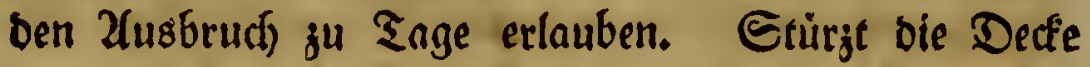
ein, fo wiro vielleid)t ein Eee entffeţen : frurgt aber eine Seitenwanb ein, fo miro ein Theil bes 2 Baffers mit Ungeffinme herausftroimen, aber immer wiro nod)

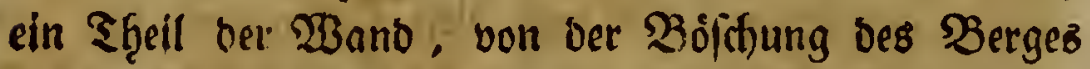
geffủrzt, gatrưubleiben, es wirb alfo eine betrádflidie

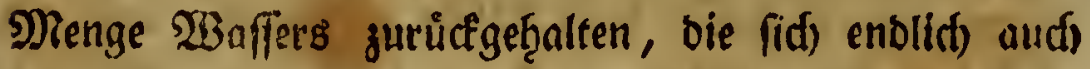

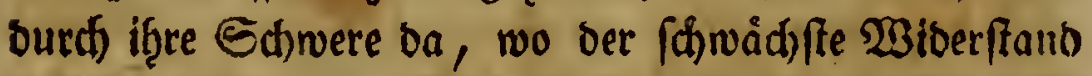

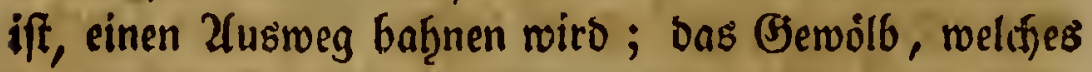

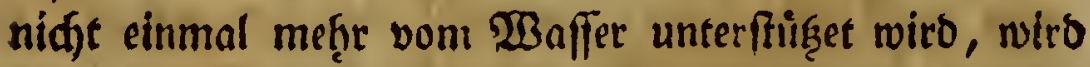
Gier und bort nadffinfen, einige ₹̧̧eile werten fich) gar lostrennen, f̧ier und bort mirt ein Treil biefes Einf. werfes gar einfturzen, und es ift gewißs, oaß biefes offt fotion furg vor oem 2lusbruche bes $\mathfrak{B a f f e r s}$ ges

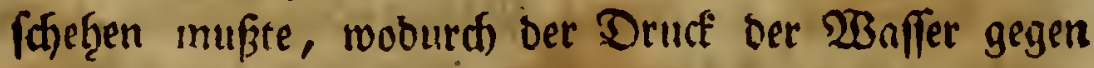
bie SBánbe bain nur noch mehr verftarfet warb; Daf̧er mukisten fo viele Unebenţeiten foroogl auf sem 
Boben, als an ber Derfe uno ben ßainden biefer J̧îflen entffeţen. Gonberbar ift es immer, aber aus biefer Theorie leidf) begreiflid), baß̧ man nod) bent. zu Sage in allen greffen Syob̨len $\mathfrak{B a f f e r}$ attriffe, bas nod) baju faft allemal bie beutlidften Ifugenzeugen

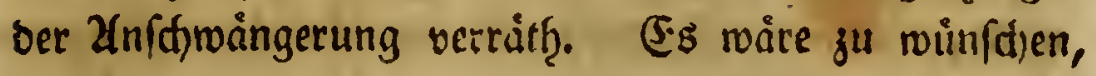

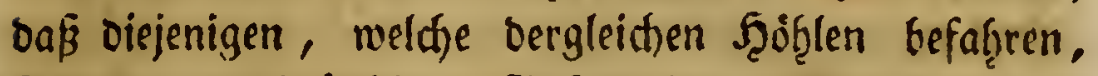
ftatt ber meitläuftigen $\mathfrak{B}$ efajreibung ber felffamen Stalactitenformen uns mit ben Seftandtbeilen ber in biefen Wereffatten ber Natur vorgefunbenen $\mathfrak{2 B} n=$ fer befannt inadjen wollten, id) bin verfidjert, baß allemal if̧r vorjüglidffter Seftanotreil eben. ber fryn rerbe, der bie Şauptmaffe des 'Bergez felbft ausmadit.

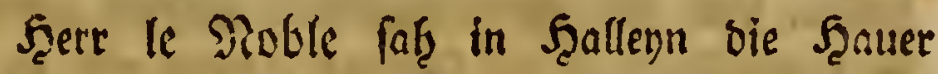
(Eifennuird)er) meiftens nacteno arbeiten; er foteint

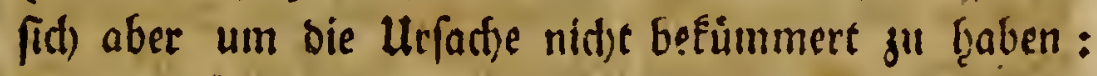

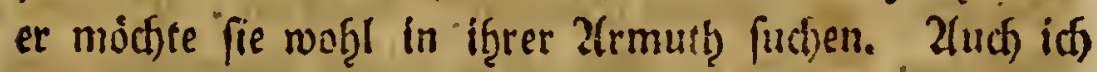
fat fie in $\mathfrak{B e r d}$ tesgnoen nidte anders, uno verlangte Die Ulrfactye zu wiffen; bie alnfwort war foịt befriesi. geno, fie thaten es um ben Edyueip fo visl moglith zu verḩinbern, der mittels des Ealzfaubes, welther fiit) ourd) vie S.ciber gleidjwotêl nid) Ģinlänglid) $a b=$ ḩalten lieffe, iţre গ̧aut angreifen múrbe, fo máren fie aber bem Edjweiffe viel meniger ausgereset, uns sugleid) im Etanse fict) Den Etaub von Beit zu. ลeit vom Keibe ju mifden.

Zuffer bem Eteinfalje mürbe Serdfergaben gleidinoogl nidft olgne eigentşumlidfes Sals fenn. 
$2(m$ Wege nad) oer Biffjofswiefe, nidft weit binter. ber fogenannten (Smunonerbrúcfe, ùber bie man nad) Sufţ̧eim, einem fürftlicfen sufffóloffe, fòmmt, quillt ein Waffer am Fuffe eines lanbberges ţerbor, bas 24 Mobel bृailt, aber bem ungeadjtet nidje benuf̧et wiro, weil bas Steinfalz viel befrảdjelidjern Gerwinnn abrwirft.

Zflle bergleidjen Salzquellen feģen abermal naturtlidfe Sinfwerfe, bie fict) beftänbig erweitern, aber enslid) einftürzen und verfiegen muifen, boraus.

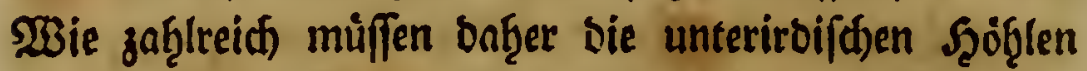
fenn, bie bie Natur fdion peit Jaf̧rb̧unberten auss gräbc ! Und, alles zufammengenommen, wie wab̧r. fdifeinlid ift es nidst, Daß ber ganze Ersboben allents

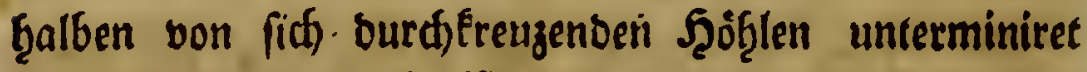
fey) ! und ifts Dann ein $\mathfrak{B}$ unber, wenn wir in ber (5e) f(f)id)se bie traurigften Begebenţeiten bon geborftenen Bergen, verfunfenen Stäbten, Märtten, Dörfern,

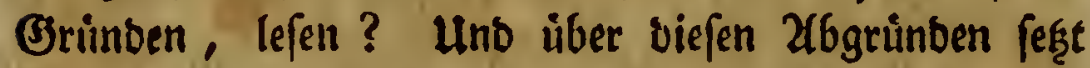
fid) ber Menfa), bas verwegenfte aller Brefajopfe, ḩin, mad)t weitläuftige Entwürfe für fommenoe Beneras tionen, ober fpottet woh̨l gar ber Bjotţ̨eit, untergräbt frembe ₹ugeno uno Ȩ̧re! - Wie mandffältig firto

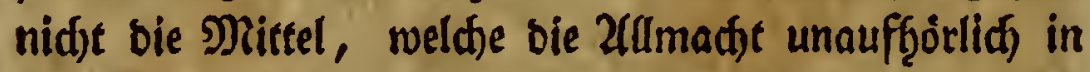
J̧anben ţat., Den Unfinn ber Men/d)en zu ftrafen!

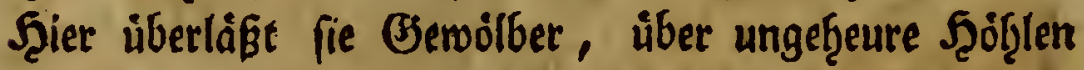
gefpannt, ifrem eigenen Bemidte; ; Dort fenbet fie J̧eere von Ş̧eufdrecten Ģin,

die vor fich) blübenbe ffluren,

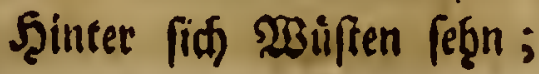


\$3แrghaujen, Den r 9. Jảnn. 1784. 241

ein anbermal verję̧rt eine gefráfige Raupe io wenigen

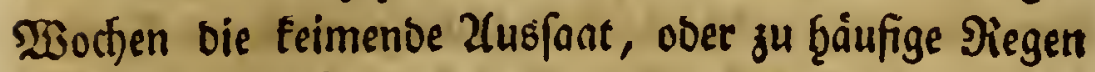
fròmen Sănberberberben, unb f̧unger, und Sied)。 ţ̧uाn நृerab.

Id) ùberlaffe mid) fef̧r gerne foldten Betrad). tungen. Die (Beffhidfte ber Natur, wenn man nidft bloß bely trocfenen Namenverzeidyiffen, ober magern faftlofen Befdreibungen einğelner Rörper ftę̧en bleibt; hat viel Seelenfebenios, uno füḩtet auf zaf̧llofen

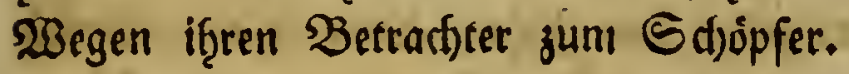

Dod) Shlnen fino biefe fanften Siuthrungen fo

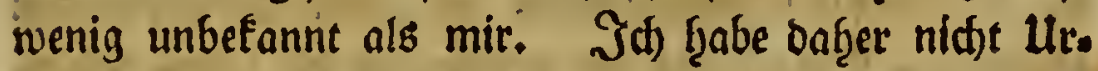
fact)e, Gie erft saran zu erinnern, fonbern fablieffe oen $\mathfrak{B r i e f}$ mit oer 2 erfiderung $u$. f. f.

\section{Sdirant.}

\section{Funfaefunter SBrief.}

\section{Surghaulen, den 4. J̧orn. x784:}

Exfte Allpenteife. Buriftung za Derfelben. Berge maiz. Şóbenrandy. Gdhneibftein. Sortfdreis: ten Der Segetation. Emiger Schnee.

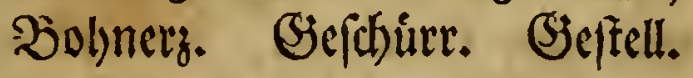

S fen erften Julius trat ich mcine erfte Jieife in das Jeathgefirg an; fie gieng viber bie $\mathfrak{B e r g}$. mais, oie Rrauscafamais, Den Jinnar, ons Rünigs. thal, ben Rinigsberg, bis an den Edfneibltein an ber falgburgifden Örầnge, von ba gieng id) zurưd Finturbifi.zsiefe 1. Band. D. में 


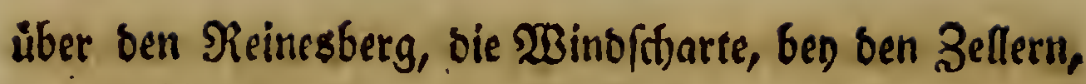
bas (J)efd)ürr, neben bem Sanfilaberge nad) ber Mitter. buitte, von ba ciber bas Beffell nach bem Kauffelbe

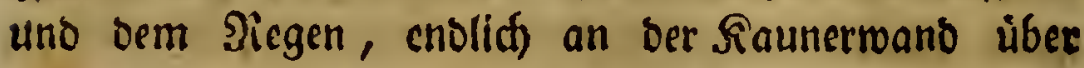
bent Raunerfeige an ben Rònigsfee, wo id) mid nad) Et. Saartfgolomàus ủberfdiffen ließ̧, um von ba

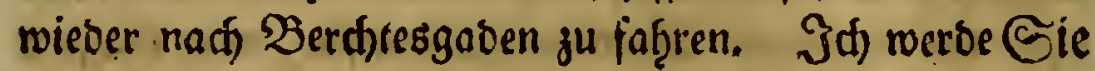
in Diefem sBriefe weber mit ben Dflanzen, noch mit ben Thieren, bie mir auf oiefer פieife auffieffen, unfer: Ģalten; leztere follen ben Jinţalt eines eigenen $\mathfrak{B}$ riefs ausmadien, und von ben erfern werbe id Shyen

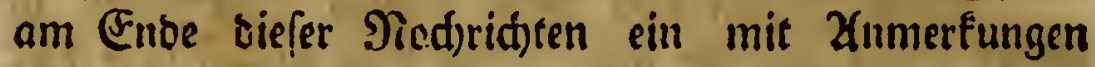
begleitetes Berzeidgniß̧ zu úberjenden Die Erg̨re б̧aben.

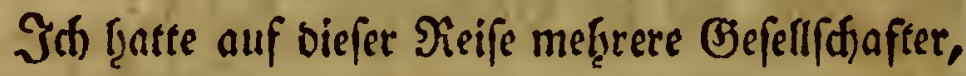
unter meld)en fith ein Srriger befand., ber nebft ben Sebensmitteln mod) einen Rik söfdpapier, einige mit

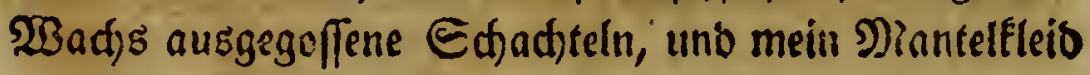
zu tragen ḩatte, erffere jum Zuftrocfnen ber \$finizen, uno Zuifbenafirung ber Jufeften, leştercs aber, um midh wiser bie $\mathfrak{R a d}$ tfrófte ju bemaḩren. Diefe $\mathfrak{B o r}$. fid)e mar. nid)ts weniger als ùberfiuffig: ob itf) gleid ùber einer $\mathfrak{B}$ efte bon Beug einen tidsenen Siode an. byatte, fo wäre mir bennod) bie Bebirgluft an ben Zabenben nad) einer tagelangen, faft beftanbigen $\mathfrak{B e s}$ roegung ofgne ein Mantelfleio eben fo beftowerlid) ges mefen, als in ben wirtlidy falten Etunden bes be. ginnenden Morgens, น́ber bieß meinem saget von Şeu aud bes Nind)ts rortrefflicte

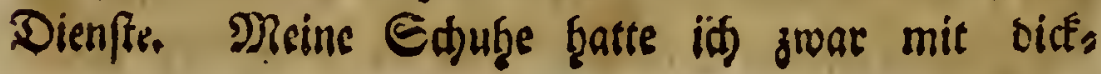




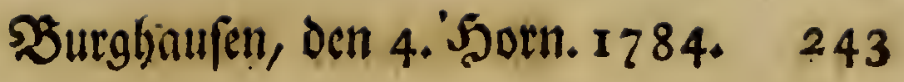

foligten Binsfduufen von Suften vertaufd)t, aber vergeflen, fie mit fpizfópfigten Nágeln befdlagen zu lafs jen, eine Unact)tfamteit, sie mir auf biefer গieife viele Be(fimerben verurfactite, unb bie id mid in ber Golge abermal zu begę̧en forgfáltig நূưtete.

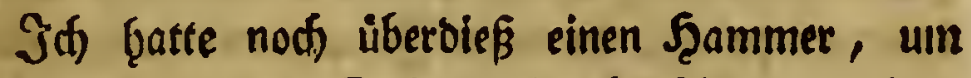
\$2robeftưfe von ben Steinen herabjuld/lagen, einen Brief mit Etecfllabeln, unb ein Ssangzeug für bie Ins retten zu mir geffectit. (Eine blecherne Siudfe, wie id fie in meiner 2fnleitung bie Naturgefdichte zu ftubiren

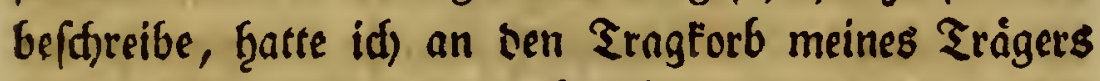
ongergänget; id fand es aber balb bequemer, fie ba, wo idf feinen Fall ju befürd)ten ḩatte, felbft zu tragen.

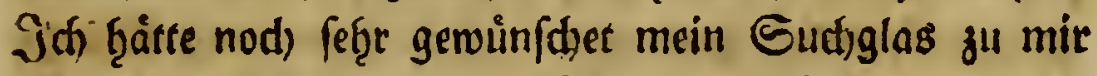
zu frecten, allein fomob̧l bie Jurdt) baffelbe zu verlieren, als ble $\mathfrak{B e t r a d ) t u n g , ~ w i e ~ u n n u ́ z ~ e s ~ m i r ~ f e n n ~ m u ́ f f e , ~}$ on id ouf vergleidyer Rieifen weber Gelegenbeit, nod)

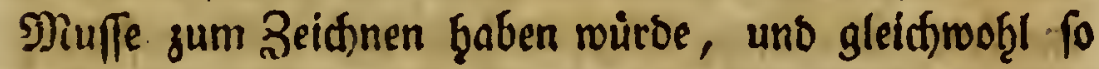
fleine (Segenffande, bie fid) burd) baffelte entbecten lief: fen, nid)t woofl fortbringen fönnte, bielten mid foroof̧l bei) biefer als ben folgenten Sieifen $a b$, es zu ţ̧un. zlber es ift 3eit, mit biefer 2(us/finveifung abzu. brecjen, um auf bie Reife felbft zurúcf zu fommen.

Wir maren ffion einige Stunben immer bergn gegangen, als wir auf bie $\mathfrak{B e r g m a i s}$ gelangten, eine

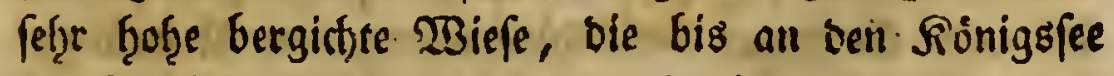

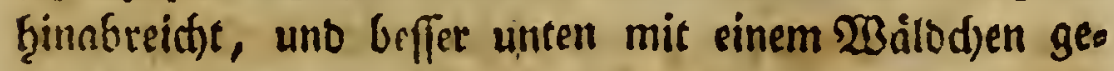

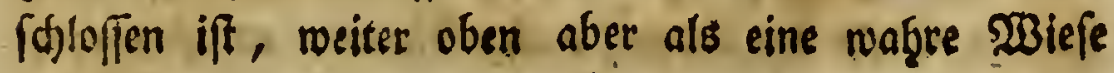

$$
\text { a } 2 \text { bea }
$$


bef̧nnbelt nitb. Das 2 bort Mrais bebeutet fier to gut, als in einigen Begenoen Baierns onz einen Drt, von bem man ef̧ef̧in bie $\mathfrak{B a l}$ aung abges trieben, um if̨n zur Cultur bequemer ju machen, onb̧er eini.je Dorfer und Marftflecten ben Mamen Perlesteut (Berd,toldsteut), Bifdofstcut, Sodenniais u. f.f. bee fomment traben, in berer Bufammenjerzung gemeinig, (id) ver erfte Ţ̧eil Denjenigen beoeutet, auf beffen Befest ober $\mathfrak{B}$ eranlaffung bie 2lbtreibung nes $\mathfrak{W a l b e s}$

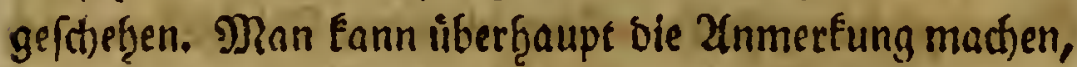
Dnß bie meiften Flecten und Dorfer, in felbit anfegrn.

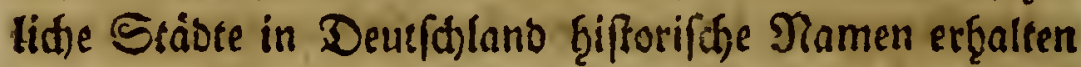

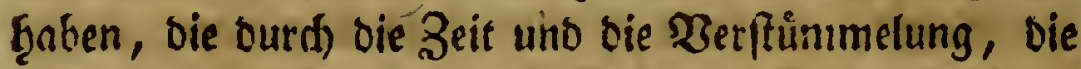
fid) oie Nadyfommenfdafft exlauber, nad) uilo nads gang unfenntlid) geworben. (J) hatie nidst urfacbe

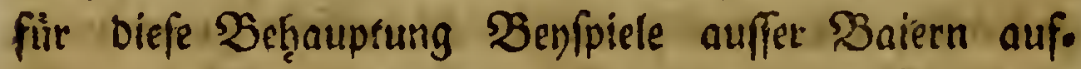
zufut)en, Miunthen, Sanbef̧ut, Riegensburg (ons in altetn Edfriften inmer Nieginesburg beift), Etabt

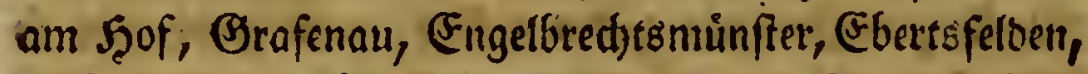
(Eberbarfsfelden), SBurmannsquicf, bie Tirfen, Zell,

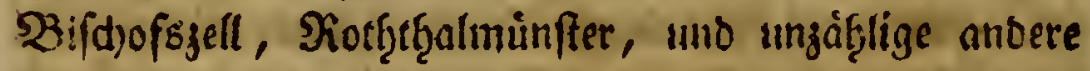
Derter, bie jebem leicht beiffallen, uns augenfderinlitife Barwife bavon, felbft die Berge, bie iđ) auf viefer uno meiner folgenten Reife befudjet liabe, ber Rönigse

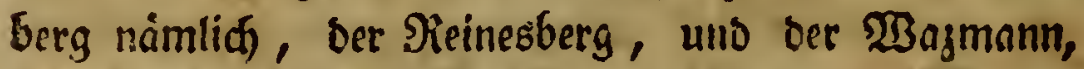
ob fie gleic) unberwofint finb; unb efieber nod) weit rauhęer gemefen fenn mägen, ffeinen baf̧er zu gef̧oren.

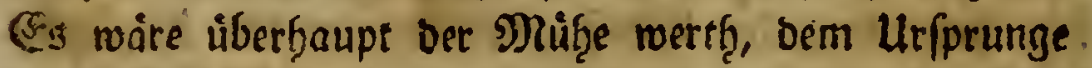
folther $\mathfrak{B}$ elienurungen, bie nicht gleidh 2unfangs eine launige Brille nnénben, madjzufpuren; es ift maf̧r, 


\section{Burshaulen, Dem 4. Sorn. I 784. 243}

Siufęen wưrbe biefe arrbeit menig gewáf̨ren, aber boch immer eine 2 dbranonolung de Calceis Veterum um vieles aufwiegen.

Meine Begleiter waren bereits mube, und rie: then nir an biefem Sorte ausgurutgen, weil wir nod

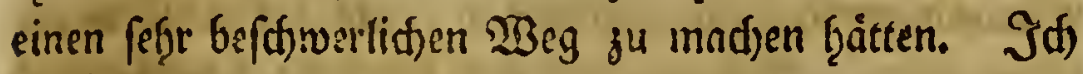
füblte zivar nitfte eben bie 9iothwendigéeit biefes zu thun, bie fie antriet, mir biefen Fiaț zu erţ̨eilen; ba

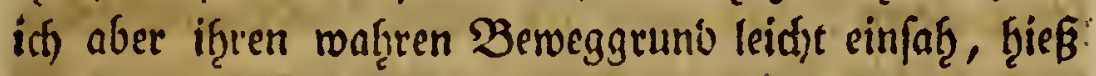

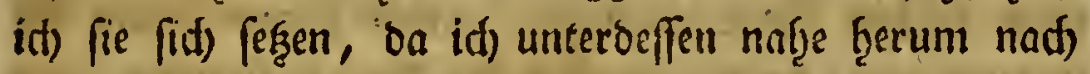

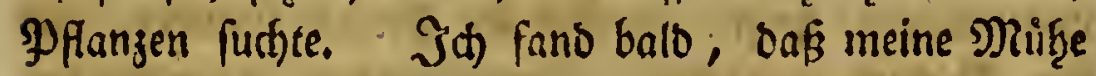
vergeblid) fen, weil bie $\mathfrak{B i e f e}$ vor nicht gar langer Beit abgemaiget worben, baher idf) auth faft nidfts, "als bas Satyrium mit ber braunen Zlef̧re barnuf antraf. Jđ feşte mid) alfo gleidffalts, und hatte Utrfnche, meinen Begleitern vielen Dank zu wiffen. Die Sergmais

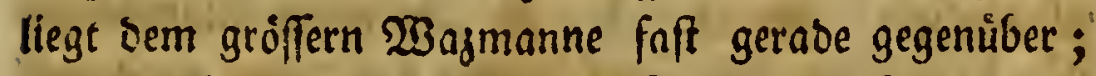

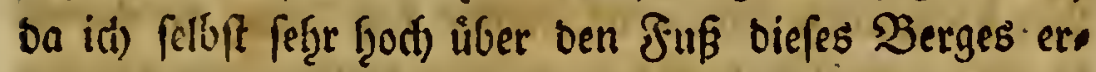
baben mar, da nur gany unten am Jufse viefer groffen $\mathfrak{B}$ ergmiefe ein fleiner $\mathfrak{B}$ alb ben Fußs biefes $\mathfrak{B}$ erges bebectte, fo hatte id biefen ungef̧euren Berg faft gang in (Jiefictece, und gerabe fo naf̧e, als es nơthig mar;

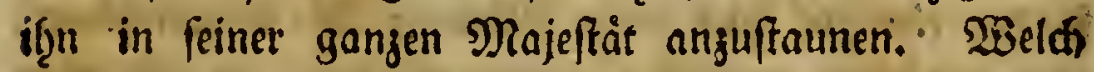

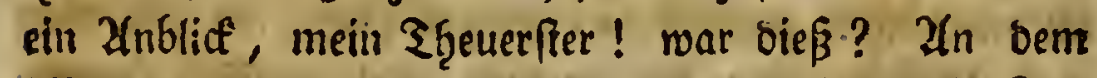
atbhange eines hohen Berges gelagert; beffen Fuß bis an ben Eee finabreidte, faht idf gegenüber cine

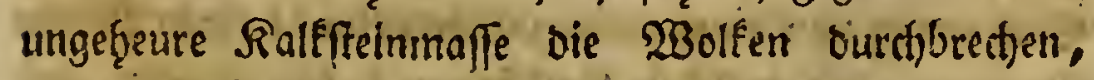
uns fich bis jum Seimmel aufthuirmen. Da ocr Menf(s) immer geneigt iff, alles nad) fich abjumeffen, to frelfie curth id in biefem Xugenblicfe eine unwill.

$$
\text { จ. } 3 \text { füఠ̆lide }
$$


fứf̧rlide Bergleidfung meines eigenen Sörpermaffes mit biefem Berge an, verfatwand baben, wie es natürlid) war, in meinen eigetren 2lugen, uno ein wirflid) angenef̧mer Taumel bemeifterte firf) meiner fo

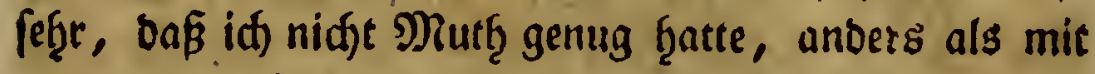
verwanbtem (B)efid)te aufzuftefzen, als bie Tieife meiter gef̧en follte. Das war bie erfte ţerrlidse, feelenţebende Scene, sie mir bie Bebirge Darboten, für fid) allein

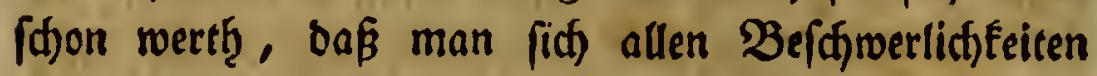

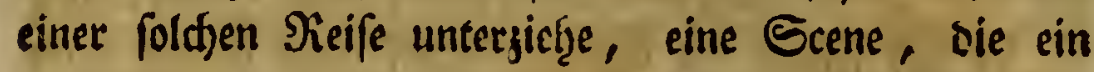
unnembares, grofles Gefuifl im \$eerzen emporet, bas fein Didfter mit allen feinen farben ausjubrůden vermag.

Bon ba aus giengen wir nath ber Rrautcafa,

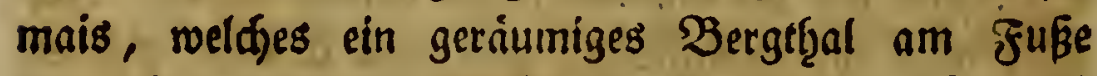
bes Säners ift, oer fid) bennahe fegelförmig ùber oaf. pelbe erf̧ebt, eeime Beffalt, bie fę̣r vielen zalpenbergen

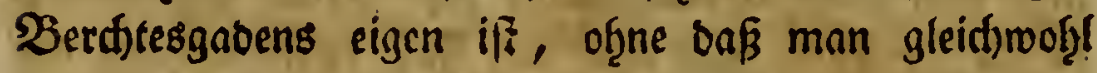
bie geringfte Urfache ḩătte, einen ausgebrannten $\mathfrak{B u l f a n}$

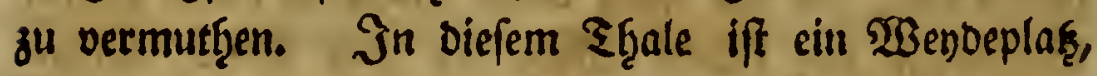
aber et roar noch nidft benuf̧et, wir fanoen baf̧er Die Şuttte leer, uno fein พ̉affer ǵerzugeleitet; mir fanben für gut Ģier unfer Mittagmaḩl einzuneţmen; idf) Durdf(ud)te gugleid) ben ganjen. Plak, of fidh nid)t audi) Befrieoigung fïr meinen viel gróffern Scunger nad) Naturfórpern fänoe, uno traf. zugleid in einer Entfernung yon einer fleinen $\mathfrak{B i e r t e l f f u n d e ~ e i n e n ~ f l e i n e n ~}$ Bađ) an, Det zwiffjen Ralffteinen Daf̧in riefelte, unō uns nebfi bem vortrefflidien $23 e i n e$, ben uns bie Eorgs

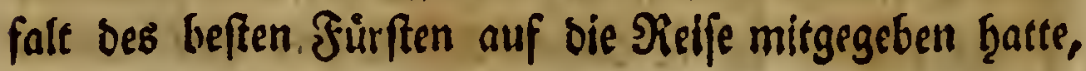
feg̨t gute Dienfte leiffete.

श(ad). 


\section{BZurghnufen, Den 4. Şorn. I 784. 247}

Nadjoem roir uns f̧inlänglid gelaber ḩatten, giengen wír ùber ben Jåner nads bem ßönigsberg fort, wo id unterwegs einen verfallenen (Balmes)/f(burf antraf, aber zugleich baran war, baf wir balb in bas Rónigsthal fommen mo̊chten, no wir umfer शiad)tlager zu nef̧men fdoon efzer befdiloffen bुatten. Die Urfache meines Eilens war ein herangief̧endes Dunnerwetter, wie mir bafür bielfen; ons idf) nidjt silft Gatte unter frinem Stimmel, no id) uno meine Begleiter bie gróften ablcitenten Riorper maren, aus. zuhalten, befonbers ba id) allenthalben feidjte uno f(t)male Gurctien in ben Ralefteinen antrof, bie wie mit einem Meiffel gemad)t zu fenn (d́)ienen, uno mob̧t aud) eines anbeen Uriprunges fenn moidsen, mir aber als beutlide Spuren gemaltiger Donnerftralen vor. fämen. Wir hatten ben folgenden Iag eben bie Ero

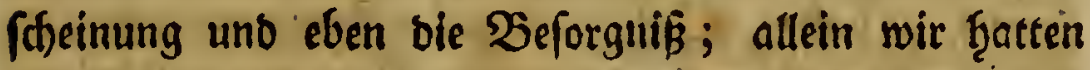
uns bentemale in unferer Nielnung betrogen; bas verméyntlid)e Donnerwetter war nid)ts weiter als ber eben bamals faft burd) gang (Europa verbreitete Jeób̨enraud), ber aber auf bem (Bebirge viel bưnner, uno nit in groffen Entfernungen redfte fidtbar war; er verfd)want allemal am 2(bend wieser, uns wat niemal bes Miorgens eb̧er zu feţen, bis bie Eonne

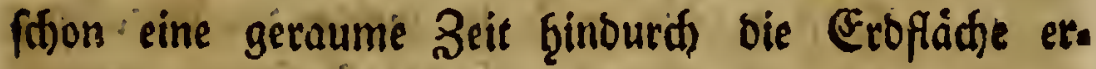
wärmet ḩatte.

Эक) hatte biefe Rebelerf(f)einung oas erftemal am 24. Jum., a!s idh von Jjern maín Burgḩaufn

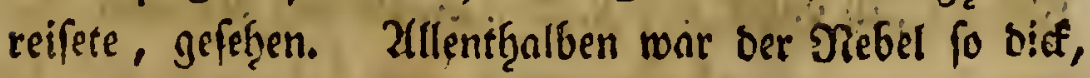

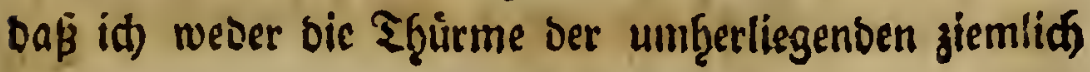

$$
24 \text { nab̧en }
$$


nab̧en Rird)en, nod bie Eleinen $\mathfrak{W a}$ ilber, bie mir im Woege lagen, in gar nid)t groffen Entfernungen fef̧en fonnte. Es bुatte bie vorţergef̧ende Wodbe ftarf ges regnet, aber id) badje nod) nidjt Daran, bafi biefe Piegen bie Urfacte biefes Drebels fenn burften, bis idf naci) Marftl fam. Şier fand id ben Innffrom fo ausgetreten, thaß er biz an bas lezte j̦aus biefes Marftes reidfe, , uno id) von dempelben bis an bie

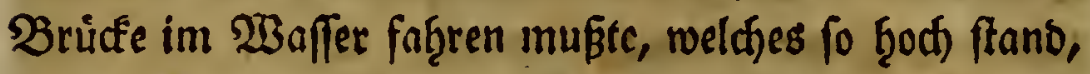
oaßs linf́s neben meinem $\mathfrak{B}_{B}$ agen (red)ts fiatte id) Den eigentlitien Rimnfaal bes Fluffes) tleinere Nachen vorbenfub̧ren, ouf weld)ell fid) Seufe, bie úber bie Bructé ḩergefommen waren, überféşen lieffen. Bon nun an ftano id) Eeinen 2(ugenblite meḩr an, bie Utr fache bes Rebels in einein ju f(f)nellen Sd)melzen bes, Edjnees auf ben Eisgebirgen, ber Wiege bes Inn, froms, zu fuchen. Sth warb auf meiner gegenwairtigen (3iebirgreife noch) meligr in meiner Meunung beftåttiget, als id) an einem ganz ţeitern Mlorgen einem feţe fiog̨en $\mathfrak{B}$ erge (bem $\mathfrak{B}$ rette) naf̧e genug, und gerabe gegenúber ftand. Zluf biefem .Berge lagen nod) bier uns bort groffe Sdjneefelber, bie, fo balb fie von ber Sonne befchienen rourben, einen biffen Nebel $a b=$ Dưnfteten, ber balb barauf bie Şeiterfeit bes . Miorgens, trübte. Dießs: madtse mid) in meiller Miutlyinaffung fo feft, Daßs id allemal auf bie 2 nfragen meiner Jreunbe biefes Nebels wegen zurúdfichrieb, id) wäre ath ber $\mathfrak{B}$ Biege biefes $\mathfrak{P g}$ ąnomenons gewefen, fie fönnten fid) baf̧er auf meinen 2 (usfprud verlaffen. 


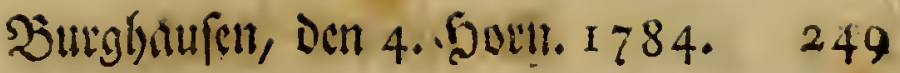

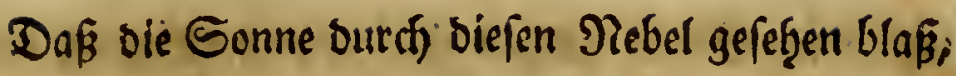

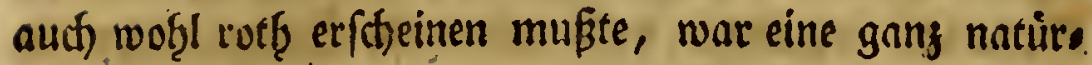
liffe Eact): aber eben fo wenig befremoeno fonnte es

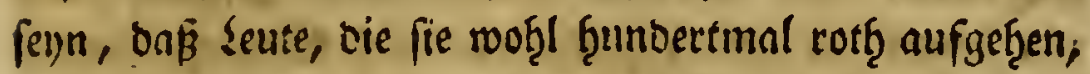
roth untergeţen fab̧en, of̧ne firf) jemnal um bie Utrfache Diejer Erróbeinung ju befümmern, fíd) wunbern mußs? ten, fie fo am vollen Tage zu erblicient. Saureno antere Erfdeinungen, bie bie Gadje erft fffrectbaret madten, waren bie Folgen incer Erftaunung: fie fab̧eur ftair Ģin auf ben roţ̧en Eonnentôrper, ber fie mit feinen Stralen nidst mȩ̂r blentete : was war

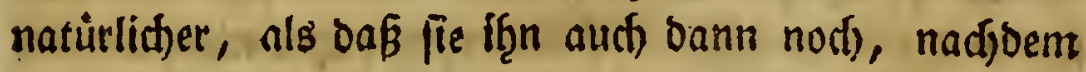
fie ifgre Zugen weggemenbet hatten, befonders weun fre biefelben nach) weiffen (Segenffänoen ridteten, erblicfen, fogat in Berwegung., und nad) und nath) unter vere fdjiebenen Farben erblicfen musten, wie biefes allemat gefdbief̧t, wenn man einen leutfitenden Rörper lange Deit Gintereinanber ftart. angefêten ந̧at. Berzerte Etralen, eine fleine in bas aluge tretenbe Ş̧ràne u.o.gl. fonnten bann leidit Silber von Rreujen, uno was fonft bergleidgen fojrectlidjen Zeugs feyn mag, Ģerbor: bringen.

Diefe leute faḩen alfo wirflich, was fie su fef̧en glaubten; aber bie Begenftainte waren nidbe auffer ingnen; fie waren im Zugue felbft, fie maren Sortfégung ber einmal zu hefftig gewordenen Sdiwin.

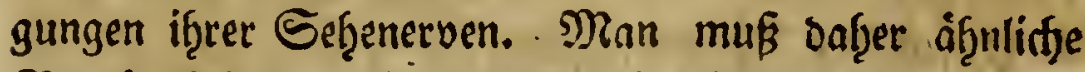
Begebentheiten, bie uns die Bef(tiid)te aufbervatiret,

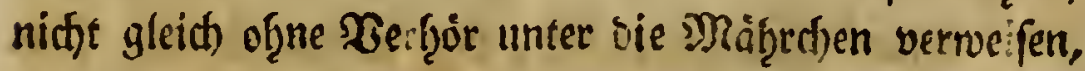
wie ber $\mathfrak{B e r f a f f e r}$ bes grand Theatre hiftorique

$$
25
$$


bey einer Stelle geffran ḩat, in weldjer er uns bie Nactbridst erţ̧eilet, Daß man im Jaf̧re 1667 in Entroaben zwo Sontren im Sinmpfe gefef̧en ḩabe, voun Denen bie Ģellere (bie eigentlidhe Sonne) Riugeln auswarf, bie bie farbe verinderten, je nadjoem fie fiid) von ingr entfernten. Der Beitraum zuiffhen 1667 und 1783 betraigt 116 , weld)e Saḩl fich) ourd) 19 (bie 2inzaḩl ber Jab̧re, nad) meldyen ber Mono pein groffes Jaḩr vollbradt) ḩat, uno in gleithen Tagen gleidfe Stellungen beţaupter) bis auf ben Eleinen Bruch $\frac{2}{19}$ Jab̧re $=38$ Tage unb einige Etunben genau bivibiren läßjt. Eollte man baher niad)t benfen,

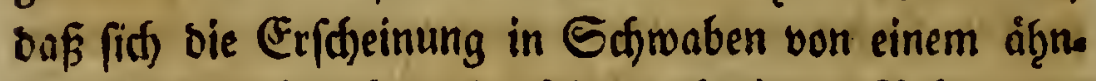

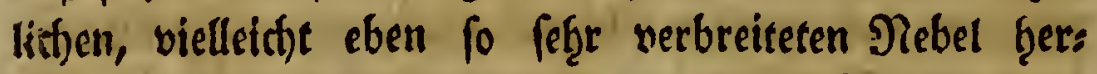
fotreibe, uns baß fie viele von uns im Jaf̧re 1802 abermal ḩaben werben? Sft es boch) porwoghl aus Er. faģrung als aus Theorie gervifí, oak bie Etellung bes Monbes, bie auf unfere Meere einen vorzuglidfen Einfluß̧ ḩat, einen nod) weit vorjůglichern auf unfere Ztmosphąare, uno oie bamit verbunderie 53 itterung biabe. Es fann nber nod) anbere Nebenurfad)en ge: ben, bie einen folden Sçdhenraud) nur in cinzelnen (B)egenten ḩervorbringen, ober aud) ben allgenieinen in einzelnen sänbern berGinbern, gerabe wie fids bie Meeresflut, ourd befondere Umftànoe aufgeţalten ober beforbert, nid)t.überall genau nad) ben abgefonberten Begriffen ber Ţ̧̧eorie ridstet.

Einen Edimefelgerud), ben einige järtlide Pet. fonen beis diefem Эiebel serfpuret baben wollten, babe 
Surghaufar, Den 4. Scorn. 1784.251

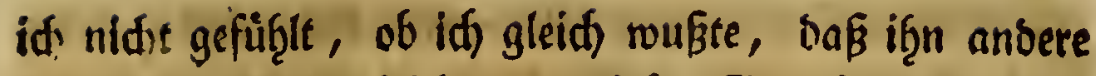
füblten. Wermutf̧lid) war biefer (J)erud) mef̧r elects

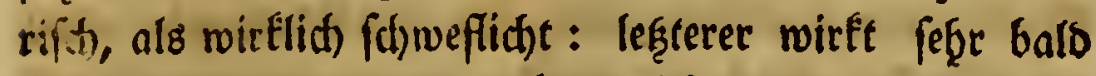
all meine $\mathfrak{B r u f t , ~ j e n e n ~ f e n n e ~ i d f ~ b l o k ~ a u s ~ B u ́ d j e r n , ~}$

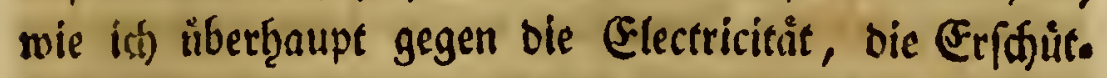
terung ausgenommen, zientlid) unempfinslid, bin.

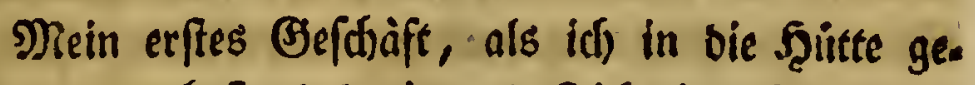
fommen war, beftand barinn, Daß idf bie gefammelten Y) flangen in meine Papiere cinlegte, bas Ma afet oben uno unten mit einem Pappenbecfel belegte, uno feft

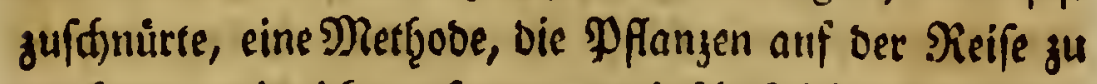
trocfnen, oie id) unferm gemeinfdinftlid)en greunbe, Şerrn Dr. Panjer zu Nürnberg, ju verbanfen f̧abe.

Den folgenden Tag giengen wir ben Rönigsterg auf einer nnoern Seite ḩinauf, uno bem Sd)neibfteine zu. Diefer $\mathfrak{B}$ erg, ber nod) jegt ferge frod) iff, fobeine mof̧l ef̧er nod) viel ḩöb̨er gemefen ju renn. "Ëegenúber hat er bas SBrett, einen ansern nodh fiófeern $\mathfrak{B e r g}$, an beffen Jufe wir vorbengegangen waren. Swifden bey.

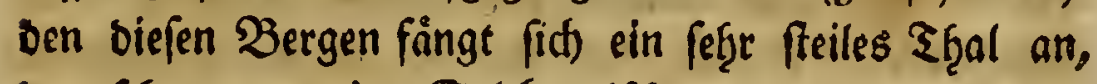
Das fajon gang im Salzburgiffien liegr.

Id) werbe ein anbermal wieber Bselegenfeit f̧as ben, Des Brettes Erwäf̨nung ju thiun. Befrombens war mirs aber, baßz id) felbft auf ber Spige bes Edfmeibfteins auf lauter lofem (Sefteine einf̧ergieng.

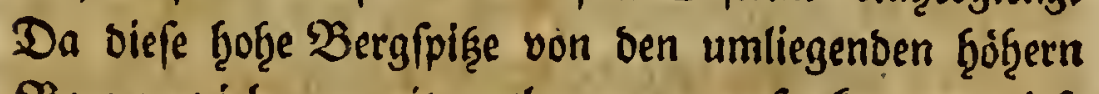
Bergen viel zu weit entlegen war, fo Eonnten biefe Trůmmern unmóglia erff bariber bergefallen fenn. 
allein biefe Erffdeinung wirro leidft begreiflid, wenn

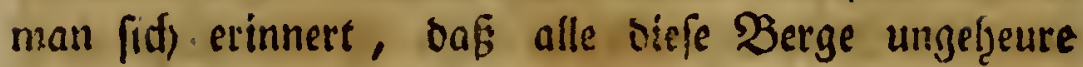
Maffen von Ralffífein fenn ; ber Ralffein wiro aber

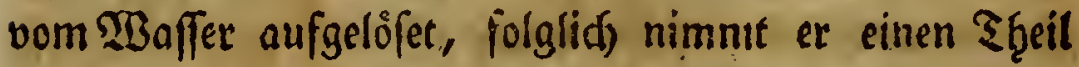
Deffelben in fein Junnerffes auf: Diefes frieret im Winter; und zerfprengt Den Etein, Daf̧er aud bas. Rradjen und Donnern fómmt, bas man zuweilen im

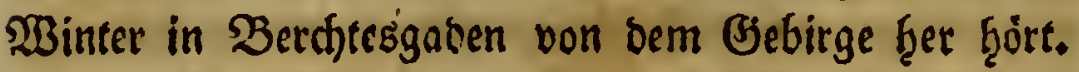

Eine anbere mertfoúroige Errftheinung genåhrten

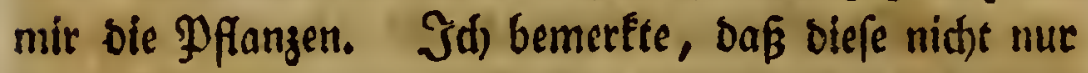

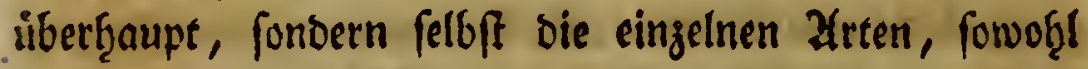

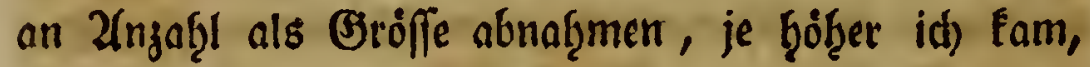
to Daß ber $\mathfrak{B e r g}$ am Ende faft gang fab̧l war, nut feine Jelfentrummern fint mit einer Siledffenart gleidffam übermalet, bie aber fo bưne iff, uns allents b̧alben fo feft am Eteine flebt, oaß idy fie anfänglich gang verfanute, umo Den Etein für eine feg̣t artige

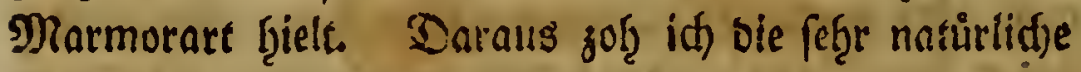

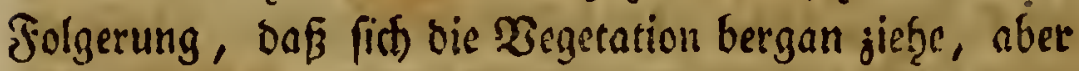
Die Edritte, bie fie madit, firto áufferft langfam, uno

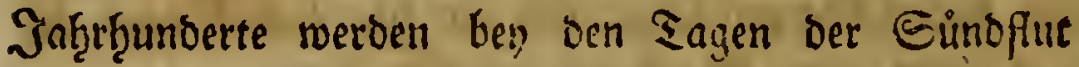

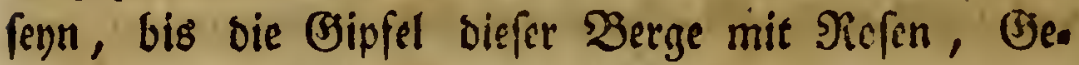

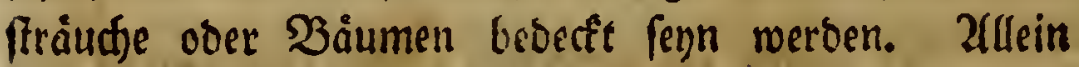
ic) muß Sie mit einem now weit merfnuiroigern Berge befannt madjen, elge id mir bie Efare geben fann, Şlonen alle bie groffen fosmograpţifiden Bors

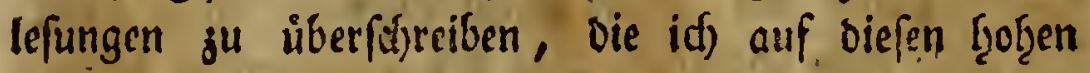

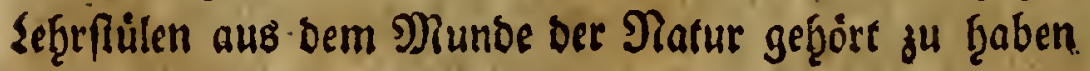
glaube. 


\section{Şurghaufen, Den 4. Sorn. 1784. 253}

Bom Edrneibfrein foment wir an ben Rieines. berg, wo wir bem chitacn Edunce gegeniber fanden.

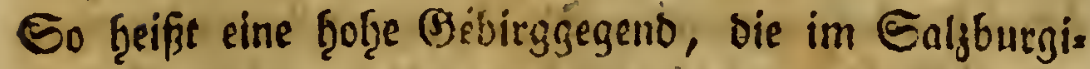

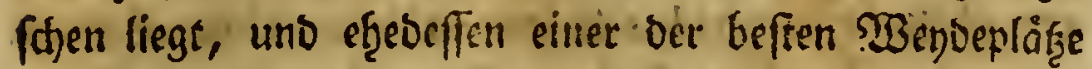
genefen fenn foll. (EB ift gang noturlich), bấ fid) bुofge Begenben unter sewiffen umftänden, bie nur burd) bie Zeit berbengefintret werben, in (Bletfder

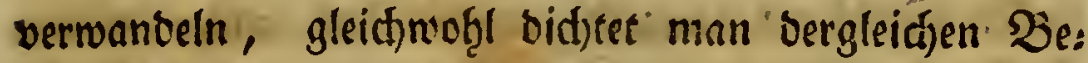
gebenf̧eiten gerne eine übernaturtlid)e Urfart)e an, und eine foldte mufite nun aud) bie fenn, weldse bem sivis gen Edjuee fein erftes Dafunt unmittelóar gegeben

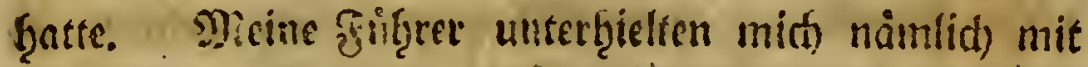
Dem 9) Juthimillen ber efgemaligen Eennerinnen biefer

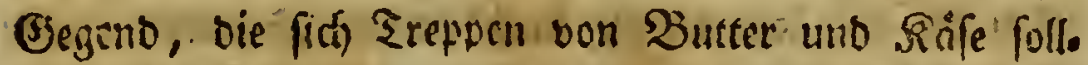
ten angeleget hraben, um bequemer auf bem Bsebirge

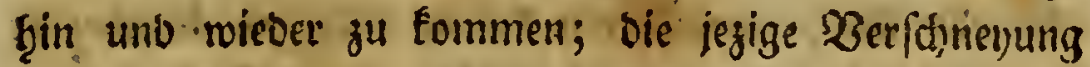

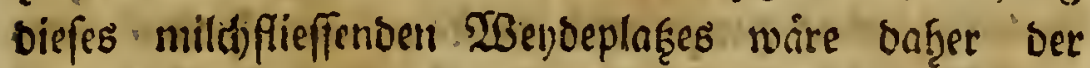
Eage nath nidts anters als cine geredte Etrafe biefes fünof̧aften Mnutfimillens; allein bie Gad)e modite

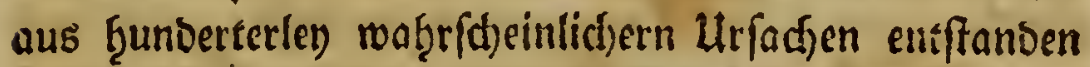
fenn, feģters fie hingu, uno man fano vielleicht erft in ber Folge für gut, weil man fie nidjt erflären fonnte, biefes Măberden zu erbidjten.

Bir famen unterbeffen in bie $\mathfrak{B}$ Bind/ffarte, ein rautfes felfengebirg, bas nod) ganz neuterlic) mit Edthee bebeuft gervefen zu fenn foneinet. Zuffer oaß

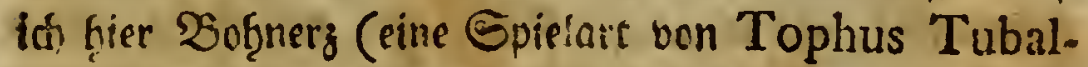
caini Lin, Die flach georüdt, länglidst, bon ber Bgróffe und Beptalt einer gebrücten Bob̨ne, aber aud fleiner 
unb bon unregelmábiger, bod) allzeit geboưdfer Jigut iff: Minera ferri fubaquofa Waller. -y)ineralfipfr.

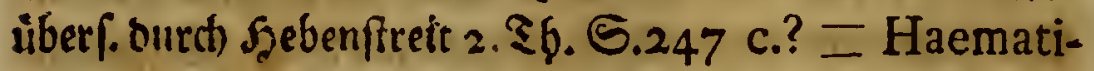
tes globularis nitidus fufcus tritura rubra. Select. ex amoenit. acad. C. Lin. Graecii I 769 . p. 256 .

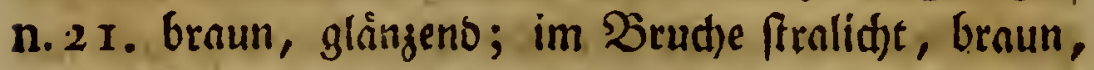
mit Dofer unternif(d); am Etridje gelbbraun, im naffen Strid)e braungelb) unmittelbar unter meinen Jüfifen in einer enffestilidjen $\mathfrak{W}_{\text {Beite }}$ von allem $\mathfrak{Z}_{\mathfrak{B} a f f e r}$ zufammengetefen fratte, fant id nidfts, was eine Bemerfung verbienet hăatte. $\mathfrak{B}$ Bir giengen . fogar eine lange Streefe auf einem Edfneefelbe, auf weldem ids einige berffogene Infeften antraf, sie erffarret Dalagen. $\mathfrak{B}$ on ba aus famen wir nadt einigen Stunben in bie Gegend bell Den Bellern eine grar viel tiefere Gegent, als alle bie, weldfe wir biefen Morgen -burdreifet ḩatten, aber raube unb wüffe; wir Gielten uns

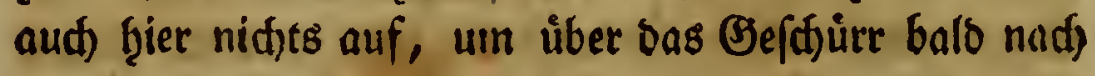
ber Mitctergutte ju fommen.

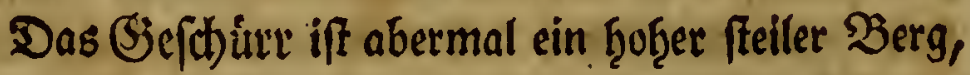
Der nod) allentf̧alben mit tieferm Sdinnee bebect' war. $\Im$ G mir, menn idf nod) bie Derter feefen wollte, bie wir uns zu befegen vorgenommen faatten, fo feen biefer $2 B e g$ unumgäuglid ju madjen. Id f́letterte alfo, fo gut idfs vermodite, Ģinan, uno war fdoon ziemmlich weit gefommen, als idf ausgliff(d)te, uno ben $\mathfrak{B}$ erg wieber

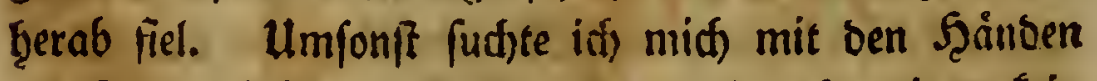

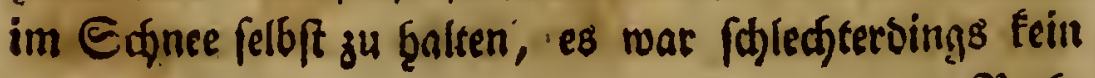




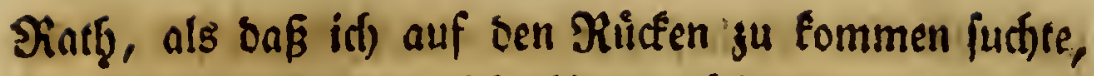
um menigftens vor mid) biin. ju fȩ̣en, was id) ju

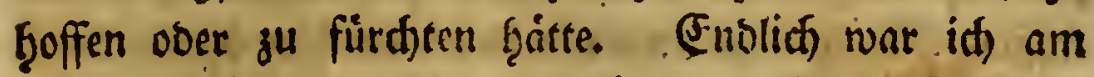
Enbe bes Edfnees uno jugleidy am folüe beż Berges;

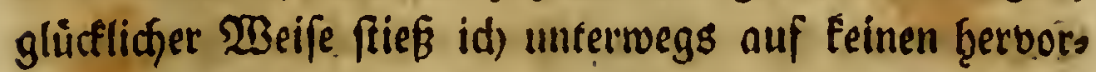
ragenben Etein, fonft mürbe ich) vielleid)t ben Tag bor meiner abreife woḅl Den leg̨ten Bud)ftaben in meinem seben gefdrieben ţaben.

Şier fran' id, nun tief unten am Berge, jwar weniger von ineinem Jalle erfdrect, als meine J̦ingrer, aber bod verlegen uno mutḩlog. Enolid) magte idfs abermal, aher buth) Llmwege, Ginouf ju fommen, uno diefinal gieng bie Eache gang gut, bis wir nad) einein meḩr als frunbenlangen $\mathfrak{B}$ eg an bie andere Seite bes Berges Émen, Die mir wiever binabfreigen musten. Jih mar im J̧inauffeigen gefallen, uno jejt follte id über biefen Schneeruiden abmärts gehen! Mein ß̧erg.

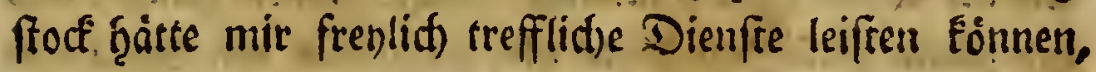
nber ben nuffe id) nidit ju gebranchen. - Untera

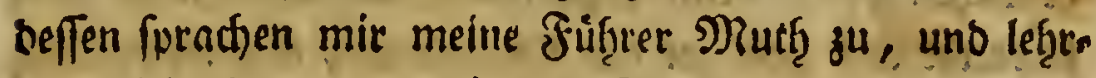
ten mist) einen gan, eigenen (Jang, ber mir in ber Solge audh auf andern freilen, obgleid) berradyfenen, Bergen feţr bienlid, aber freslid) von hem, ben man in ber Tanjfdule lernt, fefgr verfdieben war.

Wir Ģatten biefen Morgen wof̧] bren bis vier Stunden lang auf Sdineegefiloen gewandelt, unb waren won ben befd)werlidjen $w_{\text {egen }}$ abgemattet, eg war Dageer Beit an einen Drt ju gelangen, wa wir unfere Firáfte wieder exţolen fönnten. So balb wir 
unis alfo im Şgale, bas unterm Sicftelle liegt, bes fanben, nabemen wir auf fcton feit einiger Beit ver. miß̈ten Siafen unfer Sager, uno bielten Giier offene Safel. Mid) wiegten bald bie lauern sufftchen in einen fúffen Edflummer, ben id) auf Bottes Eroboben lingeftredt' föftlider fano, als Rónige in feibenen Beften.

WBir ḩatten nur nod) orey Efunben weit bis an ben Drt ju gef̧en, wo wir unfer Nadftlager aufo zufd)lagen beftiminet ţatten. Jit) beforgte baher nad meinem (Erad)en nod) an eben ber Stelle meine ge: fammelten Pflangen, uno bann féften wir unfern $\mathfrak{W}_{\mathrm{B}} \mathrm{g}$ über bus Oeffélf naç bem kauffelde, uno loon da

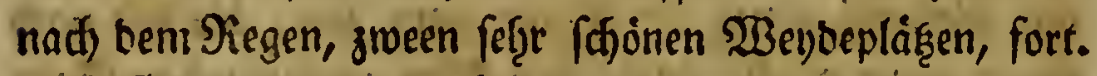
Das Seftell ift ein úberaus feiler Berg, on beffen Eeite ein fo fdjmaler Steig fortgelgt, baß $\mathrm{B}$ er gar offt

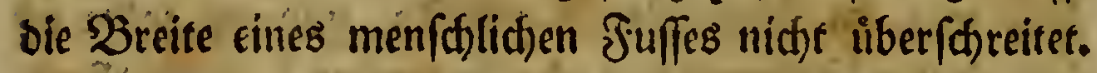
2fn ber Eeite fief̧e man auf fpişige Jellen ţinab, uno ben jeben Salle wurbe es hier unfehifbar um bas leben geffref̧en feyn : benn obgleid) bie alogrünoe nidft gamz

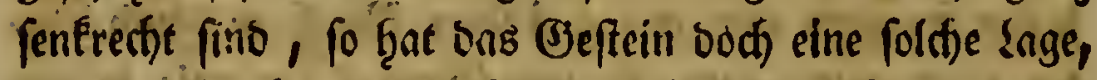
baß man fid an beinfelben nicft ḩalten fönnte, uno

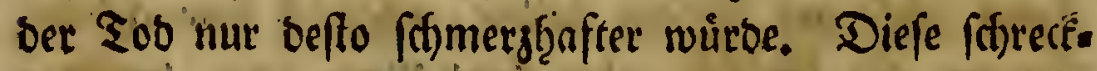
lide $\mathfrak{B o r f t e l l u n g , ~ u n o ~ n o d ~ d ) ~ m e t ̧ r ~ b i e ~ a ̈ u f f e r f t e ~} \mathfrak{B}$ eforg.

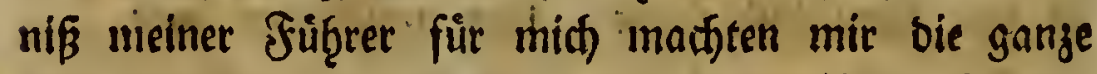
Beit Ginourch, als idf mid) auf biefem $\mathfrak{B}$ ege befand, fȩ̣r bange. Şier war '́eine Z̦wergfidjte, an beren

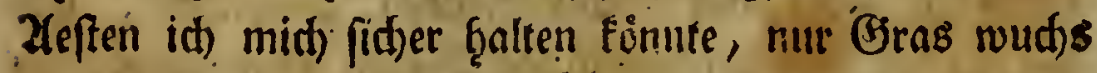

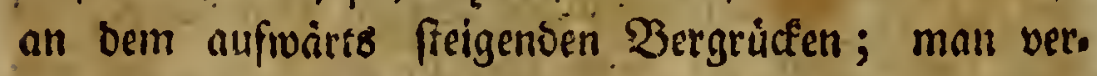

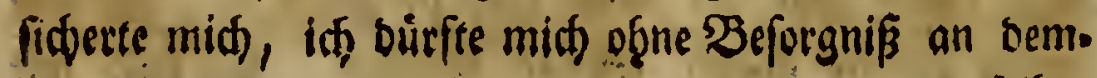




\section{Burghaufen, den 4. Sourt. I784, 257}

felben bुalten, nur folfte id) es tief unten an ben $\mathfrak{B u t .}$ zeln greifen, oaben aber weite, bod) bedactitfame E(t)ritte mad)en, uno midh bư̆ten, bely bern ungemofinten (Błange mit sem einen fuffe an ben andern zu feffer. Durch biefe Belef̧rungen unterridjeet. legte iø) biefen $\mathfrak{B}$ eg

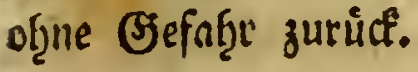

Den folgenden Sag giengen wir immer abroàtts bis an bie Raunerwano, wiber bie man an bas ufee oes Rönigfeez ḩerabfo̊nmt, ein viel gefăb̧rlicherer Eteig, als bie hisher genannten, nicht fo foffer megen ber Gefaḩr zu fallen, als weil fich) ber $\mathfrak{X}$ eg beftintig bin und wieber faflángelt, und ba belj jebem Tritte Eteine ůber bie $\mathfrak{Z}$ ano berabrollen, jeber Ģof̧erer

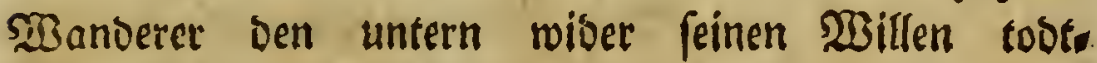
fhlagen foonnte.

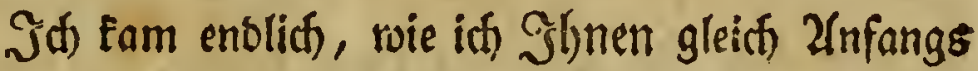

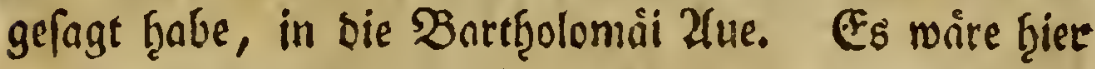

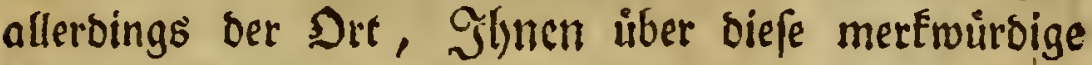

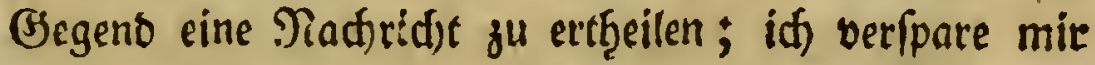

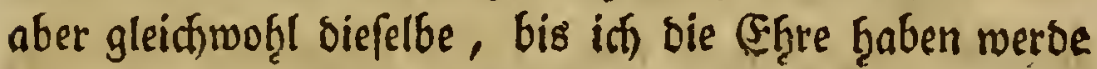
Sie ibberbaupt von ben Eeen in Berdjtesgaben zu unterţalten; bis baţin $\mathfrak{H}_{0} \mathcal{f}$.

Schrank.

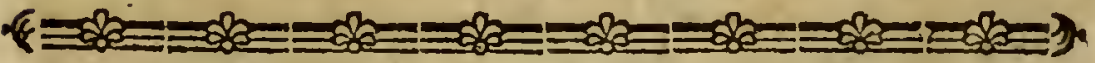

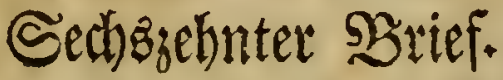

Surghaufer, ben S. J̧orn. I784.

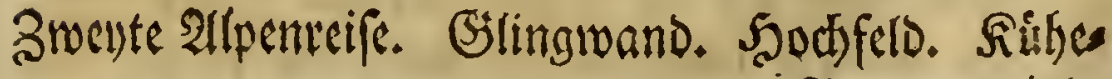
Faturbif Sriefe I.Sand. $\Re$ taind. 
raino. 20 azmannfdiarte. Eimfturzen der $\mathfrak{B}$ erge. Gortgang Der Zegetation uno Des Eifes. Fernere Sieife.

o mir gleid) auf meiner vorigen Reife tảglich - ein Donnerwetter brofice, fo war nidtes befto.

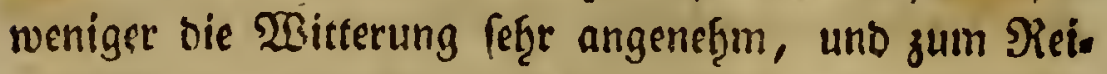
fen bequem, ein (Blinck, bas id) bey meiner zwenten Zl(penreife nidyt genop, aber aud) nidft Uurfad)e f̧atte zu vermiffen, weil id) dafür burd) Bemerfungen ver.

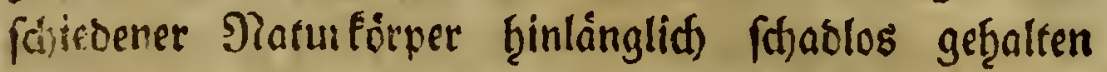

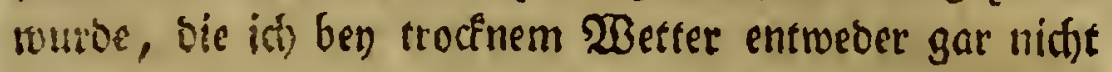
oser nitlyt fo bequem múroe traben beobactiten fönnen. STie in ber Sictur alles gut ift, vom rilligen Etiere,

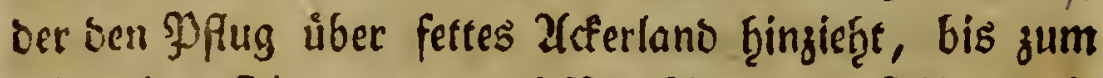
grimmigen ₹iger, ber ouf Menfthen uno Ţ̧iere mü. thend cabeinflürt, fo giebt es aud) an biefem groffen Melferfturffe ber 2t!lmadit feine Eeite, bie bem $\mathfrak{B} e=$

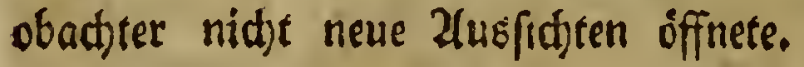

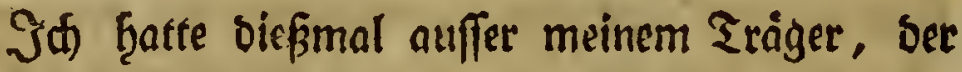
zugleid) mein Ssuistrer war, feinen Menfden bey mir, weil id) ouf meiner erfen Recife gelernet bৃatte, Daß

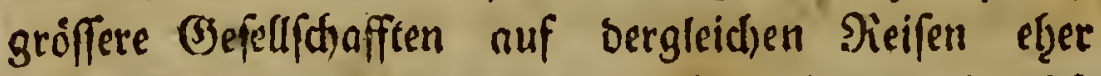
hincerlich als vortţeif̧̧aft fenen. 'Wir giengen ziemlid) frưţe von hacufe aus, uns famen gleid)wob̧l erft gegen Mittag im Serrenraino an, einem SWendeplatęe, ber

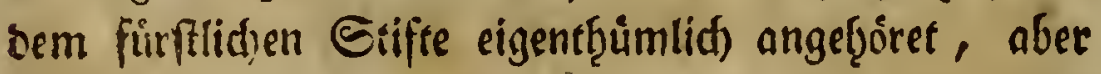
iejt verpadset iff. Die Eennyzutte war bुier nidft von $\mathfrak{S c o l}_{2}$, und, wie bie meiften übrigen ùbel besect; fie war vielmeģr burdjaus gemauert, und fo gut ein. 


\section{B̈urglaufen, Den 8. J̧om. I 784. 259}

geridtet, baß fie bequemer, als mantfes Bauernf̧aus,

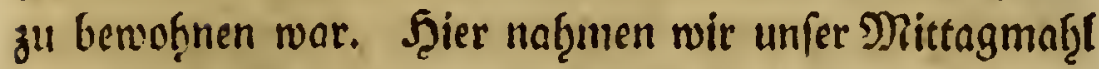
ein, bas mir weit beffer wúrbe gefd medfet b̧aben, wenn id) biefen Morgen reidher an Naturförpern, ober Demerf́ungen über biefelben geworben waire, als es wirflid) gefdjang.

Man formmt, nadjoem man einmal bie be. woḩnten (jegenden zurưcfgeleget hat, ourd) einen ben fdywerlidhen Steig an Der Rlingwano ţię̧er, uno muß

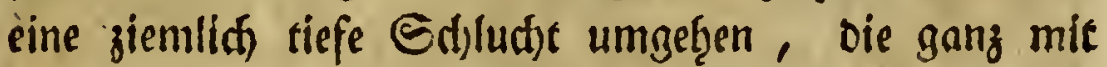
ben Trummern ber einfallenden Geitell becedft ift. Der Eteig, von bem idf) eben (prar), zietgt fich an oer Slingmand fort, uns iff zientich fteil, aber fo breit, Daß man bem Sdwwindel fefer ftarf ergeben feun múfite, wenn man einen Erurg in bie Ed)ludft tḩun follte; unterbeffen bzat man ond) Urfactje bef̧utfam zu gelgen, weil man auf einem fo farfen Zlbhange, ber gang mit lofen Ralffeinsrummern bebecft iff, fefre

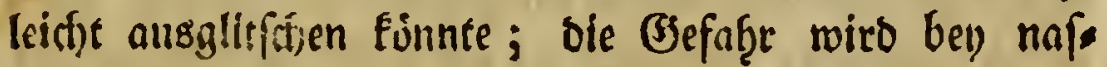
fem $\mathfrak{B}$ efter betråct)tlidjer, weil es, wie mid) mein Souffrer nochmals verfid)erte, groffe lettigte Etellen giebt, bie Durd) bie Jeudttigfeit fohlupprig gemadtet werben.

Diefer Setten, ben id) bey ber trocftnen $\mathfrak{B B}_{\text {ittes }}$ rung niđht waf̧rnafim, mag nun von ciner Natur fenn, von weld)er man will, Miergel voer \$lgon, (waḩr. (theinlid) if er Nergel), fo ift er bemerfenswerth genug, Daßj id nid) einen 2(ugenblide babey aufa balte.

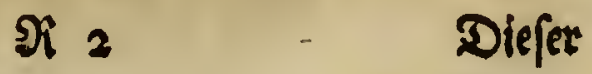


Diefer Setten feret weber in bie Tiefe nod) in bie W̧and fort, fondern übersecter blos an einigen. Etellen

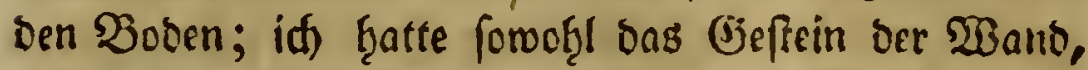

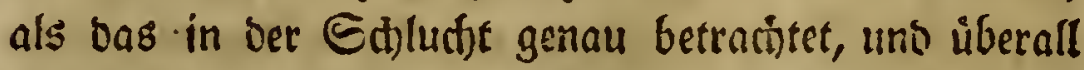
eitel brid)igen Ralfftein gefunben; Der Setten fdeint baher nidfts weiter zu fenn, als ein Mlergel, ber nus Dem Șantfteinftaube, unter welden fid) vegetabiliffe uno thieriffije $\mathfrak{B}$ eftandţeile gemifd)et ḩatten, ents franbet war. Sth ţatte eine ganz áf̧nlide Errfheis nung an ber Etrafie bon Ealjburg nach Burghaujen, als int am 27. Julius nad) Şaufe reiftete. Ber=

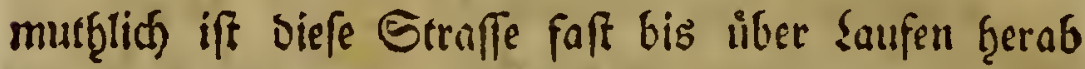
mit lauter Salffteintrummern befdututtet, caber fie aud in ber Eonne fefgr blentend weisgrau iff. Thun Whatte es aber bren Tage lang fef̧r frarf geregnet, uns id) fant bie jezt mieber trocinente Etraffe fajmierig, uno ben Roth gefáidft fid) einigermaffen bilten zu laffen, eine Eigenffinft, bie nur berm ₹y̧one uno ben Damit verbunbenen Erbarten zuf́oimmt. Diȩ́ erin.

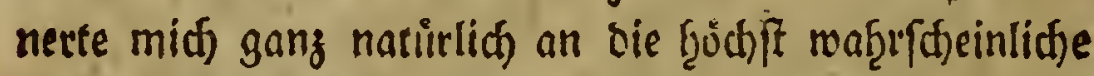

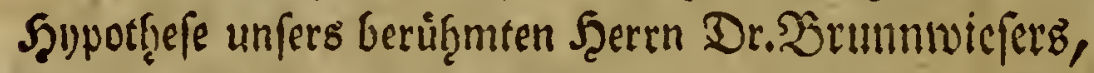

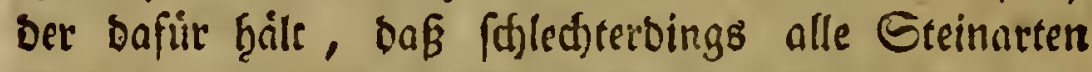
Durch Den $\mathfrak{B}$ entritt vegetabiliffter Eubftanzen in ₹̧̧on verwittern, weidfe Bef̧auptung er in ben 2(bุ̧ant). lungen ber Eurfürftiden $2\left(E_{a}\right.$ bemie zu Mủnd)en weit: láuftiger auseinander gefeget, uno für mich) ourd) $\mathfrak{B}$ or. meifung verfolienener Eteinarten, berer äuffere Dee sufft ausgeferzte Eeiten offenbar in einen meiftens

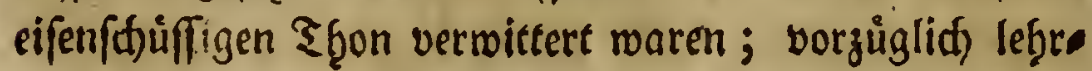
reid waren niir einige Şornfteine, bie mlt 2loern Durd): 


\section{Dourghamen, Den 8. Sorn. I 784.261}

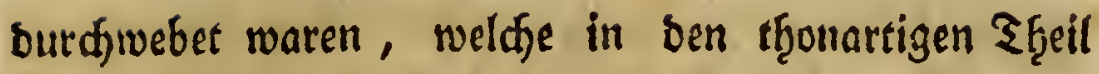
unumterbrod)en fortfeģten, zum beutlidjen Beneife, Daß̧ biefer nid)t erft anberwårtig fingugefommen, fonbern aus bem Ereine felbft entfanden fen. In biefer Şupothefe werben bie concentrifdyen Tḩonftein. rinben begreiflich, bie man auf ben fhoonen 2 gattugeln

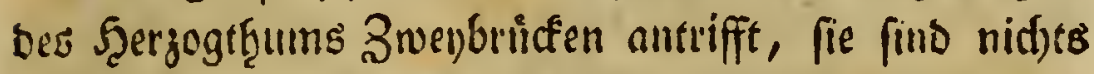
anbers, als bie aufferften 2(garfafidfen in ib̆rer Berwifferung.

Nad) eigenommenem Mittagmaf̧le giengen wir

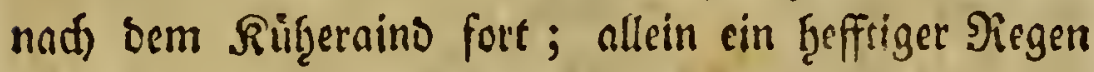
nơtḩigte uns in einer Şoljḩutte unfere 3ufludst zu neţmen, two wir einen Baucréned)t autrafen, bet ebenfalls unter biefem mirtg̨lidien Dadje das Enbe

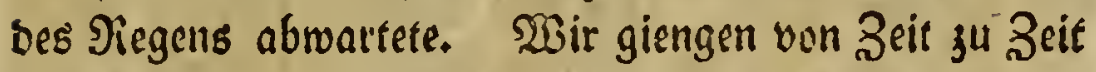
einer um ben anbern hinaus, um ž fef̧en, ob ber Biegen balo ein Enoe nef̧men mód)te, allein es ḩatte allen Zinfdein, baß or mef̧rere Tage fortoauren, uno bie fernere Sieife fithledtertings unmỏglid maden

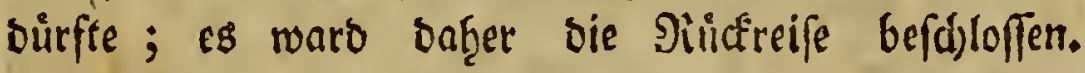
Unterbeffen verbroßs midjs gleidjwofil, biefen Bang vergeblid) gemad)t zu f̧aben. Wer Saauernfned)t

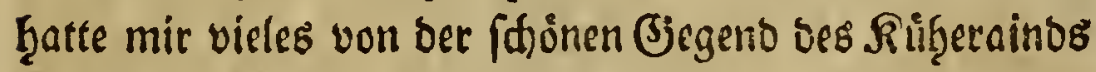
èzáf̨let, und bie Errfaf̧rung gegeben, daß man ben

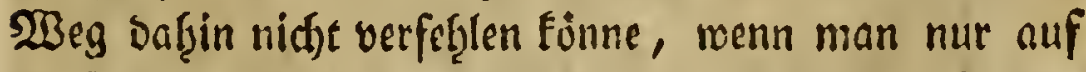
sie Sauben (Fleinere Etcine, bie auf bis groffern Fs! fen.

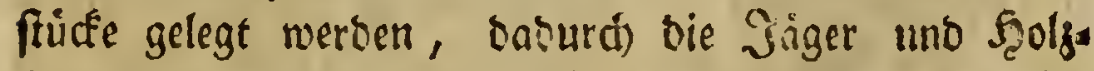

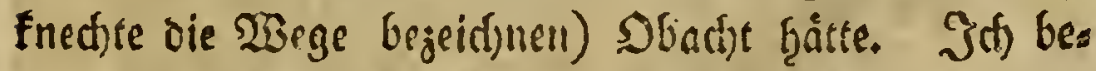
fabl alfo meinem Sráger in ser Şuttse ju verbleiben, unteroeffen ich nud bem Siutgraing mitten unterm 
Pegen fo lnnge als mirs geluftete, fortgethen wirbe;

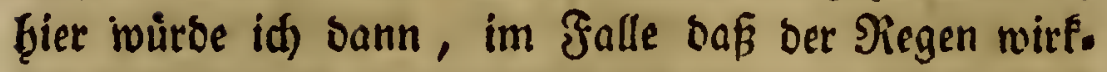
(iid) fortonutern follte, wieber eintreffen, um mit ibm nad) Şaufe zu fab̧ren; follte firt) aber inzwirften ber Şimmel mider $\mathfrak{B}$ ermutţen ausḩeitern, fo ţatte er ben Befeğl mir zu folgen.

Tdh gieng |aufånglid) ben Tauben gang forgfälltig nadf; aber bals hatte id über oen Y)fanzen bie Tauben vergeffen, und gerieth auf bas Jaotffeld, eine ę̧emals walbigte Gegeno, wo man aber feit geraumer Beit

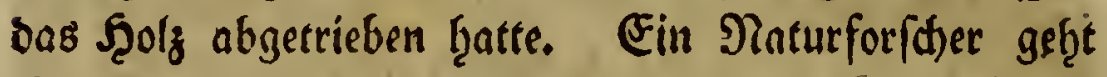
eigentlich niemals itre, unb mid) reuete es nid)t Ģieģer gerafţen zu Fenn; nur gieng ids, nactoem id mid Gier fatt gefuchet Ģatte, nads ben ₹anben zurưdf; allein

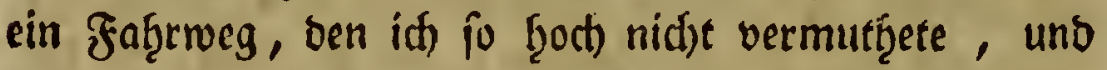
auf ben ids) midh mefgr, als auf bie Eanben verließs,

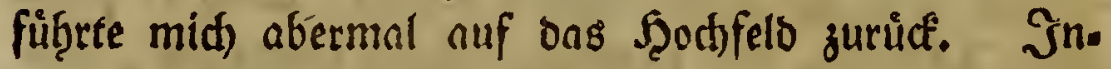

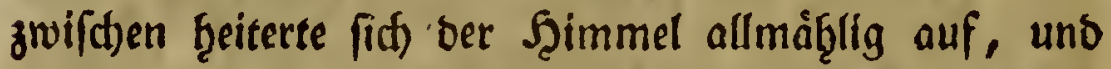

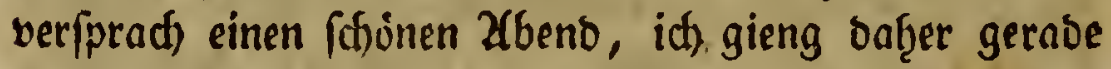

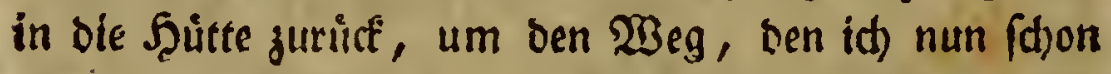
zmenmal berfeglet batte, in Gefellffiaft meines Tràgers zu madjen; allein biefer nar meinem $\mathfrak{B}$ efegte faton

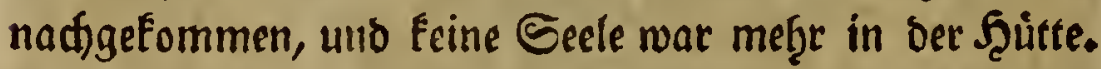
Jezt mußte in) midh alfo bequemen, meine 2lugen

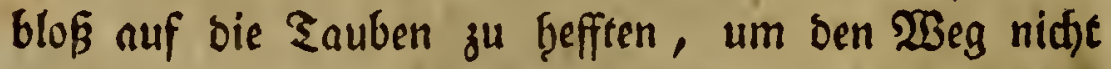

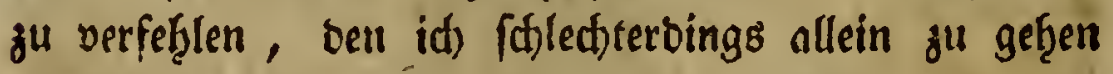
gezmungen roar; iff fam ouch wirflid über ben Neverticgel, eine angenefgrme, etwas walbigte Bies

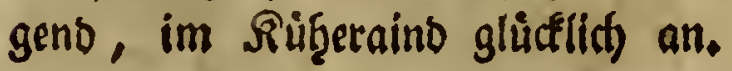




\section{SBurghaufen, Den 8.5orn. I 784. 263}

Es war bieß abermal eine beerrlidje Ecene auf ben Zllpen; ein ţeiterer Şimmel l̈ber nir, um mid)

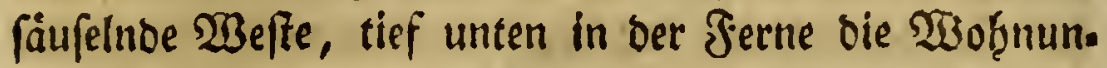
gen uno Sorgen ber Menfd)en, und zu meinen Jüuffen ein Jachendes (S)rume, an bem bie Siegentropfen now Giengen, in benen fint bie fheibende Sonne fpiegelte; zueen Menfdjen beis mir, mein Träger und ein כauernfned)t, ber bie 2(nffalten für ben morgigen ₹ag

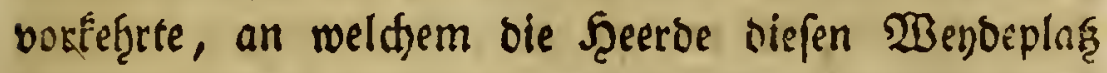
bejieḩen follte; Der Mann Der Szeerbe war aud fidon vorausgegangen, uno weybete bie Erftlinge reines funftigen Bebietes ab. Das ift, meine sieben! rief id) anf, bas ift hererlid)! Mir war ḩier fo wob̨l, fo in. niglid) zufrieben war mein $\mathfrak{S e r z}_{\text {, baf }}$ id) Diefen 2 (beno unter bie vergnnigteften Stumben meines lebens zătilen

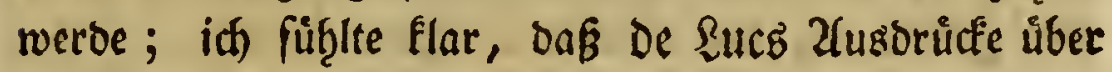
bie gofeje fanfte Sthwermuth, bie er und feine Befells (d)affterinn bृod) oben auf bem Sura füb̨lten, nichts

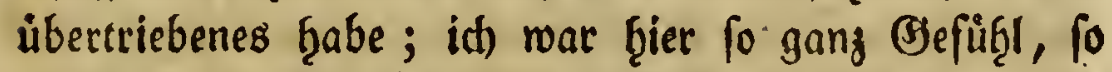
ganj im Taumel ber Wonne, baf id auf alles ver:

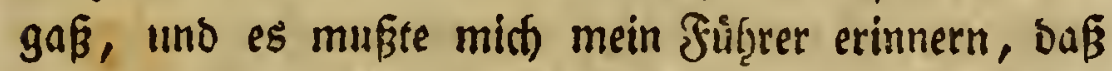
ber Zlbent fortruicfe, und mcine Pflanzen noch unver. forget feven. Jđ gieng baf̧er nod) ein wenig Ģerum, ob id) nidst etwas meţrers finden fơnnte, uno beforgte bas gefuntene, fo gut es mo̊glich war. Sánolithe (Jes fprăche am Generḩeerbe fủrzten uns bie Etunben bes bunflern 2(bends, uno id) füḩlte aud) bier in oen

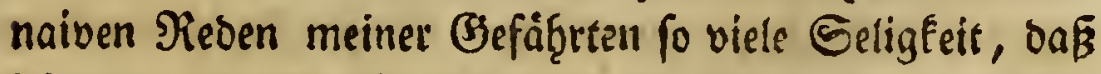
id) mir orbentlid) Mlůf̧e gab mid if̧nen fogar in ser Epradbe gleid zu madjen. 
Der Rúf̧eraino mad)t mit dem f̧errenraino eine Gortfeģung bes fleinern $\mathfrak{X a g m a n n e s ~ a u s ; ~ b e n d e r ~}$ F̧at fid forwolel, als ber bajwifden liegenden (begenben

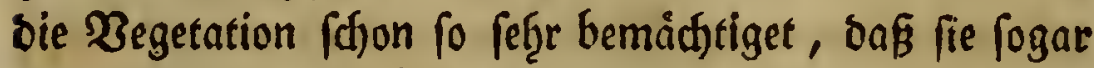
burd) f̧errlict) 23 nloungen getrennet werben, bie meis ftens aus Tannen uno Sifften beftelien, von benen belyse

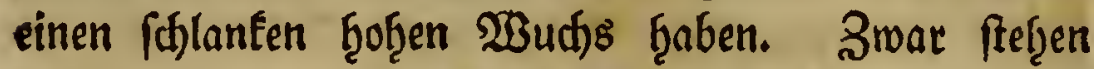
nod) Gier uno on eingellue nadfe Felfen ůber bie Sber. fläche ḩervor, bie nur in if̧ren 2 ertiefungen mit nies

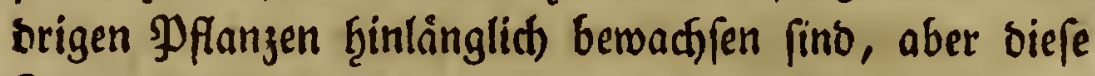
Jelfen fino fo fparfant, baß man fie auf oem fetten, mit

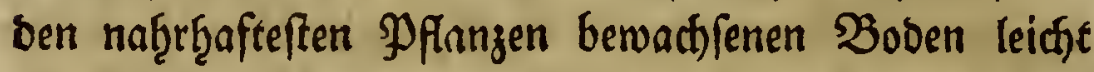

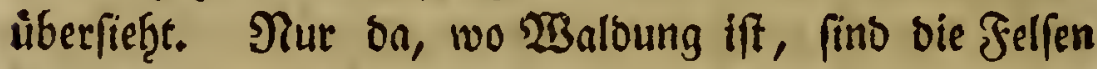

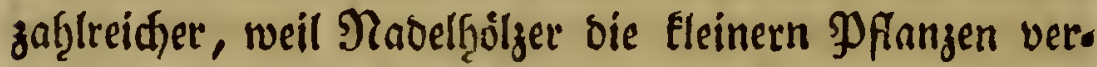
brången, und sufft und SBitterung weniger frey an Der Rerftórung ber Steine arbeiten Éonnen.

Den folgenben Tag giengen wir nach bet $\mathfrak{B} a$ tas mannfd)arte fort; fo tieipt ein ungeţeures Tḩal, bas oben, wo fich) bie Spizen biefer benden Berge befinden, anfångt, tief hinab reidft, uno meines Erad)tens Ģătte man von feiner grỏßten Tiefe bis an bie Epişe roof̧l brely bis vier Stunben zu fteigen. Das war aber für mich niffte notb̧wentig ! weil wir jiemlich weit oben ḩerborfamen; gleidjwoḅl Eam id) nidft ef̧er als in andertf̧alben Stunben Ginauf, und, ob idj) gleidh abs wärts viel fanneller gieng, fo ḩatte int bennod) eine

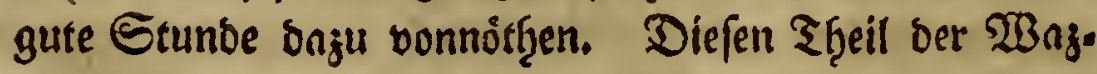
mannfharte werbe id in ber Folge allemal burn bie

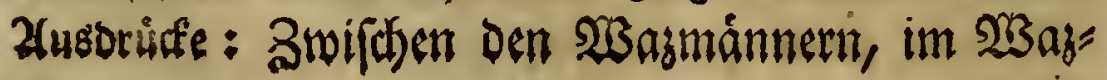




\section{Burghaulen, Den 8.55orn. 1784. 265}

manntbale, in Der $23 a$ ammunnflyarte bezeidjuen, weil

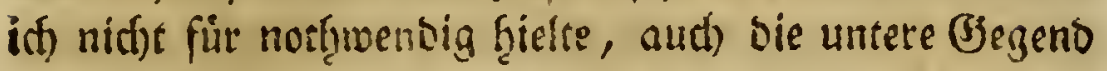
zu unterfudgen, beforibers ba id) ocutlide Epuren fand, Daß Bief ta genendet f̧ate, uno baf̧er meine Miůf̧e wenig wưrbe belofgnet worben. feunn.

Der SO!an meiner Ricife war anfänglich nidft Darnad) eingerid)et, Daß id) biefes lange, felfichte Sergthal Ginauf gef̧en follte, aber ber Zlnblidf beffelben hatte fo viel hoghes für mid), uno verfarach mir fo visle

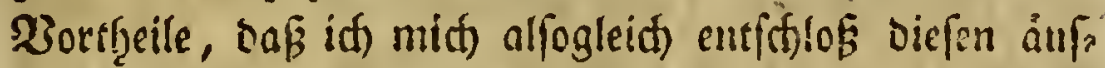

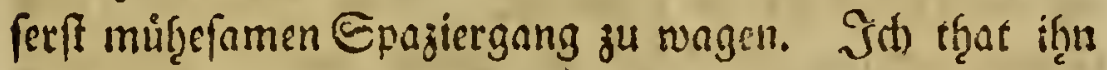

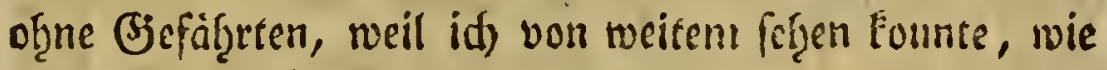

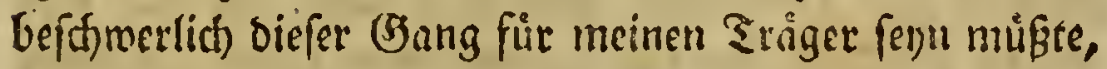
Der mir aud) mit feinem Trageorbe an mandfen (b) ginben zu folgen follectiterbings nicht múrbe im Etante gewefen fenn; zugleid) war es nidge miglitts zrwifhen Den beyben $\mathfrak{B}$ ergen irre zu gef̧en, oier fiid) ju vers

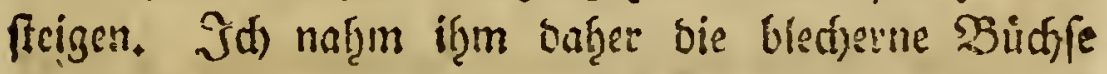

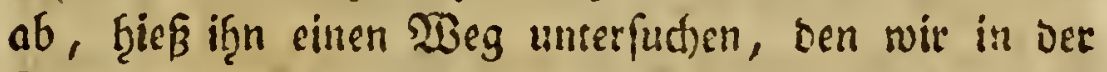
Jolye ju getgen Gătten, uno ben man mir als gea

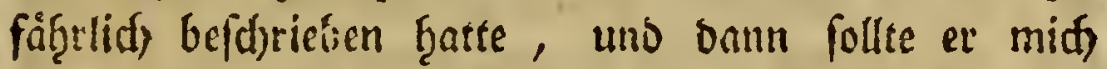
bem Sote, wo infifgn verlaffen tratte, gezeniber erivarten.

Etellen Sie firs jmo ungef̧etre SGainte von Ralfftein vor, bie to gernoe fins, als bie 23 indoe Şlues Bimmers, jwifchen biefen $\mathfrak{B}$ innoen ein Thal, bas now) viel fteiler bergan lainft, als ber fteilfte Santberg, uno mit oen ₹rümmern nngefültes if , Die

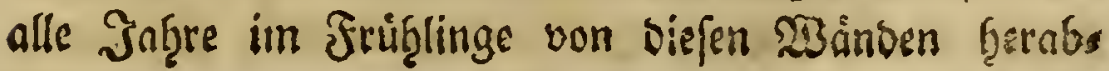




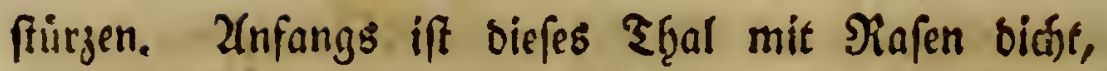
wie eine WBiefe, berwadfen, llad) uno nad) neţmen

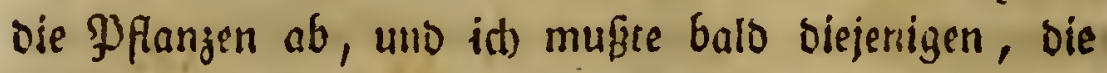
id) fammeln wollte, zwifhen ben Hleinern Eteinen ḩeraus b̧olen. alltmåb̨lig verlieren fid, aud) biefe meţ̧

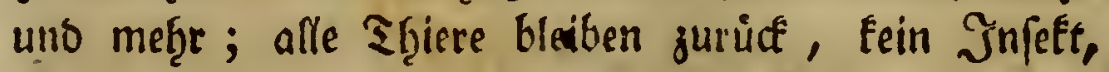
nitht einmal eine frliege fómmt melger baḩinan, unter. beffen die feelfentrummern immer groffer baliegen, uno jejt ḩat bie ganze Botanif ein Ende, weil feein Pflänza d)en, fein Brósdien (eine unbeutlid;e Eteinflethte auss

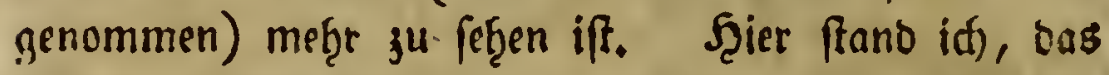

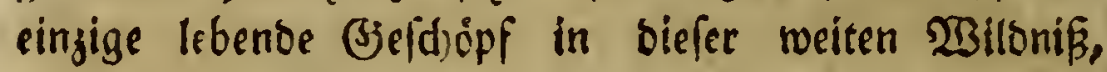
zroiften ben Trümmern zweener. Berge, faf̧ vor mir

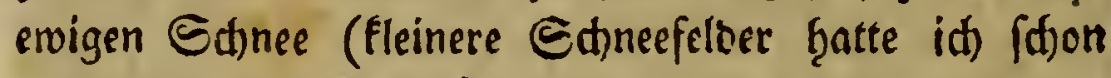
unterwegs zurùcfgelegt), unt weithin ḩinter mir an. gebautes Sand ; runo um mid) Bilber Des Tobes, Berippe beftånoig nadffurzenoer $\mathfrak{B a r g e . ~}$

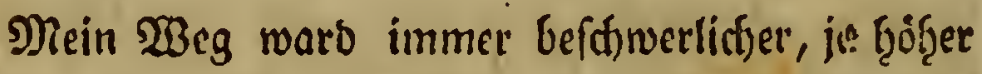
id) fam; id) mufte mid) balb jwifden zween nafie

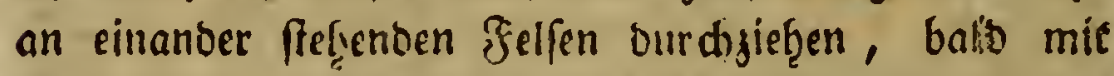
allen Bieren auf biefelten Ginnuftletseln, um curf ber anoern Seite nod) weit mixbefamer binabzufteigen.

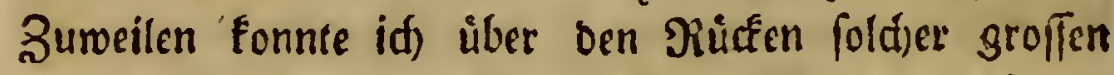
Jelfentrummern fortgelien, und von einem! auf ben

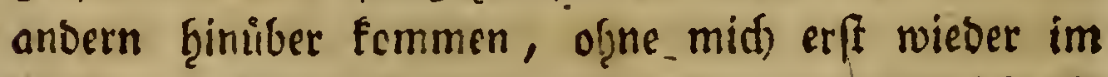
Eteigen gentbt zu haben; aber allemal mubite ia) bie

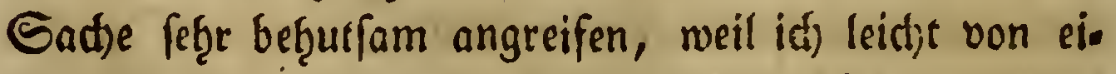

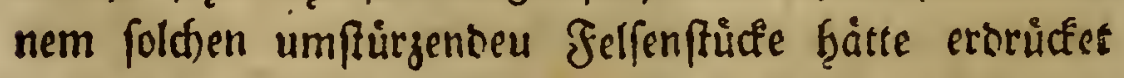
werben fonnen: denn Sic müffen wiffen, daß berley- 


\section{Burghaufen, Den 8.50rn. 1 784. 267}

groffe Srimmern offt ouf Eleinern, oben zugerundeten, aufiegen, sic banu jebes (3zevicht, oas mall an bent Seiten nnbrad)te, umftủrzen wirtoe. Sd) warb in biefen Ericfe muirflití) Durd) bie (Erfaf̧rung unterrid)= tet ; iff wollte eben von einem foldhen Jelfenftücte auf ein anters Gimibertteten, bas it) feiner (Jjröffe reegen feft gemiry om 2joben glaubte, um midt tragen ju

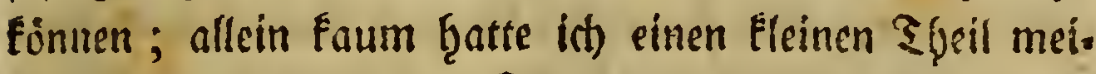

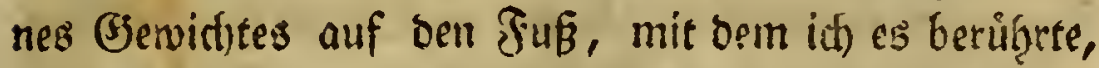
finfen laffen, als bas fellfenftutaf nach allen Eeiten ju

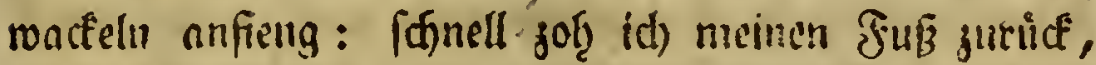
fucte ber Steinmaffe burd) cinen Hunweg beysutom= men, um iţren (Struno ju unterfurtien, uno faint, Das fie auf einem andern groffen felferifticke, das in ben

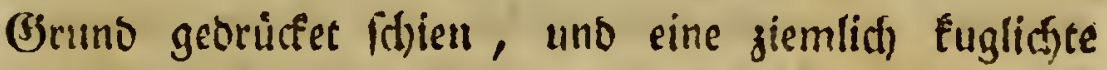
(b)eftalt ţatte, auflag, mitf̧in oaffeltbe nur in ber Mitte, uno in gar wenigen Puncten berífintte.

Ernfthaft uno (f)autbervoll, aber bod) angeneşm, war mein Etand Ģod) oben in diefem fohrectlichen Ţale, jwifd)en ben ungeheuren Srimmern her Berge. Der Zlublicf biefer grauenvollen Ecene fzatte für mith fo viel Broffes, to viel Erţabenes, das id) itgn gerne fruncenlang fortgeferget baatte; aber ein Tebel, ber zmilähen ben befdinenten Bsipfeln fdonell und mád)tig mifitieg, uno mids ein balbiges Donnerwetter ver.

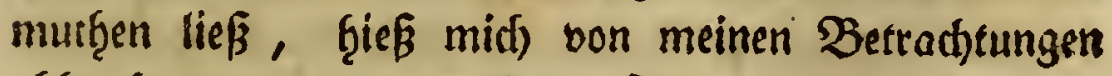

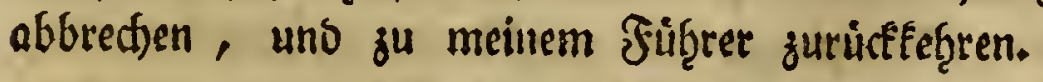

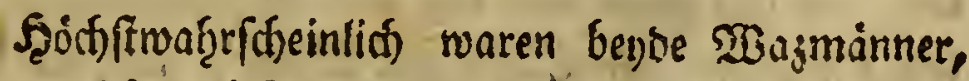
zroildfen weldgen siefes ungeţeure $\mathfrak{B}$ ergtţal mitten inne 
liegt, efecteffen ein einziger aber feftr fteiler $\mathfrak{B} e r g$, oef-

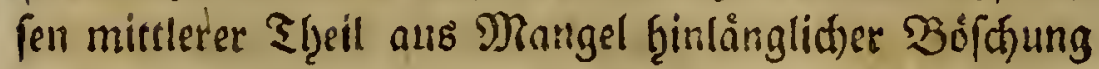

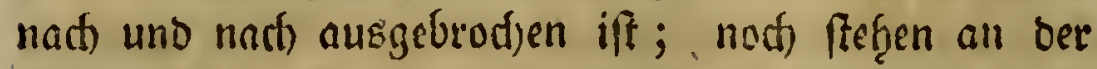
Epige diefes Tzales einige Ueberbleibfel diefes 3wi=

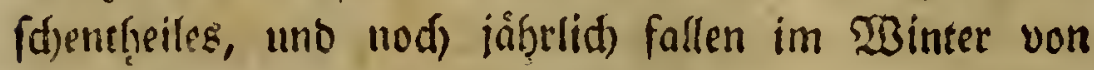
ben benden 2 Sånden einige Etticfe herunter. Mithts ift ǔbrigens natürlicter, als biefe Begevenfeeit: benn nuffer bem, Daßj foun ber $\mathfrak{B}$ egriff einer $\mathfrak{B}$ and jeoe Böfdjung ausfdifieft, fo oringt Sdinee uno. Siegen in bie Epalten biefer Feljengebirge unaufgeorlid) cin; frieret biefes, fo fpringt biefelben nod) melzr, uno madt eine Menge Trummern log, bie bely ben milt: beftem Zlnlafie. herabfallen. Zluf biefe Zlet entftano

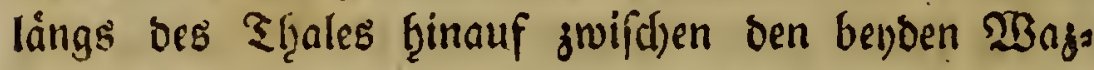
månnern ein neuer Sergricfen alls lauter berabges frürgren Felfanfticien, Der, wie Gic fich leidit denfen

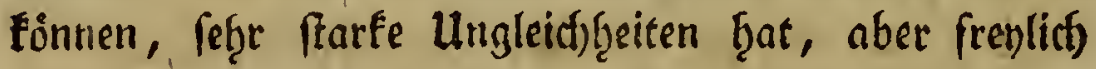
mit feinen benden Erzeugern fo lange in feine 2 Ber. gleidfung foummen Fann, bis biefe cinmal gröpsten= theils eingefrutrzet fenn werben.

Mir fiel bey diefer Belegenţeit ber $\preccurlyeq$ brief ein, ben Gulpitius an sen Cicero liber ben Tob friner Sod)ter gefdrieben ţatte. Sd) fat bier Berge fferben, bie einer Eroigfeit trogęen ju fönnen fdienen, - unto wir trauern untrojftidy an bem Earge einer geliebten Perfon, bie fdjon oamal zu ferben anfieng, ta fie geboghren warb. Berge verwefen, wie शationen baf̧infthwinben, und von ben einen uns oen anbern bezeitsinet ber fpán tere Ėeograph bie Etelle mit Müfze, aud jebem von 


\section{Şurghaufen, Den 7. Şorn. I 784. 269}

uns ift gleidfes Soos befthieden, aber unfere Dauter

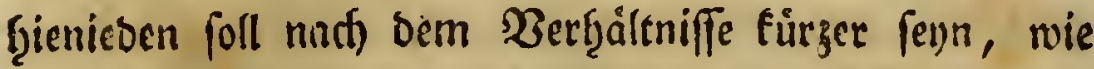
unfere Maffe fleiner ift, als bie der Nationen uns Berge; unauff̧òrlidge $\mathfrak{B}$ Biberwårtigéteiten follen, wie Wetter uno Sälte an ben BSebirgen, an unferm Leben nagen; bis endlid) nath) sen weilen Naturgeféçen, bie ber anbetensmüroige Sctiojpfer von Erwigfeit ḩer bes ftimmet ḩatte, ber Tag fömmt, Daß jene gånjlith

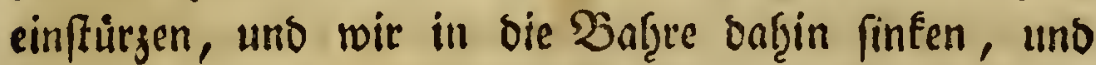

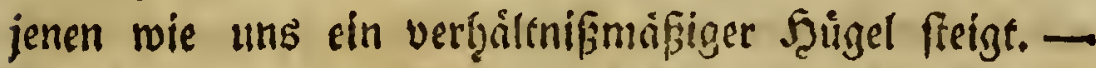
Eo frábilte idy bies an biefem groffer Edhaufpiele mein f̧erg wider alle Stitrme, bie unvermuţ̧ete ßerainbe. ranigen auf Daffellbe mact)en burften, und mußre nid)t, Daß id nad) einigen Monaten bey bent Sob. falle einer geliebten Ectwoefter bieje Borbereitung notţig f̧aben follte.

WBas if Shnen nealiif) von bem Jortgange ber Begetation zu fdrreiben bie Estre Ģatte, fand if Gier in einem viel grôffern $\mathfrak{B}$ ejirfe vollfommen be. ftättiget. (Fs war allgenef̧m zu feţen, wie fid) ber 2llprauff, und bie nejförmige Siseibe um die Jelsfteine ந̧erumfdhlangen, und fie manchntal ganj einf̧ưllten, wie if̧re abgefforbenen Zwoeige eine fleine Edjicft von Dammerie auf ben Edjeiteln biefer Felfentrủmmern

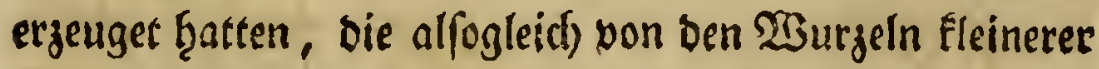
PPflangen wieber befeftiget warb. Freylid) geht bie Begetation bey afle bem feftr langfam aufroatts; felbft

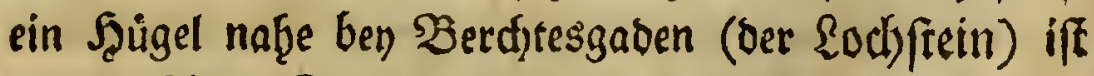
nur in feinen Epalten, und ba, wo er flache Steller

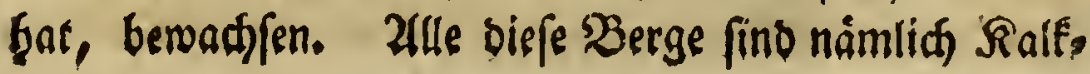


gebirge, bie beftänbig von Sufft unb $\mathfrak{B}$ etter angegriffen werten; ber Siegen waifd)t bie aufgélö̧ten Theile augenblicflid) meg, unb bie bünnen Flecíten, bie fict)

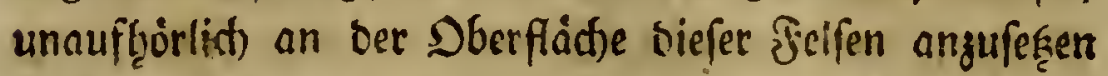
fuctern, fơnnen fie nid)t Gignlänglid) bor ber Berfiurung

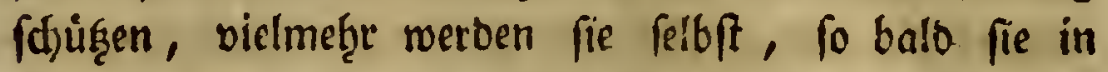
Bermef:nng geben, mit fortgeriffen, uno in bie şäler

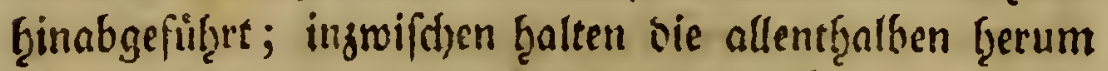
liegenden Eteine, und tie baburd) verurfadjen $\mathfrak{l} n$ s ebenţeiten inmer viele Dammeroe auf, uns fo rüct aller Berfiorung ungeact)tet bie Begetation gleidwwobl

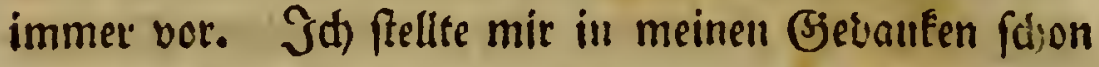
bie Jalgrthunterte vor, in weld)en biefe jez̧t raubeen Berge bis an if̧re, frenlid) betráchtlich 'erniedrigten, Gianfer beroobnet fenn muirben; fal ta goldene Felber, wo id) jezt fúmmerlid) cinige alpenpfianzen zmifthen ben Eteinen herausgrub : foh Da Raninichent-

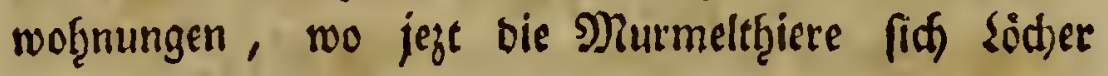
gegraben ḩatten.

Unter biefen füfen Traiumen gieng idf'an ber füblid)en Seite Desjenigen Bergrúdens, ber fid) zrwir

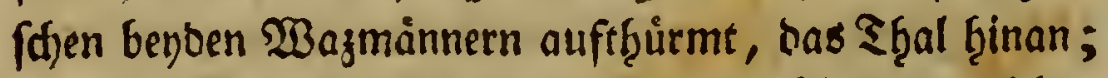
aber f̧erab naḩm id meinen $\mathfrak{B e g}$ an feiner norolid)en Eeite, uno wie roar idh) betroffen, ba id) Ģier eben fowof̧l ben frortgang bes Eifes feţen mußte, als iff vorţin ben ber $\mathfrak{B}$ egetation gefergen hatte! - Id) fanb, was id nidst vermutţete, baß fid) aud) bier Das (Fis immer weiter ḩerabzię̧e; bie Bemeife waten unlaugbar, if mufte nidht nur viel lánger auf ben oben Ed)neefelbern berabgef̧en, fondern id) fano for 


\section{Surghaufen, Den 8. Sorn. 1784. $27^{1}$}

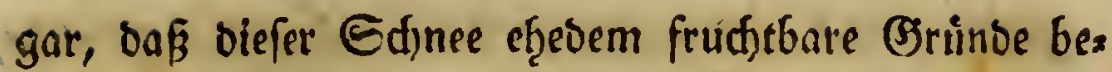

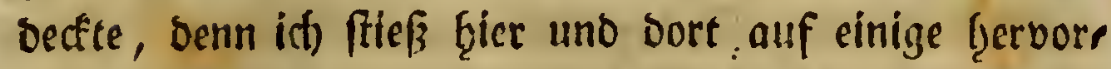
ragente Etcine, um bie fing bie fraurigen Ueberrefte

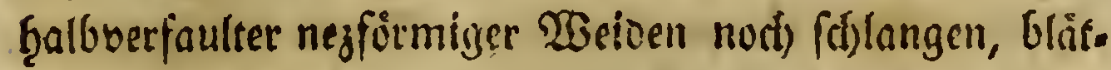

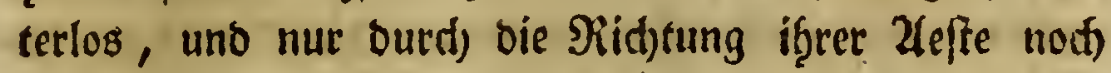
fenntlid) fdienen fie mir zu fagen: Du bgaft bidf geirret.

Diefer $\mathfrak{B}$ ergrưcfen ift bem gröfern $\mathfrak{B}$ nzmante biel nahber als oem fleinern, baḩer nuth bas norblicte Sfyal enger als bas füblidje ift; bie Eonnenftralen fönnen alfo ungeffinbert bas froblid)e Ţcile erwärmen, uno bie Feuttrigfeit bes Bobens in náb̨renoe Safte verdinnen; aber an ber Norbfeite werben fie von

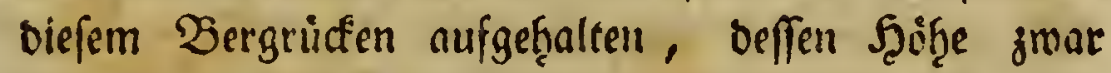
an fid) nid)t fef̧r betridetlid, aber bod groß genug

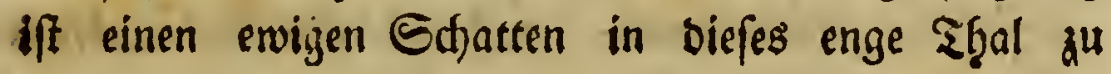
werfen.

Diefe Berlängerung bes Eifes fömmt bann im gegenwărtigen Falle von einem gang befontern $\mathfrak{U m}$

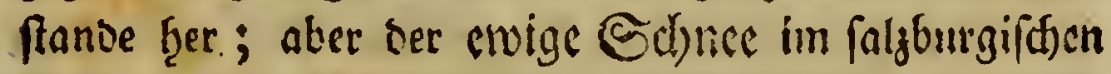
Şod)gebirge, bon bem id neulid) (t)rieb, und now) viele andere Edinneegefitio unferer 2llpen in Europa, bie ef̧emals vorfrefflitge $\mathfrak{B} e$ endeplásęe waren, fino Gachen, von benen vielleidte bie Erflärung ungleid)

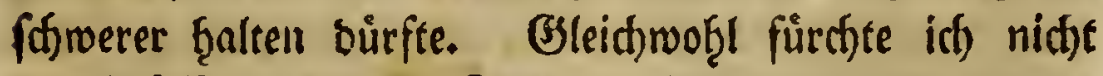

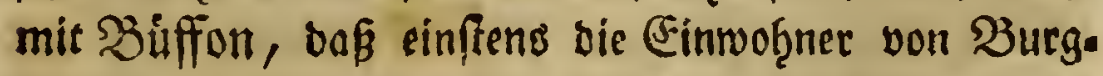
braufen gezroungen werden follten ifgre Staot ju ver. laffen, um nidft unter einem ewigen Exthnee erftarren

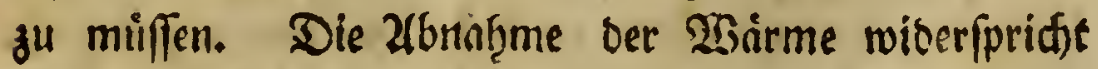
vielmef̧r ber befannteften Erfaf̧rung : Die ţeutigen 
Italiäner finben Deutfolano ben weitens fo Enlt nidjt, als bie Riomer Das alte Bjermanien fanben. Man fònnte cinwertsen, bas Zlusţauen Der WBatber in Deutfhlano iffne ber Eonne ben Butritt zur Erofflictee, unb verf̧inbere bie gar ju fithefle Berflicthitigung ber Erowärme, unterbeffen Das beutige Jtalien felbft nidje mehre fo warm ift, als es in ben Sciten bes Jultus Eaffar war. Man beḩauptet aber bas lezfere, olgne bafi man babely im Stande måre es zu erweifen, und bie Zlusteutung Der $\mathfrak{B}$ âlder in Deurfchland follte eţer bie $\mathfrak{B e r f l u d}$ )tigung ber Erowarme begünftigen als verf̧indern, menigftens mürbe fich, alles gegeneinanber abgeroogen, Bermę̧rung uno Berminterung fo jiem: lid) gegeneinander auffęeben.

Jid) glaube immer, Daß fidf alle bie neuerlid mit emigem Edynee bebecten Bjebirggegenden mob̨l ficher in Dem falle bes ellgen Thales, bas in ber 2Bazmanuffyarte zwifhen bem erft entfandenen $\mathfrak{B}$ erg. ruidfen und bem groffern STGazmanne liegt, befinoen

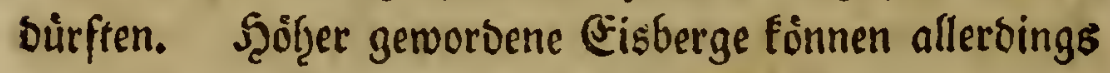

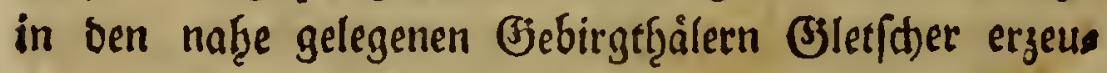
gen; aber man fhliefst, beud)t mir, zu voreilig bort Dem, was auf 2llpen vorgetit, wo bie ounnere sufte Der Sonnenwairme fef̧r wenig empfänglid) ift, auf bas, was in Ebenen gefdietgen foll; fo bals in bem offe erwäh̨nten Thale bie Sonnenftralen wieber ungef̧inoert auf bie Erbe treffen fonnen, war alles in ber ffionften Blütḩe, und idf fand wirflit) nit Dem einen Fuffe auf Exfjnee, und ḩatte mit bem anbern fagon eitre 


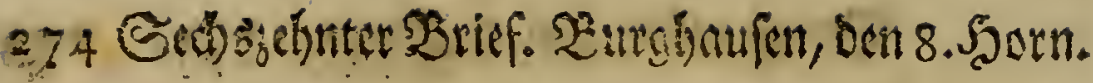

rug̣ten wir aus : bern of es gieif Mittng war , fo naḩmen wir bennowi auffer etwoas Suppe nidf)ts z" unis, weell wir uns benge nidft moghl befanben; die

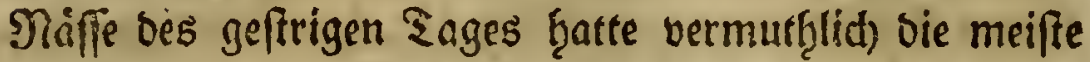
Edjuld baran. Won f̧ier gieng bie গieife immer bergab, gegen ben Sisinbact) zn, ber ba, wo wir

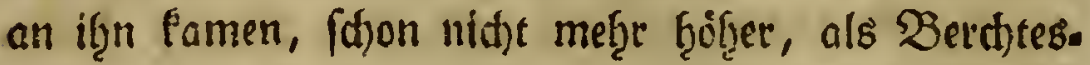
gaben felbff, liegt. Sith unterfuchte unterwegs einige Rreibenftutfe, die man jenfeits des Қaches aus bem Berge gerwinnt, uno momit einiger Şandel getrieben wiro, aber mein gsührer, ber fitf) unterbeffen auf einen

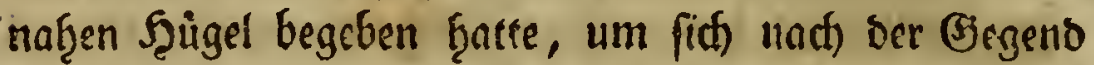

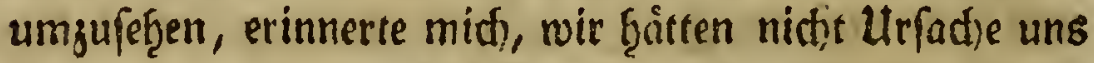
lange aufzuthalten, weil er abermal ein Donnerwetter in ser Ferne erbliaft batrte. Es war bafferbe and eţer $\delta a$, als wir es vermutḩeten, und mir muften unter einem frarfen Plaģregen nod) einen ziemliden

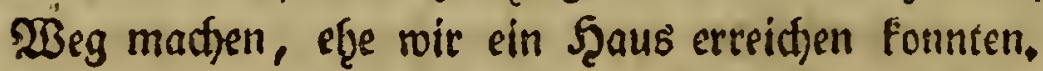

Dießs iff fo ungefáf̧r bns Tiefultat meiner gwenten Bjebirgreife, bie mir eben fo viel $\mathfrak{B}$ ergnügen ales Belef̧rtung verffiaffte. Die Eleinen $\mathfrak{L}$ nbequemlidja

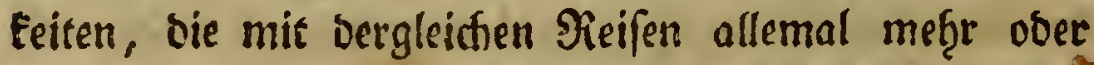
tweniger berbunber fino, entfatlen bem Geóadjeniffe? fo balb fie überffanbett find; aber bie Gerrlidgen

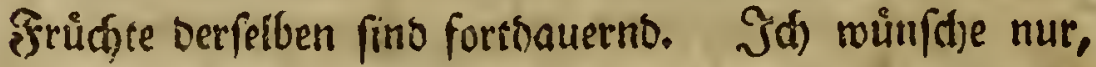
Daß̧ Sie diefe Nad)ridjt mit eben fo vielem $\mathfrak{B} e r g n u g e n$ Iefen mogen, als mir bie Erinnerung an biefe Pieife gewåg̨tre, unb verb̧atre u. . . f. 


\section{Siebenzelynter SBrief. SBurghaufen, Den 2e. 275}

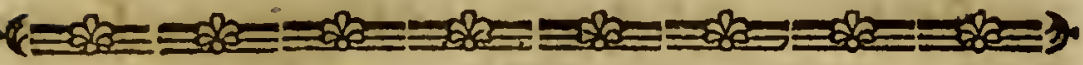

\section{Siebenzehnter 3rief.}

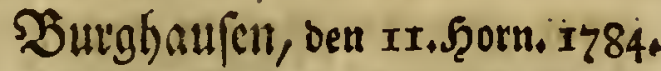

Dritte Arpenteife: nach Dem Geelyorn.

$S$ (d) habe jwar gegenwartige Rieife aus meinem - Tagebuche befonbers ausgetroben, um Iflnen onvon umftintildfe शind)ridje zu geben; id werbe aber bod) onbat) ganz Furz Fein, theils meil iff Shnen nur tas (5)efagte abermal fagen muskte, theils weil idy in ber (befffroinoigfeit, mit weldter idf biefe Rieife madte, unmoglid to viele Beobadtungen madien fornte, als wenn idf fie mit meiner ganjen Muffe an.

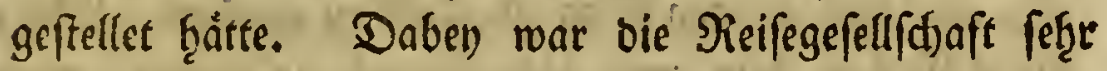
großs, uno fef̧r anfef̧nlid, aber eben bię̧ Ģinnert ben

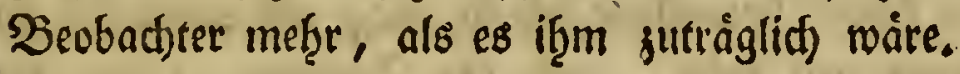

TBir fuñren Zhends ben is September nad; ber Sinmfau, befateen unfermegs einen Torfgrunt, auf reldfiem nåd)if bem Torfmoofe (Sphagnum paluftre) bie Moosbeeren, uno bie benden Sonnenthame

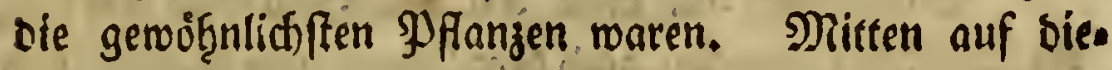
fem groffen Eumpfe fteh̨t ein Leḩen, das Moosleb̧en genannt, bas mich) gang in Erftounen feģte. Jas fonnte nur mit $\mathfrak{R}$ üf̧e, nut mit Şilfe meines $\mathfrak{B}$ ergo ftoctes, uno einer gemiffen scictrigfeit meines Rörpers, mit welder id gleid) Den $\mathfrak{B}$ afferfpinnen über bers gleiden Eủmpfe Ģingufdhreben weis, Daf̧in fommen, gleid)wobgl fand id hiier mitten im Eumpfe eilie b̧übfde Strecle, bie man als $23 i e f e$ benujte; unb fogar eini. 
gen Felbbau. Jd) fann bas Bergnugen nid)e aus. brucken, bas id allemal fübzle, mann ind ba, mo bie

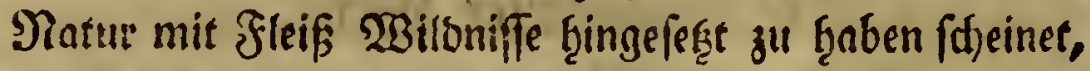

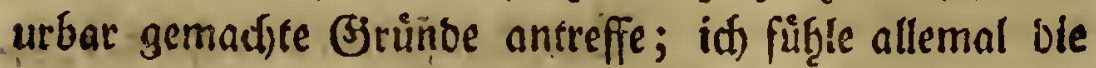

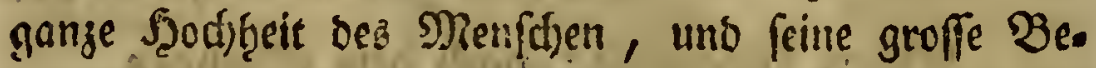
frimming, unter ber Dberḩerrfdjafft bes Edjopfers

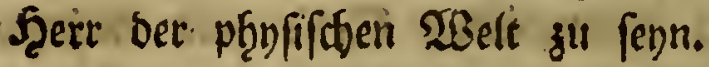

Wir bratjen fojon um 2 Uţr bes Nachts wieber

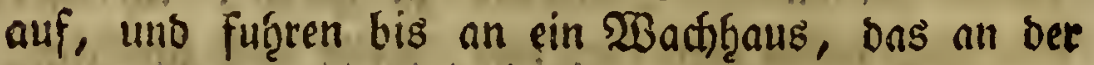

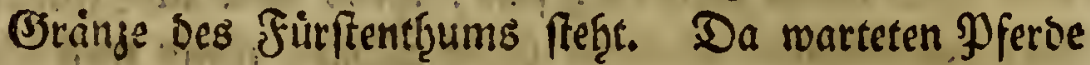
auf uns, bie uns ben úbrigen $\mathfrak{W}_{\mathrm{eg}}$, fo weit man mit Pferben fortfommen fonnte, bas ift, bis an bie

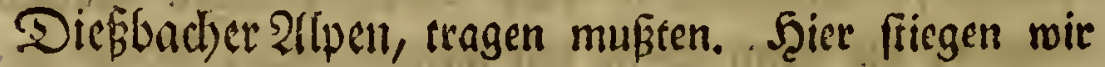

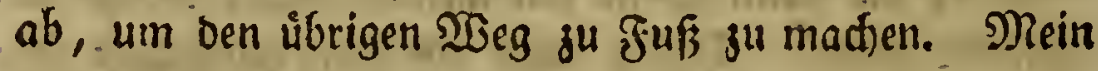
guttes (s) fitut fam mir Gier trefflid) zu fratten; vom Pferde hernb botanifirte idh fo gut, als wenn id) gut $\mathfrak{F u}$ ß gegangen wåre, uno bie Bauetn, Denen bie Pferve gef̧oitten, pflúcften mir von ben Y)fanzen, bie iit) if̧uen einmal genviefen b̧atte, meţe Etuctee, als mir felbft lieb war.

Wir Eamen auf biefe 2(rt Den Şinterfece vorbey, bie Mhiblftirze vorben, nad) bem J̄iger f̧aufe Falleck, von in über sie אältenbrunnemais, úber bie Raltens brunnir alben in bie Siefbacker allben, giengen Dann fo ziemlich am Diefbuct)e fort nad) Der Mitters liutte, den groffen uno Eleinen Sumbstod vorbey über ben bolsen Slyron, ober mie bas Ding nod fonft teifift, über bic Gticge nath ber Scochiviefe, fitegen enolid) yon biefer an bem Eeef̧orn ģinauf. Riưt. 


\section{Burghaufen, Den I r. Sorn. I 784. 277}

soarts naţmen wir eben diefen 2 Beg verfef̧rt, weil ein anberer, ben wir b̧ätten sef̧en fornnen, viel zu gefáhrlía) war.

Die Mullylfurtse ift cin Berg, beffen Spige breit, aber bunne, brenlappidst, und fo fteil iff, baß es feinem ş̧iere mòglid wiro fie ţinauf gu flettern. Eie bridft aud beftånoig ab, befonbers im Minter, uno beym Erbneefdmelzen, uns bie Srümmern liegen bis all bie Etraffe.

Saltenbrum foreint feinen Stamen von ber groffen Rålte vollfommen zu thaben, bie on Gerriffet.-

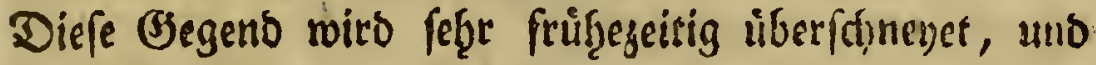
of fie gleid) bev) unfrer Z(nfunft offen (orber) war, fo

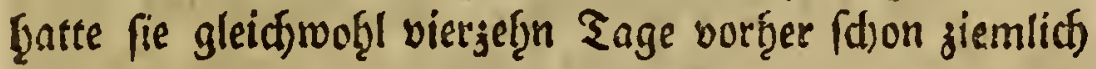
tiefen E(tnee, aud mitten im Eommer ift ḩier bie sufft fuifl, und in ben Stunben bes פTlorgens und Z(benos wirtlid) falt.

গiact) Dem, nas id ein anbermal ùber bas Wort Mais gefagt habe, follte bie Rultenbrunnermais ein ausgetrauener $\mathfrak{W}$ alo feyn. Das mag fie aud) yor ungefăficr fünf̧ig Jaf̧ren genvefen fenn, aber jezt ift

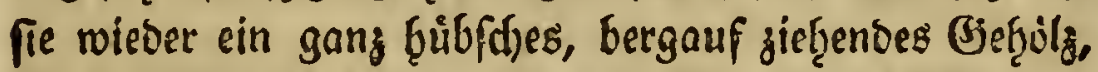

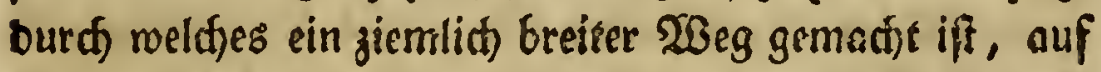
weldjem mir ḩinauf ritten. (5) war noch Nadise, sa wir uns ḩier befanden, bod bemertte idh, taf an ber

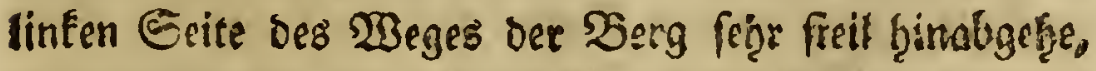

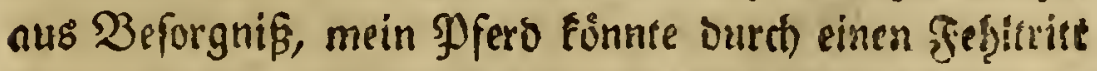

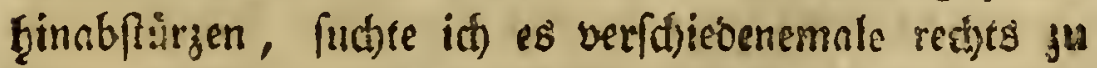
liten: allein es lenfis bem ungeaditet allomal wirver

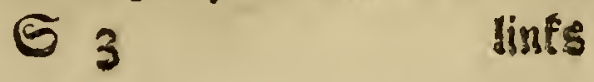




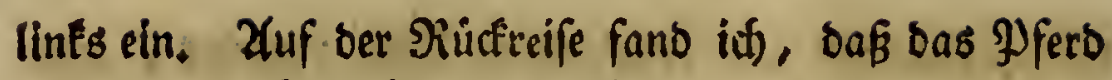
utrache seb̧abt ந̧abe mir nid)t zu gef̧orfamen: Das Edjneemaffer uno bie Siegen ḩatten bie Erbe von Der rectiten Seiten biefes $\mathfrak{W}_{\text {egeg }}$ weggeifinemmet, und eine bloffe Eteinplatte zurigelaffen, auf weldfer fich

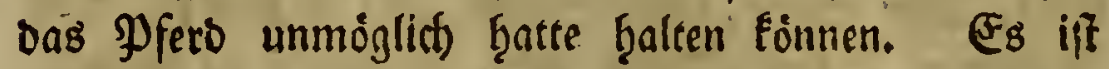
in bergleithen Fällen allerbings bey weitem bas $\mathfrak{B}$ efte, wenn man fid) bem Pferbe, bas ben $\mathfrak{W}_{\mathrm{eg}}$ fajon offt gegangen iff, ủberläpt: biefe Ş̧iere geţen in fold)en umftänoen weit fidjerer, als fie ber geldjifftefte Reiter lenfen muirbe; aber eben berowegen find $\mathfrak{B a u e r n p f e r b e}$ aus ber Begent ben berrfdaffitlidjen Parabepferben allemal meit borzuzief̧en.

Bon ber Mitterhittte, bie betråd)tliđ ţợ̧̂er liegt, offinet fid gegen Tyrol f̧inaus ein rauḩes Theater von fteilen, naften Fellengebirgen, uno im Şinter. grunbe lagen bie Ețneegevirge, bie mir fo naţe nors famen, als wairen fie nur einige Etumben entfernet; eine präd)tige Scene für einen Maler, aber ned) prär()figer für einen Phillofopḩen! So weit wir ůbrigens von biefen Styneebergen entfernet maren, fo fübiten wir Dennod bie Edsneelufft reģr ftarf.

Niकts ift gefdicfter cine erb̧izte Einbilloung von

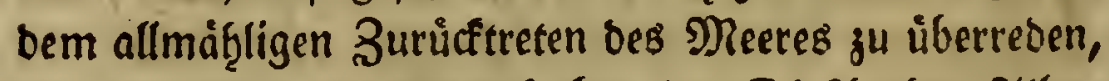
als bie (Jegend, bie fid) ben ber Dief̧bat)er 2llben anfängt, uno an ber Eplize bes Eeef̧orns iţrg Enbe erreidet. Die ganze Etrecfe bis an bie Mitterḩưtre ift felfidgt, uno zieb̨t an fid) fef̧r factse bergan, bem

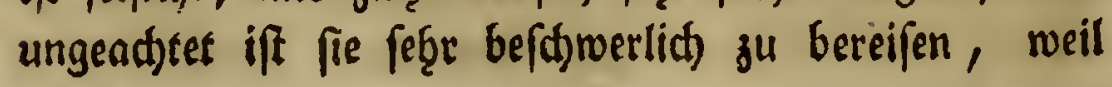




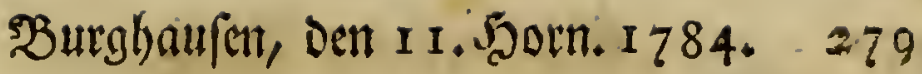

man auf biefen felfentrummern, bie fid beynaţe ftufs fenweife. erf̧eben, immer berumfteigen mufs. Der aluf. gang von ber Mitterţuitte nad) ber Şod)wicfe ift fefre

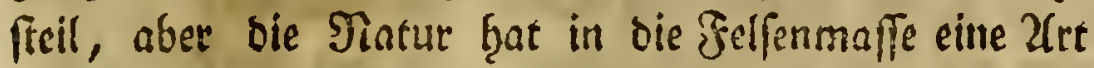
von Treppe gef̧auen, bie freyfid) nid)t für gewoofznlicje Sieufden gemad)t zu feun fheinet; unterdeffen ftiegen

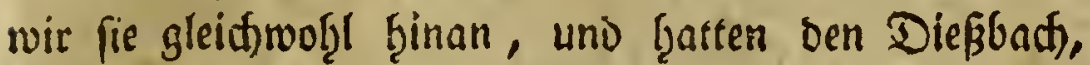
Der mit einem entfeglidien (Jerånffie liber vie Jelfen herabfrúrgt, immer jur linfen f̧and.

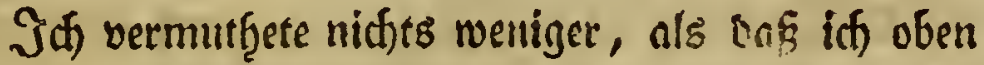
über biefer Jelfentreppe flathes, ebenes lano autreffen

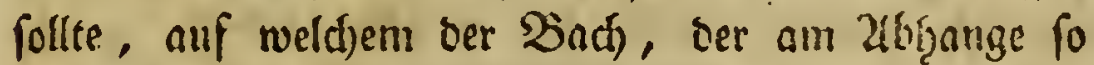

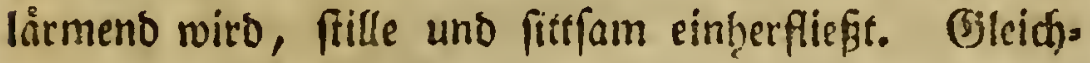

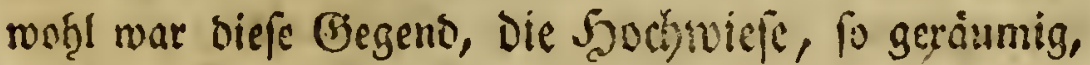
Daß fie nllein, wenn bie Bevilfermig einftens fo trod)

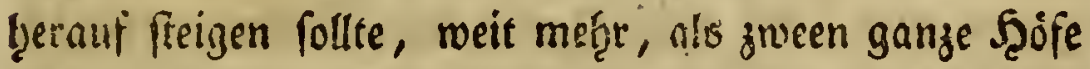
abgeben wirtoe. Unteroeffen mulfien Sic fict bey Dem Slamen ber J్hodwiefe eben feine Sitefe benfell, die einen betråd)tlict)en f̧eufd)lag geben bürfte, die Pflans zen waren alle ferger nirbrig, uno der grópte Theil bie. fer Ġebirgheide war mit bem Eteinfatutte bebecff, ben bie Edureemaffer am 2lufange des Eommers bon ben umberliegenoen Bergen mit fich) terebajüįren. Reines unferer Şaustţiere, Den Şuno vielieid)t ausgenommen,

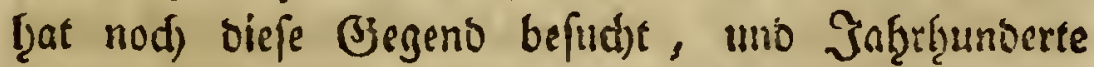

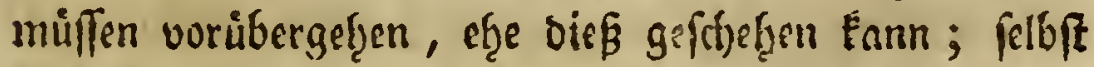
Ed)afe, bie id) ref̧r weit oben in ber $\mathfrak{B}$ agmannicharte angetroffen hatte, fommen nidht hiiejier, fie wurben

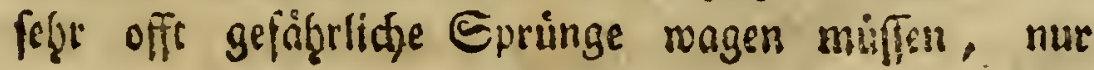

$$
54
$$

(3) 


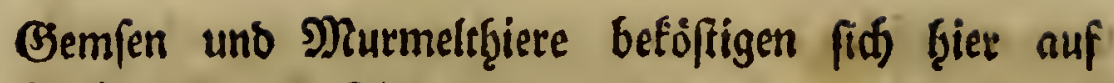
Siédnung ber flora.

Bjanz Ginten am Enoe ber Şodfwiefe fömmt man auf bas Seef̧orn ţinauf, weld)es ein $\mathfrak{B e r g}$ iff, beffen Spife man wobll in jroo Etunben, von feinem Juffe an geredinet, nicht erreider. Sith vermumberte mid) (ȩ̂re, b̧ier Ciftus Helianthemum, Das bey uns

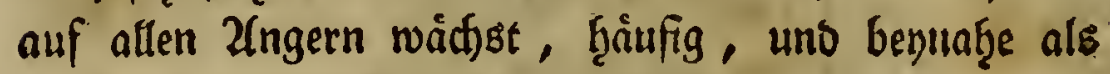
bie vorzughlidjfte $\mathfrak{P}$ flanze anzutreffen.

Diefer $\mathfrak{B e r g}$ ift ben ganzen Sommer úber offen, unb es giebt ba weit herum feinen andern, ber bew trádtlidi groffer, uno mit Edynee bebectit wäre. (Bteidf)woţl ent(pringt ziemlid) ḩod) oben an feinem 2tbf̧ange eine ber Dueflen bes Dirß̈bartes, bie nie verfieget. Boft weis es, auf weld)em Berge ber Edfnee liegt, ber bisfe Quelle mit $\mathfrak{B a f f e r}$ verfief̧t: Bielleidft ift es einer ber Edneeberge, bie Gie be. reifet ḩaben: vielleicfte einer im innern affrifa. Sie lad)en; und id) vermutḩe es felb/t nictft, baßs ber Dieß̉bad, fein Dafeun afrifanifden Edjneegebirgen follte ju banfen bzaben, aber wunbern wirbe midss nidft, menn man einmal eine å̧̄nlidje Entbectung machen follte; wer fann wob̨l fagen: Das ift un. nóglich ? Unwafirtffeinlidh mag es immer fenn, aber bod) ware nid)t bas erftemal, daßj man

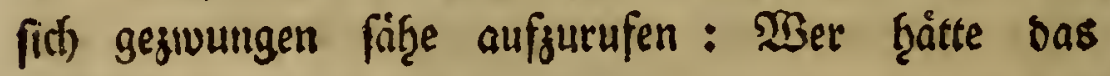
geoact) :

Sebod) meine Joee über bas innere (Jemeb unferer Exbe ift nod nidt to reit gebief̧en, baß in 
Burghaufen, Den 1 1. Şorn. 1784, 281

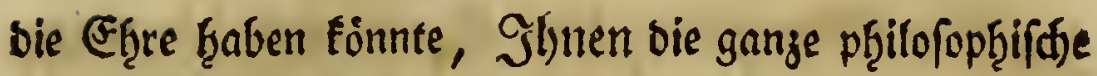
Troiumerev), wie Sic es nennen mơdten, vorjulegen. Ith frabe gegenwårtig mur bie Ef̧re Sie ju verfifjern, Daßs id) U. f. f.

\section{Surant.}

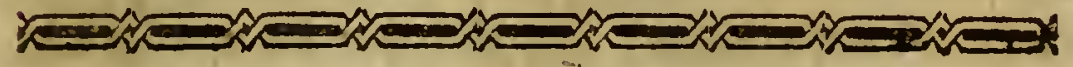

\section{A(d)tzethnter $\mathfrak{B r i e f}$.}

\section{Sourghaufen, ben 33.5 orn. 1784 .}

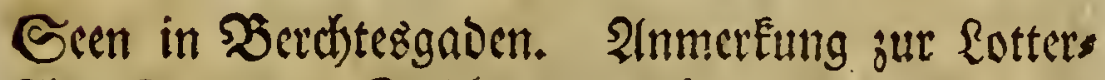
fiten Rarte von Galzburg. Rionigbfec. Şarthos lomái 2ue. Dberfec. Dic Snalat. Mitterfec.

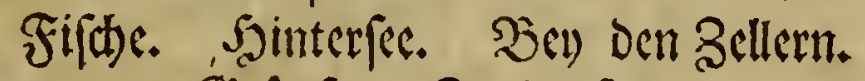
Girinfee. Sumbenfee.

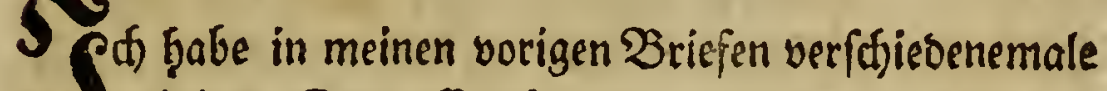
$\checkmark$ einiger Seen Errodą̧nung getţan, aber bas

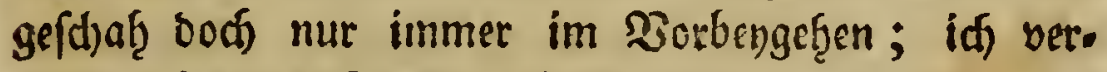
fparte mirs geflifientlidf ouf einen eigenen Brief, Sie mit ben Geen biefes sanbes zu unterţalten. Mir fino folgente Geen befannt: Der Rönigsfee, ber Mitter.

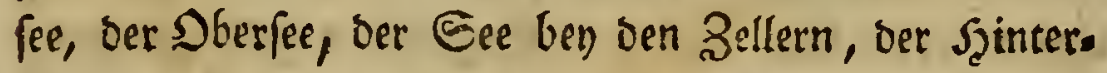
fee, Der Brizinfee, und ber Funbenfee, ber aber zur

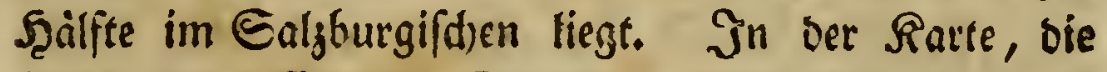
Sotter vom Errffifte Ealyburg geffochen ţat, wiro nodf ein See jwiffen der Siamfau und bem Şallftḩurm

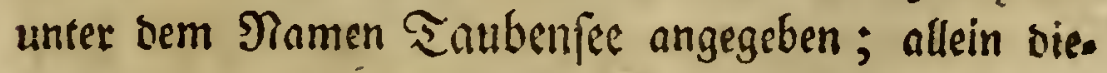
fen leştern ţabe inf reber gefetgen, nod, Dabon reben ตุôren.

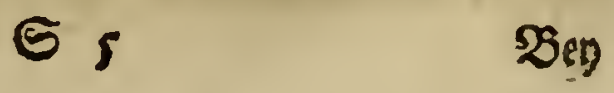


Bey) Belegentzeit biefer Sarte muß̧ ich einige Berfecten růgen, bie mir in terfelben eine ßerichtizung zu verbienen foreinen. SDetcr foget ben Rónigsberg ganz naf̧e an bell Şauptort bes Sandes, an $\mathfrak{B}$ erdjes. gaden felbft f̧in : er fdjeint dicfen Berg mit bem Edwwarzorte verwed)felt ju Ģaben, bas gerabe an bie. fer Etelle fref̧en follte, ba bingegen ber Rionigsberg bis gegen die Mitte ber offlid)en Seite bes Rönigsfecs

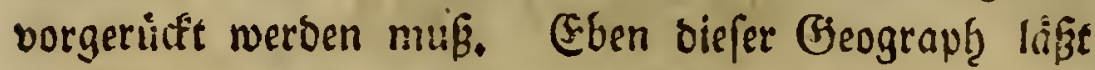
bie 2llben aus dem ફ̧interfee entftểen, bie bod) if̧re J̧auptquelle im Rónigsfee ḩat, uno butd) einige nms, sere Sadde, bie fid) Darein ergieffen, worunter ber $\mathfrak{B a d}$, weldjer bon ber Bifdjofswiefe feerfömmt, wolgt

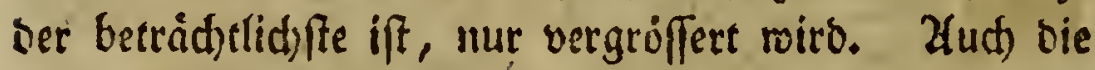
Sartholomái Zlue foll nidjt an bas weftlidfe Enve bes Eees, fondern ungefähr am Ende bes zweuten Drittḩeiles beffelben fîthen. (Zuf ber Rarte, bie eben biefer (B)eograpt vom ganzen baier(d)en Rreife ge. liefert b̧at, ift biefer Şeģicr verteffert). Daß́ biefer Drt, ber auf eben die art nie Bercteresgaten anges zeigt ift, zu proid)tig angezeiget worben, merben Gie bey) oer Bselegenbeit einfeb̨en, wemn meine Erjöhifilung Diefe merfwursige Zlue zum Biegenftand h̨aben witb.

Der bernifzmtefte unb grófte aus allen biefen Eeen ift unftreitig Der Rónigsfee, ober wie er noch fonft beif̧t, ber Bartf̧olomåi Ece. Diefen lef̧tern Pamen bৃat er von einer Sirdye, bie auf ber Zue, von ber ith furz zusor rebete, unter bem ₹itel bes beillgen $\mathfrak{B a r t b g l o m a ̊ n s ~ e r b a u e t ~ w o r b e n . ~ Z l b e r ~ b e r ~}$ erftere 


\section{Surghaufen, Den I 3. Satn. 1784.283}

erftere Name ift f(s)werlid), z"l erflăren, ba befanntlid biejes Sano niemal einem Sỏnige angeţidrig war, uno woblgl fonwerlid) jemal Rơnige b̧ief̧er getommen fegn mơjen. Bon einem Enoe zum antoern fährt man i̧̨n ungefâţer in jwo Etunoen, aber nad) ber Sueere fiat

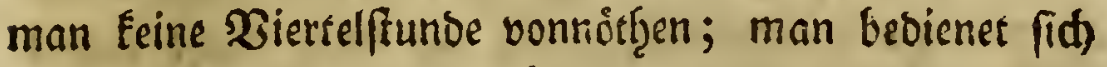

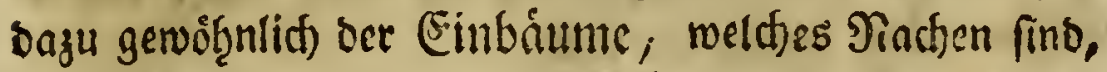
bie aus einem eingigen Etid $\mathfrak{J g}_{\text {ol }}$ gematbe werden;

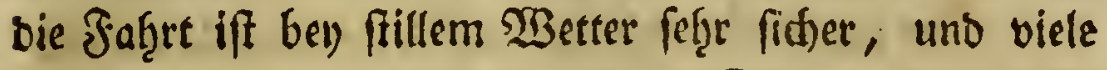
gief̧en fie auth im Eturme ber in Eafiffen vor; bod find aud einige Ediffe ba. Der Boben diefes Eeps befteht aus Ralf und fleinen Trimmern von Ralte feinen, und nirgenos, ausgenominen nabe ben feinem Z(usfluffe, und gang oben an ber Kaunerwans, fat fidf) sie Begetation anfercen fönnen; in Dem erften Drte if es cin Eaamfraut (Potamogeton), ons id) nid)t unterfutfet ḩabe, uno am lef̧tern eine Lllbe, bie id ein andermal befafreiben werbe. Die $\mathfrak{B a f f e r}$, welthe biefen Gee unterf̧alten, fins, aliffer ben (Sirundquellen, ein Sach), ber unweit feines Xusfuffes liber eine Marmorwano ferabftromt, uns eine ganz artige Ras. fave bilbet, uns nod) ein anberer $\mathfrak{B a t}$, Der an bem Ende viefes Eees von einer ungeţeuren fiellenwans

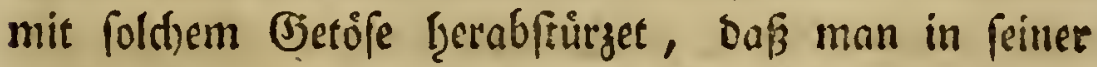

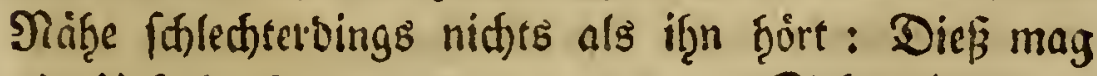

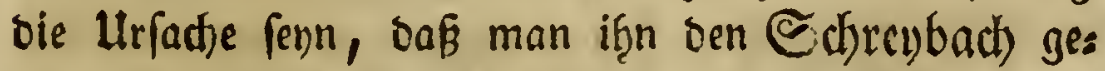

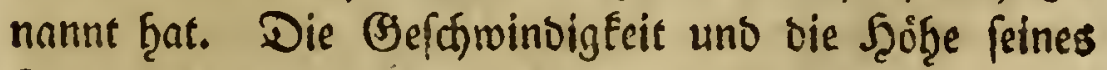

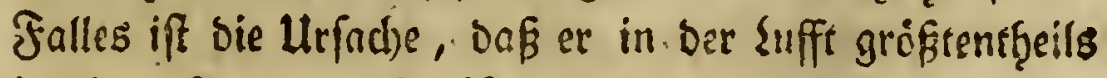
in einen Regen aufgelöfet mirt, ber bie Edjiffenben, weld)e ba vorbey) faftren, fthon in groffen Entferutungen. 
benejet; ju biefen fömmt nody ber 2tusfluf bes Sber. fees, von bem Gie bald fioren follen!, ber fich in

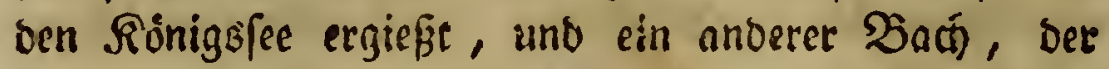
binter oer Bartbolomäi 2lue entfpringt, uno teinen fid) tbaren 2lusfluß in ben Gee beat. Z2(ber biefer $\mathfrak{B a d}$

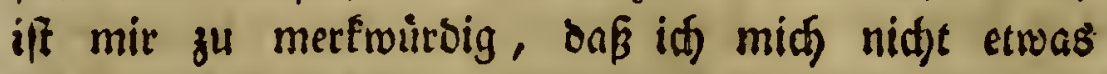
linger babey nufgalten follte.

Der ganje $\Re \delta$ nigfee ift auf feiner reffliden uns

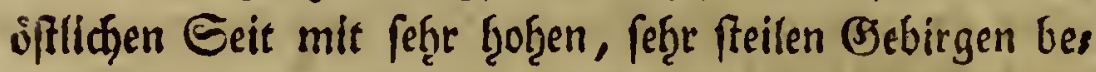
gränfet, welche groffenţ̧eils an if̧rem Fuffe to feiger

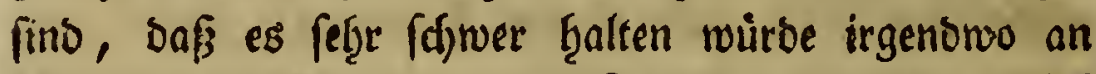
das Sano zu fommen, im Falle man bas ungliuf

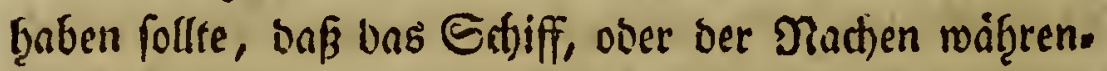
ber Fabert untergienge; nur an ver $\mathfrak{B}$ efteite mad)e

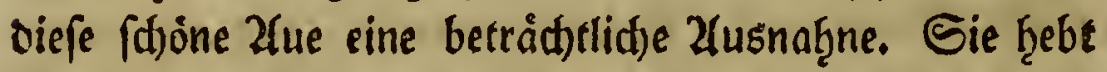
fid) fachte aus bem $\mathfrak{B a f f e r}$ Ģervor, unb jief̧t fid) in

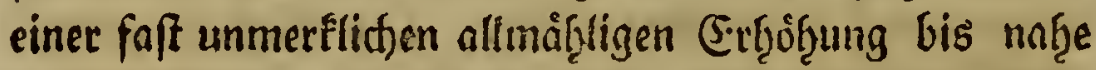

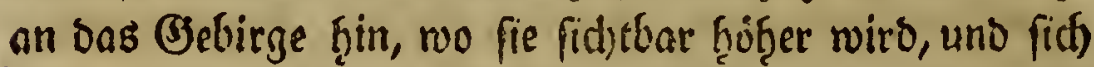

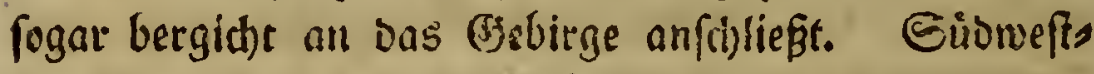
lich zieģt fich) in bas Salfgebirg eime bergan laufente,

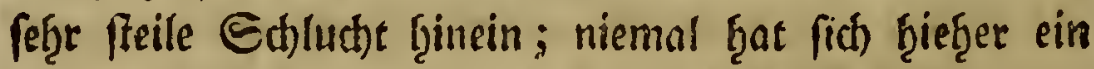
wärmenter Sonnenftral verirret, fein $23 a n b e r$ ocm: nad), wenn biefe Sd)lutft mit eroigem Elife bebectt ift; man nennt biefen Sort bie Eibłapelle, ober aud) bie gláferne Sapclie. Jd) verfucifre ifgm bengufommen, inoem id) langs des $\mathfrak{B}$ adjes, ber baherab foimmt, fortgieng; allein id́ war nod) foum eine ḩalbe Etunse weit gegangen, als mirs unmogglich warb weiter zu

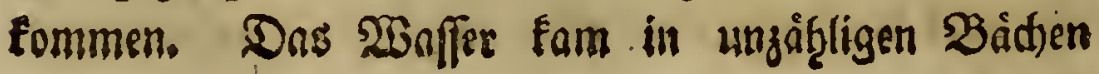


Burghaufert, Den 13. Saonr. I 784.285

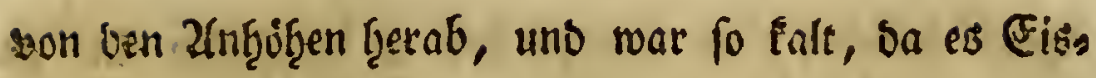
waffer war, bas idf es nidfe wagen burffe bem unts

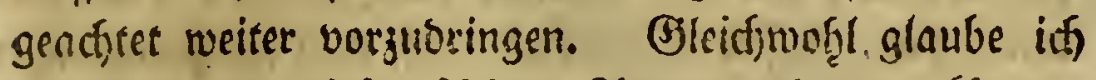

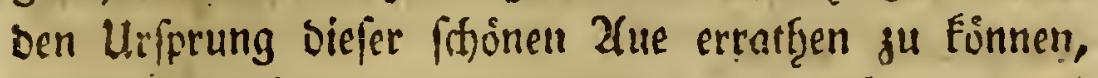

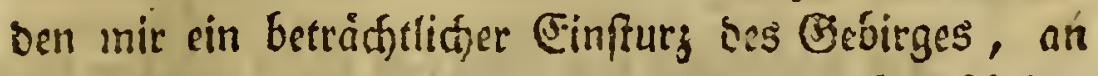

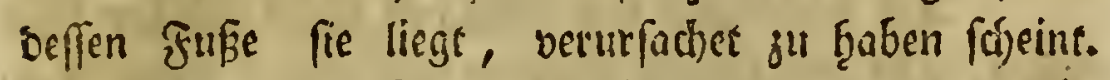

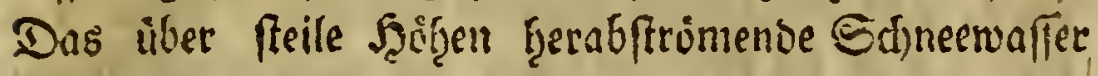
Der Eisfapelle fübrtete, was fie nodh jejt thut, ver-

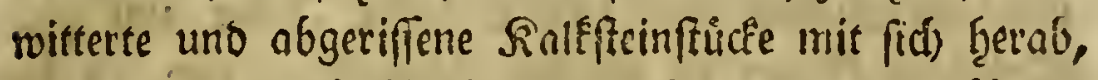

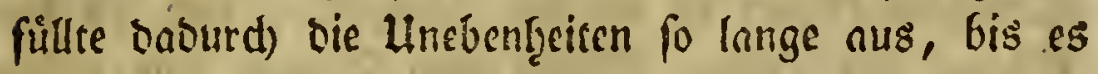

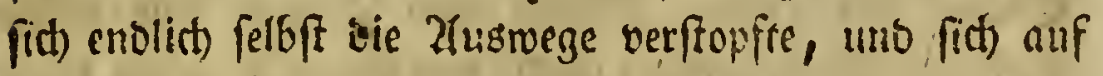
Den jezigen Sinnfaal am fibliditen Enve ber Zlue eins fóränfte. Sobald in Nieorigungeri feffes land zut

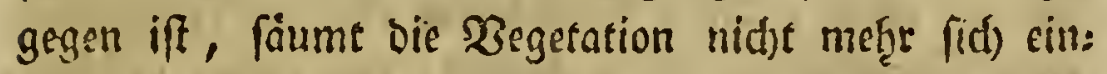
zutinoen; aber aud) biefe beffeght groffentrgeils aus

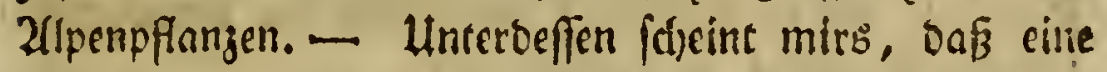
Beit fommen bưrfte, zu welther Das șGaffer biefe Zfué,

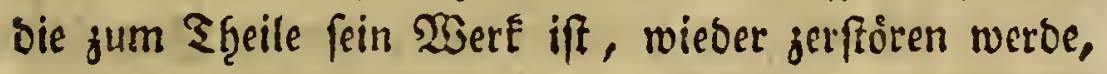

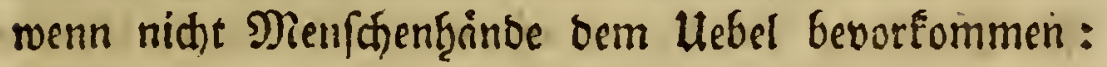
ber $\mathfrak{B} a d$, ber aus biefem Ednneervaffer enfipringt, நृat wirflid) fhon jegt fein eigen Beft gefdroffen, und verlieret fidi) am Enve unter bem Ennbe und Den Eteinen, bie er mit fidf fortroflte. Fiod) f̧at er

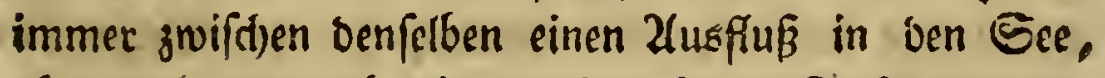
aber nad) und nad, wiro aud biefer berffopfet merben, uno das. Waaffer wirb bie ganze ţerrlide Zlus in einen Sumpf verwandeln.

Das fürfflitife Suffedsló, bas eingige Gebainbe

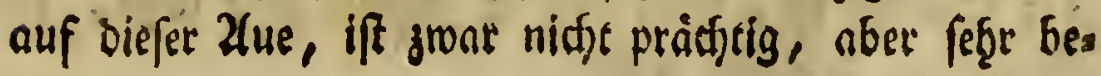


quem gebauet; es befinbet fid) an bemfelben eine ar: tige Rird)e, bie ben গamen vom ḩeiligen Bartf̧olo. máus ḩat, beffen Martertoo auf bem Şodjaltarblatte vorgeftellet wirb.

I(m nơroblid)en uno füblidien Enbe ift ber See offen, und mit Feinem Jiebirge begraingst; feinen 2lus:

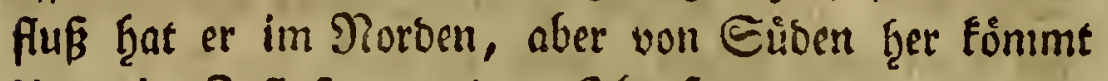

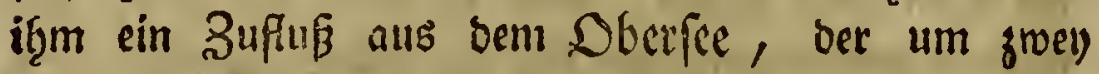
Drittbeile fleiner ift als ber Kónigsfee, und an ben Eeiten von Sften, Suben und $\mathfrak{B}$ eften mit fteilen Sialfgebirgen begrinjet wird, von benen er theils burd, Fleinere Baicl)e, theils burch Den Siettenbach rein Waffer ertialt; ; biejer Band) bilbet ober ber Silfthunfel, einer fübweftlid)en (J)ebirggegens, eine ḩob̧e, prädtige Cafcabe, bie es berbienete den ginfel des vornef̧miten sandfdaffetmaiers zu befdajtigen.

Die Giegend zwilden biefen beyben Seen ḩeif̧ bie Snalat, und fojeint nir unter bie neuern Erwers bungen bes feffen Sandes zu geţören, bie es bem (Fin:fturzen ber Ȩebirge zu verbanten : ḩat; fie fteigt auf Det 2 efffeite feb̨r ḩoch aufwaitts, uno ift gang mit grooffern uno Eleinern Felfentrưmmern bebect, und baherer fef̧e uneben, aber bennod) ḩat bie Begetation fíton feģr viel getwonten.

$\mathfrak{I}_{n}$ ber Stffeite ber Saalat befindet finf ber Xusfluß̧ bes Dberfecs. Diefes $\mathfrak{B}$ affer bilbet aber unterwegs abermal einen See, ber now fleiner als ber Dberfer ift, uns weil er mitten inne liegt, ber Mitterfe genannt wiro. 
Burglaufen, oen I 3.50 m. I 784.287

Diefe orel Seen entf̧alten auffer ben Salblingen, roovon idf ein anbermal reben werbe, ben Iad)sforellen, Jecditen, Znpaffen (fo freiffen f̧ier unfere Flufbarfe, Perca fluviatilis Lin.); uno Truffon (Эiutlyen Gadus Lota Lin.) feine andere $\mathfrak{Z}$ rt.

Der Şinterfec liegr ungefäber eine Etunte von

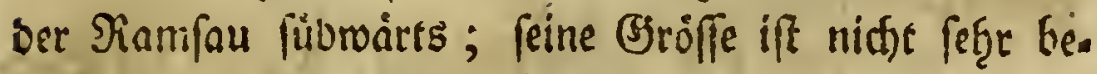
traidtlid, fie war es aber yormals in einem fiobers (Brabe, Denn ber See byac fich nocí) auf eine ftunben. lange Etrecfe, bie jezt trocfen liegt, verbreitet. Evr liegt am Jufie eines byofen, aber brichigen Berges, Deffert Tramen mir entfallen ift, und von bem die ab. gefallenen Fuimmarn, bie allenţ̧alben fyerum liegen,

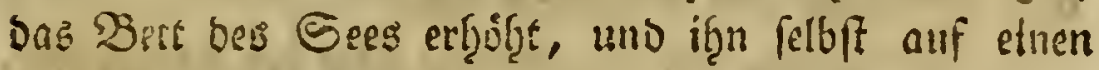
viel flcinern Begirte eingefकloffen ju ḩaben fácinen. Jat) weis nicht, ob biefer Eee auffer ben Iffrillen uns den ficrerliden Ealbingen nod) eine anbere Sifthart enţ̧alte.

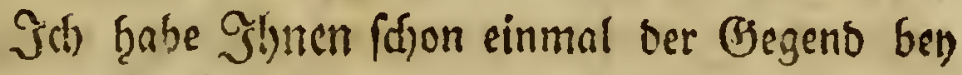
Den Zellern Ervadţnung gethan, Der Eee biefer (Bjegent, ber weber fonderlid groj, nod) feg̨r tief ift, fotheint rein $\mathfrak{W a f f e r}$ biof yon bem Ed)nes ju befommen, ber

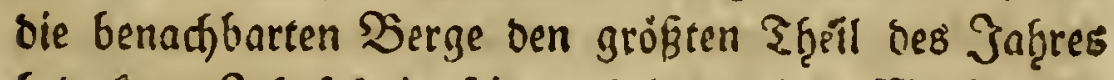
bebect. Jath fah in feinem ţellen, reinen 23 affer weit Ginein jedes Gteinthen, aber feinen ऊifh, uno meine Süugrer wukten unir eben fo wenig ju fagen, of er

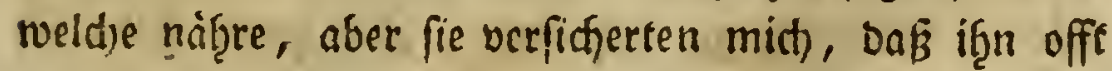

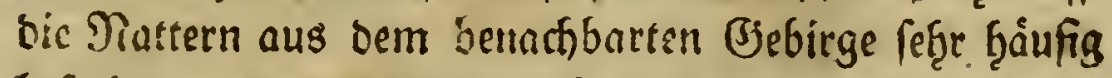

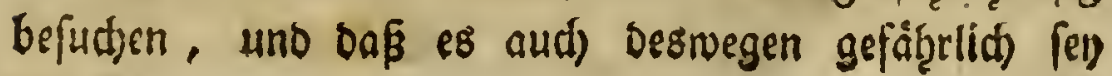




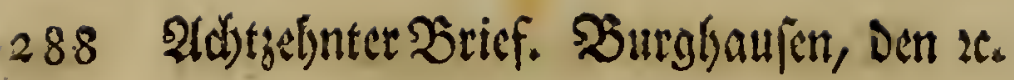

fid) in bemferben gu baber. Jat wünfafte ferge; biefe- Inattern gu fergen; benn mir ift es nid)t maḩts

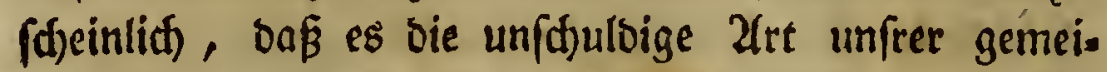
nen Saattern fenn follte; allein bie sufft war bamal

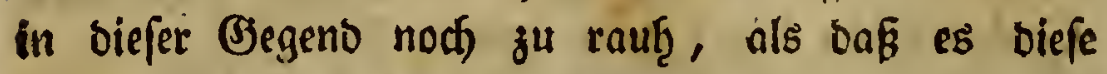

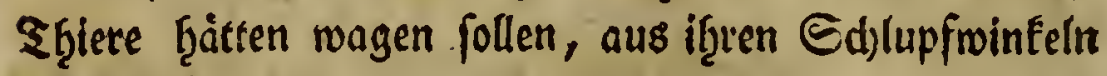
நृervor żufommen.

Zluf bem ferge froţen Bsebirge, bas an ber Suib.

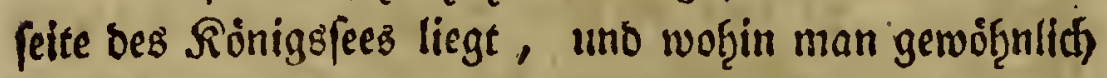
burdf einen f(f)malen, fáauberbollen Eteig gelanget, won bem man immer bie frenffe 2 (usifidit gerabe auf ben See herab ḩat, befinbet fíd der (Grümfee, Det

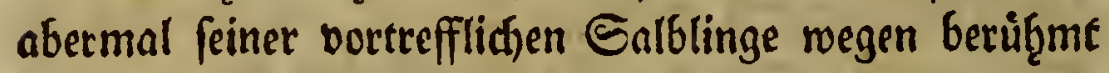
iff, bie aber hizer eingeféget fint.

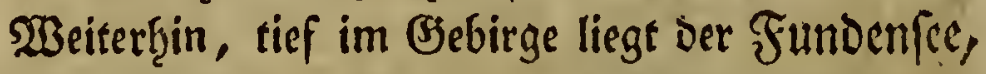
an ber falizburgiffden Grànze, bie mitten burch ben.

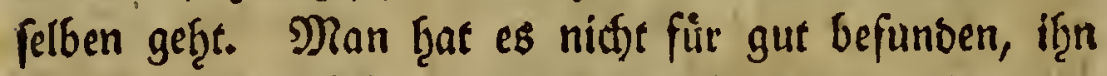

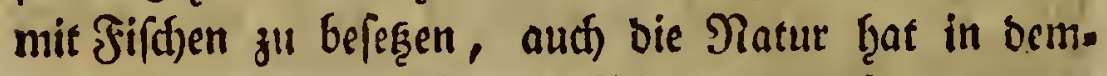
fetben teine andere $2 \mathfrak{f r t}$ als $\mathfrak{P}$ frillen eingeféfęet, benen niemano in biefes unvegfame Bebirg nadjuguef̧en fůr gut befindet.

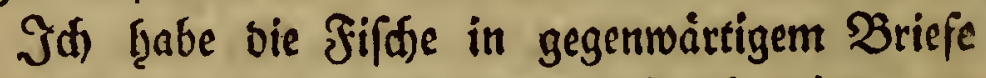
nur im $\mathfrak{B o r b e n g e f g e n ~ a n g e z e i g e t , ~ w e i l ~ i f f ~ m i r s , ~ u m ~}$ bie $\mathfrak{B B i e b e r t h o f u n g e n ~ z u ~ v e r m e i b e n ~ v o r g e n o m m e n ~ h a b e , ~}$

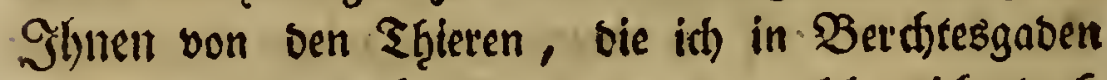
entroeber felbft beobactstet, ober won weldsen idf bods

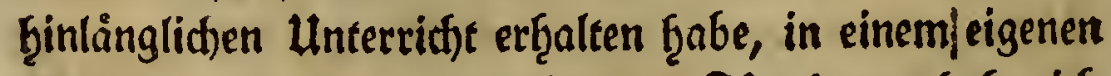

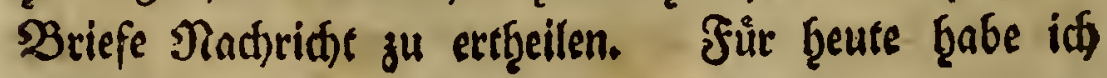
bie Eॄ̧re u. f. . .

Sabrank 
Peunzeclnter Brief. Burglaufen, Den 2 . 289

\section{Seutisebnter \$rief.}

Burghaufeu, ben x6, 5̧orn. I784:

Der Gdimargort. Der Rablfiein. Der hohe

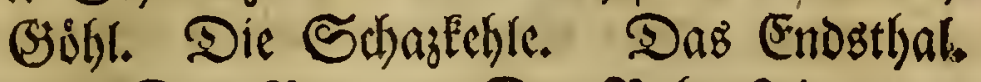

\section{Das Brett. Der Siabenfein. \\ Der Soipel.}

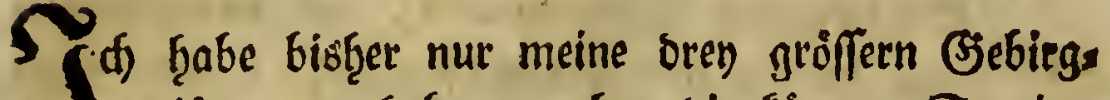
3 reifen ausgetroben; aber bie fuirzern Spaziergónge naw) den niebrigern Bjegenden waren mir eben

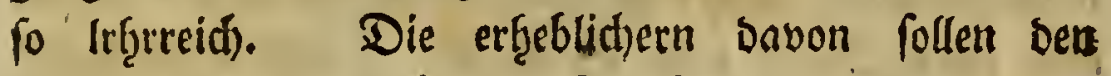
Snf̧alt des gegenwårtigen Briefes ausmadjen.

Der Sdywarzort, beffen idf ffien neulid Ers

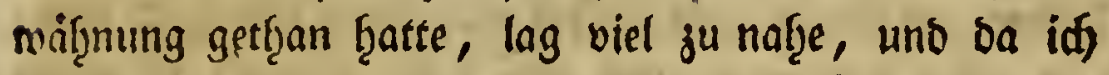

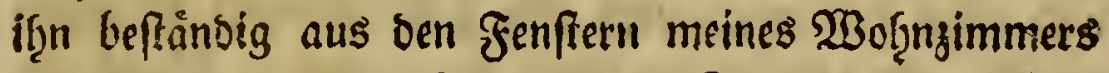

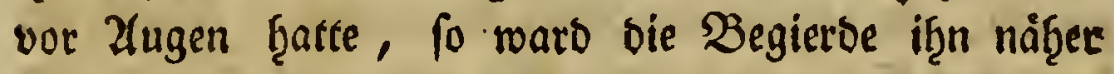

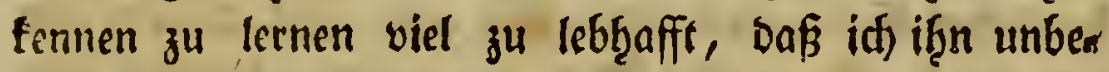

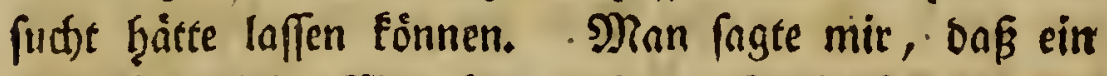
ganz fenntlidger $\mathfrak{2}$ Beg bis an feinen Bipfel fứfre, undo

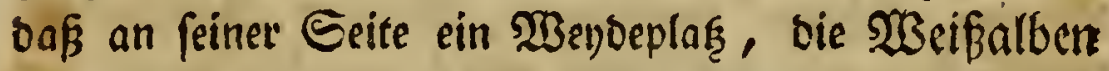
genannt, liege, Der eben abgeweendet werbe: biés twar mir fdjon genug, onf id eines ₹nges, von feiner Eeele begleitet, nur in Geferlffodafft meiner blecternen

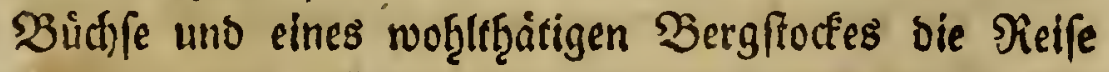
Daf̧ín antrat. Es war f(t)on giemiid fpăt im ḩerbfte (ben 24. Eeptember), als iff baḩin gieng, und idy

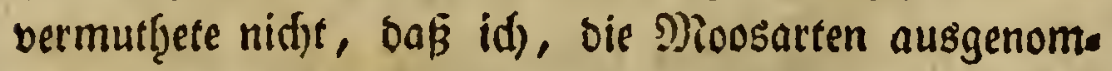
men, nod) einige Ffanzen finden follte; aber eben

ETaturbif Zriefe I.ذand. 
bie Moofe waren Urfacte, baß̧ idh gernobe biefen

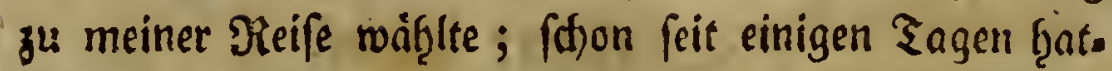
ten wir feuct)es, neblicttes 2 Better mit untermi (t)tem

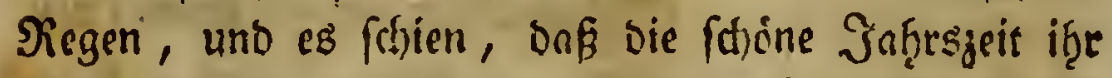

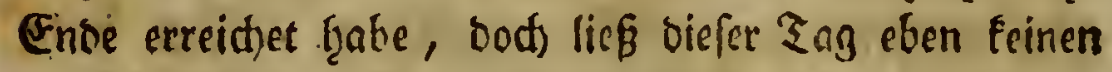
Regen f̧offen. Das maren nun eben tie beften $U_{m}=$ ftänoe für. einen botaniffhen Epajiergang nad) 9roofen.

Eo lang ber $2 \mathfrak{B a l b}$ bauerte, mit wela)em sies, fer $\mathfrak{B e r g}$, ber mit jum Şudigebirge geţort, bernathfen

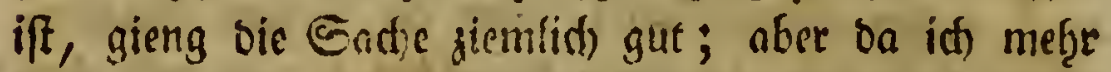
nat) Pflanzen, als nach bem 5 Bege umfath, fam iff

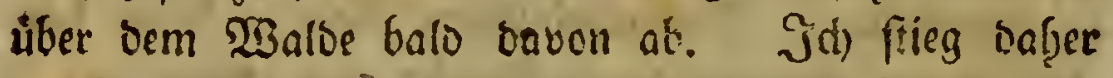
ben Berg serabe Jinauf, ein $23 e g$, ber zienlich bes fámerlich, aber ob̧ue Befaf̧r ift, weil man allents

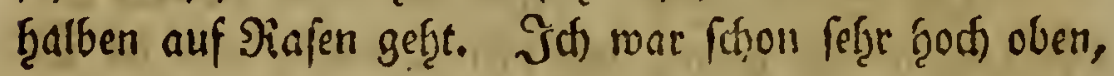
uno baătte nur nod) etroa eine f̧albe Ettunbe ju getgen ge(babt, als id) mid) ungefáţr unmendete. S23cld) ein

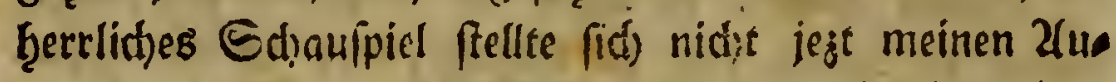
gen bar! Ein groffer Ţ̧̨eil bes ganzen niebrigen Lans bes lag vor meinen afugen mit allen feinen f̧äufern; Bäđten, Şügeln, Sanobergen, Wiótern, Zecfern uno Wiefen ba, wie auf einer topograpfifidjen $\Re_{\text {Rarte lag }}$ alles biefes yor mir, uns id) fonnte mid) an bem Znblicf biefer reizenoen lanofdjafit nidjt genug fättis gen, als plöglich unten in ber Niestrigung sin Rebel vom $\mathfrak{B}$ inde berbengeführet murbe, ber mir wie ein Borthang die anmuthige Ecene verbedfte.

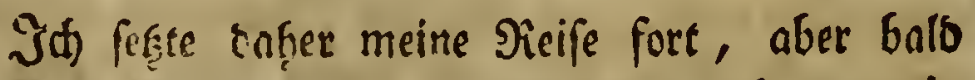
(batte fid) Der Diebel allgemein verbreitet, id wars in 


\section{Bumghaufen, ben 1 6.Scorn. I 784. 29:}

if̧n eingef̧ưltt, und faţ nidjt tíber einen Steinmutf weit bon mir weg. Ţ̧eils aus Jurcht auf einem uni: befannten $\mathfrak{B}$ ege, auf bem mich nun nid)t einmal bie 2fusfid) meţr leiten fonnte, auf 2logrünoe ju gee raţ̧en, ooer bod) irre zu gef̧en, ţ̧eils weil id bie nod) weit prädtigere 2(usfid)t von feinem Bsipfel bis

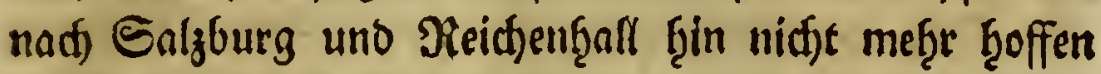

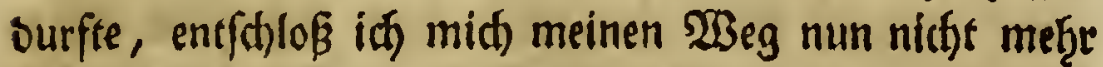
bergan fortzuleşen. Jif) gieng onb̧er quer burd) ben Walb sem. Weydeplatęe ju, wo id) enolidh gegen

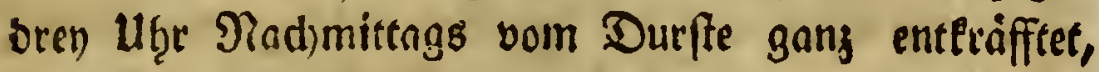
weil id) unternegs feine singige Suelle antreffen lonnte, anfam; aber ein Ericf Butterbrod, uno ein gutee

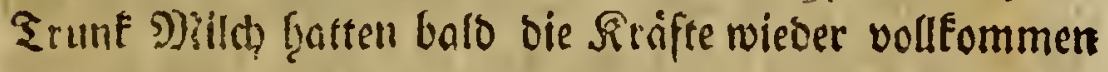
thergeffellet, uno es war mir nod Dazul lieb, nid)t

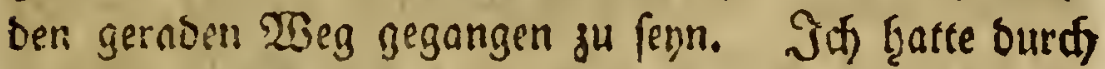
mein Srren ben Serg Defro genauer zu unterfucten Bietegenḩeir getrabe, unt mittels einer nnoern Sieife, bie id fdon im Eommer nad bem (Enosthale ges mactit f̧atte, war id) nun faft gang um benfelben. ḩerum gefommen.

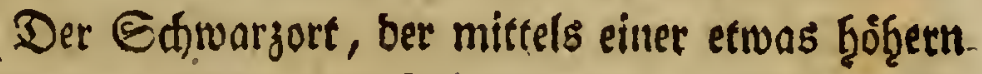
Forffescung, weldye ber Rethlftein (

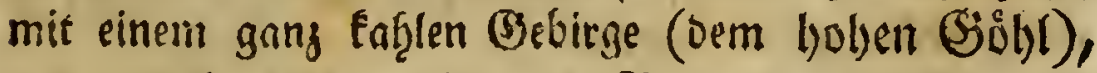
und burch biefes mit Dem Srette zufammenfzingt,

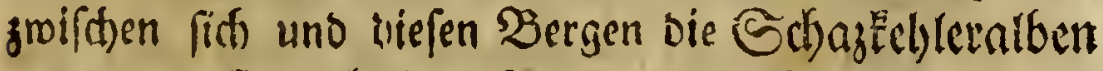

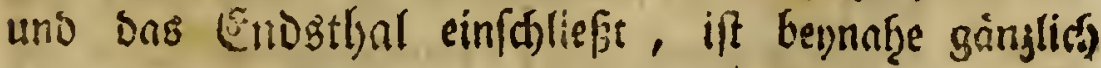
vorm fernern Einfturze gefidert : faine Sooffoung ift.

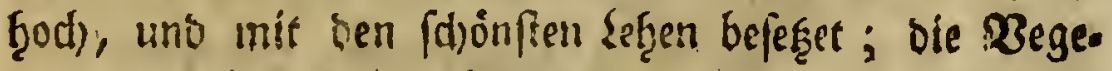

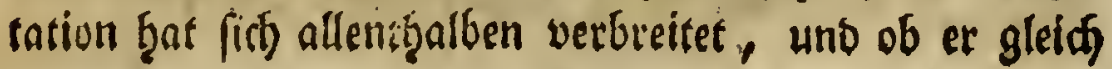


nur bis über feine פgitte mit $\mathfrak{2 B a l b u n g}$ befleibet iff; fo fangen bod) aud) liber berfelben bie Baiume fdon an fith feftzufegen, uns auf feinem Bipfel ift wieber ein

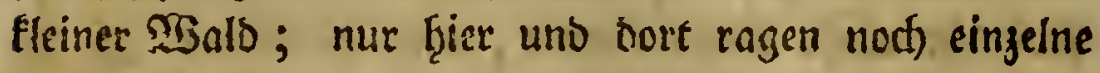
Jelfen heertor, bie von ben Pllangen nod) nidft wiber Den Einfluß ber SBitterung gefidert fino, aud liegt

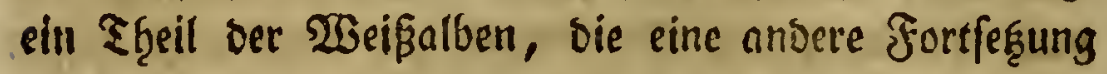
biefes Berges ift, oben auf einer ungemein freilen SBanb, uno es ift in oer That ein fürdferlicf)er $2(n$. blice, wenn man von unten binauf fief̧t, uno $\mathfrak{M e n}$. fả)en und ßief̧ auf ber ziemlith ableángenten flàdje, oie über oiefer $\mathfrak{B}$ and liegt, erblictet.

Man fann nod), wenn man ni(d)t zu furdtrfam ift, vom Safwarzorte auf oen Sisklftein oḅne Brefaf̧e Einauffommen, und biéz ifte eigentilit) ber Sort, welder

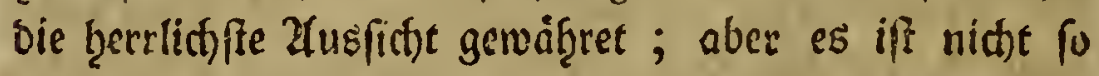

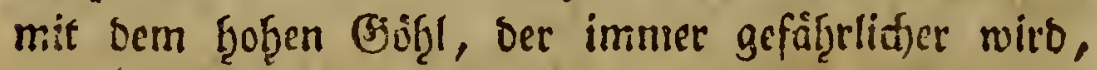
uno ten Eent ju Sage nur genibre Jigiger nod) zu bes

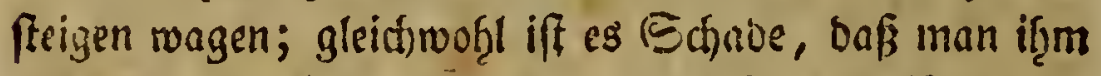
nic): wobl benfommen fann: id) thabe rohe Marmora

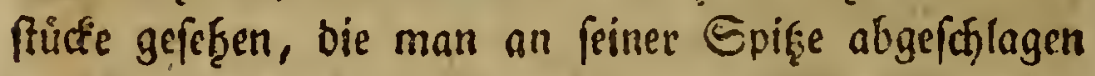
Vyar, uno oie meines Erachtens angefdyliffen ref̧r idjơne Edhauftude, uno in gröffern Maffen vortrefflictie Tafelftucfe geben ourrften.

Şinter bem Edywarzorte liegt bie Gdjajtetle,

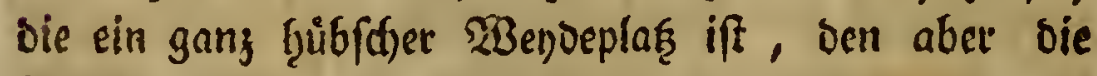
Berge ringsum umgingeln; mitten burrh fließ̧ ein fleiner $\mathfrak{B a c h}$ firtfam fort, ber aber fogleid) ftürmeno wird, fo balb fein $\mathfrak{B e g}$ bergab gef̧e, und Eroe und 


\section{S3urghaufen, Den 16.50rn. I 784. 293}

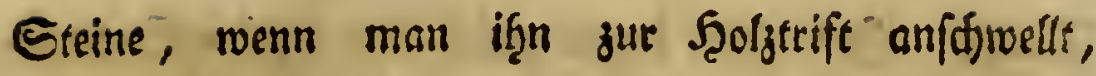
mit fortreist.

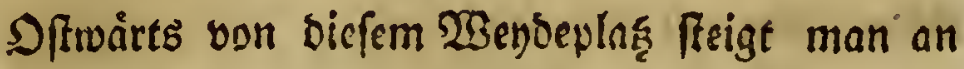
einem berwadffenen, aber siemlid) uarvegfomen fef̧r b̧ofen Eromall hinan, uno fơmmt über benfelben burd einen ziemlich fatmalen Eingang in bas Enosthal, bas

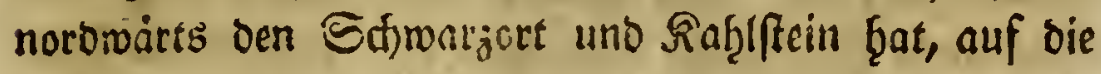
man, zwar feḩr milhefam, aus biefem Thanle an eini. gen Stellen nod) fommen formte; oftuarts ltegt Der bुoţe (Jöbll, ber auf diefer Eeite cine entfegliche Felfen. wano bilbet, auf bie es vielleid)t feine Bemte wagen bürfte zu flettern; an feinem Fupe liegt ein eroiges. Eisfelo. Eibmairts liegt bas $\mathfrak{B r e f t}$, ong fid) bis an Den Erfmeibftein fortzief̨t. Diefss క̧̧̧al verdienet

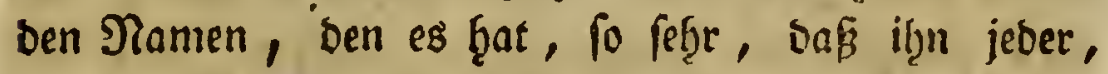
ber es fiefret, gleidd benm erften Zunblicfe bamit belegen wưrbe.

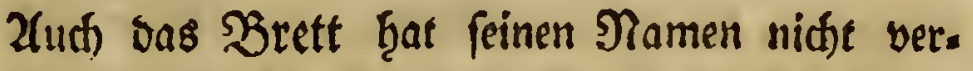
geblich erţalten. Diefer $\mathfrak{B e r g}$, Der faft allentrgalben fef̧r fteile $\mathfrak{B}$ Binbe ḩat, ift auf feinem Siucten bie gange länge fiin fefgr flad), uno ob gleid) an feinen feilen Geiten bie Begetation feţr fiummerlid fortfómmt, to verfidern hod) bie Jäger, Dap fein Rivicfen mit bem fd)onftell Rafen bebecft fen.

Meine Beforgnis ber SBitterung wegen. warb nod) all eben bem ₹age, an weld)em id vom Edymarzorte zurücfgefeţret mar, gänglid) geţoben; ber f̧immel beiterte firf vollfommen auf, uno wir

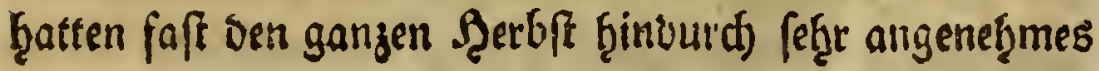


2Better. If gieng onfier gleid ben 27 ften Septem. ber nach bem Rabenftein, um aud) biefe Begeno in Zugenfdeein zu neţmen.

Der Siabenftein if ein jiemlidf Ģoţer Berg, ber an ber Seite nad) Berthteggaben ju, eine fricile Waanb ḩat, auf bem Piưfen allenţ̧alben mit einer

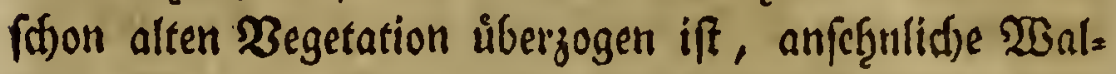
oung trägt, unb fid) gegen ben Utnterfperß, mit bem er einen faft red)ten $\mathfrak{B i n f e l}$ madft, faft in eine find)e

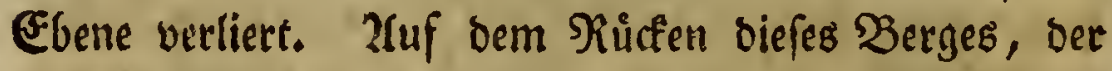
an ben Eeiten ziemlid) fteile Bôfojurigen ţat, trifft man, befonbers in ben niebrigern Jjegenden, Fef̧r troţe

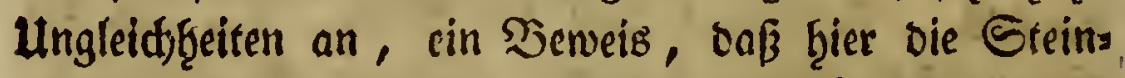
maffen ferabgerollet fino: benn gerabe fo milfte bas Tḩal zwifhen ben $\mathfrak{W a z m a n n n e r n ~ a u s f e c ̧ e n , ~ m e n n ~ t s ~}$ mit Begetation bidhte bebectit wäre. 2(n bem f̧ơhern

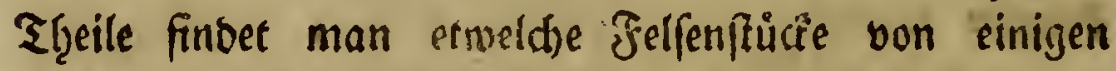
Rlafftern, bic an ben Eeiten zum Ş̧eile nod) unbes waid) fen fint; fie fáseinen wie trergefdleubert, aber

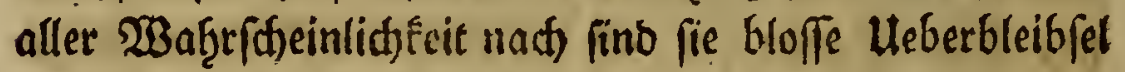
ber ef̧emaligen Ralfferinffidsten, won reld)er Steinark fie wirflid find. Bor biefem $\mathfrak{B}$ erge liegt ein anberer Heinerer, bo(t) ziemlid) hoherer, ber zwifthen fidf) uno ber fteilen 2 Bund bes Siaben/feines, bie jebnd) am

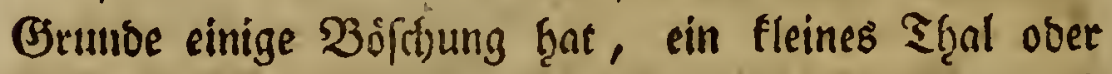
vielmef̧re cinen Braben billbet. Diefer $2 \mathrm{erg}$ ift offenbar aus ben Trimmern bes borigen entfanben, wie man aus bem (befteine beuflich abnimmt, bas auf feinein Bipfel noch nithe berwadsen ift, uno feţe unorbentliq untereinanber liegt, wie auf bem $\mathfrak{B}$ erg.

rùcfen, 


\section{Sburghauien, Den I 6. Soom. I 784, 295}

rưcfen, ber fids gmild)en ben Wajmànnern erbebt. Diefer leştere Berg ift unter feinem Bsipfel peg̨r ftart mit Begetation überzogen, aud befinoen fich ouf

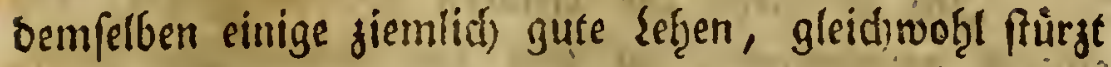
er an ben Seiten, wo $\mathrm{er}$ fteiler if, nod) immer ein, wie benn im $\mathfrak{B i n t e r ~ b e t r a ̊ d ) ! l i c t ) e ~ T r u m m e t n ~ a u f ~ o e n ' ~}$

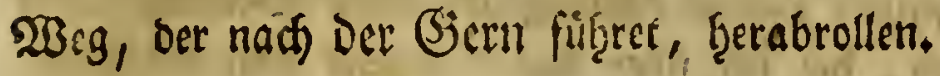

Wenn man über ben Ritfen bes Rabenffeines gegen ben Unterfperg herab geţi, fo f̧at man biefes

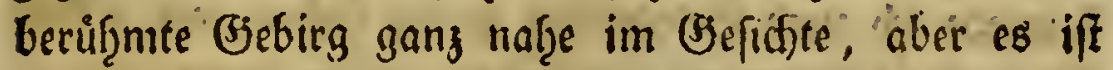
nicht leitht bon biefer Seite binaufzufommen, weil es bier fef̧r fteil ift. Man unterfdecibet auf semferben fệr beutlid) eine groffe Şöhte, bie von weitem einem

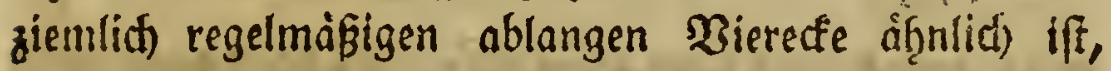
roeil bie Ltngleidfteiten in ber Entfermung vetfdyinoen. Ein Zaure, oer in feiner Jugeno bqeimlid) bem (Je: wilbe nadjgegangen war, verfidjerte mich, Daf ber

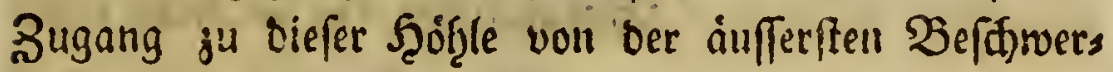

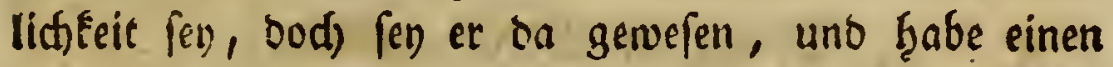
fetge ftarten suffizug gefüblet, ḩinein fer er aber nidjt ges

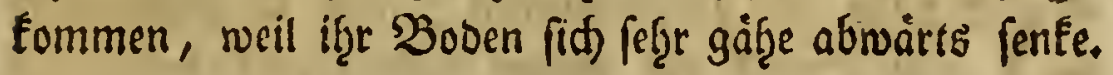

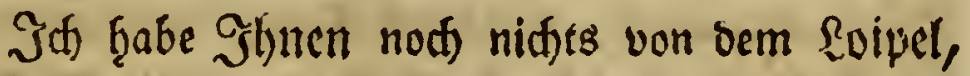
einem Şalbalpengebirge gefagt; aber id muF es be.

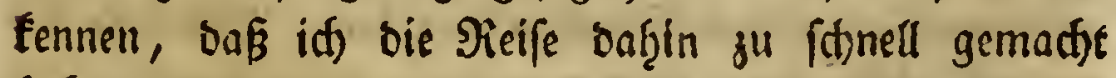
babe, als baßs ith im Etande genefen wäre, fosmo. graphifde Beobadtutungen anjuftellen; éinige Dffanzen, bie id Safelbft fanto, cine fajone Cafrave, bie bie Natur gebaut, uno einige Eticfe ciker feģr fdjơnen, feften Sicinfog̨le, bie bafelbft, nbet fef̧r arm, bridt, 


\section{Bwansigfter Bricf.}

waren alles, was ouf unfern $\mathfrak{B}$ riefroectfel einigen Bezug f̧aben fonnte.

Iđ) übergeţe bie fleinetn Epaziergänge, sie einzeln genommen, zu fẹtr ins Sleinerliçse fallen wúrben. Ia if mir balb bas $\mathfrak{B}$ ergnugen madten werbe, Slynen meine fosmograptiffthen Betradtungen vorzulegen, bie id úber ons (Sefef̧ene gemacht ḩabe, ons mir alles bas, was id). feit langer Beit liber (S)e:

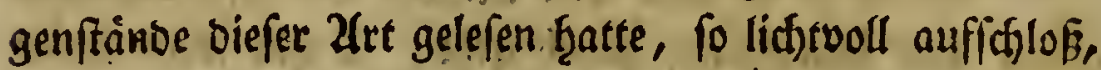
fo werbe in nod) Belegenteit genug ḩaben, bie erf̧eb.

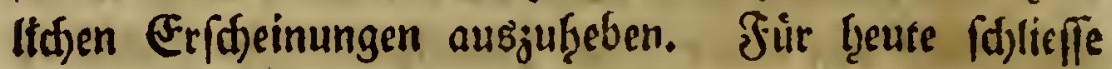
id) meinen $\mathfrak{B r i e f}$ mit ber $\mathfrak{B}$ et fitjerung u. f. f.

Edrank.

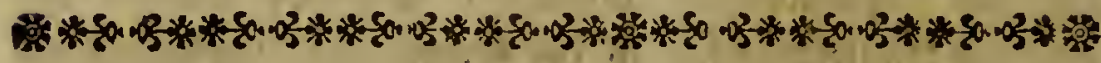

Zmansigfter Strief:

Sourgljaulen, ben $20.520 r n .1784$.

Shicre in Berdjteggaden.

$S$ Ch habe mir vorgenommen Slynen in gegenwarti= 1 gem Briefe ein Purzes $\mathfrak{B} e r z e i d n$ is Derienigen TGgiere vorzulegen, bon berec Dafeun in Berchseggaben id) theils ourdh ben 2(ugenfdeen, theils burd) glaub.

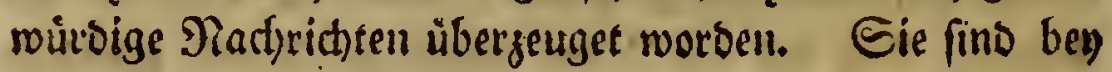
reitem nidfe alle; aber war $\mathfrak{e s}$ móglich in einer Beif von ungefäbr viertḩalb Monnţ̧en alles ju fef̧en? sisas bie Infeftell anbelangt, to habe ith bas $\mathfrak{B e t}$. zeidjnifs babon an unfern gemeinf(j)afflliden freunt,

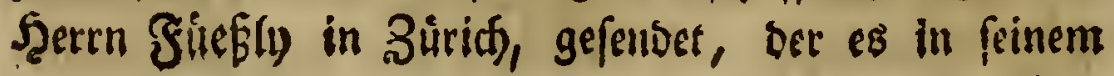




\section{Burghaufen, Den 20.57orn. 1784. 297}

Magajine befannt macten mirb. Die ůbrigen mie

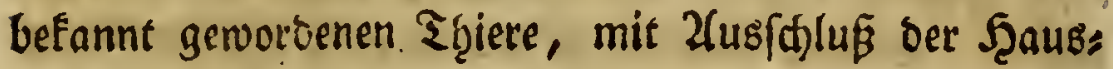
triere, fins:

1. Die groffere gemeine Filedermatts. (Vefpertilio murinus. Lin.)

Lin.)

2." Der gemetire Fudfs. (Canis Vulpes.

3.: Der \&udb). (Melis Lynx. Lin.) Er Gålt fidf auf bem Llnterfperge ouf ; ift aber nidfe zafiltreid).

4. Der $\mathfrak{B i r}$. Vrfus Aretos. Lin.) Jit in Berdftesgaden gar nid)t ju f̧aule. Mian ţat aber

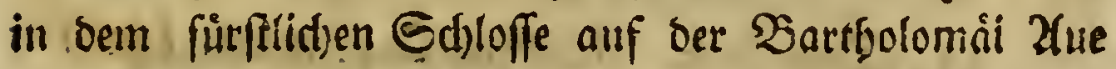
eine Tafel, auf weldyer in langweiligen Sieimen ber Streit einiger Fifther befthrieben wiro, den fie mitten im Rùnigsfee mit einem Bären thatten, ber iber ben SBajmann Gerabgefonnien war. Da man weit umfree voll, Diefem ŢGiere nicfits weis, fo ming er wobgl gar aus Syrol gefommen fein.

5. Dar Dachs. (Urfus Meles, Lin.) Nicht felten.

6. Der Şafe. (Lepus timidus. Lin.)

7. Das Nurme(t)icr. (Mus Marmota. Lin.)

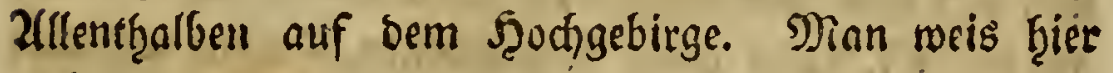
nictits von bem Şeumad)en biefer Tyiere, und bem Einfüf̧ren Deffelben, bavon uns einige Edrrifutreller to treuţerzige Nactrichten Ģinterlaffen ţaben. Die Jä. get betfiffern vielmegre, baff fie das Biras, jedes für

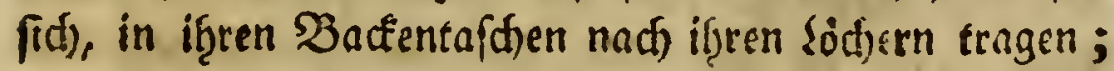
unb als id if̧nen das fufrrwerk befdrieb, deffen fie fid 
nad) (Guer, Gpon und risheler, uno anbern baju betienen follten, madjtent fie fid) fo gut, als ber Braf.

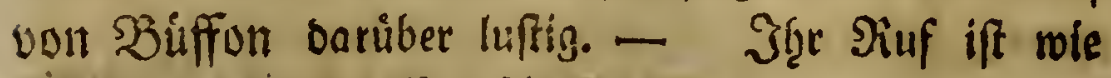
Das Peifien eines Menfhen.

8. Dic Shafetmaus. (2Bielmaus) (Mus avellanarius. Lin.)

9. Dnв Eidjhirnd)en. (Sciurus vulgaris Lin.)

I0. Der Shirfth. (Cervus Elaphus. Lin.) 2(uf

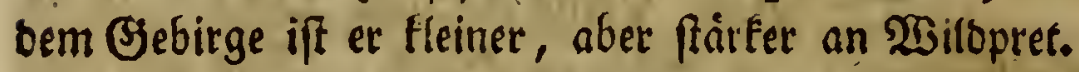

1 I. Då Sich. (Cervus Capreolus. Lin.)

I2. Die Şemie. (Capra Rupicapra. Lin.)

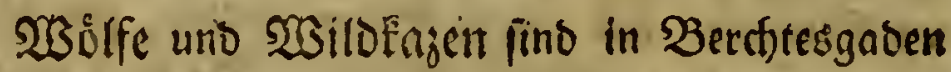
fdjon lange unbefannt; aber aud) Stter uno $\mathfrak{Z} i b e r$ fino nid)t minder fremo; bod) ift es ber erfte weniger.

13. Der Giem fengever. (Vultur barbatus.Lin.)

Diefen (j)ever Ģat Giefner unter bem গinmen Goldgyr in feinem Bogelbuche auf tem LXIII Diatte, und aus thm Gonfton (Theatr. univerf. de avibus) ouf ber VI. ₹afel unter bem Namen Vultur alpinus vel aureus, Bologener abgebiltbet. Bende 2fbblloungen fino jiemlid) gut, nur follten bie fetern, befonsers an ben Seinen, nid)t fo fohuppenförmiz yorgeftellet werben.

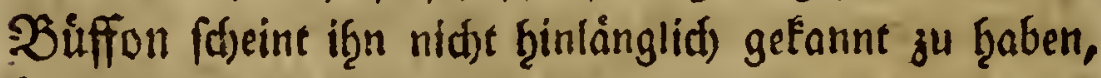
fonft múrbe er ig̨n nid)t für eine 26 banderung bes braunrotfen (3)eners, babon auf Der 16. Tafel ( Traturs gefdsichte 


\section{Bürghaulem, Den 20.5̧orn. I 7 84. 299}

gefthidfte Der Dónel der Berl. teutfden Zlusgabe in 8.) eine 2lbbiloung geiicfert wiro, gelgalten ţaben. Zfber aind) Sonftonz Vultur baeticus, uno eben defferben Vultur aureus fơnnen nid)t moḩl einerley $\mathfrak{B o g e t}$ feun, ba ber lef̧te, nicftet ber erffe, gebartet ift. I(t) ţabe Belegenţeit geţabt, einen nod) nid)t gang nus:

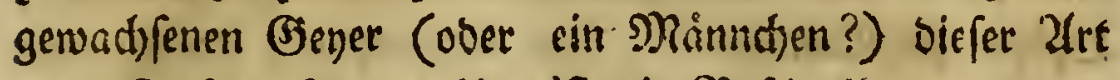

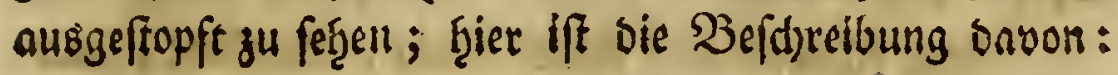

\section{1usmefinng.}

\section{Die länge vom Sdnabel bis an bie Epige}

$$
\text { des Erwange日 } 3^{\prime} 4^{\prime \prime}
$$

Dez Sd)rabels bis an bie Alugenwintel

(bie Rrůmmung nid)t mitgemeffen). . . 3" Des Ed)uabels (bie Rrimmung mit.

gemeffen) bis all bie Miafcrnourjel - $3^{\prime \prime}$. $10^{\prime \prime !}$

Der Beine bis all bie Beţen : $7^{\prime \prime} 4^{\prime \prime \prime}$

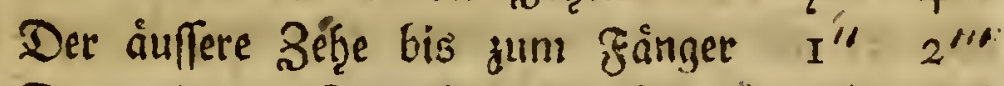
Der mittlern Zeţe bis jum f̧innger $2^{\prime \prime} 4^{\text {ut }}$

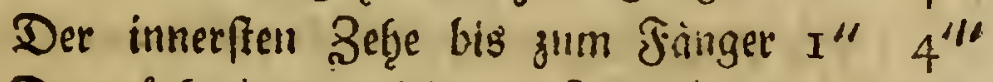
Der rúcfraatrs gerid)reten Beb̧e bis

$$
\text { zum Fringer I" } 6^{\text {oul }}
$$

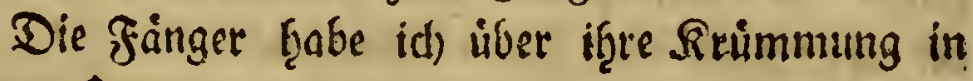
eben ber Dronung gemefien.

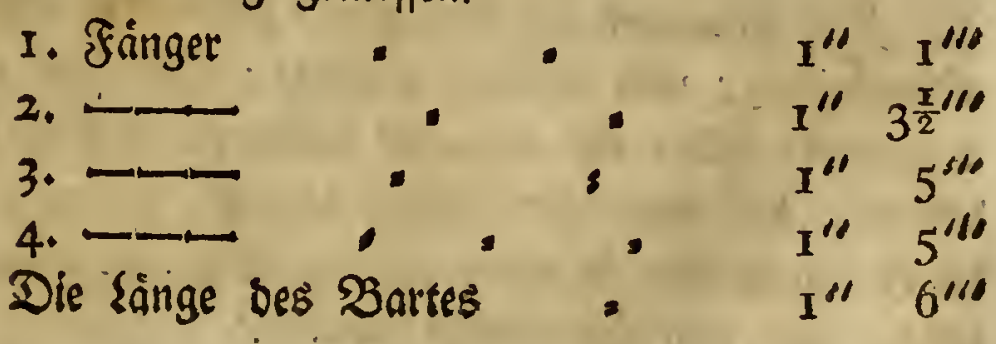


Der Dberfichnabel b̧acfenförmig, sufammengebrưcft, mit zwein faft anliegenben SBlättern auf jeber Seife, davon bas innere breiter ift, uns fid) auf bem Ravicen fanft verliert. Die Jarbe ift

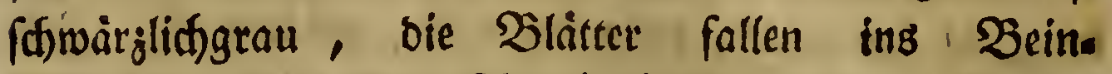
farbige. Der llnterfobnabel ift gerabe, an ber Spige zugeftumpft.

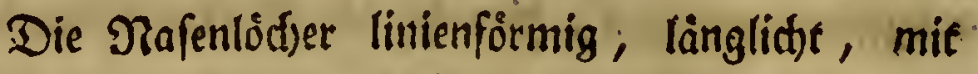
fdywarjurnunen $\mathfrak{B o r f t e n , ~ b i e ~ v o n ~ b e r ~ E d j n a b e l n u r z e l ~}$ ausgeb̨en, unt in einer vorwärts gerict)teten Etcellung froff anliegen, bebect. Zluch ber Linterfanabel ift an.

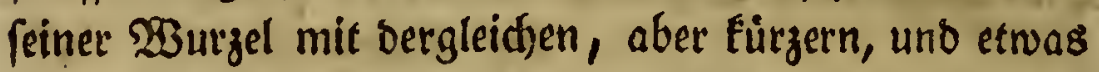
fofwarkern, Borften bebecft. Unter Dem Unterfduabel Tiget unmeit ber $\mathfrak{B} u r z e l$ ein baarigfer f(f)warger $\mathfrak{B a r t}$, wie benm Siegenbocf; bie Borften diefes Bartes find von ber Didfe uut Feftigfeit eines Pferbeţaares, abee nirffe runo, fonbern zufammengebrưcft, und am (3runbe mit einer, nur burch eifie vergröffernoe sinfe fidstbaren, Jangne berfeţent.

Die Oberfonabelmurgel becft auf ifrem Silifen: bie Unterfdnabelwurzel an ten Eeiten, uno unten Ginter ben Borften uno bem Barte, eine Fortfegéng Der Ropfţaut mit i̧̧ren Pflaumen.

Die $\mathfrak{P}$ faumen auf bem Ropfe find weiß, rơtḩlide afdenfarben, und frfwarg gemifdst: über bem obern Zugenliebe bilbet ein fdimaler fojwarzer Erreif einen Bogen, ber von ber Ednabelwurgel fómmt, fid binter ben 2lugen in einer fofiefen Sichtung aufroärts jefęt, und am Shinterfopfe verliert. 


\section{Burghaufu, Den 20. Saum. I 784. 301}

(Bleid) unter biefem Etreife fangen am Şintet. fopfe sie Pflaumen an in Febern tiberjugetren, die allmảhlig länger netben, nber am ganzen Nacten nod) immer fafectid)t bleiben, on ben Eeiten aber fofwarj find. Unter tem Edinabel fangen bie Febern gleidf an ber Regle an, uno fino bis an Den Ropf braungelb mit Utntermifthung vieler braunfahwarzen.

Die $\mathfrak{B r u f t}$ und ver $\mathfrak{B} a$ uch find fifftbraungelb, uno zief̧en faft ins Jabellenfarbene.

Die Beine fins bis auf dic Jilfe mit lan. gen abmärts ftefenden Jebern bebectit, sie bräuntidft weí̄ finto.

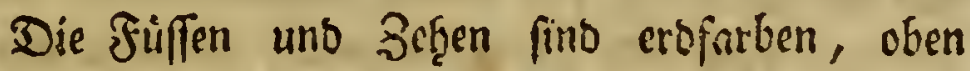
fd)upwidf, unten fȩ̣r fdfwielig; Die Fònger graulidifdoroars.

Der Siucfen ift fhroargbraun mit unterge* mifdrem formusigen $\mathfrak{W} e i \beta$, bas mef̧r ober weniger ins Sraune fált.

Die Edfwungfebern und Riuberfesern fins

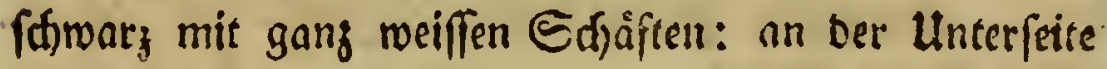
fino biefe Febern tief fdattenbraun, bie Edfáfte aber fratt ber meiffen farbe iffrer Dberfeite wectjperweife mit meislidtgrauen unb graufftwarzen ober briunlictsen geroelten Binden bemaler.

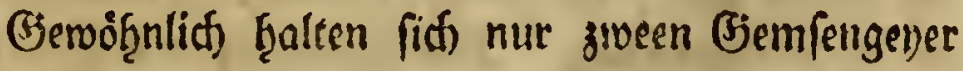
in einerlai) (Segens auf, bavon alier allemal ber eine ein Sijeibdan, ber andere ein Miannidjen ifr. 
Die Jäger geţen autf oie Jago biefer Raubviggel gegen bas Enve bes Winters aus, weil bie Bsebirge im \$inter unzugänglid) fint, im Sommer aber ben

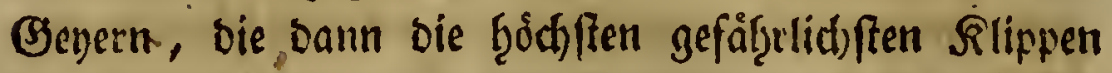
bewotgnen, nidge wof̧ bensutommen iff. Gie baren fiif) zu Dem Enve Da, wo fie miffen, bak fid) ber (J)ever auffgalte, eine Sclitfe von Baumzroigen, in weldse fie fit) verbergen, uno werfen in einiger Ent.

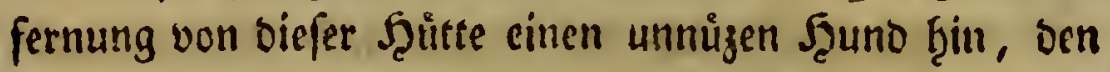

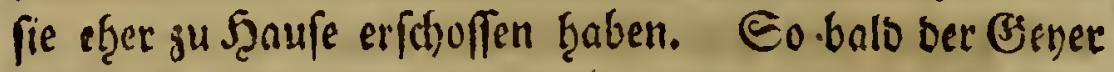

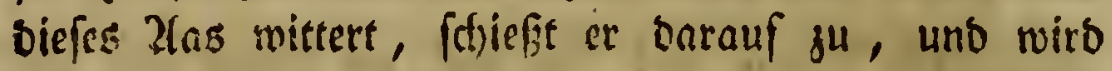
wou bem Jigner, unterbeffen er fich bamit befdjaftiget feine Sockfpeife ju verzeftren, mit finer Rugel erleget. Es ift gefígerlich fith einen (Gemfengeyer, ber nod)

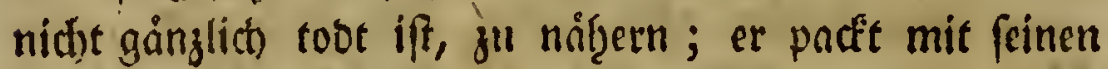
Fángern den Säger, uno wirbe feiner fogar mandje. mal aud) Meiffer werben, wemn fidi) biefe Seute in foldsem Falle nid)t burd) $\mathfrak{B o r f i d f t}$ zu helfen wüsten.

14. Der Saubenfalf. (Faillein) (Falco palumbarius, Lin.)

Accipiter palumbarius. $\mathscr{F} o n f t$. av. tab.8. Ein älterer Bogel.

Afterias. Sternf̧abidft. Fonft. av. tab. 8 . Ein jâţriger Bogel.

Der alugenring uno bie Beine fatoon limoniengelb;

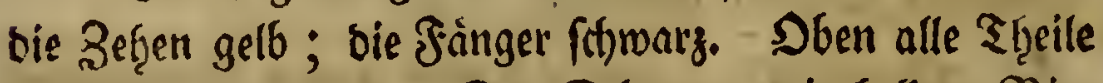
bunfelfthaftenbraun. Der Edywang mit ţellern Bin. Den unterbrocien. Ututen ift der $\mathfrak{B o g e l}$ weiß mit quer. laufenten fhattenbraunen Wellen, - Die Gróffe ciner Eactitinge.

I 5. D $\mathrm{CL}$ 
Şurghaufen, Den 20.550rn. 1784. 303

15. Der $\mathfrak{H}$ ) bo. Lin.)

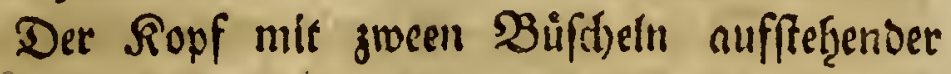
Federn; ber seib getberaun : fotwors seflammt. Eeltner.

16. Der Sinbe. (Corvus Corax. Lin.)

Tieffimarg; Det Silicfen braufdywars; ber Sd)wang getunbet. - Rómmmi boch auf bie Zlpen.

17. Die farwalge Rrailye. (Corvus Corone. Lin.)

(j)anz blauffhwarz; Det Edwwang gerunber;

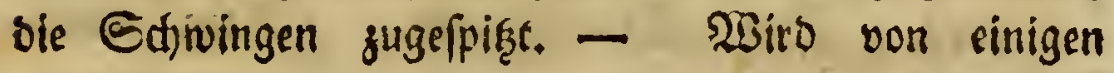
gegeffen.

18. Die Zogelérable. (Corvus frugilegus. Lin.)

Tieffrjwars; bie Stirne afdhenfårbigt; ber Stymang gerunbet.

19. Die Rebelérábe. (Daḩel) (Corvus Cornix, Lin.)

zf(jhgrau ; ber Ropf, Die Refile, bie Slugel, und ber Edjwanz fdimars.

20. Die Elfter. (Corvus Pica. Lin.) Xeiß̧ uno fdrwarz fdjectict.

21. Der ?luphel)er. (Corvus glanda. rius. Lin.)

Die Dedfedern Der flügel f̧immelblau: mit reiffen unb fdimarjen Binden; Der Rörper rơtf̧lid)t. 
22. Der Sergletyer. (Corvus Pyrrho Corax. Lin.)

Diefer Bogel ift mir nidft Ģinlänglid bes

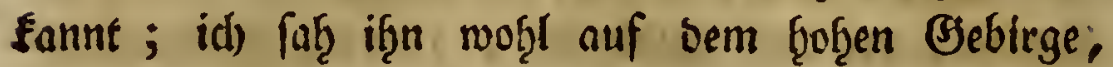
fowoḩl im Sluge als in ber Siuf̧e, aber in ju weiten Entfernungen, als baß id iḩn ḩătte genau betradjten fönnen. Er fáien mir von ber Groffe der Enatfráţe, Gråunlidft afdjengrau, mit einem fdjmusig gelblidjten Sdjnabel.

23, Der bunte Gpecht. (Picus major. $\operatorname{Lin}$.)

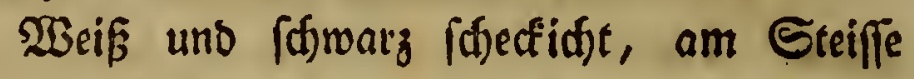
roth. paea. Lin.)

24. Der Saumlanfer. (Sitta euro-

Dic Edfwanjfotern fótwars: bie vier àufferften

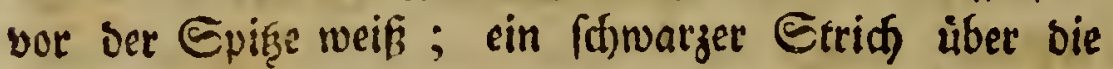
Zlugen nad) Dem Şinterfopf.

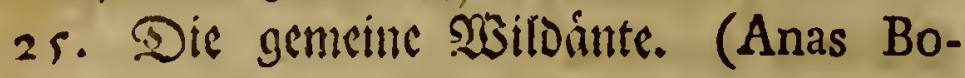
fehas. $\operatorname{Lin.}$.)

Der Sdnabel gerabe; einige Sdrwangfevern beym Mainnden aufroaitts gefraufet; ber Epies gel blau.

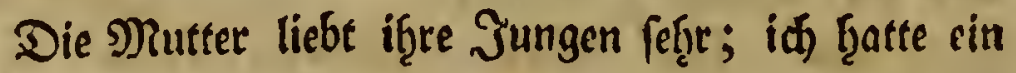

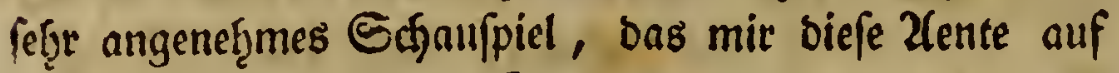
Dem Rỏnigsfee gab. S(d) wat nàmlid) jugegen, als man fid) jur suft in bem Sdjieffen junger 2lenten Iibte; als wir uns fo weit nảberten, baß bie 2fenten mit bem ßof̧re befrichen werben fonnten, trieb bie

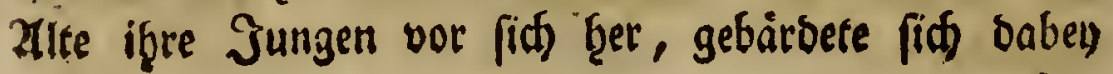




\section{SBurghaufen, Den 20. 50rn. 1784' '305}

felffam; breitete bie flighel, als wollte fie if̧re Rins

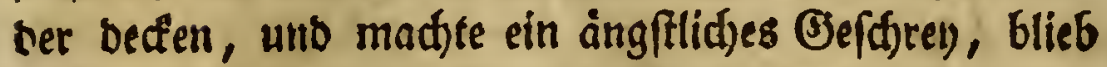
aber, ob fie fid gleid) nad Bermógen zul entfernen fuchte, immer unterm Stfuffe, unterbeffen bie Jungen pȩ̣r ruḩig fortfdsmammen. Euft nad gefdeţenem Edfuffe entfernte fie fich fonnell. Diefer aug ber Mutterliebe war auffalleno; unterbeffen glaube ids bot) nidft, baß er bas geringfte moralifde (Befúb̧

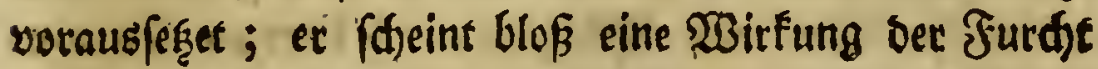
zu fenn, bie bey allem Beftreben, bie fie einflófet, fí) von bem gefürdfeten (Segenftanbe zu entfernen, sugleid bie నerven fpannt, und baburd bie Semegung hemmt.

26. Der Fifther. (Pelecanus Pifcator. Lin.)

Der Effrwang feilformig; ber Efhnabel fägesăh̨nig : Der seib weî́ : Die Gtirne uno bie Edfroingen fofrars.

Ex niffet nicft im Berdftesgabenffjen, formmt aber eingeln zurveilen in bie Seen.

27. Daв 2(uerfulyn. (Tetrao Urogallus. Lin.)

Die fúffe rauts; bet Sdjwang gerunbet; bie 2 (d)feln weißß.

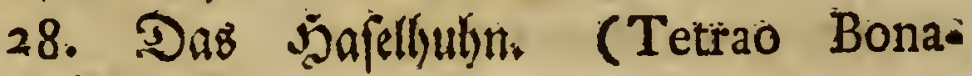
(ia. Lin.)

Die Jüffe rouf ; vie Edfwangfebern afdens grau mit fdjwarzen Puncten, und einer fdfwarzen SSinde : Die zroo mittlern of̧ne Binbe.

Datutbift.2Stiefe I. Bans. 


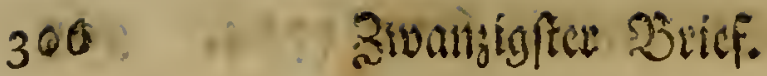

29. Das Gdfnetbulyn. (Tetrao Lagopus. Lin.)

Die fruffe raug ; die Ed)wungfetern meiß : bie Echwanzferern fatwarg mit siner weiffen Epig̨e: Die mittlern ganis meis.

30. Das @teintuthr.

3d) habe biefen $230 g e l$ nidit sefef̧en, und weis nidft, was man unter biefem গamen verftef̧e, es múpte nur das Sirglyulyn (Tetrao Tetrix Lin.) F(nn.

31. Das graue Siebhulin.. (Tetrao Per. dix. Lin.)

Die Süffe fobl, gefporrnt; ein nafter rother flect unter ben 2lugen; ber Echroung roftfarben; die Bruft rotḩbraun.

32. Die SWact)tel. (Tetrao Coturnix. Lin.)

Die Sưffe Eafil; ber Rơrper mit brandgelben langlidfen flesfen; bie 2lugenbraunen weißß; die Edtwangfebern am Siande rofffarben.

33. Die Mafferamifl. (Sturnus Cinclus. Lin.)

Sdinarg: bie Bruft weif.

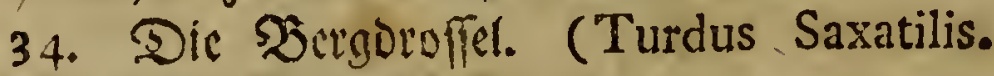
Lin. SBirglerde Berchtesgad.

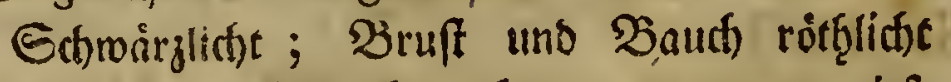
jinmetbraun : Der Edynatel oben uno unten tiefIdivarz: an ben Eeiren fdumefelgels.

Ein Durtfrree Etrcif gebet ourd bie Zlugen.Dic Grioffe des Esibenfinwanzes. 
Domghaufen, ben 20.50rn. 1784. 30\%

3of̧nt bुod auf ben Bergen, wo fdon alle WBalbung auff̧ớret.

Der Befang ift nicht unangenef̧m. Der Sdinabel ift reniger gewoilbt, als er gensofinlid bey Den Droffeln iff, uno verţâlttnismåkig oủnner; bie F̦uffe fino zum Siz̧en auf Zeften gefhidfter, als zum Bjehen. - Das ift aber alles, was id) bon biejem Bogel weís, ben id nidft genau genug ju befradten

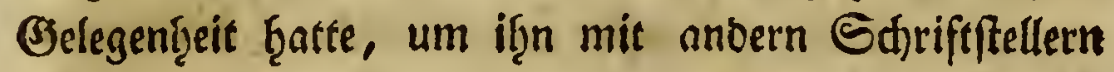
zu vergleidjer. (Seneigt wàre id) immer, if̧n für bie

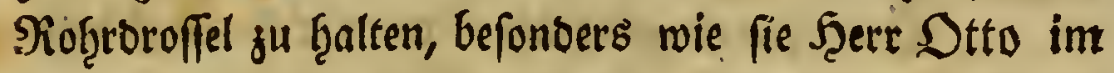

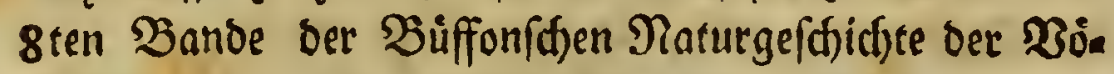
get befditeibt; aber ber $\mathfrak{W}$ ofgnort ift zu feţr entgegen.

35. Dir Rummmithabel. (Loxia Curviroftra. Lin.)

Die Etfnabelfiefer freuzmeife überein. anber gebogen.

36. Der Gimpel. (Loxia Pyrrhula. Lin.)

Die Blieomaffen fononr ; Die Deuffedern bes Sthivanzes und ber Ģintern Ruberfebern weißs.

37. Dir Fint. (Fringilla coelebs. Lin.)

Die Blieomaffen fajwarz: bie Sthwingfebern benberfeits weifs : bie bren erfiten ungeflectt; zwo Sd)wangfebern frhief meisgeflect.

38. Der Eperling. (Der Epas. Fringilla domeftica. Lin.)

30. Die weiffe $\mathfrak{B a d f f t c l j e . ~ ( M o t a c i l l a ~}$ alba. Lin.)

Sben blak fdjattenbiaun; bie Stime uno bie

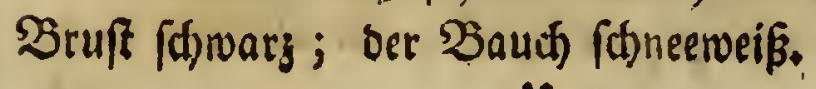

$$
\text { นI } 2
$$
40. Det 
$\therefore$ 40. Die gelbe Sadd)fetge: (Motacilla flava. $\operatorname{Lin}$.)

Die $\mathfrak{B r u f t}$ uns bet $\mathfrak{B a u d}$ gelb ; oben fittidy. gruin; Sie flügel fdywarz : weiß banbirt.

41. Dab Siotl)fthwánzchen. (Motacilla Erithacus. Lin.)

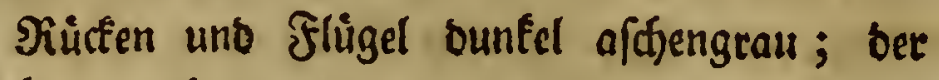
Baud) braunrotḩ.

42. Dei gethaubte Bauntúnig. (Motacilla Regulus. Linz.)

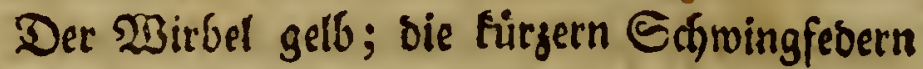
an 2tuffenranbe gelb, in ber Mitte weif.

43. Der ungebaubte Bauntunig. (Motacilla Trochilus. Lin.)

(Braugrun, bie Flluget unten, uno if̧re Dect, fesiern gelb; gelbe Zlugbrauuen.

44. Die Siandffd)malbe. (Hirundo ruRica. Lin.)

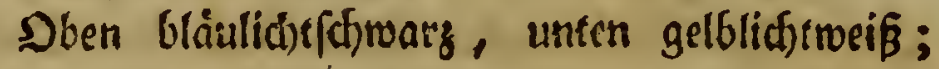
Die Sdymanzfesern meigigeflectit: bie zmo mittlern un. Beflectr ; Die Reb̨le rotḩbraun.

45. Die gemeine Srite. (Bufo vulgaris. Laurent.)

Grau blenfarben, aufgefdirnollen, mit röthy Vinfiten $\mathfrak{2}$ argen; ein nierenförmiger Sjöcfer an ben Digrengegenden.

46. Dir Galmafiertrote. (Bufo fal[us. Mibi.)

Silein, now flliner als ber grüne Saubfroffi, oben erofarbengraulidjt, warzidt; ;ie Warzen Elein, 
3tmghaufen, den 20.55orn. I784. 309

mit bem Rörper gleidffarbidft, im Mitcipuncte bur(s). boḅrt; bie Beine uno Bef̧en fá)attenbraun banbirf, unten an ber Ref̧le weiflitht ; Der Baud) meiflldtr,

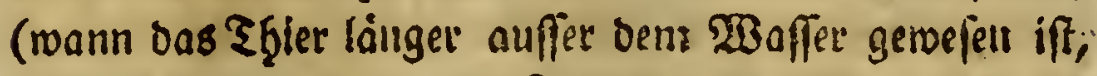

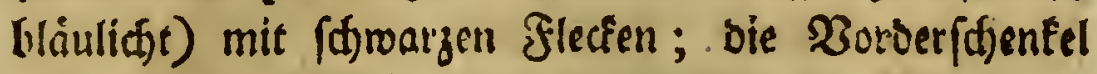
auf ber Unterfeite, unb afle Fupballen gelb.

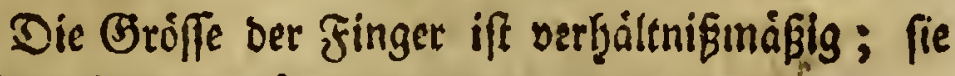
beobnatset folgenbe Dronung:

In ben Saánden: $3,4,2,1$, : welches ber Eleinfte ift; bie finger 2 und 4 find faft gleid.

2II Den Finfen: 4, 3, und 5; 2 und I; bie leştern fleiner.

Eie molynt in ffehendem $3 a$ ffer, bas aus Ealgwaffer uno Regenmaffer gemifdtet ift; fie flielit bas (ifft: : ba id fie eine Beittang in einet Sdact)tel aufbeficielt, fprang fie allemal nach ber fifattigen Geite Derfelben; niemal frieg fie an bem Ranie ber Exfaditel berauf.

Iक habe fie forwob̨l im Sommer als im Şerbfte beobactiet, uno immer von einetlen Gredfe gefunden, Daf̧er ḩalte id fie für eine eigene $2(\mathfrak{r t}$, und Ģabe mir folgende Rennzeiden abgezogen:

B. manibus tetradactylis fiffis; plantis pentadactylis fiffis : fupra terreo-olivaceus; fubtus albidus maculis nigris.

$\$ 6$ id fie gleid) viel in ben Şanden ţatte, fo

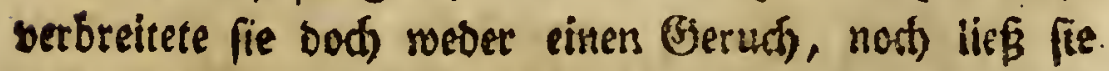

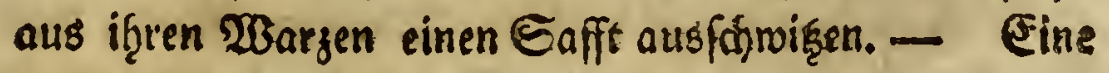
Falte on ter Rel̨le habe id nidjt gefeb̧en.

Gollte fie vielleidt Rana bombina Liva. fenn?

$u_{3}$

47. Dis 


\section{Der fumme frofd). (Rana muta.} Laurent.)

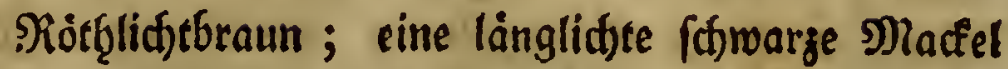

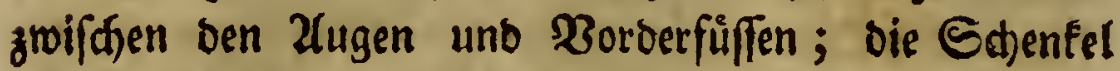
fafroirgliff banbirt. Laurent.)

In einem Braben Des Berges bor bem Rabenftein.

Er war oben fajwárglicftbraun, unten fámujig.

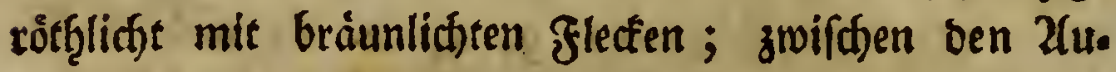
gen bis ju ben 2 orberfüfen war ein fønarzer Gtrid). Er war nur noch einmal fo grof, als ber Saubfrofक.

48. Der Eaubfrofdy. (Hyla viridis. Laurent.)

Oben grun, unten meiplidst; an ben Eeiten eine gelbe Sinie.

49. ₹ritonartiger Эroteub. (Proteus Tritonius. Laurent.)

Die Şinterfúffe jum Beţen; Der Ropf mit eis nem etroas aufgeblafenen Rropfe; auf bem Riuden eine STautfloffe.

$\mathfrak{W B O b}_{\text {int }}$ in einem Eleinen, aber nicft vere

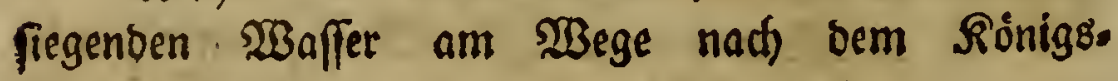
berge.

50. Sthwarzer Molta). (Salamandra atra. Laurent.)

Bians fdjwar, ungeffect.

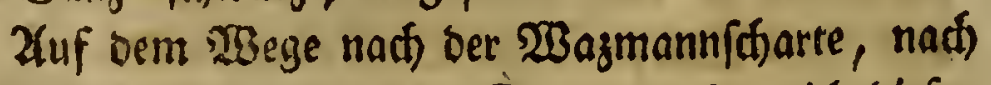
bem folse, ber Bugel, bem Stgattel, fano id biefen

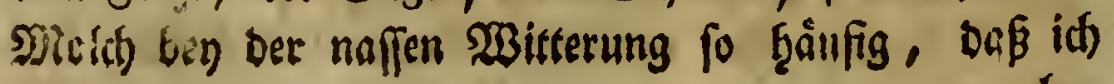


Bumblaufen, ben 20.550:n. I 784 . 3:1

ben jebem Subtritte beforgen mußre, eines biefe: Ţ̧⿻ierden zu zertreten, eine Gadbe, die mir leio getḩan ḩätte, on es ein unfónablid)es Thier betraf, Dos gang gewís um einer meipen, aber mir unbe. fannten abfidst nillen fo baufig on war.

Das Tfgier iff urbeigmb fleiner als ber geflecte Mold, uns eben fo langfam.

51. (Geflecteter 920(d). (Salamandra maculofa. Laurent.)

Shwars; gelb gefledt.

Wolynt um Jriecentécerg.

Beyde 2(rten werben von ben Berdftesgabnern Wegnarren genant.

52. (Stune (EiDed) fe. (Seps viridis, Laurent.)

Der Ţalsftragen abgefôndort, blau; bie Mittels fdilbe runblidft; Der Rorper gotozriu.

soolint in ben freinernen $B$ efrienigungen.

53. Sthwarze Eibedhle? (Seps? atra.)

Ith) ḩabe diefes Thier nid)t binlänglid) ges

fę̣en; visfleidft war es ein (fimarzer Nold); aber es war bod) f(f)nell, uno ber Edjwans fdjien mir viel bunner und lánger nls bey ben Moldten, unb bee Rörper fithntere.

Id) babe es in bem $\mathfrak{B a}$ albe unterm Sdywarz: orte auf einem faulen Baumftocte gefegen, allein als id) es greifen wollte, war ez roeg, unb nidht mefir zu finben.

54. Die B (inofdulcid)e. (Anguis fragilis. Laurent.)

Der Siórper gleicbformig, glate, etwas glán= jent, filbergrau; nuf bem Siopfe und giucten sine Makel.

Dié 
Die Săger in Serdtesgaben f̧aben mir bie Un:

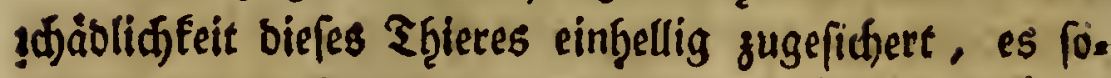
gar in meiner Begenwart ergriffen, unb eine Jeitlang fin ber ḩano fortgetragen.

55. Die gemeine $\mathfrak{P a t t e r . ~ ( N a t r i x ~ v u l g a r i s . ~}$ Laurent.)

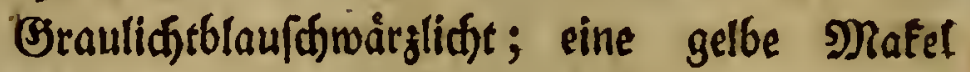
zu benben Seiten bes Şinterfopfes, binter biefer eine fobrarze.

ta. 13loch.)

56. Die Duappe. (Ruthte.) (Gadùs Lo-

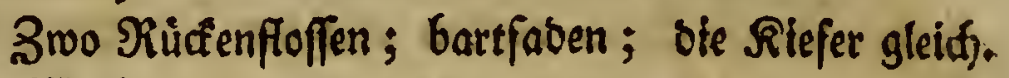
Woblyt im Rönigsfee.

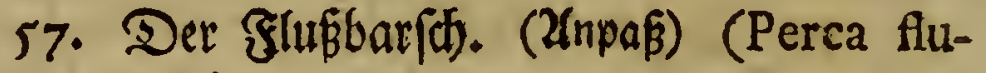
viatilis. Bloch.)

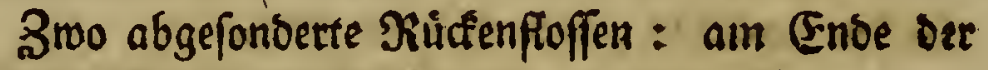
erfeten ein fdrwarzer Flect.

Boblint im Rosnigsfee, wirb aber nidft ges

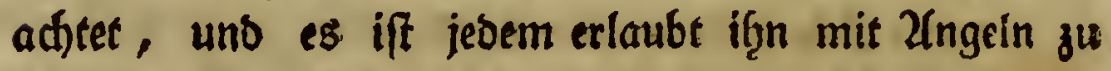

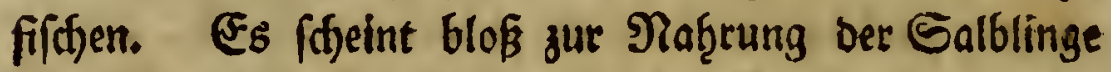

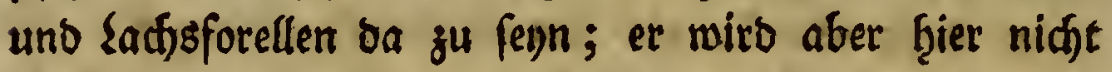

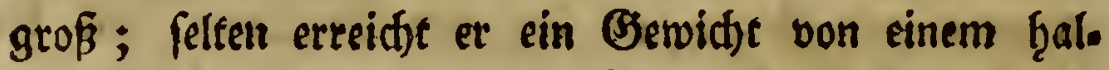
ben Pfunbe, vermegirt fid aber aufferorbentlid) ftark, uno man finbet an feidfen Stellen ganze Jeeere Diefer Biffhe.

58. Die Ead)sforelle. (Salmo Trutta. Blocb.)

Etgraarze Zlugenflecten mit einer bुellern Einfaf: fung; bie 2ffterfloffe mit eilf Stralen. SOblgnt in Ronigsfee, und Sberfee. 


\section{5urglaufen, Den 20. Sctit. $1784 . \quad 313$}

\section{Man fat verfdiesene male fef̧r groffe filfdie}

Diefer 2lut gefangen; fie wurben zum Zfnbenfen abs gebilbet, aber biefe albbilbungen fino niø)t zum beften nuggefatlen; man ḩat aber nicht vergeffen baju zu ferzen, was es für ein Fif(t) fen, uno meldhes Be. widft er gef̧abt ţabe. Şier find bie Refultate bie. fer Gemálbe :

1676. Den 20. Jun. - 29. Pf. im Dberpee.

1714. Sen 10. Eept. - 21. -

1717 . Den 8. Eept. - 24.-

1717. Den 12. Sept. - 16. -

1718. ben 13. Sept. - 22. -

1718. Den 1.Dftob. - 23.-

1719. Den I. Decemb. - 52.-

1725 . Den 12. Sept. - $16 \frac{F}{2}$. -

59. Der Salmling. (Salmo alpinus. Lin.)

In ben Seiten rothe Stectse, bie an ben Ränbern verwifdtt finb; bie bicfiten Etralen an sen Bruft - Baud) - und affterfloffen wis.

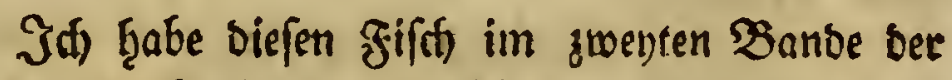
Edyriften ber berlin. naturforfdyenben Befellfolaft be= farieben. Es war bief die erfte umftánblide Diadg. ridfe, weld)e bie 2 selt von biefem eben fo fdjoinen als

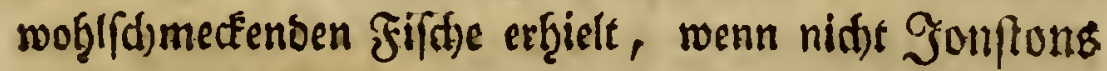
Salmarinus nid)ts meiter als eine Epielart teffelten ift,

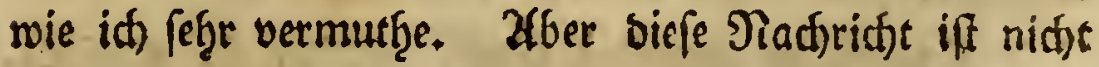
of̧ne Unrid)tigfeetten, bie id nun in einem Briefe an

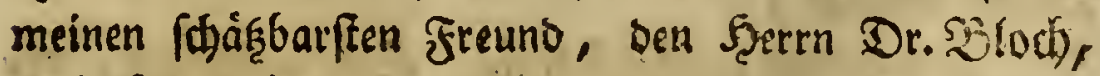
verbeffert ḩabe, Der Davon zum $\mathfrak{B}$ eften Der Nactura 


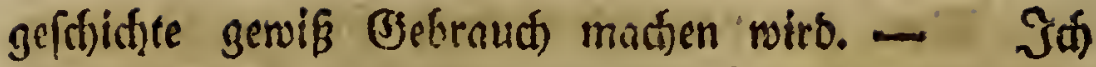
merfe bier nur nod) an, Dof cus oen vier Eeen, in weldse fich biefe firithe befinden, bie yom Rönigsfee.

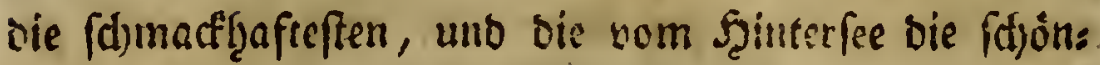
ften fino, bie jugleith Jonftonz Salmarinus am nådften fommen. - Die beffe Subereisung firt bie Tafel if, wenn fie im Ecenaffer mit einer Jcanoboll Eals abgefotten merten.

6o. Der Sced)t. (Efox Lucius. Lin.)

Die Estmauge breit uno georúctit.

6I. Die Pfrille. (Cyprinus Aphia. Lin.)

Deun Etralen in ber 2efferfitifie; Die Zugen. ringe rotfi; Der Rörper burdffacineno.

Toolint in Funoenfee, Scinterfee u. a. D.

62. Der Sicgcnourm. (Lumbricus terreftris. Miiller.)

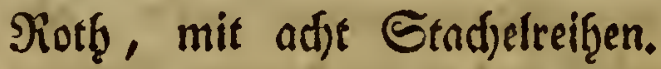

63. Dis Siobsmurn. (Lambricus Tubifex. Müller.)

Foth, Genberfeits factslidst.

Wobnt in ruhigen $\mathfrak{W}$ affern, bie nuf einem

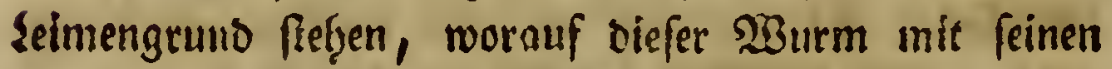

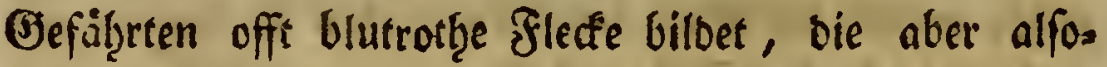
gleits verfatwinoen, fo balo man fie berifintr, ober nur feinen Edjarten barauf fommen lófir.

64. Die gemeine Egel. (Hirudo medicinalis. Muiller.)

Sainglifts, fobmaitglidgt: oben buntlinigt, une ten gelbfectidgt. 
Şurgbaufn, Den 20. Som. I784. 315 65: Die zweinangigte Egel. (Hirudo bioculata. Müller.)

sang; erofarben ; juen 2lugen. Wolynt in Baidjen.

66. Die fdruarze Sdyutefe. (Limax ater. Muiller.)

Ed)wars; runjlidst. Wolynt in feuidten $\mathfrak{B a ̈ l b e r n .}$

67. Die Elcine graule felofduncte. (Limax agreftis. Müller.)

Weißlid)t ; oie Fứfৃler fdymary.

Woljnt in Barten; auf Wiefen; in $23 a$ albern.

68. Die $\mathfrak{W a l b j}$ )necfe. (Helix Lucorum. Miiller.)

Sippe braun.

Die Edfanle fuglidjt, unburdjboģret; vie Wohnt in $\mathfrak{B}$ iloern.

69.- Die Giattenffhneffe. (Helix hortenfis. Miiller.)

Die Sdjaale fuglidjt, unourdbobint; bie Sippe weir.

.250lint in Bebuffien.

70. Die raulye Gdjnecte. (Helix hifpida. Miiller.)

Die Edfaale faft fuglinft, genabelt, raufi.

Eef̧r flein; bie grófte von ber Brróifie eines sinfe; von Farbe faft nusbraun; gails mil flesne!s $\mathfrak{B}$ orften befegest. 


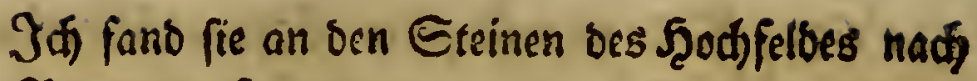
einem গ̧iegen zu Şunderten.

71. Die gefricte Erofaraube. (Helix perverfa. Müller.)

Die Eđ̆aale tḩurmförmig, braungeftreift, linfs gerounden; an Ber Deffnung eine Eleine ßinne.

Das ₹̧̧ier if ffjwars.

230 lynt im Moofe, uno an Baumftaimmen.

Dieß mären nun ungefähth bie Thiere, beret

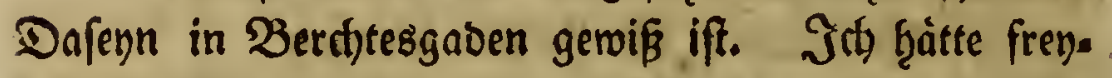
lid bas ßerzeidnif nod) um ein gutes verlängern fơn. nen' wenn iaf bie Infufionstbiere, bie man fid) in eis nem jesen \{aube felbft madfen Eann, unb bie Inteftinal= rourmer, bic man in ber Borausfeģung berjenigen

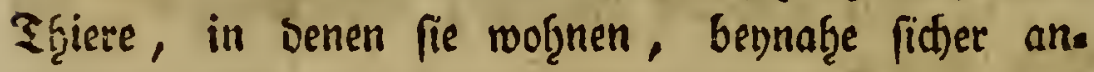
mef̧men barf, nod) f̧ätte beyfę̧en mollen; aber moza

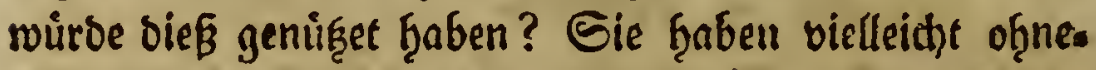

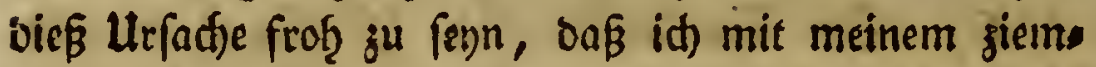
lid) trocknen Berseidyniffe am (Enoe bin; id) werbe baḩer nidfts weiter Ģingufegen, als Daßs id) mit bers jenigen Şodjactutung u. f. w.

\section{Sthrant.}

\section{(sin und ztwanzigfter SBrief. \\ B̈urghau[en, ben 24.5̧orn. 1784 .}

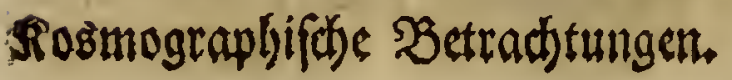

Einfturyen Det SBerge. 
5ुurghaulen, Den 24. Sorn. 1784. 3r7

Derge. Daz beutige fefte Sano ift nicht alt.

Sindflut. Etwas uiber airarat. Alter Der erften Merriften.

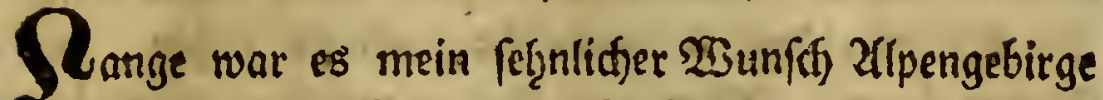
bereifen zu Énnen, nidft fo viel, um ba Pflar: zen zu fucten, in roeldfem Puncte ich hoffen burfte; von meinen freunden, bie ju berlen Rieifen mef̧rere Belegenţeit ḩaben wirben, fajablos geţalten gu wer. ten : als am on Sit unb Stelle tiber alles bab Zluffichlüffe zu erf̧alten, was id) bisf̧er in fo vielen

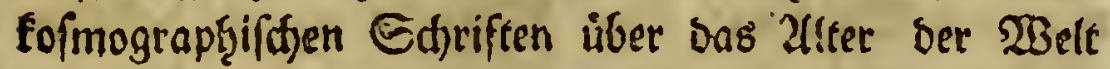
getefen ḩatte. Da fich nun bic fo lange genuinfoste Gelegenḩeit barbot, gab idf mir Miţ̧e, bie Matur

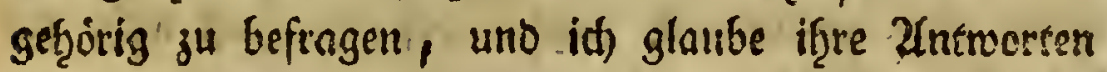
redite verftanden zu baben. Sie foulen Den Jnḩalt biefes SBriefes ausinaden.

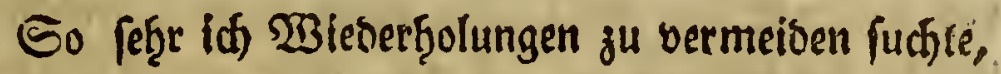
fo menig war mirs mogglict) von meinen Sieifen zu re. Den, of̧ne alle Zlugenblicfe bes Einfturrzens ber $\mathfrak{B} e r g e$

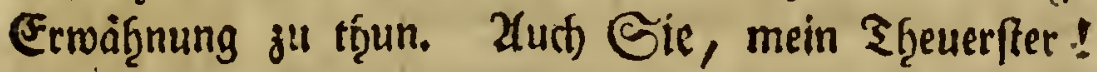
бৃaben biefes cinfturzen offt genug angemertet, uno waren felbft einmal Beuge Dabon. Dieß ift in ber F̧at auf ben meiften Bsebirgen eine ber auffallenoffen

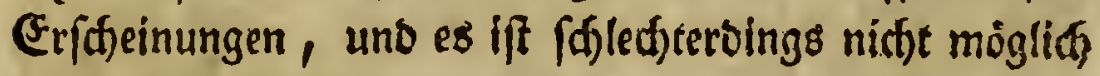
Dergleichen Bjebirge zu bereifen, ub̧ue von biefem

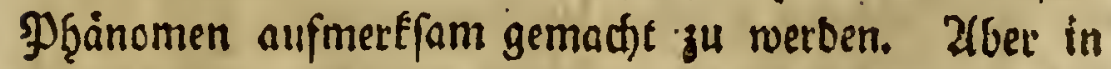
Berdtesgaben ţat man nidht einmal notf̧ruendig die Bebirge pelbft zu bereifen. Waenn man an ber seitung

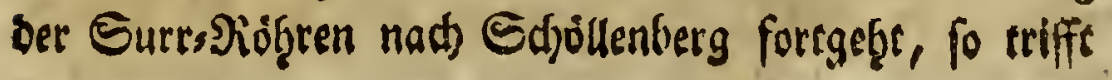


man unnelt bes Riliannaltes ungeţeure nod) ziemlid,

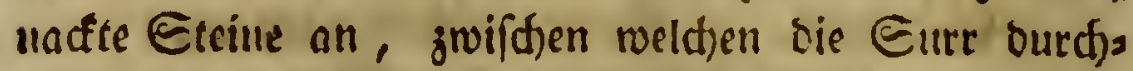
gefüfrret wirb. Man fief̧t es if̧nen gleid) beım erften Zlnblicfe an, baß̧ fie nicht ba, mo fie fref̧en, ent. ftanoen fenen. Zaber man frat jur linfeen in einiget Entfernung einen ḩoben Bèrg von gleidfem Bjeftein, bas jezt faft gang beruatjen ift; man sarf fich biefen Berg nur viel bुother benfen, als er jegt iff, uno if̧n cins

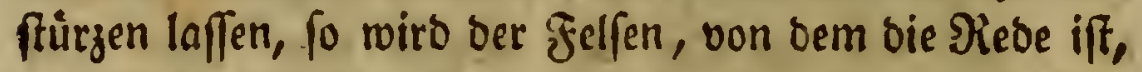

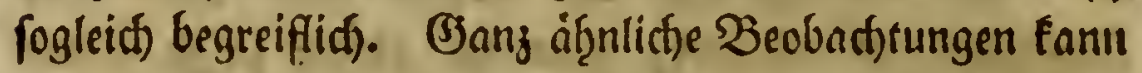
man am $\mathfrak{W e g e}$ nad) bem Sounigsfee unneit heffelben machen; aud) fier liegen mitten auf ben fifounften Wiefen einzelne, aber ungelgeure, Jelfenftücfe, an

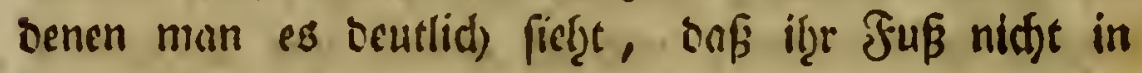
Dic Eroe reiche.

Der 乌jalinglopf if ein gemeiner Lanbberg, nun beffen Epige idf . Ginnen einer guten $\mathfrak{B i e r t e l f t u n t e}$ bis an bie Gtraffe bernbgieng, feine untere Böfduung ift ganz bervad)fen, und fogar bebauet, fein oberer Theil malbigt, fotwer zu befteigen, unb allenthalben mit herburragenben Jelfentrúmmern befergt, bie immer groffer werbell, ie weiter man linaulf fömmt, niemal aber von einer gar zu groffen Naffe fino. Da biefce Berg feinen groffern in ber Nábe f̧at, von tem ex bุâtte erzeuget werben Eònnen, fo muß̧ er boch moţh mit Den (Jebirgen gleitffen Ur.prung fraben; aber burch) fein Einfturgen ift er verflàrfect worben. Zallerbings fdeinen bie obern Steine an einer feften Maffe ange-

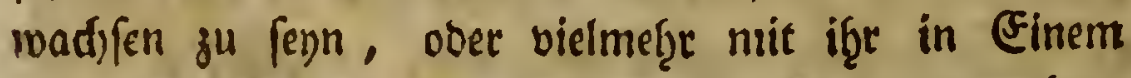
forijugểen, uno ein einziges Stủof ausjumatien. 
SBughaufm, Den 24. Sorm. 1784.319

SIGeiter unfen, sod) noth im $\mathfrak{W a l b e}$, fino oie Trum mern lofe. 2am furfe bes $\mathfrak{W a l s e s}$ if ein segen, wo

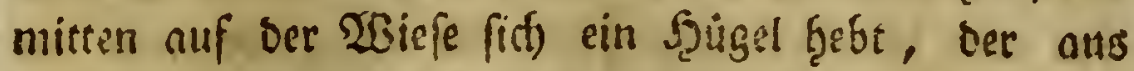
lauter untereinanber geworfenten Eteinen befént, oben mit Baiumen Ggrodjen, an ben Geiten aber faft fab̧

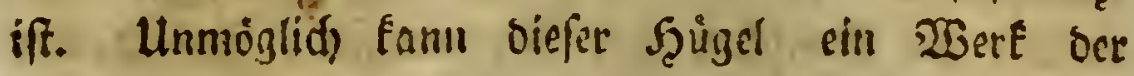
Menfden fenn; die Eteine fino zu grop, um von Menfden geworfen; uns jul fther, um hier ju= fammengetragen ju fenn. Wabreftzeinlid war biefes

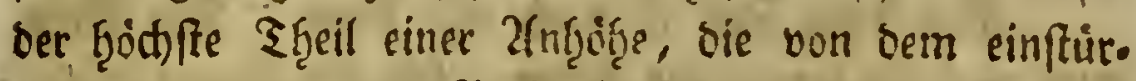
zenten Bipfel res Palingfopfes erjsuget morben; nactimals haben bie Mienfd)en bie untern Eteine nad)

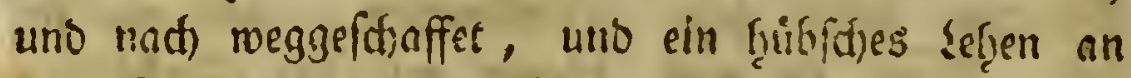
iģrer Etelle angebinut. -aber eben boburd) eridswer. ten fie fich bie Zerfforung oss oberffen Theiles, Deffen SWande nun freiler murben, und auf bas angebaute Sano berabrollen múrben, wenn fie niche bie 3egetation, befonders bie Baumwurzelin, zus. frommen Gielten.

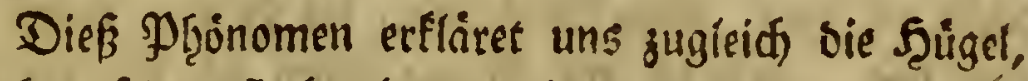
bie fid) auf bem flad)en Zande mitten auf ebenen \$iefén, oder wohfl gar in Ş̧álern erręeben.

Unterbeffen ந̧indert biefes beftindige Einftúrgen Der Berge bie $\mathfrak{B e g e t a t i o n}$ ganz ungemein; gleidjmobl ift es weife Finrid)tung ber anbetenswurbigften Bor. fidit: : Durd) bas beftánoige ફ̧erabrollen ber Eteine werben nid)t nue bie Berge niebriger : fie befommen

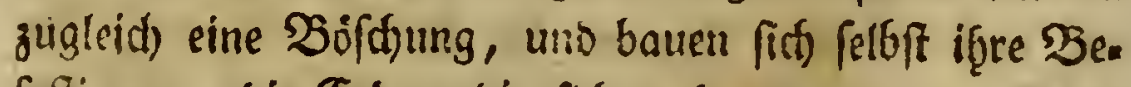
fertigung; bie Eroe, bie fid) nach und nath zwifden 
ben Steinen fammelt, uno von i̧̧nen aufgef̧alten wirb, wird buta) bie immer ftärter werbenbe Begetao" tion nod) fdnnefler vermeţret, sie Bergfeite wiro ba: ourd) immer befteiglidfer, fructitbarer, enblid) gar bemof̧nbar. Es fommt gewiß nod sine 3eit, zu

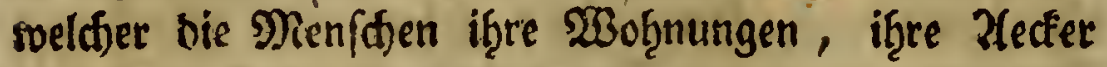
uno Bärten ţaben merden, wo jigt Biemen und Murmelthiere wensen.

Zlber biefe Zeit ift nod) fef̧r ferne. Die Eroe muß nod) Jaḩrtaufenbe ftef̧en, bis bie গatur biefen groffen Şau vollbringen wirb. Sloch ţaben sie Pflangett niddst fȩ̣e vorgeorungen, uno wo fie fino, liegen fie nod) immer mit ber Berftórung in Rample.

Dod biefes nod) fortbauternbe Einfturzen] ber Derge, Diefe Zrmuth an Begetation auf Denfelben, biefer gånglithe Mangel auf ben f̧öd)ften Spizen, was zeigen fie nnbers an, als bas bas heutige fefte sand

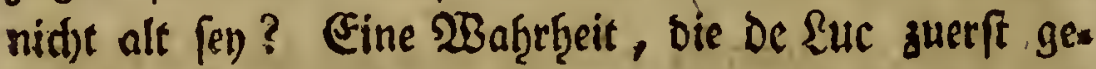
ragt ḩat, uno bie bie Nincur jeben leţret, ber if̧re Seḩren mit unbefangenem Beifte auf ben Ģebirgen amfioret. Da verfdtwinoen zugleith mit ben angebau. ten Begentoen alle bie groffen Bafilen von Gą̧rtaufens. ben, bie man aus ben unterfdjobenen, ober úbel ver:

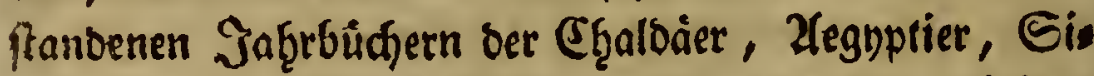
nefer to frengebig nufjabb̨t, uno alle bie wif̧igen Tråume, bie man fo gerne an feinem Pulte tríumt, um pbilofopgifdge Romane fdreiben zu fönnen, bie Der Diaturgefdichte to menig zutråglid. find, als bie

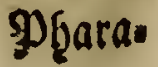




\section{Şurghaulen, Den 24. Şotn. 1 784. 320}

STharamunbe uno f̧enmannsfinber ber bürgerliffen

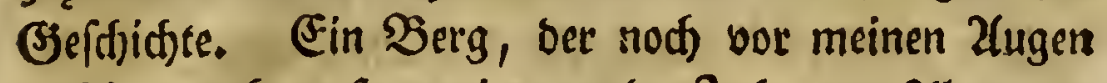
einftürzet, fann fo menig $4886_{3}$ Jaf̧re bor Zfleranber Dem $\mathfrak{M a c e b o n i e r ~ b a ~ g e w e f e n ~ f e r n , ~ a l s ~ e i n e ~ S ̧ u ̈ t t e ~ z u r ~}$

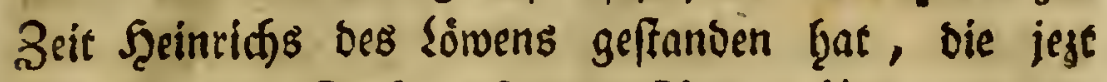
ùber meinem $\Re o p f e$ zufammenftürzt. Uut bie 3eugen; bie man zu Bergauptung biefer Jafien

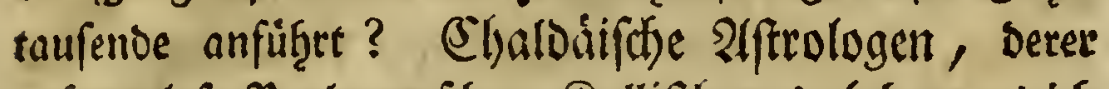

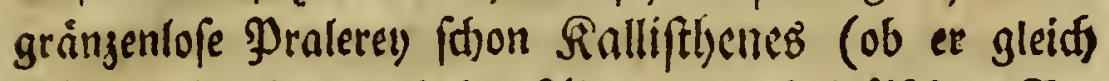

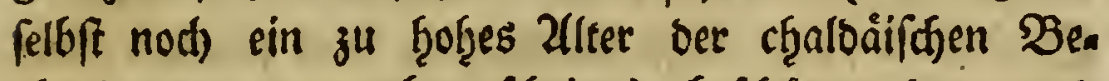
obadftungen anjugeben fafeint, bef(d)ämet f̧at, uns Derer $\mathfrak{B o r g e b e n}$ Den búrgerliden und affronomiffen

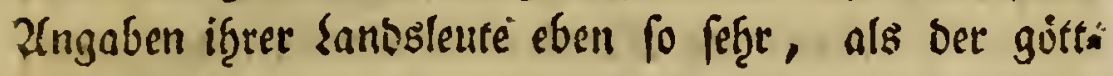

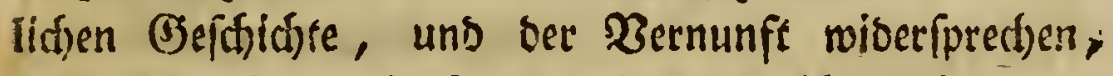

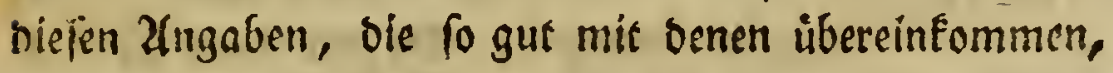
Die wir von LXX Dolmerf(dern ţaben: 2legyptifthe afftrologen, ober überţaupt Afeguptict, bie if̨re

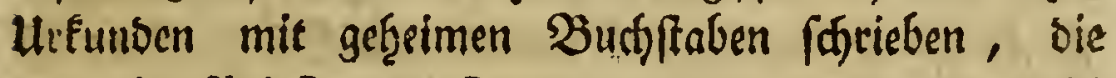
nur bie פrieffer verfanben, uns berer Renntnifs

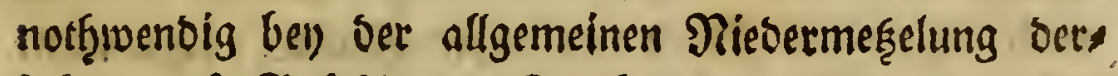

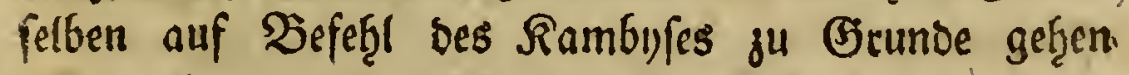

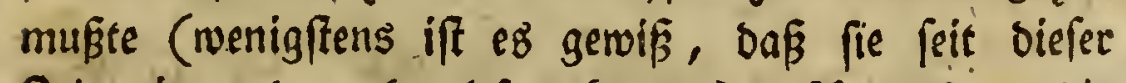

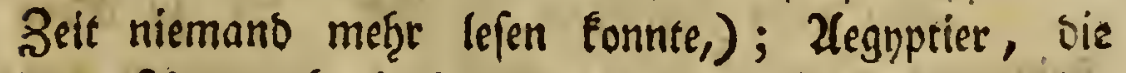
į̧re Şimmelsbeobadfungen 48863 Jaḩre vor alle: kanber mollen angefangen faben, uno binnen biefer

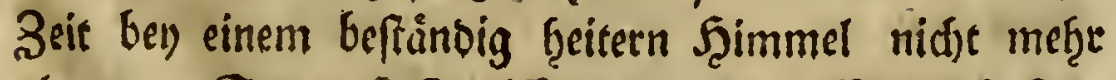
als 373 Eormenfinfterniffe, und 832 Monisfinfters

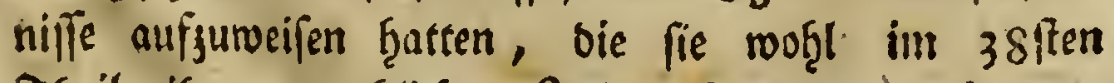

Theile ifrer angeblitfen Safretaufende gang bequem

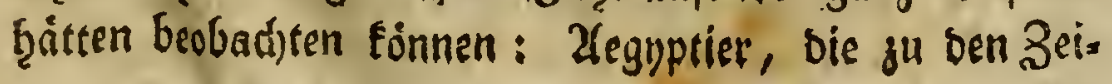
Eaturbift. Briefe I.Band. 
ten bes Shales in ber Gseometrie, ber unentbeţre lidfften Scilfşriffenfstaft für bie affronomie, fo un. miffeno waren, onß fie von biefem Philofophenen exft

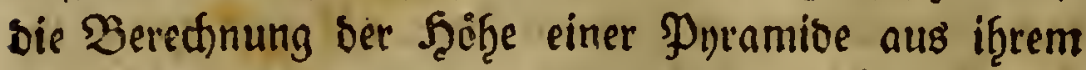
E(t)atten lernen mußzien; Sincfen, berer Ehronolo:

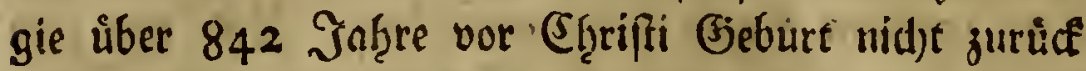
gețr, uno berer (Ecffhichte über bie Zeiten bes Yas Ginaus, bas ift, was bie (seffhidfte Der (Striecten vor bem trojanifthen Rriege war; Arrander, von weldeen SvidittB erzåh̨lt, was ein neuerer, fonft giemlid) Ģarts

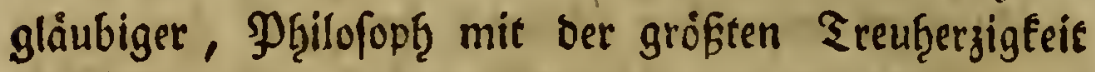
nadbetet, uno id) mir forgenbermaffen iber feģt habe: Nod) vor Rronions (Jjeburt, fagt man, onß 2lrfaber traben

Sinber bemolgint, uns vor bem Dafeun des Fondes (*;

aber Sie miffen bodt), was fur Ţ̧iere in 2(rfabien zu Şaufe gef̧ören; uno wollten wir Dvids Zeugníß annef̧men, was mùßsten mir nicht glauben! ben. nod) fagt felbft Dotd in biefer Stelle: fagt man (feruntur).

aber man begeidinet bie Beit, bie ju Bern fteinerung erforbert wirb, nad) ben. Trimmern Der Brưcen, von berer Erbauung bie (Befd)id)te Mela

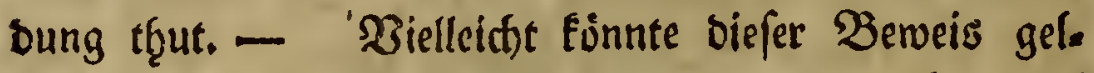
ten, wenn fī bie Berfteinerungen allentţalben nuf

(*) Ante Jovem genitum terras habitaffe feruntur Arcades, et Luna gens prios illa fuit. 
einerley 2 Urt verthielten; aber eben bief ift eine Satt)e, bie man niemal wiro berveifen soinnen; es ift fogar gemis, baßi mand)mal eine fére geringe Zeit baju geţơre, wie id leifft Dartḩun fơnnte, wenn ifj aus, fidweifen wollte.

Die Unvolffommenţeit unferer Rünfte uno Wififenfdaften ift allerbings eill guter Beneis für bie Jugeno bes Menfdiengefalechtes, uno Maillct, Der if̧n zu entfráften fuct)te, if babur(⿻) in unge. reimtb̧eiten verfallen, ůber bie jeber Echüler ladht, ber einige $\mathfrak{B o r t j e n}$ lang ben Borlefungen über bie

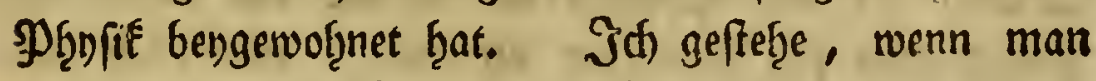
es verlangt, den Sinefen die Ef̧re zu, bafí fie man. des fdoon feit Jaḩrḩunderten wuß̧ten, was wir erff geftern entbecfet ḩaben, aber biefe alte, biefe meḩx als 60000 Jaf̧re alte Nation, Die man uns immer vorwirft, glaubte vor nod nidt viel Jah̨ren, baß̧ ber Mlono : mit einem Dradjen ju faimpfen ţabe, wann fie eine Sinfternißz faf̧; bie Berechnung oer Bervegungen Merfurs war ganj ùber if̧re Rräfte; felloft bie bes Moubes fannte fie nid)t Ģinlainglich, bie erfte Uḩr, meldye bie Jefuiten Daf̧in bradjten, Egielt man in Gina für ein ₹̧̧ier. - Mit einem Worte: Eina if eben fo gut ein nod nidte frbe als tes Land als Europa, und bie Runfte uno 2 Biffens fahaften fino menigftens nid)t über bie unfrigen finin aus. Umfonft vertheiviget Raillet feine Zltten unb fidf mit einer vorgeblichen Bicnuglamifeit an nùs. lid)en. Renntniffen; umfoult bef̧auptet er, unfere ḩeus 
tigen Renntniffe fenen meţr vormig̨ig als nüs̨lich: mag es fo fenn - aber es war von bem sie Sifoe nicft; bas f̧atten fie renigftens vor 100000 Jaf̧ren foton fenn follen; Prunt ift allemal bie riofrige folge Des Uleberfuffes am Notfimentigen, unt ter empor. ftrebende đJeift bes Menftien ift niemal mit sem blok núşlidgen zufrieben, was er befiģet.

Die geringe 2(nzaḩl ber Menfdhen ouf bem gefammten Erolreife ift ein neuer Seweis bes nod,

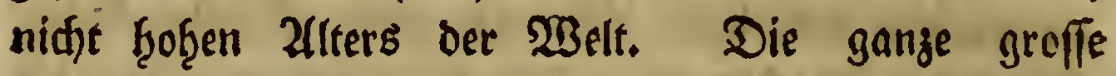
Tataren if eine $\mathfrak{B}$ uffte, in welder bie ftreifertoen Fatarf̧orben fidf gleidfan berlieren; aber nod) weit einleudftenber ift ber entfestlidfe $\mathfrak{B u f f s m a n g e l}$ in 2(merifa, wo groffe Familien fir Nationen gelten, und wo ungeţeute Jెegenden, gegen bie unfere f̧erz

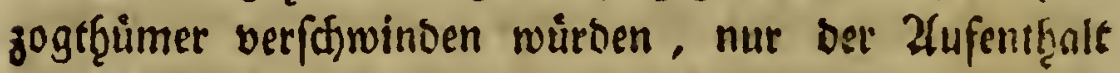
woilder Ş̨iere uno ber Infetten finb.

(B)eicfinof̧ mus auf ber Erbe feit if̧rem $\mathfrak{D a}$. fenn eine fef̧r ftarfe Rrevolution vorbengegangen fern. Die verffeinerten Eeetgiere, bie man allenţ̧alken, fos wof̧l ţod) auf ben Bipfeln ber Bebirge, als tief in Det Eroe findet, fagen es uns laut, baß unfer gatr. jes Ģeuriges feftes Lano eţeoem Seegruno sewefen fev. Unterbeffen fafweigt bie Beffojidte, ein paar Uteberfofwemmungen ausgenommen, bie eingelne Sanver betrafen. Thur bie ,geilige (Jefdrid)te, bie

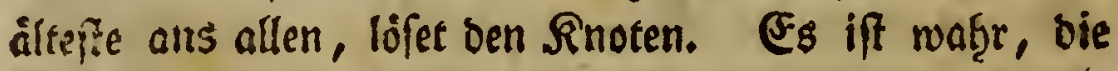

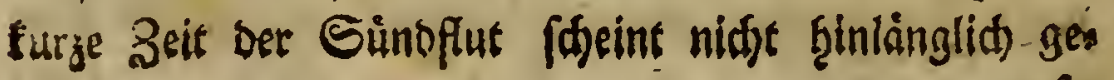
wefen 
wefen zu fenn, um alle bie \$irfungen ந̧erborgubrito gen, bie wir auf unferm ḩeutigen sanbe als 2 Berfe

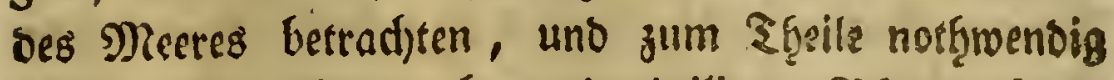
betradten miffen; aber bie beilligen Büher fagen fein $\mathfrak{W o r t}$ bavon, daf bas kano, bas mir heute be. wofinen, eben bas felbe fen, welches 210am uns Seth

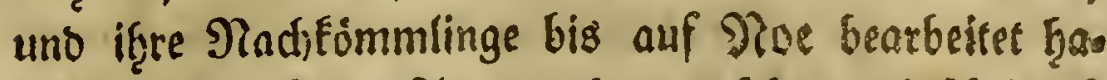

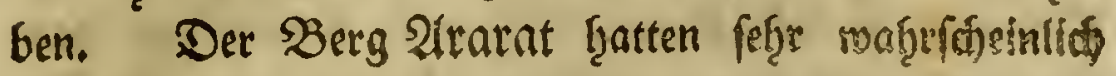
Damal, als biefer neue Etanmoater ber Menfdyen aus ber 2 (rd)e hererborgieng, nod) teinen গamen, et

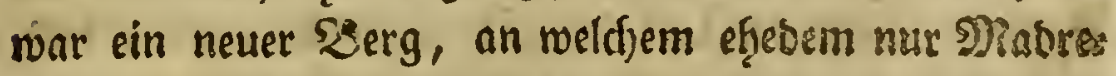
poren unt Zuftern gervof̧net Ģatten.

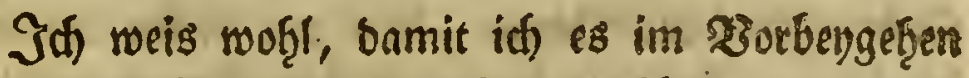
fage, Daß Ziratat luftig madten: weil feine Swifge nath Sournes forts Beridjen gans unbefreiglid ifs, forsobst feines

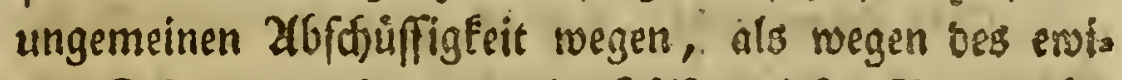

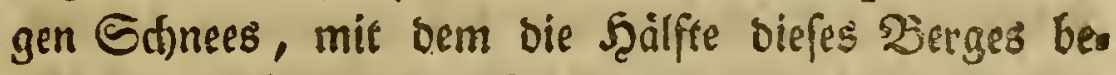

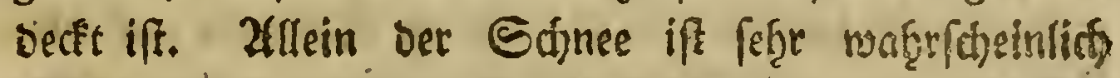
viel jünger, als bas Dafenn biefes Berges, und un. fers ganjen feften lanbes, fo wie es ber Stines alles (bjletfder ift. - Diefer Edfnee, ber bey feinam immer.

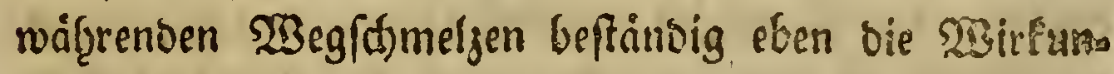
gen ţerborbringt, bie bie Berge Berciteşzaveng alle

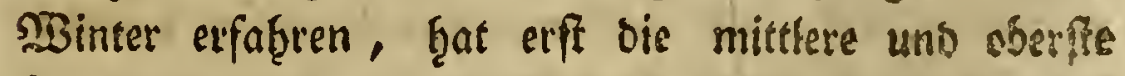

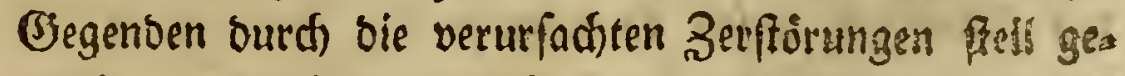
madte, und wiro bamit fo lange noch fortfafzres?, fis fid ber $\mathfrak{B e r g}$ nach und nat) fetbff ernizbriges. 2tbet wet f̧at jemal (bie unwiffensen Zrmeniet auss 
genommen) als ausgemadit augenommet, baß̧ bie 2frde gerabe auf biefem Berge, bem wir heure ben Namen Zlarat geben, gerubet Ģabe? Die beffen Ed)riftausleger balten mit bem 2 erfaffer ber 2 uls, gate bafúr, Zurarat fey ber alte Name Jrmeniens, uno in Zfrmenien giebt es auffer biefem Pुicf nod andere Berge. Der heebrailfde Tent fot)eint felbft biefe Mennung zu befrififtigen, indem er 2lrarat von ben Bergen unterideidet : nuf Den Şergen 2rarats (in. ftatu conftructo) gerabe wie wir fogen : auf Den

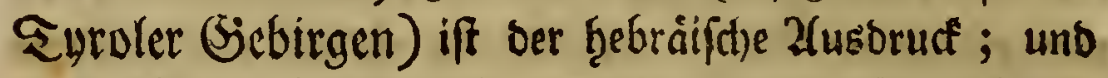
nad) Zfrarat (moḩ nid)t in bie elenden Sennf̧utten, bie Tournefort antraf, ober auf bie unmirtblidsen Sdnneefelder des Berges, bem mir biefen Namen ges ben) Gaben fidh bie benden Göhne Gennaderibs nad) ber Ermoroung if̧res Baters gefluddztet.

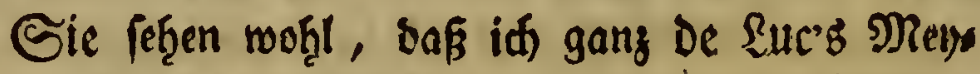
nung benpflictie, fowoḩl ibas bon efremaligen Buftans

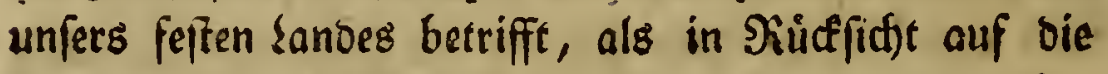
Zeit, zu meld)er biefe Sievolution vorgegangen; abet

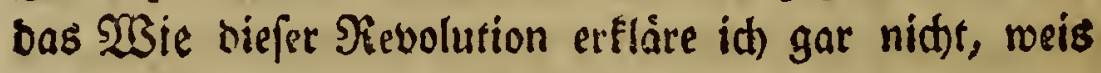
es nidft, uno beţaupte breufte, daß es niemano wiffen

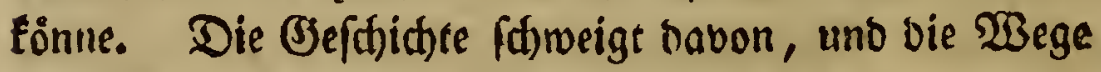

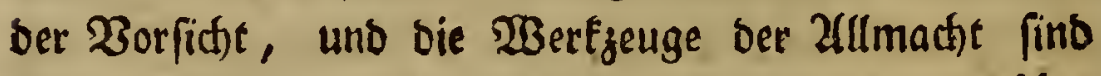
zu mandfältig, als onś wir jemal etrọas barúber

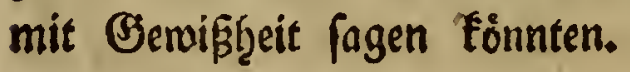

Borausgefeget allo, bon jur Beit ber allgemeis nen Eủnoflut bie groffe ßerånderung des Meergrunbes 


\section{SBurghaufen, Den 24. Şorn, 1784. 327}

borgegangen, baß̧ fcit berfelben ßeit bie eţebeffen be.

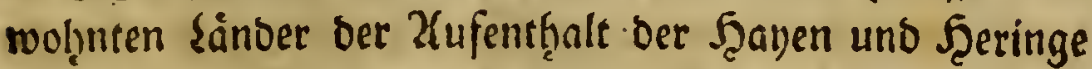

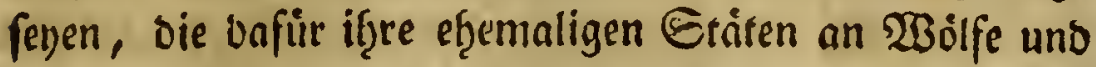
somminge abgetreten, fo folgt fitson aus biefent, baß Das Meer nid)t to allmåţlig zurisfgetreten, wie uns B̧uffon, Julti uno Maillet gerne úberreben

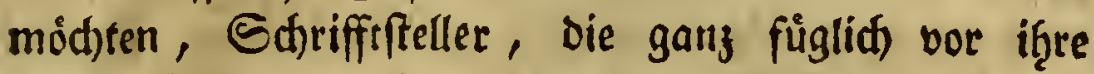
fosmograptil(t)en Ed)riften aus Dvid bas Motto b̧ătten regęen follen :

In nova fert animas mutatas difco tormas. Corpora u. f.f.

Da ber Jnḩalt berfelben eben fo gut ein Gemifo

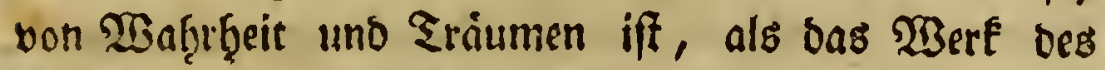
Didfters von Eulmo, bas er mit biefen Wjorten ants fángr. Dem allmábligen Buricftreten bcB Meeres wiberfprechen fofledfterbings alle Bebirge in Berthtesz gaden, wiber(prid)t (d)led)erbings Tourneforts 2(rarat, wiberfpredsen raufend andere Bjebirge; ein fanfter

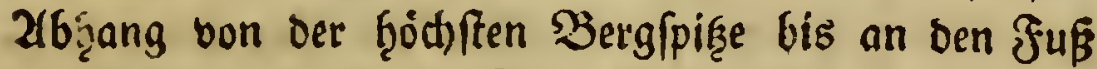
Der (J)ebirge mürce die Folge Dabon fenn, ba wir jejt allentḩalben fehre fteile Maffen erblicfen, bie bas Meet: mitten in feiner hefftigften $\mathfrak{B}$ ewegung verlaffen zu bृa ben fheint. Doch id will mid) über diefen Bregen. fano nid)t långer aufb̧alten, oa de suc in feinen Briefen mit eben fo vieler Brunditsfetit Bifffons Beweife miberleget hat, als fiegreid) feine eigenen Beweife fint, bie er bawiber weitläufig anfüf̧ret. (

aber

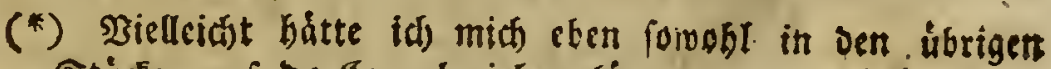

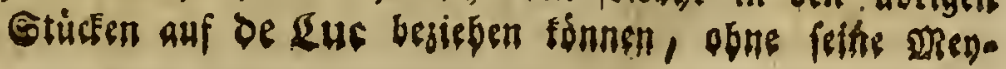


atber bariun fann id biefem groffen Mianne nicht beutretten, wenn er bie Menfften fid) von ben (J)ebirgen auf bie Plainen verbreiten liât. Die Be.

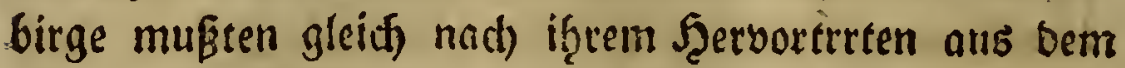
Waffer ber $\mathfrak{B e g e t a t i o n ~ n o t ) ~ r e i t ~ w e n i g e r ~ e m p f a ̈ n g l i d o ́ ~}$ gewefen feinn, als fie 6 je jezt find; und jejt fino fie es nod) wenig-genurg, mic Sic gefergen ţabent. Frellid) fheinen bie SJlairen eben fo wenig zur Bervof̨nung faflefliti) gewefen zu fenn; bas waren Savannen, bie mit bem Edjlamme bes $\mathfrak{M}$ iceres bis in eille groffe Ziefe bebect, lange Beit vonnoitţen ţatten, um auso gufrocinen, und roobll für Rrooten unb Binfen, aber

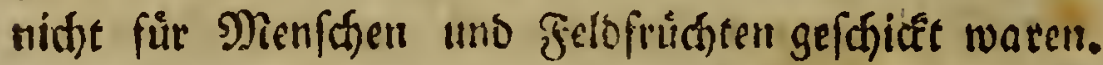
alber Mittelgebirge, bie weber fo fafl! als bie. Şocf)e gebirge sa lagen, nod) fo unreinlid) und ungefuns, als bie gllánen waren, módfen fid) bie erften $\mathfrak{B}_{e}$

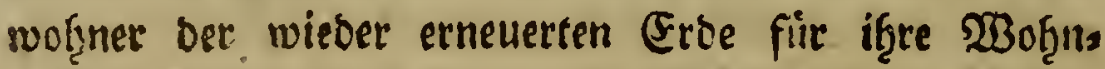
pläge ausgefurset ţaben. Die nach uns nach nuls. getrochineten Ebenen, auf Denen bie Begetation notge. wenoig

numgen grofientheils mir cigen zu maden; ober id batte viellidit Diefat ganjen srief wobl ganj weglaffen fơn: nell. - 2lecr mail bat io off sic finnteidfen Srinme Drs franzöliffen Dlinius (Der Die Eigenfalffen Des romiforn fidi in mebr als cittem Stide fo eigen gemads bat) madịctroumet, oaf es nidit ủberfiufing feyn fann,

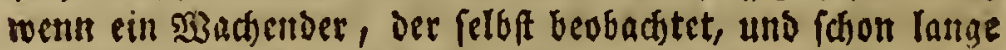
gelernet bat, wie man bie siatur befragen måfe, nods ciumal fagt, was ein grofferer $\mathfrak{B} e 0 b a d j t e r$ fajon vor ifin

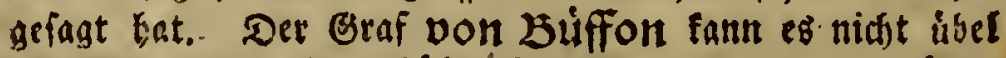

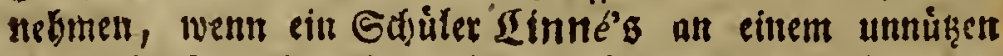
fartenbaule noch incit meniger $\mathfrak{b e b a g e n ~ f i n d e t , ~ a l s ~} \mathrm{cl}^{2}$ att ben erleidtermben, und fogar mirflid) belebrenden Metfobert ber von ifm pogenantiten ఇamenfammler fand. 


\section{Surghouren, Den 24.55orn. 1.784, 329}

wenbig måchtiger fyeranmudos, als auf oen biel man gerern Bergen, locfen fie endida) gleicfiwofil vọn 16 .

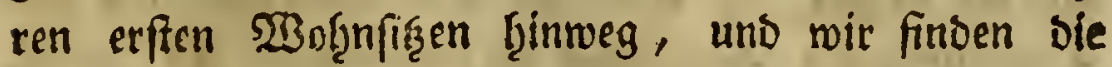
Menfden balo in ben Ebenen Eennaars verfammelt, um fich ba anzubauen.: grreylid) muste ben immer.

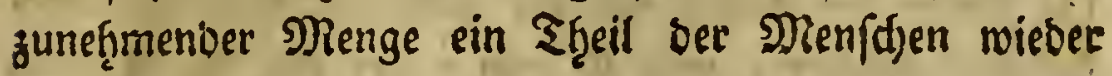
bergnufwäts zief̧en.

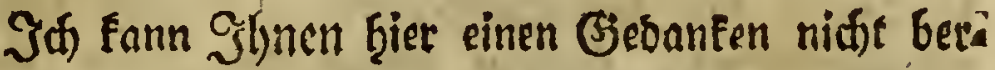

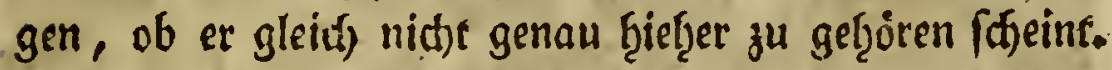

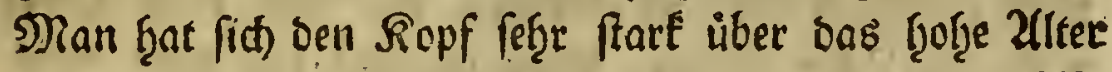
Der antesiluvianifáen Bäter, uns bie fd)nelle $\mathfrak{Z}(\mathfrak{b}$ a naf̧me ber Eebensjaf̧re nari) ber Eünofut zerbred)en. İt) glaube, bie Eache fiatte eben to viel Ropfbrechens nidft vounotthen gef̧abt. Man ret)inet gea woifgnlid) fiebenmal fo lange Beit für bas ganje leben

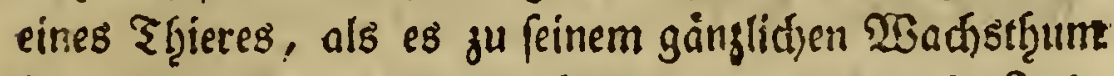
braucf). Da ber Menffi bis in sas zwnnzigfte Jafe fortwad)sf, fo mürben Dann 140 Jag̨re bie Jabje feines Lebens fenn. Zllein biefe Regel ift betriglid);

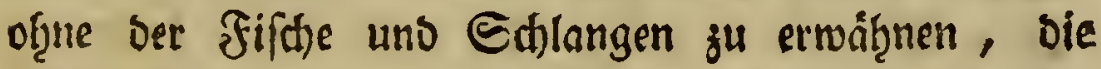
viefleid)t of̣ne Unterla $\beta$ wadfen, fo traf meine $\mathfrak{B}_{\text {ea }}$ rertimung bey Durd)lefung ber büffonfden Thier. gef(hid)se felten genau mit ben Beobad)tungen úber

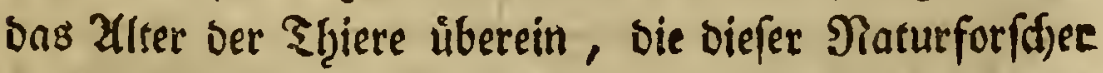

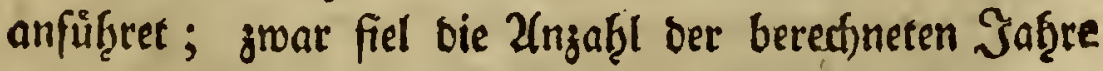
mandfmal ju großj. aus, aber meiftens war fie zu gering; Daju fómmt nod,; Daßj eż vielleidt) ben allen

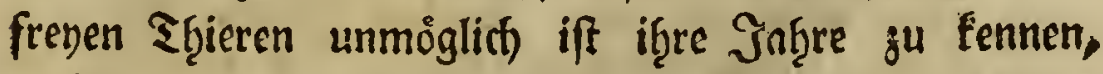
naøbem fie einmal über cine gewiffe periobe Ginaus 
find; und adjet man nod, , wie man woht foltte, auf bie $\mathfrak{B o g e l , ~ f o ~ u ̊ b e r t r i f f t ~ i f ̧ r e ~ s e b e n g g e i t ~ o i e ~ m i t ~}$

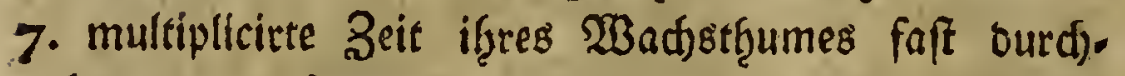
gefenenos zwey : brey) bis viermal. Daburd) werben uns Die Gaf̧re ber Båter vor ber Suinoflut fófon betràd)t. Iiđ) begreifictjer, weil fie nun wiber eine Riegel ftreiren, bie man willeủrutrlid angenommen ḩar. Seģett

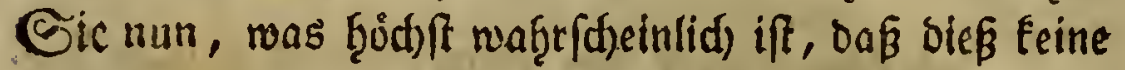
aftronomifonen, fonbern burgerlidje Iafgre gerwejen fenen, von Zlernte zu Zlernte, unb oáá diefe Bàter soofll eines ber sanber, berer es aud) jejt viele giebt, bewobnet baben, in weldhem fie binnen einem aftrono. mifcten Safir zmenmal arnten fonnten, fo fommen 900 Jaţre ftion auf 450 berab, bie beil ben bas

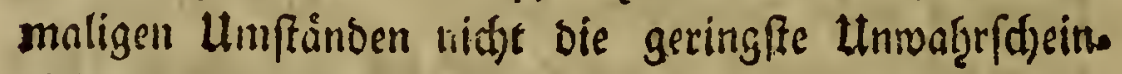
lidféfit meţr ţaben.

- Nach ber Güroffut lebten bie Menffen nut nod) I 20 Jaf̧re, aud) wob̨l Jaf̧re yan 2lernte ju Zlernte (benn anfänglid) fonnten fie wob̧ feine gea übten Xffronomen fegn), aber biefe Jafgre famen mit. ben afronomiftsen Gafren fo jiemlid úberein. In bem sanbe ingrer erften Nieberlaffung (2(rmenien) fonns

ten

(*) Sth max Dodh aegen meine Eegner folir frengcbig, dab

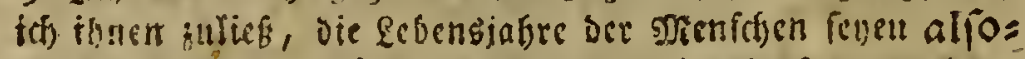

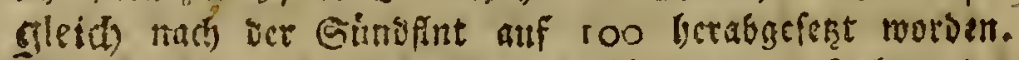

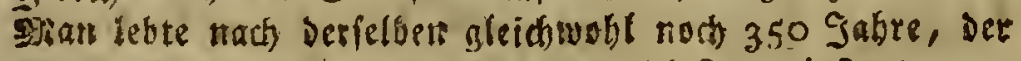

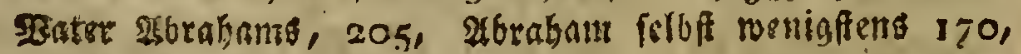

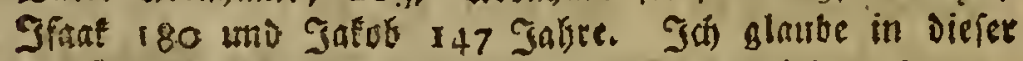

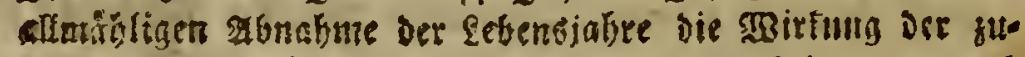

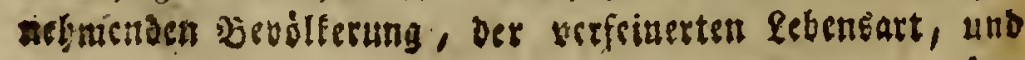




\section{Burghaufen, Den 24. Sorn. I784. 331}

ten fie nidft meţr zmentral bes Jaf̧rs ărnten, uno einmal ein நृalber Monat ju vief, ein andermal ju wenig, je nadjoem bie fruidjte reiften, moth ten sie

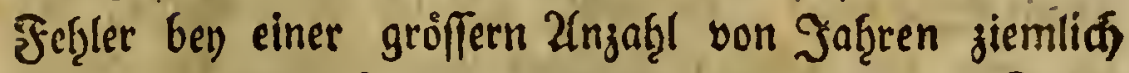
gegeneinanber auffeben. Bleidfwohl find I 20 Jaţre nod) immer werig, wenn man fie gegen 450 ḩätt; aber fonnte bief wohll anbers fenn? Die Menfchen bemobyten eine neue Erbe, bie feit ber Edopyfung unter $\mathfrak{W}$ fffer gelegen ţatte; urtheilen Sic felbft, wie nactitheilig biefe mit faulen Dimften angefülte sufe Dem thierifdgen leben múffe geworben feinn. Swar verbefferte fie fid in ber Folge, aber bie Manfafen wourben aud) zafilreicher, bilbeten groffe (jefellfofhaften, Jegten Etäbte uno Rönigreidte an, verfieffen if̨re erfte

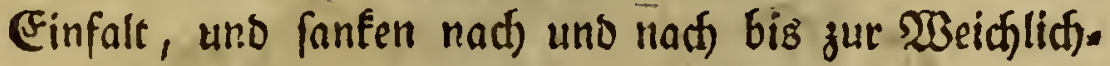
feit tzerab, ober mußten, da Das (Eigenţ̧umstedfte fid) allmäblig mit allen feinen zmenbeutigen Solgen einfdlid, Darben. Der Genufs des freifdes, eine

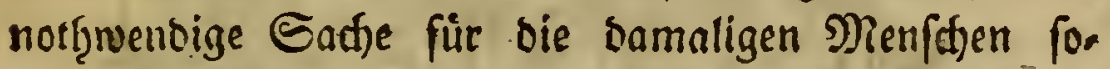
wob̧l als füs uns, modtete das Eeinige aud baju

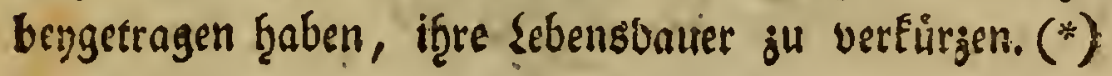

$$
\text { Y) } 2
$$

$\mathfrak{d} 6 \mathrm{et}$

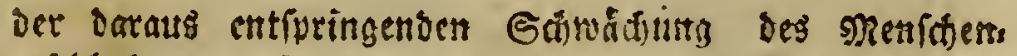
geidflectes ju fehen. Sielleidht liegt bier noch ein (5)

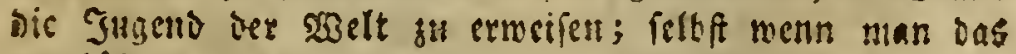

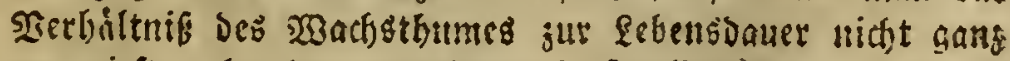
megnirft: aber Daun werden mir freylid sicht 20 mit 7 fordern nach und nach), mie wir biber binaufrusfee?, 30 ,

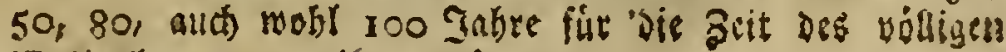

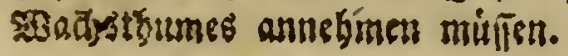




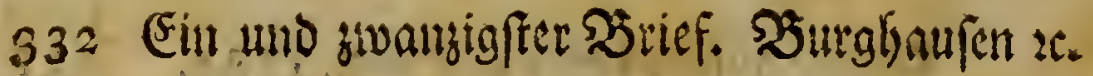

2(ber es ift Beit, baß̧ id) abbred)e. ŞBie es angenefrm ift, roenn man einem Freunbe fatreibt! Wie ta unbemerft bie Gtunven vorúber eilen! seben Sic mońl, fo woh̨l, als id) es münfdae, ber iid mit ber ganzen fodjactstungswollen Jreunofd)aft unabanberlid bin u. f. f.

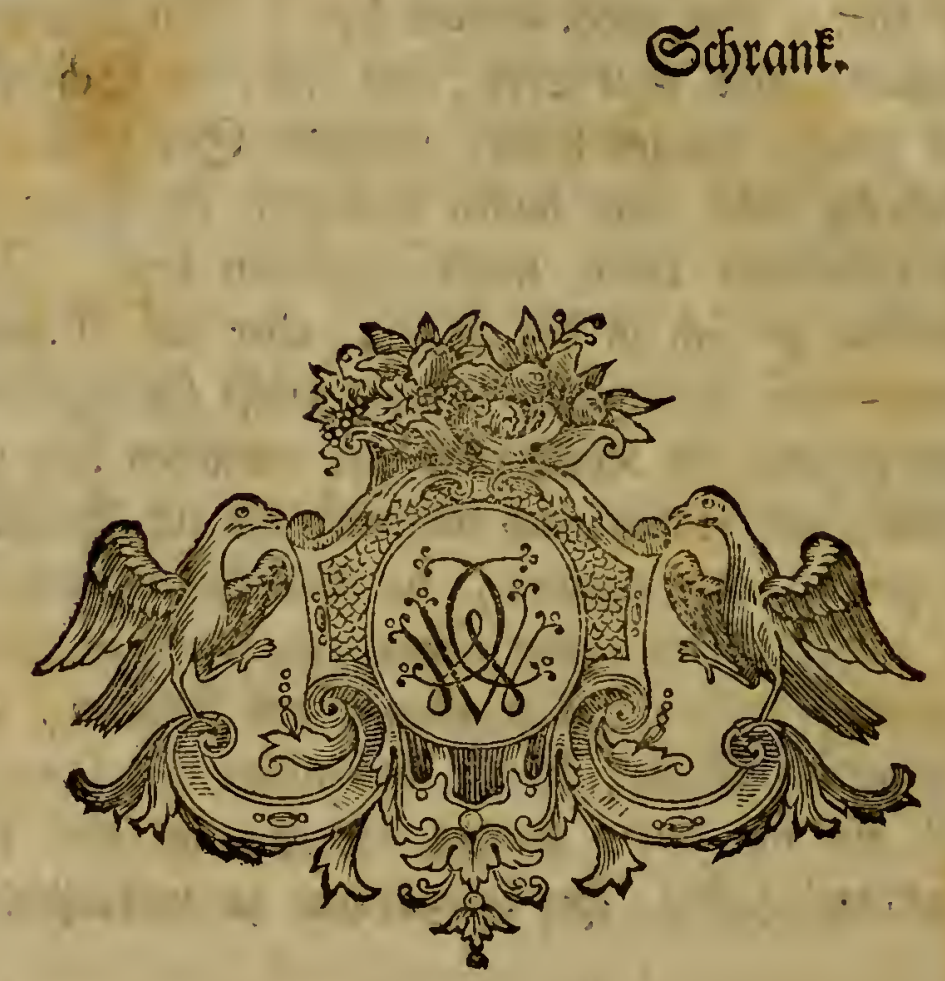




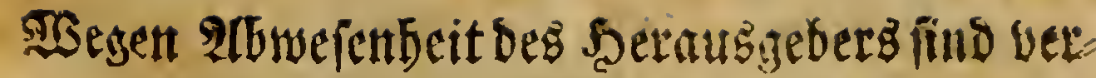

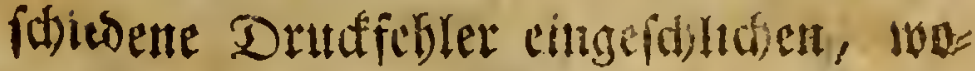

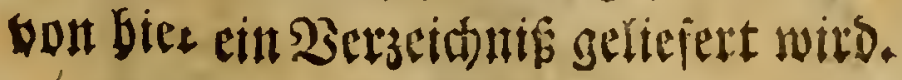

Scite כrile

lise

lies

323 tetradititi tetradaltyli.

9 7 Toxcicodenónum: in icodendrum,

$x 6$ I4 feq. Plantanus

2216 . rifite

245 Difcutella Bifcutella.

- I7 murben mirve.

$31 \quad 22$ Acipenfes Acipenfer.

40 I6 Sindergruitde Bintergrunde.

41 I7 maifgen máfigen.

- 24 2isiongnera 2istpmern.

- 25 feinen

$42 \quad 19,20 \quad$ Dns

- $25 \quad$ ficfing

43 IO सisgint

Feinte

DeE

$44 \pi$ Goliters

finfing.

$45 \quad 12$ Ervenawen

aizbidil.

$46 \quad 23$

$47 \quad 3$

fpicat

Sisutwiefe

$-24$

$48 \quad 8$

$-17$

Eroen

eine

23eg

49 II

fraugțale

Galitters.

- 16

514 rugofutis

5348 weisen weilen.

54 vorles. gaben gebert.

5621 annebmen asnetyma

- 30 feime fein.

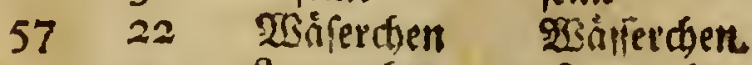

- 27. farmebe farmebit.

5823 Jenebad כembad.

6019 Dem Den.

687 Ergeuguiffen Erzeugniffe.

- 27 Dál. Nid. 
Eeite Zcile für lies

69

4

Shorben

Echoor

Edjortlet.

- 6

כittergruno Zillerstund.

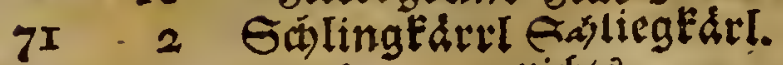

II $\quad 2 \quad$ nid) nidbtỉ.

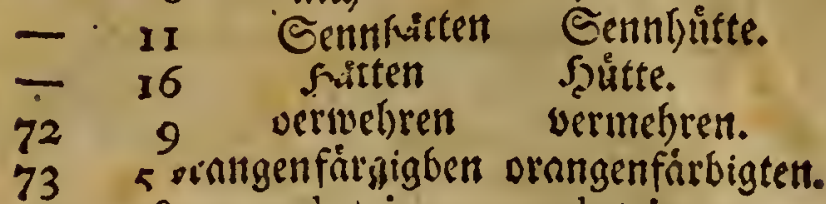

2 is clytris elytris.

74 lezt. ibren ibje.

80 6 Gewand 5ramand.

- 7 Gisongial Gajonbial.

- 22 frivere Mianke Edbmeremanks.

- 26 Dicfen Diefem.

833 Ghenpiat Gds̊nbidsl.

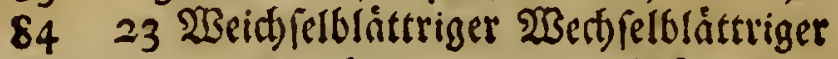

8524 DeIm

89 vorles. zugleid zimlich

90 vorleq. queerúbert. queeriber.

9118 in nuf.

93 sotleg. beteite bereitet.

9520 von vor.

- 21 Berg rủden 3̧ergruffen.

$96 \quad 15$ Gerlesberger Gevlosberger.

- 22 Gerlooftein Gerlosftein:

fügclden fögeldhen.

- - כiggader Śippadjer.

1004 Sturide Etunden.

- 15 . Martel Marbel.

$\mathrm{YO}_{2} 4$ Danans Danaus.

- 27 find fand.

I03 I2 elytis elytris.

I05 5 :

106 I6 Der Die.

з10 20 meteorologifinen entomologiftek.

III 19 aușmadyen ausimadje.

112.18 2lbre

1145 Demielben Denfellen. 
Geite 3eile für

líes

$123 \quad 18$ Etcingruts

$126 \quad 17$ Diefe

- 18 Decfe

- 26 baben

$127 \quad 17 \quad$ Hmt

$135 \quad 12$ Ieljercitfer

$\begin{array}{ll}23 & \text { DDeis } \\ 20 & \text { geflinget }\end{array}$

$x 360$ geflingert

13820

15118

$\begin{array}{ll}153 & 17\end{array}$

1603

165 I

$168 \quad I$

$17^{2} \quad 23$

$173 \quad 17$

fordortonte.

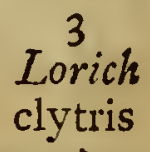

mun sen

ictericius

Rnx.

II.

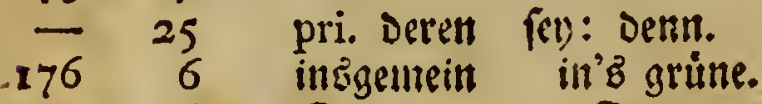

$177 \quad 16$

Dennt

Dann.

1823

1945

mittbeilent

Dich)

bier nocts

197 Iegt.

Den

$200 \quad$ I 3

tweil

- 21 กแร

$208 \quad 19$

(el)

9 S3ngntautts

$21 \mathrm{I}$

261 II

eigenontmen

Melis

eintbeilen.

ich.

Dicfe.

bebent.

ittit.

Jelytreicher.

claio.

geflimpert.

fonserbar.

Dct.

Laich.

elytris.

Inn zu Dent.

i尺tericus.

尺rt.

III.

297. 7

327 Io animas mutatas difco tormas

nidbt nod.

Desె.

weif.

nuf.

feyn.

Waintuttis.

eingenommett.

Felis.

animus mutatas

dicere formas.

E.65.3.21. múp die Stelle: ift Iinfs vom Gaul $x$. fo verandert werden: ift linfb vom Gaul, oem Sgents farte und Ygentgletf(her, ser fid, wenn man von Bell nach Diefen (jiegenden fielt, in Seintrgrunde bes $\mathfrak{d r o :}$

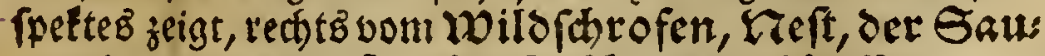
toano, uns dem baufergletfder eingefdioffent.

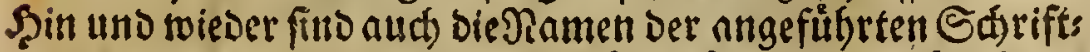

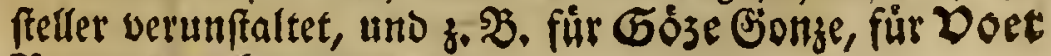
Sont geditufet toorden. 


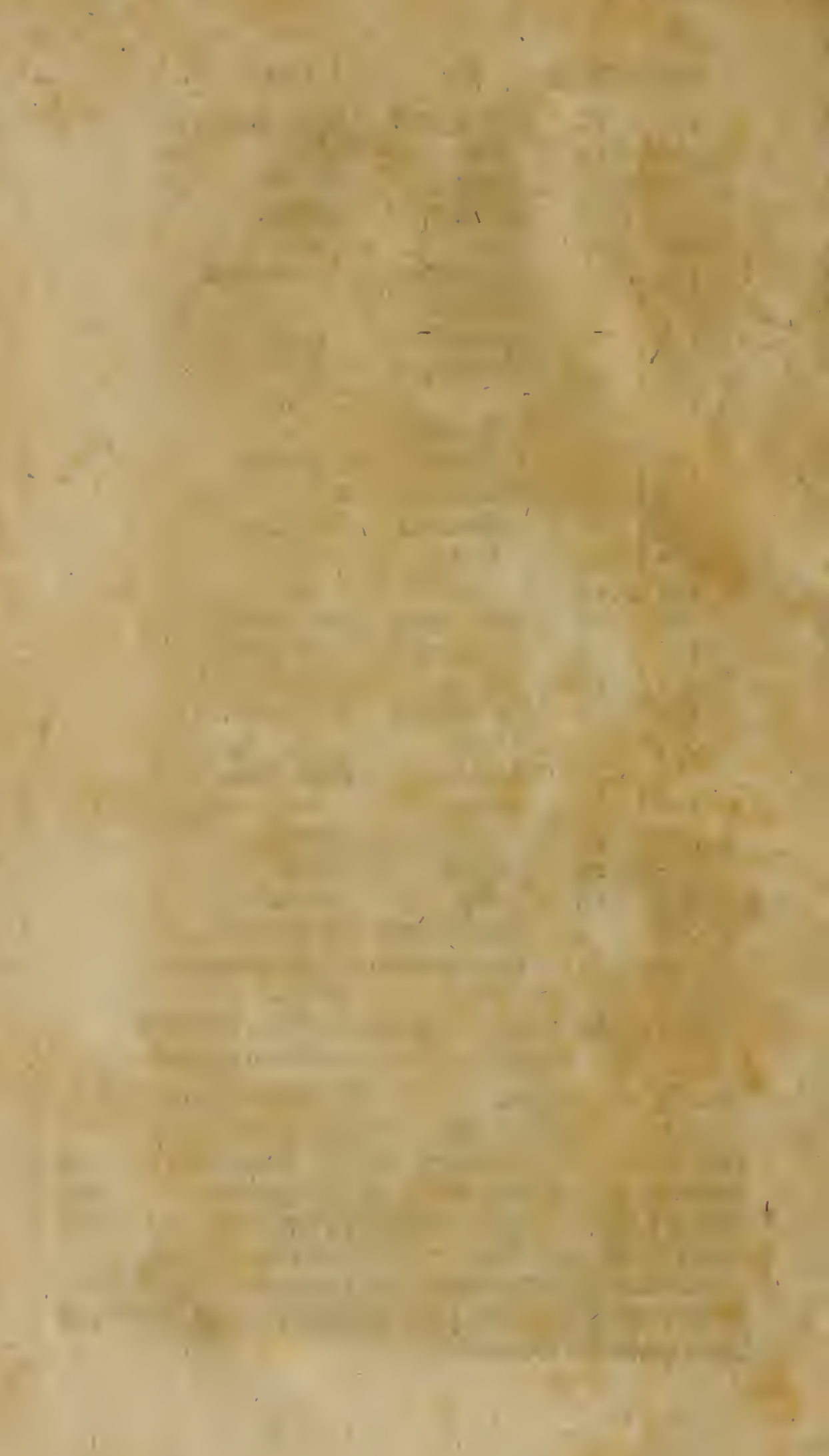




\section{INSERT FOLDOUT HERE}




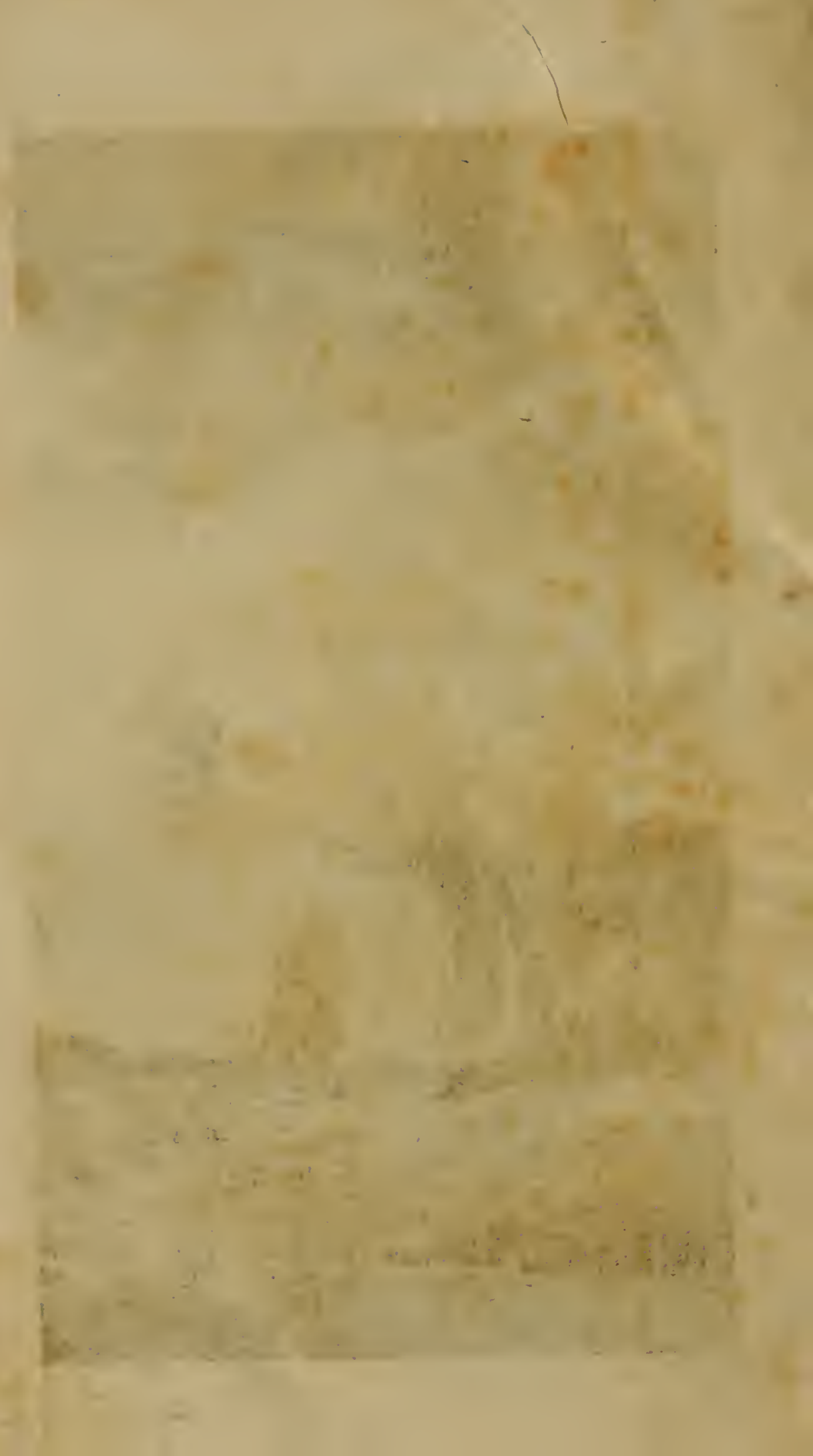





